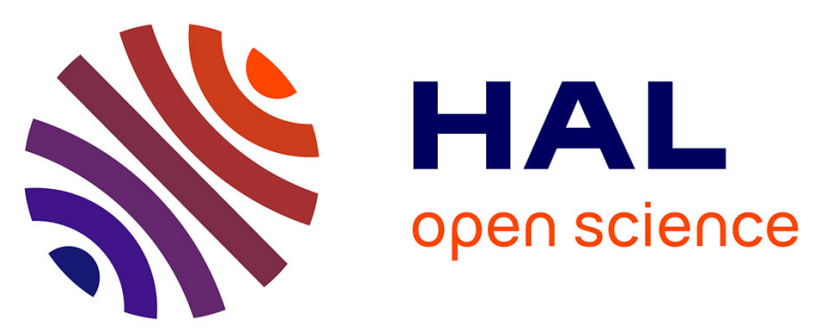

\title{
Enjeux de la transition écologique
}

Laurent Audouin, Emmanuelle Baudry, Céline A Bellard, Elsa Bonnaud, Laurent Bopp, Pascale Braconnot, Francois-Marie Breon, Raphael Brett, Thierry Brunelle, Jean-Christophe Bureau, et al.

\section{To cite this version:}

Jane Lecomte, Jean-Michel Lourtioz, Sophie Laval-Szopa (Dir.). Enjeux de la transition écologique. EDP Sciences, pp.428, 2021, 978-2-7598-2662-9. 10.1051/978-2-7598-2661-2 . hal-03461012

\section{HAL Id: hal-03461012}

https:

\section{//hal-universite-paris-saclay.archives-ouvertes.fr/hal-03461012}

Submitted on 7 Dec 2021

HAL is a multi-disciplinary open access archive for the deposit and dissemination of scientific research documents, whether they are published or not. The documents may come from teaching and research institutions in France or abroad, or from public or private research centers.
L'archive ouverte pluridisciplinaire HAL, est destinée au dépôt et à la diffusion de documents scientifiques de niveau recherche, publiés ou non, émanant des établissements d'enseignement et de recherche français ou étrangers, des laboratoires publics ou privés.

\section{(ㅇ)(1) $\$$}

Distributed under a Creative Commons Attribution - NonCommercial - NoDerivatives| 4.0 
Sous la direction de Jean-Michel LOURTIOZ,

Jane LECOMTE et Sophie SZOPA

assistés de Catherine EVEN et Guillaume ROUX

\section{Enjeux de la transition}

\section{écologiaue}

Enseigner la transition écologique aux étudiants de licence à l'université

universite 



\section{Enjeux de la transition écologique}

\section{Enseigner la transition écologique aux étudiants de licence à l'université}

Sous la direction de Jean-Michel LOURTIOZ, Jane LECOMTE et Sophie SZOPA assistés de Catherine EVEN et Guillaume ROUX 
Cet ouvrage a bénéficié du soutien financier du programme «Investissement d'Avenir».

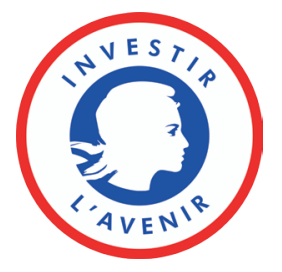

Illustration de couverture : Terre sur rocher moussu dans la forêt, iStock, @ ooyoo.

ISBN (papier) : 978-2-7598-2661-2

ISBN (ebook) : 978-2-7598-2662-9

ISBN (e-pub) : 978-2-7598-2716-9

https://doi.org/10.1051/978-2-7598-2661-2

Cet ouvrage est publié en Open Access sous licence creative commons CC-BY-NC-ND (https://creativecommons.org/licenses/by-nc-nd/4.0/deed.fr) permettant l'utilisation non commerciale, sans modification, la distribution, la reproduction du texte, sur n'importe quel support, à condition de citer la source.

(C) Les auteurs, 2021 


\section{Les auteurs}

Laurent Audouin, Enseignant-chercheur, Laboratoire des 2 infinis Irène Joliot- Curie (IJCLab) Université Paris-Saclay

Emmanuelle Baudry, Enseignante-chercheuse, Professeure, Laboratoire Écologie, Systématique, Évolution (ESE) Université Paris-Saclay

Céline Bellard, Chercheuse au CNRS, Laboratoire Écologie Systématique Évolution (ESE) Université Paris-Saclay

Elsa Bonnaud, Enseignante-chercheuse, Laboratoire Écologie Systématique Évolution (ESE) Université Paris-Saclay

Laurent Bopp, Directeur de Recherche au CNRS, Département de Géosciences, École Normale Supérieure (ENS) Université de Paris Sciences \& Lettres

Pascale Braconnot, Directrice de Recherche au CEA, Laboratoire des Sciences du Climat et de l'Environnement (LSCE) Université Paris-Saclay

François-Marie Bréon, Directeur de Recherche au CEA, Laboratoire des Sciences du Climat et de l'Environnement (LSCE) Université Paris-Saclay

Raphaël Brett, Enseignant-chercheur, Institut d'Études de Droit Public (IEDP) de la Faculté Jean Monnet, Université Paris-Saclay

Thierry Brunelle, Chercheur au CIRAD (Centre International de Recherche Agronomique pour le Développement)

Jean-Christophe Bureau, Enseignant-chercheur, Professeur à AgroParisTech, Université Paris-Saclay

François Chiron, Enseignant-chercheur à AgroParisTech, Laboratoire Écologie Systématique Évolution (ESE) Université Paris-Saclay

Franck Courchamp, Directeur de Recherche au CNRS, Laboratoire Écologie Systématique Évolution (ESE) Université Paris-Saclay

Charlotte Da Cunha, Enseignante-chercheuse, Laboratoire Culture-EnseignementArctique-Représentations-Climat (CEARC) Université de Versailles Saint-Quentinen-Yvelines

Marc Delmotte, Ingénieur de Recherche au CNRS, Laboratoire des Sciences du Climat et de l'Environnement (LSCE) Université Paris-Saclay 
Nicolas Delpierre, Enseignant-chercheur, Laboratoire Écologie Systématique Évolution (ESE) Université Paris-Saclay

Nathalie de Noblet-Ducoudré, Directrice de Recherche au CEA, Laboratoire des Sciences du Climat et de l'Environnement (LSCE) Université Paris-Saclay

Catherine Even, Enseignante-chercheuse, Laboratoire de Physique du Solide (LPS) Université Paris-Saclay

Aude Farinetti, Enseignante-chercheuse, Institut d'Études de Droit Public (IEDP) de la Faculté Jean Monnet, Université Paris-Saclay

Nathalie Frascaria, Enseignante-chercheuse, Professeure à AgroParisTech, Laboratoire Écologie, Systématique Évolution (ESE) Université Paris-Saclay

Jeanne Gherardi, Enseignante-chercheuse à l'Université de Versailles SaintQuentin-en-Yvelines, Laboratoire des Sciences du Climat et de I'Environnement (LSCE) Université Paris-Saclay

Pierre-Henri Gouyon, Professeur au Muséum National d'Histoire Naturelle de Paris et à l'Institut d'Études Politiques de Paris

Christine Hatté, Directrice de Recherche au CEA, Laboratoire des Sciences du Climat et de l'Environnement (LSCE) Université Paris-Saclay

Frédéric Lantz, Professeur à L'École nationale supérieure du pétrole et des moteurs (ENSPM, nom de marque IFP School)

Catherine Larrère, Enseignante-chercheuse, Professeure émérite à l'Université Paris 1 Panthéon-Sorbonne

Paul Leadley, Enseignant-chercheur, Professeur, Laboratoire Écologie Systématique Évolution (ESE) Université Paris-Saclay

Jane Lecomte, Enseignante-chercheuse, Professeure, Laboratoire Écologie Systématique Évolution (ESE) Université Paris-Saclay

Yves Levi, Enseignant-chercheur, Professeur, Laboratoire Écologie Systématique Évolution (ESE) Université Paris-Saclay

Harold Levrel, Enseignant-chercheur, Professeur à AgroParisTech et chercheur au Centre international de recherche sur l'environnement et le développement (CIRED)

Jean-Michel Lourtioz, Directeur de Recherche émérite au CNRS, Centre de Nanosciences et de Nanotechnologies (C2N) Université Paris-Saclay

Valérie Masson-Delmotte, Directrice de Recherche au CEA, Laboratoire des Sciences du Climat et de l'Environnement (LSCE) Université Paris-Saclay

Christian Mougin, Directeur de Recherche à l'INRAE, UMR Écologie fonctionnelle et écotoxicologie des agroécosystèmes (ECOSYS) 
Jean-Eudes Petit, Ingénieur de Recherche au CEA, Laboratoire des Sciences du Climat et de l'Environnement (LSCE) Université Paris-Saclay

Gilles Ramstein, Directeur de Recherche au CEA, Laboratoire des Sciences du Climat et de l'Environnement (LSCE) Université Paris-Saclay

Yorghos Remvikos, Enseignant-chercheur, Professeur, Laboratoire CultureEnseignement-Arctique-Représentations-Climat (CEARC) Université de Versailles Saint-Quentin-en-Yvelines

Franck Richecœur, Enseignant-chercheur, Professeur à Centrale-Supélec, Laboratoire Énergétique moléculaire et Macroscopique Combustion (EM2C) Université Paris-Saclay

Guillaume Roux, Enseignant-chercheur, Laboratoire de Physique Théorique et Modèles Statistiques (LPTMS) Université Paris-Saclay

Marielle Saunois, Enseignante-chercheuse à l'Université de Versailles SaintQuentin-en-Yvelines, Laboratoire des Sciences du Climat et de l'Environnement (LSCE) Université Paris-Saclay

Jérôme Servonnat, Chercheur au CEA, Laboratoire des Sciences du Climat et de l'Environnement (LSCE) Université Paris-Saclay

Rémy Slama, Directeur de Recherche à I'INSERM, Institut de Recherche en Santé Publique (IRESP)

Sophie Szopa, Directrice de Recherche au CEA, Laboratoire des Sciences du Climat et de l'Environnement (LSCE) Université Paris-Saclay

Cécile Tran Kiem, Chercheuse doctorante à l'Institut Pasteur

Jacques Treiner, Professeur honoraire à Sorbonne Université, Shift Project

Améline Vallet, Chercheuse à AgroParisTech, Laboratoire Écologie, Systématique Évolution (ESE) Université Paris-Saclay

Jean-Paul Vanderlinden, Enseignant-chercheur, Professeur, Laboratoire CultureEnseignement-Arctique-Représentations-Climat (CEARC) Université de Versailles Saint-Quentin-en-Yvelines 



\section{Sommaire}

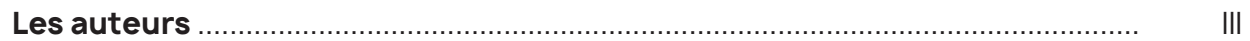

Remerciements …............................................................................. XIX

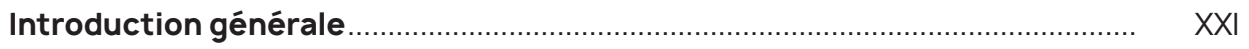

\section{Partie /}

Causes anthropiques des changements globaux: évolution de la place des humains dans la nature le système Terre, un système aux ressources finies

\section{Chapitre 1 \\ Système Terre, sociétés humaines et environnement}

1.1. Une histoire du climat avant les Humains - G. Ramstein .............................. 3

1.2. Interactions Humains climat au cours de l'Histoire - G. Ramstein ............... 7

1.3. Interactions Humains biodiversité : une perspective historique

- J. Lecomte.

1.4. Technologie, gouvernance et développement:

une réflexion sur les rapports des sociétés occidentales

avec l'environnement - J.P. Vanderlinden

1.4.1. Interrelations entre les concepts de développement, de technologie et de gouvernance.

1.4.2. Un avatar du concept de développement: l'hégémonie fordiste....

1.5. La part du Droit dans les causes anthropiques des changements globaux - A. Farinetti.

1.5.1. La souveraineté étatique comme cause des changements globaux

1.5.2. Des libertés fondamentales comme causes des changements globaux

1.6. Économie et environnement - J.Ch. Bureau et H. Levrel..

1.6.1. Propriété et biens communs 


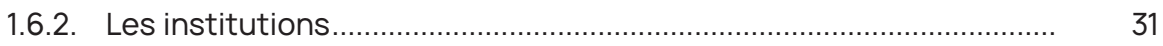

1.6.3. Les défaillances de marché........................................................... 32

1.6.4. L'actualisation............................................................................ 32

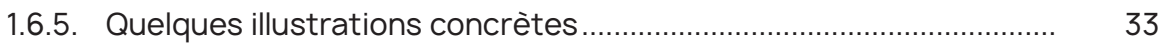

\section{Chapitre 2 \\ Énergies et pollutions}

2.1. Production et consommation d'énergie : la domination des énergies fossiles - C. Even et G. Roux.

2.1.1. L'énergie en physique ................................................................. 37

2.1.2. Chaîne de production de l'énergie : l'exemple de la voiture............. 38

2.1.3. Chaîne de production de l'énergie : généralisation ......................... 40

2.1.4. Consommation d'énergie : échelle individuelle ................................ 41

2.1.5. Consommation d'énergie : échelle mondiale...................................... 43

2.1.6. Domination des énergies fossiles .................................................. 45

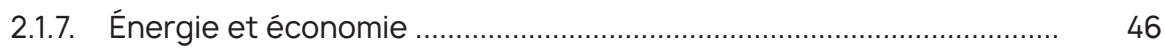

2.2. Le pétrole, enjeux économiques et environnementaux - F. Lantz .............. 47

2.2.1. Le pétrole, ses usages et ses enjeux ................................................ 47

2.2.2. La production de pétrole ................................................................. 48

2.2.3. Les réserves de pétrole ................................................................. 49

2.2.4. La transformation du pétrole et les produits finis........................... 49

2.2.5. Les ressources non conventionnelles ............................................. 50

2.2.6. Les enjeux environnementaux..................................................... 51

2.2.7. Conclusion............................................................................... 52

2.3. Pollution atmosphérique - J.-E. Petit et S. Szopa .................................... 52

2.3.1. Les enjeux liés à la pollution atmosphérique ................................... 52

2.3.2. Les polluants atmosphériques et leurs sources .............................. 53

2.3.3. Influence des polluants sur le climat ............................................. 55

2.4. Pollution de l'eau - Y. Lévi........................................................................... 58

2.4.1. De quelles eaux parlons-nous ? ...................................................... 58

2.4.2. De quelle pollution parlons-nous? .................................................. 60

2.4.3. De quels effets et de quels risques parlons-nous ?.......................... 62

2.4.4. Savons-nous analyser ces contaminations? ..................................... 64

2.4.5. Comment sont traitées les eaux d'égout avant de les rejeter dans le milieu naturel? 
2.4.6. Existe-t-il une réglementation? .................................................. 66

2.4.7. Conclusion................................................................................... 66

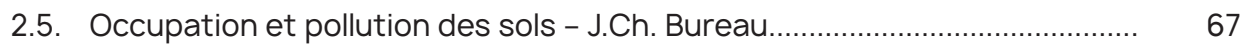

2.5.1. Diversité des sols ......................................................................... 67

2.5.2. Les surfaces agricoles et forestières ......................................... 67

2.5.3. L'érosion des sols ........................................................................ 70

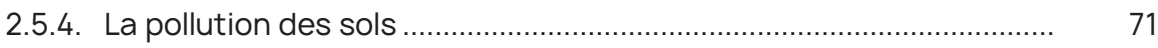

2.6. Échanges de carbone dans les sols - Ch. Hatté ....................................... 72

2.6.1. Cycle du carbone et activités humaines ........................................ 72

2.6.2. Stockage du carbone dans les sols ............................................... 73

2.6.3. Comment augmenter le stockage du carbone dans les sols? ........ 75

2.6.4. Retour sur les modèles de carbone dans les sols ............................ 77

\section{Chapitre 3}

\section{Empreinte écologique et démographie}

3.1. Une introduction à la comptabilité environnementale :

l'empreinte écologique - C. Even et G. Roux .............................................. 81

3.1.1. La «naissance » du développement durable ..................................... 81

3.1.2. Qu'est-ce que l'empreinte écologique plus précisément? .............. 83

3.1.3. Comment calculer l'empreinte écologique? .................................... 85

3.1.4. Calcul de l'empreinte carbone ........................................................ 88

3.1.5. Déficit écologique mondial ............................................................ 89

3.1.6. Limites de l'analyse basée sur l'empreinte écologique.................... 92

3.1.7. En guise de conclusion? ................................................................ 92

3.2. Combien serons-nous en 2050? - J. Treiner et G. Roux ................................. 93

3.2.1. Combien avons-nous été dans le passé? ......................................... 93

3.2.2. La transition démographique ............................................................. 95

3.2.3. Et après la transition démographique? ............................................ 97

3.2.4. La pyramide des âges .................................................................... 98

3.2.5. Le «Jeu de la population » .............................................................. 100

3.2.6. Comment ça marche ?..................................................................... 102

3.2.7. Pour conclure? ............................................................................... 104

3.2.8. Annexe 1: l'espérance de vie ............................................................ 105

3.2.9. Annexe 2 : Questions à propos du contrôle des populations et des migrations 


\section{Partie /l \\ Érosion de la biodiversité}

\section{Chapitre 4 \\ Les différentes facettes de la biodiversité}

4.1. Qu'est-ce que la biodiversité? - J. Lecomte et P.-H. Gouyon......................... 111

4.1.1. Petit historique et définitions - J. Lecomte ...................................... 111

4.1.2. Une dynamique plutôt qu'un état - P.-H. Gouyon ............................. 115

4.2. La biodiversité du point de vue de sa préservation - J. Lecomte................. 117

4.3. Lien entre biodiversité et fonctionnement des écosystèmes:

l'exemple des forêts - N. Delpierre .......................................................... 120

4.4. Les éthiques environnementales - C. Larrère ............................................ 125

4.5. Les services écosystémiques: nouvelle métaphore instrumentale des relations Humains-Nature - A. Vallet.

\section{Chapitre 5}

\section{Les menaces sur la biodiversité}

5.1. Changements d'usage des terres et des mers - H. Levrel.

5.1.1. Évolution de l'usage des sols au cours de l'Histoire .......................... 133

5.1.2. La révolution verte et l'usage hyper-intensif des sols..................... 135

5.1.3. L'usage des mers, pêche extensive et aquaculture ......................... 137

5.1.4. Changements récents dans les usages des terres et des mers ..... 138

5.2. La surexploitation des ressources naturelles - E. Baudry ............................ 139

5.2.1. Définition de la surexploitation ....................................................... 140

5.2.2. Conséquences directes de la surexploitation: l'exemple de la pêche à la morue au large de terre-neuve......

5.2.3. Conséquences directes et indirectes de la surexploitation des ressources, l'exemple de l'île de Pâques

5.2.4. Prélever de façon durable ............................................................... 143

5.3. Le changement climatique - P. Leadley.................................................... 143

5.3.1. Impacts du réchauffement climatique sur les écosystèmes marins

5.3.2. Impacts du réchauffement climatique sur les écosystèmes terrestres.

5.3.3. Impacts du réchauffement climatique sur les écosystèmes d'eau douce. 
5.4. Impacts des pollutions sur la biodiversité - C. Mougin ............................... 148

5.4.1. La pollution lumineuse..................................................................... 149

5.4.2. La pollution chimique.................................................................... 150

5.4.3. Conclusions.................................................................................... 152

5.5. Espèces exotiques envahissantes - E. Bonnaud et C. Bellard ..................... 152

5.5.1. Processus d'introduction d'une espèce exotique ............................ 152

5.5.2. Conditions pour qu'une espèce exotique introduite devienne envahissante .............................................................................. 154

5.5.3. Les invasions biologiques .............................................................. 155

5.5.4. L'exemple de la Perche du Nil ........................................................ 156

5.6. Dynamique des populations à faible effectif - E. Bonnaud

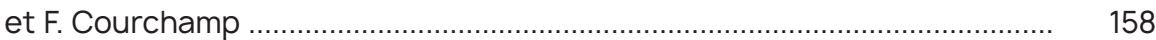

5.6.1. Conditions conduisant à la rareté d'une espèce .............................. 158

5.6.2. Difficultés rencontrées par une espèce rare..................................... 159

5.6.3. Croissance de populations à «densité-dépendance négative»..... 160

5.6.4. Croissance de populations à «densité-dépendance positive» ...... 160

5.6.5. Exemples d'espèces chez lesquelles a été observé l'effet Allee...... 161

5.6.6. Surexploitation et effet Allee Anthropogénique .............................. 163

5.7. Les mécanismes d'homogénéisation en cours - F. Chiron............................ 164

5.7.1. Origine et définitions .................................................................... 164

5.7.2. Causes, mécanismes et conséquences............................................. 166

Chapitre 6

Suivi de la biodiversité, scénarios pour l'avenir, relations entre climat et biodiversité

6.1. Les outils et dispositifs d'observation de la biodiversité:

l'apport des sciences participatives - F. Chiron

6.1.1. Le nouvel essor des sciences participatives en écologie.

6.1.2. Des programmes et des processus collaboratifs entre scientifiques et citoyens

6.1.3. Un exemple de programme de sciences participatives ................... 174

6.2. Scénarios et modèles d'évolution de la biodiversité - P. Leadley.................. 175

6.2.1. Contexte général des scénarios et modèles..................................... 175

6.2.2. Modèle de «niche» - Application au chêne sessile ........................... 176

6.2.3. Exploitation des grandes bases de données .................................... 178

6.2.4. Conclusions............................................................................................. 180 
6.3. Les synergies et antagonismes climat et biodiversité - P. Leadley 180

6.3.1. Les impacts du changement climatique sur la biodiversité

6.3.2. Impact des mesures d'adaptation et d'atténuation du réchauffement climatique sur la biodiversité

6.3.3. Solutions fondées sur la nature

6.3.4. Actions sur les causes communes du changement climatique et de la perte de biodiversité

6.3.5. Conclusion.

6.4. Comment les arbres répondent aux changements saisonniers?

- N. Delpierre.

6.4.1. Les effets du réchauffement climatique sur la feuillaison des arbres

6.4.2. Adaptation des arbres au réchauffement climatique?

6.5. «Mesurer le souffle» des écosystèmes - N. Delpierre

6.5.1. Bilan carbone des forêts

6.5.2. Mesure du «souffle» d'un écosystème forestier.....

6.5.3. Importance des écosystèmes forestiers face au changement climatique.

\section{Partie III \\ Le changement climatique}

\section{Chapitre 7}

\section{Le système climatique}

7.1. Les composantes du système climatique et leurs interactions,

les forçages naturels et anthropiques - M. Delmotte.

7.1.1. Les composantes du système climatique

7.1.2. Les forçages climatiques et la composition de l'atmosphère.

7.1.3. La notion d'effet de serre au premier ordre...

7.2. Bilan radiatif, effet de serre et gaz à effet de serre - F. Raffin et M. Saunois

7.2.1. Le bilan radiatif de la Terre

7.2.2. L'effet de serre additionnel lié aux activités humaines.

7.3. Les émissions de gaz à effet de serre par secteur et par région 
7.4. De quelles façons notre gestion des écosystèmes terrestres contribue-t-elle au changement climatique? - N. de Noblet-Ducoudré.....

7.5. Les indicateurs du changement climatique aux grandes échelles

- M. Delmotte 214

7.5.1. Météorologie, climat et systèmes d'observation............................... 214

7.5.2. Observation des changements en cours............................................ 216

7.5.3. Archives paléoclimatiques et observations d'aujourd'hui

Chapitre 8

\section{Impacts des changements climatiques}

8.1. Manifestations du changement climatique en France et ailleurs dans le monde - S. Szopa.

8.2. Comment le climat impacte-t-il les écosystèmes terrestres?

- N. de Noblet-Ducoudré

8.2.1. Les changements de température

8.2.2. Les changements de précipitation

8.3. Acidification des océans et impacts sur les écosystèmes marins

- L. Bopp

8.3.1. Augmentation de la concentration de l'atmosphère en $\mathrm{CO}_{2}$ et acidification des océans

8.3.2. Conséquences de l'acidification sur les organismes marins

8.3.3. Autres causes de dégradation des océans.

8.3.4. Des solutions pour lutter contre l'acidification des océans?

8.4. Impacts des changements climatiques sur la santé humaine

- R. Slama et C. Tran Kiem

8.4.1. Température et santé humaine - R. Slama....................................... 235

8.4.2. Maladies à transmission vectorielle - C. Tran Kiem .......................... 239

\section{Chapitre 9}

\section{Reconstitutions paléoclimatiques, modèles et projections climatiques}

9.1. Reconstitutions paléoclimatiques de la variabilité climatique naturelle

- J. Gherardi

9.1.1. Rappels sur le fonctionnement du système climatique aux différentes échelles de temps

9.1.2. Les archives climatiques et leur importance pour les modèles climatiques. 
9.2. À quoi sert un modèle climatique? - J. Servonnat ...................................... 252

9.2.1. Présentation des modèles climatiques............................................ 252

9.2.2. Variabilité interne et variabilité forcée de ces modèles ................... 254

9.2.3. Évaluation des modèles .................................................................... 256

9.3. Scénarios futurs et résultats - P. Braconnot ............................................. 257

9.3.1. Rappels sur les éléments constitutifs de la modélisation climatique

9.3.2. Construction des scénarios ............................................................. 258

9.3.3. Projections futures des scénarios et modèles climatiques............. 260

9.4. Combien de carbone nous reste-t-il à émettre? - S. Szopa........................ 261

9.4.1. Objectif de réchauffement global.................................................... 261

9.4.2. Estimation du carbone restant à émettre ....................................... 263

9.4.3. Différentes options pour ne pas dépasser les seuils prescrits....... 265

9.5. Présentation du GIEC et des rapports spéciaux - Équipe GIEC ................. 265

9.5.1. Qu'est-ce que le GIEC? ............................................................... 265

9.5.2. Comment sont produits les rapports du GIEC? .............................. 266

\section{Partie IV}

\section{Comment répondre aux changements globaux}

Chapitre 10

\section{S'adapter aux changements, de la réduction de la vulnérabilité} à l'augmentation de la résilience

10.1. L'adaptation: déclinaisons et enjeux - J.P. Vanderlinden.

10.1.1. Stimuli climatiques, vulnérabilité, capacité adaptative et transformation

10.1.2. Le processus d'adaptation dans un contexte d'incertitudes et de certitudes

10.2. L'adaptation en tant que transformation profonde - Y. Remvikos

10.2.1. Le concept d'adaptation et ses différentes acceptions

10.2.2. Le réchauffement climatique, un défi auquel l'humanité tout entière doit faire face

10.3. L'adaptation en tant que réduction des vulnérabilités à la variabilité climatique actuelle - J.P. Vanderlinden

10.3.1. Vulnérabilité et exposition

10.3.2. Conceptualisation d'un risque climatique. 
10.3.3. Identification des facteurs de vulnérabilité et des leviers d'actions, l'exemple d'Ho Chi Minh Ville

10.4. Planification territoriale de l'adaptation - Ch. da Cunha

10.4.1. Historique des plans d'adaptation et de leur mise en place.

10.4.2. Mise en place des stratégies locales d'adaptation.....

10.4.3. Cas d'étude: New-York city

10.4.4. Défis restants pour la planification de l'adaptation au niveau local

10.5. Enjeux de gouvernance - R. Brett.

10.5.1. Décision politique et protection de l'environnement

10.5.2. L'importance de la participation de tous à la prise de décision .........

10.6. Domaine de la santé: adaptation aux températures élevées - R. Slama ...

10.6.1. Situations à New York et en France durant les dernières décennies.

10.6.2. Pratiques à mettre en œuvre pour l'avenir .

10.7. Domaine de l'agriculture : adaptation aux sécheresses - J.-C. Bureau........

10.7.1. Le problème des ressources en eau

10.7.2. S'adapter aux sécheresses grâce à de nouvelles cultures et des cultivars.

10.7.3. Des changements de culture à l'agroforesterie, l'exemple du Sahel

\section{Chapitre 11}

\section{Agir sur les causes des changements globaux}

11.1. Réduire la pression anthropique sur les écosystèmes:

limiter la dégradation et la fragmentation des habitats

- J. Lecomte et N. Frascaria

11.1.1. Permettre la libre évolution

11.1.2. Accompagner la libre évolution

11.2. Agir sur le levier agricole: agroécologie, système alimentaire et réduction du gaspillage - J.-C. Bureau et T. Brunelle.

11.2.1. Réduction des gaz à effet de serre dans l'agriculture.

11.2.2. L'agroécologie et l'agriculture bio.

11.2.3. Modes de consommation alimentaire et réduction de gaz à effet de serre

11.2.4. Gaspillage alimentaire et émissions de gaz à effet de serre. 
11.3. Solutions techniques pour réduire les émissions individuelles de gaz

à effet de serre- F. Richecœur

11.3.1. Les technologies pour chauffer et isoler l'habitat individuel. 318

11.3.2. Les technologies pour les transports

11.3.3. L'hydrogène, le combustible de demain pour l'énergie et les transports?

11.3.4. Les technologies pour l'agriculture ................................................ 324

11.3.5. Conclusion.................................................................................. 325

11.4. Produire une énergie décarbonée: les énergies renouvelables (EnR)

- J.-M. Lourtioz

11.4.1. Les différents types d'énergie renouvelable

11.4.2. Place des énergies renouvelables en France et dans le monde

11.4.3. Limites au développement des nouvelles énergies renouvelables..

11.4.4. Focus sur la conversion photovoltaïque de l'énergie solaire

11.4.5. Conclusion.

11.5. L'énergie nucléaire, une autre source d'énergie décarbonée:

principes, avantages et limites - L. Audouin

11.5.1. Principes de l'énergie nucléaire

11.5.2. Centrales nucléaires

11.5.3. Une énergie à faible émission de $\mathrm{CO}_{2}$.

11.5.4. Radioactivité et déchets nucléaires.

11.5.5. Le nucléaire, une source modeste à l'échelle mondiale; et demain?

11.6. Changer nos modes de vie : sobriété, efficacité et substitutions dans notre consommation énergétique - F.-M. Bréon

11.6.1. Sobriété dans nos modes de vie

11.6.2. Gain d'efficacité dans le résidentiel et les transports

11.6.3. Une meilleure efficacité dans notre consommation d'énergie : le tout électrique?

11.7. Émissions négatives de $\mathrm{CO}_{2}-\mathrm{C}$. Even.

11.7.1. Géo-ingénierie : traitements directs et indirects du réchauffement climatique

11.7.2. Émissions négatives par capture du $\mathrm{CO}_{2}$ atmosphérique. 


\section{Chapitre 12 \\ Aller vers une transition écologique juste}

12.1. Qu'entend-on par «transition écologique juste»?

12.2. Inégalités distributives: un juste partage des externalités positive et négative de l'environnement?

356

12.2.1. Responsabilités d'émissions de gaz à effet de serre.

12.2.2. Impacts et conséquences des changements climatiques

12.2.3. Efforts pour préserver la biodiversité et les services écosystémiques

12.2.4. Bénéfices reçus du fonctionnement des écosystèmes.

12.3. La dimension participative dans les prises de décision en politique environnementale

12.3.1. L'exemple des négociations internationales sur le climat.

12.3.2. L'exemple de la gouvernance des services écosystémiques....

12.4. Le rôle de l'action publique contre les inégalités environnementales

12.4.1. Des incitations économiques pour lutter contre le changement climatique?

12.4.2. Agir avec des outils réglementaires pour la préservation de la biodiversité?

12.5. Grands principes d'une transition écologique juste et perçue comme juste? 



\section{Remerciements}

C'est avec grand plaisir que nous avons préparé et harmonisé les textes issus des enseignements des Enjeux de la Transition Écologique à l'Université Paris-Saclay au cours de l'année universitaire 2020-2021. Cet enseignement est l'un des premiers sur le sujet à être déployé à grande échelle, auprès de plusieurs milliers d'étudiantes et d'étudiants de licence à l'université. La préparation du présent ouvrage a été l'occasion d'échanges de grande qualité avec l'ensemble des auteurs de cet enseignement. Nous les remercions pour leur engagement dans l'enseignement, la qualité de leurs contributions et leur même engagement dans le présent ouvrage.

Nous exprimons notre plus grande reconnaissance à Isabelle Demachy, VicePrésidente Formation innovation pédagogique et vie étudiante de l'Université Paris-Saclay ainsi qu'à toute l'équipe de la Direction de l'innovation pédagogique, en particulier Hugues Cazin et Aurélie Rousseau, car cet ouvrage comme l'Unité d'Enseignement sur les Enjeux de la Transition Écologique n'aurait pas pu se faire sans leur impulsion de départ et leur soutien constant depuis plus de deux ans.

Nous exprimons aussi notre vive reconnaissance à la Direction de l'École Universitaire de Premier Cycle Paris-Saclay (EUPS), nommément Pascal Aubert et Véronique Mathet pour leur implication efficace dans l'organisation de l'Unité d'Enseignement, ainsi qu'à l'ensemble des responsables de licence de l'Université pour leur appui et leur concours volontaire à l'organisation et au déroulement de cette unité d'enseignement.

Toute notre gratitude va à Anaïs Antoine de l'EUPS pour sa contribution à la brochure de synthèse réalisée à l'issue de cette première année de l'Unité d'Enseignement, brochure qui a été le point de départ décisif pour que nous engagions l'écriture du présent ouvrage.

Nous remercions aussi l'équipe de Julien Sempéré à la Direction des bibliothèques, de l'information et de la science ouverte à l'Université Paris-Saclay, en particulier Nathalie Barnault Lac et Cédric Mercier pour leur accompagnement tout au long de l'écriture et de la préparation de cet ouvrage.

Jean-Michel Lourtioz Jane Lecomte Sophie Szopa 



\section{Introduction générale}

Le présent ouvrage fait écho au cours «Enjeux de la Transition Écologique» dispensé à plusieurs milliers d'étudiants de licence de toutes disciplines à l'Université Paris-Saclay depuis la rentrée universitaire 2020. Ce cours s'inscrit dans les objectifs de l'Université en matière de développement soutenable ${ }^{1}$ et témoigne de sa volonté de sensibiliser à terme tous les étudiants à cette problématique. Plus généralement, il fait partie de l'ensemble des formations aux enjeux planétaires globaux (Fig. 1), qui sont produites à destination non seulement des étudiants dans leur diversité mais aussi des professionnels et du grand public.

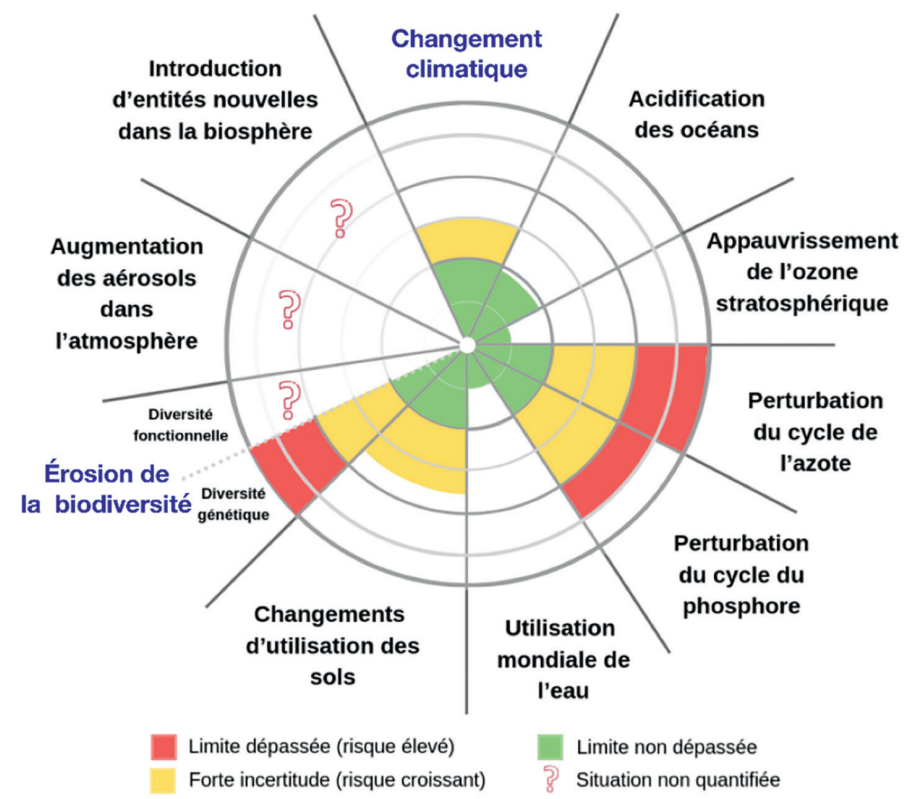

Figure 1: Enjeux planétaires globaux. Le concept des limites planétaires définit un espace de développement sûr et juste pour l'humanité, fondé actuellement sur neuf [1] processus biophysiques. Des seuils quantitatifs ont été définis pour sept des neuf limites. En 2009, J. Rockström indiquait que trois d'entre elles étaient franchies (changement climatique, érosion de la biodiversité, perturbation du cycle de l'azote). Lors de la révision du modèle conceptuel [2], de nouveaux seuils ont pris en compte le niveau régional et l'hétérogénéité des processus. En dépit de débats encore suscités à ce sujet, le concept est reconnu et adopté aux niveaux européen et international. Figure adaptée de [2].

1. Charte de Développement soutenable de l'Université Paris-Saclay adoptée le 6 juillet 2021. 
Le principal objectif de l'ouvrage est de faire comprendre les mécanismes et conséquences liés au réchauffement climatique et à l'érosion de la biodiversité ainsi que leurs relations avec nos modes de vie et de consommation. Plus généralement, il vise à apporter une vision transversale pour appréhender les changements globaux, notamment en lien avec les objectifs de développement durable de l'ONU et le concept de limites planétaires [1].

Plus de 40 chercheurs et enseignants-chercheurs d'horizons différents ont contribué à l'ouvrage, allant des sciences exactes aux sciences humaines et sociales en passant par le droit, l'économie, la gestion et la médecine. Le contenu de l'ouvrage s'articule en quatre grandes parties, elles-mêmes divisées en chapitres et sous-chapitres.

Dans la première partie, le lecteur découvrira d'abord les évolutions du climat et de la biodiversité sur notre planète depuis ses premiers âges jusqu'aux changements accélérés des derniers siècles en mettant en parallèle le développement de notre société, les problématiques environnementales et leur gouvernance, leur lien avec l'économie et leur régulation par le droit. Sont ensuite illustrées les principales perturbations de notre environnement en relation avec notre consommation énergétique et nos modes de production. La manière de quantifier «l'empreinte écologique» des humains et celle permettant de prévoir l'évolution démographique à l'échelle mondiale sont également explicitées. Cette partie met en lumière la nature interdisciplinaire des questions soulevées par les changements environnementaux globaux, leurs causes et leurs interdépendances.

La deuxième partie rentre de façon plus détaillée sur les mécanismes d'érosion de la biodiversité. Elle rappelle d'abord ce qu'est la biodiversité, sa dynamique, ce qu'elle représente comme valeurs, les services que nous tirons des écosystèmes et les menaces que, par contre, nous faisons peser par nos modes de production et d'exploitation de la nature. Sont également expliquées les méthodologies qui permettent de suivre son évolution, celle des espèces qui la composent, rares ou abondantes, et de placer les enjeux à court et long termes avec les enjeux climatiques.

La troisième partie décrit le fonctionnement du système climatique, les mécanismes d'effet de serre et les indicateurs d'évolution du climat. Si les manifestations du réchauffement climatique sont déjà sensibles, cet ouvrage montre la manière dont son aggravation entraînera, s'il n'est pas maitrisé, une multiplication d'événements extrêmes et des impacts importants à la fois sur les cultures et la végétation, sur les océans et leurs écosystèmes et sur la santé humaine. Est expliquée la façon dont les modèles climatiques sont construits pour nous permettre de projeter les évolutions possibles du climat futur et estimer la quantité de $\mathrm{CO}_{2}$ restant à émettre pour ne pas dépasser des seuils critiques.

La dernière partie traite d'abord des transformations de société et de gouvernance que suppose la mise en place de processus d'adaptation aux changements globaux. Elle décrit ensuite les actions possibles pour préserver la biodiversité et 
atténuer le réchauffement climatique : comment faciliter la libre évolution des entités de la biodiversité, agir sur l'agriculture et nos modes d'alimentation, comment agir sur l'habitat, les transports, les modes de consommation et la production d'énergie, comment agir directement sur les émissions de $\mathrm{CO}_{2}$. Les compromis à faire dans nos choix technologiques et sociétaux sont abordés. Cette partie se termine par la notion d'une «transition écologique juste» qui nous interroge sur la possibilité d'agir face aux changements globaux tout en garantissant la justice sociale.

Jean-Michel Lourtioz Jane Lecomte Sophie Szopa

[1] J. Rockström, W. Steffen, K. Noone, Å. Persson, F.S. Chapin III, E. Lambin, T.M. Lenton, M. Scheffer, C. Folke, H. Schellnhuber, B. Nykvist, C.A. De Wit, T. Hughes, S. van der Leeuw, H. Rodhe, S. Sörlin, P.K. Snyder, R. Costanza, U. Svedin, M. Falkenmark, L. Karlberg, R.W. Corell, V.J. Fabry, J. Hansen, B. Walker, D. Liverman, K. Richardson, P. Crutzen, J. Foley. 2009. Planetary boundaries: exploring the safe operating space for humanity. Ecology and Society 14 (2), 32. http://www.ecologyandsociety.org/vol14/iss2/art32/

[2] W. Steffen, K. Richardson, J. Rockström, S.E. Cornell, I. Fetzer, E.M. Bennett, R. Biggs, S.R. Carpenter, W. de Vries, C.A. de Wit, C. Folke, D. Gerten, J. Heinkel, G.M. Mace, L.M. Persson, V. Ramanathan, B. Reyers, S. Sörlin. 2015. Planetary boundaries: Guiding human development on a changing planet. Science 347 (6223), 1259855. DOI: 10.1126/ science. 1259855 



\section{Partie I}

\section{Causes anthropiques}

des changements globaux : évolution de la place des humains dans la nature le système Terre, un système aux ressources finies 



\section{Chapitre 1}

\section{Système Terre, sociétés humaines et environnement}

Dans ce chapitre, nous découvrirons les évolutions du climat de notre planète depuis les premiers âges jusqu'à nos jours. Nous verrons qu'en très peu de temps, l'humanité a étendu son influence à la planète tout entière en ayant désormais la capacité d'agir sur le climat, comme elle a aussi assujetti les non-humains à ses intérêts. Ces changements globaux, du climat, de la biodiversité et de notre environnement en général, font en retour peser des menaces sur l'avenir de l'humanité. Nous mettrons ces changements globaux en parallèle avec le développement de nos sociétés occidentales, en analysant en particulier les mécanismes sociotechniques, juridiques et économiques qui gouvernent leur fonctionnement et leur rapport à l'environnement.

\subsection{Une histoire du climat avant les Humains - G. Ramstein}

Quels sont les processus physiques qui régulent le climat de la Terre? Nous nous plaçons ici à des échelles de temps que l'on a beaucoup de mal à imaginer. Nous passerons ainsi successivement en revue le rôle que joue la variation d'intensité de la luminosité du soleil à l'échelle du milliard d'années, et l'influence de la tectonique des plaques à l'échelle de quelques dizaines de millions d'années.

Déjà, prenons un peu de recul du point de vue spatial. Par exemple, la Terre est à une distance du soleil qui en fait une planète «habitable». Mais si on regarde une de nos proches voisines, Vénus, par exemple, c'est un four. Non pas parce qu'elle est plus proche du soleil, mais parce que son atmosphère est extrêmement dense en gaz à effet de serre, ce qui fait qu'à sa surface, il fait presque $450^{\circ} \mathrm{C}$. Donc, elle est relativement inhabitable. Si on regarde notre autre voisine, Mars, qui est un peu plus loin du soleil, elle n'a, au contraire, qu'une très légère atmosphère. De ce fait, il y a de très grosses différences de températures entre le jour et la nuit et il y fait 
globalement assez froid. Elle est donc, elle aussi, relativement inhabitable. Pourtant, toutes ces planètes, la Terre, Mars, Vénus et les planètes géantes sont baignées par la même énergie solaire.

Sur Terre, comme sur Mars ou Vénus, 99\% de l'énergie nous vient du soleil. Donc, l'histoire du soleil est fondamentale. Comme vous le savez sans doute, le système planétaire est né il y a 4,6 milliards d'années. C'est le moment où le soleil s'allume, où les planètes se forment et donc, c'est très important de savoir quelle est l'histoire du soleil lui-même, car c'est lui le grand pourvoyeur de notre énergie. En fait, son histoire est relativement simple à connaître. Pourquoi ? Parce que notre soleil est tout à fait ordinaire. Notre soleil n'est juste qu'un réacteur nucléaire dont on connaît très bien l'évolution. Comme on le voit sur la figure 1.1, sa luminosité augmente de 7\% par milliard d'années. Faisons un calcul simple : 4,6 milliards d'années pour l'émergence de notre système planétaire, 7\% par milliard, cela fait qu'il y 4,6 milliards d'années, le soleil était beaucoup moins puissant qu'aujourd'hui, de l'ordre de $30 \%$ moins puissant. Cela aurait dû suffire pour que sur Terre, il fasse $0^{\circ} \mathrm{C}$. Donc, finie la vapeur d'eau, finies les trois phases de l'eau comme on les connaît: la Terre aurait été une planète gelée.

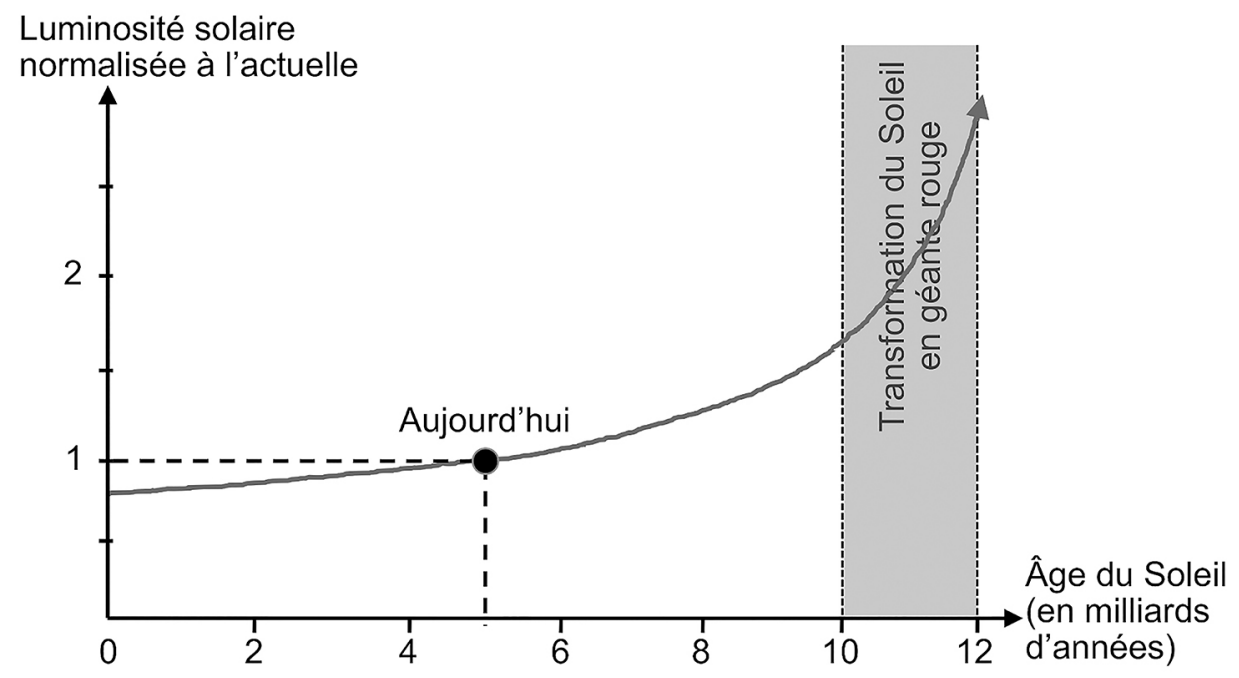

Figure 1.1: Évolution de l'intensité lumineuse du soleil depuis l'origine de la Terre. D'après [1].

Or, sur Terre, on sait grâce à des données que l'on n'a jamais été dans cette situation, ce qu'en anglais on appelle «the Paradox of the Faint Young Sun» (le paradoxe du soleil jeune et faible). Comment se fait-il qu'avec un soleil jeune et blafard, la Terre qui aurait dû se couvrir de glace, ne l'a pas fait? Forcément, dans le système, il y avait quelque chose de différent par rapport à ce que nous connaissons. Eh bien, ce qui était différent, c'était l'atmosphère de notre planète. À des échelles de temps de plusieurs milliards d'années, l'atmosphère de la Terre était beaucoup plus riche en 
$\mathrm{CO}_{2}$ et peut-être aussi en méthane, et ce sont ces couvertures gazeuses, par leur effet de serre, qui nous ont protégés et qui ont fait que la Terre ressemble à ce que nous connaissons aujourd'hui. Cette relation entre notre atmosphère et notre climat est donc tout à fait fondamentale.

Voyons maintenant ce qui se passe à l'échelle de dizaines de millions d'années. Là, c'est la tectonique des plaques qui va être le facteur forçant du climat. Pourquoi dix millions d'années? Parce que c'est le temps nécessaire pour que la position des continents change par rapport aux océans de notre planète de manière visible. Le visage de la Terre, c'est-à-dire les positions des continents, change sans arrêt. Sauf qu'à l'échelle de nos vies, cent ans, on ne s'en aperçoit pas car leurs déplacements ne sont que de quelques centimètres par an. En revanche, sur plusieurs centaines de millions d'années, on voit les continents dériver sur la lithosphère, cette pellicule de surface la plus proche de nous. Initialement collés les uns aux autres, ils se séparent, puis tendent à s'assembler à nouveau, etc.

Arrêtons-nous sur un point un peu plus complexe. II s'agit de ce que l'on appelle le cycle exosphérique du $\mathrm{CO}_{2}$. Qu'est-ce qui régit la teneur en $\mathrm{CO}_{2}$ dans l'atmosphère à l'échelle des temps géologiques? Si aujourd'hui la source principale de $\mathrm{CO}_{2}$ atmosphérique est sans aucun doute l'ensemble des activités humaines, à l'échelle des temps géologiques, c'est le volcanisme qui émet le $\mathrm{CO}_{2}$ dans l'atmosphère. À l'inverse, ce qui va extraire le $\mathrm{CO}_{2}$ de l'atmosphère pour le remettre dans l'océan, puis dans le manteau terrestre (la pellicule sous la lithosphère), est ce qu'on appelle l'érosion silicatée. Comment cela se passe-t-il?

Les précipitations sont toujours légèrement acides à cause du $\mathrm{CO}_{2}$ atmosphérique qui se dissout dans les gouttes d'eau. Cette acidité permet d'éroder chimiquement les roches silicatées et sera donc neutralisée pendant ce processus avant de rejoindre l'océan. Finalement, ceci permet de réduire l'acidité de l'océan; ainsi cela favorise la précipitation des carbonates qui vont piéger le carbone durant des durées géologiques. Eh bien, ce cycle là (Fig. 1.2), qui est extrêmement lent et permet donc de retirer du $\mathrm{CO}_{2}$ de l'atmosphère sur des temps très longs, est très important.

Divers scientifiques ont joué un rôle prépondérant dans la compréhension de ces phénomènes. Le premier d'entre eux, Alfred Wegener avait compris, dans la première moitié du siècle précédent (vers 1920), que les plaques dérivent. II avait aussi très bien compris que dans leur dérive avec le temps, les continents peuvent changer de latitude et donc de climat.

Ce qui avait été moins bien compris, c'est la relation avec le $\mathrm{CO}_{2}$ atmosphérique. En effet, quand tous les continents montent vers le pôle nord ou le pôle sud, il fait extrêmement froid sur Terre, mais aussi extrêmement aride. En conséquence, il ne pleut plus et le flux de $\mathrm{CO}_{2}$ qui est lié à l'érosion ne fonctionne pas. Mais le volcanisme, lui, fonctionne encore. II injecte du $\mathrm{CO}_{2}$ dans l'atmosphère qui n'est pas retiré, et la teneur en $\mathrm{CO}_{2}$ augmente donc dans l'atmosphère. II fait froid, mais le $\mathrm{CO}_{2}$ augmente et va conduire à un réchauffement de l'atmosphère. Inversement, quand toutes 


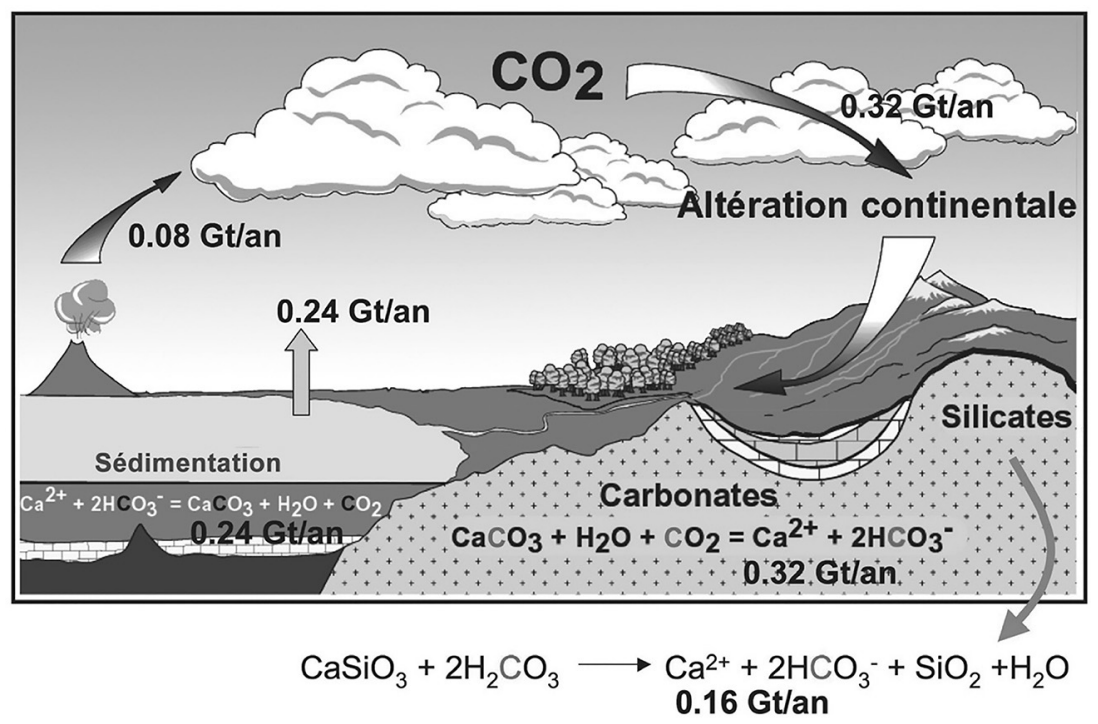

Figure 1.2: Cycle exosphérique du $\mathrm{CO}_{2}$. D'après [2].

les plaques sont dans les zones tropicales et équatoriales, il pleut beaucoup, ce qui entraîne beaucoup d'érosion, la teneur en $\mathrm{CO}_{2}$ de l'atmosphère diminue et donc l'atmosphère se rééquilibre à des taux de $\mathrm{CO}_{2}$ plus faibles. II pleut beaucoup, il fait chaud, mais comme le $\mathrm{CO}_{2}$ baisse, cela entraîne petit à petit un refroidissement de l'atmosphère. Ce mécanisme est fondamental car il permet de comprendre les liens très intimes entre notre atmosphère et ses gaz à effet de serre à toutes les échelles de temps.

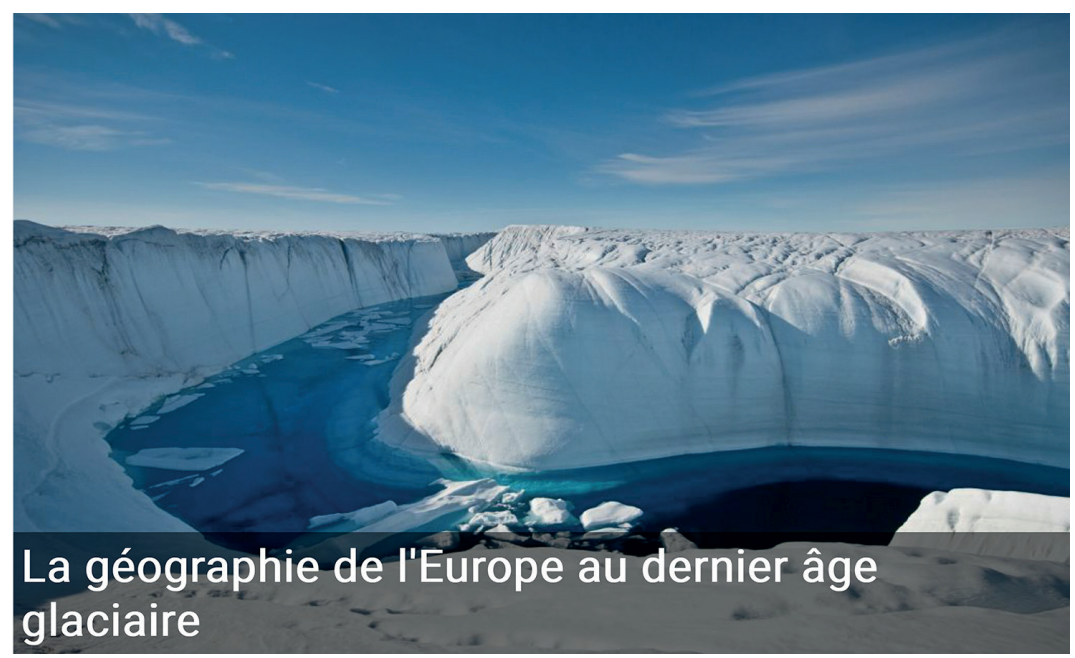

Figure 1.3: La calotte glaciaire du Groenland - Eaux de fonte. Source: ( lan Joughin / Université de Washington. 
Après ces climats très contrastés mais au fonctionnement relativement simple, nous allons voir ici le climat dans lequel les humains et leurs ancêtres ont évolué et nous allons nous intéresser ainsi au climat à l'échelle du million d'années. En fait, c'est un climat assez particulier. Pourquoi? Parce que contrairement aux premiers milliards et aux dizaines de millions d'années de la Terre durant lesquels, la plupart du temps, il n'y avait pas de calotte de glace, sur les derniers millions d'années la Terre possède deux calottes pérennes: le Groenland qui est là depuis environ 3 millions d'années (Fig. 1.3) et l'Antarctique qui est là depuis environ 34 millions d'années. Deux calottes de glace: une dans chaque hémisphère.

\subsection{Interactions Humains climat au cours de l'Histoire - G. Ramstein}

Le dernier million d'années est une période assez extraordinaire, durant laquelle les variations du climat terrestre ont été importantes et ce, bien avant qu'Homo sapiens ne colonise la planète, qu'il invente l'agriculture et se sédentarise. Nous reviendrons, bien sûr, un peu plus loin sur le moment où les premiers changements climatiques d'origine anthropique se sont fait sentir, en particulier sur les changements fulgurants qu'ont produit les activités humaines depuis le début de l'ère industrielle, c'est-à-dire à l'échelle de la centaine d'années.

Depuis un million d'années, les variations du climat terrestre se manifestent par des oscillations massives des calottes de glace. Aujourd'hui, nous avons le Groenland et l'Antarctique. Mais, il y a seulement 20000 ans, une énorme calotte de glace se trouvait sur l'Amérique du Nord et le Canada, qui mesurait 2500 m de hauteur. Et une calotte de glace semblable se trouvait aussi sur l'Europe du Nord. Or, depuis un million d'années, la situation oscille entre des situations interglaciaires, dans lesquelles nous vivons actuellement avec deux calottes de glace seulement, et des situations glaciaires, avec quatre calottes de glace si massives que le niveau marin a pu baisser alors de $120 \mathrm{~m}$. En fait, le climat varie de façon plus ou moins périodique entre un interglaciaire qui dure quelques milliers d'années et auquel succède la croissance des calottes de glace dans l'hémisphère nord. Elles se construisent pendant à peu près 100000 ans puis fondent en à peu près 10000 ans, revenant à un état interglaciaire; et ainsi de suite. Ce régime de cycle climatique est celui de la Terre depuis un million d'années.

Quelle est l'origine de ces variations? Nous avons vu que le moteur des variations à l'échelle du milliard d'années est le soleil. Celui des variations climatiques à l'échelle de la dizaine de millions d'années est la tectonique des plaques. À l'échelle du dernier million d'années, où les cycles sont finalement assez rapides, des dizaines de milliers d'années seulement, ce qui change est la quantité d'énergie reçue au sommet de l'atmosphère. Elle change à cause de trois paramètres, appelés paramètres orbitaux, qui sont illustrés sur la figure 1.4. Pourquoi ces paramètres changent-ils? Parce qu'ils dépendent de l'orbite de la Terre autour du Soleil, de l'influence de la Lune et des autres planètes. 
Le premier paramètre est la précession climatique, qui résulte des interactions gravitationnelles avec le Soleil et la Lune et varie selon un cycle d'environ 20000 ans: l'axe de rotation de la Terre tourne lui-même autour d'un axe perpendiculaire au plan de l'orbite terrestre. La précession climatique est la combinaison de la précession des équinoxes, c'est-à-dire de l'axe de la Terre, et de la précession du périhélie, c'est-à-dire de l'orbite (Fig. 1.4), ce qui induit des modulations des saisons, lesquelles n'arrivent pas toujours au même moment et ont des intensités différentes sur des échelles de temps relativement longues. Par exemple, dans la période actuelle et dans l'hémisphère nord, l'été se produit lorsque la Terre est la plus éloignée du Soleil, ce qui se traduit par des contrastes saisonniers peu marqués, alors qu'à l'inverse, il y a 10000 ans, l'été avait lieu lorsque la Terre était la plus proche du soleil et les variations saisonnières étaient bien plus marquées. Cette précession change donc le climat à l'échelle de 20000 ans.

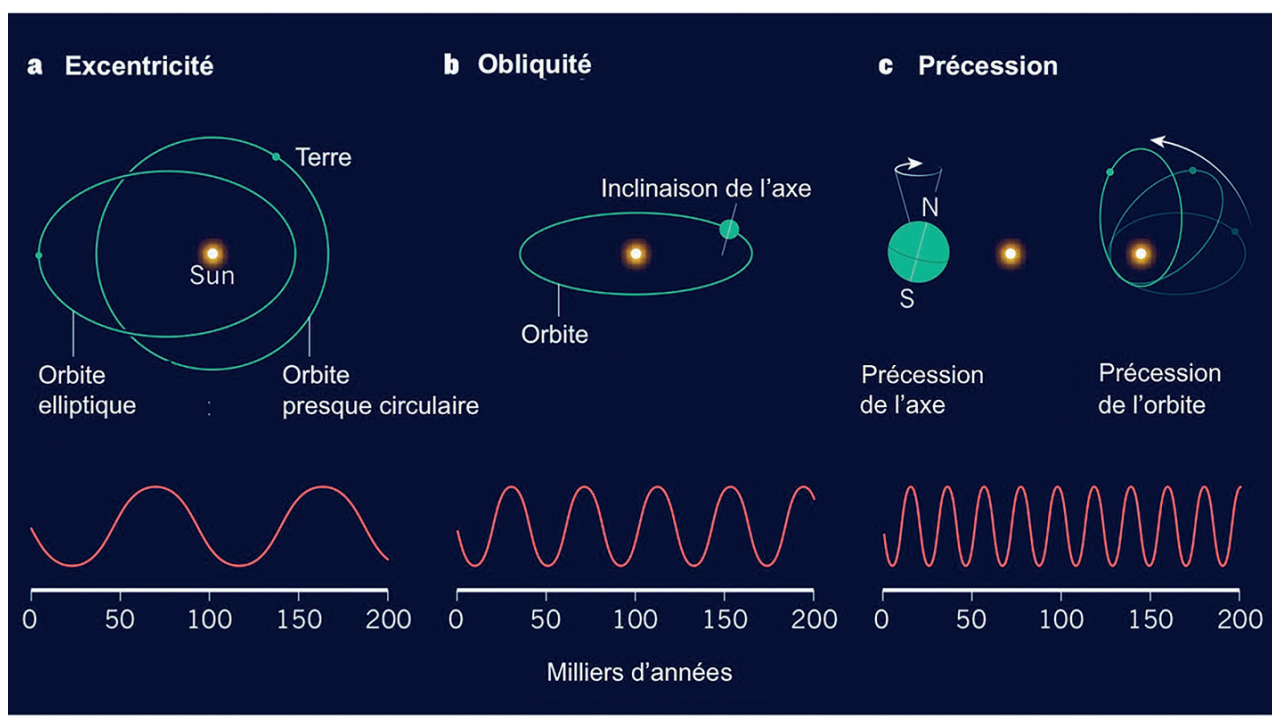

Figure 1.4: Mouvements de la terre qui affectent le climat aux échelles de dizaines de milliers d'années. D’après: Mark Maslin, Nature, Vol. 450, pp. 208-210, 8 décembre 2016 [3].

Ensuite, et c'est un paramètre fondamental, il s'agit de l'obliquité terrestre. L'obliquité, c'est l'angle que fait l'axe de rotation de la Terre et le plan dans lequel elle se meut. En effet, la planète Terre se déplace autour de son Soleil, un peu penchée: c'est cette inclinaison que l'on nomme obliquité. Les grecs de l'Antiquité avaient bien compris l'importance de cette inclinaison, puisque justement en grec, inclinaison se dit «climat». Donc, cette obliquité varie avec une période de 40000 ans. Chose très particulière, c'est le fait que le satellite de la Terre, la Lune, joue sur les paramètres orbitaux, en particulier sur l'obliquité, en la stabilisant. Ainsi, l'obliquité de la Terre ne peut varier que dans un intervalle angulaire très réduit, entre $22,1^{\circ}$ et $24,5^{\circ}$, ce qui est extrêmement favorable à la régulation du climat. 
Le troisième paramètre est l'excentricité orbitale. II s'agit en fait du caractère «aplati» de l'ellipse que décrit la Terre autour du Soleil. La trajectoire de la Terre n'est pas tout à fait circulaire : il y a ce que l'on appelle une petite excentricité, c.-à-d. un caractère un peu aplati, qui varie sur des périodes de l'ordre de 100000 ans à 400000 ans.

Que vont faire ces trois paramètres? En fait, ils vont changer l'énergie qui arrive au-dessus des régions polaires, là où peuvent se former des calottes de glace. Pas énormément, mais suffisamment pour que, dans certaines périodes, cela produise des changements majeurs. Ces paramètres orbitaux sont donc le déclencheur du changement sur ce dernier million d'années et on sait les calculer très précisément sur des dizaines de millions d'années grâce à la mécanique céleste.

Comme nous l'avons indiqué, les variations des paramètres orbitaux se traduisent par des cycles glaciaires/interglaciaires (Fig. 1.5) qui font respectivement apparaître et disparaître d'immenses calottes de glace dans l'hémisphère nord, appelées Laurentide pour l'Amérique du Nord et fennoscandienne pour l'Europe du Nord. Elles sont si volumineuses que la fonte de la calotte Laurentide entraîne une hausse du niveau marin de $90 \mathrm{~m}$ à peu près. La fonte de la calotte fennoscandienne entraîne quant à elle une montée du niveau des mers de $30 \mathrm{~m}$. L'ensemble fait $120 \mathrm{~m}$ de niveau marin qui monte quand les deux calottes fondent. Pour avoir un ordre de grandeur, si le tout Groenland fondait, cela n'entraînerait qu'une montée de 7 m du niveau marin.

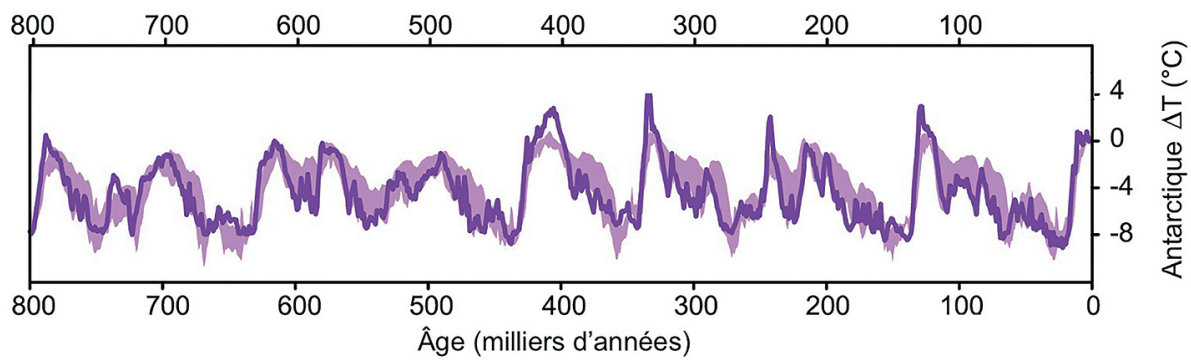

Figure 1.5: Évolution de la température antarctique reconstruite sur les 800000 dernières années à partir de données de 7 calottes de glace. La courbe met en évidence la succession des périodes glaciaires (minima) et interglaciaires (maxima prononcés). Extrait de la figure 5.3, rapport IPCC WG1 AR5 chapitre $5^{2}$.

En fait, depuis un million d'années, le climat est marqué par ce développement de calottes, pas leur fonte rapide, et un interglaciaire un peu plus chaud. Voyons maintenant un point fondamental: quel rôle a joué la concentration atmosphérique de $\mathrm{CO}_{2}$ et ses variations dans ces cycles glaciaires/interglaciaires? Nous pouvons étudier ce rôle grâce aux mesures de $\mathrm{CO}_{2}$ présent dans les bulles d'air piégées dans les glaces. Et nous disposons, depuis la fin du siècle dernier, de mesures de $\mathrm{CO}_{2}$

2. Rapport IPCC WG1 AR5 Chapitre 5: https://www.ipcc.ch/report/ar5/wg1/

Podcasts du GIEC : https://podcasts.apple.com/fr/podcast/le-climat-en-questions/id1547948182 
pour les 800000 dernières années, grâce à une calotte de glaces de plusieurs kilomètres prélevée en Antarctique, ce qui a permis de souligner le lien très fort entre $\mathrm{CO}_{2}$ et cycle glaciaire-interglaciaire.

Qu'a-t-on découvert en analysant le $\mathrm{CO}_{2}$ dans cette calotte de glace? Le $\mathrm{CO}_{2}$ semble, en fait, très peu varier : il oscille entre 180 ppm, le minimum pour les périodes glaciaires, et 280 ppm, le maximum pour les périodes interglaciaires (Fig. 1.6). À chaque fois que les calottes se développent, le $\mathrm{CO}_{2}$ baisse jusqu'à 180 ppm et quand le $\mathrm{CO}_{2}$ remonte, les calottes fondent en période interglaciaire. Cette découverte est vraiment exceptionnelle car on connaît désormais les deux processus à la base du changement climatique sur presque un million d'années: les variations de $\mathrm{CO}_{2}$ et les variations astronomiques.

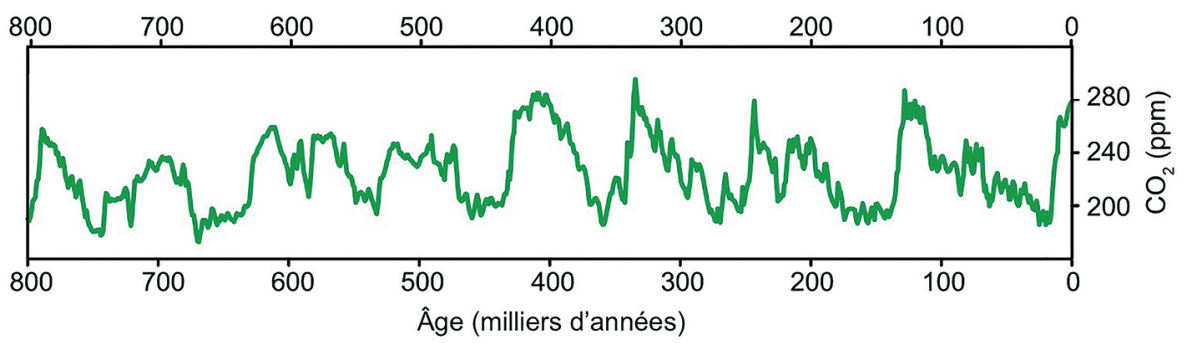

Figure 1.6: Évolution de la concentration atmosphérique de $\mathrm{CO}_{2}$ obtenue sur les 800000 dernières années à partir de mesures dans des calottes de glaces. On observe la corrélation étroite avec l'évolution de la température de la figure 1.5. Extrait de la figure 5.3, IPCC WG1 AR5 chapitre $5^{2}$.

Et les humains dans tout cela? Homo sapiens a finalement quitté l'Afrique il y a seulement à peu près 130000 ans, et en quelques dizaines de milliers d'années, les humains se sont dispersés sur l'ensemble de la planète. Cette dispersion n'aurait pas pu avoir lieu sans que l'on passe d'une période glaciaire à une période interglaciaire. Avec une habileté extraordinaire, Homo sapiens s'est déplacé, est parti d'Afrique équatoriale, est monté en Asie par le Levant et par l'Arabie Saoudite, puis a envahi l'ensemble du globe en arrivant en Australie il y a 50000 ans et même en Europe il y a à peu près 20 000-30 000 ans. Cependant, il n'a pu passer en Amérique du Nord qu'à partir du moment où la calotte Laurentide a suffisamment fondu. C'est la raison pour laquelle l'arrivée d'Homo Sapiens en Amérique du Nord est beaucoup plus tardive.

Cette population humaine restait alors très tributaire des variations astronomiques du climat. Elle était vraiment inféodée à ces changements. Nous allons maintenant aborder la manière dont les humains ont, par leurs activités, modifié en moins de deux siècles le climat global de la planète de manière aussi importante que le font la variation de luminosité solaire ou la tectonique des plaques, mais cette fois-ci à une vitesse fulgurante.

Nous avons précédemment expliqué les mécanismes du changement climatique sur des échelles de temps très importantes et qui sont difficiles à imaginer: 
le milliard d'années, les millions d'années, les dizaines de milliers d'années. Intéressons-nous maintenant à une échelle de temps plus simple à envisager où le climat répond à des forçages qui ne sont plus des contraintes liées à la tectonique des plaques, à la luminosité solaire et aux paramètres du mouvement orbital de la Terre. Le climat répond désormais à un forçages issu d'un nouveau facteur géologique: I'Humanité [4]. Pour satisfaire à nos besoins énergétiques, nous brûlons des combustibles fossiles et, lors de cette combustion, nous produisons du $\mathrm{CO}_{2}$ qui s'accumule dans notre atmosphère. Cette accumulation du $\mathrm{CO}_{2}$ est très bien documentée à partir des mesures faites par Charles David Keeling à Mauna Loa, à Hawaii (Fig. 1.7). Nous disposons d'une très longue série de mesures depuis les années 1950; des mesures qui se sont depuis lors étoffées, grâce aux apports de nombreux autres observatoires.
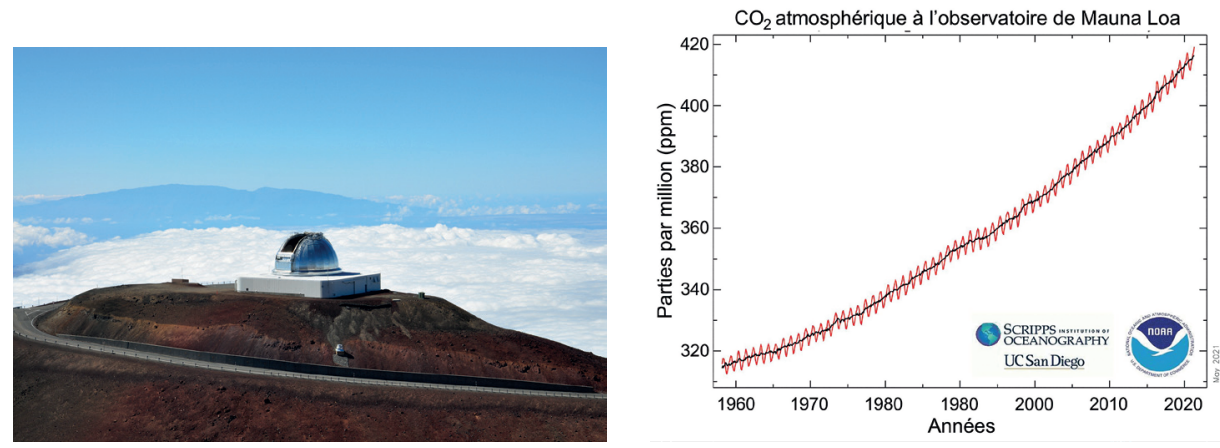

Figure 1.7: À gauche: la photographie de l'observatoire de Mauna Loa (@istock). À droite: l'augmentation de la concentration de $\mathrm{CO}_{2}$ atmosphérique (en ppm) mesurée à Mauna Lea depuis 1958 (Données de Dr. Pieter Tans, NOAA/ESRL et Dr. Ralph Keeling, Scripps Institution of Oceanography, CC BY-SA 4.0).

Si nous revenons à la température et à ses changements sur à peu près les derniers mille ans, on constate très peu de modifications avant les derniers 200 ans (Fig. 1.8). En réalité, on n'observe juste deux petites modulations. L'une est l'Optimum médiéval, époque lors de laquelle la température s'est élevée de quelques dixièmes de degré dans l'hémisphère nord. L'autre correspond à ce qu'on appelle le Petit Âge glaciaire, du XV au XVIII' siècle, durant lequel il a fait un peu plus froid (comme on peut le deviner en observant les peintures des peintres flamands de cette époque). Mais ce ne sont, encore une fois, que de petites modulations.

Si l'on prend encore plus de recul et que l'on regarde les 10000 dernières années, soit l'Holocène, l'interglaciaire dans lequel nous vivons, on peut vraiment parler d'encéphalogramme plat: le climat est très stable. C'est une chance extraordinaire, et c'est d'ailleurs pour cette raison que les humains se sédentarisent à ce moment-là. Durant le début de cette période, dans le croissant fertile, qui est une région de terres riches, l'homme a commencé à utiliser ces sols: c'est le début de l'agriculture, de la sédentarisation, de l'utilisation de l'eau pour l'agriculture mais aussi de l'usage du feu pour la céramique. C'est également le commencement de l'élevage. 


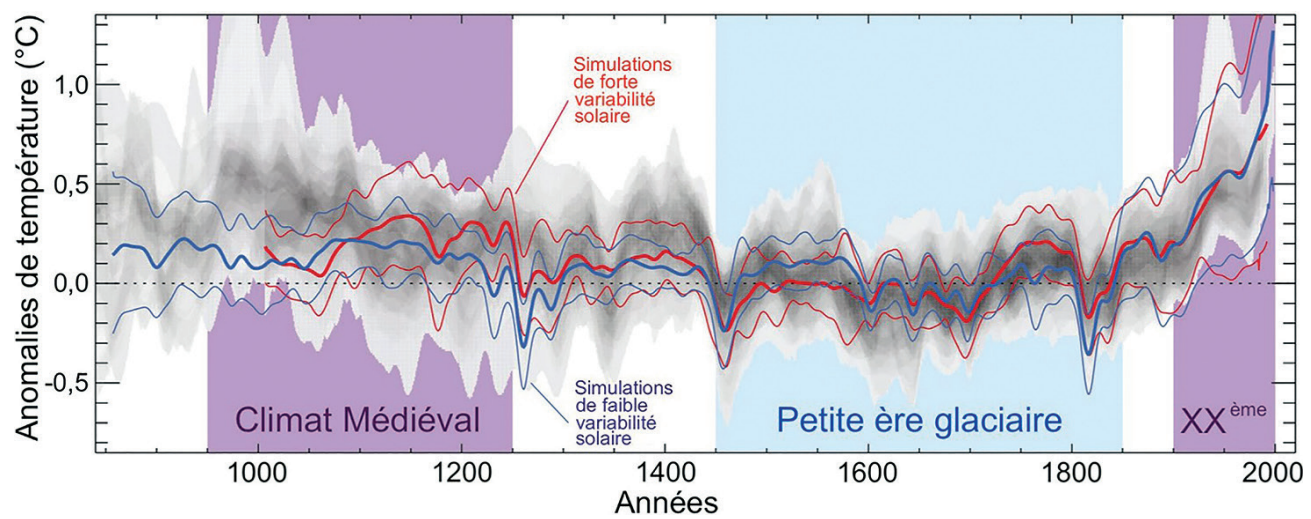

Figure 1.8: Évolution de la température de l'hémisphère nord simulée à l'aide de modèles numériques (en bleu et rouge) et reconstruite à partir d'observations (en gris) sur le dernier millénaire. Extrait de la figure 5.8, IPCC WG1 (2013) AR5 chapitre $5^{2}$.

Le changement de climat induit par les activités humaines est d'autant plus extraordinaire qu'il s'est produit dans un contexte relativement froid, à un moment où le niveau de concentration atmosphérique $\mathrm{du} \mathrm{CO}_{2}$ était bas par rapport aux niveaux qu'a connus notre planète, à un moment où celle-ci possédait deux calottes de glace. Notons que, si la quantité de $\mathrm{CO}_{2}$ atmosphérique monte, ces calottes de glace vont fondre et, de fait, augmenter le niveau marin.

Aujourd'hui, les scientifiques utilisent des modèles numériques en y incluant des processus de plus en plus complexes et grâce à des machines de plus en plus sophistiquées. Ces modèles, qui décrivent la physique et la chimie de l'atmosphère, des océans, de la végétation et de la biosphère, permettent de simuler l'ensemble des interactions et de faire des projections sur les siècles qui viennent. La figure 1.9, issue de l'un des rapports du GIEC (Groupe International d'Experts sur le Climat), illustre ainsi comment la température moyenne et le niveau des océans pourraient varier dans le siècle à venir.

Focalisons-nous sur deux scénarios du GIEC. Le premier est le scénario RCP2.6, qui suppose des émissions de $\mathrm{CO}_{2}$ telles qu'après 2050, l'on parvienne à changer la trajectoire du $\mathrm{CO}_{2}$ atmosphérique en en absorbant plus que l'on en émet. Dans un tel scénario, on contiendrait alors la hausse du niveau marin à $40 \mathrm{~cm}$ et on limiterait l'élévation des températures globales à moins de $2{ }^{\circ} \mathrm{C}$.

Maintenant, intéressons-nous au scénario RCP8.5, dit business as usual, qui suppose que rien n'est fait pour infléchir nos émissions de $\mathrm{CO}_{2}$. Dans ce cas, à la fin du siècle, on devra faire face à des remontées de niveau marin d'au moins un mètre et à une température moyenne qui pourra s'élever de $4^{\circ} \mathrm{C}$, voire plus, selon les modèles. L'avenir est donc vraiment entre nos mains dans le sens où c'est nous, les humains, qui devrons gérer, en toute connaissance de cause, le $\mathrm{CO}_{2}$ que nous allons émettre. À cause de l'emprise des humains sur la planète et de leur utilisation 
extensive et excessive des gaz à effet de serre, le $\mathrm{CO}_{2}$ atmosphérique augmente très rapidement, ce qui a des conséquences non seulement physiques et chimiques, mais aussi biologiques. Dans les 20 ans à venir, nous serons donc amenés à prendre des décisions cruciales, dans un contexte où non seulement le climat est bouleversé, mais où l'ensemble de notre environnement ainsi que les relations entre humains et non-humains sont également modifiées.
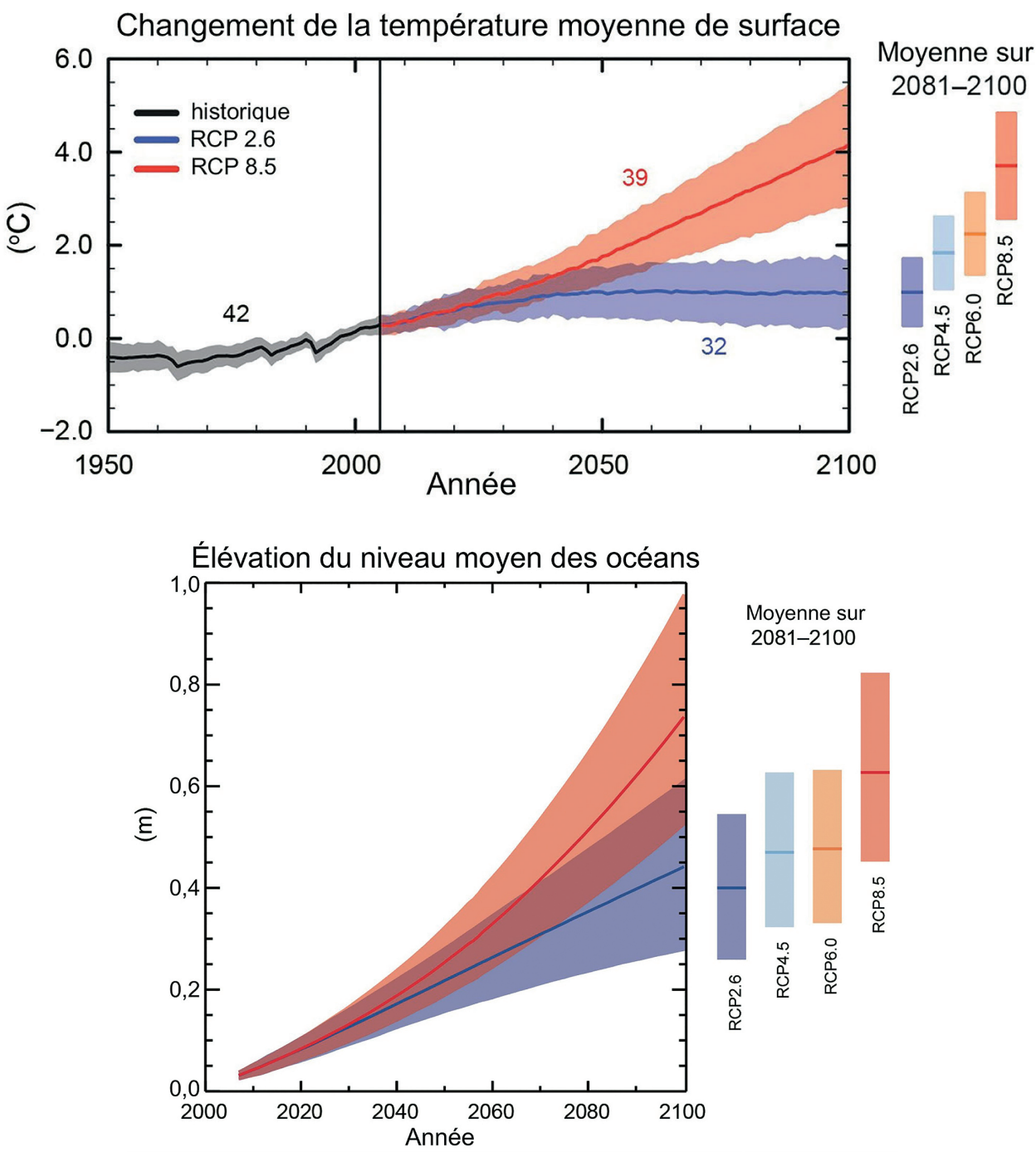

Figure 1.9: En haut, l'évolution de la température moyenne à la surface de la Terre prédite jusqu'en 2100 à partir de différents scénarios avec la période 1986-2005 prise comme référence. En bas, l'élévation du niveau moyen des océans prédite jusqu'en 2100 en prenant la même période de référence. Différents scénarios climatiques sont considérés (voir section 9.3). Source: IPCC WG1 (2013) AR5, chapitre $5^{2}$. 


\subsection{Interactions Humains biodiversité: une perspective historique - J. Lecomte}

Revenons sur la période géologique de l'Holocène, qui a commencé il y a environ 10000 ans et qui fut une étape charnière pour les humains. Durant cette période, un climat stable leur a, en effet, permis de se sédentariser. Ainsi, c'est au Néolithique, entre l'an 12500 et l'an 7500 av. J.-C., que des petites communautés humaines commencèrent à se regrouper dans des villages permanents (Fig. 1.10).

Ces communautés humaines y ont développé l'agriculture mais aussi l'élevage. Ce grand changement, qui les fit progressivement passer du stade de chasseurscueilleurs à agriculteurs et ceci en relativement peu de temps, serait apparu au Proche-Orient (Fig. 1.10) et presque simultanément en Chine du Nord, au Sahara et dans la Cordillère des Andes. Les humains qui tiraient avant cette époque leur subsistance de la chasse, de la pêche et de la cueillette deviennent dès lors des producteurs et peuvent renouveler ce qu'ils consomment par les semis et l'élevage.

Cette émergence de la sédentarisation, de l'agriculture et de l'élevage qui a été favorisée par la stabilité et la clémence du climat, a eu des conséquences sur l'organisation sociale des communautés humaines. Le besoin de protéger les cultures, les récoltes, les troupeaux et les provisions issues de la sédentarisation ont, par exemple, conduit progressivement au concept de propriété et au droit. Le Néolithique a aussi contribué à développer un autre regard des humains vis-à-vis du reste du vivant, les non-humains. Attardons-nous un peu plus sur ce point.
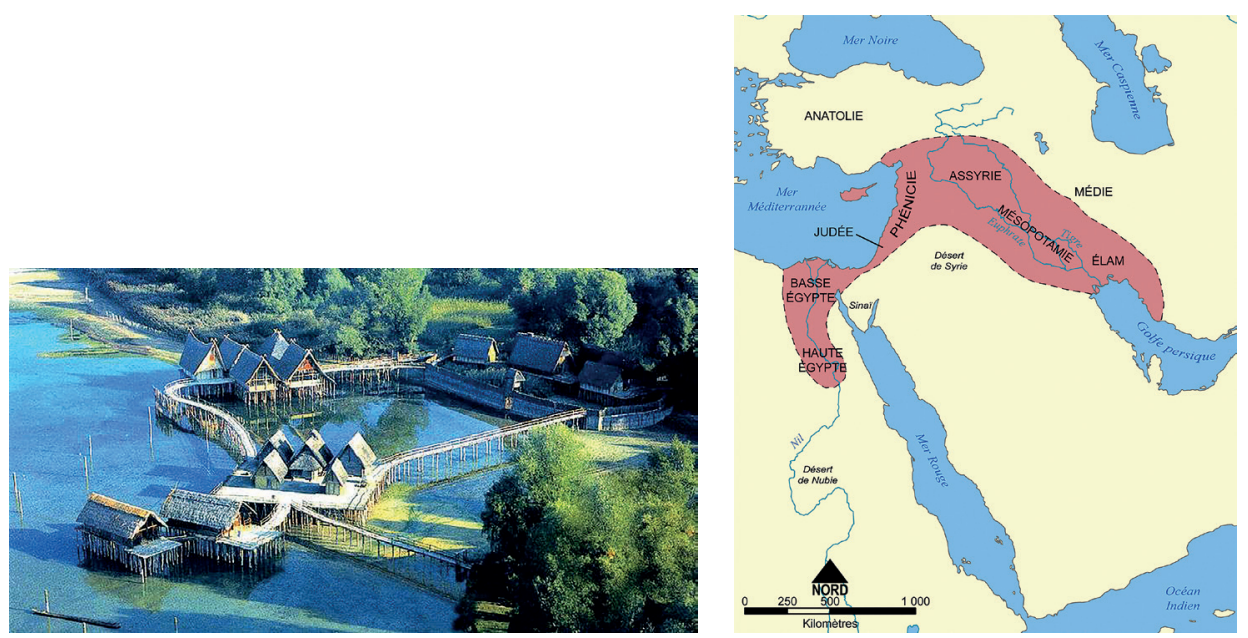

Figure 1.10: À gauche: la reconstitution de palafittes que construisaient les Européens du Néolithique vivant près des lacs en Allemagne: @Spiridon Manoliu, Wikipédia, DP. À droite: le croissant fertile, région du Moyen-Orient incluant l'Égypte antique, le Levant et la Mésopotamie, où ont commencé à se développer l'agriculture et l'élevage. Source: Wikipedia.org (CC BY-SA 3.0). 
Dans l'état actuel des connaissances, les plus anciennes domestications d'espèces animales sont celles du porc, de la chèvre, du mouton et du bœuf, mais c'est aussi probablement très tôt que les premiers chats ont été apprivoisés [5]. La proximité du chat et des humains pourrait ainsi s'expliquer par la prolifération des souris attirées par les réserves de graines autour des premiers villages permanents. Le chat mais aussi le loup, dont la domestication va conduire à toutes les espèces de chien que nous connaissons, vont ensuite se rapprocher de plus en plus des humains jusqu'à s'immiscer au sein même de leurs domiciles pour devenir des animaux dits de compagnie.

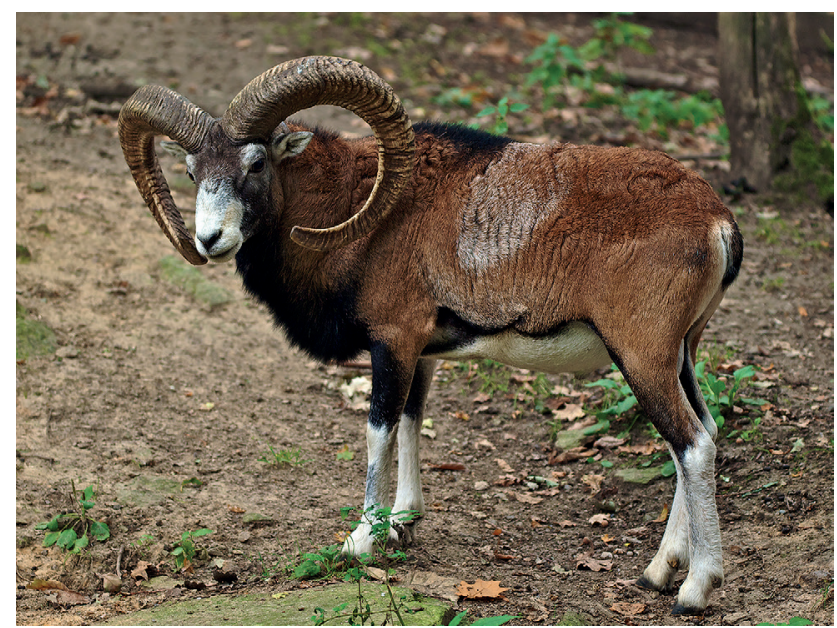

Figure 1.11: Mouflon de Corse, CC BY-SA $3.0^{3}$.

À l'inverse, certains animaux domestiques, qui s'éloignent ou s'échappent des humains, retourneront dans le monde dit «sauvage». Selon Jean-Denis Vigne, archéozoologue du Muséum national d'Histoire naturelle, les mouflons de Corse (Fig. 1.11) sont, par exemple, des moutons introduits au néolithique et redevenus sauvages.

Un clivage entre la nature sauvage et la nature domestiquée mais aussi plus largement une logique d'ingénierie du vivant par les humains a donc pu débuter à cette époque. Pour illustrer ce clivage ou dualisme entre humains et non-humains, nous vous proposons en illustration les extraits d'un livre de Buffon au XVIII ${ }^{e}$ siècle (Fig. 1.12) [6].

«Voyez ces plages désertes, ces tristes contrées où l'homme n'a jamais résidé [...]. La nature brute est sauvage et hideuse et mourante, c'est Moi, Moi seul qui peut la rendre agréable et vivante: desséchons ces marais, animons ces eaux mortes en les faisant couler, formons-en des ruisseaux, des canaux [...] mettons le feu à cette bourre superflue, à ces vieilles forêts déjà à demi consommées;

3. https://fr.wikipedia.org/wiki/Mouflon_corse\#/media/Fichier:Ovis_orientalis_LC0267.jpg 


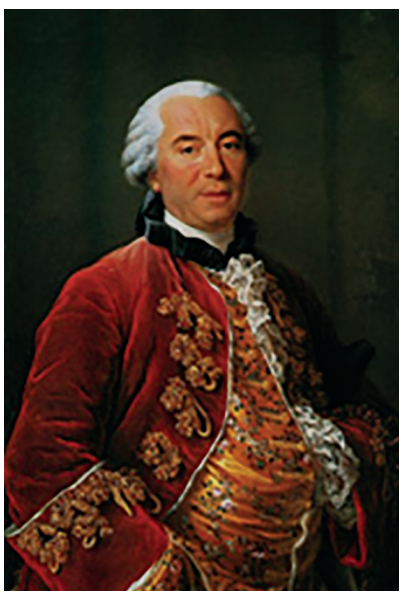

achevons de détruire avec le fer ce que le feu n'aura pu consommer [...] bientôt au lieu du jonc, du nénuphar, dont le crapaud composait son venin [...], des troupeaux d'animaux bondissants fouleront cette terre jadis impraticable [...], que le bœuf soumis au joug emploie ses forces et le poids de sa masse à sillonner la terre, qu'elle rajeunisse par la culture: une nature nouvelle va sortir de ses mains [...] Qu'elle est belle cette nature cultivée! Que par les soins de l'homme elle est brillante et pompeusement parée».

Figure 1.12: Extrait du livre "Histoire générale et particulière» (1764) de Buffon (portrait à gauche). Source: wikipedia.org: domaine public ${ }^{4}$.

Cette vision portée par Buffon est clairement une vision très anthropocentrée du monde vivant, c'est-à-dire une vision qui appréhende la réalité à travers la seule perspective humaine. Cette vision traduit le fait qu'à partir du Néolithique, les humains sont sortis en quelque sorte d'une nature à laquelle ils étaient un élément parmi d'autres. Ce faisant, ils se sont sentis alors supérieurs à elle, puisque capables de la maîtriser, en cultivant des plantes et élevant des animaux. C'est ce que nous racontent les peintures, les fresques et les sculptures, qui sont autant de livres dans lesquels se lit l'histoire de l'humanité. Après la période du Néolithique, se succède une époque où les êtres vivants sont vénérés, car ils permettent aux humains, par leur prélèvement, de manger et donc de survivre, puis, ce sont les dieux à l'image de l'Homme qui sont vénérés, d'abord plusieurs dieux, puis un seul Dieu. Dans ces religions dites du Livre, les humains tentent d'être à la hauteur d'un Dieu à leur image, donc au-dessus de la Nature (voir Cauvin, 2019) [7].

Cette diversité des rapports entre humains et non-humains a été notamment décrite par l'anthropologue Philippe Descola [8]. Dans son livre «Par-delà nature et culture», il a proposé une typologie de quatre types d'interactions humains / nonhumains selon les ressemblances ou différences en termes d'intériorité et de physicalité (Tableau 1.1). Dans le cas du naturalisme dit aussi dualisme, qui correspond à la vision occidentale la plus classique, les humains sont les seuls détenteurs d'une intériorité et ont des physicalités similaires avec les non humains. Par contre, dans le cas de l'animisme courant dans les populations autochtones d'Amazonie, dont certaines ont des modes de vie relativement proches du mode chasseur-cueilleur, il y a une continuité des intériorités, les humains pouvant «dialoguer » avec les nonhumains, mais il existe une discontinuité des physicalités, chaque non humain étant considéré comme ayant des dispositions physiques particulières.

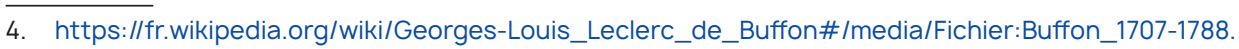
jpg 


\begin{tabular}{|c|c|c|}
\cline { 2 - 3 } \multicolumn{1}{c|}{} & $\begin{array}{c}\text { Ressemblance } \\
\text { des intériorités }\end{array}$ & $\begin{array}{c}\text { Différences des } \\
\text { intériorités }\end{array}$ \\
\hline $\begin{array}{c}\text { Ressemblance des } \\
\text { physicalités }\end{array}$ & Totémisme & Naturalisme \\
\hline $\begin{array}{c}\text { Différence des } \\
\text { physicalités }\end{array}$ & Animisme & Analogisme \\
\hline
\end{tabular}

Tableau 1.1: Typologies d'interactions entre humains et non-humains (d'après Descola, 2005 [8]).

Dans le cadre des enjeux à relever face aux changements globaux, que sont l'érosion de la biodiversité et les changements climatiques, s'interroger à propos des différentes relations que nous nouons avec les non-humains tout au long de l'histoire mais encore aujourd'hui doit nous conduire à nous interroger sur les racines profondes de nos interactions avec eux, les valeurs qui y sont associées et finalement ce qui fait le fondement de nos sociétés (Lecomte \& Sarrazin, 2020) [9].

Cette réflexion sur nos relations vis-à-vis des autres habitants de la planète pourrait être l'occasion d'opérer un changement radical pour penser le futur des non-humains comme une obligation éthique et non pas uniquement comme une nécessité pour notre propre survie (Sarrazin \& Lecomte, 2016) [10]. Mais serons-nous capables d'assumer en quelque sorte ce qui serait un dépassement de l'Anthropocène pour laisser un espace de liberté aux non-humains à l'échelle de l'évolution, et ceci au-delà des intérêts anthropocentrés?

\subsection{Technologie, gouvernance et développement : une réflexion sur les rapports des sociétés occidentales avec l'environnement - J.P. Vanderlinden}

\subsubsection{Interrelations entre les concepts de développement, de technologie et de gouvernance}

Posons-nous la question suivante:

Comment, pourquoi, et avec quelles conséquences en, somme toute, très peu de temps, quelques milliers d'années, un grand singe chasseur cueilleur en est arrivé parfois, à souhaiter conduire, dans une ville comme Paris, un véhicule de quelques tonnes, essentiellement vide, disposant éventuellement de quatre roues motrices, et capable d'atteindre des vitesse mortelles, et ainsi de menacer ses congénères 
tout en contribuant à la destruction de la seule planète à sa disposition... ? Et en plus, il en a conscience!

II s'agit en fait d'une question complexe, et nous allons tenter de proposer brièvement quelques clés pour y répondre.

Être, posséder, de la technologie à la gouvernance: Dans cette première partie, nous allons conceptualiser la technologie et la gouvernance pour envisager ensuite la notion de développement lié à l'expérience humaine.

Selon les fondements de l'économie écologique proposés par René Passet en 1996 [11], «L'Homme, comme tout organisme vivant, a pour finalité première de maintenir et reproduire sa structure, c'est-à-dire être physiquement ». Être, c'est notamment se déplacer pour se nourrir. La vitesse de déplacement et la distance qu'un Humain ou un organisme peut parcourir pour trouver de la nourriture sont évidemment critiques. Plus il est rapide, mieux il est à même de garantir le maintien et la reproduction de sa structure.

Toujours selon Passet, «Être, pour l'Humain, revêt, en fait, deux significations complémentaires: "d'une part maintenir sa structure et développer les facultés qui en découlent et d'autre part, exister socialement par rapport au groupe et à ses valeurs». Ainsi, si nous reprenons l'exemple de l'Humain en quête de nourriture, se déplacer pour se nourrir sera, pour lui, plus efficace s'il s'agit d'un exercice collectif. Autrement dit, régir et administrer collectivement les déplacements sont fondamentaux pour «l'Homme social».

C'est ainsi que la possession, le fait d'Avoir prend sa place dans son rapport à l'Être. Avoir est un moyen de garantir l'Être, biologiquement et socialement, et ceci est une spécificité presque exclusivement humaine. Reprenant notre fil conducteur, si vitesse et distance de déplacement sont critiques pour se nourrir, elles ont conduit les Humains, dans une forme de légitimité d'ordre biologique, à la mécanisation de leurs déplacements, au développement de technologies, ... de la technologie. Avoir nous permet d'Être, d'être en sécurité en ayant un abri, par exemple, de nous nourrir en ayant à la fois les moyens de nous déplacer pour chercher la nourriture, les moyens de produire cette nourriture, par exemple, une machine agricole, les moyens de nous protéger, par exemple, une arme. Avoir est un moyen pour Être, le moyen n'étant pas une fin en soi.

La technologie est ainsi l'application de savoirs aux objectifs pratiques liés à l'expérience humaine, objectifs qui, souvent, reviennent au changement ou à la manipulation de notre environnement pour la création et la possession d'objets. Par là même, la facile «dégradation» de l'Être en Avoir se prolonge ici par celle de l'Avoir à «En Avoir Plus» et du rapport à l'Autre, médiatisée par la technologie. Le rapport à un objet change, en effet, notre rapport à l'Autre tant par l'avantage compétitif que par l'avantage social qu'il procure. Le moyen devient à nouveau une fin, qui décorrèle l'objet de la réalité des nécessités de l'Être. 
Reprenons l'exemple d'une machine agricole. Si un individu s'en assure l'exclusivité, il peut devenir plus efficace que son voisin, et à terme lui racheter ses terres. Plus il aura de machines, plus il aura le sentiment d'Être par l'accumulation. Mais en réalité, personne n'a vraiment besoin de ce supplément de moyens. Le moyen (la récolte) devient une fin. Les moyens du moyen (les machines) deviennent une fin. Les moyens des moyens des moyens (l'argent pour acheter terre et machines) deviennent une fin. Mais personne ne se nourrit vraiment d'argent en trop grande quantité.

Maintenant que nous avons introduit la place de la technologie, quelle est celle de la gouvernance? Nous avons vu, par exemple, que la nécessité d'administrer collectivement nos déplacements faisait partie intégrante de leur mécanisation. Géraldine Froger (2006) a ainsi défini la gouvernance comme étant l'ensemble des moyens par lesquels les individus et les institutions, publiques et privées, gèrent leurs affaires communes [12]. La gouvernance est donc un processus continu de coopération et d'accommodements entre des intérêts qui peuvent être conflictuels. Elle est donc, en quelque sorte, une technologie, mais une technologie centrale à l'humain.

Concept de développement multidimensionnel: Reprenons les notions d'Être et d'Avoir. Nous avons vu qu'Avoir un objet devient une fin en soi au lieu de n'être qu'un moyen. Par prolongement, Avoir par un moyen d'échange facile, l'argent, devient à son tour une fin en soi. Nous nous trouvons face à une double dégradation de la quête initiale, ceci au sens de György Lukács [13]. Le concept de développement pourrait ainsi se réduire à une dimension unique qui est celle d'accumuler des capacités à posséder mais le développement peut aussi être défini comme une «croissance complexifiante multidimensionnelle».

Comment pourrions-nous définir un pays développé? Est-ce par les quantités d'argent qu'il a accumulées? Est-ce simplement un pays riche? Non, pas uniquement: c'est un pays dont les institutions fournissent également de la culture, de l'éducation, de la santé, un soutien au moins chanceux, une organisation démocratique où chacune et chacun peut contribuer. C'est également un pays où les relations sociales et économiques sont harmonieusement enchevêtrées, et non un pays «tiré» tout simplement par une industrie unique ou une minorité d'individus. Au final, c'est un pays complexe, dont le développement s'est produit à de nombreux niveaux différents (santé, culture, bonheur, économie...), dans plein de dimensions.

Essayons maintenant d'imaginer les liens entre une conception de développement et les choix de technologie, les choix de gouvernance, les objectifs poursuivis et ces choix. Si ce qui nous porte est un développement «étriqué», nos choix technologiques seront également étriqués, portés par toujours plus d’Avoir.

Si ce qui nous porte est une vision riche, multiforme, complexe du développement, nos choix technologiques et nos choix de gouvernance feront alors l'objet d'arbitrage complexe et totalement enrichissant. 


\subsubsection{Un avatar du concept de développement : I'hégémonie fordiste}

L'hégémonie fordiste et l'accumulation de biens: Maintenant que nous avons introduit à notre manière les principaux concepts, illustrons celui de développement par un exemple important du siècle dernier, exemple que l'on peut voir comme un «avatar éclairant» : l'hégémonie fordiste. L'idée ici est d'observer, notamment à l'aune des concepts "d'être et avoir», de technologie et de gouvernance, comment s'est fait ce développement et de réfléchir à ses conséquences.

L'hégémonie fordiste est une des manifestations du système capitaliste [14]. C'est une organisation du capitalisme qui a connu un certain succès durant ce qui a été appelé les «trente glorieuses». L'hégémonie fordiste peut être représentée comme un tabouret à trois pieds.

Le premier pied du " "tabouret» est lié à ce que l'on nomme un mode de production, la façon dont on s'organise pour produire des biens. Le mode de production associé à l'hégémonie fordiste est appelé taylorisme, ou encore organisation scientifique du travail (OST). Pour faire court, il s'agit du «travail à la chaîne». Combiné à la mécanisation des tâches dont l'ampleur évolue avec la technologie disponible, le travail à la chaîne permet des gains de productivité, mais ce n'est pas sans conséquences!

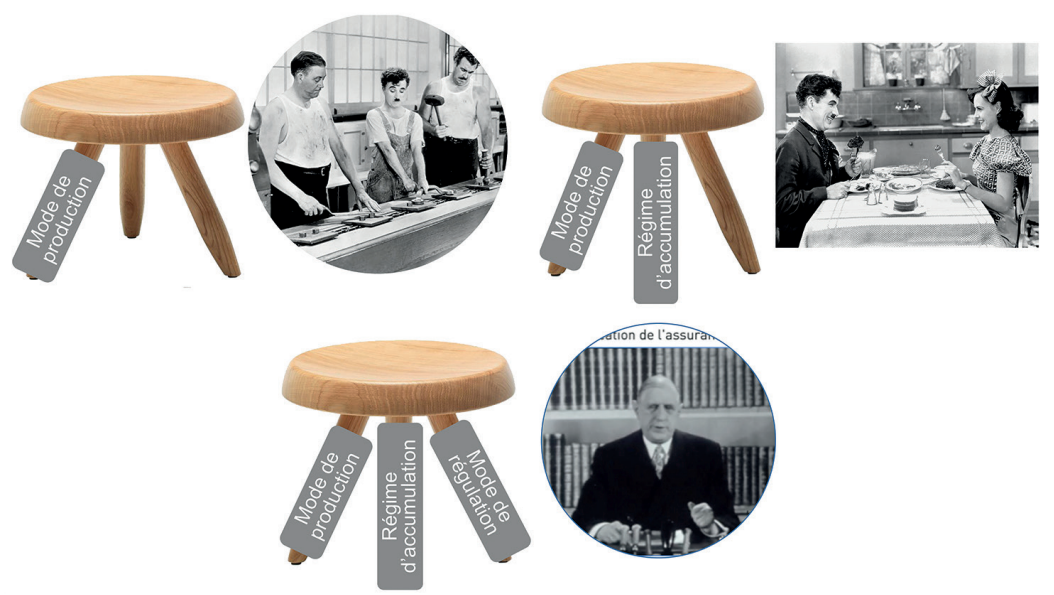

Figure 1.13 Modes de production, régime d'accumulation et modes de régulation sont les trois piliers de l'hégémonie fordiste. Les photos du haut sont extraites des Temps Modernes (Film de 1936). La photo du bas est extraite d'une conférence de presse du Président Charles de Gaulle.

Le travail à la chaîne, en aliénant le travailleur de l'objet produit, en l'en séparant, vient réduire sa qualité de vie. Dans les Temps Modernes, Charlie Chaplin en fait même une «dépression nerveuse», il devient «aliéné»! Sans compensations, ce serait insupportable. 
Les travailleurs vont alors réclamer un partage des gains de productivité - le second pied du tabouret de l'hégémonie fordiste va se mettre en place: un régime d'accumulation - les gains de productivité vont être partagés entre les détenteurs du capital et les détenteurs de la force de travail - cela sera le produit d'une négociation, parfois violente, toujours renouvelée et de plus en plus institutionnalisée.

Le mal-être lié au travail sera compensé par... du plus «avoir» - et en fait «d'avoir plus d'argent». Mais ces deux pieds ne permettaient pas de faire tenir le tabouret. En cas de crise, l'économie pouvait s'effondrer. Il a fallu attendre l'après-guerre pour que se mette en place un mode de régulation, que l'on appellera keynésianisme du nom de l'économiste John Maynard Keynes. Ce mode de régulation sera également nommé «État Providence».

Par exemple, en 1958 le président de la République, Charles De Gaulle annonce la création de l'assurance chômage - même sans emplois les gens pourront continuer à consommer et donc pour d'autres, en amont, à produire - mais il y a plein d'autres exemples.

Néanmoins, entre la fin des années 1960 et le début des années 1970, le fordisme a connu quelques hoquets, puis il a fini par s'effondrer. Que s'est-il passé? Quel pied du tabouret a donc cédé? Est-ce l'État qui devenait inopérant pour réguler par le keynésianisme? Somme toute, on avait un peu l'impression que plutôt que de réguler, l'État ne faisait que tenter d'accélérer les choses, de plus en plus. Est-ce la répartition qui ne faisait plus l'affaire? II est possible que soit les propriétaires de la force de travail, soit les propriétaires du capital, soit les deux à la fois, soient devenus trop gourmands. Peut-être que l'organisation scientifique du travail, le travail à la chaîne et la technologie connaissaient leurs limites?

En fait, l'effondrement du fordisme est attribuable à de nombreux facteurs un peu simultanés. Mais cet effondrement, et c'est cela qui nous intéresse, était contemporain d'une série d'alertes sur la dégradation de l'environnement : la «fin des oiseaux chanteurs» en raison de l'effet du DTT sur leur reproduction (voir par exemple, l'ouvrage de Rachel Carson, 1962), des inquiétudes documentées quant à la finitude de nos ressources (voir Meadows et al., 1972). II y a aussi eu le choc pétrolier, création artificielle de rareté, qui a tout de même le goût et l'odeur d'une crise environnementale, sans en être une, et qui a plongé nos économies dans la tourmente tout en montrant notre dépendance vertigineuse au pétrole.

Or, les conjonctures comme le choc pétrolier, la lame de fond en formation dans les trente glorieuses, c'est, pour faire simple, la mise en place de conditions qui allaient conduire à une série de crises environnementales de plus en plus importantes dans les années qui succédèrent. Dans l'image du tabouret, si le sol représente le monde naturel sur lequel prennent assise les systèmes humains, ce sol s'est dérobé sous le tabouret, ce sol était, en effet, grugé de façon invisible - les premiers "glissements de terrain » ont vraisemblablement eu lieu de façon un peu discrète, de façon non saillante, dès le début des années 1970. 
Si les trente glorieuses et l'hégémonie fordiste, qui lui était associée, n'étaient pas soutenables, c'est dans l'origine de la richesse matérielle produite qu'il faut en trouver la cause. Cette richesse ne trouvait pas uniquement sa source dans les gains de productivité associés à l'O.S.T. Elle était malheureusement due aussi à une exploitation irréfléchie du monde naturel. Cette exploitation a pris la forme de prélèvements inconsidérés et de pollution. À un moment, la nature n'en pouvait plus, et le modèle s'est d'abord doucement, puis plus rapidement, effondré.

Des trente glorieuses au concept de développement durable : Essayons à présent de voir en quoi ceci est lié à notre question de départ. D'abord, il est important de se rappeler que les trente glorieuses, cette époque où les gens sont devenus subitement plus riches, est devenue une référence positive. En outre, à l'époque, le modèle capitaliste de l'hégémonie fordiste était celui des États-Unis. C'est l'époque, le temps, où les pays tenant de ce modèle capitaliste étaient, pour la plupart, en guerre froide avec les pays tenant d'un autre modèle - le modèle soviétique. Le «"bonheur» à la fordiste était vendu par la propagande en appui à la guerre froide, froide pour les riches mais bien plus brûlante pour les pays pauvres où l'on se battait, et par un dispositif d'influence internationale appelé coopération au développement.

Or, ce modèle a poussé jusqu'au bout sa logique, une confusion entre l'être et l'avoir. Pour compenser le mal-être lié à l'organisation du travail, on créait du bienêtre par la possession de biens matériels. "Avoir» prenait le pas sur "être», structurellement. Avoir plus, chaque année stabilise le système, quitte à ce que cet «avoir plus» soit accompagné d'une coproduction négative, une destruction de l'environnement. Or, pour générer cet «avoir plus», les technologies deviennent orientées vers la nécessité de repousser les limites imposées par l'environnement. Finalement, même l'administration de nos affaires communes, la gouvernance, ne se tend plus que vers un but unique: stabiliser le système qui permet de continuer à augmenter les richesses à partager afin que détenteurs du travail et détenteurs du capital aient, du moins en apparence, des intérêts convergents. La gouvernance est toute tendue vers un modèle de développement unidimensionnel et simplifiant. Juste le contraire de ce que devrait être, du moins dans l'esprit de cette partie du livre, le développement.

L'hégémonie fordiste n'est plus vraiment souhaitable, et d'ailleurs les trente glorieuses ne sont sans doute pas si glorieuses. Beaucoup d'alternatives ont été proposées à l'hégémonie fordiste dès les années 1960, et surtout durant les années 1970-1980, mais il a fallu attendre les années 1990 pour qu'une de ces alternatives prenne racine. Celle qui a pris racine est celle du «Développement durable» dont le moment fondateur est la conférence de Rio en 1992 [15]. Juste après la fin de la guerre froide, remportée par les tenants du modèle capitaliste lui-même porté par les images associées aux trente glorieuses.

Ceci nous amène à une conclusion sous la forme d'une question ouverte : peut-on dans le cadre du développement durable parler de croissance complexifiante multidimensionnelle? 
Les concepts d'être et d'avoir, de technologie et de gouvernance, d'hégémonie fordiste comme modèle sont autant d'éléments qui devraient permettent d'y répondre.

\subsection{La part du Droit dans les causes anthropiques des changements globaux - A. Farinetti}

Dans les développements qui suivent, nous nous proposons d'expliquer comment le droit a organisé, en les favorisant, les conditions de la surexploitation des ressources naturelles. II s'agit donc d'expliciter les concepts juridiques à l'origine de cette situation ainsi que leurs tempéraments.

Le droit régit les rapports de l'homme avec son environnement depuis les origines de la civilisation. Certains auteurs [16] soutiennent même que c'est de la nécessité de réglementer l'usage de l'eau et de résoudre les conflits afférents que sont nés le droit et les premières institutions judiciaires.

Le droit entretient donc des liens intimes avec l'environnement et son évolution, mais son influence doit néanmoins être relativisée. Sans des facteurs métajuridiques (démographiques, techno-industriels ou socio-anthropologiques) autrement plus puissants, le droit n'aurait exercé, hier comme aujourd'hui, qu'une influence modérée, pour ne pas dire marginale sur l'environnement. Contre eux, il est de peu de poids.

Ainsi, ce n'est que parce que les outils juridiques permettent le déploiement de moyens techniques à la fois puissants et largement répandus, au service d'un modèle de société consumériste, qu'ils contribuent à produire les conséquences néfastes que l'on connaît aujourd'hui. Parce qu'il a accompagné l'émergence de ces facteurs, qu'il les a fondés et, dans une certaine mesure, légitimés et même protégés, notre droit moderne tel qu'il résulte d'une certaine interprétation des conquêtes de la Révolution française doit prendre sa part des responsabilités dans la situation actuelle. II s'agit en quelque sorte de faire «le procès» du droit, mais également de montrer les leviers par lesquels il peut agir pour réorienter son influence, puisque le droit offre toujours des clés pour atténuer les excès auxquels il peut conduire, sans surestimer sa capacité à créer les problèmes, ou inversement à leur porter remède.

L'asservissement des ressources naturelles a pu se fonder sur des principes et des normes juridiques régissant les rapports des États entre eux, c'est-à-dire le droit international, mais également sur ceux régissant l'action des personnes au sein des États, c'est-à-dire le droit national.

Dans le premier cas, le principe qui a fourni les armes les plus efficaces à l'exploitation des ressources naturelles, et donc, qui apparaît comme la principale cause des changements globaux, est la souveraineté étatique. Cette dernière a justifié une large exploitation des ressources présentes au sein des frontières nationales, bien 
que ses effets aient été atténués par le nécessaire respect... des autres souverainetés étatiques.

Dans le second cas, l'exercice de certaines libertés fondamentales acquises par le peuple lors de la Révolution française, a également contribué dans une mesure non négligeable à la dégradation de l'environnement, quoique cet exercice ait également pu faire l'objet de tempéraments.

\subsubsection{La souveraineté étatique comme cause des changements globaux}

L'exploitation souveraine des ressources des États repose sur des principes solidement ancrés dans le droit international public. La souveraineté étatique est, en effet, ce qu'on appelle un «principe général du droit international». Sa manifestation la plus dangereuse, concernant l'exploitation des ressources naturelles, a été théorisée par Judson Harmon sous l'appellation de "théorie de la souveraineté territoriale absolue». Selon cette théorie, les États sont absolument souverains sur les ressources situées au sein de leurs frontières et ne peuvent se voir imposer de restrictions autres que celles qu'ils ont librement consenties.

Cette théorie a connu son exacte opposée, la théorie de l'intégrité territoriale absolue, interdisant aux États d'exercer des usages qui réduiraient, par là même, ceux des autres États. Appliquée aux cours d'eau internationaux, elle conduirait à consacrer au profit d'un État le droit d'exiger la permanence de l'écoulement naturel des eaux provenant d'autres pays en amont, et à lui interdire de réduire le flot naturel des eaux coulant à travers son territoire dans les pays situés à l'aval. Une telle théorie aurait donc pu être propice à l'interdiction de l'exercice d'activités dommageables.

Ni l'une ni l'autre de ces théories n'ont reçu de consécration par la justice internationale, qui leur a préféré le principe de souveraineté territoriale limitée, ou d'utilisation non dommageable du territoire. L'avis consultatif de la Cour internationale de justice (CIJ) du 8 juillet 1996 sur la licéité de la menace ou de l'emploi d'armes nucléaires consacre, dans son considérant $n^{\circ} 29$, ce principe de souveraineté territoriale limitée ${ }^{5}$.

Souverains, les États peuvent donc infliger certains dommages environnementaux, mais ils peuvent également décider, par l'exercice de cette même souveraineté, d'édicter des règles protectrices en leur sein, mais aussi de limiter leurs prérogatives en signant puis en ratifiant des traités internationaux de protection de l'environnement, dont la violation est susceptible d'engager leur responsabilité internationale. Citons à ce titre des textes emblématiques comme la Convention-cadre

5. https://www.icj-cij.org/fr/affaire/95/avis-consultatifs 
sur la diversité biologique ${ }^{6}$ ou la Convention-cadre sur les changements climatiques ${ }^{7}$, toutes deux adoptées après le sommet de la terre de Rio en 1992. Mais on reproche souvent au droit international de l'environnement de ne pas contraindre suffisamment les États.

En revanche, le système juridique de l'Union européenne (UE), fondé sur le Traité de Rome de 1957 n'encourt pas ce reproche. Les États y ont consenti d'importants transferts de souveraineté, qu'une juridiction spécifique, la Cour de justice de I'Union européenne, sera compétente pour sanctionner sans que les États membres ne puissent s'y soustraire. Notons que le droit de l'UE a donné quelques occasions de progrès considérables dans le domaine de la protection de l'environnement à travers des directives européennes, dont certaines sont très ambitieuses, comme la directive "Habitat» du 21 mai $1992^{8}$ ou la directive-cadre sur l'eau du 23 octobre $2000^{9}$. Mais ces textes ne sont applicables que dans l'Union européenne, et ils s'accompagnent d'un grand nombre d'autres poursuivant notamment la libre concurrence, ou la liberté de circulation des marchandises, des personnes, des services et des capitaux, etc..., des textes qui sont susceptibles de constituer autant d'entraves à l'édiction de règles protectrices de l'environnement par les États ou l'Union européenne elle-même, et dont les conséquences peuvent neutraliser les bénéfices environnementaux dont le droit européen de l'environnement mérite d'être crédité.

Si les atténuations aux conséquences du principe de souveraineté étatique sur l'exploitation des ressources naturelles existent donc, elles demeurent néanmoins limitées pour quatre raisons principales.

Les États demeurent, d'abord, libres de sortir des traités, comme l'a fait le Japon en sortant de la Commission baleinière internationale (Fig. 1.14) pour échapper aux contraintes qu'imposait sa condamnation par la ClJ le 31 mars 2014 (Affaire de la chasse à la baleine dans l'Antarctique (Australie $\mathrm{c} /$ Japon) ${ }^{10}$ ), décidant que «le Japon doit révoquer tout permis, autorisation ou licence déjà délivré dans le cadre de JARPA II et s'abstenir d'accorder tout nouveau permis au titre de ce programme». II faut rappeler qu'à l'origine, en 1946, la convention avait été adoptée afin d'organiser la chasse à la baleine à des fins anthropiques, et que l'interdiction n'est venue qu'en 1986 en vertu d'un moratoire sur la chasse commerciale adopté en 1982 et renouvelé tous les 4 ans. En application de ce moratoire, les baleiniers japonais ne pratiquaient plus que la chasse dite "scientifique», mais depuis que le Japon s'est retiré de la Commission, ils peuvent reprendre la chasse commerciale. On peut, bien sûr, citer un autre exemple récent de sortie d'un traité international environnemental: celui des

6. https://www.un.org/fr/observances/biological-diversity-day/convention

7. https://unfccc.int/fr/processus-et-reunions/la-convention/qu-est-ce-que-la-ccnucc-laconvention-cadre-des-nations-unies-sur-les-changements-climatiques

8. https://eur-lex.europa.eu/legal-content/FR/TXT/?uri=celex\%3A31992L0043

9. https://eur-lex.europa.eu/legal-content/FR/TXT/?uri=CELEX:32000L0060

10. https://www.icj-cij.org/fr/affaire/148/arrets 
États-Unis, sortis des accords de Paris relatifs à la lutte contre le changement climatique en 2017.

Les États peuvent aussi refuser de devenir Partie à un accord international ou encore formuler des «réserves» à son encontre, ces réserves les exonérant de certaines contraintes. Ainsi, la Norvège et l'Islande ont rejeté le moratoire relatif à la chasse à la baleine et émis des réserves à son égard, leur permettant de poursuivre la chasse commerciale.

Les États ont également la liberté de causer des atteintes jugées «raisonnables» aux territoires des autres États, en application des principes précités d'utilisation non dommageable du territoire et de souveraineté territoriale limitée.

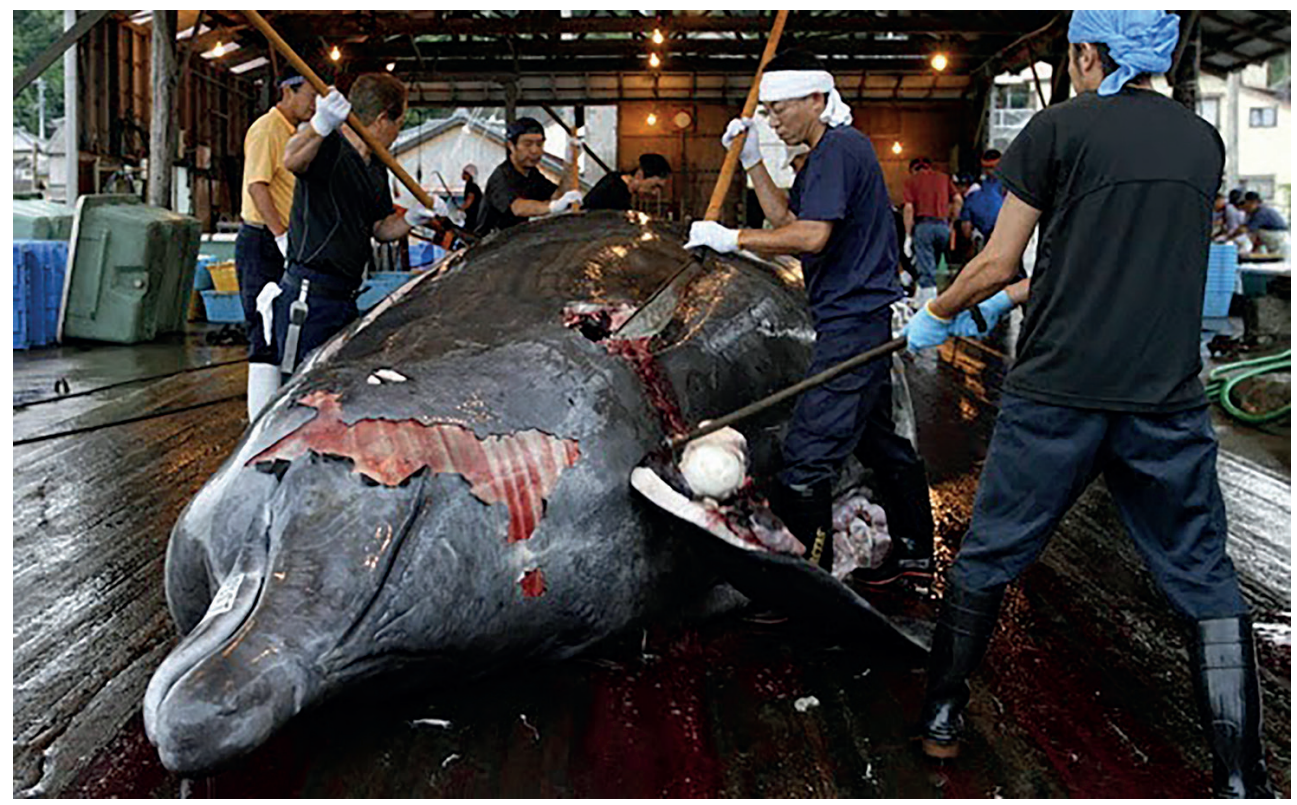

Figure 1.14: Reprise de la pêche à la baleine par le Japon en 2015. Source photo: Droits réservés.

Les États peuvent enfin refuser d'être jugés par les instances internationales (et donc d'assumer les conséquences de la violation de leurs obligations conventionnelles), l'intervention de la Cour internationale de justice étant conditionnée par l'acceptation de sa compétence par les États. Une exception concerne les États membres de l'Union Européenne qui ont, par adhésion, accepté la compétence de la Cour de Justice de l'Union Européenne (CJUE).

Les inconvénients environnementaux de la souveraineté étatique ont leur pendant à l'échelle des citoyens: l'exercice insuffisamment régulé de certaines libertés fondamentales. 


\subsubsection{Des libertés fondamentales comme causes des changements globaux}

Quelles sont, parmi les libertés fondamentales, celles dont l'exercice a pu contribuer à générer les changements globaux?

La première d'entre elles est le droit de propriété, posé par l'article 17 de la déclaration des droits de l'Homme et du citoyen de $1789^{11}$ en vertu duquel ce droit est «inviolable et sacré». De plus, notre Code civil affirme en son article 544 que la propriété est le droit de disposer des choses «de la manière la plus absolue». Ce faisant, c'est une conception absolutiste et exclusive de la propriété qui est ainsi consacrée.

Une autre liberté est la liberté contractuelle que l'on peut rattacher, elle aussi, à la déclaration des droits de l'Homme et du citoyen dont l'article 4 dispose que «La liberté consiste à pouvoir faire tout ce qui ne nuit pas à autrui ». Cette liberté est aujourd'hui affirmée par l'article 1102 de notre Code civil.

Enfin, la dernière liberté qui a largement contribué aux changements de notre environnement est la liberté d'entreprendre, elle aussi rattachée à l'article 4 de la déclaration des droits de l'Homme et du citoyen et consacrée par la loi des 2 et 17 mars 1791 (dite « décret d'Allarde»).

Pourquoi ces libertés jouent-elles un rôle aussi important dans la dégradation de notre environnement et y sont-elles condamnées?

Les libertés précitées ont valeur constitutionnelle, parce que la déclaration des droits de l'Homme et du citoyen a elle-même valeur constitutionnelle, ce qui signifie qu'elles se situent au plus haut niveau de la hiérarchie des normes de notre pays, en d'autres termes, qu'elles s'imposent à toutes les autres normes. Au besoin, le Conseil constitutionnel pourra imposer leur respect au législateur, c'est-à-dire le parlement, et le Conseil d'État pourra imposer leur respect au pouvoir réglementaire, c'est-à-dire le Président de la République, les ministres, les préfets, les maires...

Rien d'étonnant donc à ce qu'elles produisent des effets conséquents. Pourtant, leur influence néfaste n'est pas une fatalité.

Ce n'est pas une fatalité parce que ces textes prévoient toujours une possibilité de limitation, pourvu que cette limitation ne vide pas le droit protégé de sa substance. Ainsi, l'article 544 du Code civil précité nuance-t-il l'absolutisme du droit de propriété par la condition «qu'on n'en fasse pas un usage prohibé par les lois ou par les règlements». C'est la raison pour laquelle des textes viennent poser des limites à l'exercice de ces droits, par le biais de servitudes environnementales (comme la création d'un parc national) ou de polices administratives environnementales (comme la police des installations classées pour la protection de l'environnement). À ce titre,

11. https://www.legifrance.gouv.fr/contenu/menu/droit-national-en-vigueur/constitution/ declaration-des-droits-de-l-homme-et-du-citoyen-de-1789 
la reconnaissance par la loi du 10 juillet 1976 sur la protection de la nature (aujourd'hui codifiée sur ce point à l'article L.110-1 du Code de l'environnement ${ }^{12}$ - Fig. 1.15) du caractère d'intérêt général de la protection de l'environnement a constitué une étape majeure pour justifier ces interventions étatiques, mais elle avait été précédée de textes fondateurs, pour certains très anciens, tel le décret impérial du 15 octobre 1810 relatif aux manufactures et ateliers qui répandent une odeur insalubre ou incommode, et fut poursuivie par une multitude de lois et de décrets aujourd'hui codifiées principalement, mais non exclusivement, dans le Code de l'environnement.
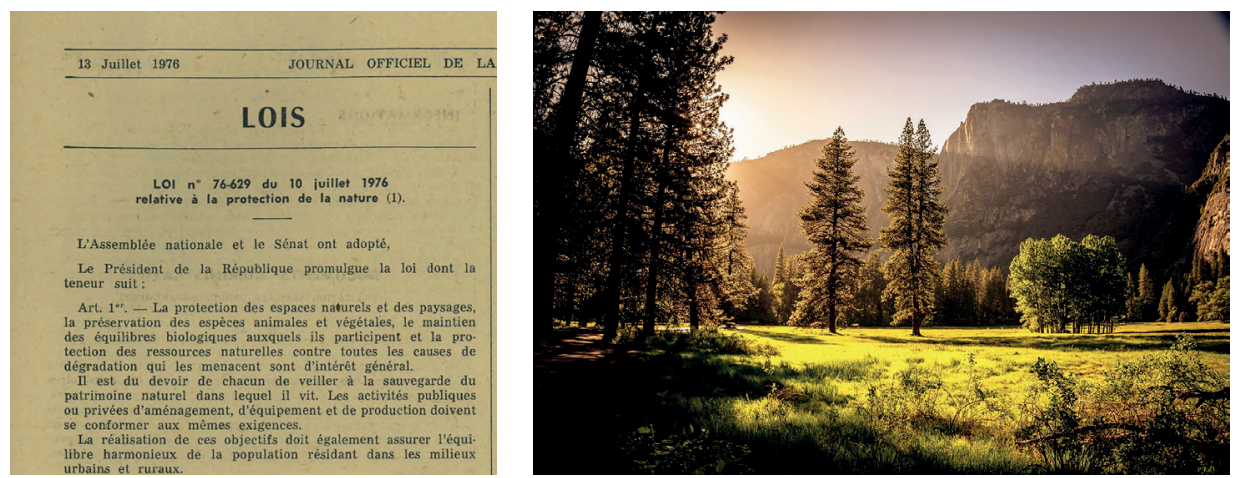

Figure 1.15: À gauche, extrait du Journal Officiel du 13 juillet 1976, relatif à la loi du 10 juillet 1976 sur la protection de la nature. À droite, image évocatrice d'un parc naturel. Photo : @lstock.

Un autre tempérament au caractère néfaste de la consécration de certaines libertés fondamentales consiste à reconnaitre le principe selon lequel l'exercice de leurs droits par certains membres d'une communauté ne doit pas entraver l'exercice des mêmes droits ou d'autres droits par ses autres membres. Ainsi, le juge judiciaire, compétent pour régler les litiges entre personnes privées, peut indemniser les victimes de préjudices environnementaux causés par les activités humaines, et ce, notamment au nom du droit de propriété.

Récemment, un texte est venu profondément remanier les équilibres en présence. II s'agit de la charte de l'environnement ${ }^{13}$ proclamée par la loi constitutionnelle du 1er mars 2005 et qui élève au même niveau juridique que les libertés précitées, c'està-dire au niveau constitutionnel, de nouvelles libertés telles «le droit de vivre dans un environnement équilibré et respectueux de la santé» (article 1er) et de nouveaux principes tels le principe de prévention (articles 2 et 3), le principe de réparation (article 4), le principe de précaution (article 5), ou encore le principe de participation en vertu duquel toute personne a le droit de participer à l'élaboration des décisions publiques ayant une incidence sur l'environnement (article 7) pour les plus significatifs.

12. https://www.legifrance.gouv.fr/codes/article_Ic/LEGIARTI000043084969

13. https://www.legifrance.gouv.fr/contenu/menu/droit-national-en-vigueur/constitution/ charte-de-l-environnement 
En conclusion, on peut dire que les causes juridiques des changements globaux n'ont rien d'insurmontable. Ainsi, la souveraineté étatique recèle un potentiel de protection de l'environnement, tandis que les libertés fondamentales nouvellement proclamées dans la charte peuvent servir de contrepoids au potentiel destructeur du droit de propriété, de la liberté contractuelle ou de la liberté du commerce et de l'industrie. Bien que puissantes, ces causes sont donc tempérées par des outils en nombre croissant, outils qui peuvent, le cas échéant, être renforcés par la délibération collective, et dont il convient surtout de garantir la mise en œuvre, pour que le droit, d'une cause, devienne un remède aux changements globaux.

\section{6. Économie et environnement - J.Ch. Bureau et H. Levrel}

Plusieurs points d'entrée aident à comprendre les approches économiques traitant de l'environnement et plus particulièrement, des changements globaux auxquels nous sommes confrontés. Le premier est l'idée de "tragédie des biens communs» qui pose le débat sur le rôle de la propriété privée, mais aussi sur l'importance d'avoir des institutions collectives de gestion des ressources naturelles qui soient efficaces. Le deuxième point d'entrée est le constat d'inefficacité du marché comme unique outil de régulation, du fait de son incapacité à prendre en compte l'environnement, les prix ne traduisant pas véritablement la valeur sociale de certains biens. Les effets négatifs, mais aussi parfois positifs dans certains cas, de cette non prise en compte sont appelés externalités environnementales. Comme troisième point d'entrée, on peut ajouter le débat sur le niveau du taux d'actualisation à utiliser dans les décisions économiques, lequel reflète la préférence des agents pour le présent plutôt que pour le long terme.

\subsubsection{Propriété et biens communs}

Le cas historique des "enclosures» qui, au XVle siècle en Angleterre, ont amené à clôturer les champs en mettant fin à une tradition de libre accès des terres pour les éleveurs (Fig. 1.16), illustre les débats qui opposent encore différentes écoles de pensée travaillant sur la nature et l'environnement. Pour certains, les droits de propriété privée sont nécessaires pour pouvoir protéger l'environnement, comme pour trouver des solutions satisfaisantes autour de conflits environnementaux. Selon eux, le libre accès aux ressources ne pouvait être soutenable que lorsque la population était très faible et qu'il y avait peu de concurrence et d'interactions entre les groupes d'humains [17]. Mais aujourd'hui, le libre accès aux ressources se traduit par la surexploitation de ces dernières, principe connu sous le nom de «tragédie des biens communs».

L'une des explications de cette surexploitation est un mécanisme très simple de théorie des jeux, une branche des mathématiques très utilisée en économie ou en écologie: le «dilemme du prisonnier». Celui-ci montre qu'un comportement optimal 
et parfaitement rationnel du point de vue individuel, peut être totalement sousoptimal et totalement irrationnel d'un point de vue collectif. Une application est, par exemple, la surexploitation d'un stock de poissons en accès libre. S'il est collectivement rationnel de restreindre ses prises afin d'avoir un stock plus élevé qui permet à chacun une meilleure pêche, il est optimal pour un individu de pêcher le plus possible, car il sait que, sinon, les autres pêcheurs le feront.

Or, il est difficile de sortir de cet équilibre dit «non coopératif», car l'intérêt individuel à trahir une discipline commune est fort dès lors que des droits de propriété ne sont pas fixés. Ainsi, certains voient dans la propriété privée un moyen de mieux gérer l'environnement et d'accroître la prospérité économique en faisant appel à l'optimisation des intérêts individuels et à l'incitation à investir qui y est associée. Le cas historique des «enclosures » est même parfois vu comme un acte fondateur du capitalisme. Selon ses défenseurs, sans ces barrières permises par la propriété privée, les animaux de chacun pouvaient paître là où un autre avait tenté de planter, ce qui mettait fin à toute incitation à investir. Si l'on suit cette idée, pour garantir un usage socialement optimal des ressources naturelles, l'État devrait fixer des droits de propriété comme une base nécessaire pour que les individus puissent ensuite négocier dans un intérêt collectif, par exemple, grâce à un marché, qui permet d'échanger ces droits ${ }^{14}$. C'est l'idée derrière la régulation des émissions de gaz à effet de serre telle qu'elle est pratiquée en Europe - ou de la régulation des émissions de dioxyde de soufre aux États-Unis.

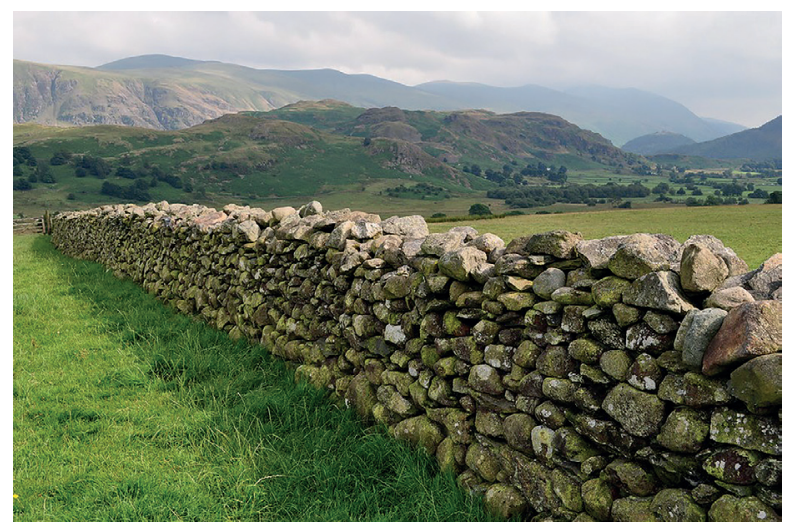

Figure 1.16: Image évocatrice des «enclosures» Photo @lstock.

14. Une illustration simple en est donnée par le confinement d'un fumeur et d'un non-fumeur dans une même pièce. L'un aime l'air pur et il lui est coûteux en bien-être de subir la fumée de l'autre. L'autre aime fumer et il lui est coûteux en bien-être d'y renoncer. Le premier accepterait un peu de fumée, mais devrait pour cela avoir des compensations, par exemple, financières. Le second accepterait de donner des compensations pour avoir le droit de fumer. Une négociation est alors possible, qui peut potentiellement être bénéfique pour les deux. Mais cette négociation n'aura lieu que si elle a pour point de départ une répartition du droit de propriété, indiquée par exemple, par un écriteau « compartiment fumeur» ou «compartiment non-fumeur ». C'est à cette condition que l'un des deux pourra entamer la négociation. 
À l'opposé, d'autres voient dans le cas historique des «enclosures» une prédation: le mouvement des enclosures a amené une classe de propriétaires à empêcher les autres d'accéder à des ressources auparavant communes et il s'en est suivi une forte pauvreté. Ils considèrent que la propriété privée n'est pas un élément déterminant de la bonne gestion des ressources. L'économiste Elinor Ostrom a donné des éléments empiriques qui montrent qu'une gestion collective soutenable d'une ressource commune était possible et même fréquente, dans un cadre non marchand. Certaines communautés parviennent à gérer des ressources de manière durable sans qu'il y ait de droits de propriété privée, et ce depuis parfois des millénaires [18]. Une partie de l'œuvre d'Ostrom a consisté à définir les conditions de succès de ces formes d'auto-organisation en y associant un certain nombre de critères: définition précise des limites de la communauté, accord sur des sanctions graduées dans la collectivité, etc. II faut souligner que l'accès aux ressources est organisé par des institutions communautaires spécifiques et que le fait de ne pas avoir de droits privés sur ces ressources n'implique cependant pas une situation d'accès libre.

\subsubsection{Les institutions}

S'il est un point où les deux conceptions se rejoignent, où convergent, par exemple, Elinor Ostrom et un autre prix Nobel d'économie, Ronald Coase, c'est l'importance d'avoir des institutions efficaces. En l'absence de sécurité juridique, de garantie des engagements pris, il est difficilement envisageable d'avoir un environnement qui bénéficie d'une protection efficace. Les pays où l'État est déliquescent ou excessivement corrompu sont ceux où une économie "extractive» s'est développée, où des rentes sont extraites au détriment du collectif, comme le montrent de nombreux cas concrets [19]. Dans ces pays, les ressources sont souvent surexploitées, et l'environnement est dégradé au détriment du plus grand nombre. Les pollutions chimiques, minières ou pétrolières en Afrique, ou le trafic de déchets toxiques y compris dans certaines régions européennes en sont l'illustration.

Mais même dans les États aux institutions plus efficaces, le processus de décision publique est soumis à des groupes de pression, et l'État fait des arbitrages en fonction de critères qui peuvent diverger de l'intérêt général. Une branche de l'économie, l'économie politique, décrit de nombreux mécanismes en ce sens. Elle explique comment certaines procédures de représentation politique telles que les modalités de vote peuvent favoriser l'organisation optimisée de catégories socio-professionnelles ou la formation de groupes de pression avec des «objectifs propres» pour aboutir à des lobbies qui pèsent dans la décision collective et à la capture des institutions publiques par des groupes d'intérêt. Finalement, ces mécanismes amènent souvent à ce que des arbitrages se fassent au détriment de l'environnement, souvent mal représenté par rapport à d'autres groupes d'intérêt. Les travaux de sociologues comme Bruno Latour sur la représentation de l'environnement dans le processus de décision expliquent "pourquoi nous navons rien fait devant la crise annoncée» et rejoignent sur ce plan des travaux en économie politique [20]. 


\subsubsection{Les défaillances de marché}

La théorie économique a montré que le marché non régulé va souvent amener à une dégradation de l'environnement. Des concepts sont centraux pour comprendre le besoin de régulation: celui d'externalité, celui de bien public et celui de bien commun. II y a externalité lorsque le comportement d'un agent agit sur un autre. Par exemple, si une entreprise pollue l'environnement, ceci peut nuire à une autre, ou à un consommateur, sans que cette entreprise ne subisse le coût de sa pollution. On parlera ici d'externalité négative. Un bien est public si sa consommation par un agent n'empêche pas celle par un autre agent - pensons à l'éclairage public - et de plus, s'il est difficile d'empêcher quelqu'un d'y avoir accès - imaginons un paysage, des phares en mer, l'air pur, etc. Un bien commun est un bien pour lequel il est impossible d'en empêcher l'accès et pour lequel, par contre, la consommation par un agent empêche celle d'un autre agent (exemple d'un stock de pêche auquel plusieurs pêcheurs ont accès).

Les biens publics et communs ne sont pas correctement valorisés par les systèmes de prix. Ainsi, celui qui produit du bien public ou du bien commun ne sera pas rémunéré à hauteur de son effort, car il ne pourra pas empêcher qu'une tierce partie s'accapare les bénéfices de ce produit, son accès n'étant pas restreint. On peut alors montrer aisément que le marché, où les prix véhiculent normalement un signal de rareté ou d'abondance des biens, ne permet pas d'atteindre un niveau socialement optimal de production. Spontanément, il y aura beaucoup de biens produits, qui sont polluants, et pas assez de biens publics. On parle de défaillances de marché.

\subsubsection{L'actualisation}

Dans le calcul économique, par exemple, la planification d'un projet, on valorise en général davantage un bénéfice présent qu'un bénéfice futur. Ainsi, un agent préfèrera en général un euro maintenant à un euro l'an prochain.

Le taux d'actualisation est le taux t pour lequel cet agent sera indifférent entre un euro immédiatement et $(1+\mathrm{t})$ euro dans un an. Si cette approche est rationnelle et aboutit à l'utilisation généralisée d'un taux t supérieur à zéro, lorsque l'on a des bénéfices ou des coûts à très long terme, elle aboutit, en fait, à peu les valoriser. Avec un taux t de $2 \%$, le bénéfice supplémentaire à 30 ans sera faible par rapport au bénéfice immédiat, de l'ordre de moitié environ ${ }^{15}$.

De plus, ce type de calcul, qui compare les recettes immédiates à des coûts futurs, amène à ignorer les irréversibilités, les changements de régimes ou autres «points de basculement». Ces deux mécanismes amènent souvent le calcul économique à

15. Pour une présentation détaillée des taux d'actualisation et des débats y afférant: http://temis. documentation.developpement-durable.gouv.fr/docs/Temis/0069/Temis-0069675/19163.pdf 
négliger les coûts à long terme, notamment les dégâts dus au changement climatique, et à ignorer le fait que des perturbations majeures peuvent changer radicalement la donne (modification de la circulation thermohaline, déplacement du Gulf Stream, etc.).

\subsubsection{Quelques illustrations concrètes}

Dans certains cas, la dégradation de l'environnement peut être assez directement liée à la tragédie des communs, et l'absence de droits de propriété est en cause. Ainsi, dans un des États où l'environnement est peut-être le plus dégradé au monde, Haïti, les origines juridiques de l'érosion des sols et de la déforestation sont évidentes ${ }^{16}$. L'absence de cadastre rend extrêmement difficile toute action de conservation des sols. La plantation d'un arbre sera dissuadée, car un autre le transformera en charbon de bois; chaque langouste sera pêchée au stade juvénile alors même que les entretiens montrent que les pêcheurs ont parfaitement conscience de l'irrationalité collective du geste. Des exemples montrent que la fixation de droits de propriété, par exemple, en restreignant l'accès à un stock de poisson ou en attribuant des contingents de pêche stricts, peut donner d'excellents résultats (stocks de morue en Norvège) alors qu'ailleurs la surexploitation semble avoir condamné les stocks à jamais (banc de Terre Neuve). C'est aussi la fixation de droits de propriété (quotas d'émission) qui permet au système européen de limiter les émissions de $\mathrm{CO}_{2}$ de certains secteurs industriels assujettis à ces droits, échangeables sur un marché sans que le plafond global soit dépassé.

Mais l'attribution, ou la capture par la force ou la corruption, de droits de propriétés sur des ressources communes a pu aussi déstructurer des équilibres ancestraux que des communautés avaient su gérer. La privatisation de terres au profit d'industries extractives ayant une vision à court terme a laissé des dommages considérables. Ainsi, au Sénégal, des sols en latérite sont devenus stériles après leur surexploitation par des cultures de rente pour l'arachide. En Haïti, l'exportation de toute la terre arable pour extraire de la bauxite a laissé à la population locale un milieu dégradé et stérile. En Mauritanie, on a assisté au pillage des ressources halieutiques suite aux concessions données à des flottes industrielles. Un autre exemple, celui du « dust bowl» aux États-Unis dans la première moitié du XXe siècle, illustre qu'une société peut, de manière consciente, détruire des millions d'hectares de terre par des pratiques visant des profits de court terme et mettant en œuvre la mécanisation et la surexploitation des sols [21]. Ces pratiques semblent se répéter dans de nombreux pays pour les ressources forestières et halieutiques (Fig. 1.17 et 1.18).

16. Le droit coutumier s'est substitué aux titres de propriétés établis au début du XIX' siècle. L'ensemble des terres est en indivision après des transferts non enregistrés entre générations, les transactions notariales étant trop coûteuses. La conséquence en est l'accès libre à chaque parcelle donnée par un grand nombre de descendants. 

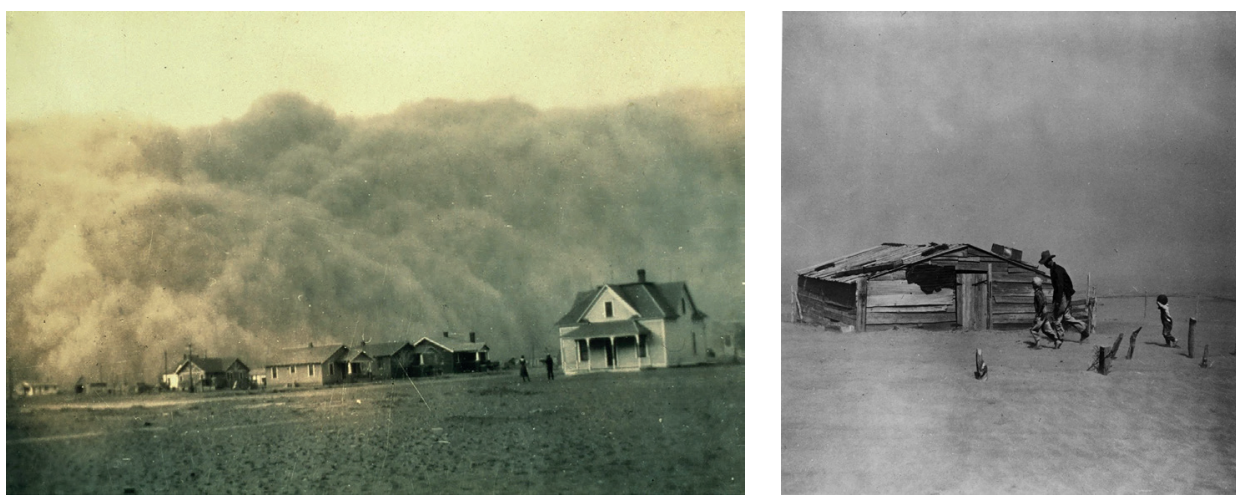

Figure 1.17: Illustrations de la dégradation des terres aux États-Unis dans le « dust bow/» au cours des années 1930. À gauche: l'arrivée de la tempête de poussière. Source: wikimedia.Commons. À droite: le paysage après le passage d'une tempête de poussière. Source: wikimedia.Commons.

Le fait que le coût des externalités négatives ne soit pas supporté par ceux qui les créent est un facteur puissant de dégradation des milieux: les «dead zones» eutrophisées dans le golfe du Mexique par les effluents agricoles du Missouri ou de l'lowa, à des milliers de kilomètres, sont directement liées à ce que les agriculteurs de ces États n'ont pas à payer pour les dommages faits aux pêcheurs de Louisiane, et a fortiori à la faune et à la flore. II en est de même pour les émissions polluantes dans l'air qui, en France, engendrent des coûts de santé faramineux et qui ne sont en rien supportés par les industriels responsables et seulement faiblement par les automobilistes. Enfin, le fait que la dégradation du capital naturel ne soit pas prise en compte dans la comptabilité ni des entreprises, ni des États, incite à sa dégradation si celle-ci est source de revenus. Les principaux indicateurs de prospérité économique, comme le produit intérieur brut (PIB), ne prennent pas en compte cette dégradation, ce qui permet difficilement au décideur politique de prendre en compte lui-même cette réalité ${ }^{17}$.

17. Le PIB est un indicateur qui mesure la création de richesses dans l'économie. ll est l'objet de critiques depuis plusieurs décennies, en particulier car il ne porte que sur des activités marchandes. Par exemple, une femme qui épouserait son jardinier salarié, lequel continuerait son activité hors de la sphère marchande, ferait baisser le PIB. Ce dernier ne tient pas compte des coûts non marchands. Ainsi, le coût de la pollution de l'air en France a été estimé jusqu'à 140 milliards d'euros annuels, mais seule une partie de ces coûts, qui font l'objet de transactions monétaires comme les coûts d'hospitalisation, se trouve comptabilisée, et elle l'est sous la forme de création de richesse par un secteur économique. Le PIB ne prend pas en compte le fait que certaines créations de richesse se font en épuisant un stock de capital naturel non valorisé, comme la production légumière en Andalousie qui accroît la richesse de l'Espagne, mais en épuisant une nappe phréatique non renouvelable. Les défenseurs du concept répondent néanmoins que le PIB, malgré ses défauts, est très corrélé aux indicateurs de bien-être indiscutables: mortalité infantile faible, espérance de vie élevée, alphabétisation, etc. et que les diverses tentatives pour lui trouver des alternatives comme celles de la Commission présidée par Joseph Stiglitz et proposée à l'initiative du président Nicolas Sarkozy ont abouti à des résultats décevants. 
Enfin, utiliser un taux d'actualisation strictement positif dans des questions impliquant le changement climatique ou l'érosion de la biodiversité amène à négliger l'intérêt des générations futures et pose dans ce cadre un grave problème éthique. C'est pourtant ce qui est fait dans la plupart des grands projets et les choix de politiques économiques. L'hypothèse implicite est de laisser aux générations futures le soin de trouver des solutions.

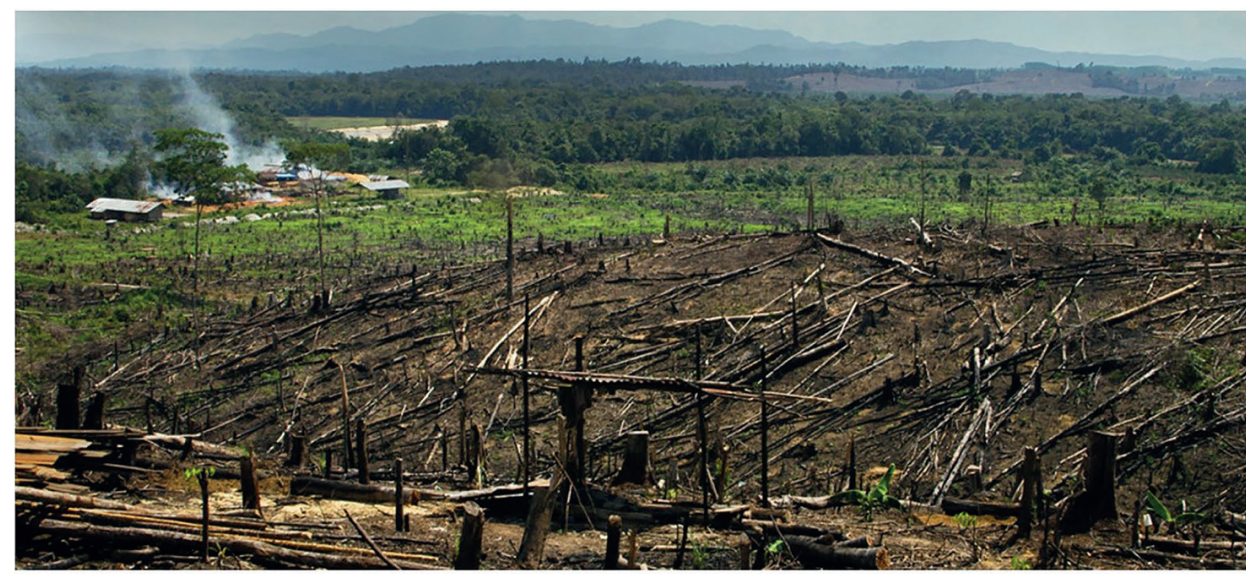

Figure 1.18: La déforestation pour la plantation de palmiers à huile à Bornéo (Indonésie). Source: UNEP.

\section{Références}

[1] G. Ramstein. 2015. Voyage à travers les climats de la Terre. Éd. Odile Jacob.

[2] J.-C. Duplessy, G. Ramstein. 2013. Paléoclimatologie. Enquête sur les climats anciens. CNRS Éditions.

[3] M. Maslin. 2016. Forty years of linking orbits to ice ages. Nature 450, 208-210.

[4] G. Ramstein, S. Huet. 2020. Le climat en 100 questions. Éd. Tallandier.

[5] J.-D. Vigne. 2012. Les débuts de l'élevage. Édition Le Pommier.

[6] G.-L.L. Buffon. 1764. Histoire générale et particulière. De la Nature. Première vue. Imprimerie royale.

[7] J. Cauvin. 2019. Naissance des divinités. Naissance de l'agriculture. CNRS Éditions.

[8] P. Descola. 2005. Par-delà nature et culture. Gallimard.

[9] J. Lecomte, F. Sarrazin. 2020. Repenser nos relations au vivant dans un contexte de changements globaux. Nos Futurs. Eds Aline Aurias, Roland Lehoucq, Daniel Suchet, Jerome Vincent. Éditions Actu SF.72.

[10] F. Sarrazin, J. Lecomte 2016. Evolution in the Anthropocene. Science 351 (6276), 922-923. 
[11] R. Passet. 1996. L'économique et le vivant (2 édition). Economica, Paris.

[12] G. Froger. 2006. Significations et ambiguïtés de la gouvernance dans le champ du développement durable. Mondes en développement (4), 11-28.

[13] L. Goldmann. 1963. Problèmes d'une sociologie du roman. Cahiers Internationaux de Sociologie 32 (Janvier-Juin 1962), 61-72.

[14] A. Lipietz. 1992. Towards a New Economic Order: Post-Fordism, Ecology and Democracy. Oxford University Press, London, New York.

[15] Conférence des Nations Unies sur le développement et l'environnement. Déclaration de Rio sur l'environnement et le développement. New-York: Nations Unies (1992).

[16] J.-C. Lasserre. 1995. «Des fleuves et des hommes». Ecodécision, 22-26.

[17] J. Diamond. 1999. "The worst mistake in the history of the human race". Discover magazine. https://www.discovermagazine.com/planet-earth/the-worst-mistake-in-thehistory-of-the-human-race. Voir aussi : Y. Noah Hariri. 2015. "Sapiens, une brève histoire de l'humanité». Albin Michel. Ces deux auteurs décrivent une situation où la faible densité de population permettait l'utilisation des ressources naturelles sans avoir recours à l'agriculture et sans réelle concurrence entre les individus face aux ressources disponibles.

[18] E. Ostrom. 2010. "La Gouvernance des biens communs: Pour une nouvelle approche des ressources naturelles». De Boeck, 2010, Édition anglaise 2003.

[19] D. Acemoglu, J. Robinson. 2015. «Prospérité, puissance et pauvreté: Pourquoi certains pays réussissent mieux que d'autres». Éditeur Markus Haller.

[20] B. Latour. 2015. «Face à Gaïa». Édition La Découverte.

[21] D. Worster. 2004. Dust Bowl. The Southern Plains in the 1930s. Oxford University Press, New York, NY, USA. 


\section{Chapitre 2}

\section{Énergies et pollutions}

Dans ce chapitre, nous discuterons du rôle de l'énergie dans nos sociétés pour aborder ensuite les pollutions qu'engendrent nos modes de vie et de consommation énergétique. L'énergie est un facteur essentiel du développement économique des sociétés. La domination actuelle du charbon, du pétrole et du gaz comme ressources d'énergie contribue de façon majeure au réchauffement climatique, comme nous l'analyserons plus en détail dans la partie III de cet ouvrage. Mais, l'exploitation de ces ressources fossiles, donc non renouvelables, participent aussi d'un développement économique qui se fait aux dépens de l'environnement, et donc d'autres ressources essentielles que sont l'air, l'eau et les sols. Nous verrons à travers ce présent chapitre que la qualité de l'air, l'accès à l'eau pour tous et la préservation des sols pour la sécurité alimentaire sont les conditions d'un développement durable pour l'humanité, c'est-à-dire d'un développement «qui permette de satisfaire les besoins des générations présentes sans compromettre la capacité des générations futures à répondre aux leurs».

\subsection{Production et consommation d'énergie : la domination des énergies fossiles - C. Even et G. Roux}

\subsubsection{L'énergie en physique}

L'énergie est une grandeur physique, qui a été introduite par les physiciens pour pouvoir effectuer des bilans lors de l'évolution des systèmes [1]. À la différence d'une position, d'une vitesse ou d'une température, c'est un concept abstrait que l'on peut voir comme une unité comptable. Imaginons qu'on tienne une balle dans une main puis qu'on la lâche, elle va tomber à cause de la gravité, et elle prend de la vitesse au cours de sa chute. 
Lorsqu'elle est dans la main, la balle est déjà attirée par la Terre, par gravitation: on dit qu'elle possède une certaine énergie potentielle de gravitation. Quand elle tombe, cette énergie potentielle diminue parce qu'elle se rapproche de la Terre. En même temps, elle prend de la vitesse et on associe une énergie dite "cinétique» à cette vitesse; et cette énergie cinétique augmente au cours de la chute.

On a ainsi une transformation d'énergie potentielle de gravitation en une énergie cinétique associée à la vitesse, transformation au cours de laquelle l'énergie totale (énergie potentielle + énergie cinétique) se conserve. C'est la conservation de l'énergie. Retenons l'idée suivante: «l'énergie est une unité comptable qui intervient dans toute transformation d'un système, c'est-à-dire dans tout changement d'état de ce système».

L'énergie possède sa propre unité, le Joule, mais pour des raisons pratiques, nous allons utiliser le kilowattheure (kWh) qui est l'unité utile pour les questions de société. Cette unité est associée à la puissance des machines, qui s'exprime en watts. La puissance est l'énergie par unité de temps, une forme de débit d'énergie. Un kWh correspond à la consommation d'énergie d'une machine de 1 kW, c'est-à-dire $1000 \mathrm{~W}$, pendant $1 \mathrm{~h}$. Les factures d'électricité ou de gaz contiennent une estimation du nombre de kWh consommés.

L'énergie se retrouve sous de nombreuses formes. Citons les plus courantes pour notre vie de tous les jours: énergie cinétique associée à une vitesse, énergie potentielle de gravitation, travail mécanique associé à la pression, chaleur, énergie électrique, énergie chimique, énergie associée au rayonnement...

\subsubsection{Chaîne de production de l'énergie: l'exemple de la voiture}

Dès lors que l'on parle de machines et du rôle de l'énergie dans la société, nous prenons un point de vue anthropocentrique qui va attribuer une certaine valeur subjective aux mots employés et aux définitions. On appelle source d'énergie un système physico-chimique à l'origine d'une chaîne de transformations qui, au final, satisfait un besoin humain.

Afin d'illustrer cela, décomposons toutes les formes d'énergie et les transformations qui interviennent dans l'utilisation d'une voiture à essence. Le cœur du système est le moteur, au centre sur la figure 2.1. Le moteur comporte plusieurs cylindres où est injecté un gaz, mélange d'air et de carburant, et des pistons, qui viennent comprimer le gaz dans les cylindres. Dans chaque cylindre, à tour de rôle, une bougie d'allumage crée une étincelle qui entraîne la combustion violente du gaz, son explosion. La pression devient alors si forte qu'elle repousse le piston et l'entraîne en mouvement. Ensuite, toute une mécanique permet de transformer le mouvement rectiligne du piston en mouvement de rotation de l'arbre moteur, ce qui fait tourner les roues de la voiture et la fait avancer. 


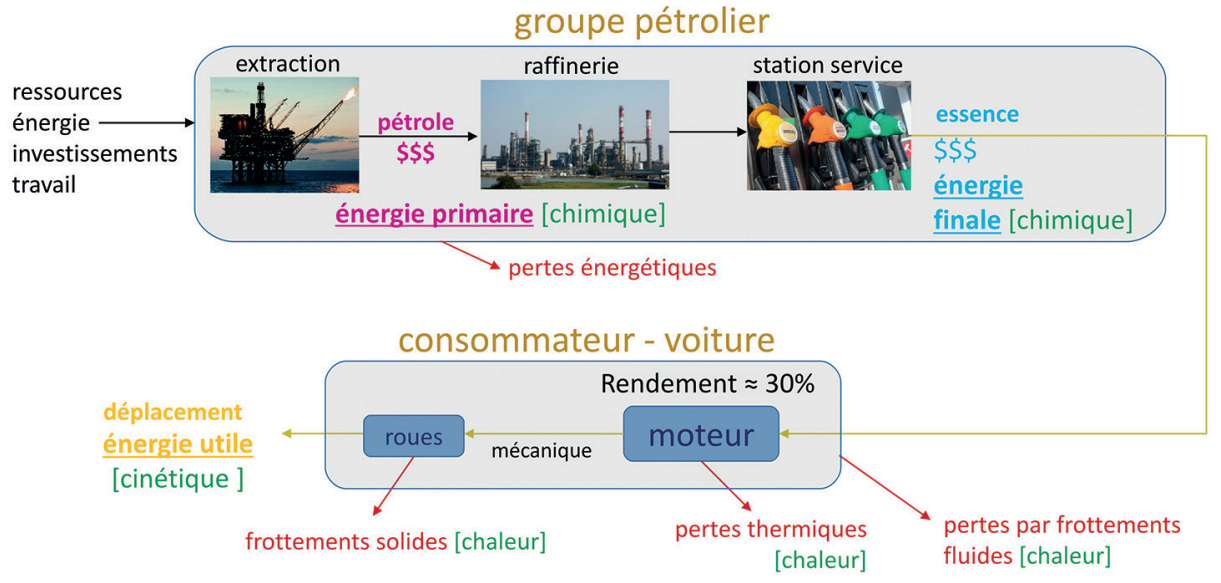

Figure 2.1: Formes et transformations d'énergie qui interviennent dans l'utilisation d'une voiture. Source: Catherine Even \& Guillaume Roux.

Si on résume, le moteur agit donc comme un convertisseur d'énergie qui transforme l'énergie chimique de l'essence en énergie cinétique de la voiture, associée à son déplacement. Déplacer la voiture, c'était bien le but, donc on appelle cette énergie cinétique «énergie utile», en jaune sur le dessin. Mais l'énergie utile n'est jamais mesurée en pratique, c'est plus une notion qui intéresse l'ingénieur ou le physicien. Pour le consommateur, ce qui compte c'est l'énergie qu'il paie pour ce service, c'est-à-dire l'essence: on parle d'énergie finale, en bleu sur le dessin.

Si l'on revient sur la notion de convertisseur d'énergie de manière générale, toute l'énergie introduite dans un convertisseur ne se transforme pas en énergie utile. Le rapport entre les deux est appelé le rendement. Pour un moteur de voiture à essence, le rendement est typiquement de $30 \%$ dans la conversion d'énergie chimique en énergie mécanique sur l'arbre moteur. L'énergie «perdue» pour l'utilisateur se retrouve sous forme de chaleur: le moteur chauffe, les gaz d'échappement sont chauds, les frottements mécaniques échauffent les pièces... À chaque étape, il y a des pertes.

Remontons maintenant la chaîne de production de l'énergie, en haut sur le dessin. L'essence ne se trouve pas dans la nature. Elle est issue de l'extraction du pétrole qui est ensuite raffiné et dont l'essence n'est qu'un des sous-produits distribués par un réseau de stations-service. Du point de vue économique, le pétrole est une matière première qui contient de l'énergie sous forme chimique: cette énergie contenue dans le pétrole, qui se trouve en amont de la chaîne de production d'énergie est appelée énergie primaire, en violet sur le dessin.

En résumé, si l'énergie utile caractérise le service rendu, ce sont les énergies primaire et finale qui apparaissent dans les statistiques. En effet, elles font l'objet de transactions économiques et donc sont systématiquement comptabilisées. 


\subsubsection{Chaîne de production de l'énergie : généralisation}

Les statistiques de l'énergie sont basées sur les énergies primaires qui sont bien répertoriées. Ces énergies primaires, ce sont les sources d'énergies présentes naturellement et gratuitement dans l'environnement, en vert sur la figure 2.2. Elles se divisent entre énergies de type stock, non renouvelables à notre échelle de temps humaine, et énergies de type flux, renouvelables. Les énergies de stock sont les fossiles - charbon, pétrole et gaz, on en reparlera un peu plus loin - et les fissiles, c'est-à-dire le nucléaire - l'uranium, notamment. D'autre part, les énergies de flux renouvelables sont l'énergie solaire et ses sous-produits - hydraulique, vent, houle, biomasse -, l'énergie géothermique et l'énergie des marées.

Notons au passage que la mise en œuvre des énergies renouvelables met en jeu des dispositifs qui utilisent des matériaux non renouvelables: panneaux solaires, cuivre, aluminium, aimants, matières plastiques, béton, etc.

La distinction flux / stock se retrouve également dans les vecteurs d'énergie, qui sont des formes intermédiaires d'énergie assurant un transport efficace. L'électricité en est un exemple: elle est à la fois un flux, à la prise de courant, et un stock, sous forme électrochimique dans les batteries.
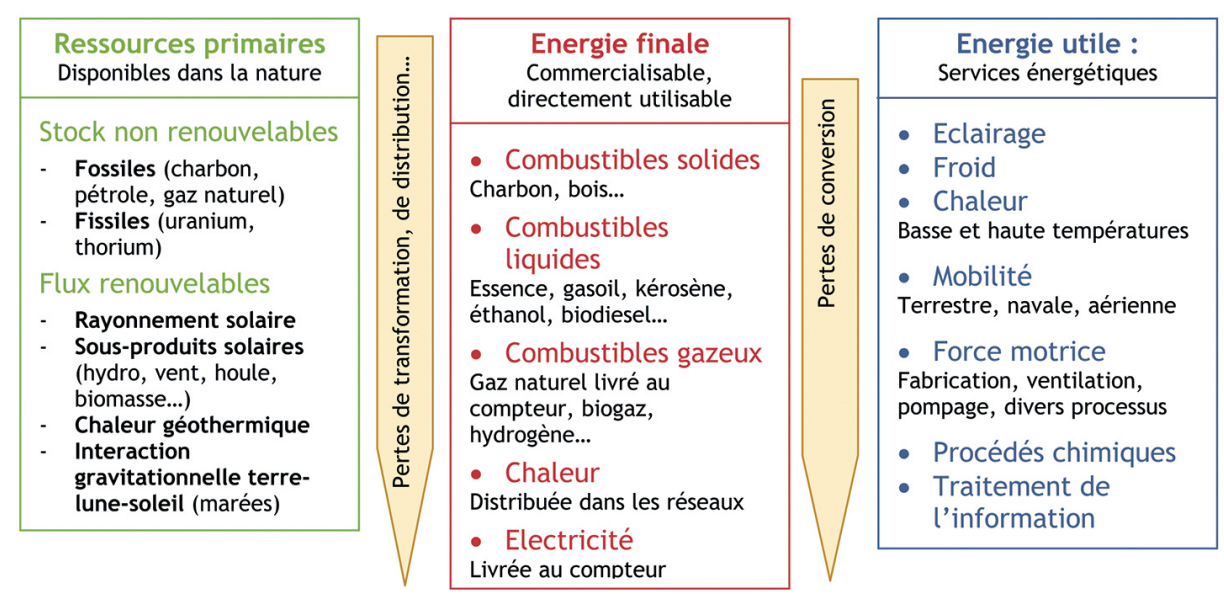

Figure 2.2: Chaîne de production d'énergie et classification des différents types d'énergie: des ressources primaires à l'énergie utile au consommateur ${ }^{18}$.

Quand on passe d'une source d'énergie primaire à l'énergie finale associée, en rouge sur la figure 2.2, il y a des pertes associées à la transformation, la distribution, etc. Si l'on reprend l'exemple précédent de la voiture, il faut transporter le pétrole

18. https://eduscol.education.fr/sti/si-ens-paris-saclay/ressources_pedagogiques/conceptschiffres-de-lenergie-comment-definir-le-rendement-dun-convertisseur-denergie 
brut depuis les puits d'extraction, puis le raffiner en essence et distribuer l'essence pour que le consommateur puisse remplir le réservoir de sa voiture.

Enfin, le passage de l'énergie finale à l'énergie utile - en bleu sur la figure 2.2 entraîne d'autres pertes lors de la conversion. C'est la notion de rendement, qu'on a déjà vue.

Insistons sur le fait que toute chaîne de production énergétique nécessite, pour exister, d'avoir une infrastructure, des investissements financiers, mais aussi de l'énergie pour en assurer l'extraction, les transformations et le transport! Ces activités ont des impacts sociétaux et environnementaux non négligeables: emplois dangereux, pollution et rejets de gaz à effet de serre, destruction de biodiversité, occupation des sols, pression sur les réserves d'eau...

\section{Intermède}

https://www.youtube. com/watch?v=S4O5voOCqAQ

\subsubsection{Consommation d'énergie : échelle individuelle}

Nous allons maintenant parler de consommation d'énergie sous différentes formes correspondant à différents usages. Nous partirons d'ordres de grandeur par personne et par jour avant de discuter la consommation à l'échelle mondiale.

Dans nos sociétés développées, toutes les fonctions essentielles comme s'alimenter, se déplacer, se chauffer, produire et travailler sont assurées à l'aide de machines nourries à l'énergie. Cela a été rendu possible en puisant dans les stocks abondants de matières fossiles - le charbon, le pétrole et le gaz - qui alimentent la grande majorité des machines, y compris les machines électriques comme nous le verrons ci-après.

En considérant l'homme comme une machine, l'alimentation lui apporte environ $3 \mathrm{kWh}$ par jour et nous pouvons fournir typiquement $100 \mathrm{~W}$ de puissance mécanique; l'essentiel étant dissipé en chaleur, ce qui permet de compenser nos pertes et de maintenir la température de notre corps à $37^{\circ} \mathrm{C}$. Une puissance de $100 \mathrm{~W}$ nous permet de pédaler sans trop forcer. C'est une puissance significativement inférieure à celle de la plupart des machines qui nous entourent. Sur une journée et après $10 \mathrm{~h}$ de travail harassant, un manœuvre fournit environ un travail mécanique de l'ordre de $1 \mathrm{kWh}$.

Les machines nous permettent de faire plus et plus vite, mais aussi des choses que nous ne pourrions faire sans leur puissance, comme faire décoller un avion. Comme l'énergie n'est pas chère, nos usages favorisent la rapidité au détriment de la sobriété: les batteurs électriques de $300 \mathrm{~W}$ sont beaucoup plus puissants que nos avant-bras. 
Reprenons l'exemple de la voiture. Un déplacement typique en région parisienne fait $12 \mathrm{~km}$ aller-retour. Vous pouvez l'effectuer en $1 \mathrm{~h}$ de vélo avec vos $100 \mathrm{~W}$ de jambes, en consommant donc en tout 0,1 kWh. Vous pouvez l'effectuer en 30 min de voiture, en consommant $1 \mathrm{~L}$ d'essence. Ce litre fournit environ $10 \mathrm{kWh}$ d'énergie finale. C'est donc environ 100 fois plus d'énergie pour diviser par seulement deux le temps de déplacement (Tab. 2.1).

\begin{tabular}{|c|c|c|}
\hline & vélo & voiture \\
\hline durée du trajet & 1 heure & 1230 mins \\
\hline $\begin{array}{l}\text { énergie } \\
\text { consommée }\end{array}$ & $0.1 \mathrm{kWh}$ & $\begin{array}{l}1 \mathrm{~L} \text { essence } \\
\approx 100 \mathrm{kWh}\end{array}$ \\
\hline $\begin{array}{l}\text { puissance } \\
\text { moyenne }\end{array}$ & $100 \mathrm{~W}$ & $20 \mathrm{~kW}$ \\
\hline $\begin{array}{l}\text { puissance } \\
\text { maximale }\end{array}$ & $350 \mathrm{~W}$ & $70 \mathrm{~kW}$ \\
\hline coût énergie & 0 & $1,5 €$ \\
\hline
\end{tabular}

Tableau 2.1: Comparaison du vélo et de la voiture en termes de consommation d'énergie sur un trajet typique de $12 \mathrm{kms}$ en milieu urbain. Source : Guillaume Roux.

Comme l'indique le tableau 2.2 (à gauche), notre consommation typique journalière d'énergie est l'addition de contributions très variées dont on donne ici quelques exemples. Dans l'addition finale, il est important de noter qu'il y a une part de consommation personnelle, mais aussi une part collective. Au total, la consommation moyenne par jour et par habitant dans le monde est de l'ordre de 40 kWh (Tab. 2.2, à droite). Cependant, on constate de grandes disparités selon les modes de vie. Dans tous les cas, l'électricité représente une fraction importante mais non dominante de la consommation d'énergie finale.

\section{Exemples de consommations journalières typiques d'énergie}

\begin{tabular}{|lc|c|}
\hline Forme d'énergie & $\begin{array}{c}\text { Conso/production } \\
\text { journalière en kWh }\end{array}$ & $\begin{array}{c}\text { Puissance } \\
\text { typique en kW }\end{array}$ \\
\hline grille pain & 0.08 & 1 \\
\hline frigo & 0.13 & 0.1 \\
\hline ordinateur portable & 1 & 0.1 \\
\hline eau chaude & 2.2 & 2 \\
\hline chauffage & 5 & 10 \\
\hline voiture & 10 & 100 \\
\hline
\end{tabular}

\begin{tabular}{lrrr}
\multicolumn{4}{c}{ Énergie moyenne consommée } \\
par jour et par habitant en kWh
\end{tabular}

Tableaux 2.2: Ordres de grandeur de notre consommation quotidienne d'énergie (pour l'estimation de ces chiffres ${ }^{19}$ ) et consommation moyenne d'énergie par personne et par jour selon le type d'énergie. Source: Guillaume Roux. 


\subsubsection{Consommation d'énergie : échelle mondiale}

Si l'on souhaite satisfaire les besoins énergétiques de tous, le simple facteur démographique va déterminer la taille des moyens de production collectifs. Leur choix est donc un enjeu politique et stratégique majeur qui doit se planifier. Prenons l'exemple de la production d'électricité. Elle est principalement assurée par les centrales thermiques qui convertissent de la chaleur issue d'un combustible (charbon, gaz, fioul, uranium, déchets, ou biomasse) en électricité à l'aide d'une turbine à vapeur (Fig. 2.3). En France, la consommation d'électricité par habitant et par jour est typiquement de $20 \mathrm{kWh}$ (Tab. 2.2, à droite), soit $20 \mathrm{kWh} / 24 \mathrm{~h} \approx 1 \mathrm{~kW}$ de puissance moyenne, ce qui correspond sensiblement à un grille-pain allumé en permanence. Pour 70 millions de Français, il faut donc disposer d'au moins 70 GW de centrales électriques, ce qui est proche de la puissance fournie par notre parc de réacteurs nucléaires. En réalité, le double de puissance de production est installé pour pallier les pertes d'acheminement et l'intermittence de la consommation et de la production.

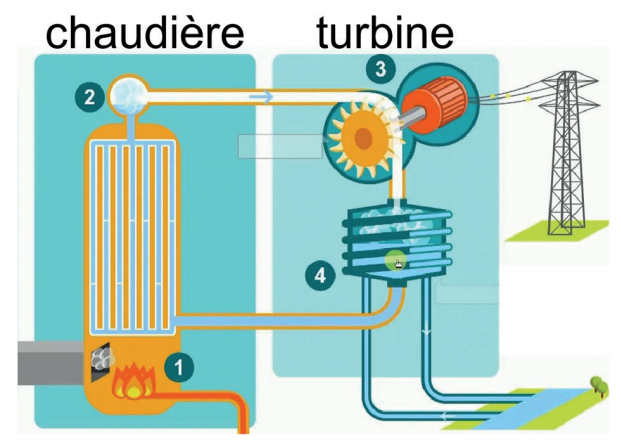

\section{consommation moyenne d'électricité $1 \mathrm{~kW} \times \mathbf{7 0}$ millions de français $=\mathbf{7 0} \mathbf{~ G W}$}

Figure 2.3: Production d'électricité par une centrale thermique et puissance électrique moyenne typiquement consommée en France (figure adaptée ${ }^{20}$ ). La puissance nucléaire installée est de $63 \mathrm{GW}$, la puissance totale installée est de 133 GW. Source : Guillaume Roux.

Discutons maintenant l'évolution historique de la consommation mondiale d'énergie. Comme on le voit sur la figure 2.4, depuis 150 ans, la contribution de la biomasse dans la consommation d'énergie par habitant diminue au profit de la contribution des énergies fossiles: d'abord le charbon, puis le pétrole et enfin le gaz naturel. Leurs utilisations se sont empilées sans se substituer. Finalement, la consommation d'énergie primaire par personne a été multipliée par presque 3,5.

20. Figure adaptée d'une figure du site web: https://www.edf.fr/groupe-edf/espaces-dedies/jeunesenseignants/tout-sur-l-energie/produire-de-l-electricite (dans sa version de 2017). Voir aussi la vidéo https://www.youtube.com/watch?v=bnF8q94T9d8 


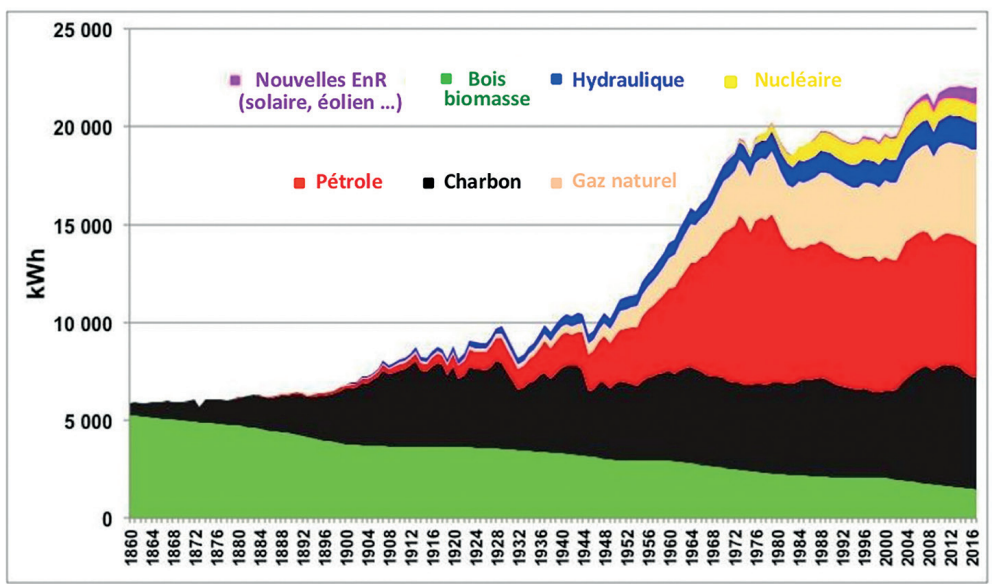

Figure 2.4: Évolution de la consommation mondiale d'énergie primaire par habitant et par source d'énergie depuis 150 ans $^{21}$. Noter l'ordre de grandeur $22000 \mathrm{kWh} / a n \approx 60 \mathrm{kWh} /$ jour. Source: Jean-Marc Jancovici.

En prenant en compte la démographie, l'énergie totale suit une augmentation très rapide (Fig. 2.5). Les chiffres moyens sont évidemment à relativiser en raison de la grande disparité, à la fois historique et géographique, de l'utilisation de l'énergie. Cette augmentation a été principalement portée jusqu'au début des années 2000 par les pays développés, Amérique du Nord, Europe, Russie et Japon. La Chine et les pays émergents tirent maintenant la production vers le haut.

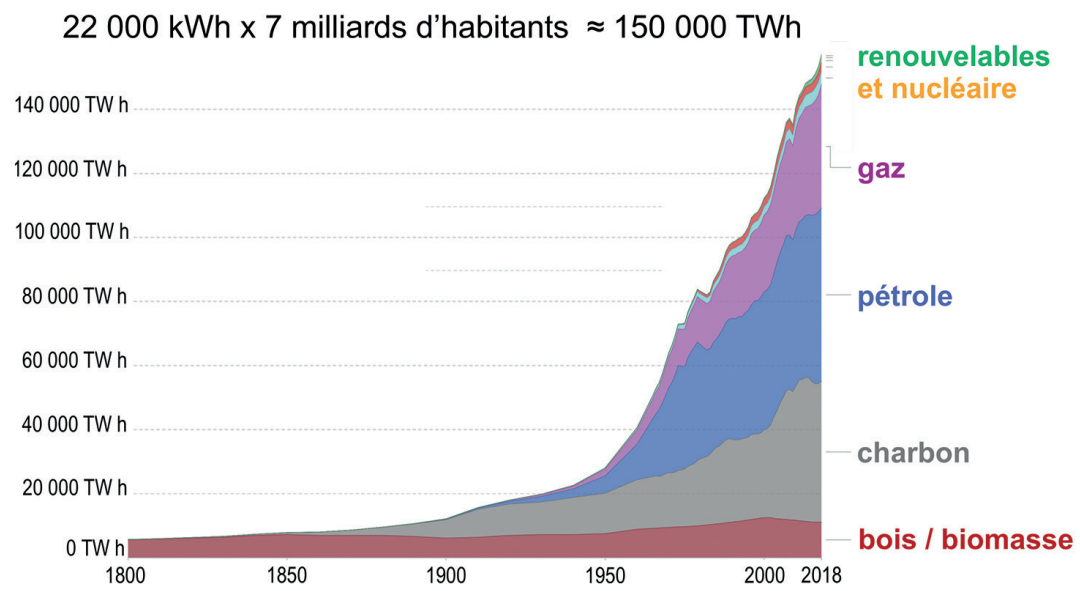

Figure 2.5: Évolution globale de la consommation d'énergie primaire par source d'énergie dans le monde. Source: Our World in data, dossier sur l'énergie: https://ourworldindata.org/energy ${ }^{22}$.

21. https://jancovici.com/transition-energetique/l-energie-et-nous/lenergie-de-quoi-sagit-ilexactement/

22. Our World in data, dossier sur l'énergie : https://ourworldindata.org/energy 


\subsubsection{Domination des énergies fossiles}

Nous allons maintenant faire un focus sur les ressources fossiles: le charbon, le pétrole et le gaz. Toutes trois proviennent de la lente fossilisation d'organismes vivants. Celle-ci produit des matières riches en carbone que l'on retrouve enfouies dans des couches géologiques après un processus qui s'est étendu sur des dizaines à des centaines de millions d'années.

Du point de vue de son utilisation, le charbon sert surtout à la métallurgie et à la production d'électricité dans les centrales thermiques. Le pétrole sert surtout aux transports. L'utilisation du gaz se partage à parts presque égales entre production électrique, usage domestique (chauffage, cuisson) et usage industriel.

Dans tous les cas, l'exploitation majoritaire des matières fossiles consiste à les brûler. Comme elles contiennent toutes trois du carbone, la réaction de combustion peut s'écrire de manière très simplifiée:

$$
\mathrm{C}+\mathrm{O}_{2} \rightarrow \mathrm{CO}_{2}+\text { énergie }
$$

L'intérêt de cette réaction est qu'elle dégage une grande quantité d'énergie. Si on reprend l'exemple de la voiture, avec un plein d'essence, soit 50 litres d'essence environ, qui se fait en quelques minutes et coûte de l'ordre de 70 euros, on a de quoi déplacer une voiture sur $1000 \mathrm{~km}$ à $100 \mathrm{~km} / \mathrm{h}$ environ.

Les énergies fossiles ont donc de grands atouts. Elles subviennent à la majorité des besoins, depuis l'échelle individuelle jusqu'à l'échelle industrielle, elles sont disponibles et peu chères, stockables et concentrées, et leur utilisation est pilotable et optimisée. Pas étonnant qu'elles représentent $80 \%$ de l'énergie primaire consommée dans le monde!

Le revers de la médaille est que la réaction chimique de combustion des matières fossiles conduit à la formation de $\mathrm{CO}_{2}$. Par exemple, si un particulier fait $15000 \mathrm{~km}$ en voiture par an, il consomme environ 20 pleins de réservoir et émet 2 tonnes de $\mathrm{CO}_{2}$. Cette quantité représente environ le quart du $\mathrm{CO}_{2}$ émis en moyenne par un français sur une année. Si l'on considère l'ensemble de nos activités, les émissions de $\mathrm{CO}_{2}$ dues à la combustion de matières fossiles représentent $95 \%$ du total de nos émissions.

Nous verrons plus précisément dans la partie III de cet ouvrage que nos émissions de $\mathrm{CO}_{2}$ sont largement responsables du réchauffement climatique actuel. Comme nos émissions de $\mathrm{CO}_{2}$ sont largement dues aux énergies fossiles, on pourra alors en conclure que l'utilisation massive de ces énergies est une des causes principales du changement climatique. 


\subsection{7. Énergie et économie}

L'accès à des sources d'énergie abondantes nous permet d'extraire, de transformer et de déplacer facilement la matière. L'énergie est donc déterminante pour les productions agricoles et industrielles. De même, le transport mondialisé de matières premières et de biens de consommation est sous perfusion du pétrole. Enfin, n'oublions pas que le secteur des services, le commerce, le tourisme, les services publics, etc., repose sur la mobilité des personnes et l'usage intensif du chauffage, de l'électricité et de la construction, ce qu'on appelle le secteur résidentiel. Même dans les pays riches, qui ont une économie fondée sur les services, on observe de très forts besoins énergétiques. Ce n'est donc pas qu'une affaire de mode de production, mais bien de mode de vie.

Notre production de richesses et donc nos revenus, mesurés par le PIB (produit intérieur brut), sont dépendants de l'énergie, et notamment du pétrole. Cela se manifeste particulièrement lors des crises économiques. Comme le montre la figure 2.6, on observe une bonne corrélation entre le taux de croissance du PIB et celui de la consommation du pétrole. D'un côté, les chocs pétroliers ont conduit à des récessions, et de l'autre, les crises financières, comme en 2008, ou sanitaires, comme en 2020, ont conduit à une diminution de la consommation de pétrole. Au final, on parle d'intensité énergétique de l'économie pour caractériser cette dépendance de l'économie envers l'énergie. À l'échelle mondiale, il faut typiquement 1,5 kWh pour produire 1 dollar de richesse.

Le fait que la croissance économique et ses bienfaits sociétaux reposent jusqu'à présent sur l'exploitation des énergies fossiles pose deux problèmes: le premier est celui de leur épuisement. Cela concerne particulièrement le pétrole qui est aussi soumis à des enjeux financiers, géopolitiques et sanitaires très forts. Le second problème est la contrainte climatique qui impose d'utiliser de l'énergie décarbonée, comme les énergies renouvelables ou l'énergie nucléaire. Ce sont là les enjeux techniques de la transition énergétique.

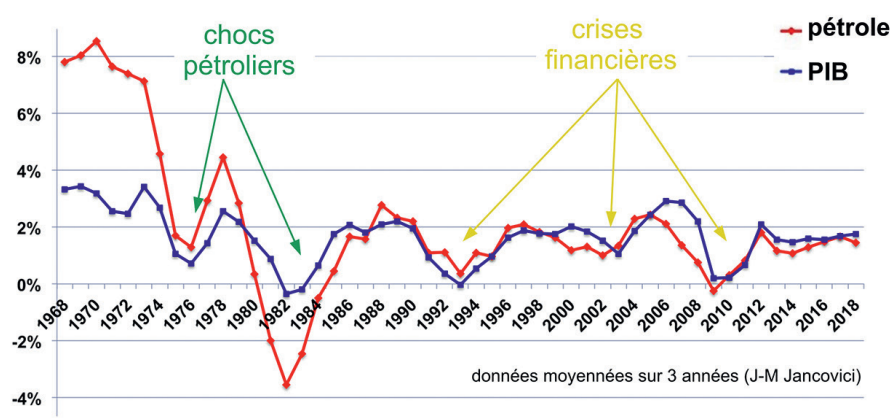

Intensité énergétique de l'économie : on utilise 1,5 kWh pour produire 1 de richesses

Figure 2.6: Taux de croissance du PIB mondial et de la consommation mondiale de pétrole. D'après Jean-Marc Jancovici 


\subsection{Le pétrole, enjeux économiques et environnementaux - F. Lantz}

\subsubsection{Le pétrole, ses usages et ses enjeux}

Le pétrole est une ressource fossile, liquide, provenant de dépôts sédimentaires constitués principalement de carbone et d'hydrogène, d'où le nom d'hydrocarbure. Il est utilisé, après transformation en un ensemble de produits pour un usage énergétique comme carburant ou combustible (85\%) ou pour un usage non énergétique (bases pétrochimiques transformées en plastiques, bitumes, lubrifiants, etc.).

Le pétrole a joué un rôle majeur dans le développement des sociétés industrialisées, notamment pour son utilisation comme carburant dans les moteurs. La progression de la consommation mondiale de pétrole est spectaculaire tout au long du XXe siècle et dépasse 4500 millions de tonnes par an en 2018 [2].

Le pétrole a progressé en termes de quantités utilisées, mais sa part dans l'approvisionnement mondial d'énergie s'est réduite pour représenter un tiers de celui-ci après avoir culminé à $48 \%$ de l'approvisionnement mondial d'énergie en 1973.

À cette époque de rareté de la ressource, la concentration des réserves est principalement dans les pays de l'Organisation des Pays Producteurs de pétrole (OPEP) et la demande se situe dans les pays industrialisés. Des tensions sur les marchés et des tensions géopolitiques vont apparaître. La substitution du pétrole va s'amorcer dans ses usages fixes tels que le chauffage ou la production d'électricité, et sa demande va venir pour l'essentiel du secteur des transports. Ce secteur représente $66 \%$ du pétrole consommé sachant que près de $96 \%$ de l'énergie utilisée dans les transports provient de produits pétroliers.

Il faut noter qu'on parle de pétrole en tonne (en masse) mais aussi en litre ou en baril (en volume). Au dix-neuvième siècle, le pétrole a été transporté des champs de pétrole vers les raffineries, où il était transformé en produits pétroliers, grâce à des barils qui avaient auparavant servi au transport de boisson. Le baril correspond à une mesure exacte en pouce cube et fait... 159 litres. Le baril reste aujourd'hui la mesure utilisée dans les transactions de pétrole. Une tonne de pétrole correspond approximativement à 7,34 barils.

Nous aborderons plusieurs questions majeures concernant cette ressource au travers de sa production, sa transformation et de son utilisation dans les produits pétroliers. Nous aborderons ensuite le problème des réserves et de leur épuisement. Pour finir, nous aborderons la question des ressources non conventionnelles (huile de schiste) et des défis environnementaux liés à l'usage du pétrole. 


\subsubsection{La production de pétrole}

La production mondiale de pétrole, qui a presque doublé entre 1970 et 2018 $(+90 \%)$, est réalisée dans quelques régions du monde avec trois grands acteurs, les États-Unis d'Amérique (16\% de la production mondiale en 2018), l'Arabie Saoudite (13\%) et la Fédération de Russie (12\%) (Fig. 2.7). La Communauté des États Indépendants (CIS) représentée sur le graphique inclut les anciens pays limitrophes de la Russie. La part des pays de l'OPEP dans la production a fortement augmenté dans les années 1970 (51\% en 1974). À la suite des crises pétrolières de cette période, la production en mer (off-shore) est développée en mer du Nord, dans le Golfe de Guinée et dans le golfe du Mexique, réduisant le poids de l'OPEP qui garde néanmoins plus de $40 \%$ de la production mondiale.

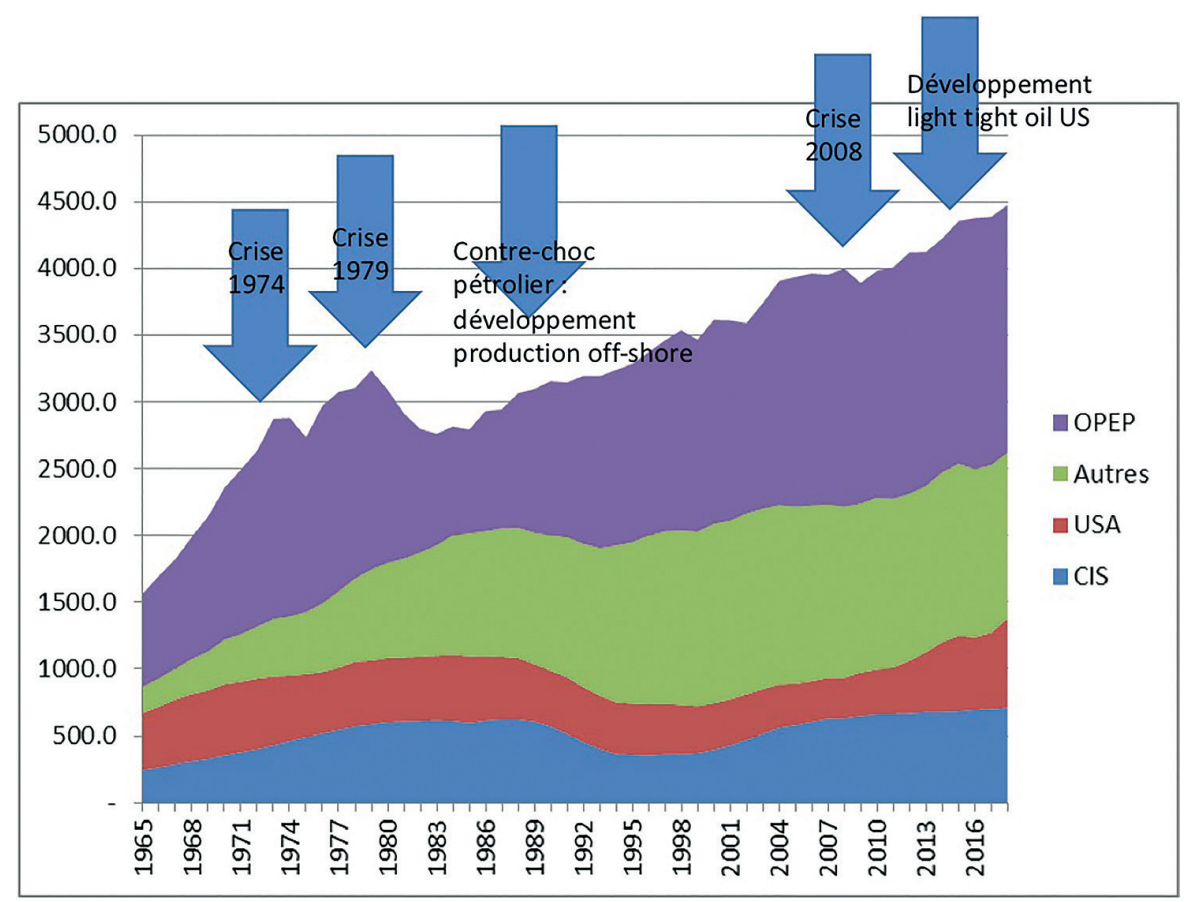

Figure 2.7: Évolution de la production mondiale de pétrole brut, mesurée en millions de tonnes. Source: BP Statistical Review of the World Energy (2020), 68 p. www.bp.com/en/global/corporate/ energy-economics/statistical-review-of-world-energy.html.

Les pétroles bruts sont de qualité très différente suivant les champs pétrolifères. Les principales caractéristiques qui permettent d'apprécier celles-ci sont la densité (degré API), sa teneur en soufre et ses rendements en essence et distillat dans le processus de raffinage. Un pétrole léger et peu soufré comme le brut algérien est donc mieux valorisé que le pétrole extra-lourd vénézuélien. 


\subsubsection{Les réserves de pétrole}

Les ressources en hydrocarbures sont des ressources épuisables dont la quantité disponible se réduit au fur et à mesure qu'elles sont consommées. Ainsi, avant que ne soient posées les questions sur les émissions de gaz à effet de serre liées à la consommation de pétrole, la question de l'épuisement des ressources a été soulevée notamment par Hubbert (1956) [3]. Dans la théorie du pic pétrolier (peak oil), la production pétrolière d'une zone géographique donnée connaît une première période de croissance avant d'atteindre un pic puis de décliner.

Quand on parle de réserve, il faut avoir à l'esprit que les ressources ne sont qu'estimées et que les réserves exploitables dépendent du niveau de technologie. On parle ainsi de réserve prouvée lorsqu'on a une probabilité de $90 \%$ de récupérer l'huile. Ainsi, comme le montre le graphique de la figure 2.8, ces réserves augmentent régulièrement en raison principalement du progrès technologique dans la production off-shore et la récupération de pétrole brut.

Les plus grandes réserves se situent au Vénézuela, en Arabie Saoudite et au Canada (Fig. 2.8). Avec un total estimé de plus de 1700 milliards de barils, les réserves correspondent à 50 ans de consommation de pétrole au rythme actuel.

Principaux pays détenant des réserves

\begin{tabular}{|l|r|r|}
\hline Venezuela & 303.3 & $17.5 \%$ \\
\hline Arabie Saoudite & 297.7 & $17.2 \%$ \\
\hline Canada & 167.8 & $9.7 \%$ \\
\hline Iran & 155.6 & $9.0 \%$ \\
\hline Iraq & 147.2 & $8.5 \%$ \\
\hline Fédération Russe & 106.2 & $6.1 \%$ \\
\hline Koweit & 101.5 & $5.9 \%$ \\
\hline Emirats Arabes Unis & 97.8 & $5.7 \%$ \\
\hline Etats-Unis & 61.2 & $3.5 \%$ \\
\hline Libye & 48.4 & $2.8 \%$ \\
\hline Unit: Milliards de barils, Source : BP Stas
\end{tabular}

Evolution des réserves prouvées de pétrole dans le monde (milliards de barils)

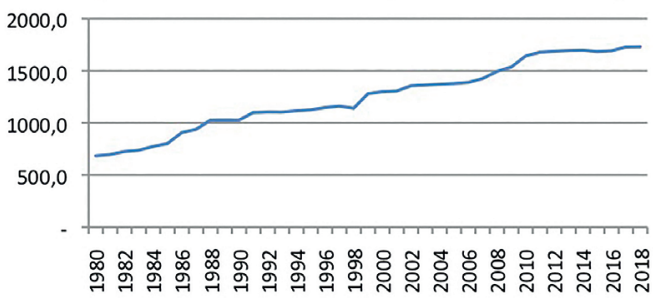

Figure 2.8: Réserves de pétrole brut dans le monde. À gauche: Principaux pays détenant les réserves (d'après BP Stats [2]). À droite: Évolution des réserves prouvées de 1980 à 2018 (d'après BP Stats [2]).

\subsubsection{La transformation du pétrole et les produits finis}

En fait, le pétrole n'est pas utilisé directement. II va être raffiné en produits pétroliers, en carburants pour le secteur des transports, en combustibles ainsi qu'en d'autres produits pour un usage non énergétique (plastiques, bitume, ...) (Fig. 2.9). On part d'une ressource, qui a des caractéristiques différentes (densité, teneur en soufre) pour la transformer en un produit dont les qualités doivent être homogènes [4]. II y a longtemps qu'on ne se plaint plus d'avoir une essence de mauvaise qualité qui fait tomber le moteur de sa voiture en panne. 
Au fur et à mesure des crises pétrolières et de la montée des préoccupations écologiques, les produits pétroliers ont été de moins en moins utilisés comme combustibles. Désormais, près des deux tiers de la consommation finale de produits pétroliers répond aux besoins du secteur des transports (route, aviation, navigation). Comme on le voit sur le graphique de droite de la figure 2.9, la consommation du secteur routier correspond à environ la moitié de la demande de produits pétroliers. C'est donc dans ce domaine que l'effort principal doit être porté pour réduire notre dépendance au pétrole pour les transports de marchandises et de passagers.
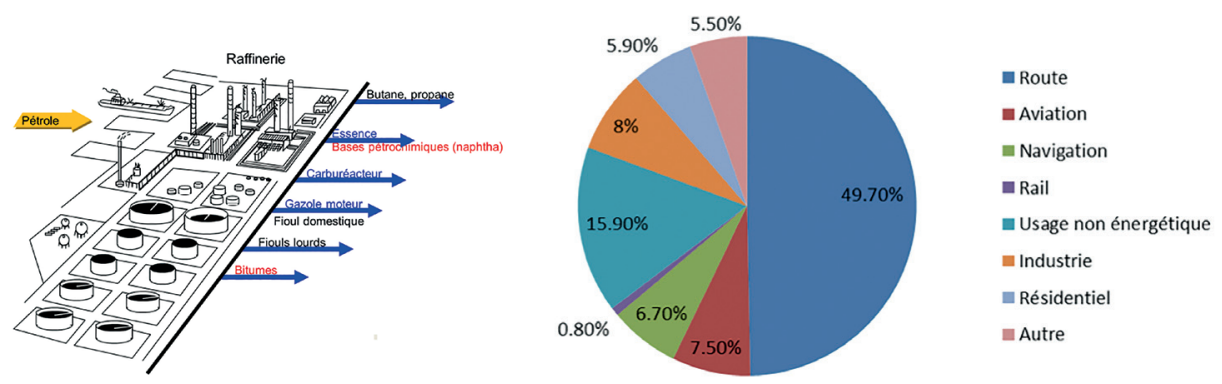

Figure 2.9: Raffinage du pétrole (à gauche) et répartition de la demande finale de produits pétroliers (à droite). (d'après BP Stats [2]).

Notons que de très grands efforts ont été réalisés dans l'amélioration des carburants, en particulier en Europe avec la très forte réduction de la teneur en soufre de l'essence qui est passée de 150 parties par million (ppm) jusqu'en 2005 à 10 ppm depuis 2009. Nous reviendrons sur cette question environnementale plus loin.

\subsubsection{Les ressources non conventionnelles}

L'épuisement des ressources pétrolières, qui sont des ressources fossiles épuisables par nature, s'est vu contrebalancé par l'exploitation de ressources dites non conventionnelles, dont les ressources en place sont du même ordre de grandeur que les ressources conventionnelles [5].

Les ressources non conventionnelles de pétrole regroupent des pétroles de natures très différentes: light-tight oil, brut extra-lourds et sables bitumineux. Les schistes bitumineux (shale oil), sont quant à eux très peu exploités. Le pétrole lourd résulte de l'oxydation bactérienne de brut conventionnel qui a été piégé dans le réservoir, et ses caractéristiques physico-chimiques sont généralement de moins bonne qualité que celles des pétroles conventionnels avec une viscosité plus élevée et des taux supérieurs de métaux lourds, de soufre et d'azote.

Le Venezuela et le Canada sont les deux pays clés en termes de réserves, le premier pour le brut extra-lourd et le second pour les sables asphaltiques (Fig. 2.10). La répartition des réserves de pétrole non conventionnel au niveau mondial montre que ces 
ressources modifient profondément l'équilibre des marchés pétroliers. Les light tight oil qui sont exploités aux États-Unis ont eu un très fort impact sur le prix du pétrole.

L'exploitation de ces ressources non conventionnelles a été rendue possible par la maîtrise de deux techniques: le forage horizontal et la fracturation hydraulique.

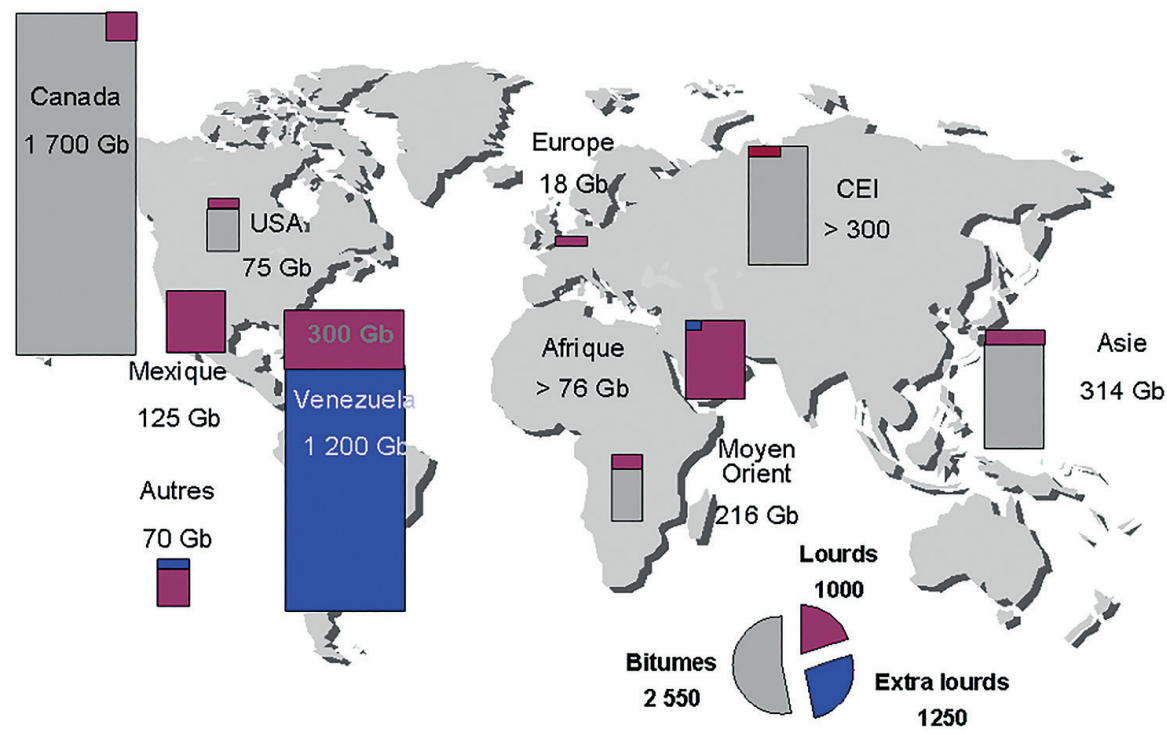

Figure 2.10: Répartition des ressources en place de pétrole brut lourd, extra-lourd et de bitume, estimées en milliards de barils (Gb). Source : D'après: [6].

\subsubsection{Les enjeux environnementaux}

L'utilisation du pétrole a été marquée par de très nettes évolutions liées aux enjeux environnementaux. Outre la suppression du plomb dans les essences, un des plus anciens challenges environnementaux a été la réduction de la teneur en soufre des produits pétroliers, considérée comme responsable des pluies acides (Fig. 2.11). La notion du temps est importante dans la mise en œuvre des politiques de protection de l'environnement. Le protocole des Nations Unies sur la réduction des émissions de soufre date de 1979. La dernière phase avec mise en place des spécifications des fiouls utilisés par les navires n'a eu lieu qu'en 2020 [7].

Les produits pétroliers sont au milieu de nombreux enjeux environnementaux, que ce soit en termes de recyclage des plastiques ou bien de réduction des émissions de gaz à effet de serre. II faut avoir en tête que c'est un facteur de l'ordre de 3 qui relie les consommations de carburant et les émissions de $\mathrm{CO}_{2}$ qui proviennent de leur usage (Fig. 2.11). Autrement dit, la combustion d'une tonne de carburant produit environ trois tonnes de gaz carbonique dans l'atmosphère. 


\begin{tabular}{|c|c|}
\hline Produit pétrolier & $\begin{array}{c}\text { Masse de } \mathrm{CO}_{2} \text { émis } \\
\text { par kg de carburant }\end{array}$ \\
\hline Essence & $3,066 \mathrm{~kg}$ \\
\hline Diesel & $3,150 \mathrm{~kg}$ \\
\hline Fioul lourd & $3,276 \mathrm{~kg}$ \\
\hline
\end{tabular}

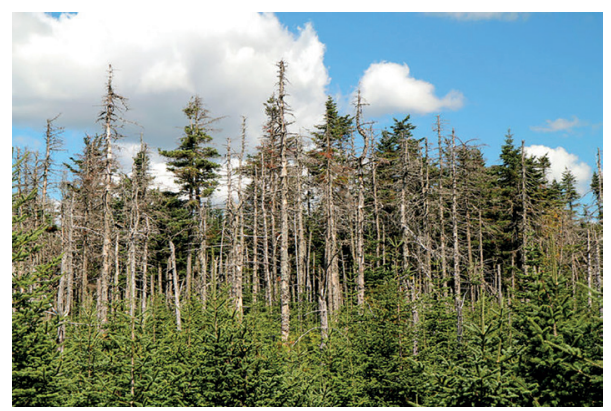

Figure 2.11: Problèmes environnementaux du pétrole: émissions de $\mathrm{CO}_{2}$ dues à la combustion des carburants (à gauche) et forêts victimes des pluies acides dues à la teneur en soufre des produits pétroliers (à droite). (Photo @istock).

\subsubsection{Conclusion}

En résumé, le pétrole fournit des produits qui sont utilisés dans de nombreux domaines comme les carburants ou les combustibles ou dans des usages non énergétiques. Les chocs pétroliers et la montée des enjeux environnementaux ont poussé à leur trouver des substituts.

C'est le secteur des transports qui reste très largement dépendant des carburants issus du pétrole et qui correspond à la plus grande part du pétrole consommé dans le monde. C'est donc dans ce secteur qu'il faut envisager des substitutions.

L'épuisement des ressources conventionnelles est contrebalancé par la découverte de ressources non conventionnelles. Si les réserves globales ne peuvent que s'amenuiser au fur et à mesure de l'extraction du pétrole, les questions environnementales se posent de manière plus pressante, que ce soit au niveau de sa production que de son utilisation.

\subsection{Pollution atmosphérique - J.-E. Petit et S. Szopa}

\subsubsection{Les enjeux liés à la pollution atmosphérique}

La pollution atmosphérique est une altération de la qualité de l'air dont les enjeux ont d'abord été perçus à l'échelle des villes avec des évènements aux conséquences immédiates et dramatiques. Ce fut notamment le cas du smog de Los Angeles dans les années 1950 ou de l'hiver 1952 à Londres, qui a vu plus de 4000 morts en une semaine en raison d'un épais brouillard acide. Ce n'est qu'un peu plus tard que l'on a pris conscience du caractère continental, voire intercontinental, de la pollution atmosphérique, en particulier avec les pluies acides. Cette pollution est liée à des composés présents en très petites quantités dans l'air, de l'ordre d'une molécule sur un million ou un milliard, mais toxiques pour les humains et/ou les écosystèmes. 
Les polluants atmosphériques présentent, en effet, des dangers pour notre santé. L'Organisation Mondiale de la Santé (OMS) lui attribue 4 millions de morts prématurées dans le monde chaque année, avec une très grande partie de ces décès dans les pays en voie de développement. En France, on estime qu'environ 50000 décès prématurés par an sont liés à ce type de pollution ${ }^{23}$.

La surveillance de la qualité de l'air en France est confiée à des associations régionales, indépendantes et agréées (ASQA). Ces ASQA ont l'obligation réglementaire de publier annuellement le bilan annuel de la qualité de l'air et de le mettre à disposition du grand public. Pour ce faire, les ASQA gèrent un réseau de stations de mesure fixes réparties sur leur territoire, des camions de mesures mobiles pour des campagnes spécifiques et des modèles de chimie-transport. En Île-de-France, Airparif est chargé de cette surveillance ${ }^{24}$. Airparif publie en temps réel les cartographies des principaux polluants atmosphériques que nous présenterons dans le paragraphe suivant 2.3.2. La qualité de l'air est considérée comme acceptable dès lors que les concentrations de polluants ne dépassent pas des valeurs critiques, exprimées en $\mu \mathrm{g} / \mathrm{m}^{3}$ et fixées par des normes européennes. Airparif est également tenu à réaliser des cartographies annuelles pour l'ozone, le dioxyde d'azote et les microparticules en estimant la superficie et la population résidente concernées par un dépassement des valeurs réglementaires ${ }^{24}$.

\subsubsection{Les polluants atmosphériques et leurs sources}

Certaines pollutions sporadiques liées, par exemple, à des accidents industriels peuvent relarguer dans l'atmosphère de grandes quantités de composés très spécifiques et présenter transitoirement des risques importants pour notre santé selon la nature des composés émis. Cela étant, sur la durée, ces pollutions restent extrêmement marginales en termes de bilan des masses de composés émis et des impacts sanitaires globaux. Majoritairement, la pollution de l'air résulte des activités impliquant de la combustion (véhicules, usines thermiques ou feux de biomasse), de certains processus industriels et notamment avec usage de solvants, et des activités agricoles. Toutes ces activités conduisent à l'émission de gaz tels le monoxyde de carbone (CO), les composés organiques volatils (acronyme: COV), les oxydes d'azote (NOx), l'ammoniac $\left(\mathrm{NH}_{4}\right)$, le dioxyde de soufre $\left(\mathrm{SO}_{2}\right)$, les composés aromatiques polycycliques, etc. ou de particules en suspension - les aérosols - comme par exemple les particules de suie. On caractérise souvent particules et aérosols par leurs tailles; les abréviations PM10, PM2,5 correspondent, par exemple, à des tailles de particules inférieures à 10 et $2,5 \mu \mathrm{m}$, respectivement. La figure 2.12 représente les parts respectives des secteurs d'activité dans les émissions de polluants d'origine humaine pour quatre polluants majeurs.

23. https://agirpourlatransition.ademe.fr/particuliers/pollution-lair

24. https://www.airparif.asso.fr/surveiller-la-pollution/bilan-et-cartes-annuels-de-pollution https://www.airparif.asso.fr/. 
Tous les polluants émis vont eux-mêmes conduire à la formation d'autres polluants (ozone, aérosols acides, etc.) par l'intermédiaire d'une chimie complexe. L'ozone $\left(\mathrm{O}_{3}\right)$ est l'exemple même de la «chimie intense » qui a lieu dans l'atmosphère. Sur ce point, il faut distinguer le «bon ozone», la couche d'ozone qui nous protège des rayonnements UV et se forme "chimiquement» dans la haute atmosphère, du «mauvais ozone», mauvais pour notre santé et l'environnement, qui se forme «chimiquement» dans la basse atmosphère, notamment par transformation chimique des oxydes d'azote et des composés organiques volatils.

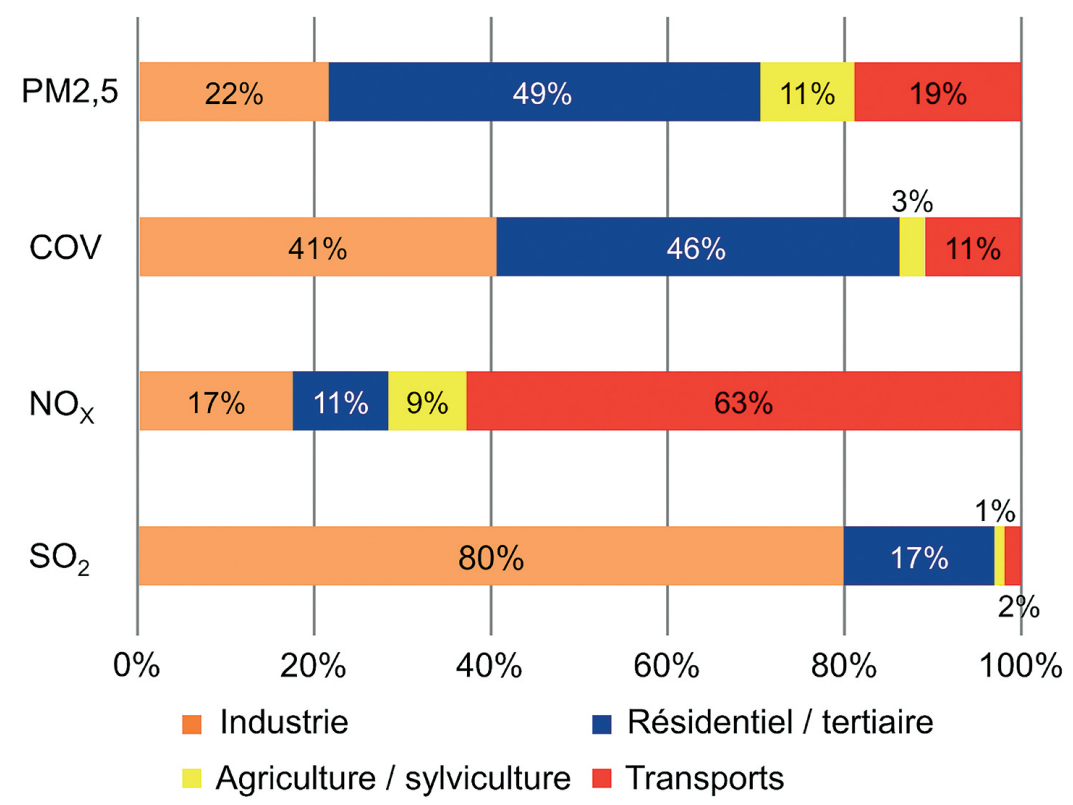

Figure 2.12: Parts respectives des différents secteurs d'activité dans les émissions de certains des principaux polluants atmosphériques. Sources: CITEPA et Annales de Mines [8].

De manière générale, la pollution de l'air a diminué ces trente dernières années en France, mais la France reste au-delà des seuils recommandés par l'OMS, notamment pour l'ozone, les oxydes d'azote et les particules. Elle a d'ailleurs été condamnée récemment par la Commission Européenne pour ces dépassements ${ }^{25}$. Cependant, la nature chimique des polluants évolue au gré des développements de nouveaux procédés industriels, d'une part, et des normes réglementaires décidées pour limiter tel ou tel polluant, d'autre part. Ainsi le dioxyde de soufre, polluant majeur des années 1970-80 à l'origine de fortes pollutions acides, a été strictement réglementé et sa concentration ainsi que celles des sulfates, qu'il produit, ont fortement baissé en Europe et aux États-Unis au cours de ces dernières décennies. La même diminution 
est en train de se produire en Asie. Néanmoins, l'application de normes, visant à la réduction des particules émises par les moteurs thermiques durant ces dernières années, a conduit à l'apparition d'autres formes de pollution avec des particules ultrafines plus difficiles à détecter mais aussi plus invasives pour le système respiratoire.

En Île-de-France, le chauffage résidentiel au bois et le transport routier sont des contributeurs majeurs aux particules en suspension et aux oxydes d'azote.

Les sources anthropiques des polluants chroniques sont, pour beaucoup d'entre elles, les mêmes que les sources de gaz à effet de serre que nous aborderons dans une autre partie de cet ouvrage (voir section 7.3). Trois grands types d'activité émettent, à la fois, des polluants et des gaz à effet de serre:

- La production d'énergie par la combustion de fuel fossile, comme c'est le cas des centrales thermiques de production d'électricité, ou d'autres activités industrielles principalement sources de $\mathrm{CO}_{2}$ qui n'est pas, à proprement parler, un polluant mais un gaz à effet de serre;

- Les transports routiers, fluviaux, maritimes ou aériens;

- L'activité agricole, source majoritaire d'ammoniac et qui émet aussi du protoxyde d'azote et du méthane, comme gaz à effet de serre.

Si ces sources font l'objet de réglementation, par exemple pour décarboner la production d'énergie dans le cadre de la limitation du changement climatique, cela aura donc aussi des bénéfices pour la pollution de l'air ${ }^{26}$.

\subsubsection{Influence des polluants sur le climat}

Certains des polluants influencent le climat en interagissant avec la lumière du soleil, le rayonnement infrarouge terrestre et les nuages (voir section 7.2 sur le bilan radiatif par Florence Raffin et Marielle Saunois). Les interactions sont diverses, avec certains polluants qui réchauffent l'atmosphère, et d'autres la refroidissent. On parle alors de forçages radiatif respectivement positif ou négatif.

Ainsi, les aérosols ont un pouvoir refroidissant. Faut-il, pour autant, conserver les aérosols dans l'air pour lutter contre le réchauffement climatique? On s'en doute, la réponse est non. Et même un double non. D'une part, parce que les aérosols, qui ont un effet refroidissant, sont des aérosols acides comme les sulfates, dont la toxicité pour les organismes vivants, les humains entre autres, et les écosystèmes est importante. Pour cette raison, ils ont été fortement régulés et leur effet de «masquage» du changement climatique s'est fortement atténué au cours de ces dernières décennies. D'autre part, parce que le climat ne se résume pas à l'élévation ou l'abaissement de la température et que les aérosols ont également modifié les précipitations et sont, par exemple, à l'origine de la sécheresse au Sahel dans les années 1980.

26. https://www.metropolegrandparis.fr/fr/plan-climat-air-energie-132 
Il arrive néanmoins que des phénomènes naturels injectent une grande quantité de ces aérosols dans la haute atmosphère, notamment lors des éruptions volcaniques, comme celle du Pinatubo dans les années 1980. Ces quantités phénoménales de matière bloquent les radiations solaires depuis la haute atmosphère, comme on peut le voir sur la figure 2.13. Mais cet effet ne dure généralement qu'assez peu de temps (1-2 ans). Plus récemment, les feux exceptionnels en Australie ont conduit à un refroidissement dans l'hémisphère sud, mais là encore de courte durée.

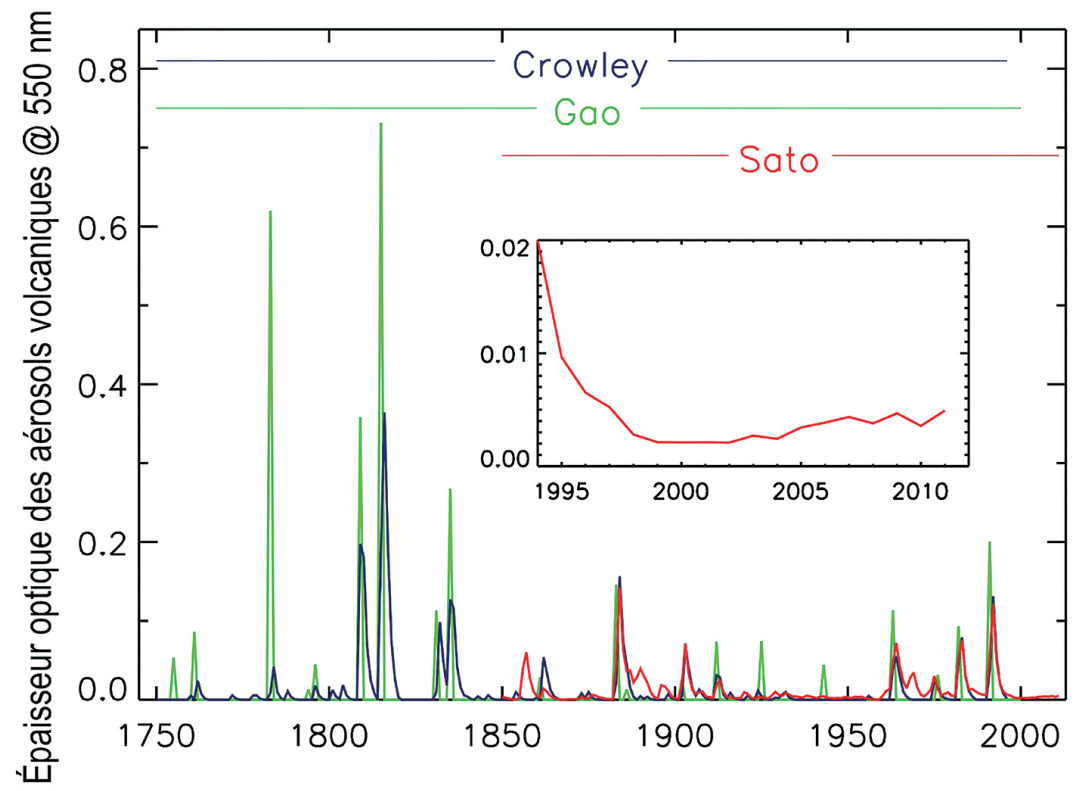

Figure 2.13: Évolution de l'épaisseur optique des aérosols volcaniques, reconstruite de 1750 à nos jours, celle-ci caractérisant l'atténuation du rayonnement solaire lors des éruptions. Les courbes en vert (Gao) et en bleu (Crowley) ont été reconstruites à partir de mesures dans des carottes glaciaires, celle en rouge (Sato) a été reconstruite à partir de données de surface et de mesures par satellite. Source : IPCC - WGI AR5 Chapter 8.

Les polluants, dont le temps de vie dans l'atmosphère est court, voient leur répartition fluctuer de manière importante dans le temps et dans l'espace. Ces changements peuvent être initiés par différents facteurs. Tout d'abord, les sources d'émission, les activités humaines notamment, sont celles qui permettent, en premier lieu, l'injection de polluants dans l'atmosphère. Mais aussi les conditions météorologiques qui vont, par exemple, favoriser la formation de pollution secondaire, et donc de nouveaux composés chimiques. Indirectement, ces conditions météorologiques influencent la temporalité de certaines sources: par exemple, plus de chauffage en hiver, lorsqu'il fait froid, ou plus de trafic routier en automne, à la sortie des «beaux jours», synonyme de forte reprise des activités. Le seul levier d'action pour diminuer cette pollution se situe au niveau des sources (Fig. 2.14). 


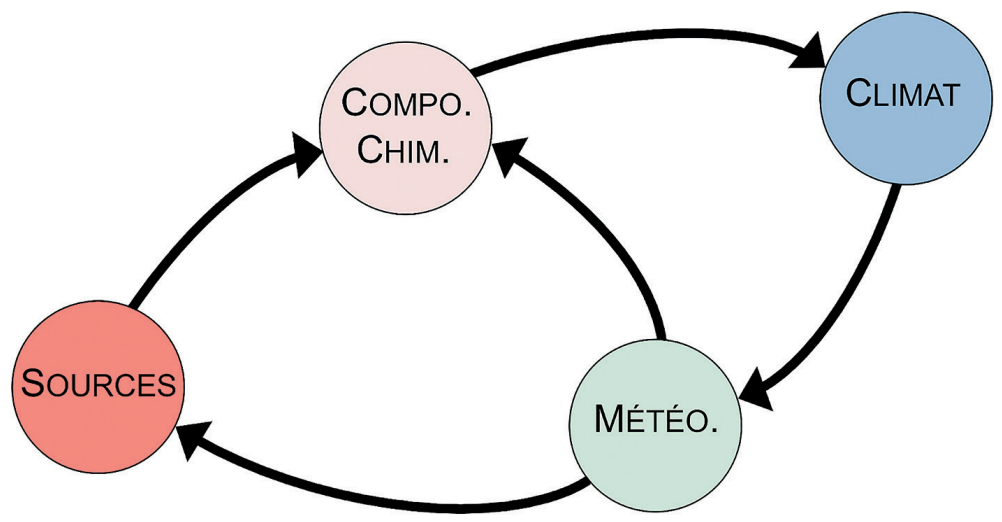

Figure 2.14: Diagramme montrant les interactions entre les sources de pollution atmosphérique et le climat. Source : Jean-Eudes Petit.

Que peut-on retenir de l'épisode de baisse des émissions de polluants liées à la pandémie?

Les effets d'une réduction peuvent être surprenants. Pour illustrer cela, prenons l'exemple du confinement lié à la pandémie du SARS-COV-2 de 2020. Nous avons tous observé une baisse très forte du trafic automobile dans la plupart des zones urbaines en France, mais aussi ailleurs en Europe et dans le monde. Cette baisse du trafic est directement reliée à une baisse des aérosols et des concentrations d'oxydes d'azote de plusieurs dizaines de pourcents. C'est donc un bénéfice net du point de vue de la pollution. Néanmoins, la chimie de l'ozone est une chimie complexe. Les oxydes d'azote participent à la formation d'ozone, mais aussi à sa destruction dans les zones très polluées! Dès lors, si la réduction des oxydes d'azote a entraîné une baisse de l'ozone en moyenne dans le monde, elle a pu localement induire une augmentation des concentrations en ozone. Cela illustre toute la difficulté à mettre en place des politiques pertinentes à toutes les échelles spatiales.

Pour résumer, la lutte contre la pollution de l'air est indispensable pour permettre de soustraire une grande partie de la population mondiale (90\% selon l'OMS) à une qualité de l'air dégradée. Cette lutte doit être menée même si, paradoxalement, la présence de certains polluants comme les aérosols peut entraîner un bénéfice involontaire en refroidissant légèrement le climat et en masquant le réchauffement climatique "de fond». Parce que souvent, les sources de $\mathrm{CO}_{2}$ sont en même temps des sources de polluants atmosphériques, les mesures de décarbonisation s'accompagnent nécessairement de bénéfices au profit de la qualité de l'air. Cela étant, nos efforts de décarbonisation ne doivent pas nous affranchir de politiques spécifiques de lutte contre la pollution, ne serait-ce qu'en raison des effets non linéaires de la chimie atmosphérique et de la formation de polluants secondaires qui en résulte. 


\subsection{Pollution de l'eau - Y. Lévi}

\subsubsection{De quelles eaux parlons-nous?}

La pollution d'origine anthropique des eaux concerne malheureusement toutes les ressources car les humains ont, depuis toujours, compris que l'eau était la voie leur permettant d'évacuer leurs déchets au plus loin. Les contaminations chimiques ou biologiques touchent donc tous les types de ressources en eau. Les constructions des villages, des sites industriels et des villes se sont toujours réalisées près des eaux pour les usages d'alimentation, d'hygiène, d'énergie, de sécurité et de transport. Les eaux superficielles continentales (rivières, fleuves, lacs) sont donc les plus polluées mais les eaux souterraines les moins protégées le sont aussi. Les plus profondes, mieux protégées, sont en général moins contaminées mais peuvent contenir des éléments naturels indésirables pour certains usages (fer, manganèse, calcium, arsenic...). II en va nécessairement de même pour les eaux littorales saumâtres ou marines qui récoltent ce que les fleuves déversent. Ainsi, la planète n'a jamais connu dans son histoire une pollution chimique d'origine anthropique aussi considérable et diversifiée telle qu'elle est observée depuis les années 1950.

Parler des eaux revient donc à évoquer des eaux douces superficielles, des lacs, des rivières, des fleuves, des glaciers et des eaux souterraines qui sont toutes les masses d'eau contenues dans le sol. II en va de même avec les eaux saumâtres des estuaires et les eaux salées des mers et océans. Ces masses d'eau sont en interaction les unes avec les autres ainsi qu'avec la pollution atmosphérique et la pollution des sols. Toute pollution déversée par les activités humaines finit par arriver, à un moment ou à un autre, dans les eaux.

Deux cycles liés à l'eau sont classiquement décrits: le grand cycle naturel et le cycle des usages ou «petit» cycle.

- Le cycle naturel (ou "grand» cycle) de l'eau commence avec l'évaporation depuis les surfaces de la planète sous forme de vapeur, puis, selon les conditions atmosphériques, la vapeur se condense en précipitations (pluies, neige, grêle) qui tombent au sol. Ensuite, l'eau de pluie va lessiver les sols et être captée par la végétation. Une partie est renvoyée dans l'atmosphère par les plantes ce que l'on appelle l'évapotranspiration. Le reste diffuse dans les sols et sous-sols et le cycle se boucle par le retour de l'eau aux rivières, aux fleuves et à la mer (Fig. 2.15). La pluie peut déjà se contaminer avec la pollution atmosphérique. De nouvelles contaminations se produisent par le lessivage des sols, le passage sur toutes les surfaces, notamment les surfaces imperméables, les routes, les toitures, etc. 


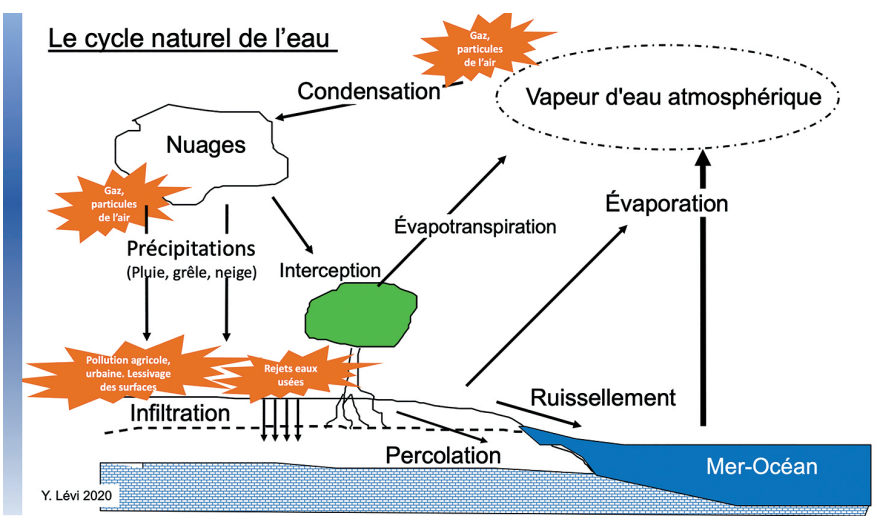

Figure 2.15: Cycle naturel de l'eau (ou «grand» cycle) et sources de contaminations. Source: Yves Levi.

- Le cycle des usages de l'eau («petit» cycle) (Fig. 2.16) se décrit de la manière suivante: après prélèvement dans la nature, l'eau est traitée pour fabriquer de l'eau potable (pour les pays qui en ont les moyens) qui est ensuite distribuée dans des canalisations pour alimenter les populations. Après usage, l'eau usée est collectée dans des réseaux d'assainissement (égouts) et transportée vers des stations d'assainissement où elles sont traitées avant rejet au milieu naturel. Ce schéma est extrêmement variable selon les moyens économiques disponibles. Dans la majorité des pays, il n'existe pas ou peu d'assainissement et les eaux usées se dirigent directement vers les milieux aquatiques. Tous les rejets industriels, urbains, ou agricoles partent dans l'environnement de manière ponctuelle (rejet d'égout, déversement volontaire ou accidentel, etc.) ou diffuse (déversements de pesticides dans les champs, pollution urbaine générale...). Ainsi, l'humain émet, chaque jour, des masses considérables de polluants, qui finissent, à un moment ou à un autre, par rejoindre les masses d'eaux.

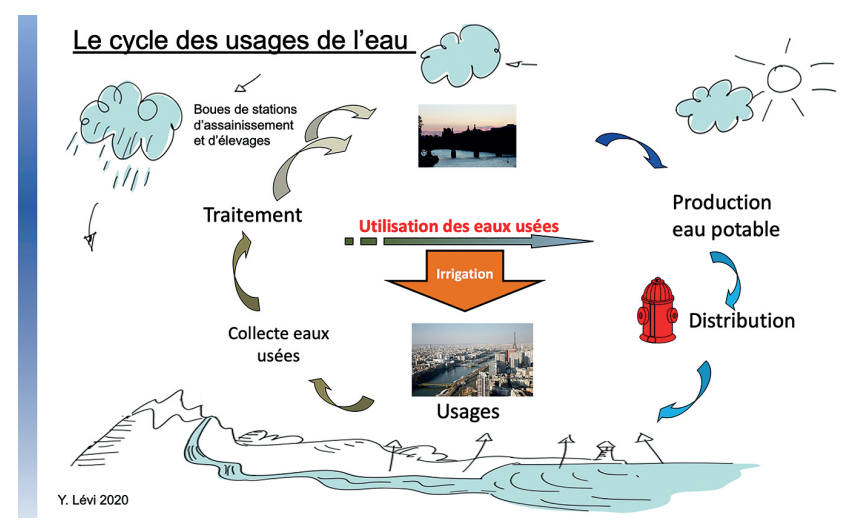

Figure 2.16: Cycle des usages de l'eau ou «petit» cycle des eaux. Source: Yves Levi. 
Globalement, pour étudier la qualité des eaux de ces deux cycles imbriqués, il faut raisonner par bassin versant (Fig. 2.17). Celui-ci constitue la surface «entonnoir» qui récupère toutes les eaux qui se dirigent pour constituer un cours d'eau (ruisseau, rivière, fleuve) et donc constitue la zone géographique à l'origine des contaminations observées.

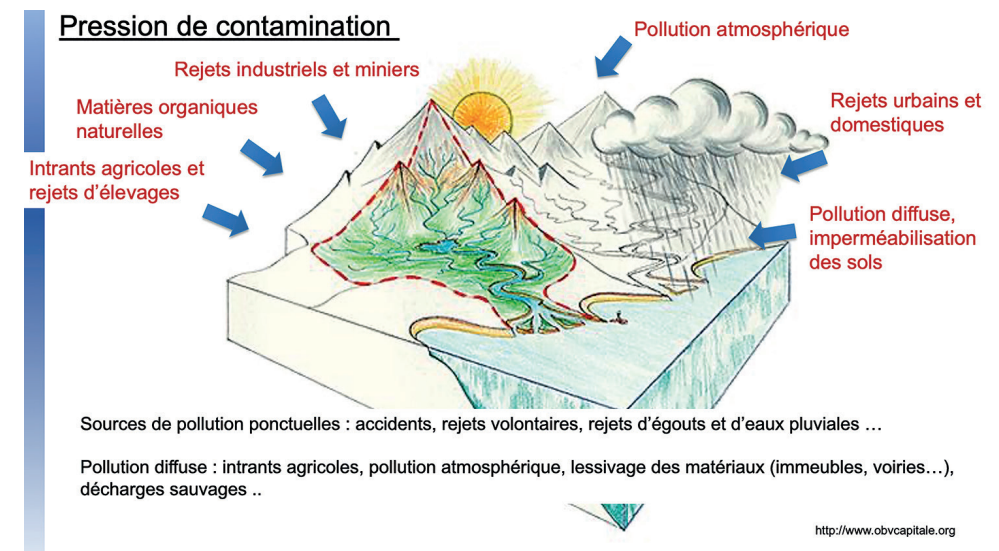

Figure 2.17: Notion de bassin versant. D’après: Organisme des bassins versants de la Capitale (OBV Capitale).

\subsubsection{De quelle pollution parlons-nous?}

Les contaminations de l'eau peuvent être de différentes natures ${ }^{27}$.

II existe une contamination naturelle de certaines eaux souterraines par des éléments natifs provenant des sols (arsenic, fer, manganèse...). Dans certains pays comme le Bangladesh ou le Vietnam, le pompage intensif des eaux souterraines a attiré de l'arsenic naturel dans les eaux destinées aux populations. À trop pomper, les populations risquent de ne plus avoir d'alternative. Impossible de boire directement l'eau des rivières et mal gérer les eaux souterraines induit des risques si elles ne sont pas traitées.

La pollution provient en grande partie des activités humaines: industries, agriculture dont l'élevage, transports, urbanisation, imperméabilisation des sols, etc.

27. La pollution des milieux aquatiques:

- Site Service public de l'information sur l'eau Eaufrance: https://www.eaufrance.fr/les-impacts-de-la-pollution-de-leau

- Agence de l'eau Seine Normandie: http://www.eau-seine-normandie.fr/les-cycles-de-l-eau

- Portail technique de l'Office français de la biodiversité: https://professionnels.ofb.fr/fr/node/322

- Rapport de la banque mondiale sur la pollution de l'eau: https://www.worldbank.org/en/news/feature/2019/08/20/quality-unknown

- Association GRAIE : http://www.graie.org/portail/ 
Outre les rejets directs ou les rejets après épuration, le lessivage des sols par la pluie conduit à des pollutions diffuses (engrais et pesticides) et ponctuelles (rejets d'égouts et de déversoirs d'eaux d'orage).

Les polluants contaminants peuvent être chimiques (pesticides, plastifiants, hydrocarbures, solvants, résidus de médicaments, engrais, etc.), microbiologiques (déchets humains et animaux), physique (chaleur en aval des centrales de production d'électricité), radiologique (déversements tels que ceux provenant de la centrale nucléaire de Fukushima) (Fig. 2.18). Tous ces éléments, la plupart du temps en mélanges complexes, constituent des dangers. Les dangers induisent, selon les doses et les organismes, des effets biologiques pouvant être toxiques pour l'environnement (écotoxicité animale et végétale) et pour la santé humaine (boisson, baignade, irrigation des cultures vivrières...).

Depuis les années 1950, la diversité des produits chimiques inventés par la chimie moderne est prodigieuse. Ceux-ci ont apporté nombre de bienfaits pour l'humanité, car porteurs de progrès, mais la contrepartie est la présence de polluants chimiques partout. Outre les pesticides qui sont déversés volontairement, les plastifiants, les retardateurs de flamme, des hydrocarbures, des résidus de médicaments... sont trouvés partout dans l'environnement, souvent à très faible trace.

Comme décrit au paragraphe 2.2.4 ci-après, pour les détecter, il existe des méthodes analytiques, dont les progrès ont été considérables ces 20 dernières années. Il est possible de quantifier des traces de polluants chimiques inférieures au nanogramme par litre $\left(10^{-9} \mathrm{~g} / \mathrm{L}\right)$. Par ailleurs, sont aussi bien détectés les polluants eux-mêmes que leurs produits de dégradation, ce qui augmente le nombre de polluants connus.

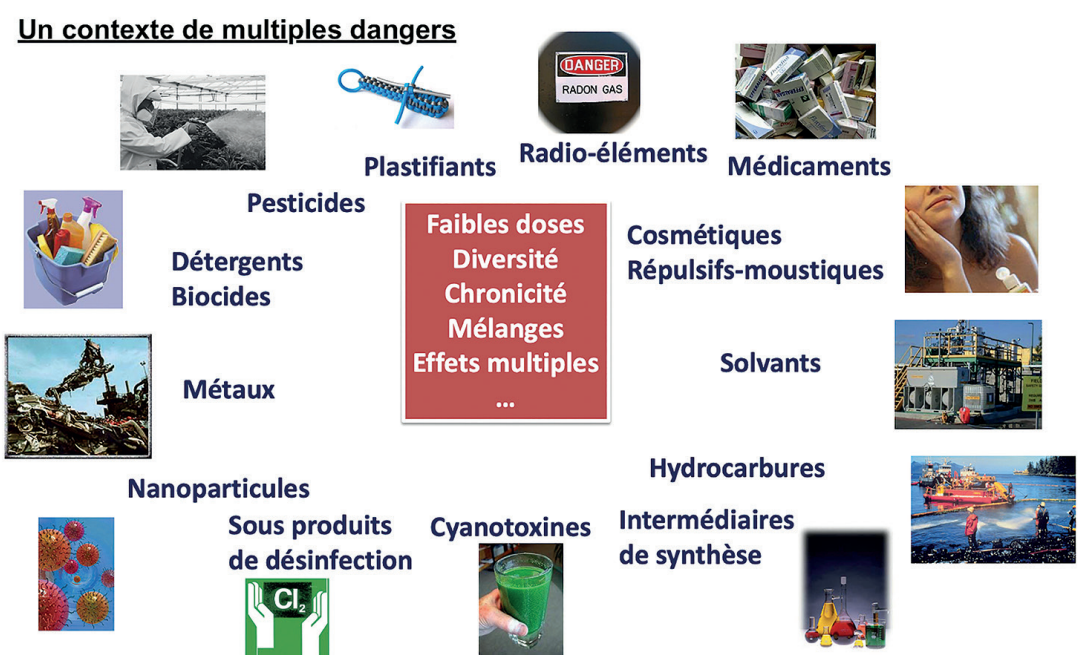

Figure 2.18: Exemples de la diversité des familles de contaminants chimiques présents dans les eaux. Source: Yves Levi. 


\subsubsection{De quels effets et de quels risques parlons-nous?}

La pollution chimique est rarement constituée de polluants isolés, mais il s'agit en général de mélanges de tout ce qui est déversé dans l'environnement. Face à cette diversité, il faut s'interroger sur les effets et tenter de quantifier les risques pour l'environnement et pour la santé humaine.

Parmi les effets biologiques, sont identifiés:

- des perturbations du système endocrinien par des polluants et mélanges de polluants qui agissent comme des hormones ou qui inhibent l'action des hormones naturelles (cancers, troubles de la croissance et de la reproduction, diabète...);

- la toxicité cellulaire dégradant le fonctionnement des cellules et pouvant conduire à leur mort, à celle des organes et donc à celle des organismes;

- la génotoxicité qui agit sur le patrimoine génétique et conduit à des mutations et des cancers;

- le stress oxydant, etc.

De nombreuses pathologies humaines sont ainsi attribuables aux eaux contaminées, sachant que l'humain est aussi exposé aux contaminants de l'air, des sols, de l'alimentation...:

- les effets à court terme (12 à 72 h), les maladies étant essentiellement liées aux microorganismes: choléra, gastro-entérites, salmonelloses, légionellose (infections pulmonaires causées par la respiration de gouttelettes contaminées à proximité de tours aéro-réfrigérantes ou dans les douches), cryptosporidioses et giardiases qui sont des diarrhées violentes dues aux protistes Cryptosporidum et Giardia, ...;

- les effets à moyen terme (quelques mois), les maladies pouvant être de nature microbiologique, par des contaminations chroniques, et de nature chimique;

- les effets à long terme (plusieurs années) dues à des contaminations chimiques qui peuvent induire des cancers (arsenic, chrome Vl, perturbateurs endocriniens, ...), des troubles du développement, des baisses du quotient intellectuel...

Les effets toxiques sur la faune et la flore ${ }^{28}$ sont évidemment de même ampleur. Ils concernent directement tous les organismes végétaux et animaux vivant dans les milieux aquatiques pollués ou dans des espaces qui subissent des irrigations par des eaux contaminées. Ils peuvent aussi être observés lorsque des matières organiques sont déversées dans les eaux, conduisant à une réduction de leur teneur en oxygène

28. Impacts de la pollution des eaux sur la biodiversité:

- Portail « eau et biodiversité » : https://www.documentation.eauetbiodiversite.fr/ 
et à un dérèglement écologique (eutrophisation). Ces effets toxiques peuvent évidemment aboutir à une mortalité directe, végétale et animale, mais ils peuvent aussi conduire indirectement à la contamination de toute la chaîne alimentaire dès lors que des espèces se nourrissent d'organismes aquatiques.

Certains contaminants chimiques sont bio-accumulateurs ce qui leur donne la propriété de remonter toute la chaîne alimentaire. Ils sont alors détectés chez des animaux vivant dans des zones extrêmement reculées de la planète, où l'industrie chimique est absente, car ils auront franchi toutes les barrières alimentaires. On a donc affaire à un risque global de pollution chimique pour la planète de même niveau de gravité et de globalité qu'est le changement climatique. Le concept «One Health-une santé » illustre en particulier l'interconnectivité et l'interdépendance des humains, des animaux et des écosystèmes en termes de santé. II s'agit d'une bonne santé de l'environnement, des animaux et des humains, sachant que l'Humain est un animal parmi les animaux (Fig. 2.19).

Identifier les effets toxiques des produits chimiques à partir de leur structure est un premier préalable pour évaluer le niveau de risque d'une pollution des eaux due à ces produits. La deuxième étape est de quantifier les niveaux de toxicité des produits par des analyses et mesures sur des cellules ou des animaux (essais in vitro et in vivo). L'étape complémentaire est de déterminer les quantités de produits présentes dans les milieux aquatiques ou dans l'eau potable ainsi que le niveau d'exposition des organismes susceptibles d'être contaminés. C'est l'ensemble des données effet - dose - exposition, qui permettra de calculer plus précisément le risque pour prendre les mesures de gestion adéquates visant à améliorer la qualité de l'eau et de l'environnement. Le risque est une probabilité qu'arrive un effet biologique à la suite d'une exposition à un ou plusieurs danger(s) (microorganismes, molécules, métaux, rayonnements...).

Globalité des expositions et des effets : One health - Une santé

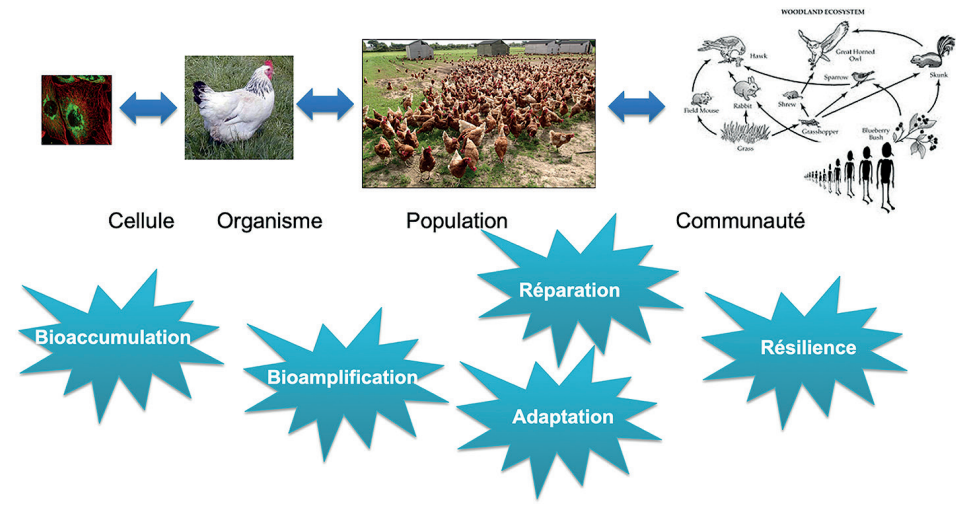

Figure 2.19: Illustration d'un volet du concept «one health-une santé» dans le continuum des relations entre santé animale et santé humaine et exemples de facteurs induisant des aggravations ou des améliorations de la situation sanitaire. Source: Yves Levi. 


\subsubsection{Savons-nous analyser ces contaminations?}

Les méthodes en chimie analytique ont fait des progrès considérables et le couplage des chromatographies en phase gazeuse ou liquide avec la spectrométrie de masse permet de doser des traces infimes de molécules même dans des matrices aussi chargées en matières organiques que sont les eaux d'égout. C'est pourquoi, la diversité incroyable de la pollution chimique est bien révélée, mais encore faut-il pouvoir l'interpréter en termes de risques toxiques biologiques.

En microbiologie, les analyses bactériologiques se font soit selon des méthodes de culture sur milieux nutritifs (in vitro) soit par biologie moléculaire. La première a l'avantage de permettre de dénombrer des bactéries réellement viables alors que la seconde détecte aussi des bactéries mortes mais dont le génome est encore présent. Pour les virus ou les protistes, les méthodes de culture sont plus longues et difficiles et parfois impossibles.

Pour les effets biologiques globaux, des essais sont menés in vitro sur des cellules, des bactéries ou des embryons ou in vivo sur divers types d'animaux allant des crustacés aux souris. L'avantage des essais biologiques est de donner une réponse concernant les effets toxiques liés aux mélanges réels tels que présents dans les eaux à analyser.

\subsubsection{Comment sont traitées les eaux d'égout avant de les rejeter dans le milieu naturel?}

Dans la majorité des pays, les eaux usées urbaines ou industrielles sont directement rejetées dans les rivières ou la mer sans aucun traitement. Même dans les pays équipés, il existe des déversoirs d'orages qui évitent que les eaux d'orage se dirigent vers la station d'assainissement et nuisent à son efficacité, elles sont alors rejetées sans traitement dans la nature.

Très succinctement, dans les pays où il existe des réseaux d'égouts reliés à une station d'assainissement, les eaux usées sont d'abord collectées, puis amenées à la station d'assainissement (Fig. 2.20) ${ }^{29}$. Les eaux brutes sont alors passées sur des grilles pour retenir les solides de taille significative. L'eau est ensuite débarrassée de son sable par simple décantation gravitaire au fond d'une fosse, puis les huiles et

29. Assainissement des eaux usées:

- Ministère en charge de l'environnement:

http://assainissement.developpement-durable.gouv.fr/

https://www.ecologique-solidaire.gouv.fr/assainissement

- L'assainissement des eaux usées vue par le Centre d'information sur l'eau (Organisation privée) https://www.cieau.com/le-metier-de-leau/ressource-en-eau-eau-potable-eaux-usees/ assainissement-eau-usees/

- Les grandes usines d'assainissement des eaux usées en île-de-France : Syndicat Interdépartemental d'Assainissement de l'Agglomération Parisienne:

https://www.siaap.fr/equipements/les-usines/ 
graisses sont remontées en surface par injection de fines bulles d'air et éliminées. L'eau ainsi traitée passe ensuite dans un premier ouvrage de décantation (un décanteur) qui va laisser sédimenter les particules. Celles-ci vont former une première boue qui sera ensuite traitée.

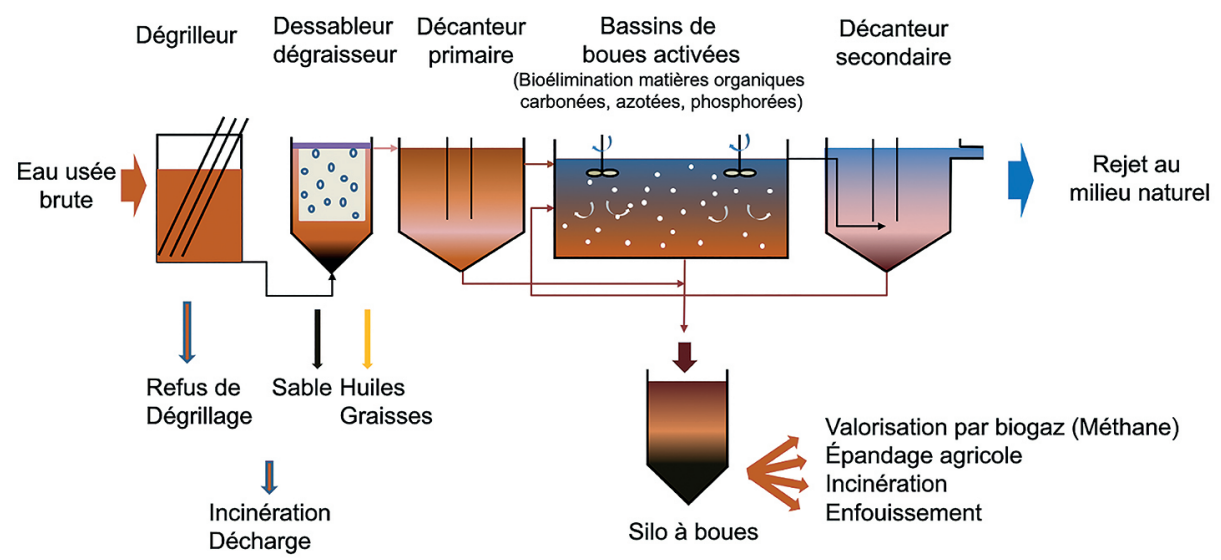

Figure 2.20: Filière-type de traitement des eaux usées dans les stations d'assainissement et de traitement des boues. Source: Yves Levi.

Une fois éclaircie, l'eau passe dans des bassins de boue activée avec une forte concentration de bactéries qui vont consommer les matières organiques biodégradables (élimination du carbone). Certaines usines sont équipées de bassins capables d'éliminer les matières azotées et phosphorées. Après cette (ou ces) étape(s), l'eau repasse par un décanteur qui va récupérer les boues bactériennes et l'eau peut être rejetée dans le milieu récepteur en ayant perdu au moins $90 \%$ de sa charge en contaminants chimiques et microbiologiques. Toutefois, certains contaminants peuvent ne pas être suffisamment éliminés.

Les boues produites sont ensuite déshydratées puis, si elles ne sont pas trop contaminées, elles sont épandues dans des champs comme engrais agricoles. Cependant, la diversité des polluants est devenue telle que certains pays, comme l'Allemagne, envisagent d'interdire totalement l'usage de ces boues. De même, en raison de la présence de nombreux polluants résiduels dans les eaux traitées, la Suisse a récemment décidé d'ajouter, après les traitements «classiques», une étape d'oxydation par le gaz ozone, qui casse certaines molécules, et un passage sur du charbon actif qui fixe les micropolluants. II s'agit d'une purification onéreuse et très complète, mais très protectrice pour les milieux aquatiques. Notons aussi que dans les zones "fragiles», par exemple, à proximité de parcs à huîtres, des procédés de désinfection sont mis en œuvre pour ne pas contaminer l'environnement et les produits destinés à l'alimentation. 


\subsubsection{Existe-t-il une réglementation?}

En Europe une directive cadre impose aux États membres une politique conduisant au bon état chimique et biologique des eaux et chaque pays doit faire des investissements pour tenter de redonner aux eaux continentales une meilleure qualité. Les grands pays économiquement développés ont bâti des politiques visant à restaurer et protéger la qualité des ressources, mais les intérêts économiques influencent largement leurs mises en œuvre. Par exemple, la pollution des ressources en eau par les nitrates d'origine agricole est un problème qui dure en France depuis plus de 30 ans sans véritable réduction. La pollution par les pesticides partout dans le monde est une constante.

Toutefois, une grande partie des nations du monde polluent les eaux sans véritables contraintes et sans moyens pour investir dans l'assainissement.

\subsubsection{Conclusion}

La chimie moderne a apporté d'immenses bienfaits pour l'humanité, mais aussi largement pollué l'environnement. Les eaux sont malheureusement le réceptacle final de toutes nos pollutions et nombre de ces molécules se dégradent très peu dans l'environnement et contaminent les eaux potables, les aliments irrigués. II s'agit d'un problème global, car les transferts de polluants se font à travers les continents et au sein des mers et océans. La chaîne alimentaire contribue à contaminer des animaux vivant dans des zones très protégées par les polluants organiques persistants (POPs). La planète n'a ainsi jamais connu une telle diversité de pollution dont les effets toxiques impactent les organismes vivants.

Parallèlement, la pollution microbiologique est toujours significative par les rejets humains et animaux et nécessite des désinfections.

Pour améliorer la situation, il faut réduire les déversements de polluants vers les eaux continentales et marines et cela ne se fait que par la réduction des rejets, la réglementation pour interdire la vente de produits les plus à risque, la collecte et le traitement efficace des eaux usées et une bonne gestion des boues des stations d'assainissement. Cela doit s'accompagner d'une surveillance des rejets avec les pénalités pour les pollueurs. Plus largement, les sociétés doivent en concertation et de manière globale, ce qui est encore loin d'être le cas, mettre en œuvre une politique de développement durable qui intègre la réduction des usages des molécules et produits les plus polluants.

La situation de la pollution chimique des eaux est grave en raison des nombreux effets biologiques induits par les mélanges complexes, même aux très faibles concentrations, et doit être considérée comme une pression globale sur le vivant aussi importante et globalisée que celle liée au changement climatique. C'est un investissement global d'importance qui est à réaliser sur l'ensemble de la planète afin de protéger nos ressources en eau. 


\subsection{Occupation et pollution des sols - J.Ch. Bureau}

Nous proposons de présenter brièvement les différents types d'occupation des sols sur notre planète en montrant, à partir de quelques exemples, les problèmes d'artificialisation, de dégradation et de pollution auxquels ces sols peuvent être soumis.

\subsubsection{Diversité des sols}

Les surfaces terrestres couvrent 13 milliards d'hectares, un hectare (ha) représentant un carré de $100 \mathrm{~m} \times 100 \mathrm{~m}$, soit $10000 \mathrm{~m}^{2}$. À titre de comparaison un terrain de football de Ligue 1 occupe environ 0,7 ha. Environ $13 \%$ sont utilisés pour les cultures, $28 \%$ pour la forêt et $35 \%$ sont constitués de pâtures et de savanes. Malgré de fortes disparités entre les pays, les pays les plus pauvres sont globalement dotés de $22 \%$ de la surface terrestre pour $38 \%$ de la population, alors que les pays les plus riches ont environ $25 \%$ des surfaces pour $15 \%$ de la population [9].

En Europe de l'ouest et centrale, les forêts, les pâturages et les terres arables représentent des proportions équivalentes des terres, soit un peu plus de $30 \%$ chacune, mais il faut souligner la part croissante des terres artificialisées, qui représentent désormais environ $4 \%$ des surfaces au niveau mondial.

En France, cette part atteint 9,5\% et progresse rapidement; l'accroissement est de $13 \%$ sur la dernière décennie, alors que la population n'a crû que de $5 \%$. Cet accroissement résulte principalement de l'extension des infrastructures de transport et de la construction immobilière. Sur une période de 10 ans et à l'échelle de la France métropolitaine, les surfaces nouvellement artificialisées sont évaluées en moyenne à 137000 ha, soit plus que la superficie d'un département comme le Val-d'Oise ${ }^{30}$. Cette artificialisation se fait essentiellement au détriment de terres agricoles, et le potentiel de production pourrait être affecté significativement si ce rythme ne diminuait pas. D'autre part, l'artificialisation des sols se traduit à la fois par une imperméabilisation des surfaces, qui accroît les dégâts des crues, par une baisse de la biodiversité qui souffre terriblement de la construction pavillonnaire et de la multiplication des infrastructures (routes, lignes TGV, etc.) et par la formation de microclimats (îlots de chaleur) tout en limitant la capacité globale des sols à stocker du carbone.

\subsubsection{Les surfaces agricoles et forestières}

Concernant les surfaces agricoles, leur mesure est très sensible à la définition puisqu'il y a un gradient entre terres peu productives et déserts. La FAO (Organisations des Nations Unies pour l'agriculture et l'alimentation) a une définition qui lui est propre, en termes de capacité de production d'un panier de biens, et classe de

30. Voir l'atlas régional de l'occupation des sols en France. Ministère de l'environnement, de l'énergie et de la mer (2016). https://www.statistiques.developpement-durable.gouv.fr/sites/default/files/2019-01/ datalab-2-atlas-regional-de-loccupation-des-sols-en-france\%20\%28clc\%29-octobre2016.pdf 
plus les terres en fonction de leur qualité. On voit sur le graphique de la figure 2.21 que la surface de terres agricoles productives est élevée en Amérique du Nord et en Europe, pays de l'ex-URSS inclus. La carte de la figure 2.21 illustre la diversité des systèmes de production agricole dans le monde.

\section{Excellente Bonne Médiocre}
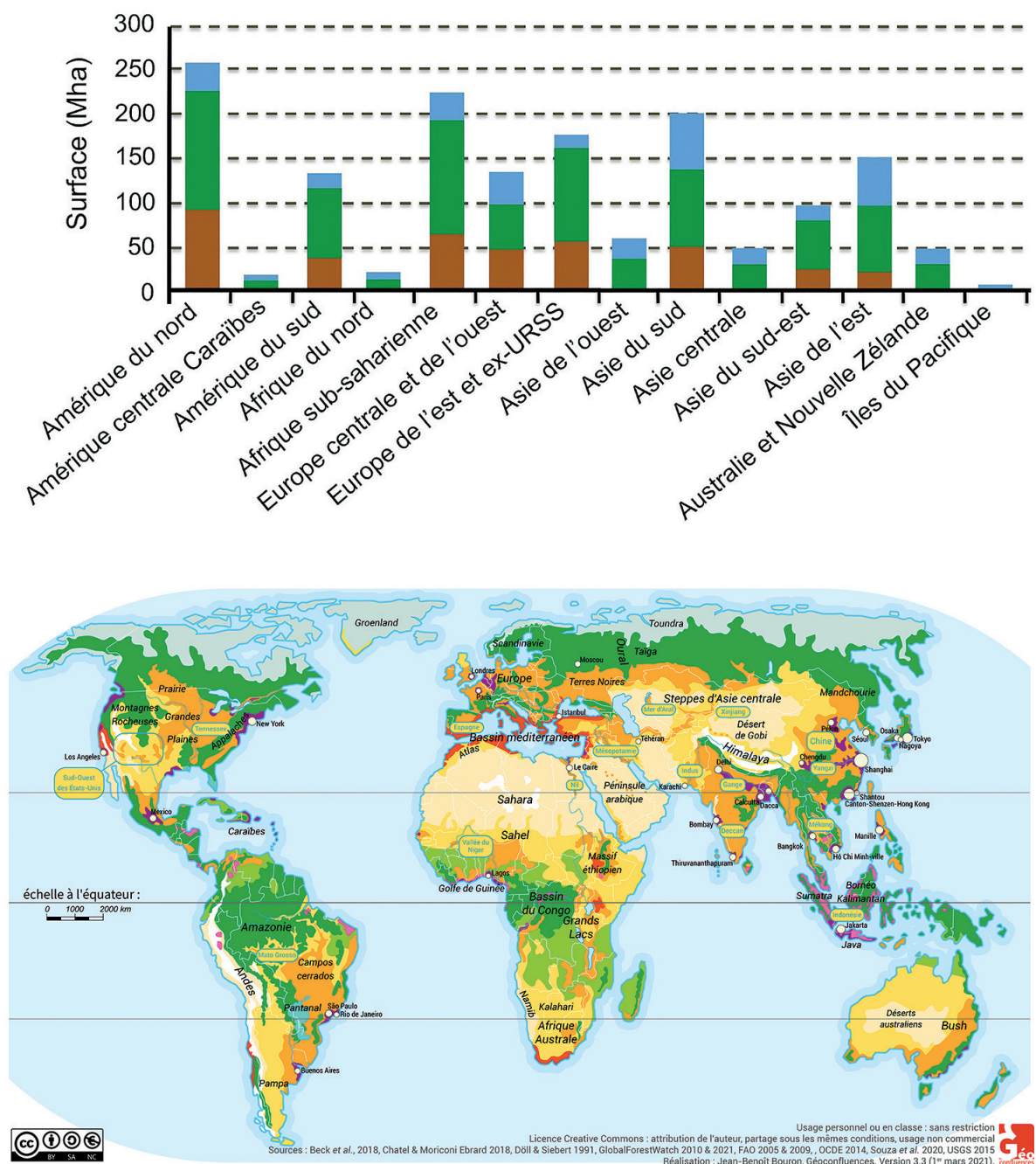

Terres arables ; $\square$ Systèmes méditerranéens ; $\square$ Plantations ; $\square$ Systèmes périurbains ; $\square$ Grands périmètres irrigués Forêts ; Savanes ; $\square$ Prairies ; Déserts ; $\square$ Montagnes ; $\square$ Milieux arctiques ; $\square$ Grands biomes humides

Figure 2.21: En haut: graphique montrant la qualité des sols dans le monde. En bas: carte des systèmes agricoles. Source: Creative commons, http://geoconfluences.ens-lyon.fr/informationsscientifiques/a-la-une/carte-a-la-une/systemes-agricoles-monde. 
Au niveau mondial, durant les 50 dernières années, la superficie cultivée nette a augmenté de $12 \%$, principalement au détriment des forêts, des zones humides et des prairies. Les savanes arborées et les forêts tropicales sont les principales sources de nouvelles terres agricoles et disparaissent rapidement, en particulier, les forêts primaires. D'ici 2050, en raison de la pression démographique, du changement de régime alimentaire et de la demande de biocarburant, la demande de nouvelles terres agricoles devrait augmenter d'environ 50\% [10]. II est très probable que les forêts tropicales seront la principale zone d'expansion et qu'une nouvelle déforestation est à prévoir sur des sols propices à une érosion. Ces estimations sont néanmoins dépendantes de l'intensification de l'agriculture à venir, ainsi que du commerce mondial. Les mécanismes sont complexes puisque l'on peut imaginer, par exemple, que la protection de certains écosystèmes à un endroit donné pousse à des importations qui occasionnent une dégradation ailleurs sur la planète [11].

Parmi les facteurs qui déterminent le potentiel agricole et forestier d'un sol, la capacité à retenir les nutriments est importante. Dans les sols des régions de forêts tropicales, en particulier dans de nombreux pays d'Afrique subsaharienne, au Brésil, en Australie ou dans les pays nordiques d'Europe, c'est la principale contrainte à la production agricole. En exportant des nutriments, l'exploitation de ces sols par une production agricole ou forestière les appauvrit rapidement. Ainsi, la transformation d'une forêt tropicale en plantations agricoles épuise souvent rapidement la matière organique. La faible profondeur des sols est une autre contrainte forte pour l'agriculture, par exemple sur le pourtour méditerranéen, par opposition aux très profondes terres noires d'Ukraine ou certains sols de plaines en Amérique centrale. La mauvaise capacité de drainage au nord de la Russie et de l'Europe centrale, par exemple, et les forts dénivelés en Asie du sud-est et dans les pays andins sont également des contraintes.

Les sols sont sujets à de nombreuses agressions anthropiques. La salinité croissante au sud des États-Unis, au Moyen-Orient, au Bangladesh ou sur les pourtours de l'Australie, est surtout provoquée par l'irrigation qui vide les nappes phréatiques et y fait entrer des eaux salines. Dans le pourtour méditerranéen, en Espagne par exemple, des terres devenues plus salées deviennent impropres à la culture. Plus généralement, dans les terres exploitées par une agriculture intensive, la diminution de la teneur en matière organique carbonée est un phénomène mondial, qui fait que le sol en perd sa fertilité et que sa structure et sa porosité se modifient. II retient moins bien l'eau et perd sa capacité à filtrer et à recycler les effluents et les polluants et devient plus sensible à l'érosion. 


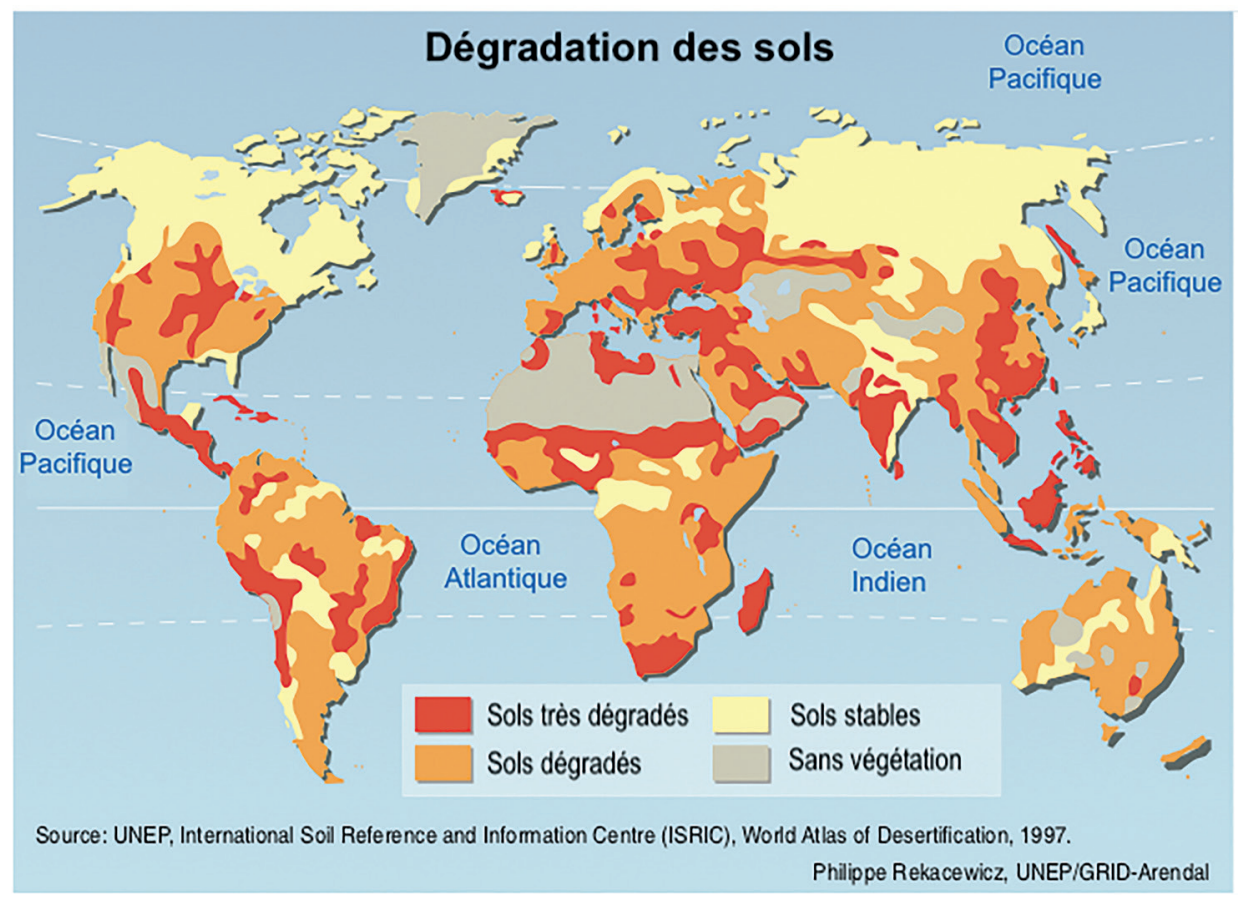

Figure 2.22: Dégradation des sols dans le monde. Source: United Nations Environmental Programme - GRID.

\subsubsection{L'érosion des sols}

L'érosion est une menace majeure. La FAO estime que près du quart des sols agricoles dans le monde est hautement dégradé (Fig. 2.22) et que cela constitue une menace majeure pour la sécurité alimentaire, en particulier dans les pays sous forte pression anthropique. Ainsi, l'Inde représente $18 \%$ de la population mondiale alors qu'elle ne dispose que de seulement $2 \%$ des terres agricoles. Des estimations portent à près de $80 \%$ la surface des terres agricoles mondiales touchées par une érosion modérée ou sévère [12]. En France, une étude de l'INRAE montre que de l'ordre de $18 \%$ des sols cultivés sont sujets à des problèmes d'érosion, de faibles à graves.

Les conséquences sont importantes, car des sols érodés sont moins productifs. Des pertes de rendement dues à l'érosion de l'ordre de $20 \%$ à $40 \%$ ont été mesurées aux États-Unis. De plus, les sols érodés ont une plus faible capacité de rétention d'eau et précipitent des coulées de boue et favorisent les inondations. L'érosion des sols limite aussi la capacité de l'agriculture à s'adapter au changement climatique et réduit fortement la biodiversité qu'ils contiennent. Or, ces sols ne se régénèrent que très lentement, sur une échelle de plusieurs siècles, voire millénaires. 
Les causes de l'érosion des sols varient selon les régions et n'appellent pas à une seule réponse. Dans certaines régions, elle est liée à un pâturage trop intensif par rapport à la capacité de régénération des milieux (pourtour méditerranéen). Les pratiques culturales comme le labour, la monoculture, et plus généralement l'exportation de nutriments par les cultures dans des sols peu profonds appauvrissent et érodent les sols de nombreux pays. Dans d'autres pays, c'est l'érosion éolienne, causée par le vent, qui est en cause, en particulier dans les régions de grandes plaines cultivées d'Amérique du Nord. L'érosion par l'eau peut être aussi une source de fuite des sols, comme en témoignent les rivières chargées de particules. La France n'y échappe pas. On observe notamment des coulées de boue et du ravinage même dans des régions de plaines comme le Bassin parisien, le Nord et la Normandie. Au niveau mondial, la destruction des couvertures végétales de type forêts ou savanes est souvent en cause dans l'érosion par l'eau.

\subsubsection{La pollution des sols}

La pollution des sols est très variable selon les pays, et souvent en relation avec la pollution de l'eau. Ainsi au Bangladesh, la gigantesque pollution à l'arsenic, des puits, des eaux de surfaces et des nappes phréatiques, mais aussi des sols, a pour origine la remontée de cet élément présent naturellement dans une roche souterraine, par les puits et forages (voir section 2.4.2). Cette pollution met en péril direct la vie de 30 millions d'habitants. La pollution au Radon, un gaz radioactif qui touche de nombreuses régions, y compris en France, a également une origine fossile. Diverses pollutions des sols, en particulier par certains métaux lourds, ont pour origine l'activité volcanique, même si elle est relativement distante.

Néanmoins, les graves pollutions des sols ont majoritairement une origine anthropique [13]. Sont notamment en cause des activités industrielles qui engendrent des pollutions aux métaux lourds, aux solvants ou aux effluents toxiques. Y contribue aussi le traitement, ou plutôt, l'absence de traitement des déchets, qui entraîne des pollutions par des matières organiques (lisiers, déjections humaines, eaux sales) ou par des éléments toxiques et radioactifs. Les activités d'extraction, de minerai et de pétrole, ne sont pas en reste, non plus, avec des pollutions qui se diffusent par l'eau et par l'air.

Parmi les pollutions d'origine agricole, les pollutions chimiques liées aux produits phytosanitaires peuvent être, dans certains cas, tenaces et difficilement réversibles. La pollution des sols à la chlordécone dans les Antilles en est un exemple emblématique. La chlordécone est un insecticide organochloré qui a été utilisé de 1971 à 1993 pour lutter contre le charançon du bananier et qui a entraîné une pollution généralisée des sols, des cultures, des eaux et des écosystèmes à l'exception de zones montagneuses où les plantations de banane n'étaient pas possibles. Or, la chlordécone est une molécule très stable et très persistante, et l'on estime que la pollution restera présente pour quelques 600 ans. La substance est cancérogène et l'on trouve des taux de cancer, en particulier de la prostate, significativement plus élevés 
que la moyenne nationale dans les Antilles françaises. Selon les résultats d'une étude de l'agence Santé publique France «plus de $90 \%$ de la population adulte» en Guadeloupe et Martinique est contaminée par la chlordécone ${ }^{31}$.

L'épandage d'engrais et de fumiers est aussi une source de métaux lourds très toxiques comme le cadmium ou le plomb qui s'accumulent dans les sols. Bien que l'on ne dispose que d'une information limitée, un récent rapport de la FAO montre l'ampleur du problème: en Australie, près de 80000 sites auraient leurs sols contaminés; en Chine, 19\% des sols agricoles sont pollués. Environ 3 millions de sites seraient potentiellement pollués en Europe...

\section{6. Échanges de carbone dans les sols - Ch. Hatté}

Pour bien appréhender la nécessité de protéger les sols vis-à-vis des phénomènes de dégradation et de pollution, il nous faut revenir sur l'importance des sols dans le cycle du carbone global et sur la capacité des sols à jouer un rôle d'atténuation dans le réchauffement climatique.

\subsubsection{Cycle du carbone et activités humaines}

Indiquons d'abord quelques ordres de grandeur (Fig. 2.23). Aujourd'hui, nous émettons environ dix milliards de tonnes (gigatonnes, Gt) de carbone par an par nos activités consommatrices d'énergie fossile. Sur ces dix gigatonnes, environ 4,5 gigatonnes s'accumulent dans l'atmosphère sous forme de $\mathrm{CO}_{2}$, correspondant à une augmentation de deux parties par million en volume (ppmv) de la concentration en $\mathrm{CO}_{2}$.

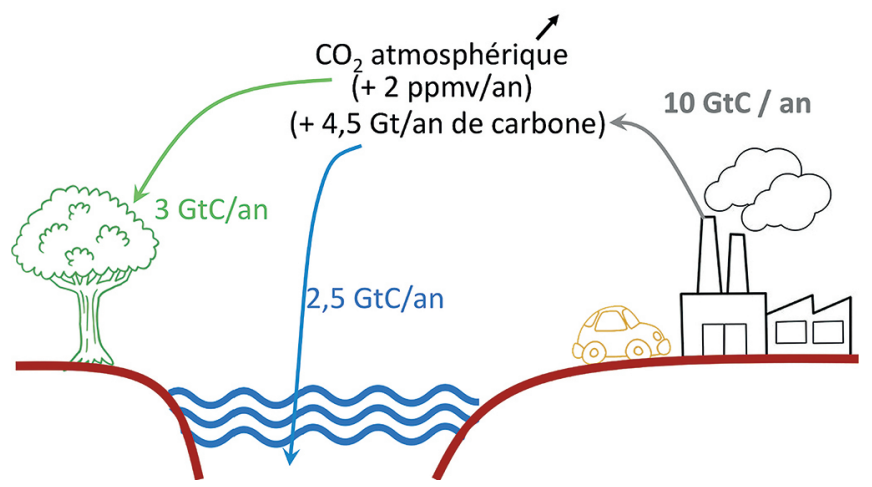

Figure 2.23: Répartition dans l'atmosphère, les océans et la biosphère du carbone émis par les activités humaines. Crédit: Christine Hatté.

31. Voir : https://www.youtube.com/watch?v=N8fTQH1qV8A.

Bien que la chlordécone ne soit plus utilisée, la population antillaise reste exposée. La molécule contamine particulièrement les légumes-racines (patates douces, carottes, ignames, etc.) en remontant dans la plante. Le lessivage des sols par la pluie conduit, par ailleurs, à suspecter la plupart des productions y compris les productions animales. Des mesures ont été prises pour réparer les préjudices économiques, notamment pour la pêche et l'agriculture. La pêche reste notamment interdite sur plusieurs zones. 
Entre les 10 Gt émises et les 4,5 Gt accumulées, l'écart est de 5,5 Gt. Ce sont ainsi 5,5 gigatonnes de carbone, qui quittent, chaque année, l'atmosphère pour être captées dans ce qu'on appelle des puits de carbone et se répartir entre l'océan et la biosphère. Environ 2,5 Gt sont absorbées par l'océan, et 3 Gt sont utilisées par la biosphère. Le flux de carbone depuis l'atmosphère vers l'océan résulte de processus physico-chimiques et d'un déséquilibre entre la concentration de $\mathrm{CO}_{2}$ dans l'atmosphère et la quantité de carbone dans l'océan. II y a davantage de carbone dans l'atmosphère que dans l'océan de surface si bien qu'un flux depuis l'atmosphère vers l'océan est possible et se produit de façon à équilibrer les deux réservoirs. Dans la biosphère, c'est la biologie qui est à l'œuvre avec le processus de photosynthèse. Ce processus, qui permet la croissance des plantes et de la végétation en général, se traduit par un prélèvement du $\mathrm{CO}_{2}$ atmosphérique. En retour, les plantes injectent du carbone dans les sols où il peut être stocké en grande quantité et pour longtemps.

\subsubsection{Stockage du carbone dans les sols}

Le carbone arrive dans les sols selon différentes voies. Ce qui est le plus connu c'est l'apport de carbone au sol par la litière qui se forme par la chute des feuilles et des écorces d'arbres (Fig. 2.24). Les feuilles sont décomposées par la faune du sol, les bactéries, les champignons, les vers de terre, etc. pour se retrouver dans le sol en tant que molécules élémentaires telles que des acides aminés, des sucres, des lipides qui vont eux-mêmes servir de nourriture de base aux microorganismes des sols. Mais cette contribution aérienne au carbone du sol n'est pas la seule. La contribution par les racines est très importante et d'ailleurs, souvent plus importante que celle de la litière. Du vivant des plantes, les racines exsudent, c'est-à-dire sécrètent des molécules, notamment des sucres qui alimentent les stocks de carbone du sol.

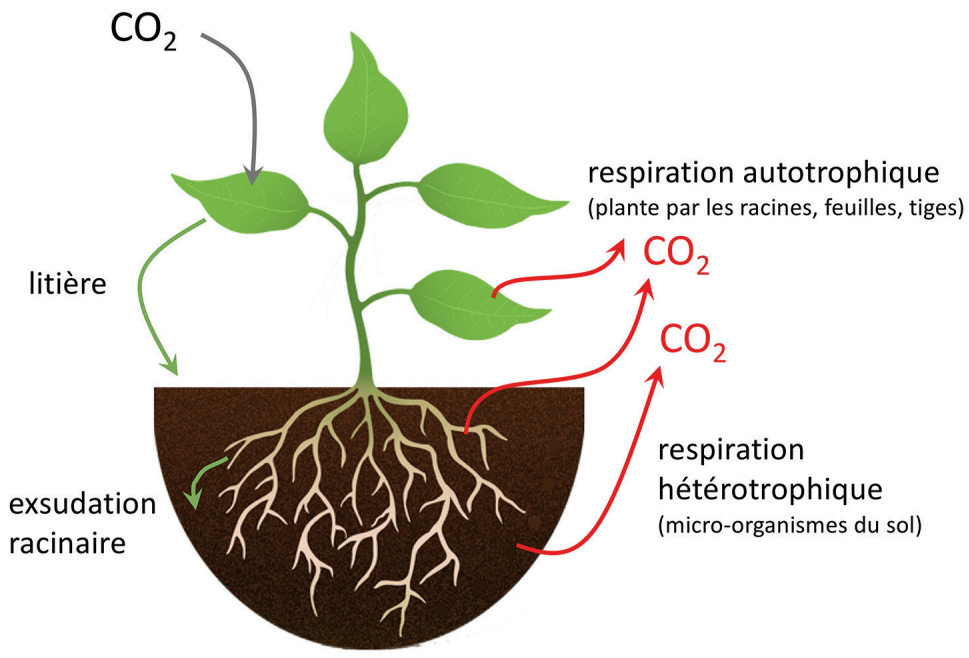

Figure 2.24: Piégeage du $\mathrm{CO}_{2}$ dans le sol et phénomènes de respiration. Source : Christine Hatté. 
Un stock, c'est un équilibre entre des entrées et des sorties. Nous venons de décrire les modalités d'apport du carbone dans le sol: par au-dessus, par la litière et par en dessous, par les racines. Les pertes de carbone, quant à elles, résultent essentiellement de l'activité microbiologique du sol. On peut aussi mentionner les pertes latérales par érosion et les pertes verticales par illuviation sous forme de matière organique dissoute, mais leur contribution respective est très variable d'un sol à l'autre. La respiration des microorganismes du sol, que l'on appelle la respiration hétérotrophique, produit du gaz carbonique. Afin de former leurs organes constitutifs, les microorganismes doivent assimiler des molécules élémentaires pour leur métabolisme, et la consommation de ces molécules pour en utiliser l'énergie se traduit par un «relargage» de $\mathrm{CO}_{2}$.

La respiration des plantes par leurs racines, respiration dite autotrophique, qui complète, pour la plante, la respiration des feuilles et des tiges, s'ajoute à cette respiration du sol. C'est cette fois le même organisme, la plante, qui capte du $\mathrm{CO}_{2}$ de l'atmosphère par photosynthèse et relargue du $\mathrm{CO}_{2}$ par respiration. Les algues présentes dans les sols en font de même. On a donc deux sources de $\mathrm{CO}_{2}$ émises par les sols: la respiration hétérotrophique des microorganismes et la respiration autotrophique des plantes. La résultante entre les entrées et les sorties de carbone nous donne ainsi accès au stock de carbone.

La répartition du carbone dans les sols n'est pas homogène (Fig. 2.25). II y a davantage de carbone en surface qu'en profondeur, mais les chiffres qui définissent les stocks sont encore mal connus. On estime à environ $700 \mathrm{Gt}$ le stock de carbone organique dans les premiers $30 \mathrm{~cm}$, à $800 \mathrm{Gt}$ celui des $70 \mathrm{~cm}$ qui suivent pour arriver à $1500 \mathrm{Gt}$ dans le premier mètre de profondeur. On évalue à environ $2400 \mathrm{Gt}$ le stock de carbone organique, si on considère les deux premiers mètres, mais tous les sols n'ont pas deux mètres de profondeur. Certains sols sont très peu profonds, quelques centimètres, quelques dizaines de centimètres. C'est, par exemple, le cas sur le pourtour de la Méditerranée.

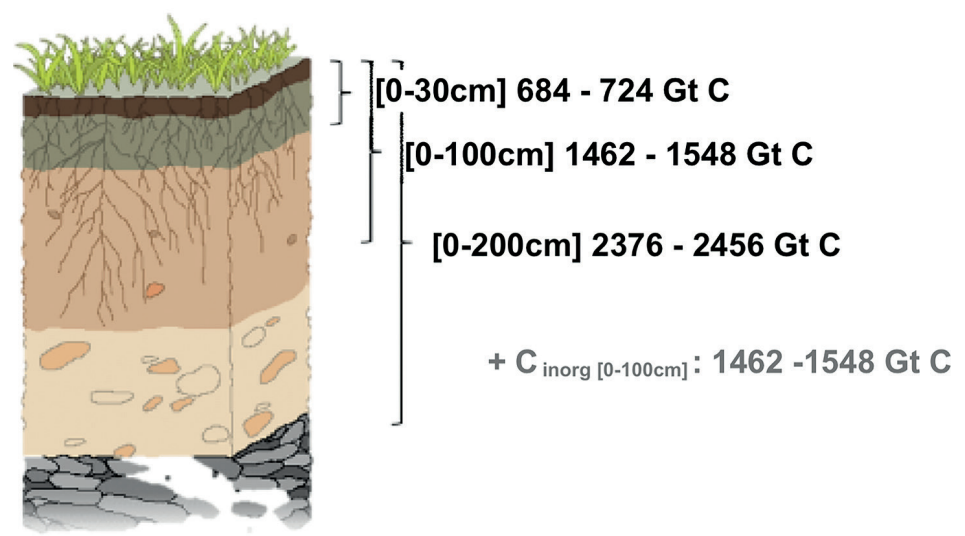

Figure 2.25: Répartition du carbone dans les sols suivant la profondeur. D’après [14]. 


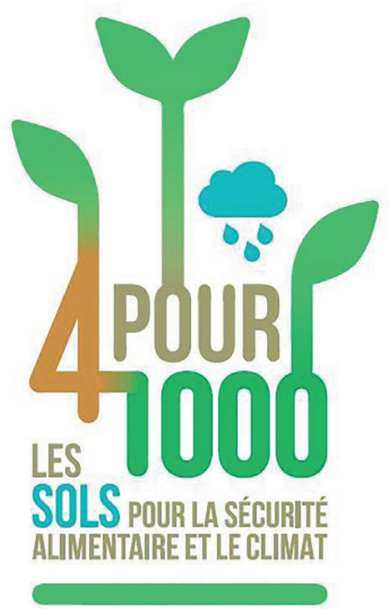

Figure 2.26: Initiative des $4 \%$ lancée lors de la COP21 à Paris en 2015 (source: IRD)
Prenons néanmoins comme hypothèse l'estimation globale de 2400 Gt de carbone dans les sols. Cette valeur représente plus de deux fois ce que l'atmosphère contient comme carbone. Si on la compare maintenant aux $10 \mathrm{Gt}$ de carbone émis annuellement par nos activités anthropiques sous forme de $\mathrm{CO}_{2}$, on réalise que ces émissions annuelles ne représentent que 0,4\% (ou encore $4 \%$ ) du carbone stocké dans les sols. Ainsi, si on était capable d'augmenter de $4 \%$ par an le stock de carbone des sols, on pourrait alors compenser totalement les émissions de $\mathrm{CO}_{2}$ d'origine anthropique. Ce calcul simple, énoncé dès 1999 par Jérôme Balesdent à l'Académie de l'Agriculture [15] a été à l'origine de l'initiative internationale des $4 \%$, lancée par la France au moment de la COP21 à Paris: «les sols pour la sécurité alimentaire et pour le climat» (Fig. 2.26).

\subsubsection{Comment augmenter le stockage du carbone dans les sols?}

Stocker plus de carbone dans les sols, a un double avantage. D'une part, on obtient des sols plus fertiles grâce à un meilleur stockage de l'eau et un meilleur apport d'éléments nutritifs aux plantes. D'autre part, on déplace les équilibres dans le cycle du carbone en limitant l'accumulation de $\mathrm{CO}_{2}$ dans l'atmosphère et en le stockant dans les sols.

Les sols constituent, de fait, le seul "grand» réservoir de carbone sur lequel on peut agir pour limiter l'accumulation du $\mathrm{CO}_{2}$ atmosphérique et atténuer le réchauffement climatique. En effet, on peut, par des pratiques vertueuses, jouer sur les stocks de carbone dans les sols alors qu'on ne peut pas moduler les équilibres physico-chimiques de l'océan. Bien évidemment, cela ne nous empêche pas de rechercher en parallèle des pratiques industrielles et individuelles plus économes en énergie.

Les stocks de carbone sont très différents selon les types de sols et l'usage que l'on en fait (Fig. 2.27). Les sols des forêts tropicales sont les plus riches en carbone. Sous nos latitudes, les forêts tempérées sont aussi les écosystèmes les plus riches en carbone, notamment par un stockage important en profondeur. À l'autre bout de l'échelle, les vignobles correspondent aux sols les plus pauvres. Dans les sols de culture, les stocks y sont très variables, mais souvent faibles par rapport à ce qu'aurait été le stock dans un sol situé au même endroit et en équilibre avec la végétation naturelle. Cela étant, les teneurs en carbone dépendent surtout des pratiques de culture, aussi bien actuelles que passées, car l'on paye sur des décennies ce qui a été fait pendant quelques années. On peut néanmoins revenir à une situation 
acceptable des sols de culture en changeant de pratiques et en prenant les parcours agronomiques adaptés.

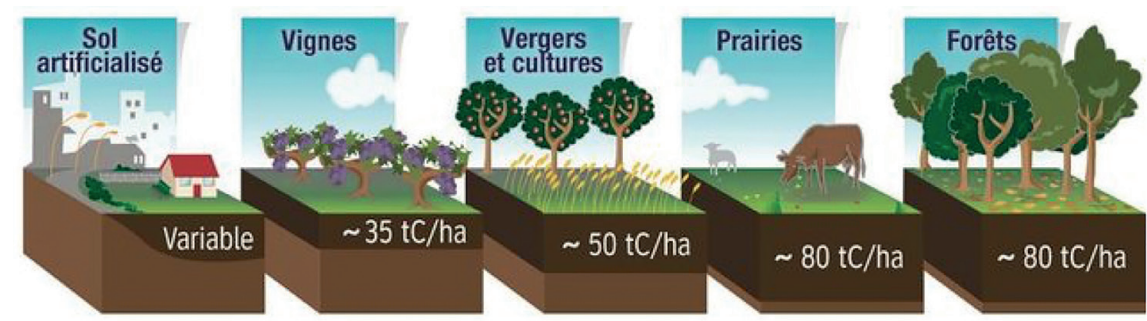

Figure 2.27: Estimation du stock de carbone (en tonnes par hectare) dans les 30 premiers centimètres de sol pour différents types de sols. Source: ADEME Gis Sol 2013.

Les pratiques d'agriculture intensive mises en place ces dernières décennies ont conduit à une perte massive du carbone dans les sols. Les parties aériennes des plantes n'étaient plus rendues aux sols et les labours jusqu'à $30 \mathrm{~cm}$ de profondeur ont contribué à une respiration massive des sols (par oxydation) ainsi qu'à une perte de biodiversité. La perte de carbone s'est alors traduite par une déstructuration des sols qui sont devenus encore plus sujets à l'érosion. Stopper ces pratiques, favoriser le couvert permanent, limiter le labour et alterner les cultures pour plus de biodiversité et plus d'apport de nutriments aux sols, tout cela est propice à une réduction des pertes en carbone et donc à un stockage accru du carbone dans les sols.

Les sélections agronomiques ont également conduit à une modification de la répartition du carbone entre les organes des plantes, que l'on appelle aussi les puits de carbone de la plante. De tout temps, on a sélectionné et, plus récemment, on a modifié génétiquement les plantes afin de favoriser le grain aux dépens des racines et des parties foliaires. Un retour à un équilibre plus naturel entre ces puits de carbone pour la plante pourrait contribuer à des apports de carbone plus importants aux sols, sans pour autant trop diminuer les rendements au point que les cultures ne soient plus rentables.

De ce point de vue, un nouveau mode d'évaluation de la rentabilité des cultures peut être un facteur clé pour l'avenir. Prendre en compte le bien-être de l'agriculteur, et pas uniquement les flux financiers entre coûts des intrants et gain par la vente des produits, permettrait d'avoir une vision plus juste, plus intégrative du système agricole. Chez les semenciers, considérer les flux financiers vers les actionnaires serait aussi un moyen de mieux considérer l'agriculture de conservation, l'agroécologie par rapport à l'industrie agricole intensive (voir section 11.2.2 plus loin dans l'ouvrage).

Aussi que ce soit par les parcours agricoles, les choix agronomiques ou les modèles économiques, tout concourt à une vision positive de l'agriculture qui serait à la fois favorable à la sécurité alimentaire et à la limitation du réchauffement climatique. 


\subsubsection{Retour sur les modèles de carbone dans les sols}

Le monde des sciences des sols est en pleine révolution. II redéfinit la vision qu'il avait du carbone dans les sols. Dans les années 1980s, on imaginait que le carbone dans les sols était divisé en trois catégories: le carbone labile qui resterait assez peu de temps dans le sol, en moyenne une année entre le moment où il y pénètrerait et le moment où il en ressortirait, le carbone stable qui resterait dans les sols pendant plusieurs siècles, voire plusieurs millénaires, et le carbone intermédiaire avec des temps de résidence moyen de l'ordre de la décennie. Pendant longtemps, on a essayé de mettre en évidence le type de matière organique à l'origine de ces différents réservoirs, la taille de molécule, le type de molécule, la densité des molécules, mais rien n'était conclusif.

De nouveaux modèles conceptuels sont alors nés et l'imagerie a confirmé ces modèles grâce à des images de plus en plus résolues. Selon ces modèles, le stockage de carbone dans les sols est régi par des interactions entre les particules minérales (les argiles) et les molécules organiques (riches en carbone), et non plus uniquement entre les molécules organiques. Le modèle le plus consensuel est celui dit de Kleber [16], selon lequel les molécules organiques et les particules minérales s'organisent en structures multicouches. La figure 2.28 l'illustre dans le cas d'une particule d'argile (au centre, en marron). Une première couche de molécules organiques, dite de contact, se forme par interaction chimique avec l'argile. Viennent ensuite s'empiler d'autres couches moléculaires (du centre vers l'extérieur), l'intensité des interactions diminuant au fur et à mesure que l'on s'éloigne de l'argile centrale. On retrouve, au final, les mêmes molécules dans la couche externe et dans la couche de contact. Dans un sol en bonne santé, les trois couches sont bien développées alors que dans un sol dégradé, les couches externes et intermédiaires, voire même les couches de contact, sont fortement réduites.

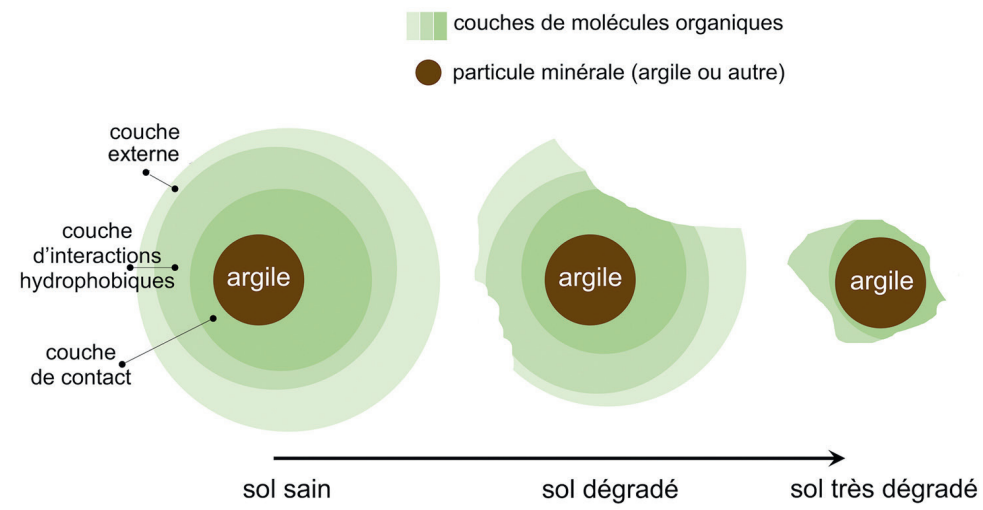

Figure 2.28: Structure multicouche associant une particule d'argile et des molécules organiques riches en carbone, selon le modèle de Kleber [16]. La réduction des couches organiques, externes, intermédiaires, voire de contact, conduit à des sols de plus en plus dégradés (de gauche à droite). Source : Christine Hatté 
Un modèle complémentaire émerge aujourd'hui, celui de la stabilisation du carbone par des interactions aux très petites échelles entre structures minérales et molécules organiques. Les molécules organiques seraient à l'origine d'une modification structurelle des surfaces minérales et se combineraient alors avec des groupements minéraux actifs pour former des ensembles stables.

Quel que soit le modèle, une modification des conditions environnementales, comme le changement de $\mathrm{pH}$ ou de quantité d'eau, en lien avec des paramètres climatiques, agricoles (labours) ou agronomiques (changement de culture) impacte le type d'interaction entre molécules et l'énergie qui y est associée. Les couches extérieures du millefeuille peuvent se désagréger et dans les cas les plus pénalisants, l'empilement complet peut être dégradé. Libérer les molécules les rend davantage accessibles aux micro-organismes des sols, qui vont les surconsommer. Elles participent aussi davantage aux processus de respiration physique ou de «lessiviation». Dans ce dernier cas, les molécules quittent le sol en partant en solution.

II est toujours compliqué de s'écarter de conditions d'équilibre qui n'existent vraiment que pour les prairies pérennes et les forêts. De ce point de vue, l'agriculture doit être l'art du meilleur compromis.

Parmi les fausses bonnes idées, l'afforestation, c'est-à-dire planter une forêt là où, naturellement, il ne devrait pas y en avoir, peut se révéler dommageable sur la durée. En effet, l'exsudation racinaire est déjà constituée de sucres qui sont la source d'énergie des microorganismes des sols. En ajoutant des sucres par une exsudation exacerbée, les populations de microorganismes augmentent et s'attaquent alors à des molécules qu'ils délaissaient jusqu'alors. L'apport de sucre au sol a donc bien pour premier effet d'augmenter le stock du carbone dans les sols en lui apportant davantage de molécules carbonées synthétisées par les microorganismes. Cependant, il s'agit de molécules simples, facilement assimilées ou respirées par d'autres microorganismes, et finalement le carbone ajouté ne restera pas longtemps dans le sol. Par ailleurs, les molécules, que les microorganismes délaissaient jusqu'alors et qu'ils se sont mis à consommer, étaient en dehors du cycle du carbone actif depuis un moment, peut-être des décennies, voire des siècles.

En définitive, la notion de durée du nouveau stockage est primordiale quand on souhaite augmenter le stock de carbone dans les sols.

\section{Références}

[1] R. Mosseri, C. Jeandel. 2013. L'énergie à découvert., CNRS Éditions.

[2] BP. 2020. BP Statistical Review of the World Energy, 68 p. www.bp.com/content/dam/bp/ business-sites/en/global/corporate/pdfs/energy-economics/statistical-review/bp-statsreview-2020-full-report.pdf.

[3] K. Hubbert. 1956. Nuclear energy and the fossil fuels. Shell development Company, Publication n 95, 57 p. http://www.energycrisis.com/Hubbert/1956/1956.pdf. 
[4] Environmental Protection Agency. 2015. Petroleum refining, in Air Emission Factors and $A P$ 42, 5 ${ }^{\text {th }}$ Ed., 21 p. https://www3.epa.gov/ttn/chief/ap42/ch05/final/c05s01_2015.pdf.

[5] R. Vially. 2012. Les hydrocarbures non conventionnels: évolution ou révolution?. Panorama 2012, IFP Energies nouvelles, 14 p. https://inis.iaea.org/collection/ NCLCollectionStore/_Public/43/095/43095221.pdf.

[6] F. Lantz, D. Bouchonneau, A. Saniere. 2012. Analyse économique des hydrocarbures non conventionnels, rapport CFE n 55.

[7] C. Dumas. 2018. La réduction des émissions de soufre dans le transport maritime: défi économique et technologique. IFP Energies nouvelles, 9 p. https://www.ifpenergies nouvelles.fr/article/reduction-des-emissions-soufre-transport-maritime-defi-economiqueet-technologique.

[8] La pollution de l'air. Annales des Mines 96 (Octobre 2019).

[9] Données: Encyclopedia of land use, land cover and soil sciences. Edited by W.H. Verheye. Chapitre 5, G. Fischer, et al. "Agro-Ecological Zones Assessments", 2010.

[10] J. Bruinsma. 2011. By how much do land, water and crop yields need to increase by 2050? FAO, Rome, Italy. http://www.fao.org/fileadmin/templates/esa/Global_persepctives/ Presentations/Bruinsma_pres.pdf.

[11] E.F. Lambin, P. Meyfroidt. 2011. Global land use change, economic globalization, and the looming land scarcity. PNAS 2011108, 3465-3472. https://www.pnas.org/content/108/9/3465.

[12] D. Pimentel, A. Burgess. 2013. "Soil erosion threatens food production". Agriculture 3, 443-463. https://www.mdpi.com/2077-0472/3/3/443.

[13] FAO. 2018. Soil pollution, a hidden reality. http://www.fao.org/3/19183EN/i9183en.pdf.

[14] N.H. Batjes. 1996. Total carbon and nitrogen in the soils of the world. European Journal of Soil Science 47 (2), 151-163.

[15] J. Balesdent, D. Arrouays. 1999. Usage des terres et stockage de carbone dans les sols du territoire français. Une estimation des flux nets annuels pour la période 1900-1999. Compte-rendu de l'Académie de l'Agriculture 85, 265-277.

[16] M. Kleber, P. Sollins, R. Sutton. 2007. A conceptual model of organo-mineral interactions in soils: self-assembly of organic molecular fragments into zonal structures on mineral surfaces. Biogeochemistry 85, 9-24 (2007). DOI : 10.1007/s10533-007-9103-5. 



\section{Chapitre 3}

\section{Empreinte écologique et démographie}

L'empreinte écologique est souvent reprise dans les médias sous la forme du «jour de dépassement de la Terre», date à laquelle l'humanité a consommé l'ensemble des ressources que la planète peut produire en une année, l'humanité vivant donc à crédit après cette date. Ainsi, le jour de dépassement de la Terre tombait le 20 décembre en 1971, le 23 septembre en 2000, le 29 juillet en 2019 avant de se décaler exceptionnellement au 22 août en 2020 en raison de la baisse de notre consommation de ressources due à la pandémie de la Covid-19. Dans ce chapitre, nous verrons, de façon plus précise, que l'empreinte écologique vise à quantifier l'interaction entre humains et biosphère au travers des flux de production et de consommation issus de celle-ci. Notre consommation globale n'est évidemment pas sans rapport avec la population humaine, qui est passée d'environ 1,13 milliards, au début de l'ère industrielle vers 1800, à 7,8 milliards en 2021. Nous aborderons ainsi les mécanismes à la base de l'évolution démographique dans la deuxième partie de ce chapitre en montrant que pour une grande part, l'accroissement récent de la population n'a fait que refléter l'accroissement de notre espérance de vie.

\subsection{Une introduction à la comptabilité environnementale : l'empreinte écologique - C. Even et G. Roux}

\subsubsection{La «naissance » du développement durable}

Historiquement, la notion d'empreinte écologique s'est inscrite dans le concept de développement durable. Pour faire court et un peu caricatural, le développement 
durable est attaché à la vision onusienne du développement humain ${ }^{32}$. Après la seconde guerre mondiale, la volonté d'améliorer le bien-être humain à l'échelle mondiale s'est traduite par la création d'agences soutenant la lutte contre la faim, le contrôle de la démographie, l'éducation, la santé et la paix. Le développement économique est alors vu comme le principal levier d'amélioration des conditions de vie. Pourtant, les limites environnementales en termes de pollutions et de ressources se manifestent d'autant plus que la pression anthropique s'accroît.

Afin de tenter de concilier développement humain et maintien des conditions de vie, une commission mondiale sur l'environnement et le développement voit le jour au début des années 1980 et publie à son issue "Notre avenir à tous» ou "rapport Brundtland» du nom de la présidente de la commission. Ce rapport a servi de base à la préparation du sommet de la Terre à Rio en 1992, qui est généralement considéré comme une avancée majeure dans la prise en compte de l'écologie au niveau international. Le «développement durable» ou «soutenable» y est alors défini comme suit: «Le développement durable est un mode de développement qui répond aux besoins des générations présentes sans compromettre la capacité des générations futures de répondre aux leurs».

Deux concepts sont inhérents au développement soutenable: le concept de "besoins», et plus particulièrement les besoins essentiels des plus démunis, à qui il convient d'accorder la plus grande priorité, et l'idée des limitations que l'état de nos techniques et de notre organisation sociale impose sur la capacité de l'environnement à répondre aux besoins actuels et à venir. Des volontés fortes s'affirment en parallèle: une volonté d'équité ou de justice intragénérationnelle, en assurant des conditions de vie décentes au sein d'une génération, mais aussi intergénérationnelle en faisant en sorte de donner aux futures générations les moyens de garantir les mêmes conditions de vie.

Dans ce contexte, un certain nombre d'économistes ont essayé, et essaient toujours, de mesurer si nos activités humaines sont ou non soutenables. Dès 1972 , avec le rapport Meadows sur les limites à la croissance économique, la plupart des indicateurs font état d'une tendance rapide au dépassement. L'empreinte écologique, quant à elle, a été introduite par les économistes William Rees et Mathis Wackernagel au début des années 1990 pour étudier les flux de consommation de ressources et de production de déchets pour la ville de Vancouver au Canada. Ceux-ci ont jeté les bases d'une méthode de comptabilité qui s'est ensuite étoffée et popularisée auprès du grand public avec la parution de leur livre Our Ecological Footprint en 1996. Leur méthode a alors été reprise par les décideurs, en particulier ceux à l'ONU, très sensibles aux approches économiques. Elle est désormais promue par

32. Encyclopédie Wikipedia: page sur le développement durable (https://fr.wikipedia.org/wiki/D\%C3\% A9veloppement_durable) et sur l'empreinte écologique (https://fr.wikipedia.org/wiki/Liste_des_ pays_par_empreinte_\%C3\%A9cologique) 
l'organisation non gouvernementale Global Footprint ${ }^{33}$, créée en 2003 et qui publie annuellement la date du jour du dépassement en collaboration avec le World Wide Fund for Nature (WWF). La date du 31 décembre correspond ainsi à une consommation soutenable de ressources au sens de l'empreinte écologique. L'empreinte écologique fait toujours, jusqu'à aujourd'hui, l'objet de travaux universitaires qui discutent les données utiles pour les calculs ainsi que les aspects méthodologiques [1-3].

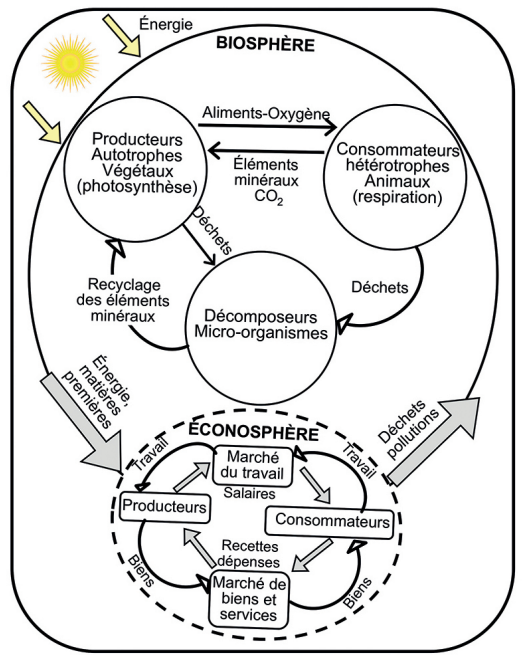

Figure 3.1: Représentation schématique de l'interaction entre biosphère et éconosphère (activités humaines économiques). D’après [1].

\subsubsection{Qu'est-ce que l'empreinte écologique plus précisément?}

Comme nous l'avons indiqué, il s'agit d'une approche comptable sous-tendue par une vision économique de la biosphère en la considérant comme pourvoyeuse d'offres en ressources naturelles, par exemple pour la production alimentaire, et capable d'assurer des services tels que l'élimination de nos déchets (voir, par exemple section 4.5). Dans ce cadre, les sociétés sont décrites par leurs activités économiques, "l'éconosphère», extrayant des flux d'énergie et de matières premières de la biosphère et y rejetant déchets et pollutions (Fig. 3.1). L'empreinte écologique se propose d'établir un bilan quantitatif de ces flux annuels. La biosphère est capable, tous les ans, de régénérer des ressources ou d'assimiler des déchets. Prenons l'exemple d'une forêt. La quantité de bois, le stock, pousse un peu tous les ans. La gestion n'est durable que si l'on prélève au maximum ce qui est régénéré en une année. Dans le cas contraire, le stock de bois diminue avec l'éventualité de disparaître un jour. Le même raisonnement se transpose facilement à la pêche. 
Une difficulté se pose néanmoins quand on considère la diversité des productions et des émissions liées aux activités humaines, car il devient difficile de suivre l'évolution parallèle de tous les bilans de ressources et de pollutions. Pour simplifier les analyses et essayer d'avoir un paramètre de suivi, voire de contrôle, les économistes introduisent habituellement un indice synthétique. II s'agit d'un nombre construit pour agréger un grand nombre de quantités et censé refléter l'évolution typique d'un système. Un des indices économiques les plus connus est, par exemple, le Produit Intérieur Brut (PIB) (voir section 2.1.7). Son but est d'estimer la production économique d'un pays sur la base de données monétaires, avec plusieurs méthodes de calcul. In fine, il agrège de multiples contributions qui, grosso modo, correspondent aux revenus que l'on peut se partager tous les ans au niveau d'un pays.

La croissance du «gâteau à partager » est actuellement vue comme le principal levier de financement dans le développement des sociétés. Cependant, le PIB qui agrège des valeurs de nature différente, oublie nombre d'activités non monétarisées et induit parfois des biais délétères pour les sociétés, comme, par exemple, le fait que des catastrophes d'origines humaines ou naturelles sont paradoxalement susceptibles d'accroître le PIB. II va en être de même pour l'empreinte écologique qui, comme indicateur agrégé, peut avoir des effets contre-productifs et omettre ou mal représenter des composantes essentielles de l'interaction entre biosphère et éconosphère. Nous reviendrons sur les limites de l'empreinte écologique un peu plus tard (section 3.1.6).

De manière synthétique, nous appellerons biocapacité l'offre en ressources naturelles renouvelables, autrement dit, la quantité des services que la biosphère est capable de régénérer. Le terme d'empreinte écologique correspondra alors à la demande en ressources naturelles renouvelables, intégrant à la fois les ressources nécessaires et la capacité à assimiler les déchets produits. II s'agit donc de la quantité de services issus de la biosphère nécessaires pour faire fonctionner la sphère économique. La comparaison des deux nous dira si les activités sont soutenables ou non, comme schématisé sur la figure 3.2. La différence entre biocapacité et empreinte écologique définit le déficit écologique:

Déficit écologique = biocapacité - Empreinte écologique

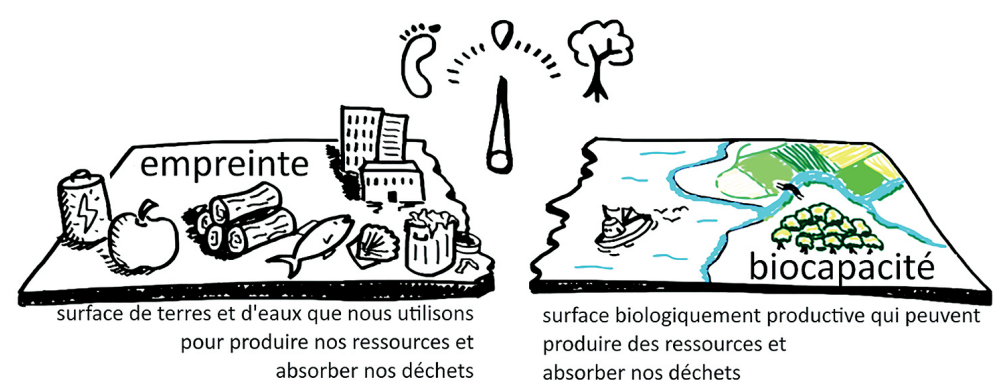

Figure 3.2: Représentation schématique du déficit écologique comme différence entre empreinte et biocapacité. D'après https://www.youtube.com/watch?v=g_aguo7V0Q4. 


\subsubsection{Comment calculer l'empreinte écologique?}

Le PIB est construit sur la base de données monétaires, qu'il faut parfois corriger de l'inflation et des taux de change. Pour l'empreinte écologique, il va falloir agréger des données de production qui n'ont a priori rien à voir entre elles, et donc trouver la manière d'additionner "des choux fleurs et des carottes». La première étape de la comptabilité est donc de trouver une unité commune dans laquelle convertir les données brutes que l'on pourra alors additionner et soustraire à notre guise. Par ailleurs, la comptabilité doit se baser sur des données accessibles et régulièrement mesurées. Pour les ressources à l'échelle des pays, l'idée est d'utiliser les grands inventaires nationaux de consommation et de production (alimentaires, énergétiques...).

Les ressources, que nous utilisons, sont, pour l'essentiel, quantifiables en surfaces de sol. Par exemple, les ressources issues des forêts et de l'agriculture proviennent de la photosynthèse, elle-même tributaire du flux lumineux incident et donc de la surface au sol qui le reçoit. Même s'il existe évidemment des différences de rendement de production, une unité surfacique est donc un bon choix. C'est ainsi que l'on introduit la notion d'hectare global, qui va jouer le rôle de monnaie pour comptabiliser l'empreinte écologique. Avant de définir plus précisément ce qu'est un hectare global, commençons par rappeler les ordres de grandeur de différentes surfaces à avoir en tête. Tout d'abord, un hectare (noté ha) représente une superficie de $10000 \mathrm{~m}^{2}$, soit la surface d'un carré de $100 \mathrm{~m}$ de côté (10 000 = $100 \times 100$ !), proche de celle d'un terrain de football, d'environ 0,7 ha.

À plus grande échelle, on exprime souvent les surfaces en $\mathrm{km}^{2}$ si bien qu'il est utile de rappeler qu'un kilomètre carré vaut cent hectares $\left(1 \mathrm{~km}^{2}=100\right.$ ha). En France, une exploitation céréalière gérée par une ou quelques personnes fait typiquement 100 ha. La ville de Paris intra muros fait 10000 ha ou $100 \mathrm{~km}^{2}$ ce qui correspond à un disque d'environ $5 \mathrm{~km}$ de rayon. La France métropolitaine fait environ $550000 \mathrm{~km}^{2}$ et comprend 100 départements, ceux-ci font donc typiquement $550000 / 100=5500 \mathrm{~km}^{2}$. Passons à l'échelle planétaire, la Terre est une sphère de rayon $\mathrm{R}=6400 \mathrm{~km}$ environ si bien que la surface totale de la Terre $\left(4 \pi R^{2}\right)$ est de $510000000 \mathrm{~km}^{2}=510 \mathrm{Mkm}^{2}$ = 51 Gha où les lettres $M$ et $G$ correspondent respectivement à Mega (million ou $10^{6}$ ) et Giga (milliard ou 109). Le plus grand pays, la Russie, s'étend sur une surface de $17 \mathrm{Mkm}^{2}$, les autres grands pays, le Canada, les États-Unis, la Chine, le Brésil, l'Australie et l'ensemble des pays européens (jusqu'à l'Oural) ont des surfaces de l'ordre de 7-10 Mkm².

Si on regarde maintenant la surface typique par habitant, pour les 66 millions de français, elle est d'environ 0,8 ha par personne ! La densité de population est simplement l'inverse de ce chiffre, soit 1,2 personne/ha ou 120 personne/ $/ \mathrm{km}^{2}$, ce qui est d'ailleurs proche de la moyenne mondiale. C'est sur ces 0,8 ha que nous devons tirer nos ressources renouvelables (bois, cailloux, alimentation, eau, etc.), c'est-à-dire un terrain de football par personne! En réalité, il s'agit d'une moyenne, car nous savons 
tous que la population est inégalement répartie sur le globe avec de très fortes densités en ville (2 millions de parisiens sur $100 \mathrm{~km}^{2}$, soit 20000 personnes $/ \mathrm{km}^{2}$ ) et des densités beaucoup plus faibles à la campagne. Cela souligne la forte dépendance des villes en termes de surfaces productives.

À ce stade, il va nous falloir relier les productions aux surfaces et c'est ici qu'intervient la notion d'hectare global. Les surfaces directement utiles au sens économique sont appelées surfaces bioproductives. Les productions issues des surfaces exploitées par l'homme sont très diverses et difficilement comparables: bois, céréales, élevage, pêche, énergie, etc. Le point clé est donc de définir une «utilité» relative de ces différentes surfaces pour les humains. Pour l'empreinte écologique, nous allons considérer qu'un hectare de céréales a une plus forte utilité productive qu'un hectare de pâturage, de pêche ou de forêt, car il produit une alimentation de base pour la population ou le bétail. L'hectare global (en abrégé hag) permet de tenir compte de la valeur bioproductive de l'hectare considéré (ha) en le comparant à un hectare typique de référence. Autrement dit, la superficie d'une surface bioproductive, exprimée en hag, s'obtient en multipliant la surface réelle (en ha) par un facteur, appelé facteur d'équivalence, qui traduit son utilité relative par rapport à une surface typique de référence:

\section{Surface bioproductive $($ hag) $=$ Facteur d'équivalence [hag/ha] x Surface en hectares [ha]}

La liste des facteurs d'équivalence est rapportée dans le tableau 3.1. Comme nous l'avons mentionné, un champ cultivé possède un facteur d'équivalence supérieur à 1 et supérieur à celui de pâturages ou de forêts. Ces facteurs d'équivalence sont basés sur des calculs de l'organisation des Nations Unies pour l'Alimentation et l'Agriculture Food and Agriculture Organisation (FAO).

\begin{tabular}{|c|c|}
\hline Type de surface & $\begin{array}{c}\text { Facteur d'équivalence } \\
\text { [hectare global par hectare] }\end{array}$ \\
\hline Champs cultivés & 2,51 \\
\hline Forêts & 1,26 \\
\hline Pâturages & 0,46 \\
\hline Pêcheries & 0,37 \\
\hline Surfaces urbanisées & 2,51 \\
\hline
\end{tabular}

Tableau 3.1: Facteurs d'équivalence en 2013 des surfaces qui permettent la conversion d'hectares en hectares globaux. D'après [4].

En fait, les facteurs d'équivalence dépendent du nombre total d'hectares globaux que l'on se fixe. Le choix, qui s'est imposé, a été de prendre pour nombre total d'hectares globaux le même que celui des surfaces bioproductives effectivement 
disponibles. La figure 3.3 permet de relier les nombres d'hectares globaux aux nombres d'hectares réels pour les principales surfaces bioproductives. Le nombre total d'hectares définit la biocapacité mondiale de la planète, laquelle peut varier avec le temps. Par exemple, la désertification peut transformer des terres productives en terres non-productives. D'après la figure 3.3, la biocapacité mondiale est de l'ordre de 12000 millions d'hag soit 12 Ghag, soit environ 1,6 hag par habitant. Pour se donner une idée, cela représente un peu moins de $25 \%$ de la surface du globe. Rappelons que l'océan représente $71 \%$ de la surface du globe et que les terres habitables représentent moins de $20 \%$ de la surface.

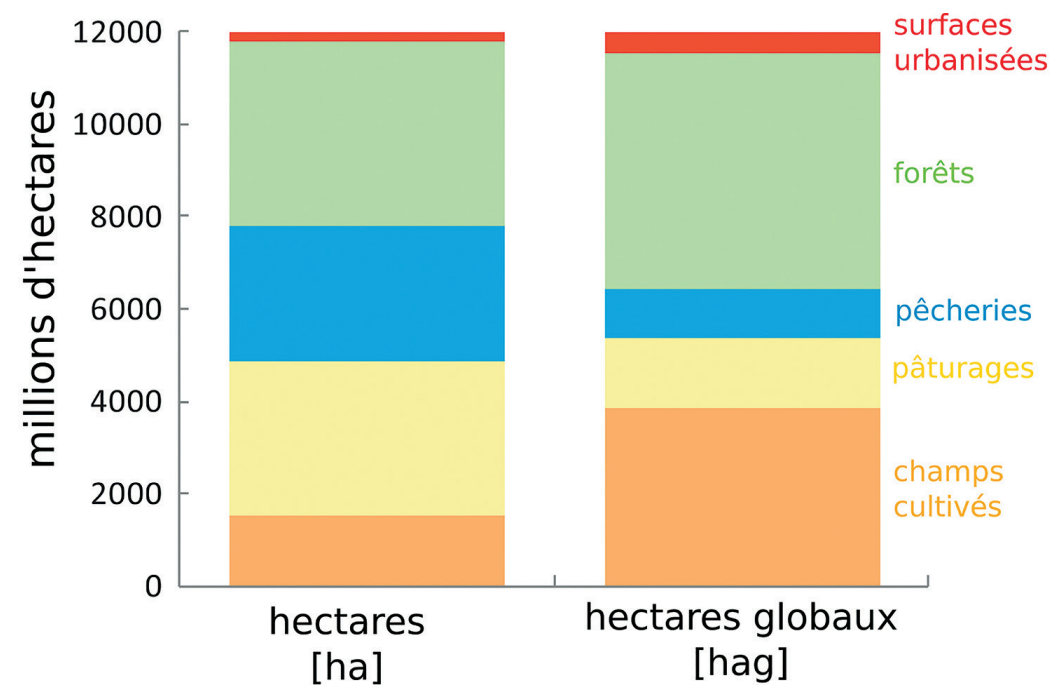

Figure 3.3: Hectares de surfaces utiles pour l'humanité et équivalence en termes d'hectares globaux. Le nombre total est le même, mais la proportion relative tient compte de l'utilité des surfaces via le facteur d'équivalence. D'après [4].

À l'échelle d'un pays ou de régions géographiques plus petites, la biocapacité va tenir compte du rendement local des productions. Par exemple, en France, les rendements de blé, c'est-à-dire, la production de blé par hectare, sont supérieurs à ceux de la moyenne mondiale. Nous prendrons cela en compte par un facteur de rendement sans dimension:

\section{biocapacité locale $=$}

\section{Facteur de rendement $x$ Facteur d'équivalence $\times$ Surface allouée}

On additionne ensuite toutes les contributions pour obtenir la biocapacité d'un pays. Notons que les terrains bâtis sont souvent comptés avec le même facteur d'équivalence que les champs cultivés. En fait, la plupart des terres urbanisées le sont au détriment de terres fertiles; en supprimant virtuellement les bâtis, le pays pourrait ainsi récupérer des surfaces à haute valeur bioproductive. Ceci explique le choix retenu. 
Venons-en maintenant au calcul de l'empreinte écologique. II s'agit de comptabiliser toutes les ressources consommées. Pour les ressources renouvelables, le comptage se fait directement, ressource par ressource. Le calcul de l'empreinte écologique sur le périmètre d'un pays nécessite de compter les besoins réels du pays en prenant en compte les importations et les exportations pour aboutir à la consommation nette :

Consommation nette $=$ Production + Importations - Exportations

On utilise ensuite le même facteur d'équivalence que pour le calcul de la biocapacité, ce qui conduit à la formule :

$$
\text { empreinte }\left[\text { hag] }=\frac{\text { consommation nette }[\mathrm{t} / \mathrm{an}]}{\text { rendement moyen }[\mathrm{t} / \mathrm{ha} / \mathrm{an}]} \times\right. \text { facteur d'équivalence [hag/ha] }
$$

Le rendement, qui intervient ici dans la formule, est le rendement moyen mondial pour la production de la ressource considérée.

Notons qu'en l'absence d'importations et d'exportations, il peut y avoir une différence entre l'empreinte écologique et la biocapacité dans le cas où un pays n'exploite pas au maximum la production renouvelable ou, à l'inverse, s'il y a surexploitation comme dans le cas de la surpêche. Cela étant, assez souvent, l'empreinte écologique associée à la consommation d'une ressource renouvelable par les humains est très proche de la biocapacité lorsqu'on applique la méthode de calcul qui est présentée. Pour les terrains bâtis, l'empreinte est, en fait, exactement la même que la biocapacité, car on utilise le même facteur d'équivalence. En revanche, une différence significative peut apparaître concernant le couvert forestier, car une forêt inexploitée apparaît dans la biocapacité alors que l'empreinte associée à la consommation de cette ressource est nulle.

\subsubsection{Calcul de l'empreinte carbone}

Nous ne détaillerons pas les spécificités de calcul pour chacune des ressources renouvelables, mais nous nous concentrerons ici sur la part la plus importante de l'empreinte écologique actuelle, à savoir l'empreinte carbone. Celle-ci est liée à nos émissions de $\mathrm{CO}_{2}$, considérées comme «déchets». La grande majorité de nos émissions de $\mathrm{CO}_{2}$ est due à l'utilisation des énergies fossiles: charbon, pétrole et gaz. Ces trois sources d'énergie ont pour point commun de contenir du carbone, d'où le nom d'empreinte carbone. Leur utilisation la plus courante consiste à les brûler, ce qui produit l'énergie dont on se sert, mais aussi du $\mathrm{CO}_{2}$, qui apparaît donc comme un sous-produit de la combustion. On peut résumer de manière très simple la transformation globale par la réaction chimique suivante:

$$
\mathrm{C}+\mathrm{O}_{2} \rightarrow \text { énergie }+\mathrm{CO}_{2}
$$


Rappelons que les trois ressources fossiles confondues représentent $80 \%$ de l'énergie primaire utilisée dans le monde: elles constituent nos sources principales d'énergie (voir section 2.1.6)!

Comment quantifier nos émissions de $\mathrm{CO}_{2}$ en termes de surface? Les ressources fossiles, qui se trouvent dans le sous-sol, et non en surface comme les arbres, n'occupent pas directement une surface de sol bioproductif. La seule solution consiste alors à estimer la surface bioproductive nécessaire pour assimiler le $\mathrm{CO}_{2}$, le déchet produit par leur utilisation:

$$
\text { surfaces fossiles }\left[\text { ha] }=\text { émissions de } \mathrm{CO}_{2}\left[\mathrm{tCO}_{2}\right] \times \frac{(1-\text { fraction absorbée })}{\text { ration de séquestration }\left[\mathrm{tCO}_{2} / \mathrm{ha}\right]}\right.
$$

Les surfaces bioproductives considérées sont des espaces forestiers censés jouer le rôle de puits de carbone, ce qui amène, dans la formule, à retrancher des émissions de $\mathrm{CO}_{2}$ celle qui sera absorbée par les océans. Le ratio de séquestration tient compte, par ailleurs, de la masse de $\mathrm{CO}_{2}$ absorbée par hectare de sol. En 2017, il était de 3,56 tonnes.

L'empreinte carbone se calcule ensuite par la formule :

$$
\text { Empreinte carbone (hag) }=
$$

Surfaces fossiles (ha) x Facteur d'équivalence forestier (hag/ha).

où le facteur d'équivalence forestier prend en compte le fait que toutes les forêts ne sont pas identiques. On aboutit ainsi à une formulation globale similaire à celle de l'empreinte écologique pour les ressources renouvelables: la quantité de déchets $\mathrm{CO}_{2}$ se substitue à la quantité de ressources consommées, le ratio de séquestration remplace le rendement de production des ressources, un facteur d'équivalence intervient dans les deux cas.

\subsubsection{Déficit écologique mondial}

Globalement, pour l'année 2019, les résultats du calcul de l'empreinte écologique et de la biocapacité au niveau mondial se résument comme suit:

- Biocapacité: B = 12,2 Ghag

- Empreinte écologique: $E$ = 21,2 Ghag.

L'écart relatif entre les deux valeurs, soit (E - B)/B, est de $74 \%$. Dit autrement, l'empreinte dépasse la biocapacité de $74 \%$. On est donc dans une situation de fort déficit écologique. On pourrait quantifier ce déficit en se disant qu'il aurait fallu $74 \%$ de surface supplémentaire pour faire face aux besoins de l'humanité, soit 1,74 planète. Faisons maintenant le lien avec le jour du dépassement de la Terre (J) dont nous avons parlé au début de ce chapitre. On utilise pour cela une simple règle de 
trois : J = Bx365/E = 210. Pour l'année 2019, il s'agit donc du 210e jour de l'année, soit le 29 juillet, qui est le jour du dépassement.

La décomposition de l'empreinte écologique suivant les différents types de ressource (ou de déchet) met en évidence une forte composante carbone de l'ordre de $60 \%$ (Fig. 3.4). La biocapacité forestière n'est pas suffisante pour compenser l'empreinte carbone qui est la principale cause du dépassement planétaire dans cette analyse. On constate, par ailleurs, que tous les secteurs d'activité économique contribuent significativement à l'empreinte écologique globale, laquelle a été multipliée par 3 en 50 ans.
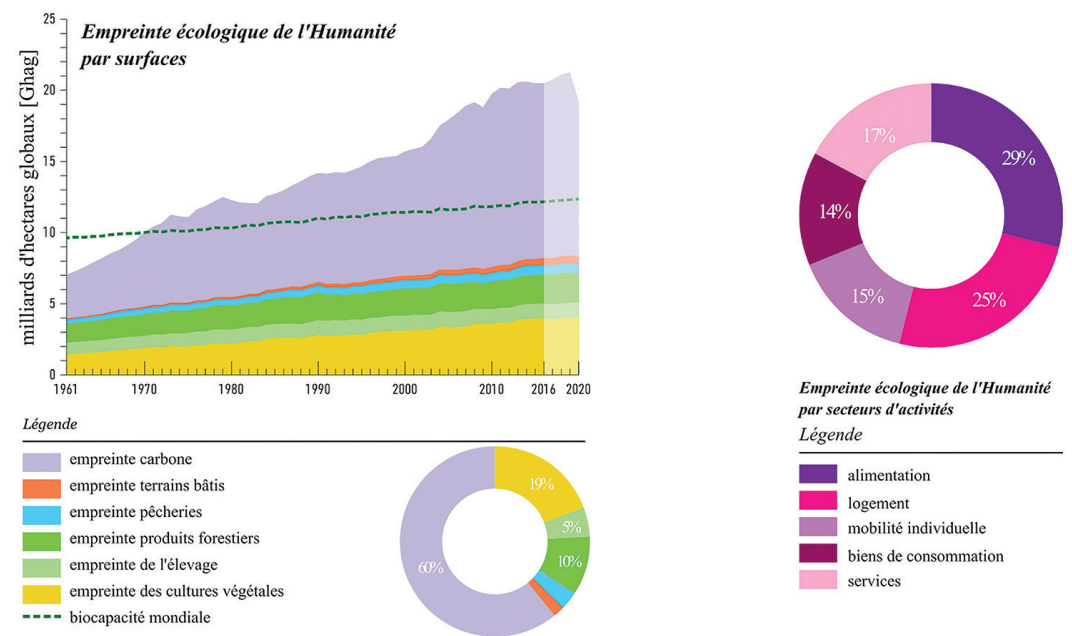

Figure 3.4: Évolution de l'empreinte écologique de l'humanité depuis 1961 par type de surface (à gauche) et par secteur d'activité (à droite). D'après [5,6].

Si l'on fait maintenant le bilan au niveau des pays, la diversité rencontrée aussi bien en termes de biocapacité que d'empreinte écologique conduit à des situations de crédit (en bleu sur la figure 3.5) ou de dette écologique (en rouge sur la figure 3.5). Les grands pays avec de grands couverts forestiers bénéficient de la biocapacité associée qui tend à les mettre en crédit pour la plupart d'entre eux (Canada, Brésil, Russie, Australie, ...). Néanmoins, les États-Unis et la Chine ont des consommations telles, qu'ils sont en déficit malgré des ressources abondantes.

Les pays (ou régions) très développés, dont les niveaux de consommation des ressources sont élevés, sont en dette en raison à la fois de leur mode de vie et de leur population (Europe, USA, Chine). Seuls quelques pays développés mais peu peuplés sont en crédit (Scandinavie, Australie, Canada, Nouvelle-Zélande). Implicitement, il y a une forte interdépendance des économies et des ressources à l'échelle internationale, avec un poids fort des importations pour les régions comme l'Union Européenne [6]. 


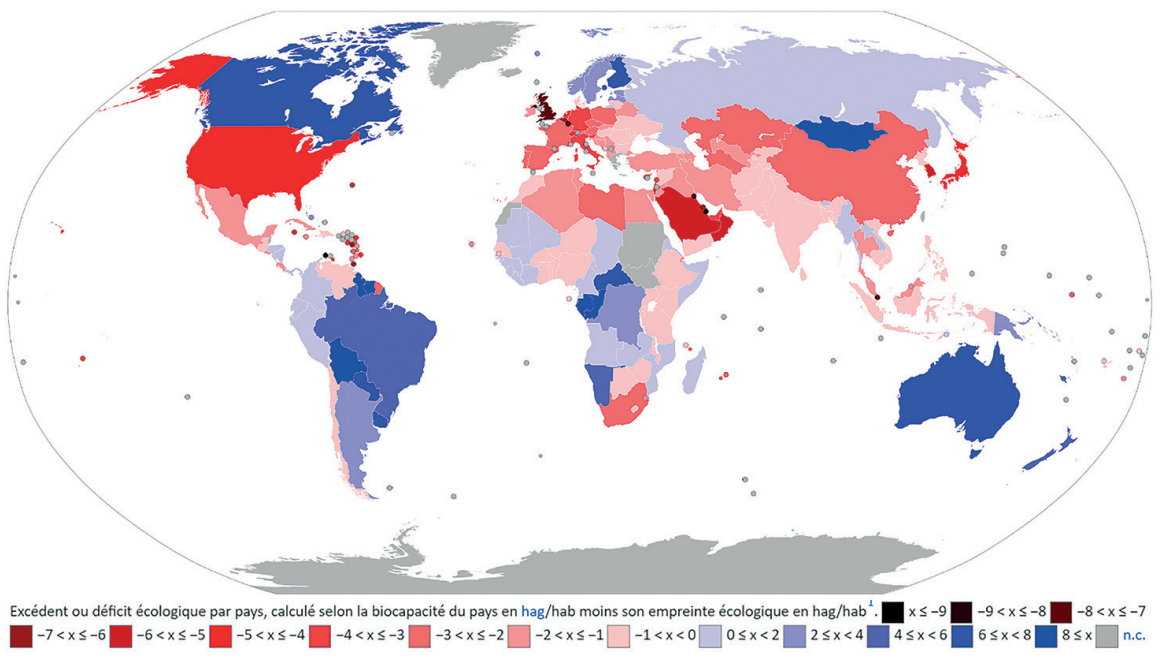

Figure 3.5: Carte des déficits écologiques selon les pays, établie à partir des données de l'année 2013. Les pays en crédit sont en bleu, les pays en dette ont en rouge. Figure adaptée ${ }^{34}$.

Bien que l'analyse présente un certain nombre de biais et de limites (voir section 3.1.6), elle fait apparaître des problématiques générales du développement durable, que l'on retrouve également à partir d'autres indicateurs qui mesurent l'impact humain sur l'état de la planète. Le mode de vie et de consommation associé à des revenus élevés est à l'origine d'un fort impact écologique, que cela soit moyenné à l'échelle d'un pays (figure 3.6) ou à l'échelle individuelle dans le même pays [5]. L'empreinte par habitant peut ainsi varier typiquement d'un facteur 1 à 6 suivant le PIB des pays, sans évoquer des cas extrêmes comme pour les pays du Golfe.

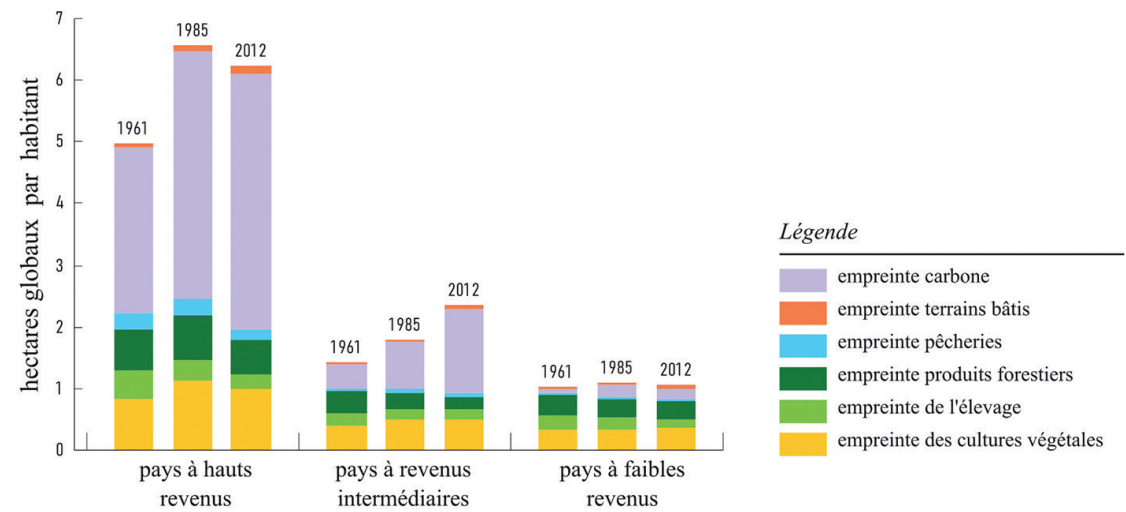

Figure 3.6: Empreinte écologique par habitant pour des pays qui diffèrent par leurs PIB ou leurs revenus. D'après [6]. 


\subsubsection{Limites de l'analyse basée sur l'empreinte écologique}

Si l'empreinte écologique a historiquement joué un rôle important comme indice de mesure de la soutenabilité des économies, elle présente néanmoins un certain nombre de biais. Par exemple, un pays peut augmenter sa biocapacité en convertissant de la forêt primaire en champs cultivés dont le facteur d'équivalence est supérieur, même si cela a un coût en termes d'empreinte carbone. Bien évidemment, dans ce choix de conversion, l'accroissement de l'empreinte carbone et la perte de biodiversité peuvent s'avérer être bien plus dommageables à long terme que le gain économique de productivité.

L'empreinte écologique ne permet pas non plus d'appréhender les liens entre les différents enjeux écologiques. Par exemple, la biocapacité peut être augmentée en améliorant les rendements agricoles par des intrants chimiques alors qu'ils conduisent in fine à de la pollution mal prise en compte dans l'empreinte écologique, les émissions de $\mathrm{CO}_{2}$ restant la pollution la plus facilement quantifiable.

Enfin, les grands cycles géochimiques (eau, azote, oxygène...) interviennent simultanément dans de nombreuses composantes de l'empreinte écologique. Cette intrication a conduit les chercheuses et les chercheurs à proposer de nouveaux outils de quantification de la soutenabilité, notamment ceux basés sur les limites planétaires [7].

\subsubsection{En guise de conclusion?}

Un dernier graphique (Fig. 3.7) permet de résumer à quel point le développement durable, ou soutenable, tel que défini en 3.1.1, reste aujourd'hui un défi majeur de civilisation. Ce graphique positionne simultanément les différents pays du monde selon leur indice de développement humain (IDH) et leur niveau d'empreinte écologique. Rappelons que l'IDH prend non seulement en compte le PIB du pays, mais aussi le niveau d'éducation et l'espérance de vie des habitants. On constate que les pays à fort IDH ont des développements non soutenables tandis que les pays à faible empreinte écologique par habitant ont des IDH à un niveau jugé non acceptable. Pour l'année 2014, date à laquelle le graphique a été établi, aucun pays ne combinait simultanément un IDH élevé et une faible empreinte écologique. 


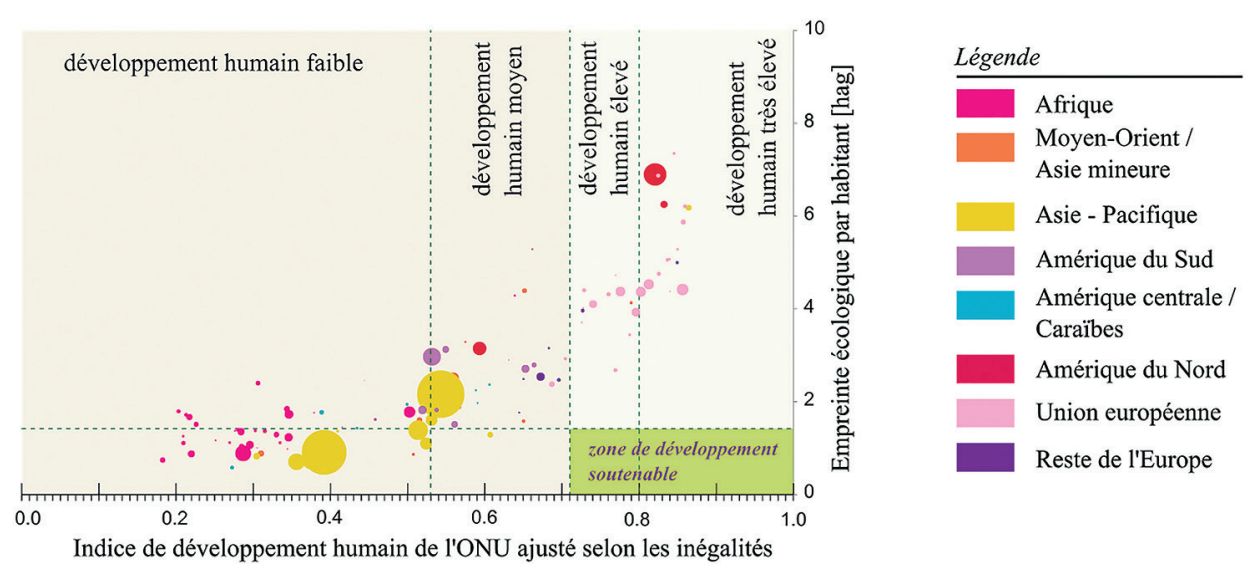

Figure 3.7: Représentation simultanée de l'indice de développement humain (IDH) et de l'empreinte écologique des différents pays du monde. L'IDH, porté en abscisse sur le graphique, a été ajusté par I'ONU selon les inégalités. Le niveau d'empreinte écologique est porté en ordonnée. À chaque pays est associé un rond dont la taille est en rapport avec la population du pays et dont la couleur indique la région du monde où le pays se situe. D’après [6], rapport de 2014. https://fr.wikipedia.org/wiki/Liste_ des_pays_par_IDH_ajust\%C3\%A9_selon_les_in\%C3\%A9galit\%C3\%A9s.

\subsection{Combien serons-nous en 2050 ? - J. Treiner et G. Roux}

L'accroissement rapide de la population humaine au cours des deux derniers siècles soulève bien souvent des peurs. Celles-ci se cristallisent principalement autour des questions migratoires d'une part, et des impacts environnementaux d'autre part [8]. Ces peurs apparaissent d'autant plus paradoxales que l'accroissement de la population est généralement associé à des progrès qui ont été recherchés et soutenus: progrès médicaux, agricoles, techniques et sociétaux. Nous visons avant tout ici à présenter les motivations de l'approche démographique, ses indicateurs et les grandes lignes de l'évolution historique ancienne et récente de la population mondiale. Nous discuterons en particulier les notions de transition démographique ainsi que les projections onusiennes pour le XXle siècle. Le but est bien de comprendre les paramètres clés qui gouvernent l'évolution de la population.

\subsubsection{Combien avons-nous été dans le passé?}

Des estimations ont été faites, avec plus ou moins de précisions, pour l'ensemble de la planète. La reconstruction globale, depuis la fin de la dernière époque glaciaire, est représentée en figure 3.8. On distingue un premier changement de régime, vers -40 000, associé à diverses améliorations techniques du Paléolithique supérieur, puis un deuxième amorcé vers -7000 , lié à la révolution néolithique et prolongé par divers progrès techniques tel le développement des premières machines (ex: les moulins), et enfin une montée quasi verticale (à cette échelle) associée à la révolution 
industrielle. Notons que les trois changements de régime ont été le résultat de progrès successifs dans l'appropriation des ressources énergétiques (outils, agriculture et élevage, sciences).

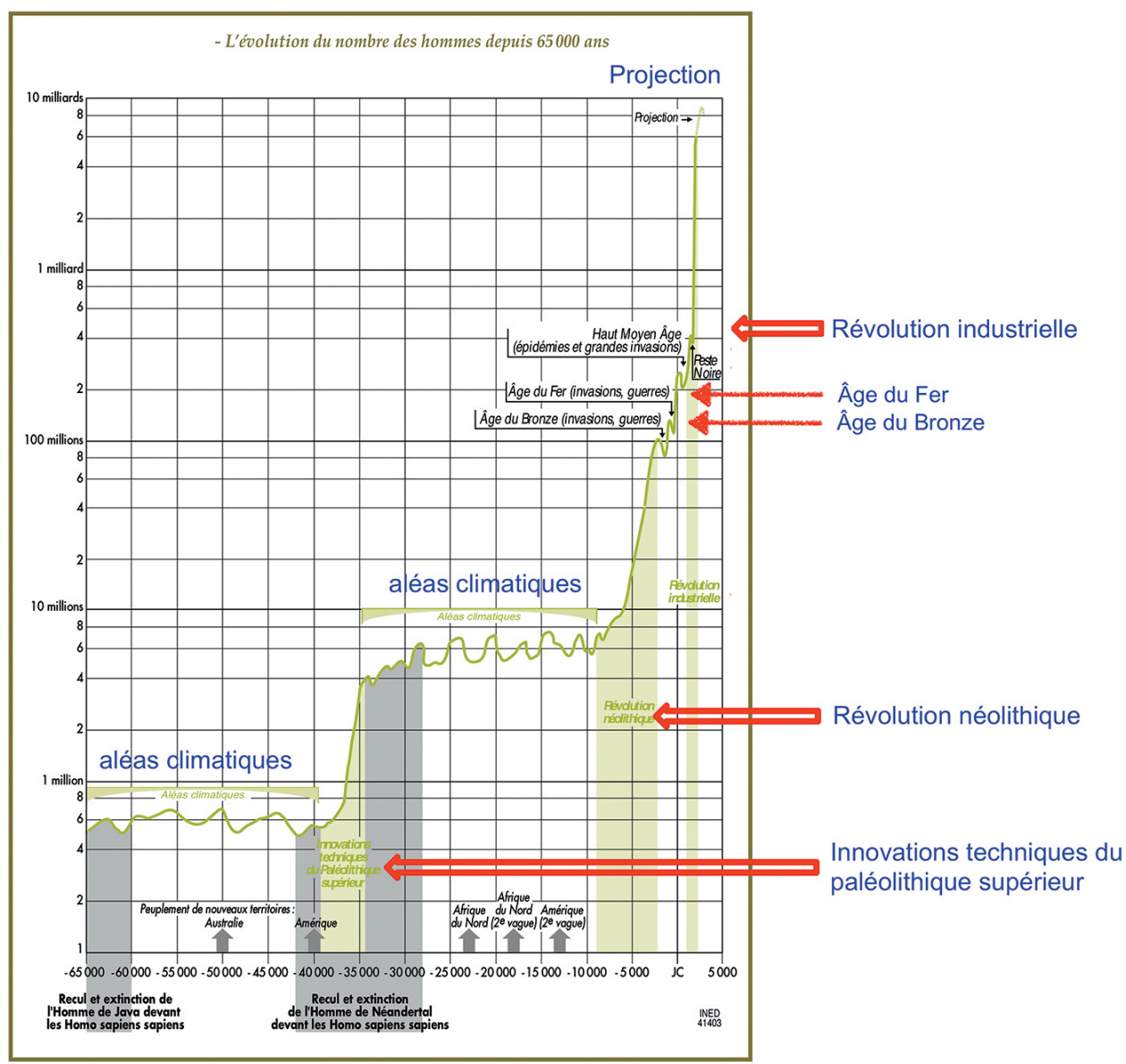

Figure 3.8: Évolution de la population mondiale, en millions d'habitants, depuis la fin de la dernière période glaciaire. D’après J.N. Biraben, Population et Société, n 394, oct. 2003 [9].

II y a quelque chose de vertigineux dans la croissance quasi verticale entamée voici deux cents ans. Pour tenter d'y voir plus clair, dilatons l'échelle des temps récents. On obtient alors le graphique de la figure 3.9, qui présente à la fois l'évolution récente et les projections de l'ONU jusqu'en 2100. Jusque vers 1950, on constate d'ailleurs que la croissance est plus rapide que celle d'une fonction exponentielle, qui correspondrait à une croissance constante. 


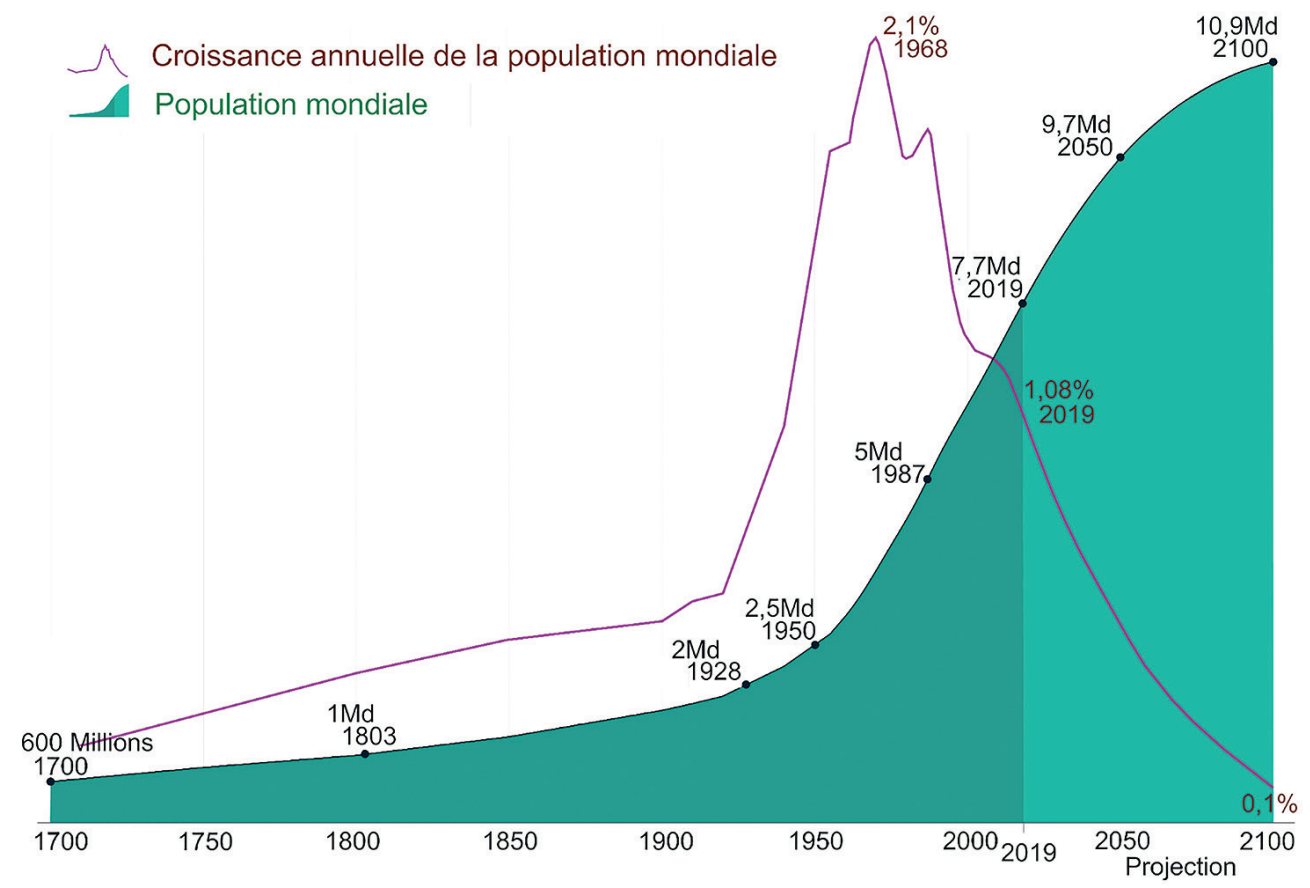

Figure 3.9: Évolution de la population mondiale, en milliards d'habitants, au cours des temps modernes ${ }^{35}$.

Typiquement, une évolution exponentielle est caractérisée par un temps de doublement qui est une constante. Autour de 1960, la population était de 3 milliards et ce temps de doublement était de l'ordre de 35 ans qui correspond à une croissance de $2 \%$. Si cette croissance avait été maintenue, la population mondiale aurait été 140 ans plus tard, en 2100, multipliée par 16, soit 48 milliards! Cette extrapolation soulevait bien des peurs à l'époque. Mais cela ne s'est pas produit. Depuis les années 1960, la croissance de la population diminue rapidement. Certes, la population continue d'augmenter, mais les projections sont aujourd'hui autour de 11 milliards en 2100. Comment expliquer cela? Pour le comprendre, regardons comment travaillent les démographes qui établissent ces projections.

\subsubsection{La transition démographique}

Pour asseoir leur opinion, les démographes s’appuient sur ce qui a été observé dans toute société qui passe d'un état peu développé - sur le plan scientifique, technique et éducatif - à un état de richesse relative; ce qu'ils appellent la transition démographique. 
Les deux paramètres qui régulent de façon globale l'évolution d'une population sont le taux de naissance et le taux de mortalité. Ces taux sont souvent exprimés en nombre de naissances ou de décès par an pour 1000 habitants. Comment dépendent-ils de l'état de la société?

Dans une société peu développée, ces deux taux sont élevés. Le taux de naissance est élevé, car rien ne vient entraver les possibilités naturelles de se reproduire. Ainsi, il n'était pas rare, au XIXe siècle ou au début du XX' siècle, qu'une femme mette au monde plus de dix enfants. Le taux de mortalité est également élevé, notamment au cours des premières années de la vie. Hygiène déficiente, soins médicaux inexistants et environnement hostile expliquent une espérance de vie réduite. Celle-ci était typiquement de 30 à 35 ans dans l'Antiquité. La mortalité périnatale reste élevée pendant longtemps au cours de l'Histoire: naître est toujours un événement à risque. Par exemple, en France, dans les années 1875, plus de $15 \%$ des bébés mourraient dans leur première année.

Si les taux de natalité et de mortalité sont élevés, le taux de croissance de la population, égal à leur différence, reste faible: la population reste stable ou augmente lentement.

Avec le développement de l'hygiène et de la médecine, la mise à disposition d'une eau propre, puis l'introduction d'antibiotiques et de vaccins, le taux de mortalité périnatal diminue. En 1975, toujours en France, le taux de mortalité périnatal n'était plus que de 11 pour 1000 , et en 2004 il était de 3,4 pour 1000 . Ces progrès accompagnent souvent une amélioration des conditions générales de vie qui diminue aussi les causes de mortalité à tout âge.

Qu'en est-il du taux de naissance? Si l'abaissement du taux de mortalité relève de progrès essentiellement techniques, en revanche, avoir des enfants a une dimension culturelle, ancrée profondément. Dans une première phase, le taux de naissance reste donc élevé. En conséquence, avec un taux de mortalité qui diminue, le taux de croissance de la population augmente rapidement, et donc la population également.

Dans une deuxième phase, l'amélioration des conditions de vie, le désir de scolariser les enfants, le prolongement de la durée des études scolaires et l'augmentation de leur coût, et surtout l'élévation du niveau culturel des femmes, tous ces facteurs conduisent à vouloir contrôler la venue des enfants par l'utilisation de moyens contraceptifs: le taux de naissance se met à diminuer. Petit à petit, un nouvel équilibre s'établit, où le taux de mortalité et le taux de fécondité sont faibles. Et où par conséquent la population n'évolue plus. La transition démographique, c'est précisément ce passage d'une situation où les taux de natalité et de mortalité sont élevés, à une situation où ils deviennent faibles. Ces différentes tendances, schématisées sur la figure 3.10, expliquent comment la période de forte croissance d'une population est en général suivie d'une stabilisation. 


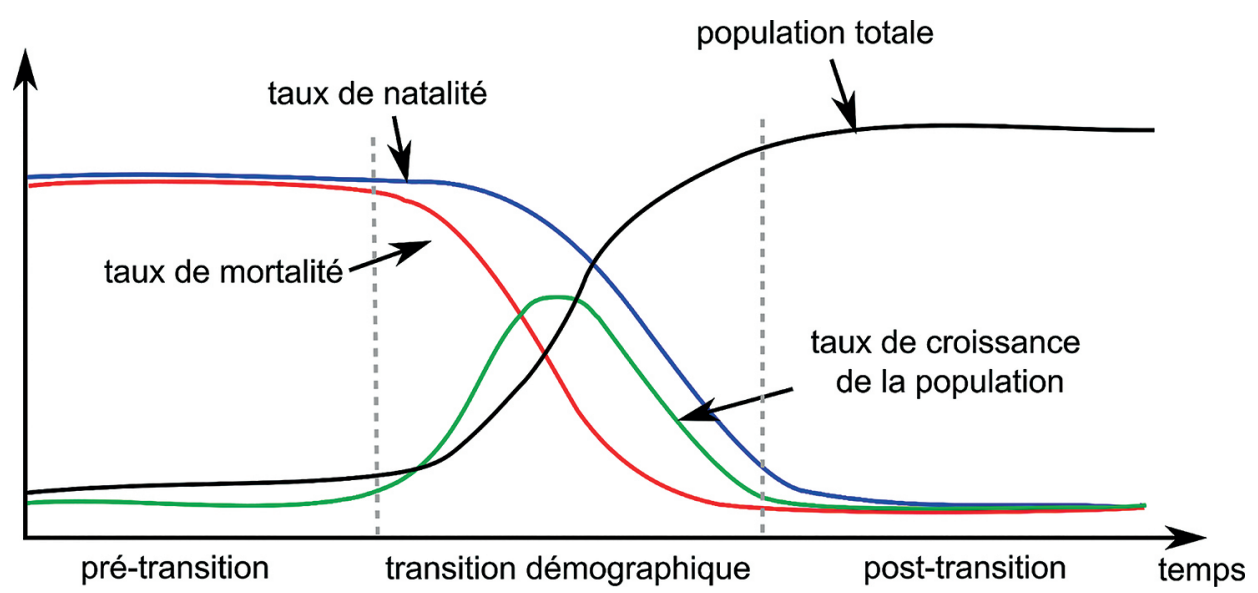

Figure 3.10: Schéma illustrant la transition démographique résultant des variations respectives du taux de naissance, du taux de mortalité et du taux de croissance de la population. La population totale atteint un palier après la transition. Seule l'allure des courbes importe ici ${ }^{36}$.

\subsubsection{Et après la transition démographique?}

La figure 3.10 illustre un cas schématique où, après la transition démographique, les taux de naissance et de mortalité s'égalisent, le taux de croissance s'annule. Par conséquent, la population atteint un palier. Mais en réalité, aucun mécanisme démographique n'oblige les deux taux à s'égaliser! II se peut très bien que, après la transition, le taux de naissance l'emporte, auquel cas la population continue d'augmenter, ou que le taux de mortalité l'emporte, auquel cas la population passe par un maximum et diminue. Ces trois cas possibles sont en fait présents dans les dernières projections de l'ONU présentées sur la figure 3.11.

II faut donc revenir à notre question initiale, et nous demander, compte tenu des connaissances que nous avons de la transition démographique dans les différents pays, ce que nous pouvons anticiper concernant la population mondiale. Un bon indicateur global est fourni par le taux de fécondité, c'est-à-dire le nombre d'enfants qu'une femme a, en moyenne, au cours de sa vie. En principe, un taux de fécondité de 2 est nécessaire pour assurer le maintien de la population à long terme. En effet, il y a en moyenne autant de femmes que d'homme, donc pour chaque femme qui meurt, un homme meurt. Pour assurer le renouvellement de la population, il faut que, en moyenne, chaque femme compense ces deux décès ${ }^{37}$.

36. https://fr.wikipedia.org/wiki/Transition_démographique

37. En réalité, le taux de fécondité doit être légèrement supérieur à 2, pour tenir compte des filles qui décèdent par accident ou maladie au cours de leur jeunesse, avant d'avoir pu avoir des enfants, ou qui sont stériles, ou dont les conjoints sont stériles. 


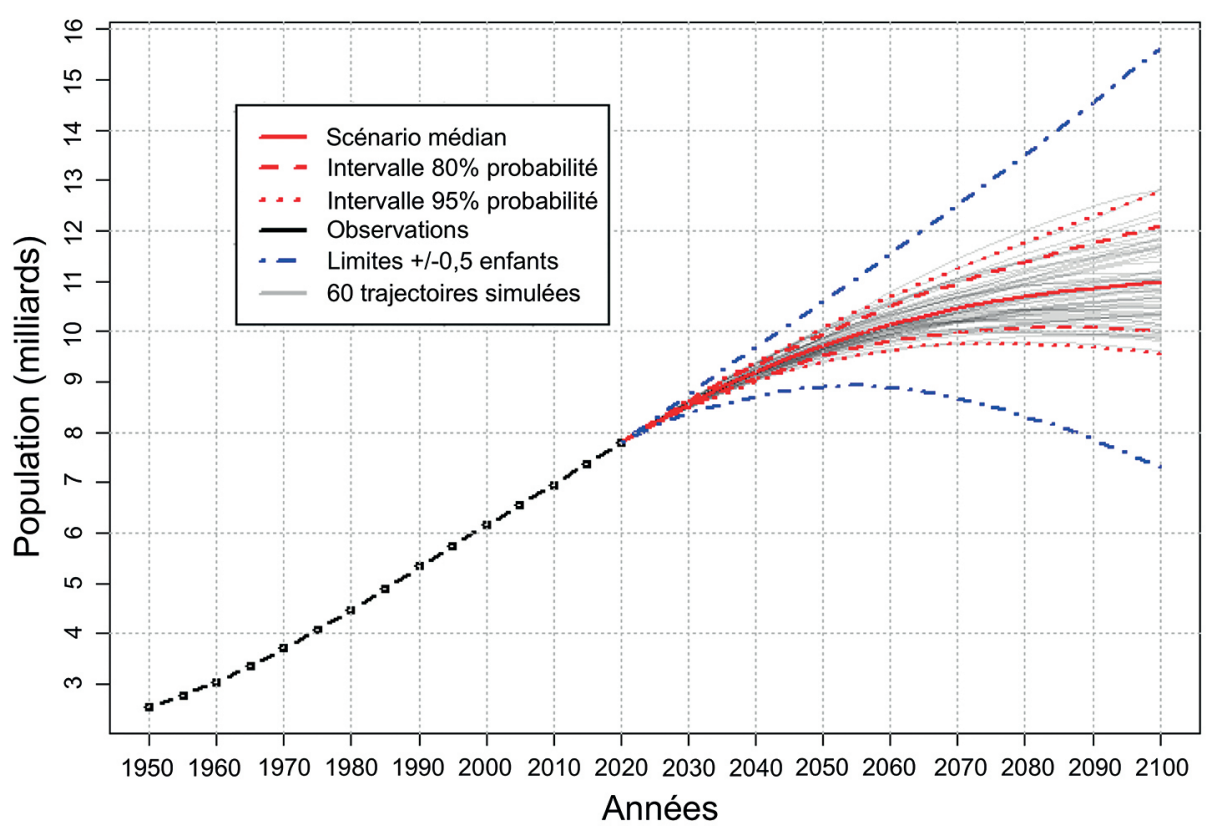

Figure 3.11: scénarios possibles pour la population mondiale. Le scénario le plus probable présente un maximum vers 2100 mais ce maximum peut se produire également plus tôt au XXle siècle ${ }^{38}$.

Dans les sociétés traditionnelles, le taux de fécondité est largement supérieur à 2. Or, on constate que la tendance générale, dans tous les pays, est à une diminution de ce taux au cours du temps. II peut même passer franchement en dessous de 2. C'est le cas, en Europe, pour pratiquement tous les pays. La France et l'Irlande ont fait pendant longtemps figure d'exception, mais les taux de fécondité viennent récemment d'y passer en dessous de 2. À l'échelle du monde entier, les disparités sont encore bien plus grandes. II convient à présent d'entrer dans le détail de la structure en âge des populations.

\subsubsection{La pyramide des âges}

Le «bon» outil pour discuter des questions de population est la pyramide des âges. L'Institut National d'Études Démographiques (INED) donne accès sur son site ${ }^{39}$ aux pyramides des âges de tous les pays du monde.

Commençons par examiner la pyramide d'un pays qui n'a pas fait sa transition démographique: l'Éthiopie (Fig. 3.12). Cette représentation indique, pour les femmes (partie droite) et pour les hommes (partie gauche), le nombre d'individus (en milliers) par tranche d'âge (ici, des tranches d'un an). Deux indicateurs supplémentaires sont

38. Site de l'ONU consacré à la population: https://population.un.org/wpp/

39. Simulateur de population de l'Ined: https://www.ined.fr/_modules/SimulateurPopulation/?lang=fr 
accessibles: le taux de fécondité: 3,95 enfants par femme en moyenne, l'espérance de vie : 67,3 ans. Cette pyramide est caractéristique d'un pays pauvre, avant la transition démographique. Le taux de fécondité est élevé, mais le taux de mortalité aussi, ce qui se voit à la pente des côtés: on ne retrouve pas, à l'âge n, le nombre de vivants de l'âge n-1.

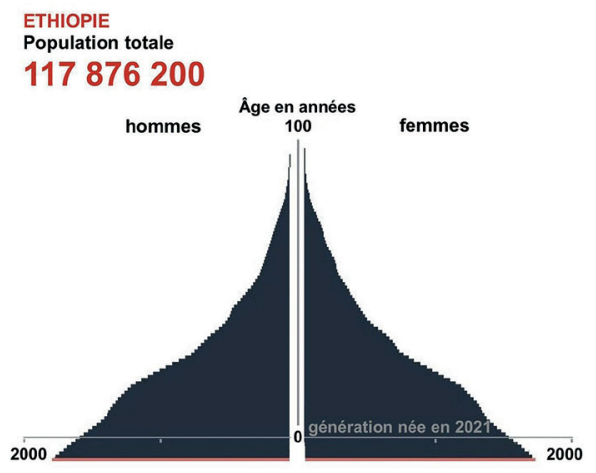

Figure 3.12: Pyramide des âges de l'Éthiopie. Source: INED.

Par comparaison, considérons le cas de l'Inde (Fig. 3.13). Le taux de fécondité est plus faible (2,16 enfants par femme), l'espérance de vie plus élevée qu'en Éthiopie (70,1 ans). De plus, on constate que, manifestement, une évolution s'est amorcée il y a une vingtaine d'années, avec plusieurs années où le taux de naissance a diminué (rétrécissement de la base de la pyramide) : c'est l'amorce de la transition démographique.

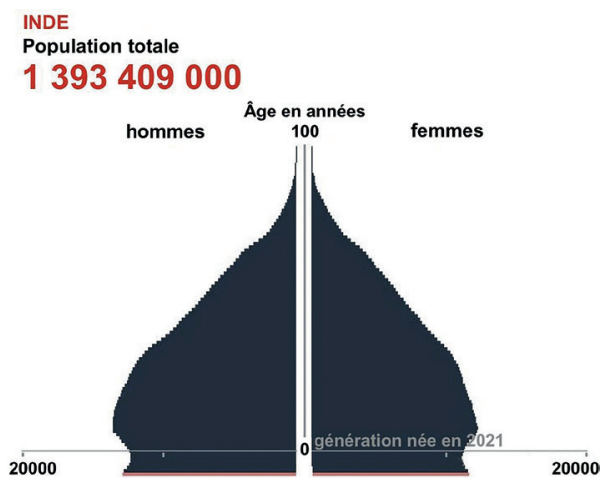

Figure 3.13: Pyramide des âges de l'Inde. Source: INED.

Voyons enfin, pour contraster, le cas d'un pays développé comme la France (Fig. 3.14). La première caractéristique, c'est que cette pyramide... n'est manifestement pas une pyramide. II s'agit plutôt du profil d'une maison, avec des murs presque verticaux, et un toit pentu. La pointe du toit, c'est l'âge maximum atteint, puisqu'il 
faut bien mourir! Cette forme indique que le poids des seniors, dans l'ensemble de la population, est bien supérieur à ce qu'il est dans les deux exemples précédents: la population, globalement, vieillit, et tous les débats actuels concernant l'âge de la retraite et le montant des pensions sont liés à ce vieillissement. L'espérance de vie à la naissance est aujourd'hui de près de 82,9 ans, sexes confondus.

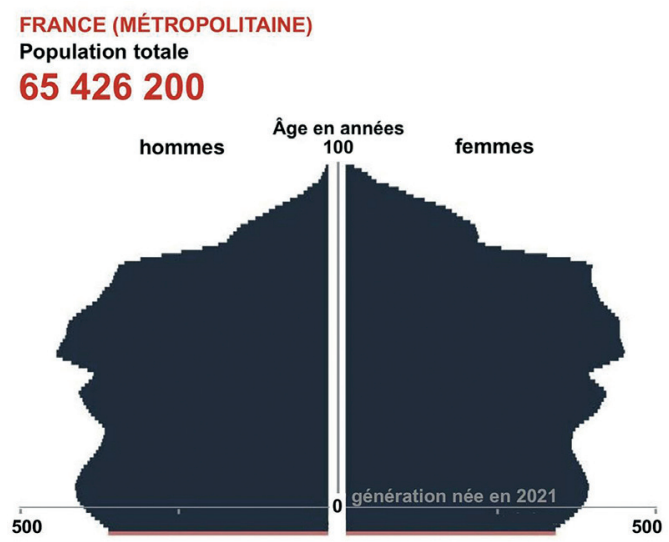

Figure 3.14: La pyramide des âges en France aujourd'hui. Source: INED ${ }^{38}$.

Les grands événements de l'histoire d'un pays se lisent sur sa "pyramide», et vous êtes invités à lire la trace des guerres et des baby booms sur les figures. Exemple: un baby-boom se traduit par une bosse dans la pyramide, laquelle se reproduit 25 ans plus tard, puisqu'il y a plus d'adultes capables de faire des enfants. Le taux de fécondité est actuellement de 1,84 en France métropolitaine ${ }^{40}$, ce qui n'assure pas tout à fait le renouvellement de la population. Mais ce taux est de 2,2 dans les départements et territoires d'outre-mer.

On ne saurait trop vous inviter à consulter le site de l'INED pour explorer pays et régions du monde.

\subsubsection{Le «Jeu de la population»}

Comment évolue une pyramide des âges au cours du temps? Gilles Pison, démographe et Directeur de rédaction de la revue Population et Sociétés ${ }^{41}$, a élaboré un outil interactif remarquable, le Jeu de la population ${ }^{38}$, qui permet de visualiser les projections de population des experts de l'ONU ${ }^{37}$. Prenons le cas de l'Inde. Les projections pour 2035 et 2050 aboutissent aux pyramides de la figure 3.15 .

40. https://www.ined.fr/fichier/s_rubrique/29847/popu_1904_conjoncture_.9.12.fr.pdf

https://www.ined.fr/fichier/s_rubrique/30029/575.population.societes.fecondite.mars2020.france. europe.fr.pdf

41. https://www.ined.fr/fr/publications/editions/population-et-sociétés/ 
INDE

Population totale

1553724000

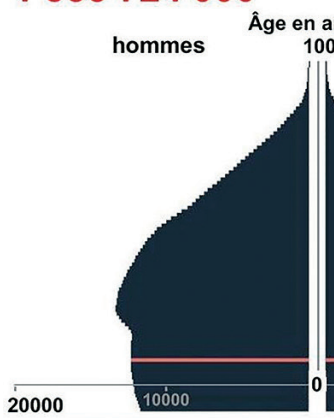

en années

femmes

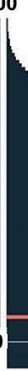

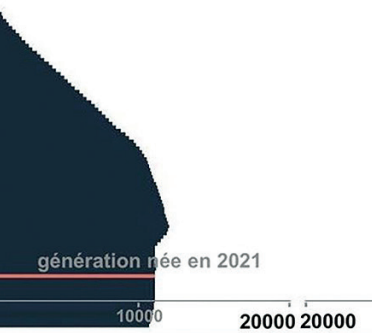

INDE

Population totale

1639176000

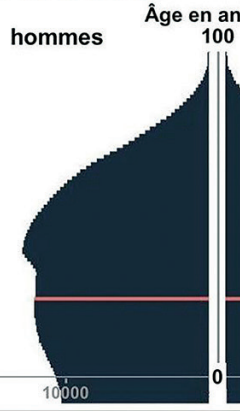

femmes

Figure 3.15: Pyramides des âges de l'Inde en 2035 (à gauche) et 2050 (à droite). Source: INED.

L'amorce de la transition démographique de la figure 3.13 se confirme au cours du siècle. Le taux de fécondité devrait passer en dessous de 2 (1,95 en 2035, 1,80 en 2050). La courbe de population totale laisse présager le passage par un maximum suivi d'une diminution, tandis que l'espérance de vie augmente et atteint 75 ans au milieu du siècle.

Qu'en est-il pour le monde dans son ensemble? Les projections de l'ONU indiquent nettement une transition démographique globale. Bien que le résultat additionne des situations très différentes les unes des autres, la projection a tout de même un sens: elle indique que la transition démographique est attendue dans tous les pays (Fig. 3.16).

Le taux de fécondité global diminue : 2,43 en 2021, puis 2,19 en 2050 (et même 1,93 en 2100). L'espérance de vie passe de 72,9 ans en 2021 à 77,1 en 2050 (et 81,9 en 2100).
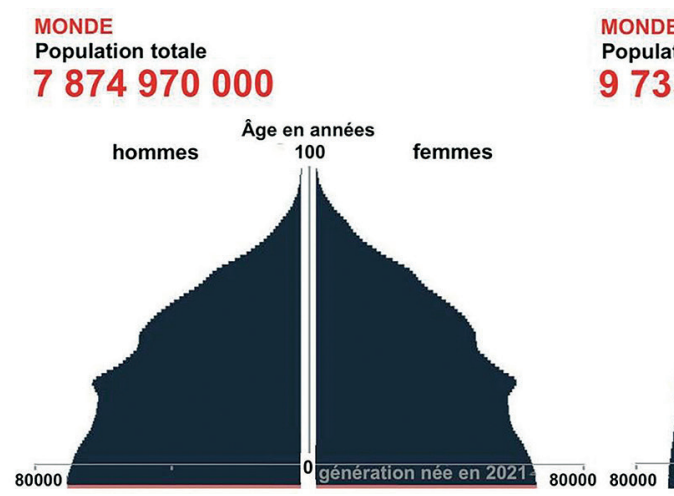

MONDE

Population totale

9735040000

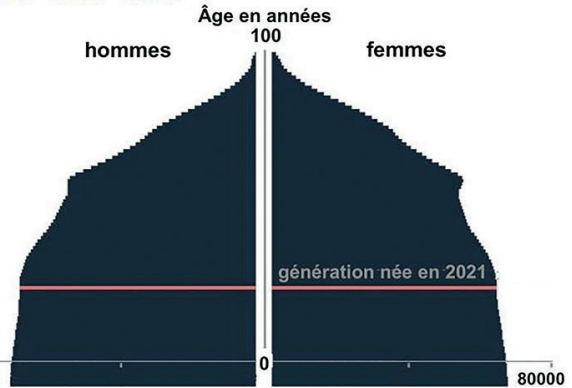

Figure 3.16: Évolution de la pyramide des âges du monde globalisé. À gauche : 2021. À droite : 2050. 
Le simulateur indique également que la courbe de population globale a sa concavité dirigée vers le bas : on s'attend donc à ce qu'elle passe par un maximum au voisinage de 10 milliards d'individus, puis qu'elle diminue. Mais quelle confiance faut-il donner à ces projections?

\subsubsection{Comment ça marche?}

Le principe n'est pas très compliqué, et quand on l'a compris, on peut soi-même, sur un tableur, se faire son propre programme et explorer ses propriétés.

Le pas élémentaire consiste à faire évoluer d'un an une tranche d'âge. II est clair que la population d'une tranche d'âge aura, l'année suivante, un an de plus - Monsieur de la Palisse le savait déjà. N'oublions pas, cependant, qu'à chaque âge, certaines personnes décèdent - accident ou maladie. II faut donc connaître le taux de mortalité par âge. Les démographes, par des études statistiques, établissent ces tables de mortalité. Elles permettent, pour chaque âge, de déterminer le nombre moyen de personnes qui vont décéder dans l'année: il suffit de multiplier le taux de mortalité de la tranche d'âge en question par le nombre d'individus qu'elle contient.

Bien. Mais cela ne dit pas comment remplir la première tranche d'âge, celle correspondant aux nouveau-nés de l'année. En effet, pour cela, il faut connaître les taux de naissance par âge. C'est à nouveau le travail des démographes que de les mesurer statistiquement. À chaque âge, entre disons 15 ans et 50 ans, les femmes peuvent enfanter. La figure 3.17 représente, par exemple, l'évolution du nombre de naissances vivantes pour 100 femmes de chaque âge, de 1991 à 2011, ainsi que celle des mortalités par âge pour 1000 femmes de chaque âge, en 1820 et en 1990 (source: Démographie, H. Leridon et L. Toulemon, Economica).
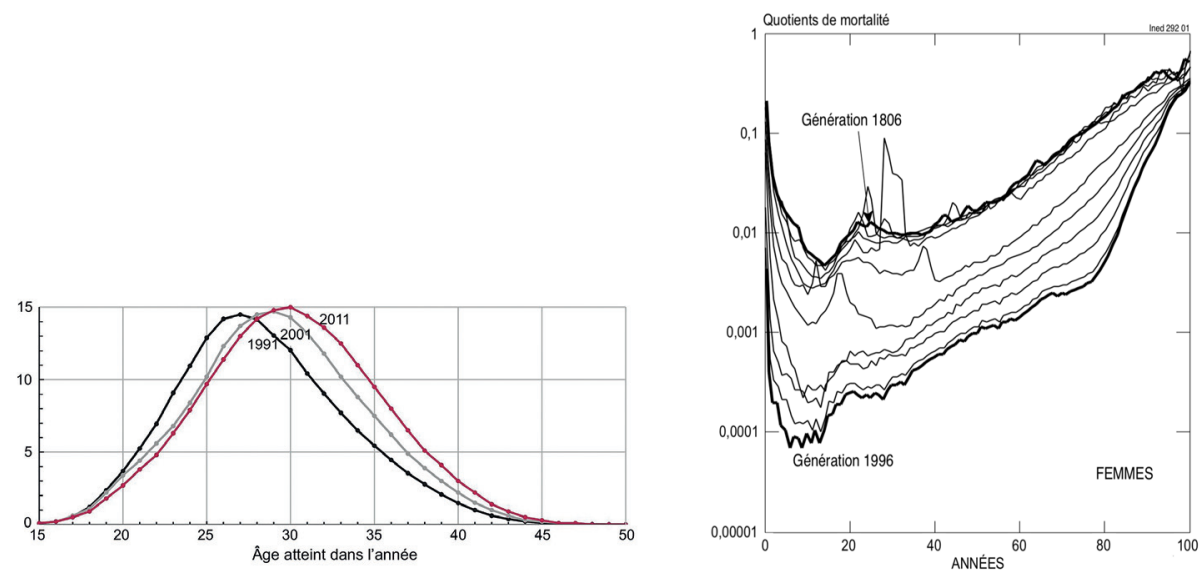

Figure 3.17: À gauche: nombre de naissances vivantes pour 100 femmes de chaque âge en France (Source: Insee, estimation de population et statistiques de l'état civil - 2012). À droite: modifications, de 20 ans en 20 ans, de la courbe annuelle de mortalité par âge pour les hommes, de 1806 à 1996 , et projection jusqu'en 2012 (Source : Ined 291 01). 
Connaissant les taux de naissance par âge, le nombre total de nouveau-nés de l'année est égal à la somme de ceux mis au monde par les femmes de 15 ans, de 16 ans, de 17 ans etc. jusqu'à ceux mis au monde par les femmes de 50 ans.

Si l'on se donne la pyramide des âges d'une année prise comme origine, nous savons maintenant comment calculer son évolution future. Dans cette discussion de principe, nous ne tenons pas compte des flux sortant et entrant : émigration et immigration. Mais on voit qu'on pourrait très bien en tenir compte.

Prenons un exemple. Pour faire simple, regroupons la population en 10 classes d'âge, et supposons que les taux de naissance et de mortalité soient ceux donnés dans le tableau 3.2 :

\begin{tabular}{|c|c|c|}
\hline âge & taux de naissance & taux de mortalité \\
\hline 1 & 0 & 0,10 \\
\hline 2 & 0 & 0,05 \\
\hline 3 & 0 & 0,06 \\
\hline 4 & 0,5 & 0,07 \\
\hline 5 & 0,5 & 0,08 \\
\hline 6 & 1 & 0,10 \\
\hline 7 & 0,5 & 0,25 \\
\hline 8 & 0 & 0,50 \\
\hline 9 & 0 & 0,80 \\
\hline 10 & 0 & 1,00 \\
\hline
\end{tabular}

Tableau 3.2: Données de départ pour «jouer» à la population sur un tableur.

Prenons, comme pyramide initiale, une répartition irréaliste mais qui permet des calculs simples pour commencer. Supposons qu'à tout âge, il y ait autant d'hommes que de femmes. Dans ces conditions, il suffit de calculer la pyramide des femmes. Supposons que toutes les tranches d'âges aient, pour commencer, 100 femmes. Comment se répartit cette population au bout d'un an?

La nouvelle tranche 2 a 100 femmes, moins celles qui sont décédées dans l'année. Comme le taux de mortalité de la première tranche est de 0,1, 10 "bébés femmes » sont morts. La nouvelle tranche 2 compte donc 90 femmes. La nouvelle tranche 3 compte $100-5=95$ femmes (taux de mortalité de 0,05). La nouvelle tranche 4 compte 94 femmes, etc. Et la nouvelle tranche 1? Les femmes des tranches 1, 2 et 3 n'ont pas eu d'enfant. Les femmes de la tranche 4 ont eu 50 enfants, celles de la tranche 5, 50 enfants, celles de la tranche 6, 100 enfants, celles de la tranche 7, 50 enfants, et les autres n'en ont pas eu. Au total, cela fait 250 enfants, dont, par hypothèse, 125 garçons et 125 filles. La nouvelle tranche 1 compte donc 125 filles. Et c'est tout ! Nous pouvons maintenant recommencer le calcul à partir de cette nouvelle répartition. Au lieu de le faire à la main, il vaut mieux utiliser un tableur. 
Le tableau 3.3 rapporte les résultats obtenus pour les premières années (en arrondissant en nombres entiers).

\begin{tabular}{|c|c|c|c|c|c|}
\hline $\begin{array}{c}\text { Année } \\
0\end{array}$ & 1 & 2 & 3 & 4 & 5 \\
\hline 100 & 125 & 115 & 107 & 99 & 98 \\
\hline 100 & 90 & 112 & 103 & 96 & 89 \\
\hline 100 & 95 & 85 & 106 & 97 & 91 \\
\hline 100 & 94 & 89 & 79 & 99 & 91 \\
\hline 100 & 93 & 87 & 82 & 73 & 92 \\
\hline 100 & 92 & 85 & 80 & 75 & 67 \\
\hline 100 & 90 & 82 & 76 & 72 & 67 \\
\hline 100 & 75 & 67 & 61 & 57 & 54 \\
\hline 100 & 50 & 37 & 33 & 30 & 28 \\
\hline 100 & 20 & 10 & 7 & 6 & 6 \\
\hline
\end{tabular}

Tableau 3.3: Évolution du nombre de femmes (ou d'hommes) calculée par tranche d'âge et pour les cinq premières années à partir du modèle simple utilisé.

On voit qu'il n'y a aucun problème à prolonger le calcul. Le simulateur de population de l'INED est bien sûr plus sophistiqué que le nôtre. Il différencie les hommes et les femmes, et surtout, il fait évoluer à la fois de taux de naissance par âge et l'espérance de vie au cours du temps. Comment? C'est là tout l'art du démographe, qui s'appuie sur ce que l'on connaît déjà de sociétés analogues à celle qu'il étudie. Mais on voit, du même coup, en quoi ces projections ne sont pas des prédictions. En effet, les ingrédients du calcul varient au cours du temps, et il n'est pas assuré que les évolutions réelles vont suivre les anticipations. Si une guerre, une pandémie, une pénurie de ressources ou une perturbation politique majeure vient modifier dans 25 ans les taux de naissance, par exemple, le démographe d'aujourd'hui n'a aucun moyen de le savoir.

\subsubsection{Pour conclure?}

Terminons par quelques interrogations. Le tableau 3.4 rapporte les projections de population de I'INED pour les différentes régions du monde et pour les années 2050 et 2100.

\begin{tabular}{|c|c|c|c|}
\hline Zones & Année 2021 & 2050 & 2100 \\
\hline Afrique & 1,37 & 2,49 & 4,28 \\
\hline $\begin{array}{c}\text { Amérique Latine et } \\
\text { Caraïbes }\end{array}$ & 0,66 & 0,76 & 0,68 \\
\hline Amérique du Nord & 0,37 & 0,43 & 0,49 \\
\hline Europe & 0,75 & 0,71 & 0,63 \\
\hline Océanie & 0,043 & 0,057 & 0,075 \\
\hline Asie & 4,68 & 5,29 & 4,72 \\
\hline
\end{tabular}

Tableau 3.4: Projections des populations (en milliards d'habitants) des diverses régions du monde. Source: INED. 
On constate la forte progression de la population africaine. Pour l'Asie, un examen plus détaillé montre que la Chine passe par un maximum au cours du siècle, et que la population de l'Inde devient plus nombreuse que celle de la Chine. Ces projections prolongent les tendances actuelles, notamment en termes de transitions démographiques, de taux d'urbanisation et d'accès aux ressources permettant ces évolutions (eau, énergie, minéraux, etc.).

La question des ressources énergétiques est particulièrement cruciale. Les 7,8 milliards de terriens d'aujourd'hui consomment environ 14 Gtep ${ }^{42}$, dont $80 \%$ sont d'origine fossile (charbon, pétrole et gaz) émettant des gaz à effet de serre. Compte tenu de l'augmentation de la population mondiale et du développement des pays émergents et pauvres, la demande énergétique devrait atteindre les 20 Gtep au cours du siècle. Comment satisfaire cette demande, tout en réduisant drastiquement nos émissions de gaz à effet de serre?

\subsubsection{Annexe 1 : l'espérance de vie}

L'espérance de vie à la naissance se calcule de la façon suivante. Reprenons notre exemple d'une population avec 10 classes d'âge, avec les taux de mortalité: 0,1, 0,05, $0,06,0,07,0,08,0,1,0,25,0,50,0,80$ et 1 (Tab. 3.2). Ces taux correspondent à peu près à ceux de la fin du XIXe siècle en France. On part d'un échantillon de 100 individus, et on les fait vieillir en leur appliquant, à chaque âge, le taux de mortalité choisi.

Ainsi, au cours de la première année, il y a 10 décès, donc il ne reste plus que 90 personnes. On leur applique le taux de mortalité de 0,05, donc environ 4 personnes vont mourir, laissant 86 personnes vivantes. Cinq, parmi elles, vont mourir l'année suivante, d'où 81 personnes restantes etc. On peut ainsi constituer le tableau 3.5.

\begin{tabular}{|c|c|}
\hline $\begin{array}{c}\text { Nombre de } \\
\text { vivants }\end{array}$ & $\begin{array}{c}\text { Nombre de } \\
\text { décès }\end{array}$ \\
\hline 100 & 10 \\
\hline 90 & 4 \\
\hline 86 & 5 \\
\hline 81 & 6 \\
\hline 75 & 6 \\
\hline 69 & 7 \\
\hline 62 & 15 \\
\hline 47 & 23 \\
\hline 24 & 19 \\
\hline 5 & 5 \\
\hline
\end{tabular}

Tableau 3.5: Évolution fictive du nombre de vivants et du nombre de décès sur dix ans pour une classe d'âge comptant initialement 100 personnes.

42. Gtep: milliard de tonnes-équivalent pétrole (tep). 1 tep équivaut à 42 milliards de Joules. 
Au bout de ce parcours fictif, 10 personnes ont vécu 1 ans, 4 personnes ont vécu 2 ans, 5 personnes ont vécu 3 ans etc. On appelle espérance de vie à la naissance la durée de vie moyenne de cette cohorte fictive. Dans le cas présent, elle vaut:

$$
\frac{1 \times 10+2 \times 4+3 \times 5+4 \times 6+5 \times 6+6 \times 7+7 \times 15+8 \times 23+9 \times 19+10 \times 5}{100}=6,53 \text { ans }
$$

Si chaque tranche d'âge fictive représentait, en fait, 8 années, les dix tranches d'âge considérées couvriraient une vie humaine de 80 ans, et l'espérance de vie que nous venons de calculer ne serait que d'environ 52 ans. En réalité, cette faible valeur correspond bien aux taux de mortalité que nous avons choisis dans l'exemple et qui sont élevés. Nous avons ainsi supposé que $10 \%$ de la première tranche décédait dès les 8 premières années, soit 30 fois le taux actuel en France!

L'espérance de vie estimée à la naissance a toute chance de sous-estimer la durée de vie réelle, car le progrès de la médecine tend à diminuer les taux de mortalité, comme nous l'avons vu précédemment pour le taux de mortalité périnatal. Elle indique seulement ce que serait la durée de vie moyenne si les conditions futures demeurent identiques à ce qu'elles sont aujourd'hui.

On peut également définir l'espérance de vie à un âge donné. Que vaut, par exemple, l'espérance de vie à trois ans dans le modèle que nous avons choisi? Le tableau 3.5 indique que 81 personnes atteignent la troisième tranche d'âge. Partant de là, nous voyons que 6 personnes vont vivre 4 ans, 6 vont vivre 5 ans, 7 vont vivre 6 ans etc. L'âge moyen atteint par les personnes qui atteignent trois ans est donc égal à:

$$
\underline{4 \times 6+5 \times 6+6 \times 7+7 \times 15+8 \times 23+9 \times 19+10 \times 5}=7,48 \text { ans }
$$

C'est, par définition, l'espérance de vie à trois ans. II est normal que l'espérance de vie augmente avec l'âge, puisque les causes de mortalité aux âges précédents ont été évitées. Autrement dit: plus on vit vieux, plus on a de chance de vivre vieux, conclusion qui, somme toute, n'est pas déraisonnable...

\subsubsection{Annexe 2: Questions à propos du contrôle des populations et des migrations}

Le grand public est généralement sensible à deux questions qui ont une teneur plus directement sociale et politique. La première de ces questions concerne les moyens de «contrôle de la population». L'idée sous-jacente est que les populations elles-mêmes agissent plus ou moins directement et plus ou moins volontairement sur les paramètres d'évolution démographique discutés précédemment. Celles-ci font généralement en sorte de diminuer leur taux de mortalité et d'améliorer leur espérance de vie, cela étant associé à de meilleures conditions de vie. Dans les pays développés, cette lutte contre la fatalité butte aujourd'hui sur un ralentissement de 
l'augmentation de l'espérance de vie [10] et la question du vieillissement en bonne santé, ainsi que sur les problématiques sociales liées au vieillissement de la population. La plupart des sociétés n'envisagent cependant pas de réduire volontairement leur espérance de vie pour réduire ou contrôler la population. L'essentiel des mesures de contrôle, historiques et actuelles, portent sur le taux de natalité et les taux d'émigration et d'immigration à l'échelle d'une région ou d'un pays.

Regardons tout d'abord la question de la natalité. Au-delà des questions médicales, les sociétés humaines ont longtemps indirectement influencé le taux de natalité par les schémas sociétaux [8]. Par exemple, reculer ou avancer l'âge du mariage joue de façon importante sur la fécondité. II arrive que le célibat puisse être forcé. Les conditions sociales et l'existence d'une structure étatique vont également jouer un rôle important : en l'absence de système de protection sociale, faire suffisamment d'enfants peut donner l'espoir qu'au moins un s'occupera de vous pour vos vieux jours. Les états peuvent mener très directement des politiques natalistes ou dénatalistes par le biais d'impôts, subventions ou même interdictions comme dans le cas de la politique de l'enfant unique en Chine qui a été imposée pendant une trentaine d'années. Cette politique a eu de profonds impacts sociaux et démographiques, comme un fort vieillissement et un surplus d'hommes, qui ont conduit les autorités à l'assouplir significativement en 2018 puis en 2021 ${ }^{43}$. De fait, les conditions économiques (une crise économique a en général un impact négatif sur la natalité) et la condition des femmes (accès à la contraception, à l'éducation et à l'emploi, planning familial, égalité homme-femme, ...) jouent de façon très significative sur la fécondité depuis la deuxième moitié du XXe siècle. Au niveau mondial, la politique de gestion de la population de l'ONU a principalement utilisé ces leviers avec une efficacité relative selon les pays [8]. La baisse de la fécondité est une tendance partagée par tous les pays, même dans des situations économiques très différentes [9].

La répartition géographique de la population joue un rôle important dans les questions démographiques. À ce titre, il a toujours existé des déplacements de populations au sein des pays et entre les frontières. Les migrations peuvent avoir des causes très variées: familiales, économiques, politiques, ou à la suite de catastrophes humanitaires (guerres, famines, catastrophes naturelles). On pourra consulter ${ }^{44}$, à ce titre, l'exemple européen depuis l'après-guerre. Elles peuvent être voulues ou subies, ou encore bienvenues ou malvenues suivant le point de vue que l'on prend. Dans un même pays, l'immigration peut être vue comme un bénéfice en apportant des travailleurs qui redynamisent la démographie et rajeunissent la population, ou comme un problème de changement culturel. Concernant les chiffres ${ }^{43}$ [11], les démographes observent que les mouvements de populations se font principalement au sein des pays, puis entre pays voisins, avant de concerner les pays du sud vers les pays du nord. Bien que le nombre et les projections ne semblent pas

43. Politique de l'enfant unique: https://fr.wikipedia.org/wiki/Politique_de_l\%27enfant_unique

44. Le Dessous des Cartes, Flux migratoires, une longue histoire. https://www.arte.tv/fr/ videos/091146-019-A/le-dessous-des-cartes/ 
représenter en soi un problème [11], la concentration des flux sur quelques passages singuliers (Grèce, Lampedusa, Canaries, Calais ou Gibraltar pour l'Europe...) crée des difficultés de gestion qui tendent à fortement politiser la question et à en amplifier la perception.

\section{Références}

[1] A. Boutaud, N. Gondran. 2018. L'empreinte écologique. Éditions La Découverte, $2^{e}$ édition.

[2] I. El Bouazzaoui. 2008. L'empreinte écologique: Proposition d'un modèle synthétique de représentation des empreintes à l'échelle "Micro» d'une organisation ou d'un projet, Thèse de l'École Nationale Supérieure des Mines de Saint-Étienne, https://tel.archivesouvertes.fr/tel-00776465

[3] M. Wackernagel, B. Beyers. 2019. Ecological Footprint. Managing our biocapacity budget. New Society Publishers.

[4] M. Borucke et al. 2013. Accounting for demand and supply of the biosphere's regenerative capacity: The National Footprint Accounts' underlying methodology and framework. Ecological Indicators 24(2013), 518-533, dx.doi.org/10.1016/j.ecolind.2012.08.005.

[5] D. Lin et al. 2018. Ecological footprint accounting for countries: Updates and results of the national footprint accounts, 2012-2018. Resources 7(3), 58. https://doi.org/10.3390/ resources 7030058 .

[6] Living planet reports, 2014, 2016 \& 2020, https://www.footprintnetwork.org/livingplanet-report/.

[7] A. Boutaud, N. Gondran. 2020. Les limites planétaires, Éditions La Découverte.

[8] J. Véron. 2013. Démographie et écologie. Éditions La Découverte.

[9] J.N. Biraben. 2003. Population et Société 394

[10] G. Pison. 2019. Pourquoi l'espérance de vie augmente-t-elle moins vite en France? Population et Sociétés 564. https://www.ined.fr/fr/publications/editions/population-etsocietes/pourquoi-esperance-vie-augmente-moins-vite-france/.

[11] François Héran. 2018. L'Europe et le spectre des migrations subsahariennes. Population et Sociétés 558. https://www.ined.fr/fr/publications/editions/population-et-societes/ europe-spectre--migrations-subsahariennes/; https://www.college-de-france.fr/site/ francois-heran/index.htm. 
Partie II

Érosion de la biodiversité 



\section{Chapitre 4}

\section{Les différentes facettes de la biodiversité}

Ce chapitre présente les différentes facettes de la biodiversité afin de mieux appréhender pourquoi et comment la préserver dans un contexte où elle est particulièrement menacée. Qu'elle se décline en termes d'espèces, de différentiation génétique au sein d'une espèce ou d'écosystèmes qui regroupent plusieurs espèces en interaction entre elles et avec leur milieu naturel, la biodiversité n'est pas une collection figée d'êtres vivants, mais est, au contraire, en constante évolution. La protéger, c'est donc préserver les processus évolutifs en même temps que, selon l'acception plus courante, c'est aussi préserver les espèces vivantes, qu'elles soient remarquables, domestiquées ou simplement ordinaires, mais aussi les espaces où elles vivent. Comme nous le verrons, les relations, que nous, humains, entretenons avec la nature au sens large, donnent lieu à différentes manières de concevoir sa préservation selon qu'on lui attribue une valeur intrinsèque, instrumentale ou relationnelle, ce qu'on appelle l'éthique environnementale. À la fin de ce chapitre, nous nous familiariserons, par exemple, avec la notion de service écosystémique, qui met en avant les bénéfices que les humains tirent du fonctionnement des écosystèmes et de la biodiversité, et qui contribuent à leur bien-être.

\subsection{Qu'est-ce que la biodiversité ? - J. Lecomte et P.-H. Gouyon}

\subsubsection{Petit historique et définitions - J. Lecomte}

Selon Edward Osborne Wilson, scientifique américain connu comme le «père de la biodiversité» (Fig. 4.1), la naissance du terme «biodiversité» date d'un colloque qui s'est déroulé à Washington en 1986 et dont l'intitulé mentionnait le terme «BioDiversity». C'est donc un néologisme issu de Biological Diversity ou Diversité biologique en français. Les communications lors de ce colloque avaient sensibilisé au 
fait que l'accélération des extinctions d'espèces était pour la civilisation une menace qui venait juste après celle d'une guerre nucléaire. L'émergence de ce terme se situe donc à un moment où les humains sont perçus comme des éléments structurants de la biosphère, moment que certains nomment l'anthropocène.

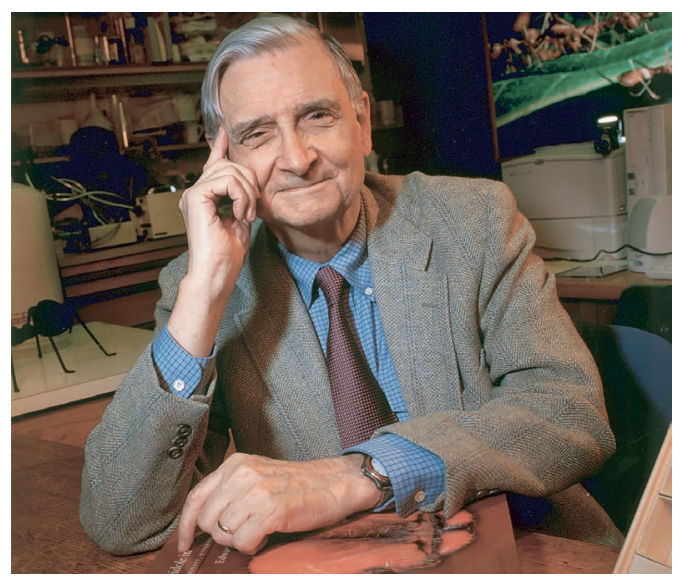

Figure 4.1: Edward Osborne Wilson (Université Harvard) en 2003. https://fr.wikipedia.org/wiki/Edward_ Osborne_Wilson (Creative commons 2.5, source : Jim Harrison).

Lors du sommet de la Terre de Rio de Janeiro en 1992, la communauté internationale rédige la Convention sur la Diversité Biologique (https://www.cbd.int/doc/legal/ cbd-fr.pdf) et les 189 états signataires s'engagent à la protéger. La diversité biologique y est alors définie comme la variabilité des organismes vivants de toute origine y compris, entre autres, les écosystèmes terrestres, marins et autres écosystèmes aquatiques et les complexes écologiques dont ils font partie; cela comprend donc la diversité au sein des espèces et entre espèces ainsi que celle des écosystèmes. Le terme biodiversité sera ensuite largement employé en lieu et place du terme diversité biologique. Dans cette définition, il est question de la diversité «au sein des espèces et entre espèces ainsi que celle des écosystèmes». La biodiversité, en tant que diversité de tout le vivant, est en effet classiquement structurée selon trois niveaux emboîtés: la diversité génétique, la diversité spécifique et la diversité écosystémique (Fig. 4.2).

Le premier niveau de biodiversité est la diversité génétique qui représente la variation génétique à l'intérieur des espèces, à la fois entre individus au sein d'une population et entre les populations d'une espèce, lorsqu'elles sont géographiquement séparées. La diversité génétique résulte du fait que les individus portent des formes très légèrement différentes de séquences d'ADN, les gènes, qui sont le support de l'information biologique. Un exemple de diversité génétique dans les populations humaines est la diversité des groupes sanguins. D'une manière générale, la diversité génétique permet aux espèces d'augmenter leur résistance aux maladies et leur potentiel de réponse à des conditions changeantes. 


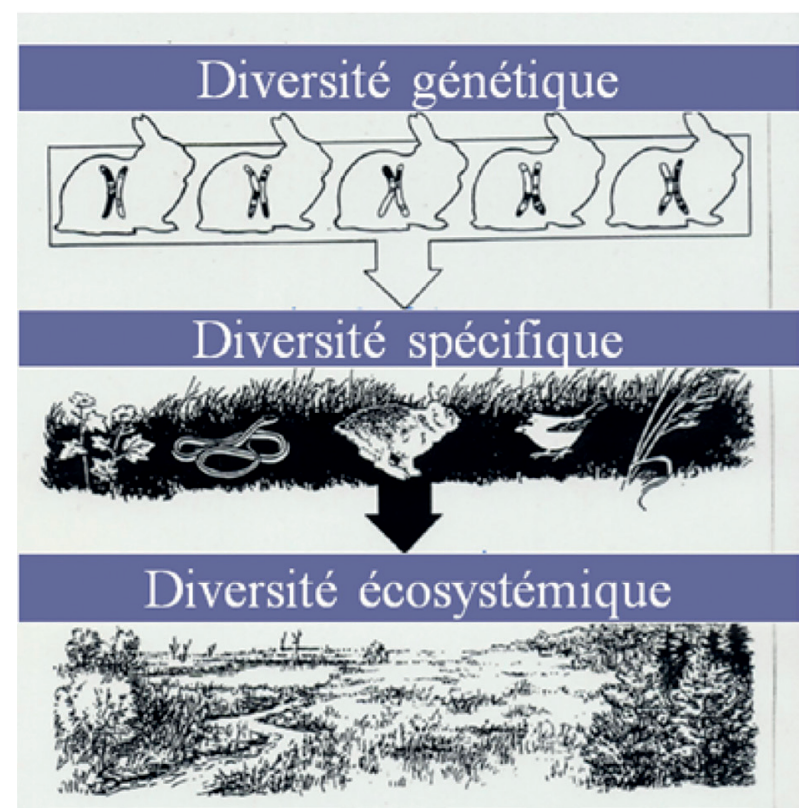

Figure 4.2: Les trois niveaux de la biodiversité: diversité génétique, spécifique et écosystémique (Source: Jane Lecomte).

Le deuxième niveau, dit de diversité spécifique, concerne la diversité entre les espèces. La diversité entre les espèces est pour une grande part la résultante de tout un ensemble d'adaptations évolutives et écologiques des organismes à des environnements particuliers. L'histoire de la biodiversité peut être représentée par un arbre phylogénétique qui retrace les relations de parenté entre des groupes d'êtres vivants (Fig. 4.3). La biodiversité est répartie entre trois grands domaines d'organismes: les archées, les bactéries et les eucaryotes avec leurs charges respectives de parasites moléculaires: virus, plasmides, transposons, etc. On y distingue les organismes unicellulaires (bactéries, archées et protistes) et les organismes multicellulaires (plantes, champignons, algues et animaux). La diversité au sein des microorganismes est une part très peu explorée alors qu'elle représente une part non négligeable de cette diversité. En termes d'ordre de grandeur, on comptabilise en 2020 plus de 2 millions d'espèces inventoriées et on estime le nombre total d'espèces à environ 8 millions (IPBES, 2019) [1].

Si la notion d'espèce peut être un des indicateurs utiles pour évaluer la biodiversité à un instant donné, il n'est pas le seul et il ne permet pas de rendre compte de la nature dynamique de la biodiversité, celle-ci étant plus un processus dynamique qu'un état (voir 4.1.2).

Le troisième niveau de biodiversité concerne la diversité écosystémique qui représente la diversité entre écosystèmes. Un écosystème correspond à des assemblages d'espèces, les communautés, en interaction entre elles (de prédation, 
de compétition, de symbiose, de facilitation, etc.) et avec les différentes conditions physico-chimiques de leur environnement (Fig. 4.4). La diversité écosystémique est donc représentée à la fois par la diversité des êtres vivants au sein de leur milieu, ce qu'on appelle la biocénose, et par la diversité des caractéristiques du milieu de vie, ce qu'on appelle le biotope.

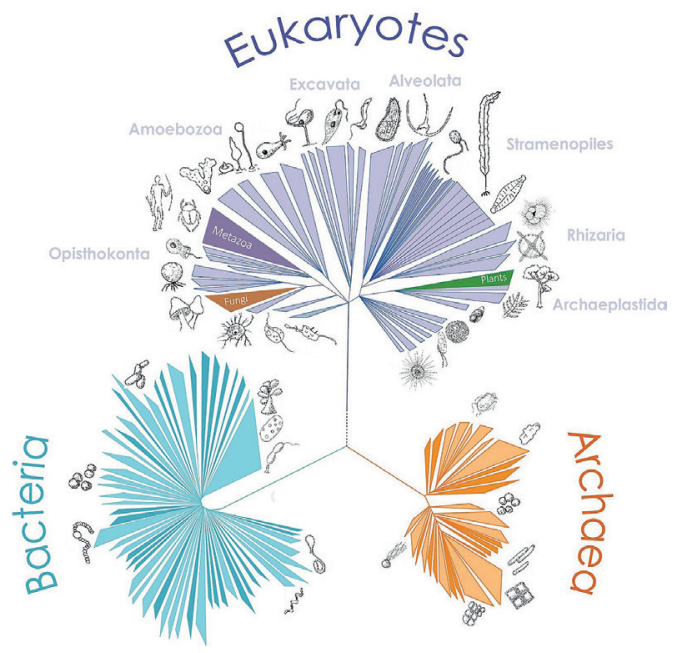

Figure 4.3: Exemple d'arbre phylogénétique. Les dessins illustrent des représentants des trois domaines du vivant (Archae, Bactéria et Eucarya) qui font partie de différents groupes d'organismes (noms en bout des «branches»-avec l'aimable autorisation de P. López-García).

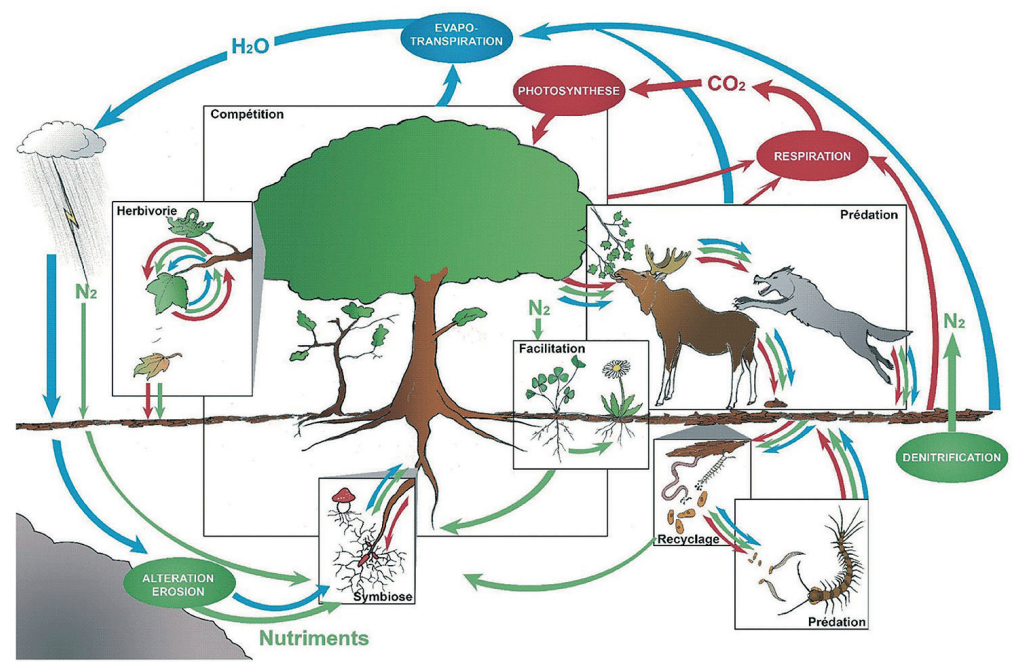

Figure 4.4: Représentation schématique des interactions et flux dans les écosystèmes [2]. Schéma I. Gounand. Source: N. Mouquet et al., Regards sur la biodiversité, SFE2, Regard R3 (2010). [https://www. sfecologie.org/regard/regards-3-mouquet/] 
Le fonctionnement de la biosphère, c'est-à-dire de l'ensemble des êtres vivants et de leur milieu physico-chimique (climat, propriétés des sols, chimie des milieux aquatiques, etc.), est basé, d'une part, sur les interactions entre les organismes vivants et d'autre part, sur les interactions entre les organismes et les milieux physico-chimiques via les flux de matière et d'énergie qui passent par le vivant (cycle de l'eau, du carbone, de l'azote, Fig. 4.4). Autrement dit, les organismes vivants dépendent de leur milieu physico-chimique mais, réciproquement, ils influencent leur milieu physico-chimique. La biosphère est donc pilotée à la fois par des flux de matière et d'énergie et par les flux d'organismes, qui se reproduisent, meurent et évoluent, ce qui influence en retour le fonctionnement de la biosphère sur le long terme [3]. De nombreux travaux montrent que l'adaptation des organismes à un environnement changeant s'exprime plus favorablement en présence d'une forte biodiversité. Le maintien d'une grande diversité (génétique, spécifique, et écosystémique) et de nombreuses interactions, entre tous les niveaux de diversité comme au sein de chacun d'entre eux, est donc essentiel pour la viabilité des entités de la biodiversité, y compris celle des populations humaines.

En résumé, la biodiversité résulte de processus écologiques et évolutifs ainsi que de flux de matière et d'énergie, qui passent par le vivant. Depuis un peu plus de 3,8 milliards d'années, la biodiversité a été façonnée par des processus évolutifs qui résultent d'interactions écologiques au sein et entre ses différents niveaux d'intégration, mais aussi avec les conditions abiotiques environnantes et développementales. La biodiversité n'est pas une collection figée d'êtres vivants, mais est issue de processus dynamiques intervenant à différentes échelles d'espace et de temps.

\subsubsection{Une dynamique plutôt qu'un état - P.-H. Gouyon}

Extraits de la vidéo UVED: Biodiversité: état ou dynamique de Pierre-Henri Gouyon, Professeur au Muséum National d'Histoire Naturelle (de 0' à 2,15' et de 6,01' à 9,08'), https://www.canal-u.tv/video/canal_uved/2_biodiversite_etat_ou_dynamique.18186.

La biodiversité, est-elle un état ou une dynamique? Le schéma de Darwin (Fig. 4.5) paru dans son livre "De l'origine des espèces» en 1859, qui décrit la diversification des formes vivantes et par lequel il explique globalement sa théorie, va nous aider à répondre à cette question. Le temps dans ce schéma s'y lit de bas en haut. C'est une habitude de géologue, mais c'est aussi le cas de certaines phylogénies qui représentent schématiquement les liens de parenté des formes vivantes, entre individus, populations ou espèces. En fait, le schéma de Darwin n'est pas une phylogénie, car l'échelle horizontale donne une mesure de la différenciation écologique des lignées. Plus des lignées sont éloignées les unes des autres sur l'axe horizontal, plus elles sont différentes sur le plan écologique. Contrairement aux phylogénies classiques où sont représentées les différentes formes vivantes en fonction de leur histoire, ce schéma tient donc compte de leur écologie. Chaque lignée à chaque instant produit des formes vivantes nouvelles, et la plupart de ces formes nouvelles s'éteignent. Mais certaines formes se maintiennent. Ce sont plus particulièrement celles 
qui sont les plus éloignées au sens écologique du terme, c'est-à-dire celles qui ont des habitats différents, car cela leur permet de réduire leur compétition. Ce phénomène répété de génération en génération, tout au cours du temps, va permettre aux différentes lignées de diverger et de produire progressivement des formes de plus en plus différentes.
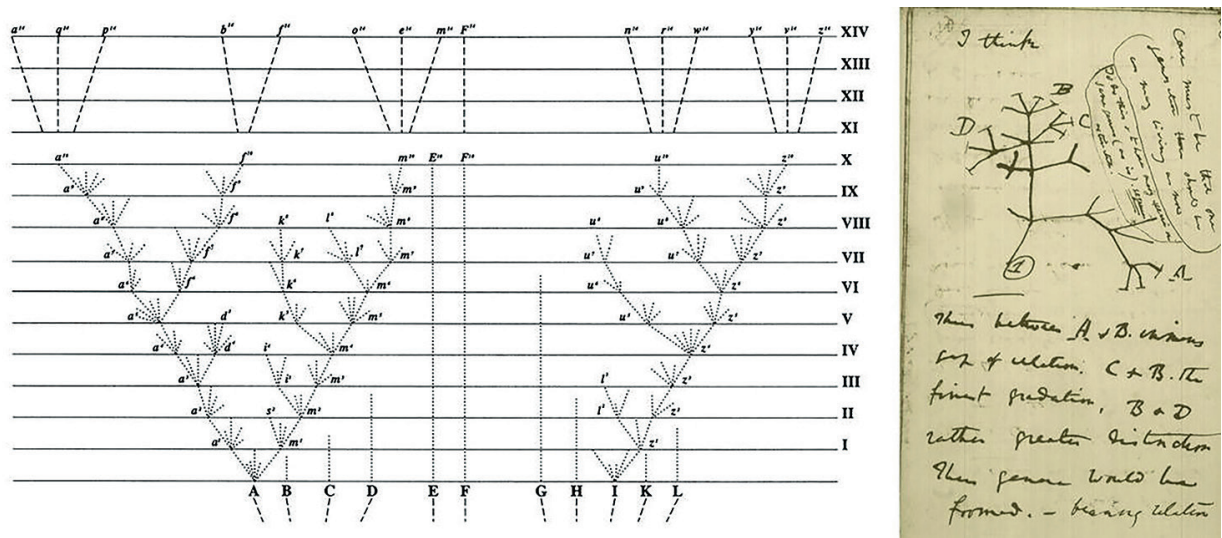

Figure 4.5: Schéma extrait du livre "De l'origine des espèces» de Darwin (1859). Chaque ligne en tiretés représente une lignée de formes vivantes, issues d'un même ancêtre. Ces formes vivantes se diversifient au cours du temps (de bas en haut) en donnant naissance à de nouvelles formes vivantes, lesquelles sont aussi susceptibles de s'éteindre (trait interrompu). La diversité des formes vivantes actuelles résultant de l'évolution de ces lignées est représentée tout en haut du schéma.

L'évolution est donc un processus permanent de production de formes vivantes issues de lignées qui se différencient, divergent et peuvent s'éteindre. La façon dont on a décrit communément la diversité par la systématique, qui est la science des classifications du monde vivant, en rassemblant les espèces à l'intérieur d'entités hiérarchisées que sont les genres, familles, ordres, classes, divisions et règnes traduit, depuis Darwin, l'emboîtement des lignées et résulte donc directement de l'histoire de la diversité. En revanche, le choix d'un niveau donné est pour le moins arbitraire, car la diversité résulte d'une différenciation continue. Deux lignées en se différenciant deviendront des variétés, puis des sous-espèces, puis des espèces par un mouvement en général progressif. Ce qu'on appelle espèce est donc un concept flou. On ne peut, en effet, nier la capacité de deux espèces de se croiser en donnant naissance à des individus eux-mêmes féconds, ce qu'on désigne par interfécondité. La séparation étanche entre espèces n'est ainsi ni un réel critère pour les plantes, ni pour bactéries, ni même pour certains mammifères. Les espèces dans le schéma de Darwin représenteraient simplement ces rameaux qui se sont particulièrement différenciés.

La biodiversité se forme et se détruit donc constamment du fait des extinctions. Dans le schéma de Darwin, on peut noter que de très nombreuses extinctions sont représentées. L'extinction n'est pas antinomique de l'idée de biodiversité. Tout au 
contraire, s'il n'y avait pas d'extinction, il n'y aurait pas de diversité. En effet, l'extinction crée en quelque sorte du vide entre deux lignées. Si jamais il n'y avait pas eu d'extinctions, il y aurait un strict continuum et donc beaucoup moins de diversité. En termes d'analogie, on peut dire que l'extinction sculpte la biodiversité, grâce au fait qu'elle est compensée par la divergence, comme la mort cellulaire sculpte nos doigts. Divergence et extinction sont donc les deux éléments de la dynamique de la biodiversité.

Il y a un équilibre de la biodiversité, mais il ne s'agit pas du tout d'un équilibre statique. Quand on parle d'équilibre, en général on pense soit à des équilibres stables, soit à des équilibres instables. La notion d'équilibre dynamique est plus difficile à appréhender et pourtant c'est un principe familier. Par exemple, il est évident pour nous qu'un vélo ne tient que parce qu'il bouge. Grâce au fait qu'un vélo bouge, on va pouvoir compenser ce qui le ferait tomber à droite ou à gauche pour le garder droit sur la route. Et on sait bien que si on s'arrête brutalement en vélo, la chute est au rendez-vous. C'est le cas aussi d'un avion. II y a un équilibre dynamique entre la pesanteur, qui le fait descendre, et la force de l'air sur les ailes, qui le fait monter. Les satellites sont de même en équilibre dynamique, ce qui fait que si jamais ils ralentissent, ils finissent par tomber. En fait, on a une perception intuitive de cet équilibre dynamique dans le cas de notre corps. On est en équilibre dynamique avec notre environnement. Le fait qu'on mange, qu'on excrète et qu'on respire, nous maintient dans cette dynamique. Et si on perd cette dynamique, on meurt. De la même manière, il faut penser la biodiversité comme une dynamique et non pas comme un état stable. On ne peut pas la conserver telle quelle et on ne peut la maintenir. On doit au contraire favoriser les mécanismes qui la gardent en mouvement. Si ce n'est pas le cas, la biodiversité et nous qui en faisons partie, sommes susceptibles d'une chute extrêmement brutale!

Le schéma de Darwin est donc la meilleure manière de représenter la biodiversité. Si on veut comprendre ce qu'est la biodiversité, il faut intégrer cet aspect d'équilibre dynamique de la vie, entre divergence et extinction. Comme disait Albert Einstein: «la vie, c'est comme une bicyclette, il faut avancer pour ne pas perdre l'équilibre».

\subsection{La biodiversité du point de vue de sa préservation - J. Lecomte}

Force est de constater que les humains, de par leurs activités, jouent désormais un rôle critique au sein de la biosphère en impactant non seulement les entités de biodiversité mais aussi, à court terme, notre propre survie. Si rien n'est fait pour inverser la dynamique des extinctions d'origine anthropique, des écosystèmes entiers seront dégradés ainsi que des populations d'espèces, voire des espèces entières qui disparaîtront à tout jamais [3]. 
D'une manière globale, les réponses à apporter face à l'enjeu global lié à l'érosion de la biodiversité ( $c f$. chapitres 5 et 6 ) doivent être issues aussi bien des champs de la recherche en écologie et en sciences de l'évolution que des sciences humaines et sociales. Elles mobilisent, en effet, un certain nombre de valeurs et sont à l'origine de l'éthique dite environnementale (cf. section 4.4).

Les espèces dites remarquables, par exemple, les pandas, les baleines, les éléphants, les tigres, etc. influencent favorablement l'opinion publique vers la préservation de la biodiversité. C'est la raison pour laquelle on les nomme aussi des espèces étendards. On comprend alors pourquoi l'association de protection de la nature, le Fonds Mondial pour la Nature (le WWF) a choisi le panda comme emblème! En mettant en visibilité de telles espèces, il est ainsi plus facile de mobiliser l'intérêt pour la préservation pour d'autres espèces moins charismatiques, comme les insectes, par exemple, qui font partie de la biodiversité dite ordinaire.

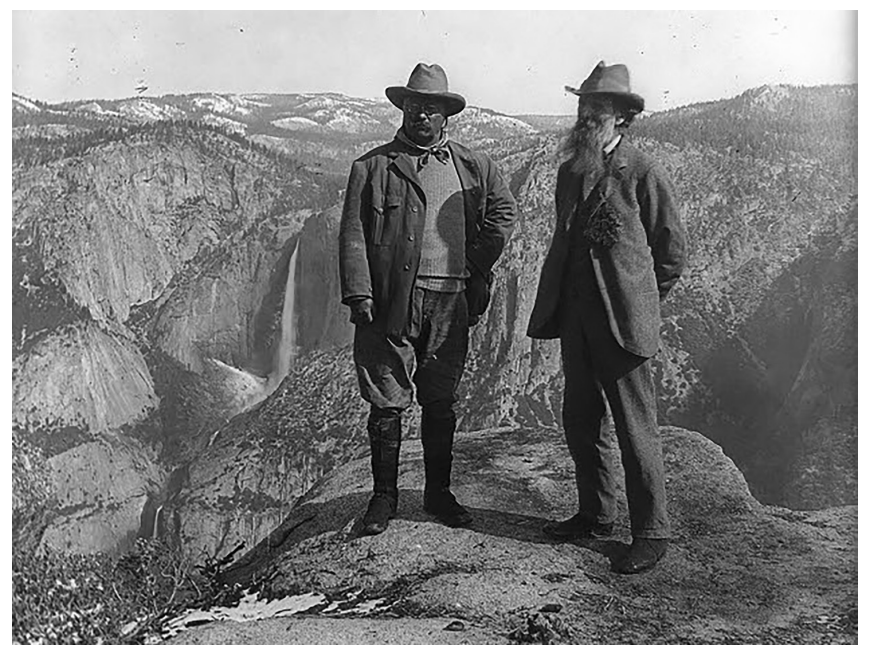

Figure 4.6: La photo, prise en 1903, représente John Muir à droite, qui amène Théodore Roosevelt, le président des États-Unis de l'époque, camper dans le Yosemite pour le convaincre de la nécessité de protéger ce site d'une exploitation grandissante. Wikimedia commons.

Les espèces remarquables légitiment aussi la création d'espaces protégés pour assurer leur survie dans les meilleures conditions possibles. En préservant les habitats d'espèces remarquables qui ont besoin de grands espaces de vie, tout un cortège d'autres espèces qui vivent dans ces habitats est aussi préservé. Pour cette autre retombée, les espèces remarquables sont aussi appelées espèces « «parapluie», car comme un parapluie, elles protègent les autres.

Les réflexions sur la nécessité de préserver la biodiversité sont relativement anciennes. En effet, dès le XVIII siècle, certains voyageurs européens identifient les limites d'une exploitation trop intense d'espaces nouvellement colonisés. C'est dans le cours du XIXe siècle qu'aux États-Unis, différentes approches d'une vision morale 
et spirituelle de préservation de la nature sont portées par Henry David Thoreau, Ralph Waldo Emerson et John Muir. Ce dernier a d'ailleurs contribué activement à la création du parc national du Yosemite (Fig. 4.6). Dans ces hauts lieux de biodiversité, il s'agissait de préserver à la fois la beauté des paysages et des espèces qui y vivaient.

Hors des espaces protégés, plusieurs milliers, voire millions d'espèces de biodiversité ordinaire sont présentes dans $80 \%$ des écosystèmes. II s'agit des populations de plantes, d'insectes et d'oiseaux qu'on a l'habitude de voire communément et qui, pourtant, disparaissent à grande vitesse. Cette biodiversité ordinaire est, en effet, menacée d'extinction dès lors que son habitat n'est pas préservé. Bien que la biodiversité ordinaire puisse apparaître moins porteuse pour mobiliser le public, car elle nous est plus commune, sa préservation est par contre essentielle pour maintenir les potentialités évolutives de la biodiversité et le fonctionnement des écosystèmes. De plus, biodiversité remarquable et biodiversité ordinaire sont issues de longs processus de sélection naturelle et leurs disparitions signifieraient de gommer d'un trait parfois des dizaines de milliers d'années d'évolution sans possibilité de revenir en arrière.

Un troisième type de biodiversité, dite biodiversité domestiquée, concerne les animaux d'élevage et les plantes cultivées. Cette biodiversité est issue des programmes de sélection génétique conduits par les humains depuis le néolithique (cf. section 1.3). Depuis peu, la mondialisation est à l'origine d'une perte de la biodiversité domestiquée qui conduit des associations et des collectifs à s'investir dans la sauvegarde de la biodiversité domestiquée et sa diversité génétique. Ainsi, les moutons d'Ouessant qui sont ces moutons noirs de petite taille (Fig. 4.7) sont sauvegardés par une association qui est l'unique organisation officiellement chargée pour toute la France de maintenir cette race de moutons.

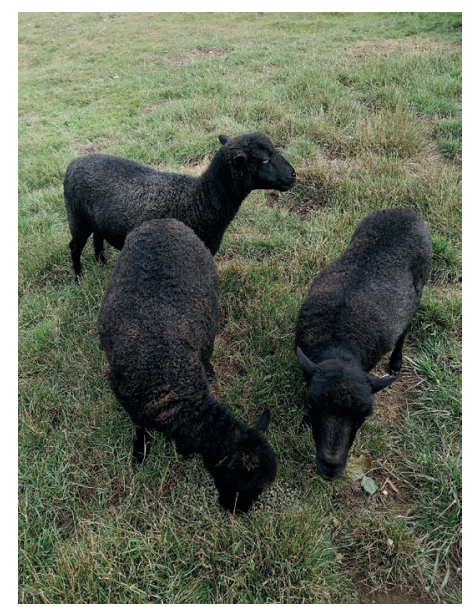

Figure 4.7: Trois moutons d'Ouessant près de Landévennec dans la presqu'île de Crozon (Bretagne). D'après Jane Lecomte. 
Pour préserver la biodiversité à l'extérieur des espaces protégés, le développement d'espaces de coexistence entre les humains et la biodiversité est au cœur de l'écologie dite de la réconciliation. Cette dernière vise à concilier des activités humaines et la biodiversité pour maintenir la biodiversité ordinaire dans les milieux «anthropisés» et de préserver les services écosystémiques (voir section 4.5). Ce type d'approche est susceptible de se renforcer, compte tenu de l'expansion actuelle des zones urbaines.

L'information et la sensibilisation aux enjeux environnementaux des populations des villes et des villages constituent l'objet de l'écologie de la reconnexion, qui s'appuie à la fois sur des éléments de la biodiversité sauvage et de la biodiversité domestiquée, présents dans ces milieux.

Finalement, les arguments peuvent être divers pour préserver la biodiversité. Faut-il privilégier la biodiversité remarquable, la biodiversité ordinaire ou la biodiversité domestiquée? Les arguments mobilisent différents registres de valeurs. Mais déjà si nous renversions la charge de la preuve quand il s'agit de la biodiversité et de sa préservation, et qu'au lieu de poser la question «Pourquoi préserver?», nous nous posions la question «Pourquoi détruire?», nous pourrions faire de plus grands pas pour lutter efficacement contre son érosion.

\subsection{Lien entre biodiversité et fonctionnement des écosystèmes : l'exemple des forêts - N. Delpierre}

Sur l'ensemble de la planète, on dénombre 400000 espèces de plantes, dont 100000 espèces d'arbres. Même si le citadin peut croiser des arbres en ville, ces arbres sont essentiellement présents dans des forêts.

Les forêts contiennent plus ou moins d'espèces. Les forêts tropicales, par exemple, présentent une diversité spécifique très importante. On y trouve de l'ordre de 100 à 250 espèces d'arbres différentes sur un hectare. Ce chiffre est à comparer avec les forêts tempérées où se côtoient 10 à 30 d'espèces d'arbres par hectare. Les forêts boréales ou taïgas présentes au nord de l'hémisphère nord ne comportent, en revanche, que quelques espèces d'arbres, parfois même une seule sur un hectare.

Mais la diversité spécifique n'est pas suffisante pour décrire les arbres, ni le fonctionnement des forêts. Prenons l'exemple d'une forêt tempérée dans le massif de Fontainebleau. Parmi les 15 espèces qui composent cette forêt, une espèce peut sortir du lot, comme le Charme, compte tenu du nombre très important de ses représentants dans la forêt (Fig. 4.8). Par contre, si on s'intéresse à la répartition en masse, une autre espèce comme le Chêne, qui ne comporte cependant que peu d'individus, représente $80 \%$ de la masse des arbres de la forêt. On dit alors que les chênes «dominent» la forêt. 
Le fait que plusieurs espèces d'arbres cohabitent dans une même forêt va entraîner des conséquences, notamment sur leur croissance. En effet, les plantes ont besoin de l'accès à la lumière pour faire leur photosynthèse et se développer. Or, seule une certaine quantité de lumière éclaire la forêt à chaque instant, et cette lumière est interceptée par les arbres les plus grands. C'est d'ailleurs la raison pour laquelle les températures en forêt sont plus modérées en été, même lors de journées fortement ensoleillées. Comme cette lumière est interceptée par les grands arbres, elle n'arrive qu'en faible quantité au niveau des arbres plus petits. On parle alors de «compétition» pour la lumière. C'est effectivement ce qui se produit, car de nombreux arbres de petite taille meurent à l'ombre des plus grands, dès lors qu'ils ne sont pas suffisamment éclairés. Cette compétition n'est pas en rapport avec le nombre d'individus d'une espèce donnée. Si on reprend l'exemple de la forêt tempérée du massif de Fontainebleau (Fig. 4.8), les Charmes y sont très majoritaires, mais $70 \%$ des feuilles sont des feuilles de Chêne, et ce sont les Chênes qui interceptent $93 \%$ de la lumière disponible.
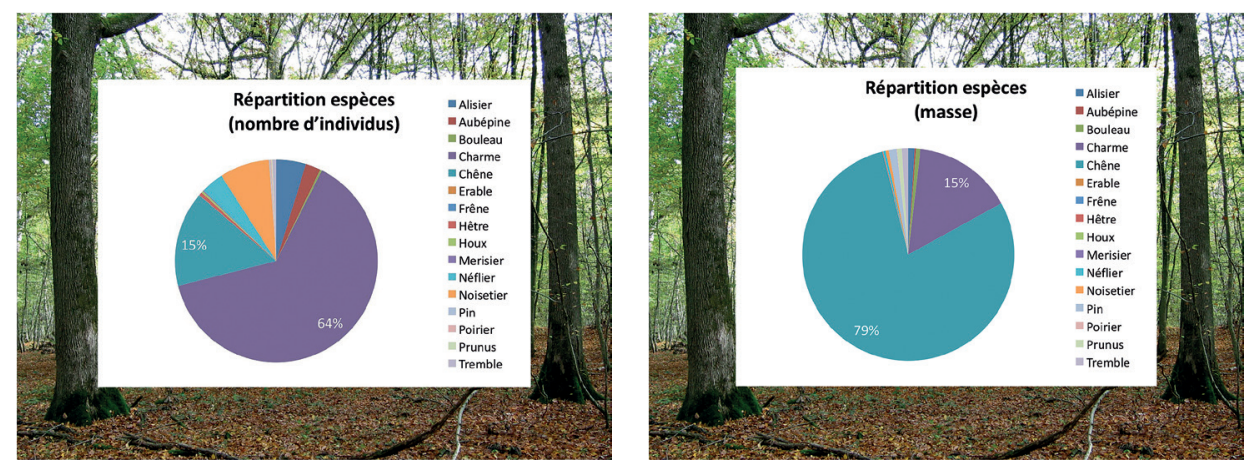

Figure 4.8: Répartition des arbres d'une forêt tempérée du massif de Fontainebleau (voir http:// www.barbeau.universite-paris-saclay.fr/). Une quinzaine d'espèces d'arbres compose la forêt. Les deux-tiers des individus sont des Charmes (diagramme de gauche). En revanche, ce sont les Chênes qui représentent $80 \%$ de la masse des arbres de la forêt (diagramme de droite). Source: Nicolas Delpierre.

De la même manière, les arbres sont en compétition pour l'eau. Le sol s'assèche par le haut, là où la densité des racines est la plus importante. Ce sont donc les arbres qui ont les racines les plus profondes qui vont donc mieux passer les périodes de sécheresse.

Lorsqu'on synthétise les données de croissance et de diversité des espèces d'arbres dans les forêts, on constate, une fois après avoir écarté les effets du climat ou de la nature du sol, que les forêts présentant le plus grand nombre d'espèces d'arbres sont également celles qui ont la plus forte croissance (Fig. 4.9). 


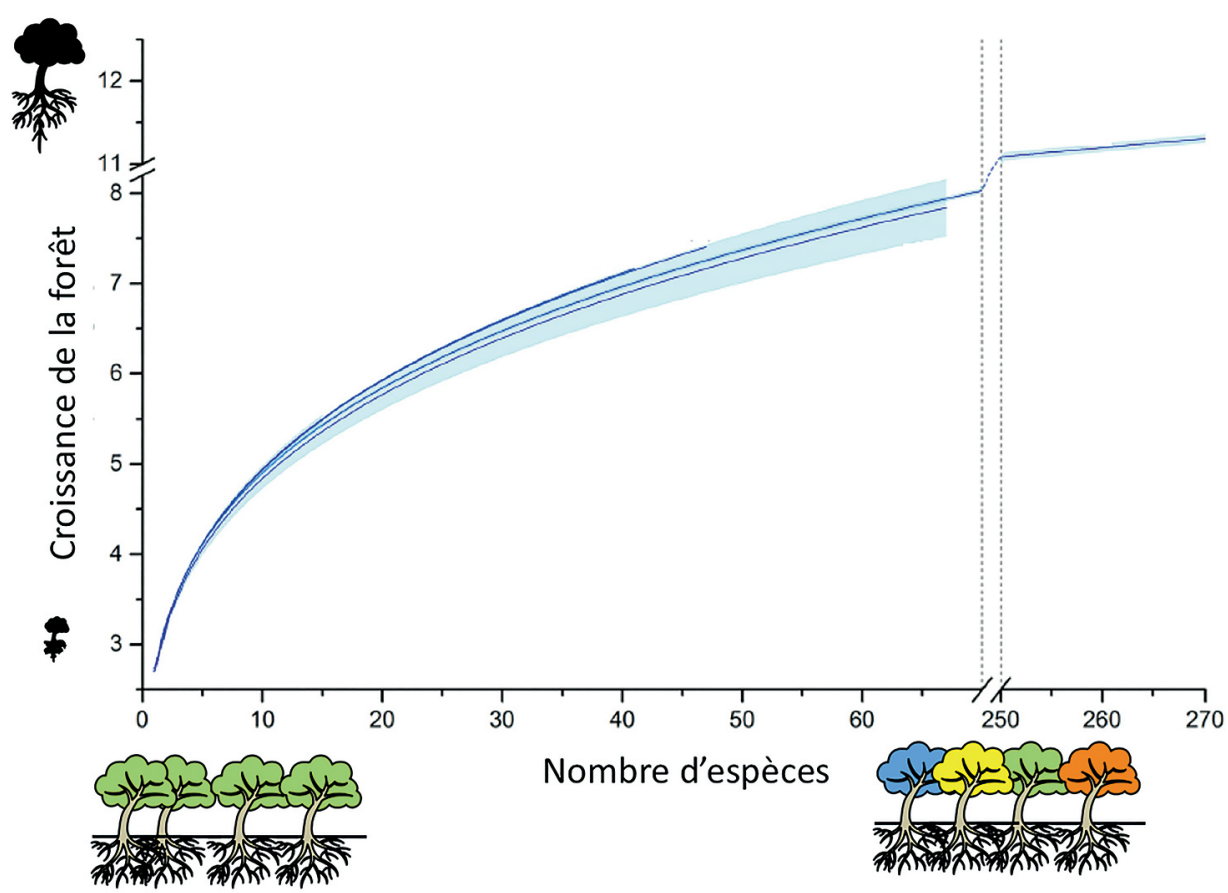

Figure 4.9: Relation entre nombre d'espèces et croissance de la forêt. D'après [4].

Le fait que la croissance augmente avec le nombre d'espèces d'arbres présentes permet d'aller plus loin dans la compréhension de la compétition pour les ressources nécessaires aux arbres (la lumière, l'eau, les nutriments du sol). En effet, si on considère une forêt avec une seule espèce, les arbres similaires entre eux vont souvent être en compétition les uns avec les autres. On parle dans ce cas de «compétition intra-spécifique».

À l'inverse, dans une forêt avec plusieurs espèces d'arbres, certains sont grands, d'autres petits, certains ont des racines profondes, d'autres ont des racines plus superficielles, et les espèces sont en quelque sorte "complémentaires», car elles ont des besoins différents. On dit aussi qu'elles ont des «niches écologiques » différentes. La figure 4.10 compare, de façon simplifiée, la situation d'une forêt à deux espèces à celle d'une forêt à une seule espèce. La compétition entre deux espèces (on parle ici de «compétition inter-spécifique») est bien présente, mais elle est relativement moins élevée que celle entre les arbres d'une même espèce ( «compétition intra-spécifique»). La croissance générale de la forêt bénéficie de cette compétition réduite. 

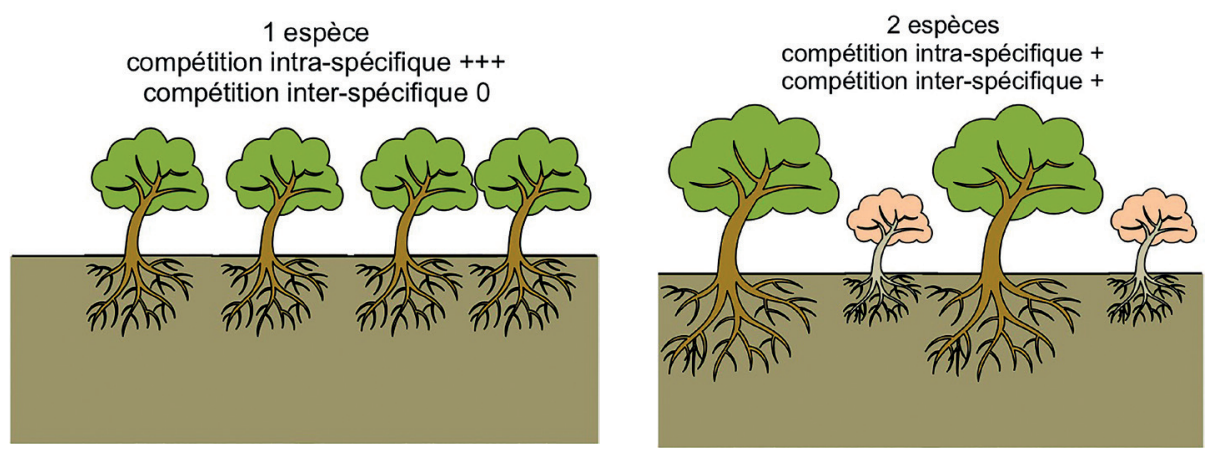

Figure 4.10: Nombre d'espèces d'arbres dans une forêt et compétition. D'après [4].

La photo de la figure 4.11, prise en automne, permet d'illustrer concrètement la réduction de la compétition intra-spécifique dans le cas d'une forêt à deux espèces. Les arbres verts sont des conifères qui restent effectivement verts toute l'année. Les autres arbres, dont les feuilles sont jaunies, sont des Hêtres, qui perdent leurs feuilles en hiver. Tout au long de l'hiver jusqu'au printemps, les conifères en mélange avec les hêtres, qui n'auront plus de feuilles, vont donc bénéficier de forts éclairements, ce qui va faciliter leur croissance.

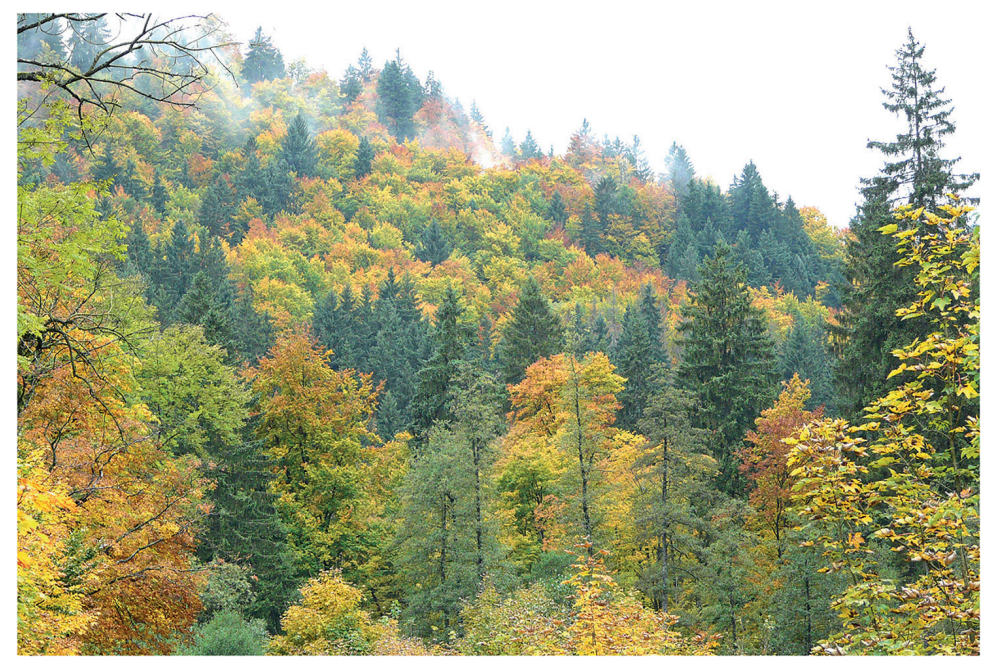

Figure 4.11: Photo prise en automne dans le parc national du Harz (Allemagne). Avec la courtoisie de Ralf Steinberg.

Le mélange de plusieurs espèces ne se traduit pas uniquement par une réduction de la compétition intra-spécifique. II permet aussi le développement d'arbres qui ont besoin du climat forestier pour se développer lorsqu'ils sont jeunes, alors qu'ils se développent mal en pleine lumière. On parle alors de «facilitation » du développement des jeunes arbres par les arbres adultes d'une autre espèce. 
Un autre exemple de facilitation est celui de l'ascenseur hydraulique (Fig. 4.12). Les plantes qui ont des racines profondes peuvent puiser de l'eau en profondeur. Elles évaporent alors cette eau vers l'atmosphère au niveau de leurs feuilles. Mais au cours du transfert de l'eau des racines vers les feuilles, dans certaines conditions, une petite partie de l'eau peut sortir des racines et ainsi humidifier légèrement le sol très sec situé en surface. Les plantes qui ont des racines moins profondes ont ainsi accès à l'eau et peuvent mieux faire face à la sécheresse.

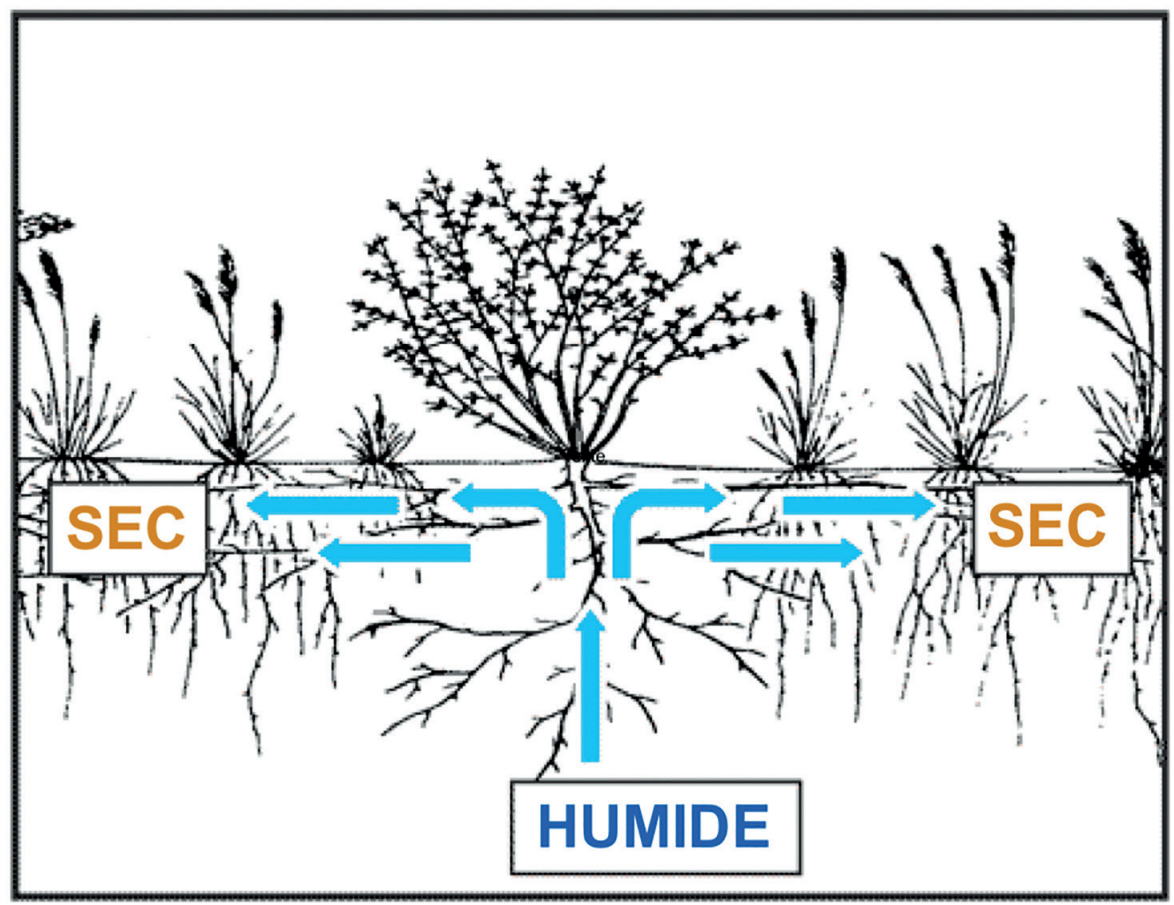

Figure 4.12: Le phénomène d’ascenseur hydraulique. Avec la courtoisie de F.C. Meinzer ${ }^{45}$.

On pense effectivement que le mécanisme d'ascenseur hydraulique entre en jeu dans la réponse des forêts à la sécheresse. Ainsi, dans une forêt monospécifique, confrontée à une sécheresse très intense, la croissance diminue fortement et ne reprend qu'après la sécheresse. Si deux espèces d'arbres coexistent, on constate que lors de la sécheresse, toutes choses étant égales par ailleurs, la baisse de croissance est moins forte. On parle alors de meilleure résistance à la sécheresse (Fig. 4.13). La croissance repart également à un niveau plus important après la sécheresse. On parle de meilleure «résilience » de la forêt. Autant d'éléments qui tendent à montrer que le mélange d'espèces est souvent associé à un meilleur fonctionnement des forêts. 


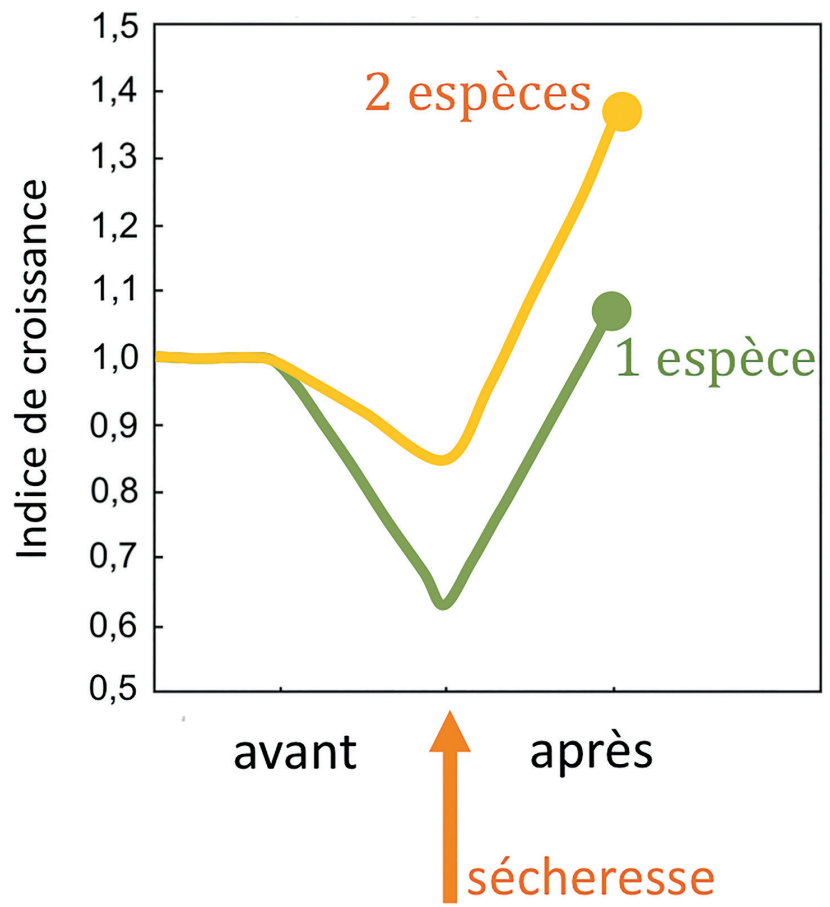

Figure 4.13: Résistance / résilience à la sécheresse et nombre d'espèces dans une forêt. D'après [5].

\subsection{Les éthiques environnementales - C. Larrère}

Vidéo UVED issue du MOOC «Environnement et Développement Durable» :

Les éthiques environnementales de Catherine Larrère, Professeur émérite de philosophie, Panthéon 1 Sorbonne (de 0,27'à 4,54' = 4,27'), https://youtu.be/vvt8BVLibjE.

Que signifie l'éthique environnementale? On désigne par là une réflexion philosophique sur les rapports des hommes et de la nature, qui s'est développée au cours des années 1970 dans les pays anglophones (États-Unis, Canada/Australie-Nouvelle Zélande/Grande Bretagne). L'idée est que nous n'avons pas avec la nature des rapports seulement techniques, mesurés par notre puissance d'action, mais également des rapports moraux: nous avons des devoirs à son égard, elle peut même avoir des droits.

Cette réflexion a donné lieu à tout un courant académique, mais, pour bien le comprendre, il faut revenir un peu en arrière, à la première moitié du XXe siècle, à l'époque où vivait Aldo Léopold, un forestier américain qui, après s'être occupé de la gestion de la faune sauvage et en avoir enseigné les règles, a écrit un livre dont la publication en 1949, juste après sa mort, a connu un très grand succès. Ce succès ne s'est jamais démenti depuis lors, et le livre d'Aldo Léopold est considéré comme un point de départ de réflexions philosophiques sur la protection de l'environnement. 
Intitulé Almanach d'un Comté des Sables, le livre retrace des épisodes de la vie d'Aldo Léopold dans la nature, au sein du domaine qu'il possédait dans le Comté des Sables dans le Wisconsin au nord des États-Unis, et également dans d'autres États américains. L'auteur y narre ses rencontres avec des animaux, ses observations, et à la fin du livre, il expose son éthique de la terre en soulignant qu'il lui "paraît inconcevable qu'une relation éthique à la terre puisse exister sans amour, sans respect, sans admiration pour elle et sans une grande considération pour sa valeur». Et, par valeur, précisait-il, il ne faut pas attendre la valeur au sens économique, mais la valeur philosophique. C'est bien pour répondre à ce type de questionnement que les éthiques environnementales se sont développées dans le dernier tiers du XXe siècle. Elles y ont apporté deux types de réponses.

La première, c'est celle de la valeur intrinsèque. L'idée est d'opposer l'instrumental et l'intrinsèque, ce qui vaut en soi et ce qui vaut comme moyen, comme instrument, dont nous nous servons. La valeur économique mesure, d'une façon ou d'une autre, l'utilité qu'une chose a pour nous. À l'opposé, parler de la valeur intrinsèque, c'est dire que les choses n'existent pas que pour nous, qu'elles ont une valeur en elles-mêmes, que tout être vivant, qui se conserve dans l'existence et cherche à se reproduire, a une valeur pour lui-même, et que cette valeur doit être respectée. Les éthiques de la valeur intrinsèque sont des éthiques du respect de la nature, d'une nature qui existe pour elle-même, indépendamment de nous.

La deuxième réponse a consisté à dire que cette opposition entre instrumental et intrinsèque était rigide et que nous pouvions très bien trouver une utilité à la nature sans pour autant la détruire comme nous le faisons quand nous consommons des produits agricoles ou tuons des animaux sauvages. Ces critiques ont fait valoir toutes les raisons diverses que nous avions de donner de la valeur à la nature, toutes les émotions qu'elle pouvait provoquer en nous tout en nous étant utile. Nous pouvons l'utiliser et vouloir la conserver. Un scientifique ne cherche pas à détruire son objet d'étude, il en a besoin! L'admiration esthétique, que nous avons pour la nature, nous inspire des pensées métaphysiques ou des sentiments religieux et nous pousse à la préserver. On peut ranger ces services culturels que nous rend la nature parmi d'autres services, dits écosystémiques, telle la pollinisation par les abeilles qui permettent aux fleurs de se reproduire. Là oú la valeur intrinsèque dit la singularité, la dignité d'une entité, son caractère irremplaçable, l'éthique de la pluralité des valeurs, insiste sur la façon dont ces évaluations diverses peuvent converger dans la volonté commune de protéger la nature.

Dans un cas comme dans l'autre, l'important est la communauté que nous formons avec les autres êtres vivants et notre souci de la préserver dans son intégrité. Telle est la règle formulée par Léopold: "Une chose est juste lorsqu'elle tend à préserver l'intégrité, la stabilité et la beauté de la communauté biotique. Elle est injuste lorsqu'elle tend à l'inverse». 


\subsection{Les services écosystémiques: nouvelle métaphore instrumentale des relations Humains-Nature - A. Vallet}

Les services écosystémiques représentent tous les bénéfices que les humains tirent du fonctionnement des écosystèmes et de la biodiversité, et qui contribuent à leur bien-être. Ce rôle n'est pas nouveau, car depuis la préhistoire, les humains ont prélevé dans la nature de quoi s'alimenter, de quoi construire leurs maisons (du bois, des pierres, les matériaux constitutifs du ciment), et de quoi s'inspirer. Les peintures rupestres, riches en animaux, et végétaux, en sont une bonne illustration.

Au XIXe siècle, des économistes comme Jean-Baptiste Say et David Ricardo soulignèrent l'importance de la nature, qui «travaille gratuitement pour nous». Ces auteurs citent, par exemple, le vent, qui fait tourner les moulins, ou le soleil qui produit de la chaleur. Le concept de service écosystémique en lui-même est relativement récent. Le terme apparaît enfin dans les années 1970 suite aux travaux de Schumacher et Ehrlich. II gagne en popularité dans les années 1990, notamment grâce à une publication de Costanza [6a] qui calcule la valeur économique des services écosystémiques à l'échelle globale, afin d'alerter sur leur importance. Cette publication sera remise à jour en 2014 [6b].

Le concept de service écosystémique est souvent représenté sous forme d'une cascade (Fig. 4.14). Cette représentation met en lumière comment les humains tirent des bénéfices divers et variés du fonctionnement des écosystèmes et de la biodiversité.

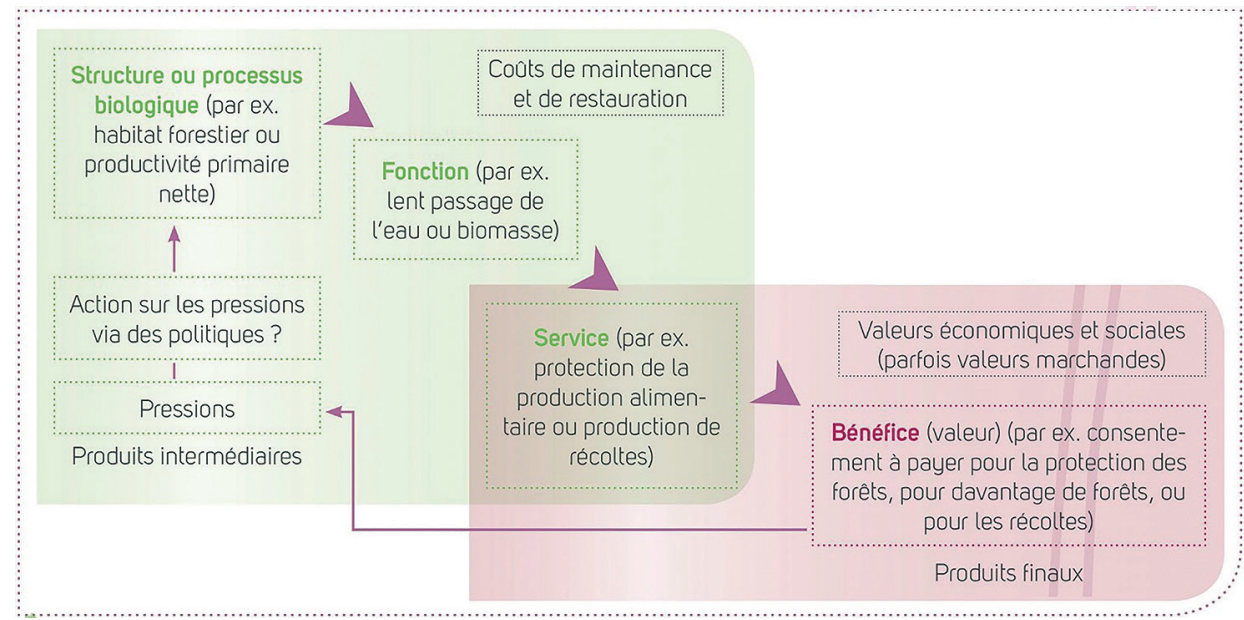

Figure 4.14: La représentation en cascade des services écosystémiques

Source: Figure 1 du rapport disponible au lien suivant: https://uicn.fr/wp-content/uploads/2016/09/ Brochure_Panorama_des_services-vol1.pdf. 
Prenons l'exemple d'une mangrove sur une zone côtière, qui permet par son fonctionnement de ralentir l'écoulement de l'eau, et celui d'une prairie en altitude, qui produit de la biomasse ou des protéines. Ces fonctions écologiques contribuent à la réalisation de plusieurs services écosystémiques. La mangrove est à l'origine de services de protection vis-à-vis des inondations et des vagues de submersion. La prairie permet la production de fourrage et l'élevage d'animaux. Ces services tirés du fonctionnement de ces écosystèmes nous apportent des bénéfices qui contribuent directement à notre bien-être, plus précisément à notre sécurité, dans le cas des mangroves, et à notre alimentation dans le cas des prairies.

La représentation en cascade (Fig. 4.14) illustre bien que les services écosystémiques reposent sur le bon fonctionnement des écosystèmes, en particulier sur les processus écologiques qui incluent les interactions entre les espèces et leur milieu ainsi que les interactions des espèces entre elles. Si le fonctionnement d'un écosystème est dégradé, alors les humains ne peuvent plus bénéficier de ses services pour leur bien-être. Lorsque les arbres des mangroves ne sont plus là pour ralentir le flux de l'eau, il n'y a plus aucune barrière aux inondations ou aux vagues de submersion causées par des tempêtes. Les populations humaines et les infrastructures environnantes se retrouvent sans protection.

La biodiversité est également importante au sein de la cascade de services écosystémiques. Une grande diversité génétique ou spécifique au sein d'un écosystème conduit le plus souvent à son «bon» fonctionnement. Si on reprend l'exemple des prairies, sa richesse spécifique, c'est-à-dire la diversité des plantes qui y sont présentes, peut contribuer à la valeur alimentaire du fourrage pour les animaux, notamment par la diversité des apports en minéraux, en acides gras et en tanins.

On distingue trois grands types de services écosystémiques (Fig. 4.15):

- Les services de régulation qui incluent, entre autres, les services de pollinisation, la régulation du climat global via la séquestration de carbone, la régulation de risques naturels comme les inondations, les vagues de submersion ou les glissements de terrain, et la régulation de la qualité de l'air.

- Les services d'approvisionnement/prélèvement qui concernent la production de biens, de matériaux, tels que le bois ou l'eau, de denrées alimentaires, tels que les fruits ou le fourrage des prairies. II s'agit aussi de la cueillette de champignons, des plantes médicinales ou aromatiques, ou encore de l'utilisation de plumes ou de fleurs pour la décoration.

- Les services culturels qui sont des services non matériels tirés des écosystèmes à travers l'enrichissement spirituel, le développement cognitif, la réflexion, l'inspiration artistique ou les loisirs. Ces services culturels nous permettent de développer et entretenir nos systèmes de savoir, nos relations sociales et nos valeurs esthétiques. On trouve, par exemple, dans cette catégorie les sports en extérieur, la chasse et la pêche de loisir, l'inspiration artistique, l'enrichissement spirituel et religieux. 

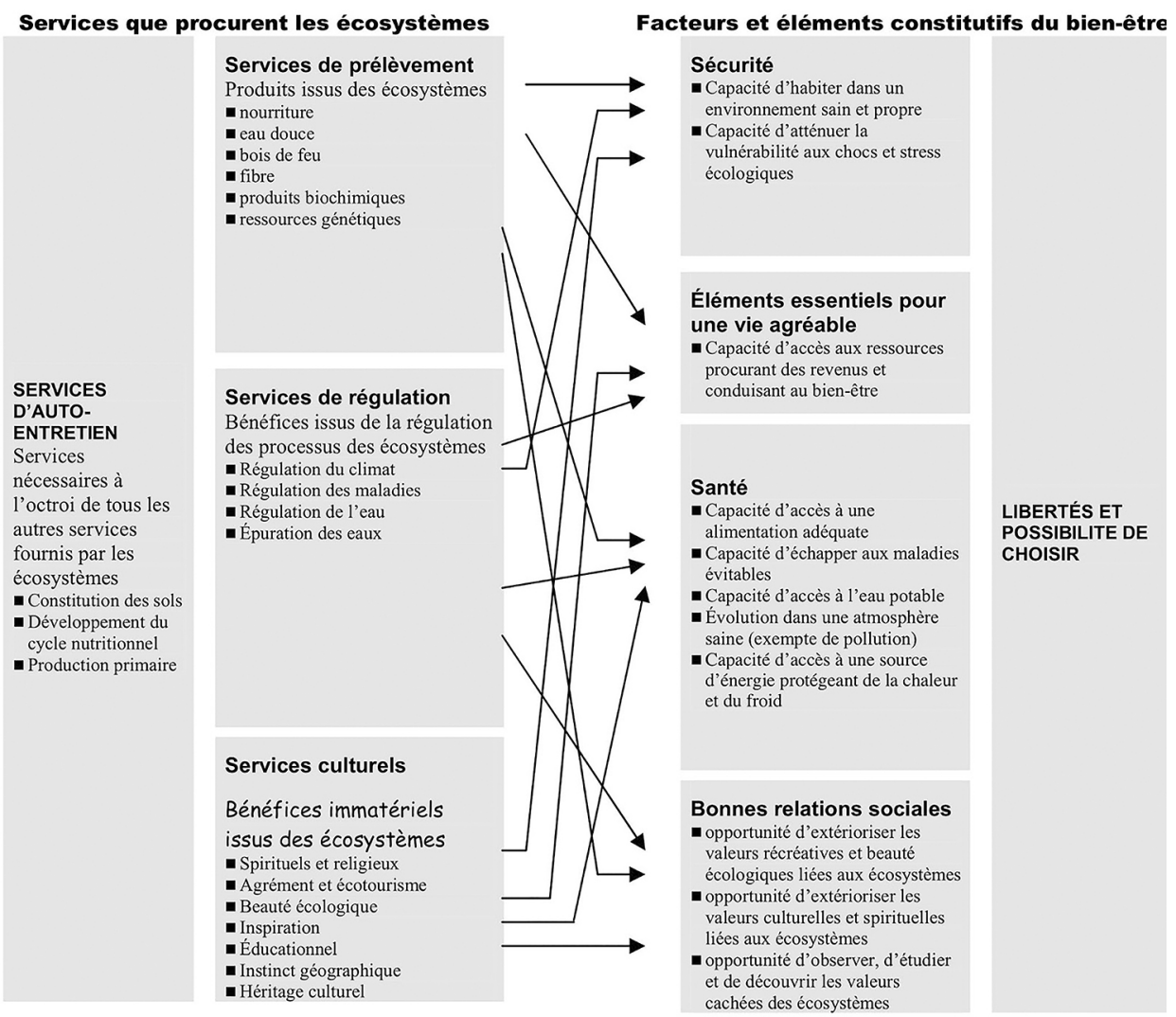

Figure 4.15: Les différents types de services écosystémiques.

Extrait de https://www.millenniumassessment.org/documents/document.6.aspx.pdf.

Les services de régulation sont des services qui découlent directement des fonctions écologiques. Les services d'approvisionnement et les services culturels ont, en revanche, un lien indirect avec les fonctions écologiques.

Une quatrième catégorie de services, dits de support et aussi appelés services d'auto-entretien (Fig. 4.15) correspond aux fonctions écologiques qui ne bénéficient ni directement ni indirectement aux humains et qui ne sont donc pas des services écosystémiques à proprement parler. Ce sont des processus de base, nécessaires au bon fonctionnement de tous les écosystèmes: cycles naturels, formation des sols, photosynthèse et cycle de l'eau.

Un territoire peut conduire à plusieurs, pour ne pas dire de nombreux, services écosystémiques. Par exemple, la forêt de Fontainebleau en Île-de-France est un lieu apprécié des sportifs pour la marche, la pratique du vélo et l'escalade, mais également pour l'observation d'oiseaux ou d'animaux ou tout simplement pour passer quelques heures au frais pendant la canicule. Cette forêt est, par ailleurs, exploitée 
par l'ONF pour la production de bois. II s'agit donc d'un espace multifonctionnel dont on tire de multiples services écosystémiques, services qui sont souvent interconnectés les uns aux autres.

La prise en compte de ces interconnexions entre services est essentielle pour la gestion des ressources naturelles. En effet, l'utilisation un peu trop poussée d'un service peut conduire à des conflits dans l'usage d'autres services. Une coupe massive des arbres à Fontainebleau, pour produire du bois, ne satisferait probablement pas les randonneurs! De la même manière, l'exploitation des mangroves pour leur bois ne leur permettrait plus de remplir leur service de protection vis-à-vis des inondations côtières.

Le concept de "service écosystémique» est de plus en plus utilisé dans les travaux de scientifiques, mais aussi par les décideurs, les aménageurs et les politiques, notamment dans le cadre des négociations internationales. Ceci s'explique par trois grandes raisons:

- Tout d'abord, c'est un concept qui se situe à l'intersection entre l'écologie, l'économie et la sociologie, et qui est donc compréhensible et mobilisable par une grande diversité d'acteurs;

- Il permet de discuter de la multifonctionnalité des territoires, des usages et des conflits qui peuvent apparaître quand on exploite les ressources naturelles;

- Enfin, il permet d'attirer l'attention sur le rôle essentiel de la Nature pour notre bien-être et la plupart de nos activités économiques. On prend souvent pour acquis le fait que la nature contribue aux services écosystémiques, mais on néglige aussi souvent le fait que nos activités (par exemple, la déforestation des mangroves) vont avoir un impact sur les écosystèmes et sur les services écosystémiques qu'ils produisent.

Le concept de service écosystémique est associé à l'émergence de nouvelles initiatives de gestion de la biodiversité et des écosystèmes, comme les mécanismes de paiement pour services écosystémiques, ou les mesures de compensation écologique.

Les limites associées à ce concept sont liées à sa définition même, mais aussi à sa mise en œuvre pratique. Citons en trois:

- C'est un concept intrinsèquement anthropocentré, car on ne s'intéresse ici qu'aux fonctions et services qui in fine contribuent au bien-être humain. II ne rend pas du tout compte des fonctions et des services qui bénéficient à d'autres espèces ou à l'écosystème en général.

- Ce concept est associé à une vision très occidentale du monde. II s'articule très mal avec les représentations du monde et les cultures de certaines 
populations indigènes, que ce soit en Amérique latine, la culture quechua, par exemple, en Asie avec le taoïsme, en Afrique ou dans îles du Pacifique sud. Ces communautés autochtones ont une vision beaucoup plus holistique des relations entre les humains et la Nature, qui dépasse de loin la notion utilitariste de «service». C'est la raison pour laquelle, I'IPBES, plateforme intergouvernementale scientifique et politique sur la biodiversité et les services écosystémiques, préfère parler plus largement de «contributions de la nature aux sociétés» plutôt que de services écosystémiques [7, 8].

- L'évaluation des services écosystémiques est souvent mise en œuvre via le calcul de leur valeur économique [9, 10], ce qui passe sous silence d'autres référentiels de valeurs comme les valeurs intrinsèques ou les valeurs relationnelles, au profit des valeurs instrumentales uniquement (voir section 4.4).

Pour résumer, le concept de services écosystémiques est une métaphore des relations humains-nature, qui offre des perspectives intéressantes en termes de protection de l'environnement, mais qui est fondamentalement anthropocentrée et utilitariste.

\section{Références}

[1] IPBES. 2019. Résumé à l'intention des décideurs du rapport d'évaluation globale de l'état de la biodiversité et des services écosystémiques. S. Díaz, et al. (eds). Secrétariat IPBES, Bonn, Germany.

[2] D. Gravel, I. Gounand, N. Mouquet. 2010. Le role de la diversité dans le fonctionnement des écosystèmes. Ciencia y Ambiente 39.

[3] R. Primack, F. Sarrazin, J. Lecomte. 2012. Biologie de la conservation. Dunod, p. 359.

[4] J. Liang, T.W. Crowther, N. Picard, S. Wiser, M. Zhou, G. Alberti, ..., P.B. Reich. 2016. Positive biodiversity-productivity relationship predominant in global forests. Science 354 (6309).

[5] H. Pretzsch, G. Schütze, E. Uhl. 2013. Resistance of European tree species to drought stress in mixed versus pure forests: evidence of stress release by inter-specific facilitation. Plant Biology 15(3), 483-495.

[6] (a) R. Costanza, R. d'Arge, R. de Groot, S. Farber, M. Grasso, B. Hannon, K. Limburg, S. Naeem, R.V. O'Neill, J. Paruelo, R.G. Raskin, P. Sutton, M. van den Belt. 1997. The value of the world's ecosystem services and natural capital. Nature 387, 253-260. https://mro. massey.ac.nz/bitstream/handle/10179/9476/Costanza\%20et\%20al\%20\%20Nature\%20 1997\%20prepublicaton.pdf.

(b) R. Costanza, R. de Groot, P. Sutton, S. van der Ploeg, S.J. Anderson, I. Kubiszewski, S. Farber, R.K. Turner. 2014. Changes in the global value of ecosystem services. Global Environmental Change 26, 152-158.

https://community-wealth.org/sites/clone.community-wealth.org/files/downloads/ article-costanza-et-al.pdf 
[7] S. Díaz, S. Demissew, J. Carabias, C. Joly, M. Lonsdale, N. Ash, .., A. Bartuska. 2015. The IPBES Conceptual Framework - Connecting nature and people. Current Opinion in Environmental Sustainability 14, 1-16. https://www-sciencedirect-com.proxy.scd.u-psud.fr/ science/article/pii/S187734351400116X.

[8] U. Pascual, P. Balvanera, S. Díaz, G. Pataki, E. Roth, M. Stenseke, ..., V. Maris. 2017. Valuing nature's contributions to people: the IPBES approach. Current Opinion in Environmental Sustainability 26, 7-16. https://www-sciencedirect-com.proxy.scd.u-psud.fr/science/ article/pii/S1877343517300040\#fig0015.

[9] E. Gómez-Baggethun, R. De Groot, P.L. Lomas, C. Montes. 2010. The history of ecosystem services in economic theory and practice: from early notions to markets and payment schemes. Ecological Economics 69(6), 1209-1218. https://www.forest-trends. org/wp-content/uploads/imported/gomez-baggethun-et-al_2009_the-history-ofecosystem-service-in-economic-theory-and-practice-journal-citation-doc-pdf.pdf.

[10] J. Smessaert, A. Missemer, H. Levrel. 2020. The commodification of nature, a review in social sciences. Ecological Economics 172, 106624. 


\section{Chapitre 5}

\section{Les menaces sur la biodiversité}

Ce chapitre décrit en détail les cinq principales menaces qui pèsent sur la biodiversité et qui sont toutes d'origine humaine: le changement d'usage des terres et des mers, la surexploitation, le changement climatique, les pollutions et les espèces exotiques envahissantes. II évoque aussi les réponses à y apporter, lesquelles passent par une nécessaire transition écologique de nos modèles d'exploitation de la nature et de ses ressources. Aujourd'hui, les menaces entrainent la réduction des effectifs des populations d'espèces, et des processus en cascade peuvent même conduire à leur disparition à tout jamais. Comme nous le verrons, on assiste aussi à un phénomène d'homogénéisation biotique qui tend à perturber le fonctionnement des écosystèmes et leur capacité à s'adapter aux changements globaux.

\subsection{Changements d'usage des terres et des mers - H. Levrel}

Le changement d'usage des terres et des mers est actuellement la première cause d'érosion de la biodiversité. La question de l'évolution de l'usage des terres et des mers renvoie à la durabilité des modes d'exploitation des espaces naturels - forêts, prairies, zones humides - ou au problème de conversion de ces derniers en espaces agricoles. Pour les milieux marins, il s'agit principalement de rechercher un niveau d'exploitation des stocks de pêche qui soit soutenable.

\subsection{1. Évolution de l'usage des sols au cours de l'Histoire}

Dès le pléistocène, l'être humain a engendré des impacts significatifs sur la biodiversité par ses usages. Ainsi, il y a près de 50000 ans, la colonisation du monde par Homo Sapiens va s'accompagner de pratiques qui vont changer de manière très importante les habitats terrestres. L'usage du feu pour rabattre les espèces, qu'il chasse, et faciliter la pousse d'espèces végétales comestibles va ainsi engendrer la disparition d'écosystèmes entiers. À titre d'exemple, on a observé en Australie un effondrement de la mégafaune entre -45000 et -43000 ans, avec la 
disparition du kangourou géant, du wombat géant, du diprotodon (un marsupial géant) ou des genyornis (oiseaux géants de près de 1,80 m de hauteur mais qui ne volaient pas).

II s'agissait d'un système d'exploitation que l'on peut qualifier d'extensif, car l'expansion démographique s'accompagnait de la colonisation de nouvelles terres une fois que les ressources d'un territoire donné avaient été exploitées. On peut parler d'une «économie de la prédation». Ce modèle va se poursuivre jusqu'à l'apparition de l'agriculture, il y a environ 12000 ans. L'espèce humaine passe alors à un nouveau modèle économique d'usages de la nature: l'«économie de la production». Les humains se mettent en effet à produire des aliments grâce aux cultures et à l'élevage. II s'agit d'un système d'exploitation que l'on peut qualifier d'intensif, qui vise à reproduire des ressources sur un territoire donné, contrairement au modèle extensif où Homo Sapiens se déplaçait pour trouver de nouvelles ressources en laissant se renouveler celles qu'il avait utilisées.

Pendant cette nouvelle phase, la croissance du rendement agricole des terres va permettre de créer les conditions matérielles nécessaires à une augmentation de la population humaine mondiale. C'est aussi pendant cette période qu'apparaissent une spécialisation des modes de production, la propriété privée, l'écriture, les empires, les civilisations, le commerce international, la monnaie, et finalement toutes les institutions capitalistes qui organisent encore aujourd'hui nos économies. C'est toujours pendant cette période que les premières villes apparaissent : Uruk, Ur, Smyrne ou Babylone.

À l'antiquité, puis au moyen-âge, on va voir apparaître les premières situations où la population humaine est telle que l'ensemble des capacités productives des écosystèmes ne suffit plus à produire assez de ressources pour accompagner la croissance démographique. C'est le cas à la veille de la chute de l'empire romain, mais aussi à la veille de l'apparition de la peste noire en Europe au XIV'e siècle.

Dans ces situations, l'occupation des sols est entièrement orientée vers la production agricole avec les terres fertiles pour la culture et les terres moins productives pour l'agropastoralisme. Le peu de forêts et de zones humides qui restent, est maintenu pour que les nobles puissent pratiquer la chasse et consommer du poisson les jours du jeûne. La moindre variabilité climatique peut générer des catastrophes humaines importantes, comme ce fut le cas pour l'empire romain notamment.

Les premières grandes explorations au XVe siècle et la découverte de l'Amérique vont offrir cependant une opportunité unique d'augmenter fortement la population mondiale avec, de nouveau, une phase de développement extensif (exploitation des ressources naturelles, puis migration une fois celles-ci épuisées), puis de développement intensif (développement de cultures d'exportation dans les colonies pour alimenter le marché européen et international). La découverte de l'Amérique permet aussi d'importer de nouvelles plantes qui vont être progressivement mises en culture 
en Europe, comme c'est le cas avec la pomme de terre, le maïs, la tomate, qui font partie aujourd'hui des principales espèces cultivées sur ce continent.

$\mathrm{Au} X \mathrm{XVII}$ e siècle, les physiocrates sont les premiers à proposer une théorie économique de l'usage optimal des terres. Cette théorie, née en France, préconise d'adopter des innovations techniques et institutionnelles pour augmenter les rendements. Parmi les solutions techniques, les physiocrates mentionnent la nécessité d'assécher les zones humides pour «bonifier les terres» et les rendre propres à l'agriculture. Parmi les solutions institutionnelles, ils proposent de privatiser les terres collectives, dont les zones humides qui auront été préalablement asséchées, pour que les propriétaires aient un intérêt à investir dans ces dernières.

En effet, si une terre est en propriété commune, les usagers de cette dernière auront, selon les physiocrates, souvent peu d'intérêt à investir du temps dans la valorisation de celle-ci puisque les bénéfices qui en découleront seront partagés entre toutes les personnes ayant accès à cette terre. Ils mettent ainsi en avant un principe, maintes fois repris depuis, de la nécessité de mettre en place des dispositifs incitatifs fondés sur une logique individuelle et privée. Ces préconisations vont être largement suivies en Angleterre, par exemple, oũ les terres vont être privatisées de manière massive (les «enclosures», voir section 1.6.1). Certes, cela va conduire à augmenter les rendements des terres, et à spécialiser la production agricole, mais l'autre effet de ces mesures est de faire exploser la pauvreté dans les zones rurales car les ressources en propriété commune bénéficiaient avant tout aux plus démunis. La conséquence indirecte sera de libérer une main d'œuvre peu coûteuse pour alimenter la croissance de l'industrie dans les villes.

Au cours du XIXe siècle, puis du XXe, les usages des terres vont fortement évoluer avec l'apparition d'instituts de recherche agronomique dans les pays occidentaux et le recours croissant à des modes de production agricole, qui s'inspirent des méthodes développées pendant la révolution industrielle: sélection des variétés les plus productives, production intensive et spécialisée, mécanisation, utilisation du pétrole, recours massif à l'irrigation, utilisation de la chimie pour fournir des engrais de synthèse et des pesticides permettant de détruire les herbes, les insectes et les champignons nocifs pour les cultures. Tout cela va mener à ce qu'on appelle la révolution verte. Le monde passe alors à un mode d'exploitation hyper-intensif des terres.

\subsubsection{La révolution verte et l'usage hyper-intensif des sols}

Un indicateur aide à prendre la mesure de la façon dont l'usage des sols a évolué au cours des âges: le nombre de personnes qui peuvent être nourries pour une surface donnée de terre. Lorsqu'Homo Sapiens était un chasseur-cueilleur, il lui fallait près de $10 \mathrm{~km}^{2}$ pour nourrir une personne. Au moyen-âge, $1 \mathrm{~km}^{2}$ pouvait permettre de nourrir 2 à 5 personnes. Aujourd'hui, $1 \mathrm{~km}^{2}$ d'agriculture conventionnelle permet de 
nourrir 100 personnes. On parle parfois des trois Malthus, dans le sens où la limite de la planète, telle qu'elle a été décrite par cet économiste au début du XIXe siècle, dépend désormais fortement des techniques agronomiques mobilisées.

La révolution verte a eu des conséquences à la fois positives et négatives. Elle a d'abord favorisé et permis d'accompagner la forte croissance de la population mondiale au XXe siècle, avec un niveau de nutrition par habitant qui s'est considérablement amélioré, même si ces gains sont restés très inégaux. La population humaine était de 1,6 milliards en 1900. Elle est aujourd'hui de 7,5 milliards. Et, en moyenne, elle souffre moins de faim.

Elle a aussi permis de ne pas détruire tous les écosystèmes de la planète. En effet, si le niveau de rendement des terres agricoles était resté celui du XIXe siècle, notre croissance démographique aurait engendré beaucoup plus de destruction dans certaines zones de la planète. La situation est, cependant, très variable selon les régions du monde. On constate actuellement une forte déforestation dans l'hémisphère sud, où des systèmes extensifs et intensifs cohabitent, et une forte croissance de la forêt dans l'hémisphère nord où le système agricole intensif et la stagnation de la population ont entraîné une forte déprise agricole et un retour de ces milieux naturels.

Un dernier point positif est que, si la population a été multipliée par 4 entre 1900 et 2000 , la consommation de production primaire de biomasse végétale n'a fait que doubler. Ainsi, Homo Sapiens s'appropriait $13 \%$ de la biomasse totale de la planète en 1900. II s'en approprie «seulement » $25 \%$ en 2000.

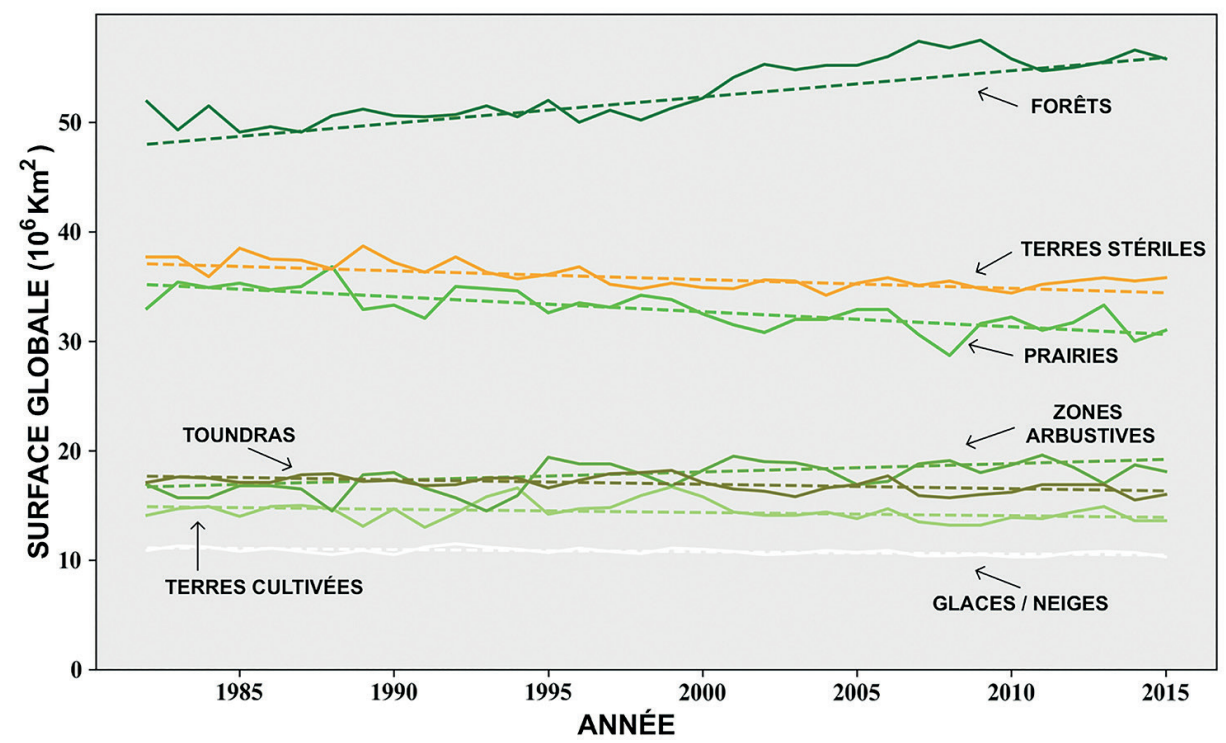

Figure 5.1: Évolution de l'occupation des sols dans le monde de 1980 à 2015. Les surfaces bâties sont passées de 0,4\% à 0,6\% dans le monde pour la période 1990-2014. D’après : [1] Creative Common 4.0. 
Le bilan, en termes d'évolution de l'occupation des terres, semble ainsi relativement raisonnable sur la période 1980-2015 (Fig. 5.1) [1]. Les surfaces dédiées à la culture semblent avoir légèrement baissé $\left(-60000 \mathrm{~km}^{2}\right)$, les surfaces forestières fortement augmenté $\left(570000 \mathrm{~km}^{2}\right)$, les surfaces de prairie fortement régressé $\left(-420000 \mathrm{~km}^{2}\right)$, les zones désertiques ont aussi décru $\left(-165000 \mathrm{~km}^{2}\right)$, sachant que les zones urbaines ont doublé sur la même période, mais qu'elles ne représentent qu'une très faible part de la surface de la planète $(0,6 \%)$.

Pourtant ces chiffres qui peuvent apparaître comme relativement positifs masquent une réalité qui est toute différente. La première remarque est que si la production agricole a pu augmenter sans faire exploser la consommation de biomasse naturelle, c'est uniquement parce qu'elle a largement pioché dans la biomasse qui s'est accumulée pendant des millions d'années dans nos sous-sols, à savoir les énergies fossiles qui sont le carburant de l'agriculture moderne, de la chimie verte et des engins mécaniques.

Par ailleurs, le recours aux produits de synthèse, que ce soit en tant qu'engrais ou pesticides, a engendré une pollution massive des terres (voir section 2.5), des cours d'eau, des nappes phréatiques et des organismes vivants (voir section 2.4) qui sont en contact avec ces produits. À cela s'ajoute une très forte érosion des sols que l'on constate partout dans le monde du fait des pratiques intensives. Ensuite, si le bilan global des forêts est bon, le contenu de ce qui est perdu d'une part - forêts équatoriales très riches en biodiversité - et de ce qui est gagné de l'autre - forêts de résineux très pauvres en biodiversité - est incomparable. Enfin, l'accent mis aujourd'hui sur les besoins en séquestration de carbone conduit à créer des plantations d'arbres qui font disparaître d'autres écosystèmes souvent plus riches en biodiversité, comme c'est le cas pour les prairies. Dans ce bilan, on voit ainsi se dessiner un monde où l'occupation du sol serait guidée par la capacité des terres à produire des ressources alimentaires, des biocarburants ou des puits à carbone, avec des conséquences catastrophiques sur la biodiversité.

Plus encore, le bilan fait l'impasse sur les zones humides qui sont difficiles à qualifier à partir des données satellites, mais pour lesquelles on sait, à partir de suivi sur site, qu'elles ont connu une très forte érosion au cours des deux derniers siècles. Ainsi il est estimé que seules $13 \%$ des zones humides existant en 1700 sont encore présentes aujourd'hui sur le globe [2].

\subsubsection{L'usage des mers, pêche extensive et aquaculture}

Concernant le monde marin, la description historique de l'évolution des pêches est beaucoup plus simple, car elle est basée sur un système extensif qui n'a pas vraiment changé depuis le début de l'histoire humaine. À mesure que les stocks de pêche ont été surexploités sur la planète, les pêcheurs ont été toujours plus loin, toujours plus profond et avec des instruments de pêche toujours plus efficaces. Le développement de bateaux usines pouvant aller pêcher partout dans le monde à 
n'importe quelle profondeur et congeler directement les produits pour les conserver, en est le résultat direct. Le corollaire de cette augmentation de l'intensité de pêche, observée sur l'ensemble des océans, est une stagnation de la biomasse totale de poissons débarquée depuis le milieu des années 1980 [3].

Depuis cette période, le monde de la pêche connaît des crises économiques et écologiques récurrentes avec des conséquences importantes pour des secteurs d'activité dans les pays du Nord et des effets beaucoup plus dramatiques dans les pays du Sud où une partie de la sécurité alimentaire dépend de l'apport en protéines issus des stocks de pêche. L'aquaculture a pris le relais de manière à augmenter la production de protéines issues des poissons à destination des consommateurs (Fig. 5.2). Cette pêche industrielle, qui utilise principalement des arts traînants (chalut), génère des effets catastrophiques sur les habitats naturels et la biodiversité marine dans son ensemble. Elle est d'autant plus nocive qu'elle est fortement émettrice de gaz à effets de serre et totalement dépendante d'un prix du baril de pétrole bas et d'exonérations fiscales sur la consommation de fioul lourd hyper toxique.

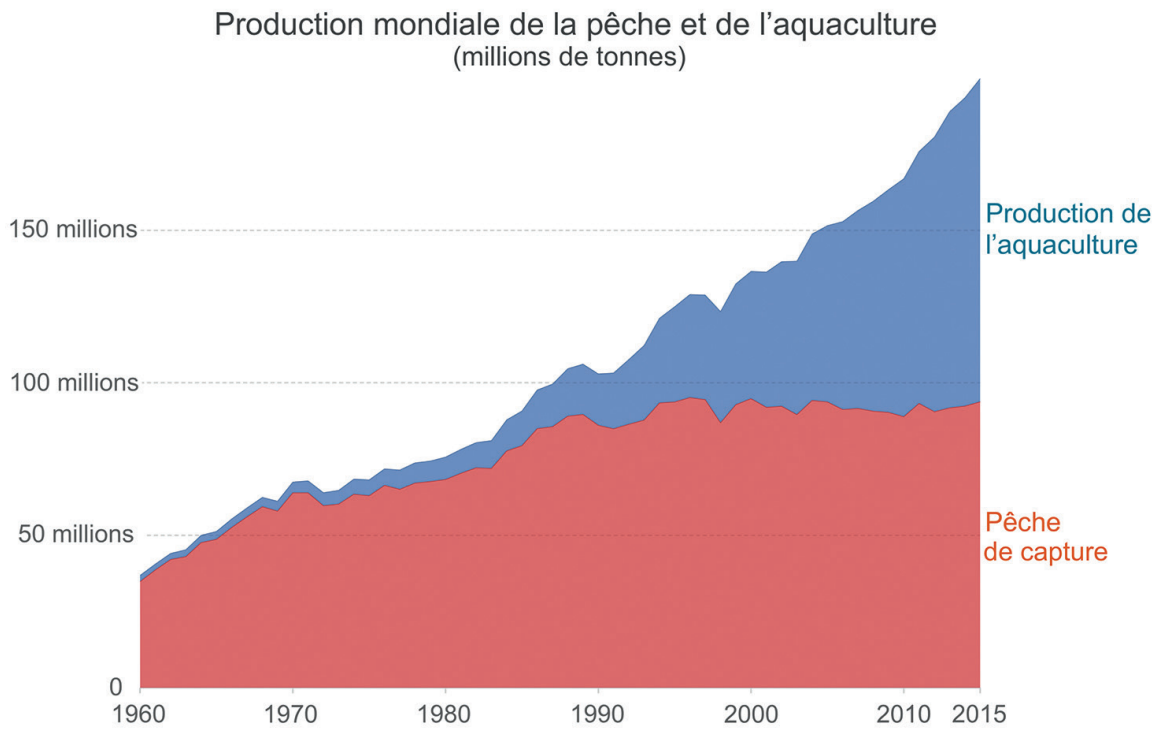

Figure 5.2: Évolution de la pêche de capture et de l'aquaculture dans la production mondiale de pêche de 1950 à 2012. Source: FAO : 2014 - https://ourworldindata.org/fish-and-overfishing (CC by).

\subsubsection{Changements récents dans les usages des terres et des mers}

Pour ce qui concerne les pratiques agricoles, on a vu ces dernières années une forte augmentation de la conversion d'exploitations agricoles en bio, en particulier 
en Europe. Ces conversions sont alimentées par deux facteurs principaux. Le premier est une augmentation de la demande des consommateurs pour des produits considérés comme meilleurs pour leur santé et moins nocifs pour l'environnement, et qui sont prêts à payer plus cher pour cela. Le second tient aux évolutions du monde agricole lui-même, qui cherche à être moins dépendant des marchés internationaux et des entreprises semencières, mais aussi à être moins en contact avec des produits dangereux pour la santé. Le taux de surfaces agricoles utiles dédiées à l'agriculture biologique en France est ainsi passé de 800000 ha à 2,2 millions d'ha entre 2010 et 2019.

Cette tendance à l'augmentation du bio est présente dans tous les pays de I'OCDE. Certes, cela conduit à une baisse des rendements, mais cela permet aussi de questionner le gâchis alimentaire tout autant que les modes d'alimentation, notamment en viande et tout particulièrement en viande de bœuf, qui sont nocifs pour le climat, la biodiversité et, au-delà d'un certain seuil, pour la santé humaine. Cela invite aussi à désacraliser la question des rendements, à «désintensifier » la production (en remettant du travail humain dans les exploitations) et à imaginer des systèmes agro-écologiques plus diversifiés au sein desquels on renonce au contrôle des dynamiques naturelles pour mieux «capitaliser» sur ces dernières.

Un autre élément positif à souligner est le renforcement, dans les pays de l'OCDE, d'une régulation de l'effort de pêche par la puissance publique, qui commence enfin à porter ces fruits. Ainsi, une étude récente sur l'évolution de l'ensemble des «stocks» de pêche dans le monde, dont $50 \%$ sont effectivement gérés, conclut à un effet significatif des mesures de régulation, notamment en matière de quotas de pêche. De nombreux stocks seraient ainsi en phase de régénération, en particulier là où ils sont bien suivis et où des quotas visant à respecter les taux de renouvellement des populations de poissons ont été appliqués et contrôlés [4]. C'est le cas pour les stocks situés en Atlantique notamment.

Ces premiers exemples d'évolution dans les usages des terres et des mers, dont on commence à percevoir les effets positifs pour les humains et la biodiversité dans son ensemble, nécessitent cependant d'être amplifiés, complétés et généralisés dans un futur proche pour espérer voir apparaître une réelle transition écologique de nos modèles économiques d'exploitation de la nature.

\subsection{La surexploitation des ressources naturelles - E. Baudry}

La surexploitation des ressources naturelles fait partie des cinq menaces qui pèsent sur la biodiversité. Elle est actuellement classée en deuxième des plus grandes menaces sur la biodiversité derrière le changement d'usage des terres et des mers. 


\subsubsection{Définition de la surexploitation}

Pour l'humanité, la biodiversité constitue une ressource incroyable en nourriture, par exemple de poisson et de gibier, et en matières premières, par exemple le bois. Ces ressources se renouvellent naturellement, mais à une certaine vitesse. On parle de surexploitation des ressources naturelles quand on prélève les ressources en question plus vite qu'elles ne peuvent se renouveler ${ }^{46}$. Nous verrons qu'il peut s'agir d'une ou de plusieurs espèces qui peuvent être concernées.

Pour avoir une idée de l'ordre de grandeur de ces prélèvements, il faut savoir que chaque année dans le monde, on capture 93 millions de tonnes de poissons ${ }^{47}$ et qu'on prélève 15,3 milliard de tonnes de bois [5]. De très grands volumes de ressources naturelles sont donc prélevés annuellement.

\subsubsection{Conséquences directes de la surexploitation: l'exemple de la pêche à la morue au large de terre-neuve}

La surexploitation d'une espèce peut avoir des conséquences directes. Si on prélève une espèce plus vite qu'elle ne se renouvelle, alors ses effectifs vont fortement diminuer. Elle risque même de disparaître, au moins localement, et les humains ne vont plus pouvoir utiliser cette espèce.

Un exemple classique de conséquences directes de la surexploitation d'une espèce est celui de la pêche à la morue en Atlantique nord (Fig. 5.3).

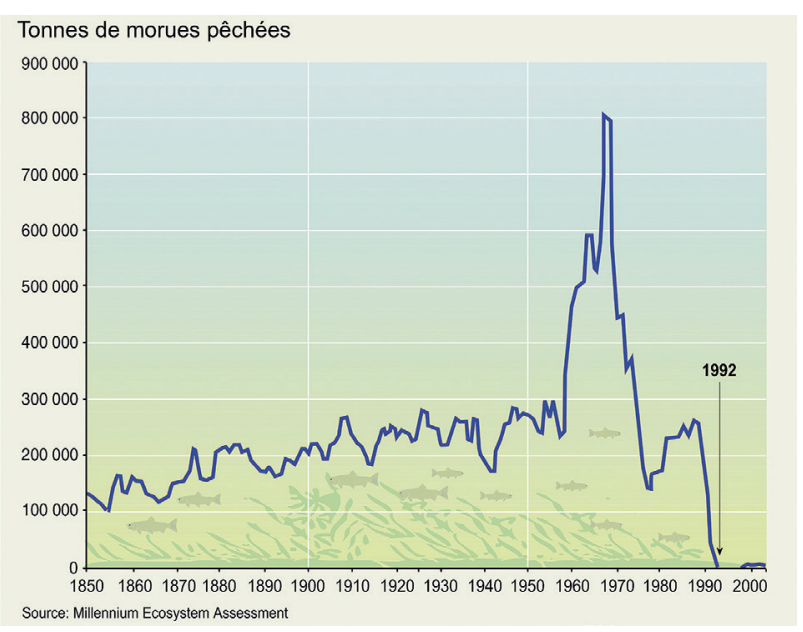

Figure 5.3: Surexploitation du stock de morue de l'Atlantique dans la zone de terre neuve (Source: Millenium Ecosystem Assessment).

46. https://fr.wikipedia.org/wiki/Surexploitation

47. Chiffres de prélèvement de poisson par an: https://ourworldindata.org/rise-of-aquaculture 
Le volume de morues pêchées au large de Terre-Neuve est assez stable entre 1850 et 1950. Par contre, à partir de la fin des années 1950, l'introduction des chalutiers de fond a entraîné une forte augmentation des captures. Ce prélèvement très important a dépassé les capacités de renouvellement de la population de morue et a entraîné un effondrement de cette population au début des années $1990^{48}$.

\subsubsection{Conséquences directes et indirectes de la surexploitation des ressources, l'exemple de l'île de Pâques}

La surexploitation des ressources peut aussi avoir des conséquences indirectes, car la disparition de l'espèce, qui a été surexploitée, peut modifier ensuite l'écosystème local. C'est ce qui est arrivé sur l'île de Pâques où la surexploitation des arbres a eu des conséquences à la fois directes et indirectes. L'île de Pâques est située dans l'océan pacifique, à 3000 km environ à l'ouest des côtes du Chili. Elle est célèbre notamment pour la grande quantité de statues monumentales très impressionnantes, les Moaïs, qui ont été érigées par ses habitants (Fig. 5.4). Ces statues sont disposées tout autour de l'île qui présente, à l'heure actuelle, un paysage très austère avec une végétation très rase, sans arbres, sans cours d'eau ${ }^{49}$.

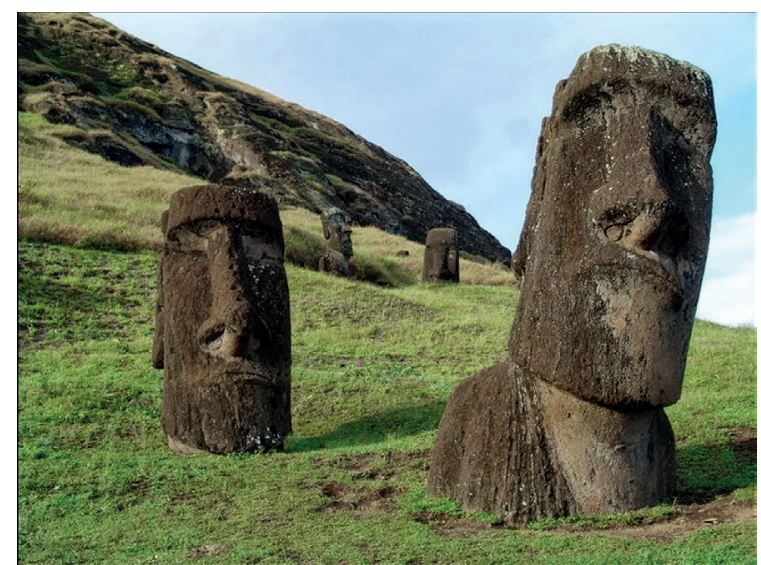

Figure 5.4: Les Moaïs de la carrière de Rano Raraku sur l'île de Pâques. Creative commons 3.0, source: Claire Provost.

L'Île de Pâques illustre, de façon très frappante, un cas de surexploitation d'une ressource naturelle, à savoir les arbres de l'île, et les conséquences assez dramatiques de cette surexploitation sur l'écosystème de l'île et donc pour les habitants. Un groupe de polynésiens aurait colonisé l'île autour de 1200. Les habitants de l'île sont ensuite restés complètement isolés, sans contact avec l'extérieur, jusqu'à la

48. https://fr.wikipedia.org/wiki/Effondrement_de_la_p\%C3\%AAcherie_de_morue_de_Terre-Neuve 49. https://fr.wikipedia.org/wiki/\%C3\%8Ele_de_P\%C3\%A2ques 
première visite par les navigateurs européens au XVIII' siècle. L'analyse des pollens fossiles contenus dans les sédiments des lacs a montré qu'initialement, l'île de Pâques était couverte d'une forêt dense avec une vingtaine d'espèces d'arbres et d'arbustes, notamment de très grands palmiers (Fig. 5.5).

Cette forêt, qui a été une ressource extrêmement utile pour les colons, a été surexploitée; les habitants ont utilisé les arbres plus vite qu'ils ne pouvaient repousser. La plupart des palmiers ont disparu dès 1450, donc très rapidement, environ deux siècles après la colonisation, et vers 1650 il ne restait plus aucun grand arbre. La disparition de la forêt par suite de la surexploitation a entraîné des conséquences dramatiques pour les habitants ${ }^{50}$.

La surexploitation des palmiers a d'abord eu des conséquences directes. Le palmier est, en effet, un arbre particulièrement utile dans la culture polynésienne. Il produit de la nourriture, beaucoup de sève, des noix, des cœurs de palmier, et du matériel pour faire, par exemple, des paniers ou des voiles de pirogue. D'autres espèces d'arbres avaient donné des fruits comestibles, des fibres pour les cordes, et, bien sûr, du bois. Le bois est un matériau très précieux puisqu'il permet de construire les pirogues et les toits des bâtiments, de faire des outils et bien sûr aussi de cuire les aliments. Après la disparition des arbres, les données archéologiques montrent que les habitants de l'île ont dû brûler des herbes pour se chauffer et pour cuire leurs aliments. Ils ont dû faire leurs outils et leur bâtiment uniquement avec des pierres et ils ont dû arrêter la pêche, car ils ne pouvaient plus construire de pirogues. Les données archéologiques indiquent qu'après la disparition des arbres, les sources principales de protéines des habitants sont devenues les rats et les poulets ${ }^{51}$.

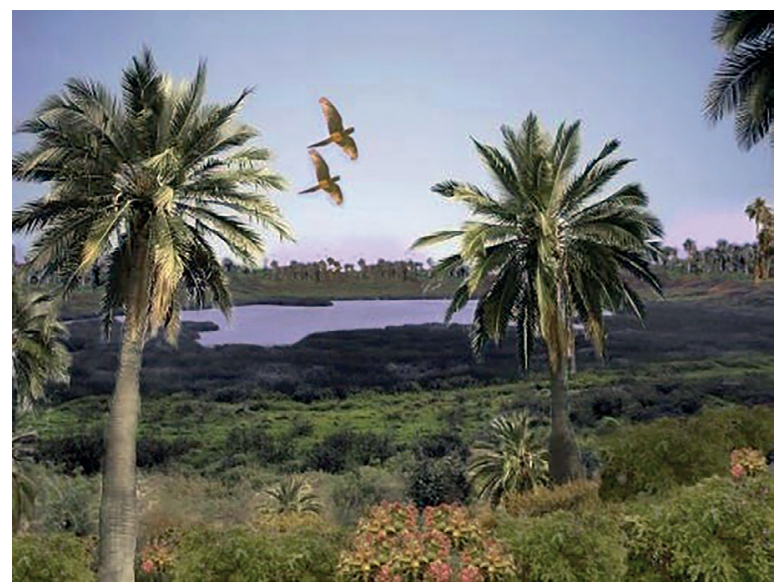

Figure 5.5: Reconstitution du paysage de l'île de Pâques au moment de la colonisation initiale, vers 1200. Creative commons 3.0, source: Rod6807.

50. http://www.kaowarsom.be/documents/PUBLICATIONS/EASTER\%20ISLAND_COLLAPSE_ TRANSFORMATION.pdf

51. https://onlinelibrary.wiley.com/doi/abs/10.1002/ajpa.22339 
La surexploitation eu également des conséquences indirectes sur l'écosystème de l'île. En l'absence de la couverture d'arbres, le sol a été exposé au soleil, au vent et à la pluie, et a subi une érosion extrêmement forte, qui a fait disparaître la couche supérieure fertile du sol sur une grande partie de l'île. Ceci a considérablement réduit la fertilité des sols de l'île et donc leur productivité pour l'agriculture.

Dans cet exemple, on voit bien que la surexploitation des arbres a eu des conséquences très négatives au niveau écologique et pour les habitants de l'île.

\subsubsection{Prélever de façon durable}

II nous faut donc éviter de surexploiter les ressources naturelles, ne serait-ce que pour pouvoir continuer à en bénéficier. Pour prélever de façon durable une espèce, il suffit de la prélever moins vite que sa capacité de renouvellement. Cela peut paraître très simple, mais on peut se trouver confronté à deux difficultés majeures: d'une part, il faut savoir estimer correctement la capacité de renouvellement de l'espèce exploitée et d'autre part, il faut aussi réussir à se mettre d'accord pour diminuer les prélèvements. Lorsque plusieurs acteurs prélèvent une ressource, il est souvent tentant pour chacun des acteurs de continuer à prélever en espérant que ce sont les autres qui feront l'effort de prélever moins. C'est notamment ce qui s'est passé au large de Terre-Neuve où il y avait des bateaux provenant de nombreux pays qui pêchaient la morue. Cet exemple de tragédie des biens communs (voir section 1.6) rend, en général, nécessaire de mettre en place des régulations pour éviter la surexploitation.

\subsection{Le changement climatique - P. Leadley}

Inspiré du MOOC UVED : Impact du changement climatique sur les écosystèmes et la biodiversité www.uved.fr/fiche/ressource/causes-et-enjeux-du-changement-climatique/30/debutant\#sequence.

Le changement climatique est l'une des principales menaces, qui pèse sur la biodiversité. De fait, le réchauffement climatique et l'érosion de la biodiversité sont deux menaces majeures sur l'ensemble de notre planète, qui ne pourront être affrontées indépendamment l'une de l'autre. Les différents aspects du réchauffement climatique seront abordés en détail dans les chapitres ultérieurs 7, 8 et 9 de cet ouvrage. Rappelons simplement ici que le réchauffement moyen sur l'ensemble de planète est estimé être d'environ $1^{\circ} \mathrm{C}$ depuis le début de l'ère industrielle. Pour le siècle en cours, les modèles climatiques prévoient des niveaux de réchauffement qui pourront atteindre 2,4 , voire même $6^{\circ} \mathrm{C}$ selon les scénarios et l'importance de nos émissions de gaz à effet de serre (voir sections 9.2 et 9.3). Ces niveaux d'augmentation sont de nature à modifier profondément les écosystèmes marins ou terrestres, sachant que les modifications en cours sont déjà largement perceptibles. Pour une grande partie des espèces, le réchauffement les oblige à changer leur aire de répartition et à migrer vers des régions plus froides ou plus tempérées. Toutes ne peuvent 
se déplacer avec la vitesse suffisante et certaines sont ainsi menacées d'extinction. Dans ce qui suit, nous illustrons ce phénomène pour les principaux types d'écosystèmes.

\subsubsection{Impacts du réchauffement climatique sur les écosystèmes marins}

Le graphique de la figure 5.6 illustre les déplacements que certaines espèces marines ont déjà effectués depuis les dernières décennies. On se rend compte qu'elles se sont effectivement déplacées, parfois beaucoup. Le déplacement le plus important est celui des phytoplanctons qui ont bougé de plus de $400 \mathrm{kms}$ par décennie pour suivre le changement climatique. Les autres espèces se sont moins déplacées, mais toutes ont essayé d'échapper au réchauffement climatique en migrant vers les pôles.

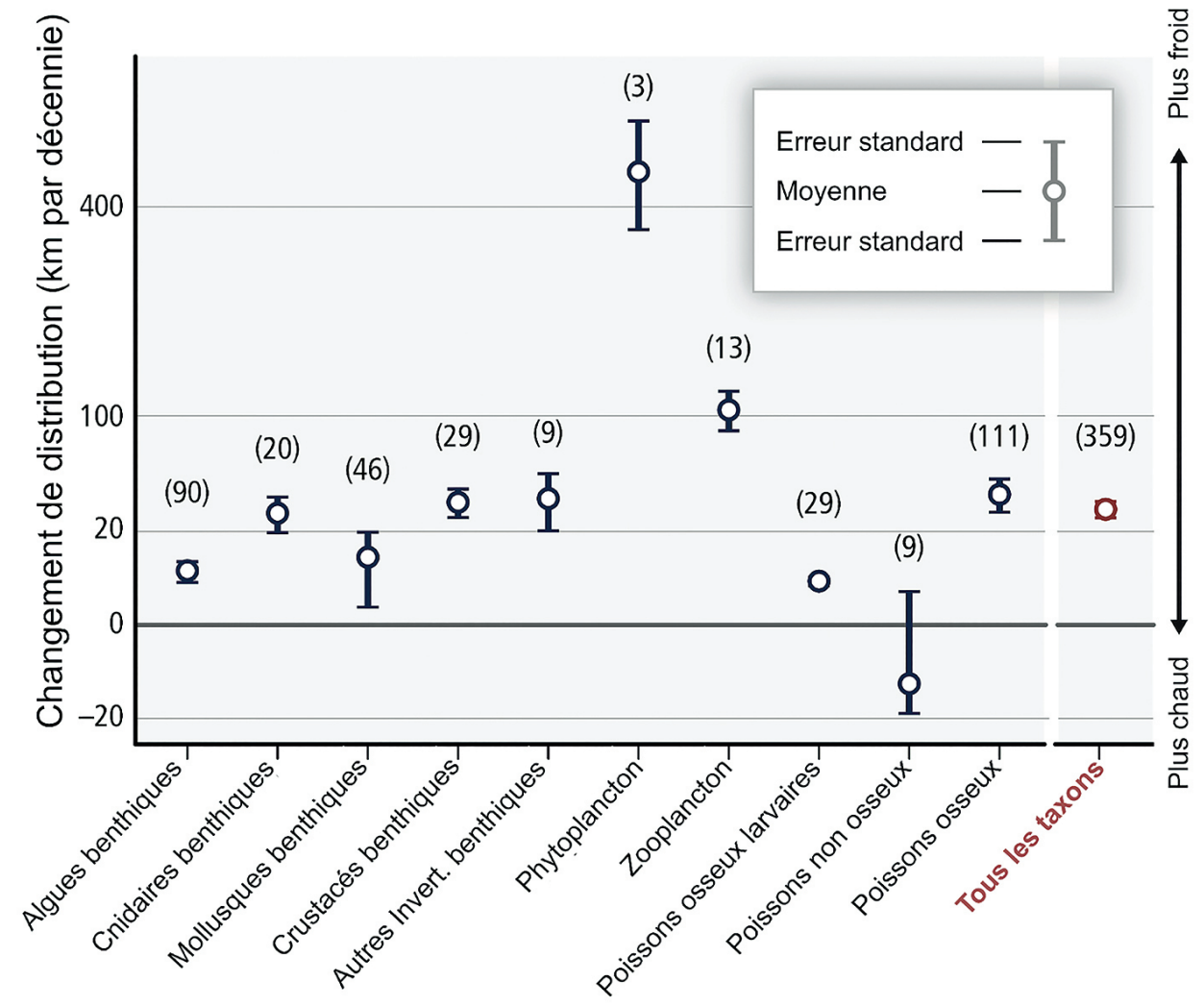

Figure 5.6: Déplacement de différentes espèces marines au cours des dernières décennies (leur vitesse de déplacement est exprimée en $\mathrm{km} /$ décennie). Les chiffres entre parenthèses indiquent le nombre d'analyses qui ont été menées pour chacune de ces espèces. Source: IPCC AR5 WGII 2014, chapitres 6, 23 et 30 . 
Il apparaît clairement que la distribution des espèces est modifiée et par voie de conséquence, le fonctionnement des écosystèmes l'est aussi. Les principales espèces touchées sont celles qui ont des difficultés à se déplacer. De ce point de vue, les écosystèmes les plus menacés sont les récifs coralliens. Rappelons que les coraux sont des petits animaux de l'embranchement des cnidaires, qui se dotent de petits exosquelettes calcaires en carbonate de calcium pour constituer effectivement un récif corallien. Le problème est qu'à partir du moment où l'eau devient acide dû aux rejets de dioxyde de carbone dans l'atmosphère, les coraux ont des difficultés à construire leurs petits exosquelettes (voir aussi section 8.3). De plus, lorsque les océans se réchauffent, les coraux ont tendance à blanchir lorsqu'ils expulsent leur phytoplancton symbiotique, ce qui peut être mortel pour ces espèces. Depuis plusieurs décennies, on constate de plus en plus de blanchiment des récifs coralliens, et pour certains récifs, comme celui de la Grande Barrière d'Australie, la couverture de coraux en bon de santé décroît constamment (Fig. 5.7). Ce sont donc des écosystèmes extrêmement vulnérables. Plus généralement, le réchauffement et l'acidification des océans ont des effets très néfastes sur toutes les espèces. Les principaux impacts en termes de ressources alimentaires concernent les pêcheries, notamment celles qui dépendent des récifs coralliens. À cela s'ajoute le fait que la présence des récifs coralliens réduit aujourd'hui les impacts éventuels des tempêtes sur les côtes, et que leur disparition fera disparaître du même coup ce rôle protecteur.
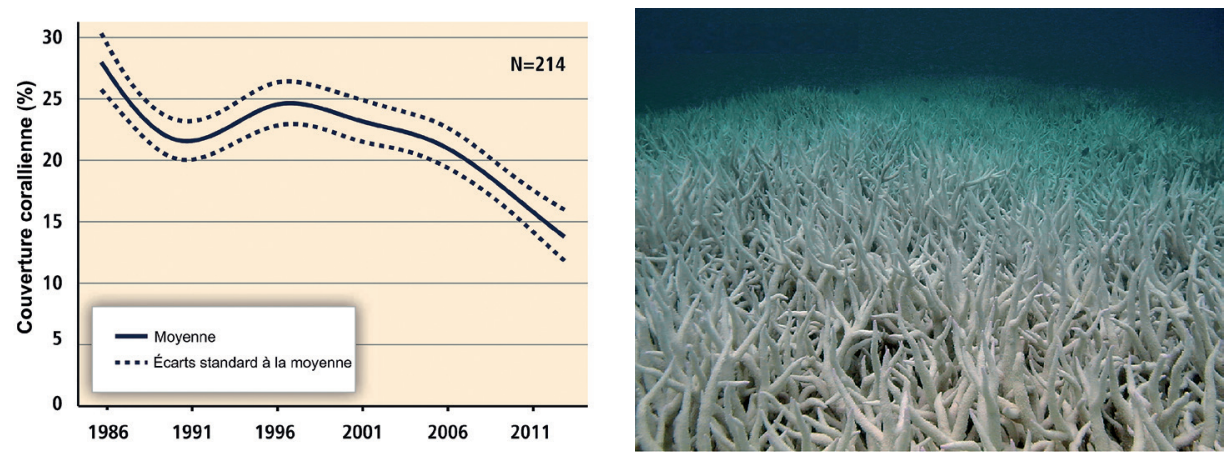

Figure 5.7: À gauche: la diminution continue de la couverture corallienne sur la Grande Barrière d’Australie ( $N=214$ récifs) au cours de la période 1985-2012. À droite: le blanchiment des coraux. Sources: IPCC AR5 WGII 2014, chapitres 6, 23 et 30 et Global Biodiversity Outlook 4, chapitre 10.

\subsubsection{Impacts du réchauffement climatique sur les écosystèmes terrestres}

Si l'on considère à présent les écosystèmes terrestres et, plus particulièrement, les espèces des milieux terrestres, on constate qu'il y a une très grande disparité dans leur capacité à se déplacer pour faire face au réchauffement climatique, ce qu'illustre le graphique de la figure 5.8. 


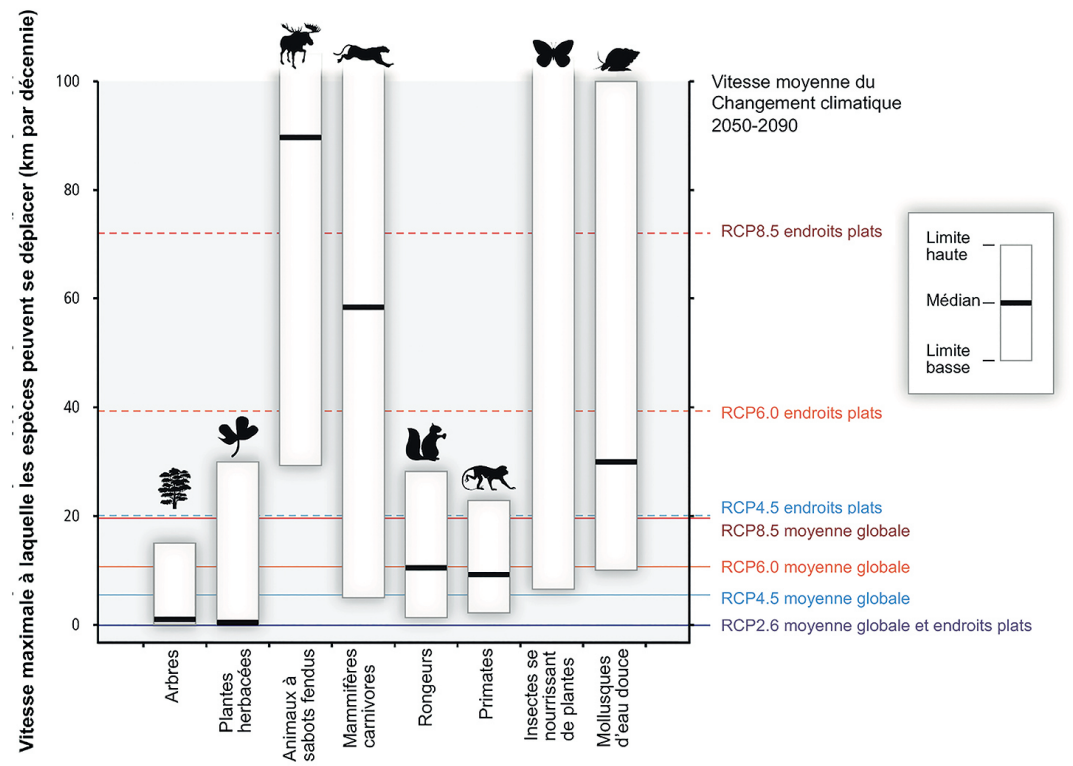

Figure 5.8: Vitesse de déplacement de différentes espèces terrestres exprimée en km/décennie. Les lignes horizontales en couleur indiquent les vitesses qui seraient nécessaires pour échapper au réchauffement selon différents scénarios climatiques et le type de topographie. Source : IPCC AR5 WGII 2014, chapitre 4.

On constate à l'évidence que les arbres et les plantes herbacées ne peuvent pas changer facilement d'aire de répartition, moins de $10 \mathrm{kms}$ par décennie, alors que les grands mammifères ont la capacité de se déplacer rapidement. Les lignes horizontales en couleur sur le graphique de la figure 5.8 indiquent les vitesses de déplacement qui seraient nécessaires aux espèces pour échapper au réchauffement climatique selon différents scénarios envisagés d'émission de gaz à effet de serre (voir sections 9.2 et 9.3). Dans le cas du scénario RCP2.6 où les émissions de gaz à effet de serre seraient très faibles à la fin du siècle, les espèces n'auraient pratiquement pas la nécessité de se déplacer. Même les arbres et les plantes herbacées pourraient s'adapter au changement climatique. Par contre, dans le cas du scénario RCP8.5 où les émissions de gaz à effet de serre seraient très élevées ou dans le cas des endroits plats (plaines étendues) ou dans les deux cas simultanément, la vitesse de déplacement des arbres et des plantes herbacées ou même des petits rongeurs et des primates, serait insuffisante pour leur permettre de s'adapter au réchauffement climatique.

Que se passerait-il donc si les plantes et les arbres ne peuvent pas bouger? Tout simplement et malheureusement, certaines espèces vont disparaître de la partie de leur aire de répartition où il fait trop chaud ou trop sec. Et c'est, effectivement, ce qu'on commence à voir pour certains arbres. Ils meurent à cause de la sécheresse et de la température très élevée. Le graphique de la figure 5.9, fait le recensement de 
l'ensemble des endroits où l'on commence à voir des dépérissements des arbres sur tout le globe.

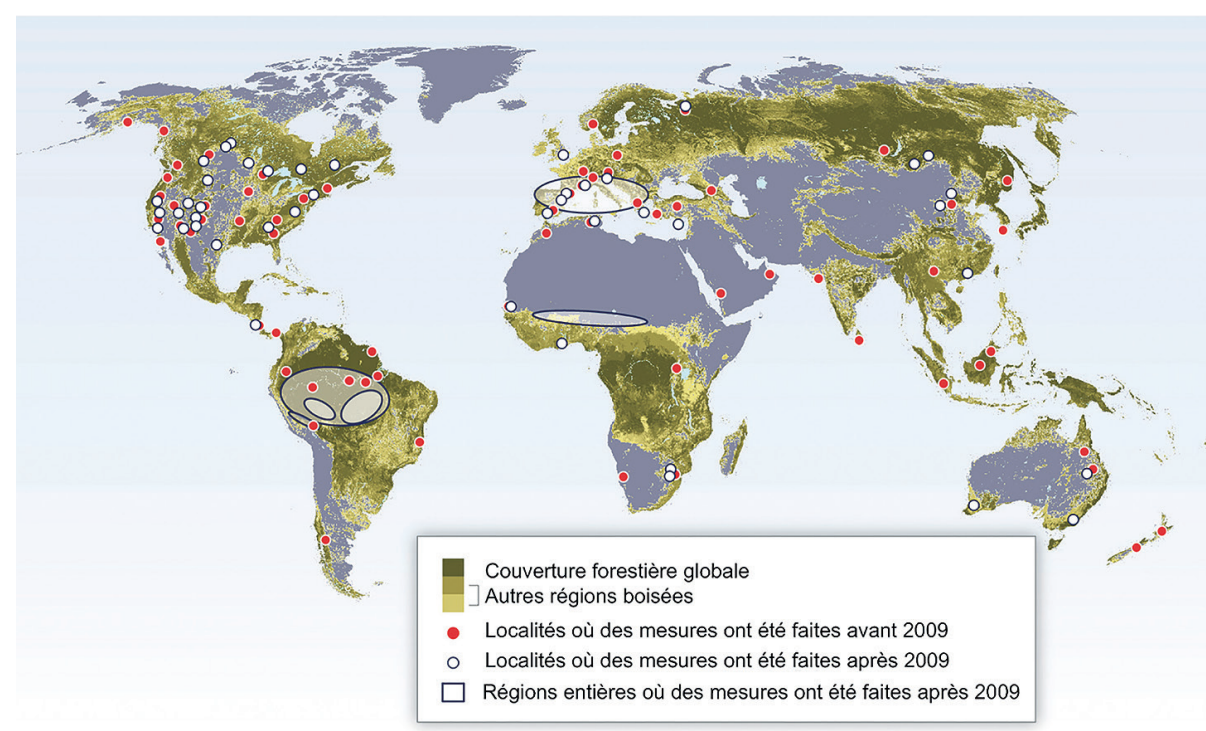

Figure 5.9: Régions du monde où se manifestent des sécheresses sévères avec mortalité des arbres. (Couverture forestière et autres parties boisées dans le monde). Les points rouges correspondent à des études locales, faites avant 2009. Les mesures plus récentes sont indiquées par des points blancs ou des formes ovales. Source: IPCC AR5 WGII 2014, chapitre 4.

Les scientifiques ont aussi conçu des modèles pour tenter de prédire l'avenir des arbres (voir section 6.2). Plusieurs modèles ont ainsi été utilisés pour étudier l'impact du changement climatique sur les arbres en France, notamment sur le pin sylvestre, car c'est une essence très importante pour la foresterie [6]. Hormis certains endroits, surtout en montagne, où le climat deviendrait plus favorable au pin sylvestre, les résultats des différents modèles utilisés dans l'étude indiquent que globalement en plaine, le climat va lui devenir très défavorable dans les prochaines années. II faut donc prendre en compte ces résultats en foresterie et probablement décider de ne plus planter de pins sylvestres, car la récolte du pin sylvestre ne se fait qu'au bout d'une cinquantaine d'années ou plus. De manière générale, le réchauffement climatique va donc fortement modifier nos forêts, non seulement les arbres, mais toutes les espèces qui dépendent des arbres dans ces forêts.

\subsubsection{Impacts du réchauffement climatique sur les écosystèmes d'eau douce}

Nous terminons ici notre bref tour d'horizon en considérant les écosystèmes d'eau douce. Ces écosystèmes sont, en effet, très sensibles au changement climatique et sont surtout des écosystèmes en haute montagne. On sait que les glaciers 
sont en train de rétrécir partout sur la planète. Un tout petit rétrécissement a plutôt tendance à augmenter le débit dans les rivières, mais un fort rétrécissement diminue leur débit. Comme l'illustre le graphique de la figure 5.10, la richesse spécifique dans les rivières diminue fortement, dès que le recouvrement d'un bassin versant par les glaciers se réduit de manière significative.

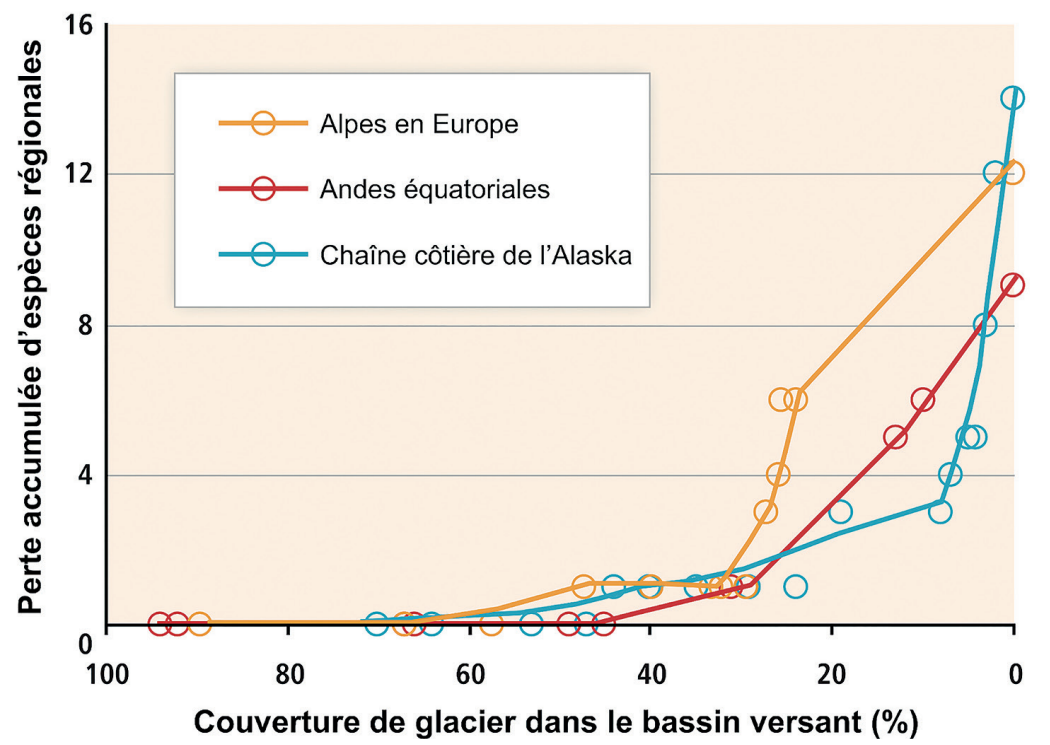

Figure 5.10: Perte de la biodiversité associée à la diminution de la couverture des glaciers sur les bassins versants. Trois régions du monde sont considérées. Source: IPCC AR5 WGII 2014, chapitre 4.

En conclusion, on constate que le réchauffement climatique a déjà commencé à faire migrer les espèces de leurs aires de répartition initiales. On constate aussi, à partir des modèles, que le déplacement des espèces va encore s'accentuer avec le réchauffement climatique à venir. Si l'on peut minimiser les émissions de gaz à effet de serre, cet effet va rester acceptable à défaut d'être négligeable. Mais si nos émissions de gaz à effet de serre se poursuivent au rythme actuel, beaucoup d'espèces ne pourront pas s'adapter et se déplacer assez rapidement pour "suivre» le changement climatique. II faut donc craindre des impacts très forts à la fois sur la diversité spécifique et la diversité écosystémique.

\subsection{Impacts des pollutions sur la biodiversité - C. Mougin}

Identifiée(s) comme la quatrième cause de perte de biodiversité sur Terre, la pollution, ou plutôt les pollutions, sont multiples par leur nature, leur étendue géographique ou persistante dans le temps, qu'elles soient physiques, chimiques, radiochimiques ou encore biologiques, toutes impactent la biodiversité. Nous nous 
concentrerons sur deux types de pollution: la pollution lumineuse dont la prise en compte est relativement récente [7], et la pollution chimique qui est plus classiquement mentionnée.

\subsubsection{La pollution lumineuse}

La pollution lumineuse englobe l'ensemble des sources de lumière artificielle, qui dégradent les cycles de la lumière naturelle (jour/nuit et saisons) et modifient la composante nocturne de l'environnement. Cette pollution croît très rapidement à travers le monde, de $6 \%$ par an en moyenne, et, dans certaines régions, l'obscurité n'existe plus (Fig. 5.11). La pollution lumineuse impacte négativement le vivant, en particulier le comportement des animaux, mais aussi les rythmes biologiques et la physiologie de l'ensemble des organismes, animaux et végétaux chlorophylliens ${ }^{52}$ [8].

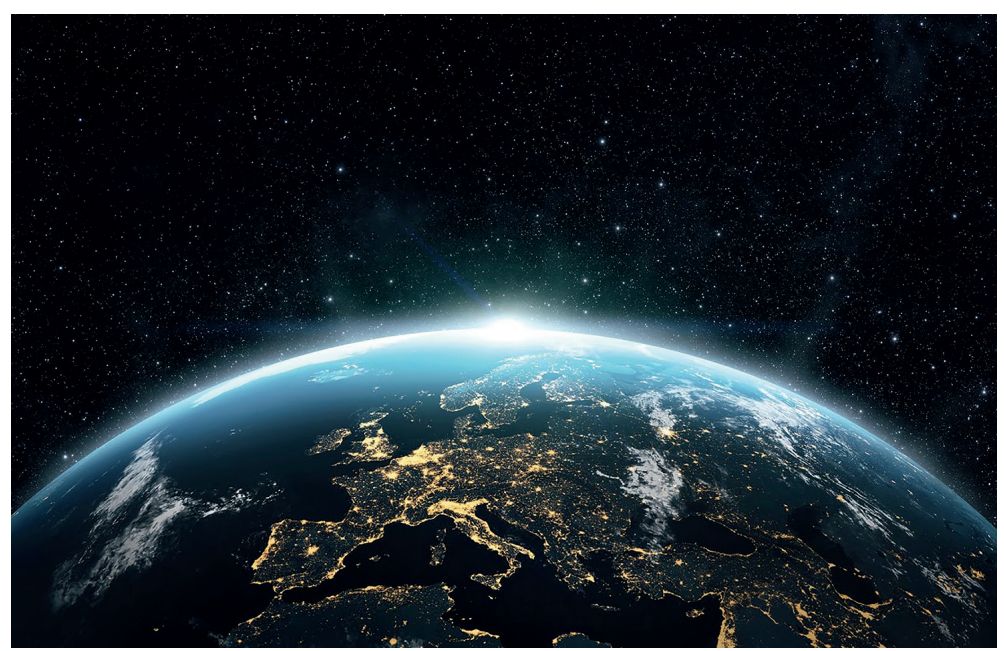

Figure 5.11 : L'Europe vue du ciel la nuit. Photo : @istock

Près de $30 \%$ des vertébrés (mammifères, oiseaux, etc.) et $65 \%$ des invertébrés (insectes...) sont exclusivement ou partiellement nocturnes. En leur envoyant des signaux lumineux contradictoires, la pollution lumineuse induit chez les animaux une représentation spatiale et temporelle dégradée de leur environnement. Par son rôle attractif ou répulsif, elle perturbe leur orientation. C'est ainsi le cas des insectes nocturnes (moustiques, papillons, mouches et coléoptères, etc.) qui se retrouvent piégés ou carbonisés sur les lampes et réverbères par lesquels ils ont été attirés. Chez les espèces bioluminescentes comme les vers luisants, la pollution lumineuse perturbe la communication entre les individus.

52. http://www.espaces-naturels.info/pollution-lumineuse-et-biodiversite-enjeu-pour-ensembleterritoire 
Les oiseaux sont également impactés. En période de nidification, adultes et juvéniles peuvent être attirés par des sources lumineuses parasites, qui les désorientent et perturbent leur retour au nid. La majorité des oiseaux migrateurs se déplace la nuit, et s'oriente grâce à la position des étoiles. En pénétrant la nuit dans les dômes lumineux qui se forment au-dessus des villes, ou éblouis à cause des lumières artificielles provenant d'édifices tels que les phares, les tours, les plates-formes pétrolières..., ils sont désorientés et subissent des mortalités importantes dues à des collisions directes.

Un autre exemple très connu d'impact de la pollution lumineuse concerne la reproduction des tortues marines. Sur les plages, après l'éclosion des œufs, les jeunes tortues ne peuvent pas retrouver la mer en se repérant sur l'horizon nocturne, ce qui induit des surmortalités par épuisement et par prédation accrue.

La pollution lumineuse impacte également l'activité alimentaire des animaux. Ainsi, les micromammifères se nourrissent moins dans les zones fortement éclairées, et s'affaiblissent. Certaines espèces de batraciens chassent avec une intensité lumineuse très faible, d'autres au contraire préfèrent des intensités plus fortes. Une homogénéisation de l'intensité lumineuse par un éclairage artificiel modifie les comportements de chasse, génère des compétitions entre espèces, ou encore modifie le rapport entre les proies et les prédateurs.

\subsubsection{La pollution chimique}

La pollution chimique est souvent mentionnée comme une cause majeure du déclin de la biodiversité ${ }^{53}[9,10]$. Par leurs nombreuses activités industrielles, domestiques et agricoles, les humains produisent, utilisent et relarguent dans l'environnement de très nombreux composés chimiques inorganiques tels que les métaux ou organiques tels que les hydrocarbures, les organochlorés, les pesticides, les perturbateurs endocriniens, etc. Les concentrations en polluant dans l'ensemble des milieux naturels marins et continentaux sont souvent faibles, sauf en situation accidentelle, mais ces composés peuvent impacter durablement les êtres vivants. L'étude de ces impacts s'appuie sur l'écotoxicologie, une discipline qui intègre la toxicologie et l'écologie, et dont les objectifs sont de comprendre et de prédire les effets des contaminants chimiques sur les communautés naturelles.

Les polluants chimiques exercent dans certaines situations une toxicité aiguë et immédiate sur les individus exposés, qui peut conduire à une mortalité rapide. Mais les mécanismes de toxicité chronique, différée, liée à l'exposition sur le long terme des animaux à de faibles concentrations de polluants, sont majoritaires et préoccupants. Ces effets sur le long terme résultent également de la contamination des milieux par des substances toxiques persistantes, qui vont être transférées et accumulées dans les réseaux trophiques. Par exemple, la bioaccumulation de 
certains polluants persistants dans les œufs a été une cause majeure du déclin des populations de rapaces [11].

Dans ce contexte, l'utilisation des pesticides par l'agriculture conventionnelle est pointée du doigt (Fig. 5.12). Ainsi, on estime que la biomasse d'insectes volants a chuté de $75 \%$ durant les 30 dernières années, et que le déclin des populations d'abeilles et d'oiseaux en Europe est essentiellement dû à l'utilisation des pesticides.

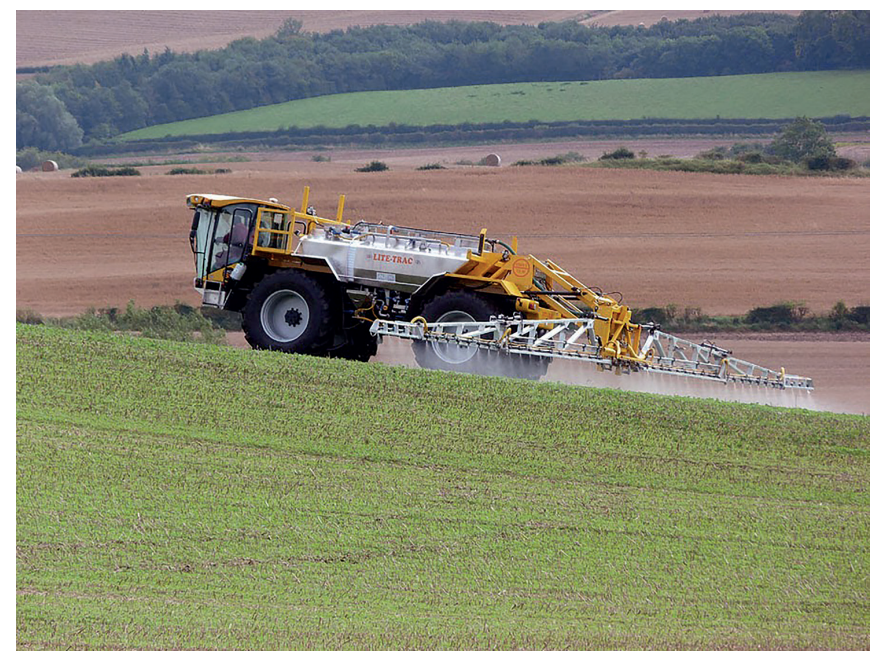

Figure 5.12: Polluants chimiques dans l'agriculture intensive. Creative commons 3.0, source: Lite-Trac.

Aux pollutions chimiques directes, nous pouvons ajouter celles, à la fois plus indirectes et plus "ordinaires», dues à l'usage intensif de matériaux issus de l'industrie chimique comme les matériaux plastiques en particulier. On estime que 24 à 35 millions de tonnes de déchets plastiques pénètrent chaque année dans le milieu aquatique ${ }^{54}$. Les organismes vivants, en particulier les animaux marins, peuvent être impactés, soit par des effets mécaniques tels que l'enchevêtrement dans des objets en plastique et des problèmes liés à l'ingestion de déchets plastiques, soit par l'exposition à des produits chimiques contenus dans les plastiques qui interfèrent avec leur physiologie. Les effets sur les êtres humains comprennent la perturbation de divers mécanismes hormonaux. Toutefois, la présence de matériaux plastiques, comme leurs éventuels impacts sur les écosystèmes terrestres continentaux, sont encore peu étudiés, mais les travaux sont en augmentation régulière depuis les 5 dernières années [12]. Dans certaines régions, des efforts importants ont été déployés pour réduire l'importance de la pollution des plastiques en libre parcours, en réduisant la consommation de plastique, en nettoyant les déchets et en encourageant le recyclage des plastiques.

54. "Predicting the future of plastic pollution and why cleanup is part of the solution" [archive] : theoceancleanup.com (18 septembre 2020) 


\subsubsection{Conclusions}

Les pollutions impactent les différents niveaux d'organisation biologique. Nous avons évoqué les impacts des pollutions sur l'orientation des individus et d'une façon générale sur leur comportement de déplacement, d'alimentation de reproduction. Mais les pollutions altèrent également le métabolisme et la physiologie des individus et plus généralement leurs performances, en exerçant de multiples effets toxiques, qu'ils soient immunodépressifs, mutagènes ou que ce soient des perturbations endocriniennes. Ces effets, observés au niveau individuel, se répercutent en cascade sur les populations, leur abondance ou leur structure démographique et/ou génétique, puis sur la structure et le fonctionnement des communautés, en termes de diversité, de dominance et de productivité. En occasionnant des effets qui vont se propager sur ces différents niveaux d'organisation biologique, sur une ou plusieurs générations, les pollutions vont in fine impacter la biodiversité.

En conclusion, les pollutions sont multiples, et affectent l'ensemble des organismes vivants. S'il est relativement facile de mettre en relation des pollutions avec des atteintes à la biodiversité, il est souvent délicat de les expliciter in natura par des relations causales. II est par ailleurs crucial de traiter la question complexe de l'exposition simultanée des organismes à plusieurs facteurs de stress (mélanges de produits toxiques, fragmentation de l'habitat, évolution de la ressource alimentaire, changement climatique, etc.), car elle représente de grands enjeux pour la recherche.

\subsection{Espèces exotiques envahissantes - E. Bonnaud et C. Bellard}

Les espèces exotiques envahissantes sont une menace majeure pour les écosystèmes à l'échelle mondiale. En effet, la présence de ces espèces entraîne des pertes de biodiversité et une modification des habitats et des interactions entre les espèces $[13,14]$.

\subsubsection{Processus d'introduction d'une espèce exotique}

Rappelons d'abord certaines définitions avant de décrire le processus qui conduit une espèce exotique à devenir envahissante [15] :

Une espèce est dite native (autochtone/indigène) quand, sur une période donnée, elle évolue dans son aire de répartition d'origine en y étant représentée par des populations qui s'y maintiennent. À l'inverse, une espèce est dite exotique (allochtone/exogène) lorsqu'elle est introduite dans une aire de répartition où elle était absente au début de la période considérée, et qu'elle la colonise par la suite.

Pour qu'une espèce puisse passer de son aire de répartition native vers une aire de répartition d'introduction, il faut un vecteur, c'est-à-dire un moyen de transport. 
Nous nous focaliserons, dans ce qui suit, sur le vecteur humain et donc sur les espèces qui sont transportées par les humains ou par toute activité humaine (Fig. 5.13).

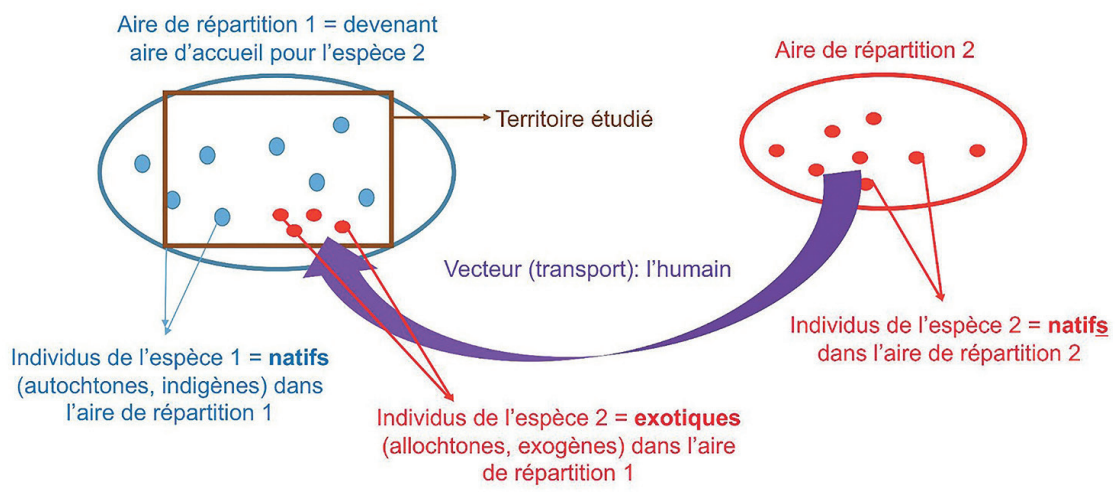

Figure 5.13: Introduction d'une espèce exotique sur un nouveau territoire.

Ainsi, dans une première phase, l'espèce exotique est introduite par les humains, de manière intentionnelle ou accidentelle, sur un territoire ou une partie de territoire où elle était jusqu'alors absente. Elle peut alors s'y établir (Fig. 5.14).

Après son établissement, l'espèce devient une espèce naturalisée, si elle rencontre des conditions écologiques favorables à son implantation durable sur le territoire d'accueil, notamment si elle s'y reproduit régulièrement et s'y maintient sur le long terme.

$\mathrm{Si}$, par la suite, l'espèce exotique prolifère et étend son aire de répartition en entraînant des impacts écologiques, économiques et/ou sociaux, l'espèce exotique devient alors envahissante. On parle aussi d'une espèce invasive, qui est un anglicisme couramment accepté.

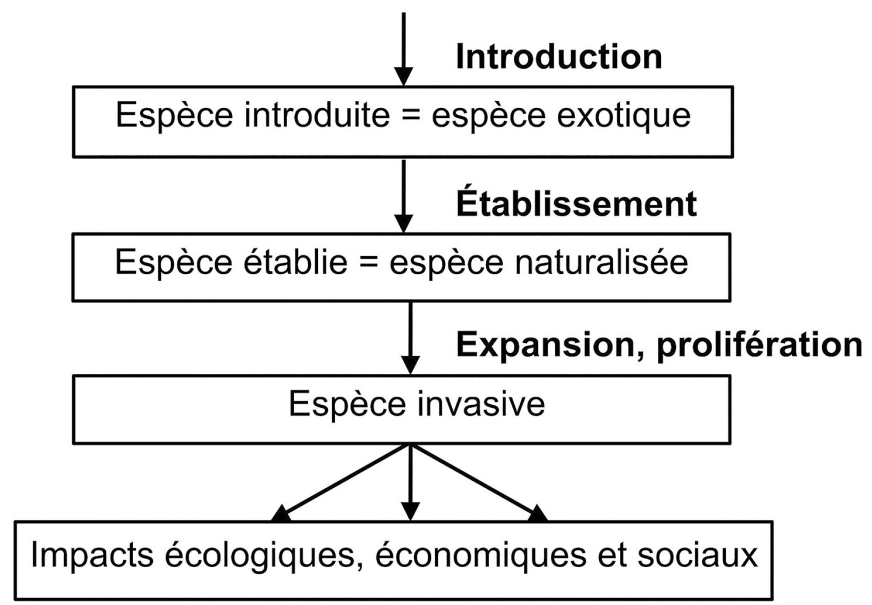

Figure 5.14: De l'espèce introduite à l'espèce invasive [16]. 


\subsubsection{Conditions pour qu'une espèce exotique introduite devienne envahissante}

De nombreuses espèces sont introduites, mais il est important de souligner qu'elles ne deviennent pas toutes envahissantes. Ce sont les espèces qui deviennent envahissantes, ou qui peuvent le devenir, qu'il faut particulièrement surveiller ou étudier pour que leur impact ne soit pas trop dévastateur dans l'écosystème d'accueil où elles ont été introduites [17].

La «réussite» d'une espèce introduite dans un nouvel écosystème reposerait sur le passage d'une série d'obstacles biotiques ou abiotiques que l'espèce doit franchir pour s'établir dans l'aire d'introduction (Fig. 5.15). Ces barrières sont aussi dépendantes de l'échelle spatiale, tant locale (disponibilité des ressources, interactions biotiques), paysagère (corridors, matrice favorable), que régionale (conditions topo-édapho-climatiques).

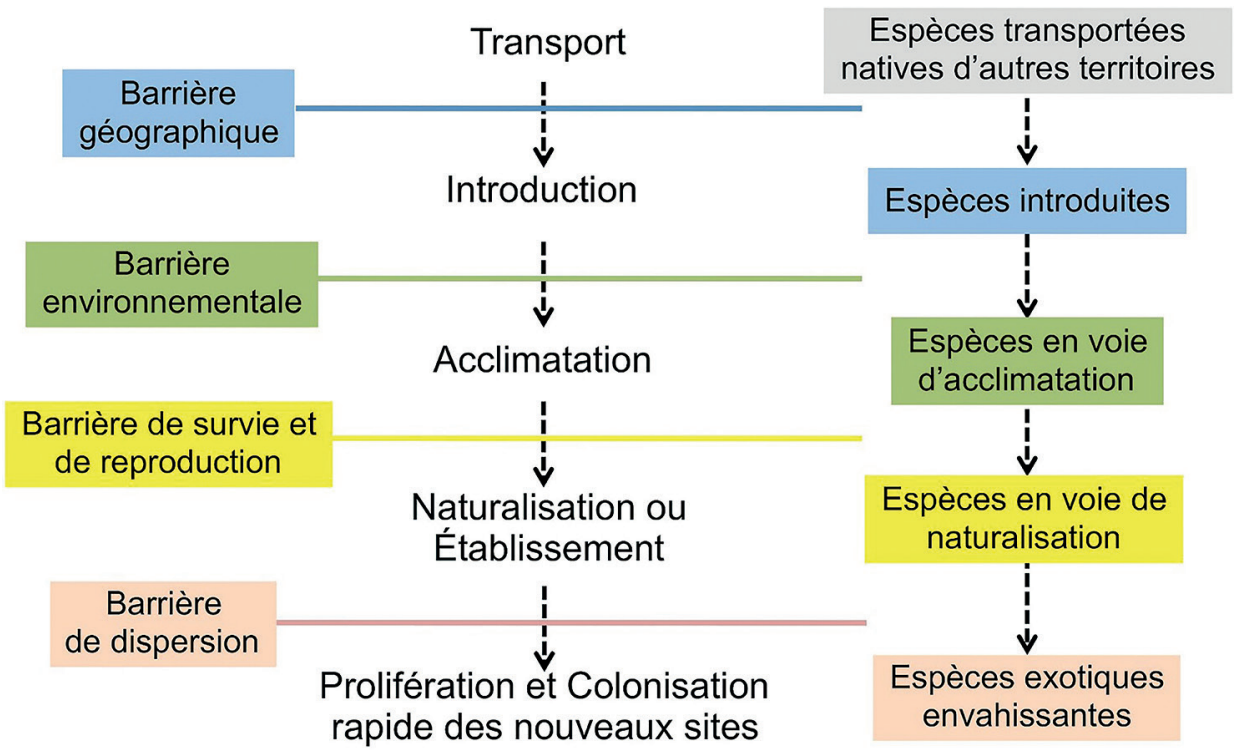

Figure 5.15: Représentation schématique des barrières que doivent franchir les espèces importées pour devenir envahissantes sur le territoire d'introduction.

Le processus d'invasion peut être divisé en une série d'étapes et à chaque étape, il existe des barrières qui doivent être surmontées pour qu'une espèce ou une population passe à l'étape suivante.

En amont, la barrière géographique, par exemple, un océan ou une montagne, peut empêcher les espèces de traverser une nouvelle zone géographique pendant la phase de transport. Les humains sont cependant souvent «efficaces» pour aider les espèces à franchir cette barrière! 
Durant la phase d'introduction accidentelle ou volontaire par les humains, il existe de nombreuses plantes et animaux en captivité et/ou en culture qui ne parviendront pas à traverser des éléments physiques tels qu'une clôture ou une haie, qu'on peut définir comme des barrières environnementales.

Pour les espèces qui réussissent à franchir ces barrières, elles doivent ensuite pouvoir survivre (barrière de survie) et se reproduire (barrière de reproduction) pour atteindre la phase dite d'établissement ou de naturalisation. En effet, les individus doivent trouver de bonnes conditions abiotiques (climat, habitats, etc.) et biotiques (absence de prédateurs et de parasites, avantage compétitif) pour pouvoir survivre et se reproduire, mais aussi établir des populations viables sur le long terme.

Pour que les individus d'une espèce ainsi naturalisée se propagent dans des endroits éloignés du point d'introduction et prolifèrent, il leur faut surmonter les obstacles qui entravent leur dispersion dans des nouvelles conditions environnementales. Une fois cet obstacle surmonté, les espèces exotiques peuvent effectivement proliférer.

La «portée» invasive est donc déterminée par les caractéristiques des environnements rencontrés, et les barrières fixent les limites de cette portée [17].

Plus le nombre d'individus est important et plus la fréquence des évènements d'introduction est élevée, plus la pression des propagules, c'est-à-dire le produit du nombre d'individus, N, par la fréquence des événements d'introduction, f, est forte, et donc plus la probabilité d'établissement sera importante.

Par ailleurs, les populations de taille plus importante ont plus de chances d'échapper à l'extinction que peuvent entraîner, par exemple, des conditions météorologiques inhabituelles ou des fluctuations démographiques aléatoires en leur sein. Dans le cas d'une stochasticité démographique plus faible, les populations sont ainsi moins susceptibles d'être soumises à des dépressions de consanguinité, à des goulots d'étranglement génétique, à des phénomènes de dérive génétique et, chez certaines espèces, aux effets Allee (voir section 5.6), c'est-à-dire à une réduction de la survie et de la reproduction dès lors que la densité de population est faible.

\subsubsection{Les invasions biologiques}

Les invasions biologiques représentent un phénomène mondial qui se produit depuis des milliers d'années, mais ce phénomène a connu un essor au cours des derniers siècles.

Le nombre d'espèces introduites depuis 1800 est en effet en constante augmentation. L'essor des transports, de la mondialisation, du tourisme ont largement contribué à de nouvelles introductions d'espèces exotiques de par le monde. Ce taux était d'environ 7 espèces introduites chaque année entre 1500 et 1800, et il a 
augmenté, depuis, pour atteindre un maximum de 585 en 1996. Seules les deux dernières guerres mondiales témoignent d'un ralentissement du nombre d'introductions. Ce taux ne montre pas de saturation ni de ralentissement sauf pour des groupes taxonomiques spécifiques comme les mammifères, qui montrent une décroissance depuis les années 50 en raison d'une législation beaucoup plus contraignante au niveau international [18].

Le phénomène d'invasion biologique, qui a lieu depuis longtemps, touche tous les pays. De nombreuses espèces ont été introduites pour la chasse, la culture ou des raisons esthétiques (ex: les plantes ornementales) ou même de manière accidentelle comme dans les cas des espèces, qui se sont échappées d'enclos, de transport au travers des eaux de ballasts des bateaux, etc. Ces phénomènes touchent l'ensemble des régions, mais plus particulièrement l'Europe, l'Amérique du Nord, l'Afrique du Sud, l'Australie et la Nouvelle-Zélande, qui ont développé des échanges commerciaux de grande ampleur durant les derniers siècles.

Les conséquences de ces introductions d'espèces exotiques pouvant devenir envahissantes sont multiples. Elles incluent la compétition avec les espèces natives pour les ressources, mais aussi la prédation sur les espèces autochtones, qui peut conduire à leur déclin jusqu'à parfois leur extinction totale [19, 20].

Les conséquences peuvent également être sanitaires comme l'introduction de certains moustiques exotiques, vecteurs de maladie (voir section 8.4.2), ou économiques comme la diminution des rendements de cultures causée par des parasites exotiques, ou encore génétiques dans le cas de d'hybridation entre espèces natives et espèces introduites dès lors que ces espèces sont proches génétiquement (voir section 4.1.2).

Enfin, les espèces envahissantes peuvent également conduire à des perturbations physiques des sols en changeant le système racinaire ou la composition des sols (voir section 2.6).

On estime aujourd'hui que les espèces envahissantes sont responsables à elles seules de $16 \%$ des extinctions mondiales et bien plus encore lorsqu'elles sont associées à d'autres menaces [21].

\subsubsection{L'exemple de la Perche du Nil}

Nous allons illustrer par l'exemple de la perche du Nil, introduite dans le Lac Victoria, ce que sont les conséquences en cascade d'une espèce introduite, devenue envahissante (Fig. 5.16).

Le lac Victoria, situé en Afrique de l'Est, est le deuxième plus grand lac d'eau douce au monde. C'est une source majeure de protéines pour trois pays: l'Ouganda, le Kenya et la Tanzanie. Ce lac est de formation relativement jeune d'environ 1000 ans. 
La famille des poissons Cichlidae, qui sont de petits poissons aux couleurs très variées, s'est diversifiée par le phénomène dit de spéciation au sein de ce lac, en raison de la structure fragmentée qu'offrent des territoires pour ces petits poissons très territoriaux. Avant 1960, il existait environ 500 espèces de Cichlidés dans le lac. Ces poissons sont surtout des herbivores et ont donc peu de valeur nutritive pour la pêche humaine.

Pour augmenter les ressources en protéines, nutritives pour les humains, la Perche du Nil, qui est un gros poisson carnivore, a été introduite dans les années 1950. II y a eu une lente progression de la Perche de 1960 à 1980 durant la phase d'installation, puis ses effectifs ont «explosé» durant la phase de prolifération. Dans les années 1980, il a été constaté la disparition de plus de 200 espèces de Cichlidés, du fait de leur prédation par la Perche du Nil. En effet, cette dernière s'était répandue dans la majeure partie du lac sauf dans quelques endroits les plus étroits.

À ces extinctions d'espèces consécutives à l'introduction de la Perche dans le lac Victoria, s'est ajouté l'abattage de nombreux arbres par les habitants de la région, car le bois s'est avéré nécessaire pour fumer le poisson qui n'était pas uniquement destiné à nourrir la population locale, mais aussi exporté dans les pays européens. L'abattage des arbres a tout d'abord entraîné une érosion accrue aux abords du lac, puis une augmentation des populations d'algues, favorisée par l'apport de matière organique et la moindre abondance des herbivores consécutive elle-même à la disparition des Cichlidés. II en a résulté le comblement du lac et un phénomène d'eutrophisation, autrement dit, une trop forte accumulation de matière organique renforçant le comblement du lac.

En 1990, 50 à 70\% du volume du lac s'est avéré sans oxygène, soit anoxique toute l'année. II s'est également produit une introduction accidentelle de la jacinthe d'eau qui, elle aussi, est une plante envahissante. L'étouffement du lac ainsi accru a entraîné en retour un déclin de la dynamique de population de la Perche du Nil, mais sans ré-augmentation des populations de Cichlidés, étant donné la dégradation écologique du lac.

L'instabilité politique de la région, le trafic et l'achat d'armes liés aux rentrées d'argent suite à la vente de perches du Nil aux pays européens, et la population humaine en forte augmentation ont accru encore le phénomène de raréfaction de ressources alimentaires dans la région du lac. Au bout du compte, la Perche du Nil n'a pas permis de nourrir les populations locales et a entraîné un désastre écologique (cf. le film «le cauchemar de Darwin»). 
Le cas de l'introduction de la perche du Nil

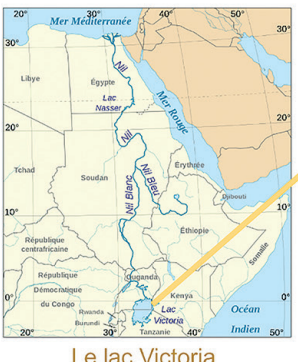

4) Introduction de la jacinthe d'eau Accentuation de l'eutrophisation du lac -> le lac devient de moins en moins propice au développement des espèces aquatiques dont la perche du Nil

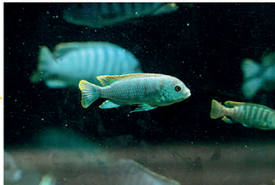

2) Extinction de 200 espèces de

1) Introduction de la perche du Nil poissons endémiques (Cychlidae)

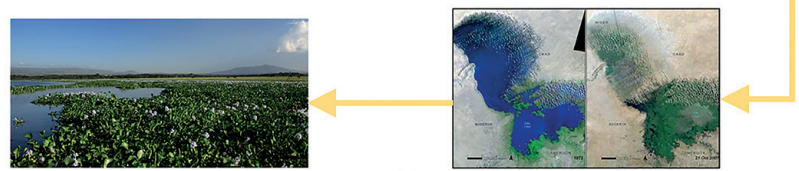

3) Eutrophisation et comblement du lac Disparition de nombreux herbivores (Cichlidae) -> Développement de algues et plantes aquatiques $\rightarrow$ Déforestation autour du lac pour fumer la perche du Nil $\rightarrow$ Érosion accrue, augmentation des sédiments

Figure 5.16: Processus ayant conduit au désastre écologique du lac Victoria par introduction de la perche du Nil. Sources: Lac Victoria (https://www.populationdata.net/cartes/afrique-nil/), Perche du Nil: Palais de la Porte Dorée - Aquarium tropical. Photo: Déodat Manchon (https://www.aquarium-tropical. fr/especes/lates-niloticus), Lac Tchad: (http://fr.wikipedia.org/wiki/tchad): Wikipedia - Cyclidae : Photo istock - Jacinthes d'eau: (https://www.afrik21.africa/kenya-moscou-finance-la-lutte-contre-la-jacinthedeau-sur-le-lac-victorial, source : Jean-Marie Takouleu).

\subsection{Dynamique des populations à faible effectif - E. Bonnaud et F. Courchamp}

Selon la liste rouge de l'UICN (Union internationale pour la conservation de la nature), les espèces rares sont souvent considérées comme menacées, car une population de petite taille a moins de chances de survivre à des catastrophes écologiques. Plus précisément, les espèces sont dites «rares», lorsqu'elles vérifient au moins l'une des deux caractéristiques suivantes:

- elles ont des faibles effectifs;

- leur aire de répartition est relativement restreinte.

Si une espèce rare est généralement endémique, le terme «endémique» fait exclusivement référence à l'habitat de l'espèce, qui peut-être ou très localisé ou très spécifique. De ce fait, une espèce endémique n'est pas forcément une espèce rare, car elle peut être abondante, même si son habitat est très localisé [22-24].

\subsubsection{Conditions conduisant à la rareté d'une espèce}

Il existe plusieurs facteurs à l'origine de la rareté d'une espèce (Fig. 5.17) :

- II s'agit d'une espèce jeune et à un stade évolutif. Les processus, qui ont conduit à son émergence, sont encore relativement récents et, de ce fait, elle n'a pas encore étendu son aire de répartition. À l'opposé, il peut s'agir 
d'une espèce apparue depuis très longtemps et dont l'habitat s'est fortement restreint au cours du temps;

- Les populations de l'espèce ont une faible variabilité génétique, ce qui entrave leurs capacités d'adaptation pour coloniser d'autres milieux dès lors que les conditions environnementales de leur milieu se dégradent.

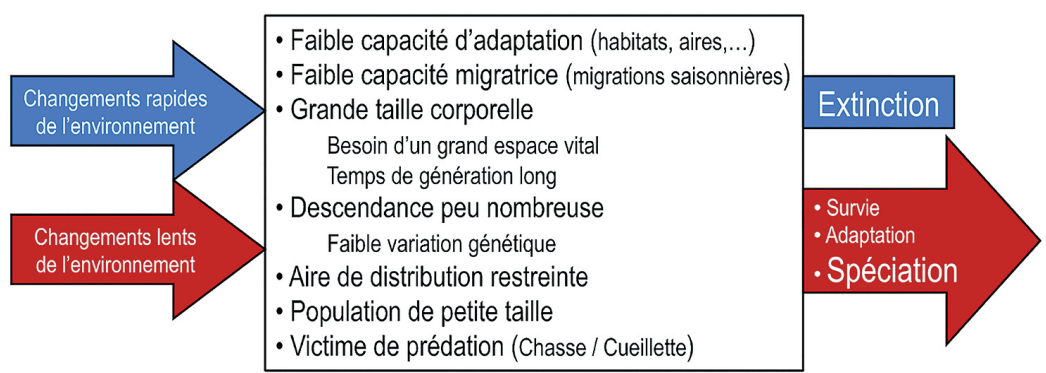

Figure 5.17: Facteurs à l'origine de la rareté d'une espèce et ses conséquences.

\subsubsection{Difficultés rencontrées par une espèce rare}

Selon le type de rareté (espèce évolutivement «jeune», pool génique réduit, faible compétition ou dispersion), les individus des populations d'une espèce rare peuvent être confrontés à un manque de partenaires pour la reproduction d'autant plus important que les effectifs sont faibles. Ceci peut entraîner un phénomène de consanguinité, une perte de variabilité génétique et aussi une moindre capacité à s'adapter aux conditions changeantes. Comme les populations des espèces rares sont, par définition, à effectif limité, les individus constituant cette population peuvent être également désavantagés vis-à-vis des phénomènes de prédation, de compétition ou de parasitisme [25, 26]. Tous les mécanismes évoqués peuvent conduire à leur extinction dans une «spirale ou vortex d'extinction» (Fig. 5.18).

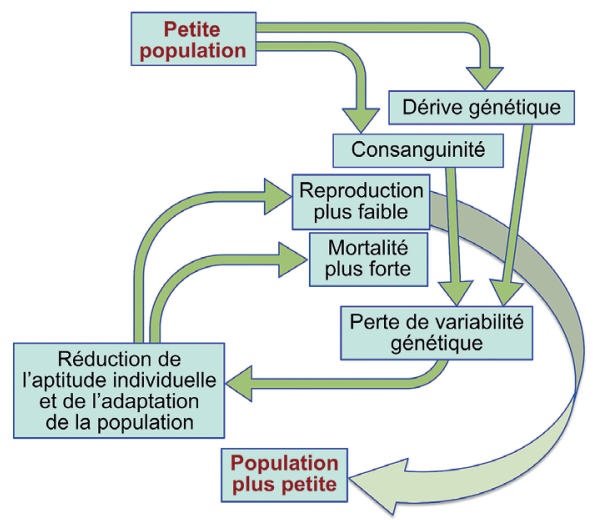

Figure 5.18: Spirale ou vortex d'extinction concernant les populations de faibles effectifs. 


\subsubsection{Croissance de populations à «densité-dépendance négative»}

On parle de densité-dépendance quand l'effectif de la population au temps «t» varie en fonction de l'effectif de la population au temps «t-1». À l'échelle de la population, le taux de croissance augmente avec la densité de population, car il y a de plus en plus d'individus au fur et à mesure que cette densité augmente (Fig. 5.19, gauche). À l'échelle individuelle (Fig. 5.19, droite), on observe un phénomène de densitédépendance négative lorsque les paramètres démographiques, survie et fécondité, diminuent au fur et à mesure que la densité de la population augmente. Autrement dit, dans ce cas, plus il y a d'individus dans la population, moins chaque individu va pouvoir se reproduire efficacement.

Prenons un exemple fictif. Si nous avons au départ une population de 10 individus avec une capacité moyenne de reproduction de 2,5 jeunes par femelle, lorsque cette population atteindra 100 individus, la capacité moyenne de reproduction par femelle pourrait se réduire à 2 jeunes.

\section{\begin{tabular}{|l|l} 
À l'échelle de la population l'échelle de l'individu \\
\hline
\end{tabular}}

Taux de croissance

de la population

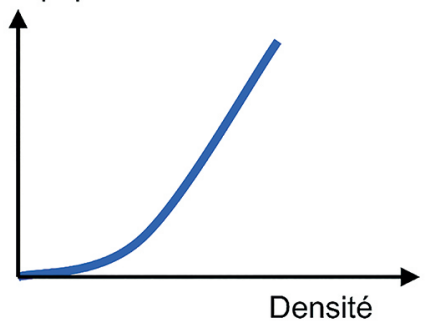

Taux de croissance par individu

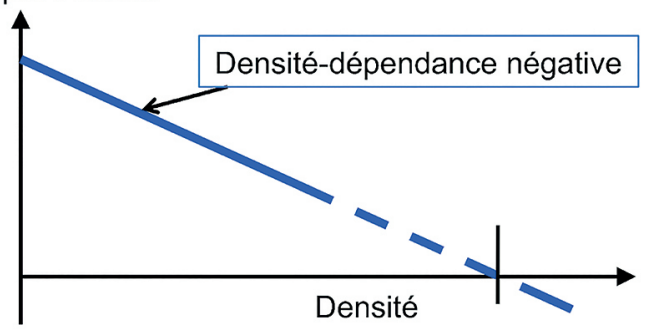

Figure 5.19: La densité-dépendance négative. À gauche: le taux de croissance pour l'ensemble de la population. À droite: le taux de croissance par individu en fonction de la densité de la population.

\subsubsection{Croissance de populations à «densité-dépendance positive »}

Dans le cas d'une densité-dépendance positive, on montre qu'à l'échelle de la population, si la densité d'individus est trop petite, les individus ne sont pas assez nombreux pour assurer la reproduction, la survie et la protection, qui permettent le maintien de la population (Fig. 5.20, à gauche). C'est l'effet «Allee», du nom du zoologiste américain M. Allee qui l'a décrit dès 1949 pour certaines espèces [27]. À l'inverse, si la densité de départ est au-delà du seuil minimal de maintien, plus la population devient nombreuse, plus la reproduction et la survie seront facilitées, 
plus il y aura de brassage de gènes favorisant le développement de la population et moins il y aura de dérive génétique avec le risque de perte de diversité génétique.

À l'échelle de l'individu, ce phénomène de densité-dépendance positive se traduit par le fait que les paramètres démographiques (survie et fécondité) augmentent avec la densité de la population. Ainsi, si nous avons au départ une population de 5 individus avec une capacité moyenne de reproduction de 2 jeunes par femelle, quand l'effectif atteindra 20 individus, chaque femelle pourrait donner naissance à 2,5 jeunes en moyenne. Le taux de reproduction est d'autant plus fort qu'il y a plus d'individus dans la population.

On note néanmoins qu'à un certain niveau de densité, le taux de croissance par individu (en rouge figure 5.20, à droite) s'infléchit et rejoint alors la courbe du taux de croissance, qui a été obtenue dans le cas de densité-dépendance négative (en bleu dans la figure 5.20 , à droite).

\section{À l'échelle de la population}

Taux de croissance

de la population

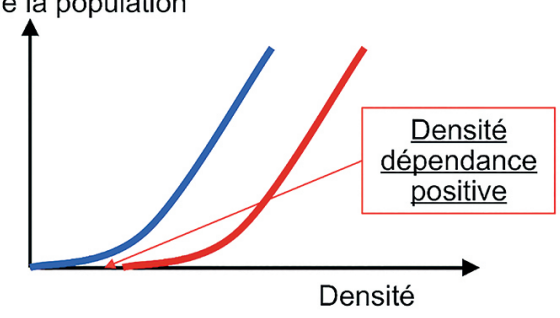

\section{À l'échelle de l'individu}

Taux de croissance par individu

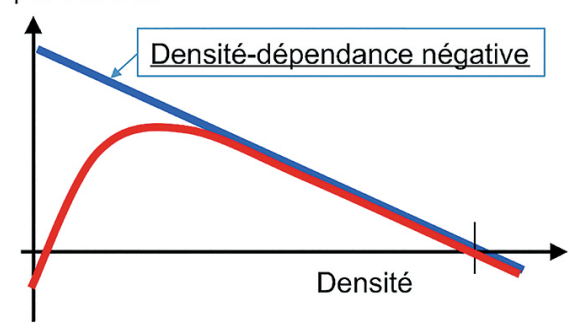

Figure 5.20: La densité-dépendance positive (courbes en rouge). À gauche: le taux de croissance pour l'ensemble de la population. À droite: le taux de croissance par individu en fonction de la densité de la population.

\subsubsection{Exemples d'espèces chez lesquelles a été observé l'effet Allee}

L'Effet Allee s'observe chez des espèces qui gèrent en groupe leur reproduction, la chasse et la protection contre les prédateurs. Les explications de l'effet Allee sont donc de nature biologique. Nous allons l'illustrer à l'aide de deux exemples très différents: les suricates et les morues.

Les suricates (Suricata suricatta), encore appelés sentinelles du désert, sont des mammifères diurnes de la famille des mangoustes, qui se nourrissent sur de longues périodes de la journée (Fig. 5.21). 


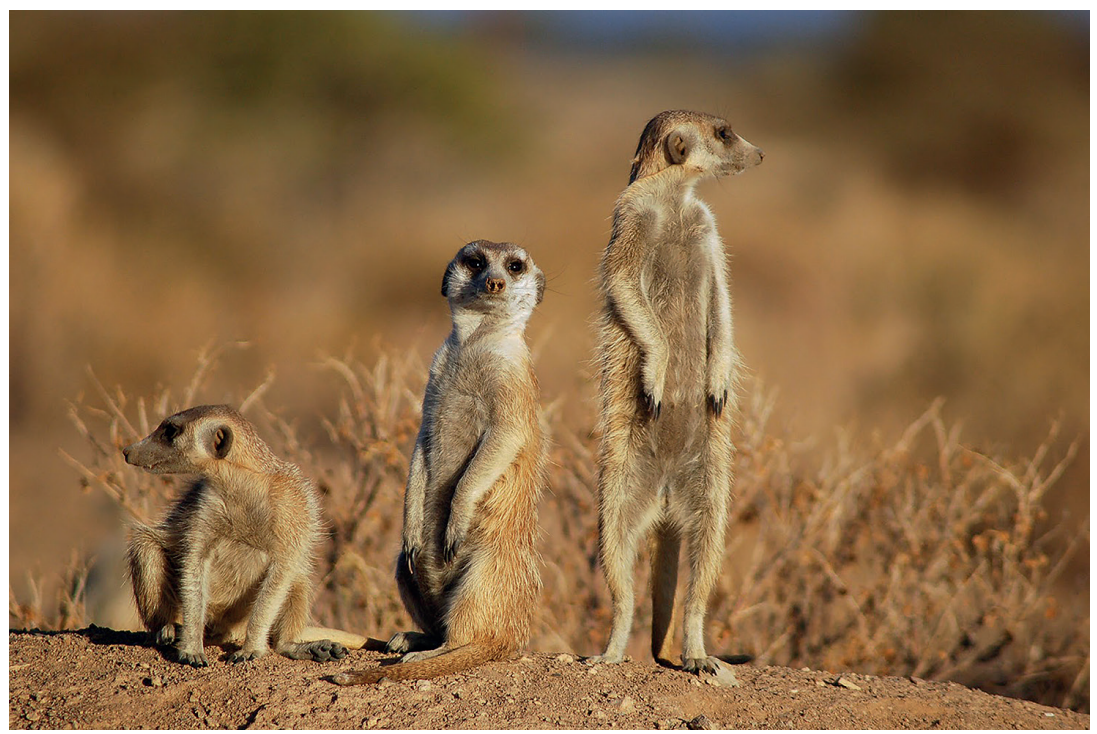

Figure 5.21: Suricates (Suricata suricatta), mangoustes namibiennes. Creative commons 2.0, source: Sara\&Joachim\&Mebe.

Leur métabolisme est, en effet, élevé, alors que leur nourriture consiste uniquement en de petits invertébrés faiblement énergétiques. Pendant qu'ils s'alimentent, les suricates tendent donc à être des proies faciles pour des prédateurs éventuels. En fait, ils limitent les risques de prédation grâce à un comportement, qu'on peut qualifier de coopératif. En présence des prédateurs, chaque membre du groupe à tour de rôle arrête, en effet, de s'alimenter pour assurer la surveillance du groupe vis-à-vis des prédateurs et prévenir ses congénères d'un danger éminent. Bien évidemment, quand le groupe devient trop petit, les individus ne peuvent plus arrêter de se nourrir à chaque fois que leur tour de sentinelle arrive, et le risque existe que le groupe continue à s'alimenter sans qu'un de ses membres ne soit suffisamment vigilant vis-à-vis des prédateurs. On assiste alors à une mortalité plus élevée pour ce petit groupe avec, en particulier, une diminution de la survie des adultes. Cela étant, les suricates coopèrent aussi pour élever les jeunes, ce qui favorise la survie juvénile, ainsi que pour chasser des proies plus grosses, ce qui favorise la survie des adultes [27].

Autre exemple, les morues vivent également en groupe et exercent le même type de vigilance vis-à-vis de prédateurs. Leur efficacité à se protéger augmente à mesure que le groupe grandit et leur reproduction est également facilitée par le regroupement des individus. On a pu observer un effet Allee dans le cas de la morue de l'Atlantique soumise à une surpêche. Le taux moyen de reproduction par individu s'est en effet réduit dès lors que la taille de la population est arrivée à un niveau très bas, ce qui a entraîné l'effondrement de la population de morues. Cette découverte empirique (par l'observation) confirme le fait qu'un effet Allee peut jouer un rôle 
considérable dans la dynamique des populations d'espèces rares ou qui se sont raréfiées, que ce soit par des causes naturelles ou humaines. En deçà d'un certain seuil de densité, les populations peuvent, en effet, entrer dans une spirale d'extinction.

\subsubsection{Surexploitation et effet Allee Anthropogénique}

II est important de bien différencier le phénomène de surexploitation des ressources et un effet Allee particulier, l'effet Allee Anthropogénique. La surexploitation se manifeste dès que l'on prélève plus que ce qui est produit par unité de temps (voir section 5.2). Dès que le prélèvement ne permet plus le renouvellement du stock d'individus, ceci entraîne une décroissance de la dynamique de population plus ou moins forte selon l'intensité du prélèvement. Cette décroissance peut conduire l'espèce considérée à devenir rare, voire à disparaître.

On parle d'effet Allee Anthropogénique lorsqu'une espèce est rare et qu'elle devient alors rentable pour les humains. De ce fait, elle est de plus en plus recherchée, de plus en plus exploitée, et devient donc encore plus rare, etc. Dans le cas d'un effet «Allee Anthropogénique», ce sont donc les humains qui par leur action de surexploitation entraînent un effet Allee [28].

Quand il y a surexploitation, il n'y a pas forcément d'effet Allee Anthropogénique. Par exemple, il peut y avoir épuisement d'une population de poissons, mais le prix de vente restant fixe, cette pêche ne devient plus rentable. Les pêcheurs vont alors se tourner vers une autre ressource de pêche dans le cas où il y a une possibilité de substitut. On observe donc un transfert des forces d'exploitation. Dans ce cas précis, il y a un phénomène de surexploitation au départ, mais par la suite, s'il y a arrêt de l'exploitation, les populations peuvent croître à nouveau et avoir une dynamique positive. Cependant, la relance de la dynamique peut ne pas être sans conséquence sur la structure génétique de la population en se traduisant, par exemple, par une perte de variabilité génétique. En effet, si ce sont plutôt des individus de grande taille qui ont été pêchés, on va alors assister à une reproduction entre des individus de plus petite taille et un phénomène de sélection va tendre à diminuer la taille moyenne de l'espèce. Ce phénomène peut se poursuivre même après la fin de la surexploitation. On peut, par ailleurs, assister à un autre phénomène, à savoir le changement de l'aire de répartition de l'espèce. L'occurrence de ce phénomène dépend de la durée, pendant laquelle l'espèce a été exploitée, de son pouvoir de colonisation ainsi que des différentes interactions qui ont pu se recréer dans son environnement.

Quand il n'y a pas de possibilité de substitut à l'espèce rare, c'est-à-dire quand aucune autre espèce ne peut être exploitée à sa place, la probabilité augmente pour l'espèce rare de subir un effet Allee Anthropogénique. Enfin, même s'il existe un substitut, le processus de surexploitation peut à nouveau se produire pour le substitut lui-même dès lors que le changement d'exploitation ne s'est pas fait de manière raisonnée. 


\subsection{Les mécanismes d'homogénéisation en cours - F. Chiron}

Le processus d'uniformisation du vivant à l'échelle de la planète, appelé aussi phénomène d'homogénéisation biotique, conduit les écosystèmes à se ressembler de plus en plus au cours du temps, du point de vue de leur composition ou de leur fonctionnement. Nous pouvons faire le parallèle avec d'autres formes d'homogénéisation ayant lieu dans les sociétés humaines, qui sont de nature culturelle, sociale ou économique. Par ailleurs, il existe bien un lien entre la standardisation de nos modes de vie et l'homogénéisation biotique avec des conséquences sur les écosystèmes.

\subsubsection{Origine et définitions}

Le concept d'homogénéisation biotique a émergé en 1999 en même temps que celui des invasions biologiques, c'est-à-dire l'introduction par les humains de nouvelles espèces dans les écosystèmes (voir section 5.5). L'introduction des espèces envahissantes à travers le monde à des fins ornementales, alimentaires ou sans le vouloir, a en effet tendance à homogénéiser les communautés locales. C'est ce que l'on nomme l'homogénéisation taxonomique. Cette manière de définir l'homogénéisation biotique mène cependant à des confusions d'interprétation. En effet, l'homogénéisation peut être alors vue comme un gain de ces mêmes espèces ou à la perte des espèces locales, ou alors à la conjonction de ces deux phénomènes (Fig. 5.22).
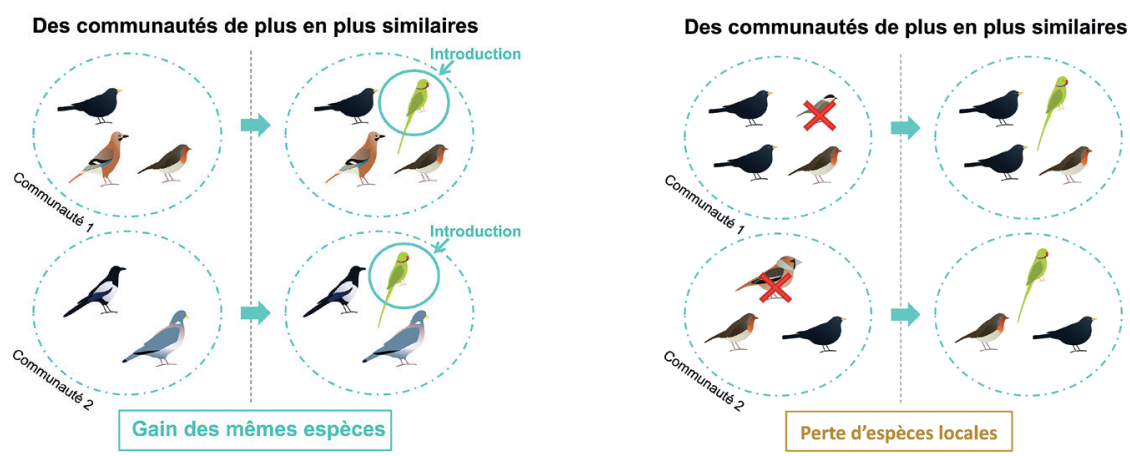

Figure 5.22: Le phénomène d'homogénéisation biotique: des communautés de plus en plus similaires par gain de mêmes espèces (en haut) et/ou par perte d'espèce locales (en bas) (Source: François Chiron).

Comme le concept d'espèces n'est pas forcément judicieux pour certains groupes taxonomiques (voir section 4.1.2), il est nécessaire de définir l'homogénéisation biotique autrement.

L'homogénéisation biotique peut être génétique, voire phylogénétique, compte tenu d'une plus grande similarité de gènes et d'allèles entre individus issus de populations différentes. 
L'homogénéisation peut aussi être fonctionnelle, c'est-à-dire définie comme l'uniformisation des caractéristiques morphologique, écologique, physiologique des espèces sans considérer leur identité taxonomique (Fig. 5.23). Ces caractéristiques, ou traits, sont impliqué(e)s dans le recyclage de la matière organique, la croissance des plantes, les régulations biologiques entre espèces, etc. Elles sont à la base du fonctionnement des écosystèmes et du phénomène de résilience. Notons que les conséquences de l'homogénéisation taxonomique sur l'homogénéisation fonctionnelle sont encore mal connues.

\section{Des communautés fonctionnelles de plus en plus similaires}

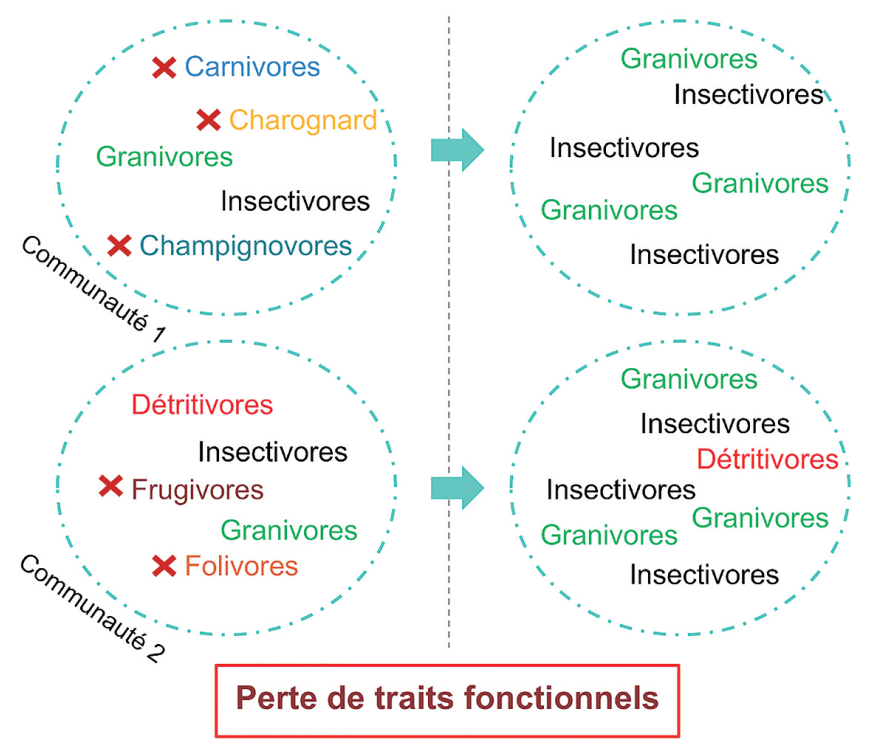

Figure 5.23: Le phénomène d'homogénéisation biotique: des communautés fonctionnelles de plus en plus similaires (Crédit: François Chiron)

À quelle échelle a lieu l'homogénéisation biotique? Localement, un jardin en ville, par exemple, peut accueillir un nombre important d'espèces, mais celles-ci sont souvent les mêmes d'un jardin à un autre et d'une ville à une autre, donc peu variées dans l'ensemble. En revanche, des pelouses sèches dans des régions biogéographiques variées accueilleront des espèces différentes, rendant ces milieux uniques et plus riches globalement.

L'homogénéisation biotique ne peut être mise en évidence que par l'observation de la faune et de la flore à l'échelle d'un pays, d'un continent, voire de la biosphère, et des différences de biodiversité entre ces espaces à l'échelle macroscopique. Ce travail fastidieux bénéficie aujourd'hui du développement des sciences participatives sur la biodiversité et de ses milliers de participants qui contribuent à cet effort d'observation (voir section 5.8). 


\subsubsection{Causes, mécanismes et conséquences}

Les causes de l'homogénéisation biotique sont, avant tout, les changements globaux liés aux activités humaines. De façon directe ou indirecte, les changements climatiques, l'urbanisation, l'intensification agricole et, dans certains cas particuliers, les introductions d'espèces transforment et modifient considérablement la surface, le type et la qualité des écosystèmes, perturbant les populations et rendant leurs conditions de vie très similaires. Par exemple, en zone urbanisée, les habitats des différentes espèces, qui sont définis par les conditions climatiques, la teneur en matière organique dans le sol et d'autres éléments biogéochimiques, ont tendance à s'homogénéiser en raison de l'uniformisation des modèles de conception des villes. La gestion des réseaux de transports, des zones résidentielles et commerciales, voire des espaces verts, est souvent identique. Cette convergence des formes urbaines à l'échelle d'un continent gomme les particularités écologiques de chaque ville et réduit mécaniquement le nombre total de niches écologiques pour des espèces différentes. Il en résulte qu'en ville on observe une homogénéisation taxonomique, phylogénétique et fonctionnelle pour les plantes, les oiseaux, les mammifères, les reptiles, les amphibiens et dans une moindre mesure, chez les invertébrés.

L'étude d'une variété de groupes a montré que l'homogénéisation biotique en réponse aux changements globaux se caractérise par la perte d'espèces spécialisées à un type d'habitat et le gain d'espèces dites généralistes, plus flexibles dans leur choix de ressources et dont les capacités cognitives, parfois étonnantes, leur permettent de s'adapter aux nombreux changements [29]. L'évolution de l'indicateur de spécialisation à l'habitat traduit ce phénomène pour les oiseaux. Depuis au moins 30 ans, le nombre d'oiseaux spécialistes diminue en France, alors que les généralistes se maintiennent, voire se multiplient (Fig. 5.24).

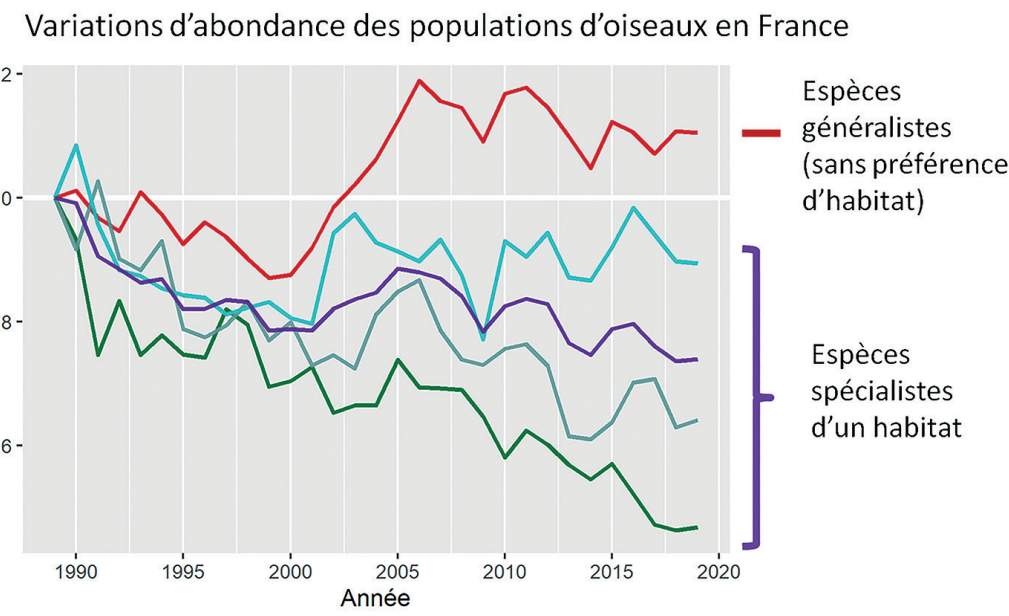

Figure 5.24: Variation d'abondance des populations d'oiseaux en France: plus d'espèces généralistes au détriment des espèces spécialistes (adapté de Fontaine et al., 2020 [30]). 
Trois mécanismes différents peuvent aboutir au remplacement des espèces spécialistes par des espèces généralistes. Tout d'abord, la destruction d'habitat entraîne mécaniquement la disparition des espèces spécialisées à celui-ci. D’autre part, les espèces généralistes plus flexibles et plus innovantes, possèdent aussi une plus grande capacité à coloniser de nouvelles niches écologiques. Face au réchauffement climatique, par exemple, les espèces tendent à déplacer leur aire de répartition pour suivre les isoclines de température. Les populations d'espèces généralistes sont capables de réagir plus rapidement, car elles tolèrent les modifications d'occupation des sols, qui représentent autant de freins aux populations des espèces spécialistes pour remonter vers le nord.

Enfin, indirectement, les changements globaux, en modifiant la qualité des habitats, modifient les interactions entre espèces. Par exemple, le changement climatique dans les forêts du nord de l'Europe augmente la compétition entre les oiseaux généralistes et les oiseaux spécialistes pour l'accès aux ressources. Ainsi, lors d'un printemps précoce, le gobemouche noir (Ficedula hypoleuca), un spécialiste migrateur (Fig. 5.25, gauche), ne peut pas anticiper sur ses quartiers d'hivernage l'éclosion prématurée des chenilles dont il nourrit ses jeunes, et ce à la différence de la mésange charbonnière (Parus major), une espèce généraliste et sédentaire, qui se nourrit de la même ressource (Fig. 5.25, droite). De plus, lors d'hivers doux, les meilleures capacités de survie des mésanges charbonnières accentuent encore leur compétition avec les gobemouches, moins agressifs dans la recherche de cavités de reproduction.
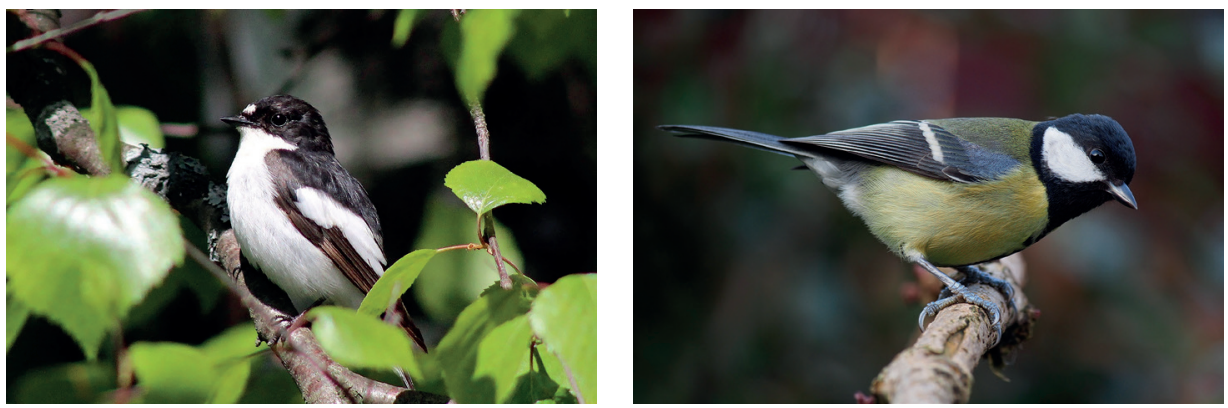

Figure 5.25: Gobemouche noir (à gauche, source : Estormiz, license CC0 1.0) et Mésange charbonnière (à droite, source: Francis Franklin, license CC BY-SA 4.0).

Ce type d'interactions, qui conduit au remplacement des spécialistes par les généralistes, reste néanmoins peu documenté dans la plupart des cas. D’autre part, il faut noter que dans des environnements naturels fortement dégradés comme en ville, les espèces tolérantes aux perturbations constituent la très grande majorité de la biodiversité. Ces espèces représentent une forme de «compensation » à la perte des spécialistes dans ces environnements qui seraient, sinon, bien pauvres en biodiversité.

La perte d'abondance, notamment de spécialistes, n'est pas l'unique conséquence de l'homogénéisation biotique. Nous avons vu (section 4.3) qu'il existait une 
dynamique positive entre la diversité biologique et le fonctionnement des écosystèmes. Cette dynamique résulte de la complémentarité des espèces entre elles à un niveau local; en augmentant la diversité spécifique, on augmente le nombre de fonctions réalisées par ces espèces. À un niveau régional, la singularité de chaque communauté entraîne une variabilité des réponses aux perturbations, stabilisant ainsi globalement tout le système. Que va-t-il alors advenir si les espèces spécialistes, qui assurent cette complémentarité et cette stabilité, disparaissent de l'écosystème et que les mêmes généralistes se retrouvent dans chaque communauté biotique? Quelles seront les conséquences sur le fonctionnement de l'écosystème et sa capacité à faire face à de nouvelles perturbations? Quelles seront les conséquences pour les humains? Ces questions font encore l'objet de nombreuses recherches en cours.

Comment répondre face à ce phénomène d'homogénéisation biotique? Nous avons évoqué à différentes reprises que la destruction et la modification des habitats par les activités humaines étaient les principales causes de l'homogénéisation biotique [31]. La première solution est donc d'arrêter la destruction et la modification des écosystèmes, même si cela ne fera que limiter la perte de biodiversité sans forcément inverser la tendance. Une autre solution est de rendre les conditions de vie plus hétérogènes pour le vivant. Lors de nouveaux aménagements de territoires, des mesures de gestion doivent ainsi être prises pour préserver les originalités écologiques locales sans répéter les mêmes solutions partout. Ce qui est bénéfique en un lieu donné peut, en effet, se révéler inefficace, voire néfaste, dans un autre environnement. Enfin, il nous faut compter sur la libre évolution des écosystèmes, une forme de «gestion inactive» trop peu pratiquée, mais un formidable moteur à la résilience et à la diversification du vivant sur le long terme (voir section 11.1).

\section{Références}

[1] H. Liu, P. Gong, J. Wang, N. Clinton, Y. Bai, S. Lian. 2020. Annual dynamics of global land cover and its long-term changes from 1982 to 2015. Earth Syst. Sci. Data 12, 1217-1243, (c) Author(s) 2020.

[2] N.C. Davidson. 2014. How much wetland has the world lost? Long-term and récent trends in global wetland area. Marine and Freshwater Research 65(10), 934-941. https://doi.org/10.1071/MF14173.

[3] F. Berkes, T.P. Hughes, R.S. Steneck, J.A. Wilson, D.R. Bellwood, B. Crona, C. Folke, L.H. Gunderson, H.M. Österblom, M. Scheffer, B. Worm. 2006. Globalization, roving bandits, and marine resources. Science 311( 5767), 1557-1558. DOI: 10.1126/science.1122804.

[4] R. Hilborn, O. Amoroso, C.M. Anderson, J.K. Baum, T.A. Branch, C. Costello, C.L. de Moor, A. Faraj, D. Hively, O.P. Jensen, H. Kurota, L.R. Little, P. Mace, T. McClanahan, M.C. Melnychuk, C. Minto, G.C. Osio, A.M. Parma, M. Pons, S. Segurado, C.S. Szuwalski, J.R. Wilson, Y. Ye. 2020. Effective fisheries management instrumental in improving fish stock status. PNAS 117(4), 2218-2224, https://doi.org/10.1073/pnas.190972611. 
[5] T.W. Crowther, et al. 2015. Chiffres de prélèvement d'arbres par an [Mapping tree density at a global scale]. Nature 525, 201-205.

[6] A. Cheaib, et al. 2012. Climate change impacts on tree ranges: model intercomparison facilitates understanding and quantification of uncertainty. Ecology Letters 15(6): 533-544.

[7] T. Longcore, C. Rich. 2004. Ecological light pollution. Front Ecol Environ 2(4), 191-198.

[8] Hölker, et al. 2010. Light pollution as a biodiversity threat. Trends in Ecology and Evolution 25(12), 682-682.

[9] C.A. Hallmann, R.P.B. Foppen, C.A.M. van Turnhout, H. de Kroon, E. Jongejans. 2014. Declines in insectivorous birds are associated with high neonicotinoid concentrations. Nature 511, 341-343.

[10] T. Gunstone, T. Cornelisse, K. Klein, A. Dubey, N. Donley 2021. Pesticides and soil invertebrates: A hazard assessment. Front. Environ. Sci.

[11] R. Carson 1962. Silent Spring. London, Penguin Classic, (édition originale)

[12] C. Mougin, I. Lamy, L. Ciadamidaro, S. Houot, J. Gasperi, R. Driss, B. Tassin. 2020. Les plastiques dans les sols: quels niveaux de contaminations et quels impacts écotoxicologiques? Dans Les sols urbains sont-ils cultivables? Mougin C., Douay F., Canavese M., Lebeau T., Rémy E. (Editeurs), Collection Matière à débattre et décider, Editions Quae, Versailles, chapitre 10, pp.182-192, ISBN 978-2-7592-3202-4

[13] E. Branquart, G. Fried. 2016. Espèces envahissantes d'ici et d'ailleurs: Synthèse sur les espèces envahissantes et présentation de 32 espèces. Éditions du Gerfaut.

[14] T.M. Blackburn, C. Bellard, A. Ricciardi. 2019. Alien versus native species as drivers of recent extinctions. Frontiers in Ecology and the Environment 17, 203-207.

[15] E. Rémy, C. Beck. 2008. Allochtone, autochtone, invasif: catégorisations animales et perception d'autrui. Politix 2, 193-209.

[16] Goudard A. 2007. Fonctionnement des écosystèmes et invasions biologiques. Importance de la biodiversité et des interactions interspécifiques, Thèse, 216 p.

[17] D. Simberloff. 2013. Invasive species: What everyone needs to know. Oxford Unviversity Press, Oxford, $346 \mathrm{p}$.

[18] H. Seebens, T.M. Blackburn, E.E. Dyer, P. Genovesi, P.E. Hulme, J.M. Jeschke, S. Pagad, P. Pyšek, M. Winter, M. Arianoutsou, S. Bacher, B. Blasius, G. Brundu, C. Capinha,

L. Celesti-Grapow, W. Dawson, S. Dullinger, N. Fuentes, H. Jäger, J. Kartesz, M. Kenis,

H. Kreft, I. Kühn, B. Lenzner, A. Liebhold, A. Mosena, D. Moser, M. Nishino, D. Pearman,

J. Pergl, W. Rabitsch, J. Rojas-Sandoval, A. Roques, S. Rorke, S. Rossinelli, H. E. Roy,

R. Scalera, S. Schindler, K. Štajerová, B. Tokarska-Guzik, M. Van Kleunen, K. Walker, P. Weigelt, T. Yamanaka, F. Essl. 2017. No saturation in the accumulation of alien species worldwide. Nature Communications 8, 1-9.

[19] C. Bellard, P. Cassey, T.M. Blackburn. 2016. Bellard, Cassey, Blackburn - 2016 - Biology letters - Alien species as a driver of recent extinctions. Biology Letters 12, 24-27. 
[20] F. Courchamp, A. Fournier, C. Bellard, C. Bertelsmeier, E. Bonnaud, J.M. Jeschke, J.C. Russell. 2017. Invasion biology: Specific problems and possible solutions. Trends in Ecology and Evolution 32, 13-22.

[21] IUCN. 2021. 100 of the World's Worst Invasive Alien Species. Global Invasive Species Database, International Union for Conservation Nature, Invasive Species Specialist Group. http://www.iucngisd.org/gisd/search.php.

[22] M.G. Raphael, R. Molina. 2007. Conservation of rare or little-known species. Page (M.G. Raphael, R. Molina, Eds.). Island Pre.

[23] S. Lavergne. 2013. Les espèces végétales rares ont-elles des caractéristiques écologiques et biologiques qui leur sont propres? Application à la conservation de la flore en Languedoc-Roussillon 8078.

[24] D. Mouillot, D.R. Bellwood, C. Baraloto, J. Chave, R. Galzin, M. Harmelin-Vivien, M. Kulbicki, S. Lavergne, S. Lavorel, N. Mouquet, C.E.T. Paine, J. Renaud, W. Thuiller. 2013. Rare species support vulnerable functions in high-diversity ecosystems. PLoS Biology 11, e1001569.

[25] I. Olivieri, R. Vitalis. 2001. La biologie des extinctions. Médecine Science 17, 63-69.

[26] A. Robert. 2011. Petites populations et vortex d'extinction. Page Regard R9 du 10/01/11.

[27] F. Courchamp, L. Berec, J. Gascoigne. 2008. Allee effects in ecology and conservation. Oxford University Press, New York.

[28] E. Angulo, A.L. Deves, M. Saint Jalmes, F. Courchamp. 2009. Fatal attraction: Rare species in the spotlight. Proceedings of the Royal Society B: Biological Sciences 276 , 1331-1337.

[29] J. Clavel, R. Julliard, V. Devictor, 2011. Worldwide decline of specialist species: toward a global functional homogenization? Frontiers in Ecology and the Environment 9(4), 222-228.

[30] B. Fontaine, C. Moussy, J. Chiffard Carricaburu, J. Dupuis, E. Corolleur, L. Schmaltz, R. Lorrillière, G. Loïs, C. Gaudard 2020. Suivi des oiseaux communs en France 1989-2019: 30 ans de suivis participatifs. MNHN- Centre d'Écologie et des Sciences de la Conservation, LPO BirdLife France - Service Connaissance, Ministère de la Transition écologique et solidaire, $46 \mathrm{p}$.

[31] IPBES. 2019. Summary for policymakers of the global assessment report on biodiversity and ecosystem services of the Intergovernmental Science-Policy Platform on Biodiversity and Ecosystem Services. S. Díaz, J. Settele, E.S. Brondízio E.S., H.T. Ngo, M. Guèze, J. Agard, A. Arneth, P. Balvanera, K.A. Brauman, S.H.M. Butchart, K.M.A. Chan, L.A. Garibaldi, K. Ichii, J. Liu, S.M. Subramanian, G.F. Midgley, P. Miloslavich, Z. Molnár, D. Obura, A. Pfaff, S. Polasky, A. Purvis, J. Razzaque, B. Reyers, R. Roy Chowdhury, Y.J. Shin, I.J. Visseren-Hamakers, K.J. Willis, C. N. Zayas (eds.). IPBES secretariat, Bonn, Germany. 56 p. 


\section{Chapitre 6}

\section{Suivi de la biodiversité, scénarios pour l'avenir, relations entre climat et biodiversité}

Ce chapitre nous montre qu'à l'aide des sciences dites participatives, le grand public et diverses associations en lien avec les scientifiques peuvent contribuer à l'observation de la biodiversité et de son évolution en apportant des données sur de grandes échelles spatiales et temporelles. En parallèle, les scientifiques développent des modèles quantitatifs ou qualitatifs de façon à prévoir les scénarios d'évolution possible de la biodiversité en réponse au réchauffement climatique. La confrontation des modèles de biodiversité aux modèles climatiques permet ainsi de pointer des solutions qui répondent à la fois à la préservation de la biodiversité et à l'atténuation du réchauffement climatique. À l'inverse, on découvrira que certaines mesures visant à réduire le réchauffement peuvent ne pas être bénéfiques à la biodiversité ou ne l'être que partiellement. Le chapitre montre aussi comment les arbres au sein des écosystèmes forestiers peuvent s'adapter au réchauffement climatique, même si les étés chauds et secs perturbent de façon notable leur fixation du carbone de l'atmosphère. De par leur rôle de puits de carbone, les forêts restent nos alliées pour atténuer le réchauffement climatique, et il nous faut donc les préserver ou même les développer.

\subsection{Les outils et dispositifs d'observation de la biodiversité: l'apport des sciences participatives - F. Chiron}

La biodiversité ne se résume pas qu'à une collection d'espèces (voir section 4.1). Son observation doit aussi rendre compte de l'abondance des populations, de leur diversité génétique et de la complexité des interactions entre espèces au sein de la biosphère à différentes échelles. 
L'importance des observations sur le «terrain » a été à l'origine des sciences dites «participatives», qui se définissent comme la collaboration vertueuse de scientifiques, de citoyens volontaires et de "parties prenantes», telles que des associations naturalistes ou des entreprises. Ce travail en commun permet de définir les questions scientifiques, les protocoles, qui seront appliqués à large échelle, puis d'analyser les résultats pour répondre aux questions posées et en soulever de nouvelles, sans oublier de financer toutes ces actions.

\subsubsection{Le nouvel essor des sciences participatives en écologie}

Les sciences participatives ne sont pas nouvelles. Les sociétés savantes du XVII au XIXe siècle ont énormément participé aux collectes d'échantillons à travers le monde, qui ont nourri les collections des Muséums d'Histoire naturelle. La relation entre les scientifiques et ces naturalistes amateurs s'est un peu distendue ensuite, même si, certains programmes impliquant des citoyens datent de plus 120 ans comme le «Christmas Bird Count» (Fig. 6.1).

Depuis deux décennies, on assiste à un renouveau des sciences participatives en écologie. Cela s'explique par le besoin de données sur de grandes échelles spatiales et temporelles, indispensables à l'analyse des changements globaux, car il nous faut comprendre et évaluer la réponse de la biodiversité au changement climatique, à l'urbanisation, aux politiques agricoles, etc. Ce renouveau dans les sciences est aussi dû au développement très rapide du numérique qui permet de massifier ces dispositifs. En France, les équipes de «Vigie-Nature» et de «65Mo», développées par le Muséum national d'Histoire naturelle, coordonnent un grand nombre de ces programmes. Enfin, il a été prouvé que les dispositifs participatifs garantissent des observations plus pérennes que ne le permettent les dispositifs professionnels financés sur fonds publics [1].

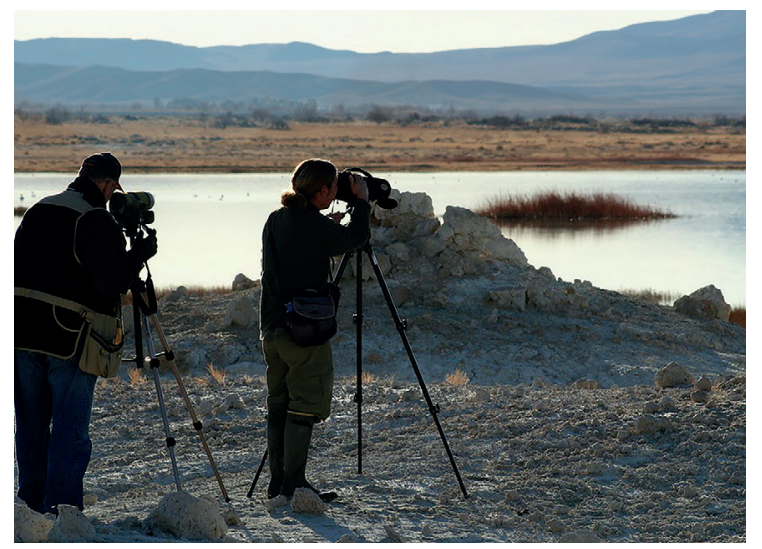

Figure 6.1: Le «Christmas bird count»: un suivi des oiseaux d'Amérique du Nord avec plus de 50000 participants (Source: USFWS Pacific Southwest Region, license CC BY 2.0, source : flickr). 
À qui s'adressent les sciences participatives? Autrefois plus orientées vers un public naturaliste, elles se sont ouvertes au grand public et aux acteurs de l'environnement. Par exemple, l'essor il y a 30 ans du Suivi Temporel des Oiseaux Communs en France s'est construit sur les réseaux naturalistes spécialistes des oiseaux. Aujourd'hui «Oiseaux des jardins», un programme de Vigie-Nature, repose entièrement sur les observations du grand public qui y trouve là une occasion d'apprendre simplement à reconnaître les oiseaux. Le programme "Florilèges prairies urbaines» est quant à lui dédié aux gestionnaires des espaces verts urbains qui souhaitent évaluer et faire évoluer leurs pratiques de gestion pour mieux conserver les plantes en ville. L'implication croissante du grand public leur vaut aussi le nom de sciences citoyennes.

\subsubsection{Des programmes et des processus collaboratifs entre scientifiques et citoyens}

On distingue, en fait, deux grands types de programmes. Les premiers sont des programmes qui ont une visée transformative de leur action sur les socioécosystèmes. Entrepris par toutes sortes d'acteurs comme des ONG, des collectivités locales, des fédérations d'agriculteurs etc., ils ont une action directe sur la société ou les écosystèmes mais n'attendent pas nécessairement une validation scientifique. Les seconds sont les observatoires de la biodiversité dont le but est de décrire à large échelle spatiale et sur le long terme son état. lls sont initiés par les scientifiques [2].

Ainsi, individus, espèces, interactions biotiques, cycles de vie, saisons sont décrits en France dans plus de 150 programmes dédiés à la biodiversité terrestre, aquatique et marine rassemblant plus de 70000 participants ${ }^{55}$. De prime abord très descriptifs, ces observatoires ont aussi une portée transformative essentielle. Les observations répétées de la nature par les participants, par exemple, un jardinier amateur qui participe à «l'Observatoire des Jardins» ou un agriculteur qui participe à «l'Observatoire Agricole de la Biodiversité», font évoluer leurs connaissances et changent leurs comportements avec des pratiques plus favorables.

Une des critiques faites aux sciences participatives, est de recruter des observateurs pas ou peu expérimentés dans le but de produire des résultats scientifiques valides. Avec du recul, on voit que la crainte de produire des données de mauvaise qualité est faible. Par exemple, les erreurs d'identification d'espèces dans l'Observatoire papillons des jardins ne sont que de $5 \%$, un taux tout à fait acceptable et comparable à celui de naturalistes confirmés. Cette fiabilité des données s'explique par l'apprentissage rapide des insectes par les participants, la validation des données par les «pairs», c'est-à-dire les experts dans le domaine, et par des protocoles simples et adaptés au plus grand nombre.

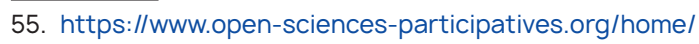


La force de ces programmes est la participation d'un grand nombre d'observateurs, qui est un objectif fondamental. La précision des analyses augmente avec le nombre de données, tandis que les incertitudes liées au hasard, et aux particularités des sites d'observation, s'estompent. Par la précision des analyses qu'ils autorisent, ces programmes jouent un rôle majeur dans les recherches en écologie fondamentale et dans les sciences de la conservation. Elles sont publiées dans des revues scientifiques internationales renommées, confirmant l'intérêt et la rigueur scientifique de ces recherches.

Mais l'intérêt des sciences participatives ne s'arrête pas là. Elles font évoluer la démarche scientifique elle-même. Pour bien comprendre cela, il est important de revenir sur l'approche classique en sciences que l'on appelle la méthode «hypothético-déductive». Celle-ci revient à poser une question - après une revue de la bibliographie existante -, établir un protocole, collecter les données, analyser et répondre à la question initiale.

Dans ce contexte, la participation des citoyens consisterait uniquement à collecter les données, sans intervenir sur les questions en amont ou la production de résultats en aval, réservées aux scientifiques. Or, les gros jeux de données, les fameux «big data», ne sont pas construits pour répondre à une question unique. Les questions émergent en fonction des données collectées qui elles-mêmes dépendent de ce que les observateurs ont rapporté. Même si ceux-ci suivent des protocoles très standardisés, il y a une part de liberté dans les lieux, les moments d'observations, les espèces, ce qui oriente le choix des questions et les résultats produits par les scientifiques.

De même, les observateurs peuvent faire part de questionnements à l'issue de leurs observations qui seront ensuite explorées par les scientifiques, voire résolues par les observateurs eux-mêmes ${ }^{56}$. Ce processus dynamique et collaboratif élargit considérablement la place des participants, et la portée des recherches en écologie. Il développe la culture scientifique et l'esprit critique des participants autant d'atouts de nos jours.

\subsubsection{Un exemple de programme de sciences participatives}

Les programmes sur la biodiversité sont nombreux mais peu sont ceux qui étudient la coexistence entre individus, c'est-à-dire leurs interactions. Birdlab (Fig. 6.2) en est un exemple très ludique. C'est un serious game qui permet d'apprendre très simplement à reconnaître les oiseaux tout en étudiant leur comportement de recherche alimentaire. Le dispositif est simple, vous reproduisez sur votre smartphone ou tablette les déplacements des oiseaux se posant sur deux mangeoires identiques que vous aurez placées au préalable sur votre balcon ou dans votre jardin. 


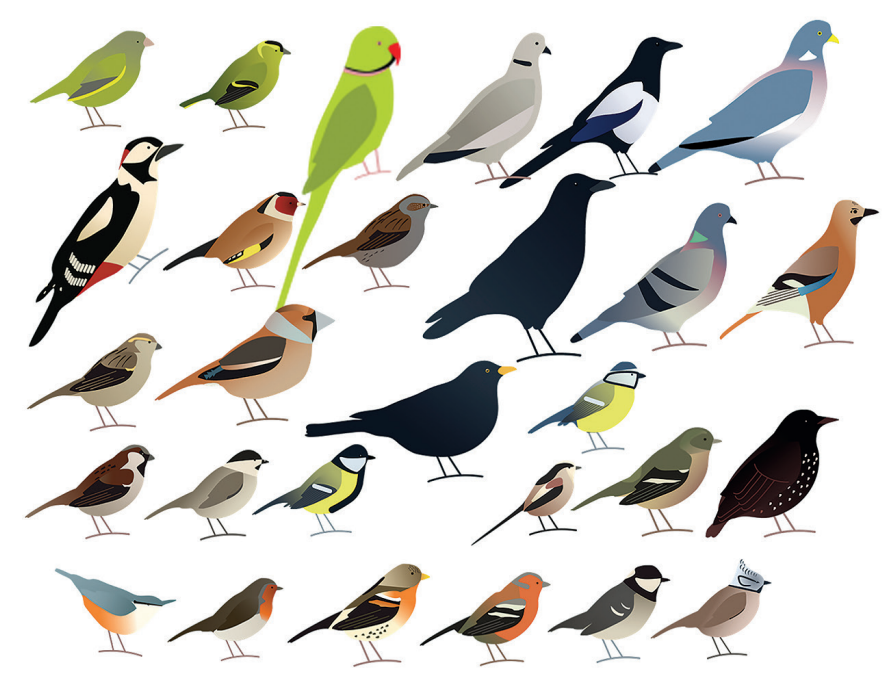

Figure 6.2: Les espèces d'oiseaux suivies dans le cadre du programme «BirdLab»; un programme de sciences participatives pour étudier le comportement des oiseaux aux mangeoires (Source: http:// www.vigienature.fr/fr/vigie-manip/birdlab).

Ce programme aide les chercheurs à comprendre combien d'espèces fréquentent les mangeoires, quelles espèces s'entraident ou s'évitent pour rechercher leur nourriture, dans quels contextes le nourrissage est-il favorable, etc. Les participants au programme peuvent ainsi apprendre relativement vite à reconnaître les oiseaux et leur comportement de façon ludique et à remporter des badges en fonction de leurs observations.

Pour les plus motivés, sur la base des outils et des démarches déjà existants (https://www.open-sciences-participatives.org/home/ et http://www.vigienature.fr/ fre), il est possible de proposer un nouveau projet de sciences participatives!

\subsection{Scénarios et modèles d'évolution de la biodiversité - P. Leadley}

\subsubsection{Contexte général des scénarios et modèles}

Les scénarios et les modèles de biodiversité sont utiles pour les scientifiques et les décideurs, ceci pour plusieurs raisons. En effet, ils permettent:

- de mieux comprendre et synthétiser un large éventail d'observations;

- d'alerter les décideurs sur les effets futurs indésirables des changements globaux tels que le changement d'affectation des sols, la surexploitation du vivant, le changement climatique, etc.; 
- de fournir une aide à la décision pour l'élaboration de stratégies de gestion adaptative;

- d'explorer les implications des voies alternatives de développement socio-écologique et des options de gestion;

- d'anticiper les changements afin d'être proactif plutôt que réactif.

Les scénarios décrivent différentes évolutions de la biodiversité et des services écosystémiques en réponse aux changements futurs des pressions sur les écosystèmes (cf. rapport 2016 de l'IPBES, Plateforme intergouvernementale sur la biodiversité et les services écosystémiques [3]). Dans ces scénarios, les causes indirectes de ces évolutions sont, par exemple, la croissance démographique humaine, l'utilisation de l'énergie ou la production agricole. Les pressions directes sur la biodiversité sont nombreuses, mais la grande majorité des scénarios traite des impacts du changement climatique et du changement d'utilisation des terres et de la pêche.

Des modèles mathématiques sont ensuite utilisés pour convertir ces pressions directes en impacts sur la biodiversité et les services écosystémiques. II existe une grande diversité dans le type et la complexité des modèles utilisés ( $c f$. rapport 2016 de I'IPBES). Pour la biodiversité, on modélise souvent la répartition spatiale des espèces, la richesse spécifique, c'est-à-dire le nombre d'espèces par unité de surface, ou l'abondance des espèces. Pour les services écosystémiques, on modélise, par exemple, le stockage du carbone ou la régulation des inondations par les écosystèmes. Les deux exemples ci-dessous illustrent la façon dont on peut modéliser les impacts associés au changement climatique et à l'utilisation des terres sur la biodiversité.

\subsubsection{Modèle de «niche» - Application au chêne sessile}

La relation entre l'abondance d'une espèce et les facteurs environnementaux reflète la "niche écologique» de l'espèce, c'est-à-dire l'ensemble des facteurs abiotiques qui sont nécessaires à la survie des individus ou des populations de cette espèce. Parmi les modèles de biodiversité, les modèles dits de niche, sont de loin les plus utilisés en termes de publications scientifiques. Ils combinent les données actuelles de répartition spatiale d'une espèce donnée avec la répartition spatiale du climat pour aboutir à un modèle statistique décrivant la façon dont l'espèce réagit à l'environnement.

Pour illustrer les modèles de niche et leur fonctionnement, nous choisissons ici une espèce d'arbre largement distribuée en Europe, le chêne sessile. Le modèle statistique utilisé permet de relier la probabilité de présence de l'espèce aux facteurs environnementaux en combinant la distribution spatiale de l'espèce avec plusieurs facteurs climatiques. Ce modèle prédit que le chêne sessile est mal adapté aux climats froids, ainsi qu'aux climats chauds et secs. II préfère les climats tempérés. 
Ceci était déjà connu qualitativement, mais le modèle statistique nous permet de quantifier cette relation. La capacité du modèle à décrire la distribution actuelle du chêne sessile est illustrée sur la figure 6.3a; la distribution obtenue (en fond gris) est en très bonne adéquation avec la répartition actuelle. On vérifie que le chêne sessile ne se trouve pas en Europe du Nord parce qu'il y fait trop froid, ni dans une grande partie de l'Europe du Sud parce qu'il y fait trop chaud et trop sec.

Mais ce qui est évidemment le plus intéressant, c'est que le modèle permet de prévoir les effets des changements climatiques futurs sur la répartition de l'espèce. Si on combine la relation statistique précédente entre la présence du chêne sessile et les facteurs environnementaux et qu'on utilise en même temps un modèle climatique qui prédit, par exemple, de fortes émissions de gaz à effet de serre, on obtient ainsi la distribution spatiale prédite pour l'espèce en 2080 (Fig. 6.3a). Le modèle prévoit ainsi que le chêne sessile disparaîtra d'une grande partie de son aire de répartition actuelle située au sud ou au centre de l'Europe (couleur rouge sur la Fig. 6.3b), car le climat y sera défavorable à cette espèce. En revanche, le chêne sessile pourrait coloniser de grandes parties de l'Europe du Nord (couleur vert foncé sur la Fig. 6.3b), car le climat lui deviendra favorable.
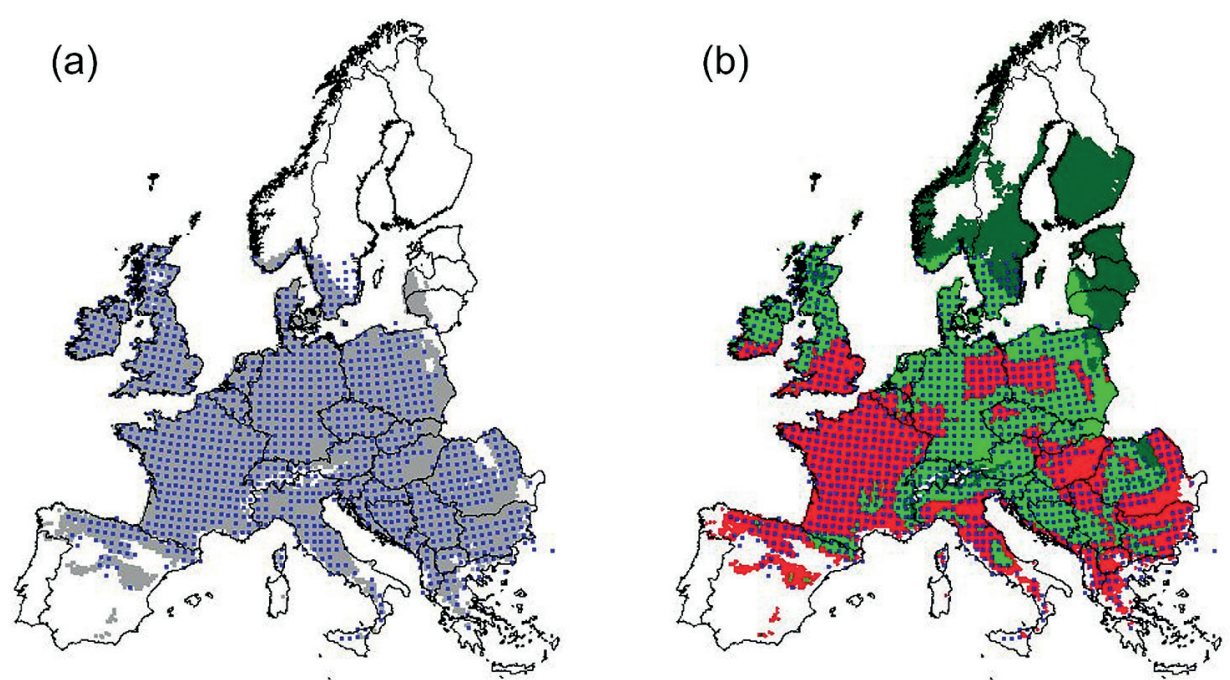

Figure 6.3: Répartition du chêne sessile. (a) : la répartition actuelle du chêne sessile en Europe (points bleus) et modélisation (gris); (b) : la répartition du chêne sessile prédite en 2080 (en vert) et sa disparition (en rouge) pour un scénario d'émissions élevées de gaz à effet de serre. Avec la courtoisie de W. Thuiller, voir [4].

Ces résultats ont d'énormes implications pour la biodiversité et le fonctionnement des forêts. Ils ont, en particulier, contribué à alerter les forestiers sur les graves dangers que le changement de climat fait peser sur les forêts. En effet, les forêts sont particulièrement vulnérables aux changements climatiques, car les arbres ont une très longue durée de vie. 
Grâce au développement de nouvelles bases de données massives, ces modèles peuvent être appliqués à un très grand nombre de plantes mais aussi à presque toutes les espèces connues d'oiseaux, de mammifères et de reptiles, vu les mesures relativement fiables de leurs répartitions spatiales dont on dispose. Ainsi, un modèle de niche du type précédent a été utilisé pour prédire les impacts du changement climatique sur 1500 plantes européennes. Le modèle prévoit une forte diminution du nombre d'espèces végétales par unité de surface (richesse spécifique) dans le sud de l'Europe avec, en contrepartie une augmentation potentiellement importante de la richesse de la végétation dans le nord de l'Europe. De nombreuses espèces vont, en effet, migrer vers le nord (Fig. 6.4).

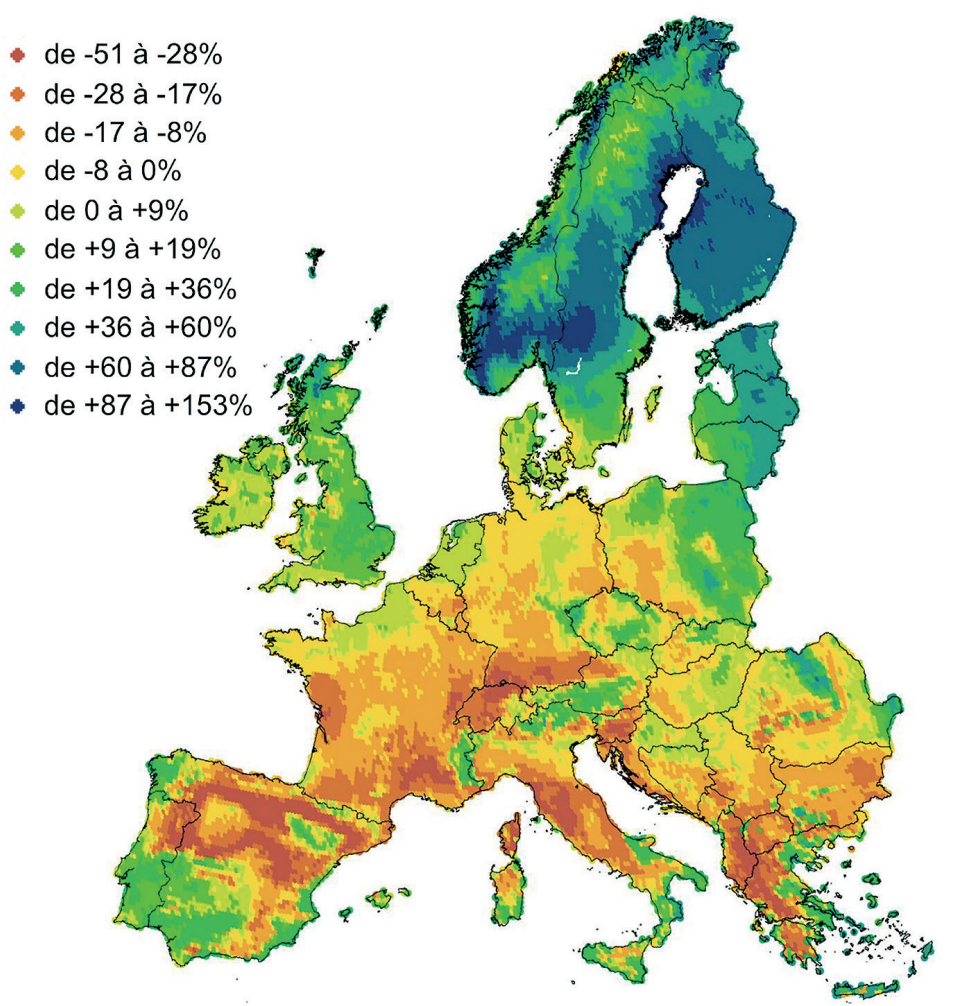

Figure 6.4: Impact du changement climatique sur la diversité de plus de 1500 espèces de plantes prédit en 2080. Avec la courtoisie de W. Thuiller, voir [4].

\subsubsection{Exploitation des grandes bases de données}

La disponibilité et l'analyse de grandes bases de données a permis d'autres percées majeures ces dernières années. Elle a notamment permis la modélisation des impacts dus aux changements d'utilisation des terres sur la biodiversité. On a ainsi pu exploiter une très grande base de données mondiales, qui compare les écosystèmes 
non perturbés avec des zones proches transformées par la sylviculture, l'agriculture ou l'urbanisation. Grâce aux modèles développés, on réalise que la transformation des habitats naturels (c'est-à-dire la «végétation primaire») en plantations d'arbres, en cultures, en pâturages ou en zones urbaines, a généralement des impacts très négatifs sur la biodiversité (Fig. 6.5).

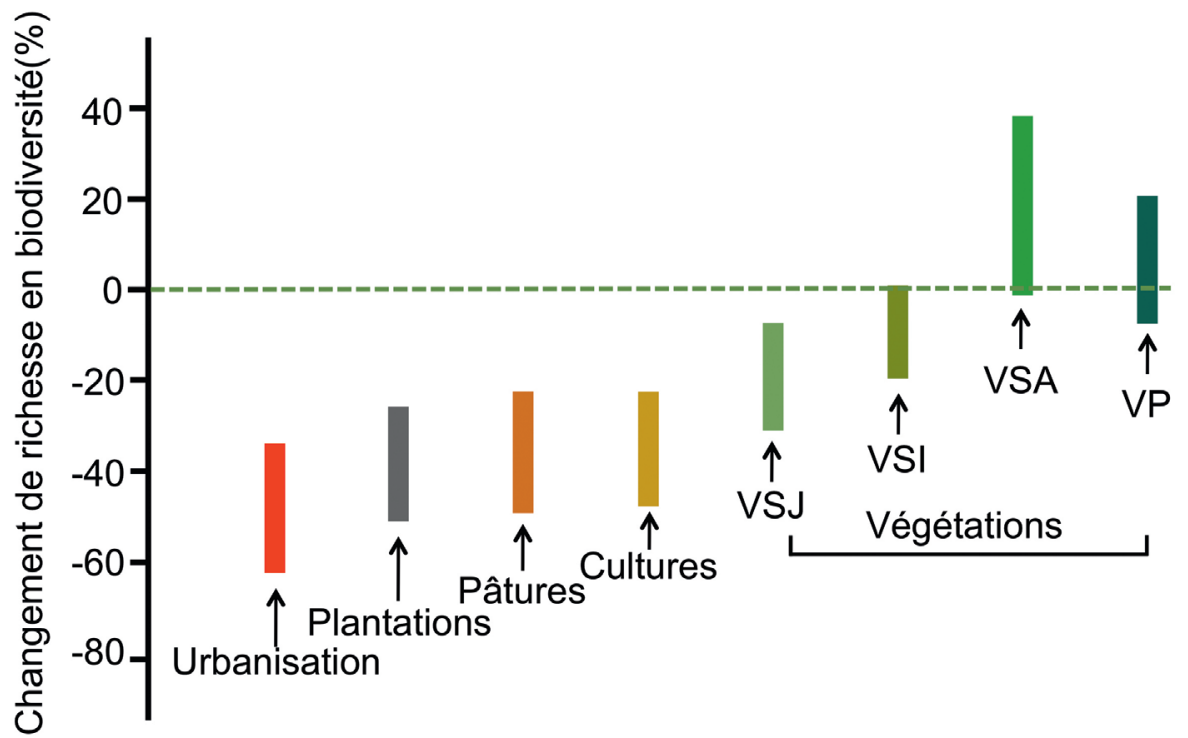

Figure 6.5: Évolution de la richesse en biodiversité, c'est-à-dire du nombre d'espèces par unité de surface, en fonction du type d'habitat et de l'intensité d'utilisation. La hauteur des barres de couleur correspond à la disparité des données selon les différentes régions du monde. VP = Végétation primaire; VSA = Végétation secondaire adulte; VSI = Végétation secondaire intermédiaire; VSJ = Végétation secondaire jeune. D'après les résultats de [5].

Ce type de modélisation exploitant des bases de données massives a été utilisé pour reconstruire les changements passés de la diversité des espèces et explorer les impacts potentiels dans quatre scénarios futurs développés par le GIEC/IPCC (Fig. 6.6). Cette analyse met en évidence deux résultats importants:

(1) les changements passés dans l'utilisation des terres ont entraîné de fortes réductions de la richesse spécifique, en particulier au cours des 100 dernières années (sur lesquelles nous disposons étonnamment de très peu d'observations à l'échelle mondiale);

(2) les changements futurs de la richesse des espèces pourraient être positifs ou négatifs, mais, étonnamment, le meilleur scénario d'atténuation du changement climatique (le scénario «RCP 2.6 », en vert) est mauvais pour la biodiversité. En effet, la bioénergie est utilisée en grande quantité dans ce scénario de façon à atténuer le changement climatique. Ceci entraîne, en retour, la perte d'habitats naturels pour faire de la place aux cultures de plantes et d'arbres exploités pour la bioénergie. 


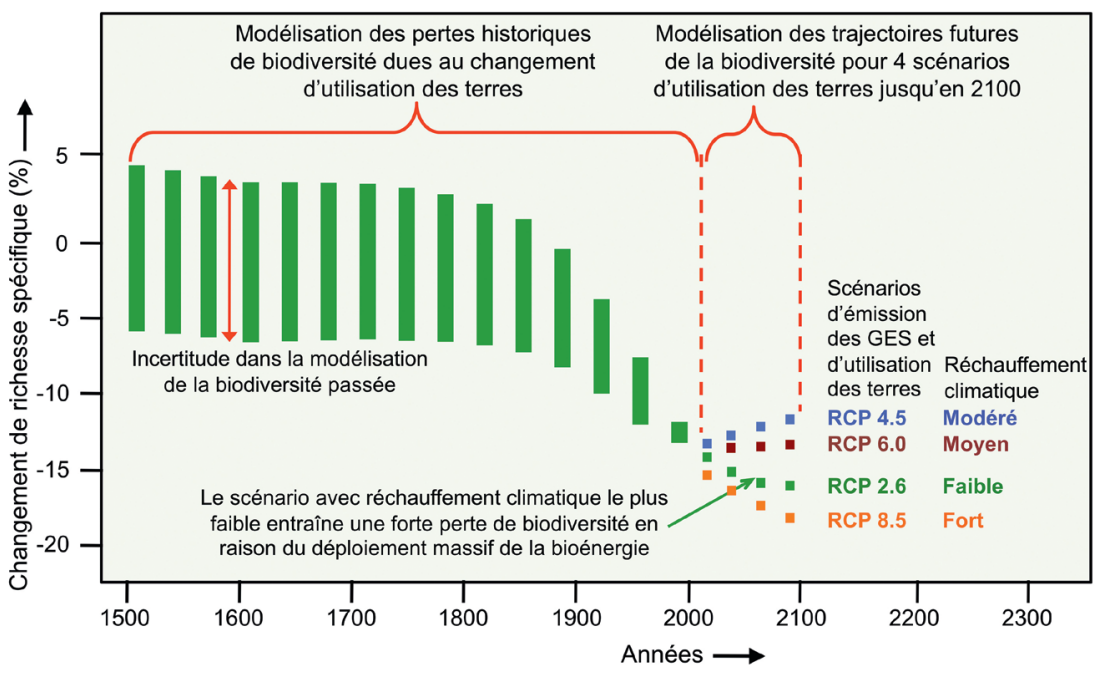

Figure 6.6: Modélisation des impacts de changement d'utilisation des terres sur la biodiversité. La partie du diagramme en bâtonnets verts correspond à la reconstruction des impacts passés en tenant compte des incertitudes de modélisation. Les courbes de couleur en pointillés (orange, vert, rouge et bleu) correspondent aux évolutions futures prédites de 2020 à 2100 à partir de différents scénarios de modélisation. D’après les résultats de [5].

\subsubsection{Conclusions}

Les développements récents en termes de modélisation de la biodiversité nous amènent aux principales conclusions suivantes:

- Les scénarios et modèles de biodiversité et de services écosystémiques sont des outils importants pour les scientifiques;

- D'énormes progrès ont été réalisés dans la modélisation de la biodiversité au cours de la dernière décennie, en partie grâce au développement de bases de données massives;

- Toutefois, l'utilisation de ces scénarios et modèles dans la prise de décision est encore trop limitée;

- II devrait être possible d'y remédier par une meilleure évaluation des incertitudes et une communication plus soutenue entre les scientifiques et les décideurs.

\subsection{Les synergies et antagonismes climat et biodiversité - P. Leadley}

L'atténuation du changement climatique et la protection de la biodiversité constituent deux des plus importants défis environnementaux du XXle siècle. Mais les efforts déployés pour atténuer le changement climatique seront-ils bénéfiques 
à la biodiversité? À l'inverse, la protection de la biodiversité peut-elle contribuer à réduire le réchauffement climatique?

Pour répondre à ces questions, il est utile de considérer les quatre liens importants, qui ont été mis en évidence entre biodiversité et changement climatique, aussi bien dans les évaluations du GIEC (Groupe d'experts intergouvernemental sur l'évolution du climat - voir section 9.5) que dans celles de l'IPBES (Plateforme intergouvernementale sur la biodiversité et les services écosystémiques) :

1. les impacts du changement climatique sur la biodiversité;

2. l'impact des mesures d'adaptation et d'atténuation du réchauffement climatique sur la biodiversité;

3. les solutions fondées sur la nature;

4. les actions sur les causes communes du changement climatique et de la perte de biodiversité.

\subsubsection{Les impacts du changement climatique sur la biodiversité}

La figure 6.7 produite par le GIEC présente, de façon illustrée, l'évaluation de l'impact du réchauffement climatique sur divers aspects de la biodiversité. Certains écosystèmes comme les récifs coralliens tropicaux et la toundra arctique sont déjà fortement touchés et le seront encore plus gravement dans le cas d'un réchauffement global à $2,0^{\circ} \mathrm{C}$ et plus.

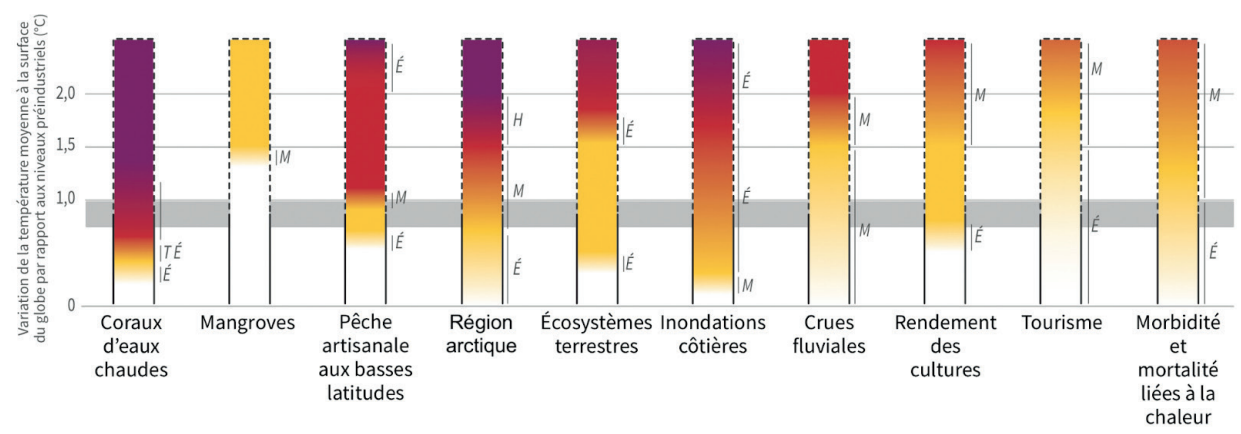

Degré de confiance pour la transition : $F=$ faible, $M=$ moyen, $E$ = élevé, $T E ́$ = très élevé

Figure 6.7: Incidences et risques du réchauffement climatique pour divers systèmes parmi lesquels des écosystèmes naturels. Les couleurs indiquent si les impacts seront a priori faibles (couleur blanche), modérés (jaune) ou élevés (graduellement du rouge au violet). Le niveau de réchauffement considéré va croissant de $0^{\circ} \mathrm{C}$ (niveau préindustriel) à $1^{\circ} \mathrm{C}$ (niveau atteint au cours du siècle dernier), puis $1,5-2,0^{\circ} \mathrm{C}$ (objectifs de l'accord de Paris de 2015) jusqu'à $2,5^{\circ} \mathrm{C}$, qui est la limite inférieure des projections actuelles de réchauffement climatique pour la fin du siècle. Figure extraite du rapport IPCC 2019 pour les décideurs ${ }^{57}$. 


\subsubsection{Impact des mesures d'adaptation et d'atténuation du réchauffement climatique sur la biodiversité}

Deux mesures clés sont proposées pour atténuer le réchauffement climatique par la gestion des écosystèmes terrestres:

- La première mesure consiste à planter des arbres en grande quantité, c'està-dire jusqu'à plusieurs milliards d'arbres. Les arbres capturent, en effet, le dioxyde de carbone de l'air et le stockent dans leurs branches, leurs troncs et leurs racines, ce qui est favorable à l'atténuation du climat. Mais trop souvent, en pratique, des peuplements monospécifiques d'espèces exotiques sont plantés sur de grandes étendues. Dans d'autres cas, les plantations sont faites dans des zones qui n'étaient pas couvertes de forêts depuis longtemps. Dans ces deux cas, les effets sur la biodiversité peuvent être dévastateurs. La plantation d'arbres est donc une bonne idée à condition qu'elle se concentre sur le reboisement à partir d'espèces d'arbres indigènes.

- La deuxième mesure proposée consiste à remplacer les combustibles fossiles par la biomasse à grande échelle, ce qu'on appelle la bioénergie. La transformation de la biomasse végétale en combustibles liquides ou sa combustion directe à des fins énergétiques peut réduire considérablement la teneur en gaz à effet de serre dans l'atmosphère, car les plantations ainsi créées captureront en retour le dioxyde de carbone émis par combustion. Cependant, la production d'une grande quantité de bioénergie nécessite de grands espaces pour les plantations, et induit donc une pression énorme sur les écosystèmes naturels, les cultures agricoles et la production alimentaire. La figure 6.8 représente le pourcentage de la biodiversité terrestre qui devrait être touchée par le changement climatique et diverses utilisations des terres en considérant deux scénarios: un scénario avec bioénergie et un scénario sans bioénergie. On réalise ainsi que la superficie des terres utilisées pour la bioénergie peut représenter une menace considérable pour la biodiversité.

\subsubsection{Solutions fondées sur la nature}

La protection et la restauration de la biodiversité peuvent contribuer à l'atténuation et à l'adaptation au changement climatique dans le cadre de ce qu'on appelle les «solutions fondées sur la nature».

Un premier exemple est l'importance de la protection des écosystèmes naturels et de la restauration des écosystèmes dégradés. En effet, les écosystèmes naturels intacts stockent une grande quantité de carbone, mais celle-ci est libérée dans l'atmosphère lorsqu'ils sont détruits. Les gouvernements ont déjà fixé des objectifs majeurs de protection et de restauration dans plusieurs traités internationaux. 


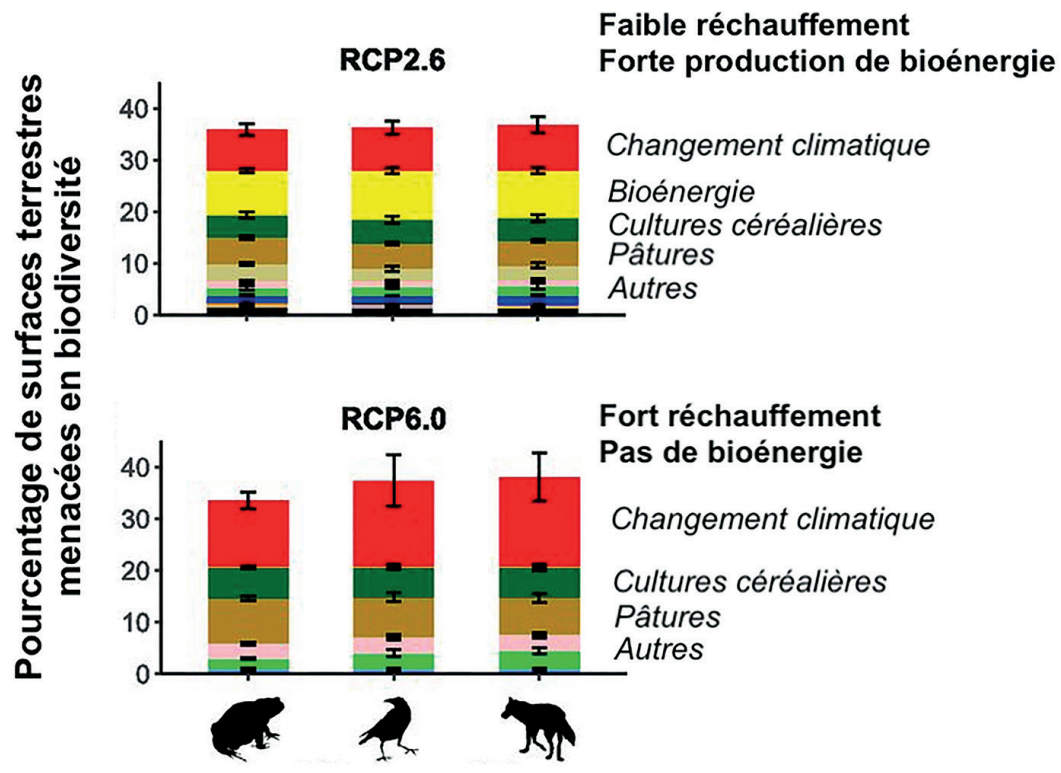

Figure 6.8: Pourcentage de surfaces terrestres qui risquent d'être perdues pour la biodiversité des amphibiens, des oiseaux et des mammifères selon deux scénarios. Dans la figure du bas (scénario sans production de bioénergie), l'impact dominant sur la biodiversité est le changement climatique, car l'utilisation des énergies fossiles conduit à des émissions élevées de gaz à effet de serre et à un fort réchauffement de la planète. Dans la figure du haut (scénario avec production massive de bioénergie), l'impact associé à l'utilisation de grands espaces pour les plantations "énergétiques » est de même ampleur que l'impact dû au changement climatique. Source : [6] CC BY-NC-ND 4.0.

Si les gouvernements respectent effectivement ces engagements, cela permettra d'éviter d'importantes émissions de carbone résultant de la conversion des écosystèmes et de stocker le carbone dans les écosystèmes restaurés.

Un deuxième exemple concerne l'importance de la protection de la diversité génétique des plantes cultivées et des espèces sauvages apparentées aux plantes cultivées. Certains cultivars et certaines espèces sauvages apparentées aux plantes cultivées sont mieux adaptés aux climats chauds et secs que les cultures actuellement utilisées en agriculture (voir plus loin section 10.7.2). Le maintien de cette diversité génétique est donc essentiel pour permettre à l'agriculture de s'adapter rapidement au changement climatique.

\subsubsection{Actions sur les causes communes du changement climatique et de la perte de biodiversité}

II existe des mesures que nous pouvons prendre pour réduire simultanément les pressions sur le climat et la biodiversité. Considérons, dans ce qui suit, trois exemples qui concernent l'agriculture et l'alimentation et qui seront développés plus loin dans la section 11.2. 
Les pratiques agricoles ou agroécologiques durables peuvent contribuer de manière significative à la fois au climat et à la biodiversité. La replantation de haies délimitant les parcelles cultivées et s'opposant, de fait, aux cultures intensives permet simultanément de stocker du carbone et d'améliorer la biodiversité. La réduction de l'utilisation des pesticides et l'utilisation d'engrais organiques peuvent également contribuer à redonner vie aux sols agricoles et à augmenter la quantité de carbone qu'ils stockent.

Les régimes alimentaires qui comprennent beaucoup de viande de ruminants comme les bovins et les ovins, nécessitent beaucoup de terre, d'eau et d'engrais, et conduisent à une forte émission de gaz à effet de serre par rapport aux régimes alimentaires qui comprennent principalement des plantes ou d'autres types de viande comme la volaille. Par conséquent, changer notre alimentation peut également contribuer, de manière significative, à la protection de la biodiversité et à l'atténuation du changement climatique. En outre, cela peut contribuer à améliorer notre santé.

De plus, environ un tiers de la nourriture produite est soit gaspillée, soit avariée. Ainsi, la réduction des pertes alimentaires contribuerait à diminuer la quantité de production agricole et donc à réduire la pression sur le climat et sur la biodiversité.

\subsubsection{Conclusion}

Pour chacune des actions envisagées, il est difficile de déterminer leurs contributions relatives à l'atténuation du changement climatique et à la protection de la biodiversité. Cela étant, le rapport conjoint GIEC et IPBES, paru en juin 2021, résume ainsi les synergies et les compromis entre ces différentes actions:

- La réduction importante de l'utilisation des combustibles fossiles, tels que le charbon, le pétrole et le gaz est absolument essentielle pour atténuer le changement climatique;

- Le reboisement à grande échelle peut apporter une contribution majeure au climat, mais doit être déployé avec précaution;

- La bioénergie ne peut apporter qu'une contribution modeste à l'atténuation du changement climatique, sinon il y a un risque élevé d'effets négatifs sur la biodiversité et la sécurité alimentaire;

- Les changements de régime alimentaire peuvent apporter des solutions majeures au climat et à la biodiversité et présentent peu d'inconvénients, mais sont plus efficaces lorsqu'ils sont associés à une réduction du gaspillage alimentaire. 


\subsection{Comment les arbres répondent aux changements saisonniers ? - N. Delpierre}

D’après le MOOC UVED : http://www.barbeau.universite-paris-saclay.fr/press.html

\subsubsection{Les effets du réchauffement climatique sur la feuillaison des arbres}

Les arbres sont des organismes immobiles. Ils vivent plusieurs dizaines à plusieurs centaines d'années, et donc tout au long de leur vie, ils sont soumis à des variations environnementales fortes. Un exemple très clair est celui de l'alternance des saisons. Sur la figure 6.9, on voit bien les variations saisonnières de température que les arbres subissent. En climat tropical, en général, cela ne pose pas de problème parce que les températures restent élevées toute l'année. Par contre, dans les zones climatiques plus froides, cela peut être problématique. Le risque principal est l'exposition des feuilles au gel. En effet, les jeunes feuilles, lorsqu'elles sont exposées à des températures en dessous de zéro, même modérément de l'ordre de -2 à $-3^{\circ} \mathrm{C}$, deviennent nécrosées et doivent être remplacées par l'arbre, car il doit créer une nouvelle cohorte de feuilles au printemps pour pouvoir faire la photosynthèse.

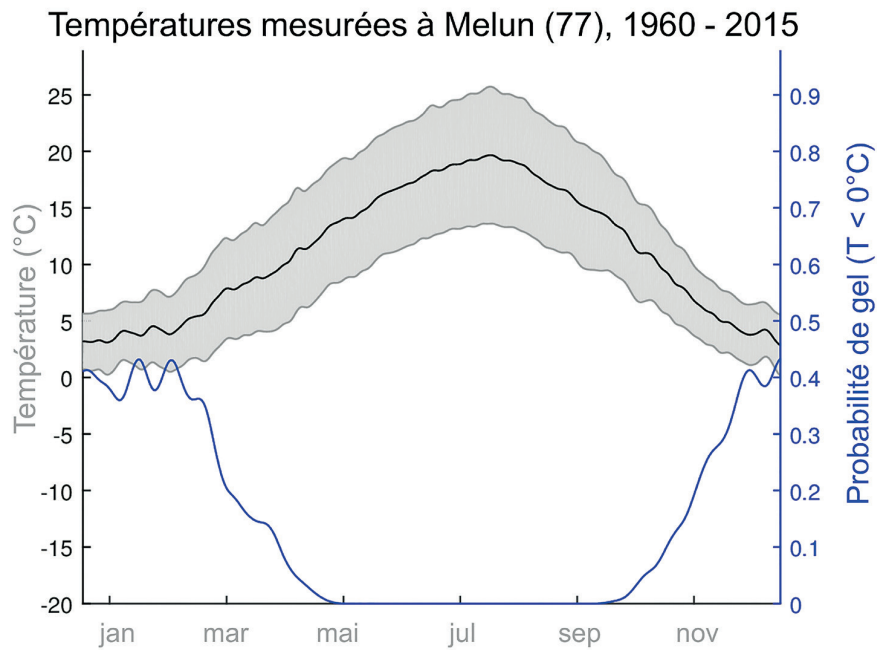

Figure 6.9: Saisonnalité des températures (trait noir) et des probabilités de gel (trait bleu) au cours de l'année sur la station de Melun-Villaroche. Données moyennées sur la période 1960-2015. La zone grisée indique l'amplitude moyenne (températures maximales et minimales) autour des moyennes journalières. Données fournies par MétéoFrance.

C'est un phénomène un peu symétrique qu'on observe à l'automne. L'arbre perd alors ses feuilles qui sont encore riches en nutriments. Chez la plupart des espèces, on observe généralement un jaunissement ou un rougissement des feuilles bien avant l'arrivée des premiers gels. La perte de couleur verte illustre la dégradation 
des chlorophylles. Les chlorophylles sont des pigments qui sont riches en azote, et durant la phase de jaunissement, les chlorophylles se dégradent et l'azote se déplace depuis les feuilles vers les branches. Cela permet à l'arbre de réutiliser l'azote au printemps suivant pour la formation de nouvelles feuilles.

Dans ce contexte, on pourrait penser qu'il est avantageux pour les arbres de concentrer leur saison feuillée durant l'été, parce que la probabilité de gel y est évidemment la plus faible. Cependant, la durée de la phase feuillée détermine la capacité de l'arbre à acquérir des ressources durant la photosynthèse. II en résulte que sa phase feuillée doit commencer assez tôt et finir assez tard pour permettre à l'arbre de pouvoir bénéficier de ressources et se développer. D'autre part, la durée de sa phase feuillée lui permet d'échapper au gel au printemps et à l'automne. Au cours de l'évolution, les arbres se sont adaptés à la saisonnalité des températures et paraissent même suivre, d'une année à l'autre, les variations de température [7]. Ce phénomène est lié à leur plasticité phénotypique, c'est-à-dire le fait que le génotype d'un individu présente des phénotypes différents selon les conditions environnementales. Les variations de phénotype dans ce cas précis sont les variations de dates d'apparition et de chute des feuilles et les variations de conditions environnementales sont les variations de température.
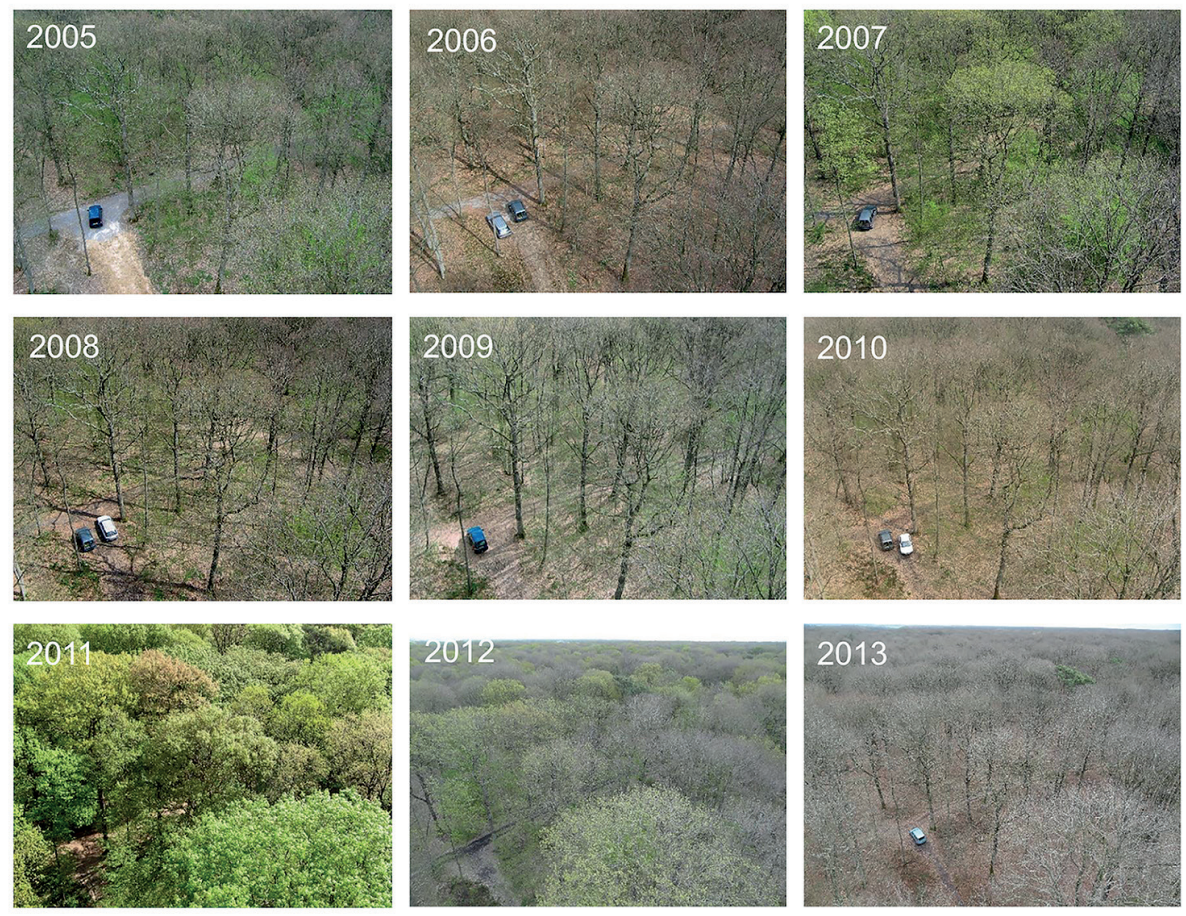

Figure 6.10: Date d'apparition des premières feuilles selon les années. Les printemps les plus chauds, par exemple 2007 et 2011, sont aussi ceux pour lesquels les dates d'apparition des feuilles sont les plus précoces. Source : Jean-Yves Pontailler et Daniel Berveiller, CNRS. 
À partir des photos de la figure 6.10, qui ont toutes été prises à la même date du 12 avril pendant des années différentes, on constate que les printemps les plus chauds, par exemple 2007 et 2011, sont aussi ceux qui présentent les dates d'apparition des feuilles les plus précoces. De façon symétrique, les automnes chauds sont associés à des chutes de feuilles plus tardives. Cette capacité qu'ont les arbres à «suivre» les températures, de par leur plasticité phénotypique, se retrouve également sur des séries de données beaucoup plus longues.

\subsubsection{Adaptation des arbres au réchauffement climatique?}

Dans le cas du Chêne pédonculé et du Hêtre, on observe des apparitions de feuilles systématiquement plus précoces au printemps sur la période récente 1980-2010, par comparaison à la période précédente 1950-1980 (Fig. 6.11). On voit le phénomène symétrique à l'automne; les feuilles chutent de plus en plus tardivement, même si le signal est un peu moins prononcé qu'au printemps. Il s'agit bien de manifestations du réchauffement climatique.

Par ailleurs, les arbres répondent de moins en moins fortement à l'augmentation des températures au printemps [8]. Ainsi, dans les années 1980, un réchauffement atmosphérique de $1^{\circ} \mathrm{C}$ entraînait une avancée de la date d'apparition des feuilles de 4 jours. Au milieu des années 2000, l'avancée n'était plus que de 2 jours et demi par degré de réchauffement. Ce même résultat marquant est observé sur de nombreuses espèces d'arbres.

La raison de ce phénomène n'est pas encore absolument claire, mais il est très probable que cette perte progressive de sensibilité des arbres aux températures élevées soit liée à un défaut d'exposition au froid, même si cela peut sembler paradoxal. En effet, les bourgeons d'où vont émerger les nouvelles feuilles au printemps, sont dans un état de dormance durant l'hiver. Cette dormance les empêche de se développer au moindre épisode plus chaud durant l'hiver. La dormance est un mécanisme subtil qui se réduit progressivement avec l'exposition des bourgeons au froid. Ainsi, on sait que les bourgeons, dont la dormance n'a pas été réduite par l'exposition au froid, vont être moins sensibles à l'influence des températures printanières élevées. En conséquence, ces bourgeons vont se développer plus lentement, ce qui conduira à une date d'apparition des feuilles qui sera plus tardive.

Avoir ses feuilles tôt pour un arbre n'est pas forcément un avantage. Considérons une année fraîche et une année chaude. Lors des années avec un printemps plutôt frais, la date d'apparition des feuilles est tardive et le début de photosynthèse est donc tardif également. Lors des années où le printemps est plus chaud, la date d'apparition des feuilles est avancée, la photosynthèse commence plus tôt et l'arbre acquiert donc plus rapidement des ressources. Mais la situation change durant la période d'été. En effet, la photosynthèse qui est un gain de carbone par l'arbre, donc un gain de ressources, se traduit aussi par une perte d'eau. 


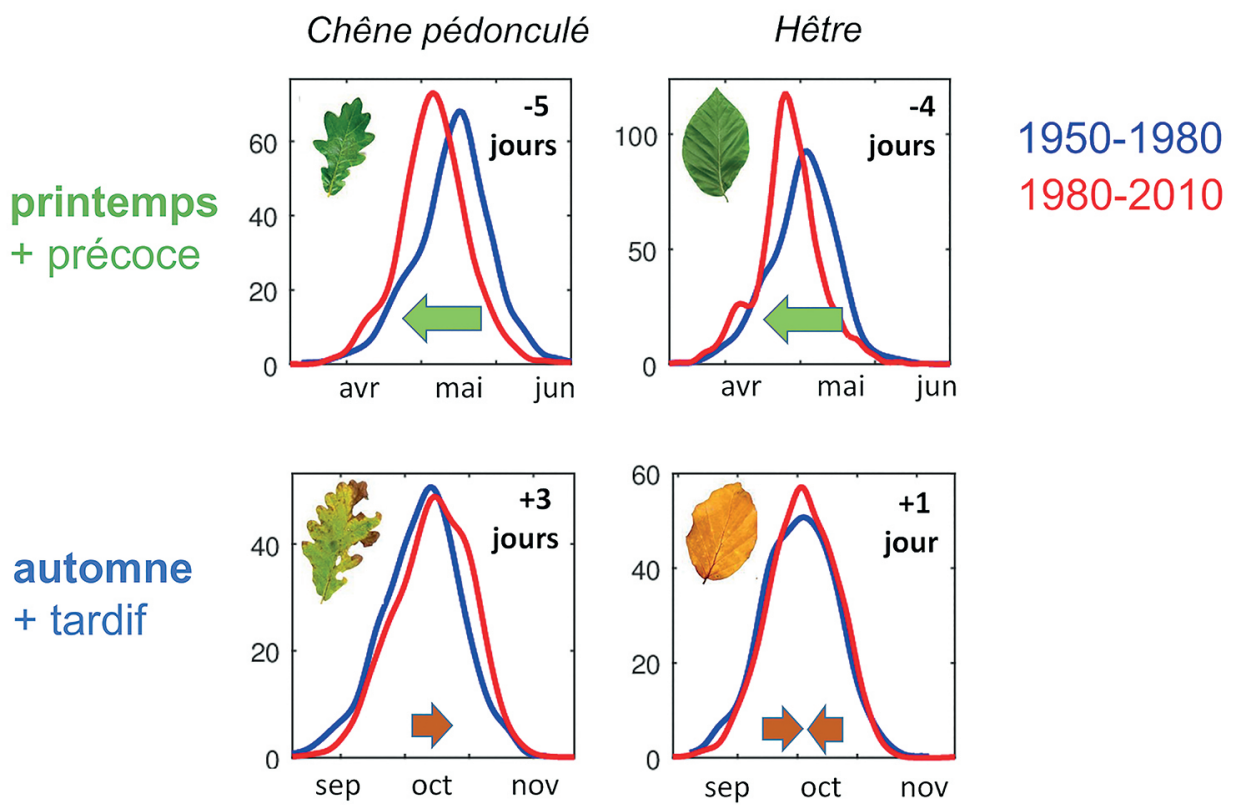

Figure 6.11: Le changement climatique a des conséquences sur l'apparition des feuilles au printemps dans le cas du chêne pédonculé et du hêtre (Données: www.pep725.eu).

Ce dernier processus s'appelle le phénomène de transpiration des plantes. Si les feuilles de l'arbre arrivent plus tôt, l'arbre assèche le sol et cela induit un stress hydrique durant l'été, qui décroît la photosynthèse. Ce comportement - apparition des feuilles plus tôt - stress hydrique durant l'été - est loin d'être systématique, mais il survient certaines années. II témoigne du fait que l'avancée de la date de feuillaison n'est pas forcément un avantage pour les arbres.

La variabilité d'une année à l'autre de la date d'apparition et de la date de chute des feuilles illustre la variabilité de la phénologie des arbres dans la durée, mais il existe aussi, pour une période donnée, une variabilité de la phénologie entre les individus d'une même population [9, 10]. Celle-ci atteint généralement trois semaines. Autrement dit, certains arbres d'une même espèce, dans une même forêt, peuvent mettre en place leurs feuilles trois semaines après les autres. Si cette arrivée tardive des feuilles avait été un désavantage, les arbres en question auraient été éliminés par sélection naturelle. Or, il n'en n'est rien, et ce peut même être un avantage lorsqu'il s'agit d'éviter un gel printanier, ou d'échapper à des chenilles prédatrices ou à certains phytopathogènes.

Pour suivre les dates d'apparition et de chute des feuilles, ce qu'on appelle la phénologie, mais aussi les dates de floraison, il existe un programme de sciences participatives, intitulé Observatoire des saisons qui est coordonné par des scientifiques et s'appuie sur des observations qui sont faites par des citoyens. 


\section{5. «Mesurer le souffle» des écosystèmes - N. Delpierre}

Les questions relatives au changement climatique et à la dégradation des écosystèmes sont intimement liées. Pour l'illustrer, intéressons-nous au bilan carbone d'un écosystème forestier, c'est-à-dire la différence entre la quantité de gaz carbonique qu'il absorbe et la quantité qu'il rejette, ce que l'on détecte en mesurant le «souffle» de l'écosystème.

\subsubsection{Bilan carbone des forêts}

Comme l'ont déjà souligné les précédents chapitres de cet ouvrage, les émissions de gaz carbonique ou dioxyde de carbone (le $\mathrm{CO}_{2}$ ) produites par les activités humaines sont, pour une grande part, responsables du réchauffement actuel de notre planète. On estime que le $\mathrm{CO}_{2}$ représente aujourd'hui $26 \%$ de l'effet de serre global (Section 7.2.1 et Fig. 7.5) et que sa concentration dans l'atmosphère a augmenté de plus de $40 \%$ depuis le XIX siècle et le début de l'ère industrielle (Section 7.2.1 et Fig. 7.6). Si cette augmentation est principalement due à l'utilisation des combustibles fossiles tel le charbon, le pétrole et le gaz, la déforestation, principalement pour création de zones de pâturage, y participe également. Toutefois, seuls $45 \%$ du $\mathrm{CO}_{2}$ que nous émettons se retrouve dans l'atmosphère, car les océans et les écosystèmes continentaux en absorbent plus de la moitié [11]. En absorbant le $\mathrm{CO}_{2}$, les écosystèmes, en particulier les forêts, atténuent le réchauffement climatique en cours, qui serait donc encore bien plus important si les écosystèmes ne jouaient pas ce rôle essentiel.

Comprendre comment l'absorption du $\mathrm{CO}_{2}$ par les écosystèmes varie en fonction du temps nous permet de mieux prédire comment cette absorption peut être modifiée par les changements globaux en cours.

Le bilan carbone d'une forêt résulte des processus de photosynthèse et de respiration, qui permettent aux arbres et aux organismes "décomposeurs» (insectes saprophages, bactéries et champignons) de se développer (Fig. 6.12).

Le processus de photosynthèse assure la formation de sucres dans les feuilles des arbres à partir de lumière, d'eau et du $\mathrm{CO}_{2}$ qui est présent dans l'air. II contribue ainsi à l'absorption du $\mathrm{CO}_{2}$ par l'écosystème forestier. Cependant, comme la plupart des êtres vivants, les arbres en forêt produisent aussi du $\mathrm{CO}_{2}$ qui part vers l'atmosphère, car ils respirent par tous leurs organes, les feuilles, les branches, le tronc et les racines. Les décomposeurs, bactéries et champignons, qui se nourrissent des débris de feuilles et de bois produisent également du $\mathrm{CO}_{2}$ qui part dans l'atmosphère. On appelle productivité nette de l'écosystème, cette différence entre photosynthèse et respiration qui peut être estimée en comparant l'absorption par photosynthèse et l'émission par respiration. 


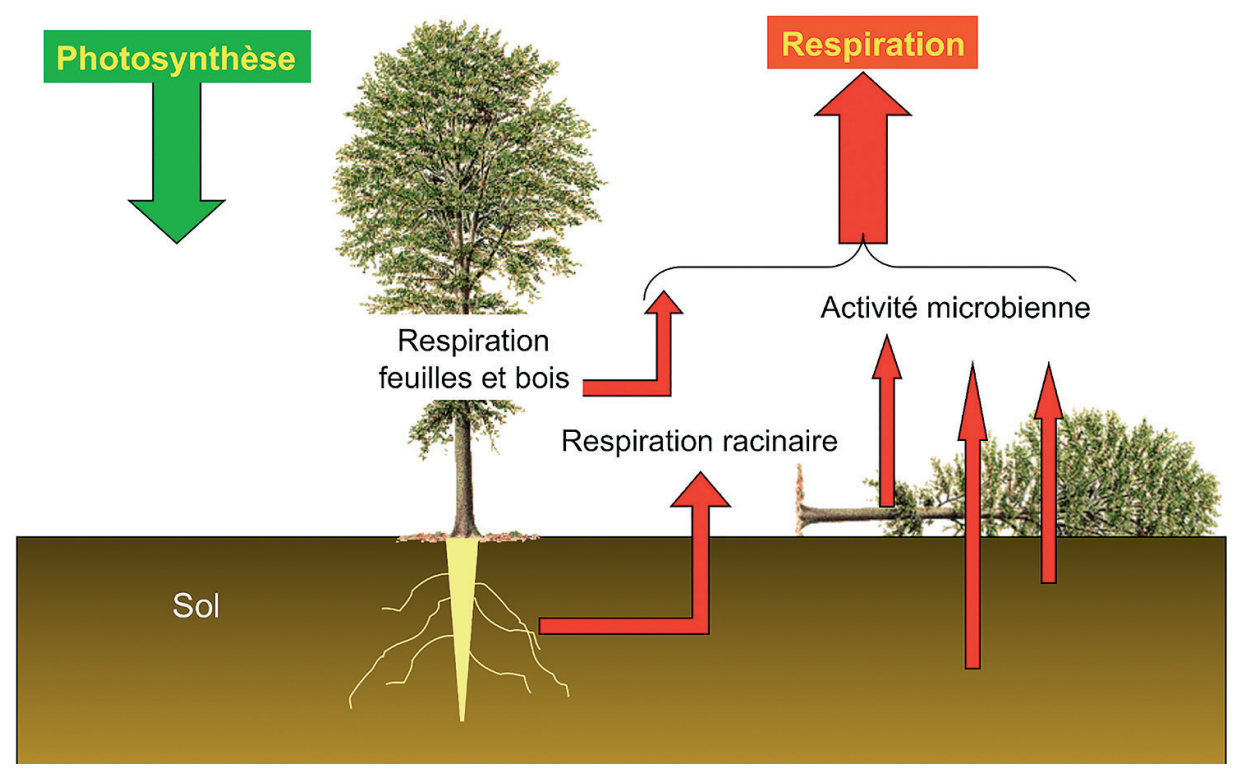

Figure 6.12: Schéma du bilan carbone d'une forêt. Source : Nicolas Delpierre.

Le «souffle» de l'écosystème est donc, en fait, cette alternance entre absorption de $\mathrm{CO}_{2}$ par photosynthèse et émission de $\mathrm{CO}_{2}$ par respiration.

Lorsque la photosynthèse est plus intense que la respiration, la quantité de carbone dans l'écosystème augmente et celle dans l'atmosphère diminue. Dans ce cas, la productivité nette est positive. On dit que l'écosystème est un «puits» de carbone, car il absorbe plus de carbone qu'il n'en émet. Dans le cas inverse, lorsque la respiration est plus forte que la photosynthèse, on dit que l'écosystème est une source de carbone pour l'atmosphère. II «fournit» du carbone à l'atmosphère. La productivité nette est négative.

\subsubsection{Mesure du «souffle» d'un écosystème forestier}

En pratique, pour mesurer le «souffle» d'un écosystème, forêt, prairie ou autres, on utilise un système de mesure ad hoc placé au sommet d'une tour, qu'on appelle «tour à flux» et qui domine en hauteur l'écosystème à étudier (Fig. 6.13). Parmi les nombreux instruments utilisés figurent, en particulier, un anémomètre qui mesure la vitesse du vent et un analyseur de gaz à infrarouges, qui mesure la concentration de $\mathrm{CO}_{2}$ dans l'air ambiant. On mesure donc simultanément le déplacement de l'air (le vent) et le $\mathrm{CO}_{2}$. Si les arbres absorbent du $\mathrm{CO}_{2}$ par photosynthèse, l'air s'appauvrit en $\mathrm{CO}_{2}$ lorsqu'il monte. Si les arbres émettent du $\mathrm{CO}_{2}$ par respiration, l'air s'enrichit au contraire en $\mathrm{CO}_{2}$ lorsqu'il monte dans l'atmosphère. 

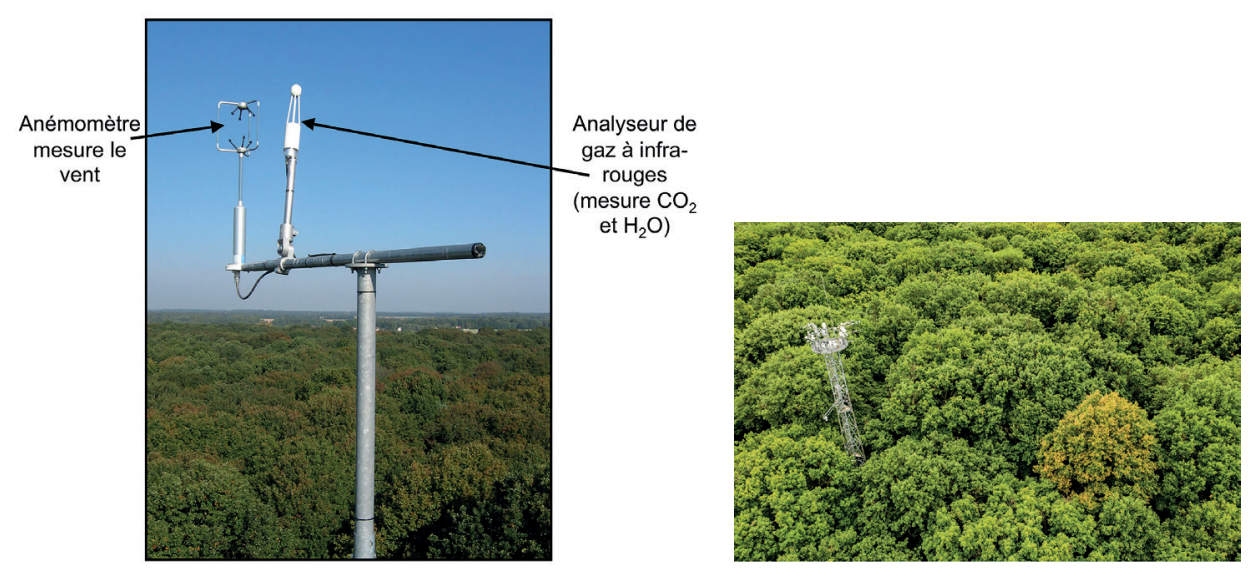

Figure 6.13: Appareils de mesures, anémomètre et analyseur de gaz à infra-rouges, placés au sommet de la tour à flux en forêt de Barbeau, tout près de Fontainebleau. La tour à flux domine la couronne des chênes sessiles qui atteignent une trentaine de mètres de hauteur. La description complète de cette plateforme expérimentale de recherche est accessible sur le site: http://www.barbeau.universiteparis-saclay.fr/index-fr.html. Source : Jean-Yves Pontailler et Ivan Cornut.

Le dispositif de mesures, comme celui déployé en forêt de Barbeau (Fig. 6.13), est répliqué à l'identique sur plus de 700 sites dans le monde [12]. Est ainsi constitué un réseau de mesures qui permet aux scientifiques du monde entier d'enregistrer les "souffles» de différents écosystèmes et de suivre leurs évolutions avec les variations du climat. Grâce à ces mesures, on constate bien que durant le jour, les forêts sont des puits de carbone grâce à la photosynthèse provoquée par le rayonnement solaire, alors que durant la nuit, les forêts sont des sources de carbone, du fait de la respiration des arbres et des organismes qui la composent (rappelons que la respiration a lieu aussi bien le jour que la nuit).

On observe également des variations du souffle au fil des saisons. Sur les forêts à feuillage caduc, la photosynthèse commence au printemps avec la mise en place des feuilles (Fig. 6.14). Ce n'est qu'à partir de ce moment que la forêt devient un puits de carbone et qu'elle absorbe le $\mathrm{CO}_{2}$ de l'air. Durant la saison froide, au contraire, la forêt est une source de carbone : elle dégage par sa respiration du $\mathrm{CO}_{2}$ vers l'atmosphère. Finalement, lorsqu'on cumule les alternances jour/nuit et les saisons, on voit que la forêt est un puits de carbone.

Sur une année entière, un mètre carré de forêt fixe environ 300 grammes de carbone (gC) [13]. Cela correspond à la quantité de carbone émise par une petite voiture pour un trajet de $9 \mathrm{~km}$. Si on étend ces calculs à l'ensemble de la forêt de Fontainebleau, qui fait environ 25000 ha, on peut estimer que l'absorption de gaz carbonique par la forêt ne compense en moyenne que $4 \%$ des émissions de $\mathrm{CO}_{2}$ produites lors des trajets routiers en Île-de-France. 


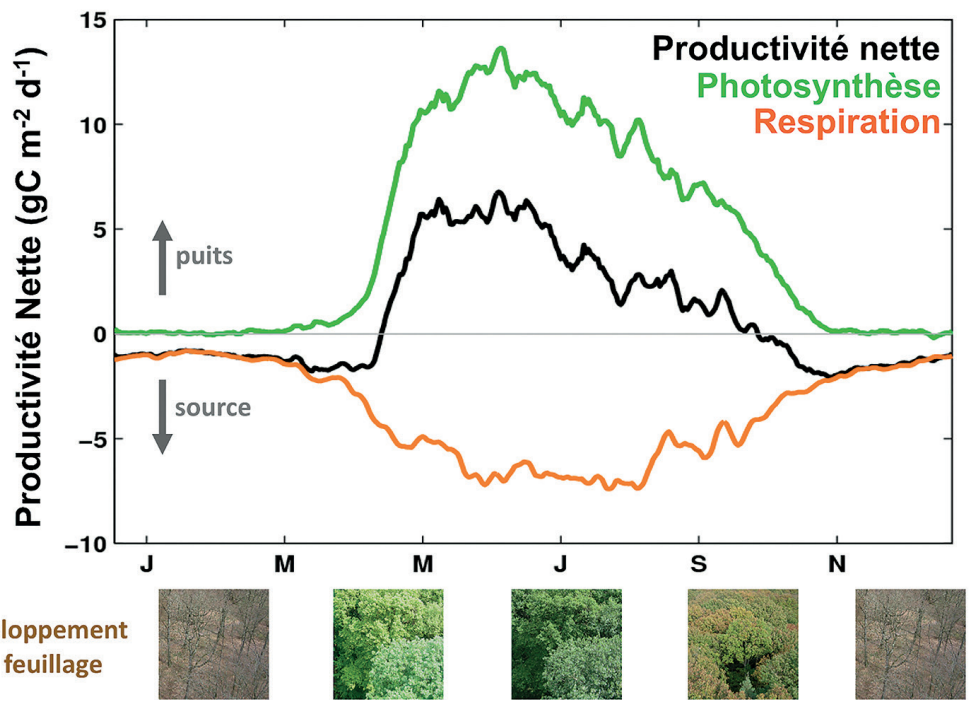

Figure 6.14: Mesures des échanges de $\mathrm{CO}_{2}$ dans la forêt tempérée et caducifoliée de Barbeau près de Fontainebleau. Source: Nicolas Delpierre.

\subsubsection{Importance des écosystèmes forestiers face au changement climatique}

La figure 6.15 met bien en évidence que le changement climatique agit sur la capacité de puits de carbone des écosystèmes. Par exemple, les printemps chauds, comme celui de 2020 , ont tendance à augmenter la fixation de carbone par les forêts; ce sont les barres vertes sur le graphique. À l'inverse, les étés chauds et secs ont tendance à diminuer la fixation de carbone par ces mêmes forêts: ce sont les barres orange [14].

Les forêts restent, malgré tout, des puits de carbone de première importance. Elles absorbent plus de $\mathrm{CO}_{2}$ dans l'atmosphère qu'elles n'en émettent et contribuent à ralentir le réchauffement climatique en cours. Elles sont néanmoins vulnérables à différents facteurs tels que les émergences périodiques de populations d'insectes ravageurs, ou encore les incendies dont la fréquence et l'intensité augmentent avec le réchauffement climatique. Le réchauffement climatique lui-même provoque des sécheresses et vagues de chaleur à la fois plus intenses et durables.

Les forêts sont également vulnérables à l'action des humains qui déforeste actuellement des surfaces considérables. En une heure de temps, c'est une surface de forêt équivalant à 530 terrains de football qui disparaît sur Terre. Si nous voulons que les écosystèmes forestiers continuent à fixer efficacement du carbone atmosphérique et à atténuer ainsi le réchauffement climatique, il faut impérativement préserver les arbres des agressions et notamment de la déforestation. Pour faire face au réchauffement climatique, les forêts sont nos alliées. Préservons-les! 


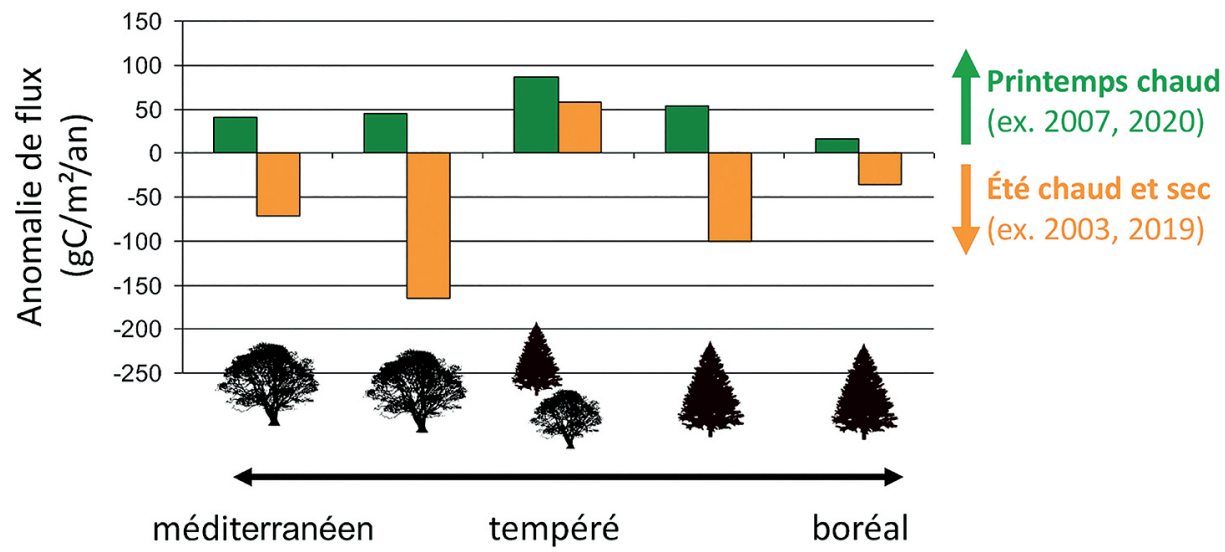

Figure 6.15: Représentation schématique des variations anormales de carbone fixé par les forêts suivant les aléas climatiques. Source : Nicolas Delpierre.

\section{Références}

[1] D. Couvet, F. Jiguet, R. Julliard, H. Levrel, A. Teyssedre. 2008. Enhancing citizen contributions to biodiversity science and public policy. Interdisciplinary Science Reviews 33(1), 95-103.

[2] D. Couvet, A.C. Prevot. 2015. Citizen-science programs: Towards transformative biodiversity governance. Environmental Development 13, 39-45.

[3] IPBES. 2016. Summary for policymakers of the methodological assessment of scenarios and models of biodiversity and ecosystem services of the Intergovernmental Science-Policy Platform on Biodiversity and Ecosystem Services. S. Ferrier, K.N. Ninan, P. Leadley, R. Alkemade, L.A. Acosta, H.R. Akçakaya, L. Brotons, W. Cheung, V. Christensen, K.H. Harhash, J. Kabubo-Mariara, C. Lundquist, M. Obersteiner, H. Pereira, G. Peterson, R. Pichs-Madruga, N.H. Ravindranath, C. Rondinini, B. Wintle (eds.). Secretariat of the Intergovernmental Science-Policy Platform on Biodiversity and Ecosystem Services, Bonn, Germany, 32 p.

[4] W. Thuiller, S. Lavorel, M.B. Araujo, M.T. Sykes, I.C. Prentice. 2005. Climate change threats to plant diversity in Europe. Proc. Natl. Acad. Sci. U. S. A. 102, 8245-8250.

[5] T. Newbold, L.N. Hudson, S.L.L. Hill, S. Contu, I. Lysenko, R.A. Senior, L. Borger, D.J. Bennett, A. Choimes, B. Collen, J. Day, A. De Palma, S. Diaz, S. Echeverria-Londono, M.J. Edgar, A. Feldman, M. Garon, M.L.K. Harrison, T. Alhusseini, D.J. Ingram, Y. Itescu, J. Kattge, V. Kemp, L. Kirkpatrick, M. Kleyer, D.L.P. Correia, C.D. Martin, S. Meiri, M. Novosolov, Y. Pan, H.R.P. Phillips, D.W. Purves, A. Robinson, J. Simpson, S.L. Tuck, E. Weiher, H.J. White, R.M. Ewers, G.M. Mace, J.P.W. Scharlemann, A. Purvis. 2015. Global effects of land use on local terrestrial biodiversity. Nature 520, 45. https://doi.org/10.1038/nature14324.

[6] Hof, et al. 2018. Bioenergy cropland expansion may offset positive effects of climate change mitigation for global vertebrate diversity. PNAS 115(52), 13294-13299. 
[7] N. Delpierre, Y. Vitasse, I. Chuine, J. Guillemot, S. Bazot, C.B. Rathgeber. 2016. Temperate and boreal forest tree phenology: from organ-scale processes to terrestrial ecosystem models. Annals of Forest Science 73(1), 5-25.

[8] N. Delpierre, J. Guillemot, E. Dufrêne, S. Cecchini, \& M. Nicolas. 2017. Tree phenological ranks repeat from year to year and correlate with growth in temperate deciduous forests. Agricultural and Forest Meteorology 234, 1-10.

[9] R. Denéchère, N. Delpierre, E.N. Apostol, D. Berveiller, F. Bonne, E. Cole, ..., G. Vincent. 2019. The within-population variability of leaf spring and autumn phenology is influenced by temperature in temperate deciduous trees. International Journal of Biometeorology, $1-11$.

[10] Y.H. Fu, H. Zhao, S. Piao, M. Peaucelle, S. Peng, G. Zhou, ..., I.A. Janssens. 2015. Declining global warming effects on the phenology of spring leaf unfolding. Nature 526(7571), 104-107.

[11] P. Friedlingstein, M.W. Jones, M. O'sullivan, R.M. Andrew, J. Hauck, G.P. Peters, ..., S. Zaehle. 2019. Global carbon budget 2019. Earth System Science Data 11(4), 1783-1838.

[12] D. Baldocchi. 2008. "Breathing" of the terrestrial biosphere: lessons learned from a global network of carbon dioxide flux measurement systems. Australian Journal of Botany $56(1), 1-26$.

[13] G. Pastorello, C. Trotta, E. Canfora, H. Chu, D. Christianson, Y.W. Cheah, ..., \& Y. Li. 2020. The FLUXNET2015 dataset and the ONEFlux processing pipeline for eddy covariance data. Scientific Data 7(1), 1-27.

[14] N. Delpierre, K. Soudani, C. François, B. Köstner, J.Y. Pontailler, E. Nikinmaa, ..., E. Dufrene. 2009. Exceptional carbon uptake in European forests during the warm spring of 2007: a data-model analysis. Global Change Biology 15(6), 1455-1474. 
Partie III

\section{Le changement climatique}





\section{Chapitre 7}

\section{Le système climatique}

Dans ce chapitre, nous présentons les principales composantes du système climatique et leur fonctionnement pour décrire ensuite, de façon détaillée, les mécanismes d'effet de serre dans l'atmosphère. Nous verrons comment les émissions des gaz, qui en sont responsables, sont reliées aux différents secteurs de l'activité humaine et comment, pour le dioxyde de carbone en particulier, elles se répartissent suivant les principales régions du monde. Parmi les secteurs d'activité, les transports, l'industrie et la production d'énergie contribuent de façon importante aux émissions de gaz à effet de serre comme on peut s'y attendre, mais nous verrons que la gestion des écosystèmes terrestres pour l'exploitation de la biomasse et les cultures agricoles en général, peut aussi participer de manière significative au changement climatique. La dernière partie du chapitre est consacrée aux indicateurs du changement climatique que l'on suit à partir de réseaux d'observations et de mesures ou que l'on extrait de différentes archives paléoclimatiques.

\subsection{Les composantes du système climatique et leurs interactions, les forçages naturels et anthropiques - M. Delmotte}

\subsubsection{Les composantes du système climatique}

Cette partie présente le système climatique de la Terre et les composantes qui lui permettent de fonctionner.

Ces composantes sont: les océans, l'atmosphère, la cryosphère, c'est-à-dire les zones englacées qui comprennent les glaciers de montagne, les calottes polaires, la banquise, et enfin la biosphère qui inclut la végétation et les animaux vivants. Ce système fonctionne parce qu'il y a une source d'énergie, un moteur, qui est le soleil. II subit des perturbations qui peuvent être d'origine naturelle, tel le volcanisme, ou d'origine anthropique, causées par les activités humaines. Les différentes 
composantes du système climatique vont alors interagir entre elles pour assurer le fonctionnement de l'ensemble.

Le système climatique n'est ni plus ni moins qu'une machine thermique dont le fonctionnement permet de rétablir le déséquilibre thermique engendré par le rayonnement solaire (Fig. 7.1). Vous verrez plus en détail la notion de bilan radiatif lié à cette machine thermique dans la partie suivante de cet ouvrage (voir section 7.2).

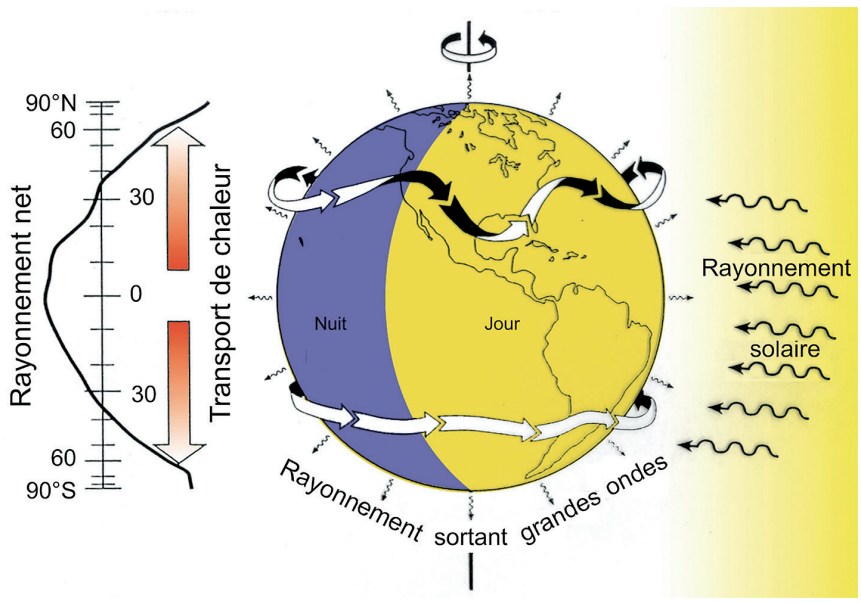

Figure 7.1: Répartition du rayonnement solaire à la surface de la Terre. Droits réservés.

Le rayonnement solaire est, en effet, loin d'être uniforme sur toute la surface de la Terre, dû à la forme sphérique de celle-ci. La quantité d'énergie reçue par unité de surface est plus forte à l'équateur, où l'incidence des rayons solaires est perpendiculaire à la surface du sol, qu'aux pôles où le rayonnement en incidence rasante intercepte une surface beaucoup plus grande. La Terre, comme tout système physique, cherche à tendre vers une température homogène et uniforme et donc le système climatique va avoir pour rôle de redistribuer l'excédent de chaleur reçu dans les zones intertropicales vers les pôles: de l'équateur vers le pôle nord dans l'hémisphère nord, et de l'équateur vers le pôle sud dans l'hémisphère sud. Pour cela, il va faire principalement appel à deux de ses composantes, qui sont l'atmosphère et les océans.

Les masses d'eau chauffées par le soleil dans les zones tropicales vont s'évaporer dans l'atmosphère et former ainsi des nuages. On parle aussi de chaleur latente. Puis ces nuages vont être transportés par les vents dominants de l'équateur vers les pôles. Au cours du transport, chaque épisode de précipitation (pluie, neige, etc.) va permettre une redistribution partielle de l'énergie emmagasinée initialement par le nuage. L'atmosphère est une composante rapide, elle réagit sur des échelles de temps de quelques heures à quelques jours, dix jours tout au plus.

De la même manière, les courants marins transportent également une partie de la chaleur, sous forme de courants chauds en surface, ou froids en profondeur. Lorsque 
les courants chauds de surface arrivent dans les zones froides, au sud du Groenland en mer de Béring par exemple, la mer gèle pour former de la banquise.

Cette banquise qui se forme, est constituée d'eau douce, ce qui implique que le sel de l'eau de mer reste dans la phase liquide de l'océan et donc que la densité des masses d'eau augmente, entraînant ainsi sa plongée vers les profondeurs. On parle de circulation thermohaline, pilotée par la température et la salinité de l'océan. La composante océanique a une très forte inertie, il faut environ 1000 ans à une masse d'eau pour effectuer une boucle complète de circulation (Fig. 7.2).

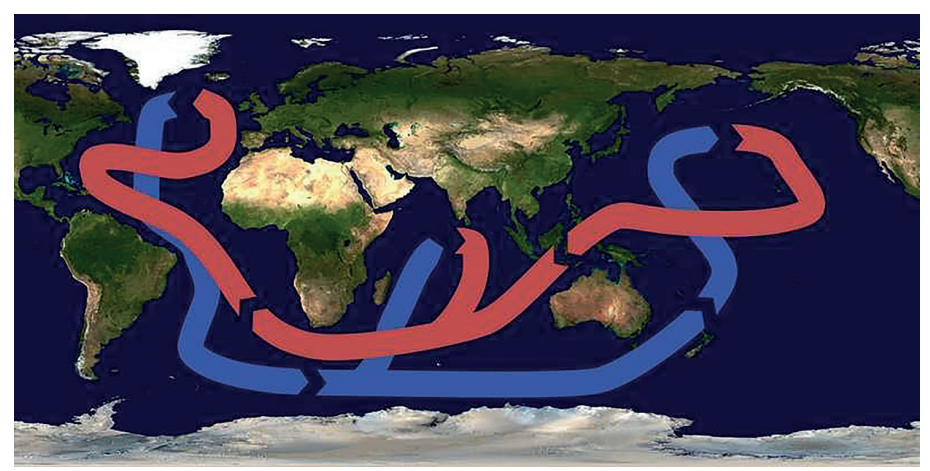

Figure 7.2: La circulation océanique thermohaline est actionnée par la différence de densité des eaux au niveau du Groenland. La plongée des eaux qui s'ensuit est le moteur du tapis roulant océanique. (C) Brisbane, Wikimédia CC-by-sa $3.0^{58}$.

La cryosphère et la biosphère jouent aussi un rôle dans cette machine thermique. On a vu le rôle de la banquise dans la circulation thermohaline, mais la cryosphère a aussi un rôle au niveau de l'albédo de la planète, c'est-à-dire sa capacité de réflexion liée à sa couleur. Plus une surface est claire, plus son albédo est élevé, et plus elle réfléchit la lumière du soleil. La cryosphère (glace et neige) contribue ainsi à maintenir des températures froides aux pôles et en altitude. Elle sert aussi de régulateur du cycle de l'eau.

La biosphère a un rôle de régulateur au sein du cycle du carbone et donc de la composition du $\mathrm{CO}_{2}$ dans l'atmosphère, tout comme l'océan d'ailleurs.

Sur des échelles de temps beaucoup plus longues, la lithosphère, c'est-à-dire la croûte terrestre superficielle, joue également un rôle. La position des continents va influer sur la circulation océanique et atmosphérique, sur la répartition de la végétation et sur la composition chimique de l'atmosphère notamment par le biais de l'activité volcanique. À grande échelle, les phénomènes d'érosion contribuent à la formation des grands réservoirs de combustibles fossiles, le gaz, le pétrole, le charbon au niveau du cycle du carbone. Nous reviendrons sur ce point plus loin en abordant la variabilité naturelle du climat. 


\subsubsection{Les forçages climatiques et la composition de l'atmosphère}

Parmi les forçages climatiques, autrement dit, les phénomènes qui tendent à modifier le climat (voir sections 1.1 et 1.2), on distingue les forçages naturels avec pour principal forçage externe, le soleil, et pour forçage interne, le noyau terrestre. À ces forçages s'ajoutent les forçages anthropiques, liés aux activités humaines. Nous reviendrons sur ces notions et sur les ordres de grandeur plus loin.

La Terre se distingue des autres planètes par l'existence de l'atmosphère, une enveloppe gazeuse autour de sa surface qui la protège et lui confère une température moyenne de l'ordre de $15^{\circ} \mathrm{C}$. La composition chimique de cette atmosphère est la suivante: $78 \%$ d'azote $\mathrm{N}_{2}, 21 \%$ d'oxygène $\mathrm{O}_{2}$, le reste étant composé de gaz sous forme de traces, dont en particulier les gaz à effet de serre qui ont un rôle fondamental dans le bilan thermique de la planète via justement, l'effet de serre. Parmi ces gaz, on peut noter par ordre d'importance, la vapeur d'eau, qui s'inscrit dans le cycle naturel de l'eau, le gaz carbonique $\mathrm{CO}_{2}$, premier gaz à effet de serre produit par l'Homme, le méthane $\mathrm{CH}_{4}$, le protoxyde d'azote $\mathrm{N}_{2} \mathrm{O}$ et les chlorofluorocarbures CFC.

Le cycle naturel de ces gaz est fortement impacté par les activités humaines qui, pour répondre aux besoins énergétiques de la société actuelle, utilisent essentiellement des combustibles fossiles et carbonés comme source d'énergie primaire: le gaz naturel, le charbon, et le pétrole, qui se produisent essentiellement par combustion du $\mathrm{CO}_{2}$ qui est rejeté dans l'atmosphère de façon massive. Le méthane $\mathrm{CH}_{4}$ est, lui, plutôt produit par l'agriculture, les rizicultures, les décharges, l'élevage des ovins et des bovins, mais aussi, de façon plus naturelle, par les zones humides.

Le $\mathrm{CO}_{2}$ qui est rejeté dans l'atmosphère s'inscrit lui aussi dans un cycle naturel: le cycle du carbone. Sur 100 molécules de $\mathrm{CO}_{2}$ anthropiques injectées dans l'atmosphère, dont $86 \%$ proviennent directement des activités humaines et $14 \%$ du changement d'usage des sols, seules $46 \%$ vont subsister dans l'atmosphère, tandis que $56 \%$ vont être réabsorbées par les puits de carbone naturels que sont les océans et la végétation. L'incertitude sur ces chiffres est de 0,4\%.

Le $\mathrm{CO}_{2}$ se dissout naturellement dans l'eau de mer, d'autant mieux que l'eau est froide, puis les microorganismes, tels le phytoplancton, le synthétisent avant d'être eux-mêmes absorbés par d'autres organismes qui vont stocker le carbone sous forme de coquilles carbonatées, la calcite des coquillages, par exemple, ou sous d'autres formes de matière organique. Lorsque ces organismes vivants meurent, ils tombent au fond de la mer et après des dizaines, voire des centaines de millions d'années, ils vont de nouveau former des gisements de combustibles fossiles (charbon, pétrole, gaz).

De son côté, la végétation va capter le $\mathrm{CO}_{2}$ atmosphérique lors de la réaction de photosynthèse qui met en jeu les feuilles vertes et la lumière. Elle va synthétiser le 
carbone sous forme de matière organique, le bois, par exemple, qui se trouve alors stocké de façon solide.

\subsubsection{La notion d'effet de serre au premier ordre}

Il peut être utile de définir et d'illustrer la notion d'effet de serre au premier ordre. Sur la figure 7.3, on voit que les rayonnements issus du soleil, visible, infrarouge, ultraviolet, vont d'abord rencontrer la surface de l'atmosphère, laquelle va directement réfléchir environ $30 \%$ de ce rayonnement vers l'espace. Le reste du rayonnement va, pour environ $20 \%$, être absorbé par la couche atmosphérique, et pour l'essentiel (50\%), va venir impacter la surface terrestre et donc la réchauffer.

Afin de maintenir son équilibre thermique, la Terre va en retour réémettre vers l'espace un rayonnement. La Terre rayonne comme tout corps chaud, mais sa température restant relativement basse, elle ne va émettre qu'un rayonnement infrarouge.

C'est là qu'interviennent les gaz à effet de serre. Ces gaz ont la propriété d'absorber les rayonnements infrarouges émis par la Terre, et donc de limiter la part de ce rayonnement qui s'échappe vers l'espace. Ils vont aussi les réémettre vers la surface terrestre, contribuant ainsi à réchauffer les basses couches de l'atmosphère. C'est ce qu'on appelle l'effet de serre naturel. Ce sont ces gaz à effet de serre qui permettent à la Terre d'avoir une température moyenne globale de l'ordre de $15^{\circ} \mathrm{C}$. Sans eux, la Terre serait une boule de glace.

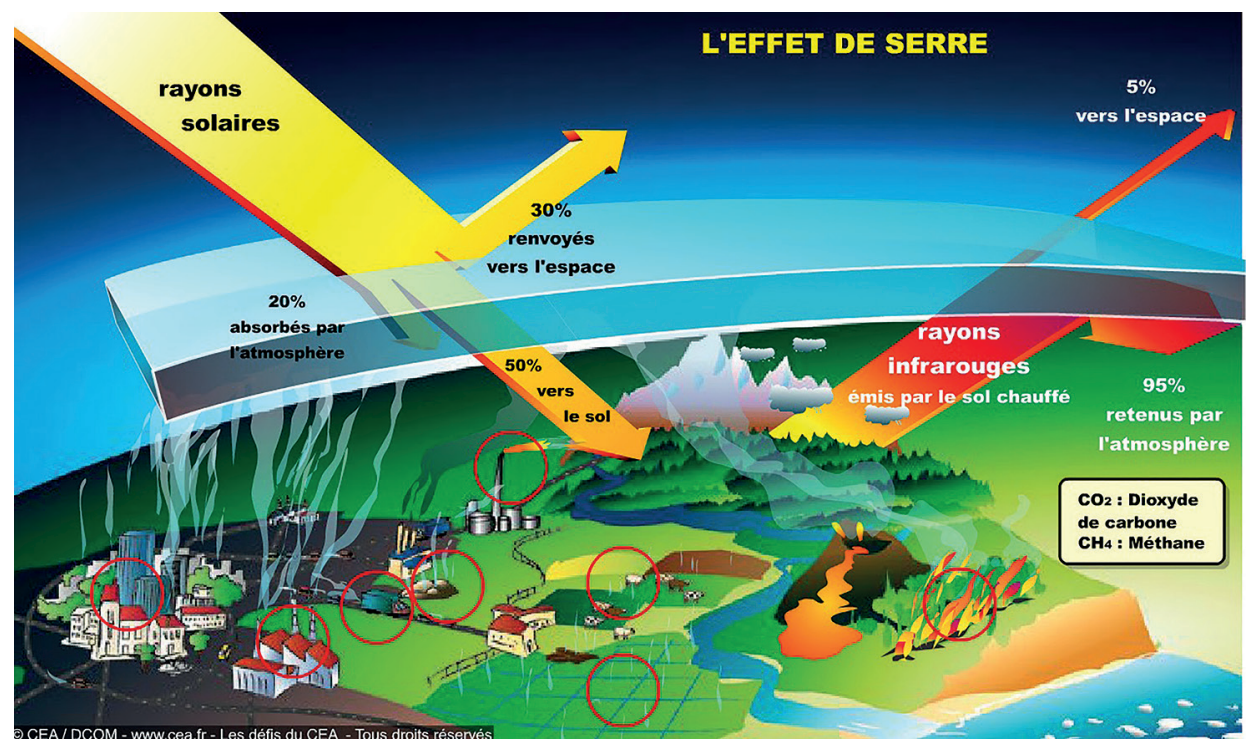

Figure 7.3: Image illustrant la distribution de l'énergie solaire à la surface de la Terre et la réémission infrarouge de celle-ci. Source: CEA/DCOM - www.cea.fr - (Les Défis du CEA) ${ }^{59}$. 
Pour mieux comprendre, je vous propose une analogie. Lorsque vous vous couchez le soir en hiver par exemple, si vous vous allongez nu sur votre lit, vous avez une sensation de froid. La raison en est que votre corps, qui est à la température de $37^{\circ} \mathrm{C}$, rayonne constamment, il perd donc de la chaleur et émet lui aussi un rayonnement infrarouge, et ce faisant, vous «chauffez » tout l'espace de votre chambre dont la température est plus proche de $18-20^{\circ} \mathrm{C}$. Pour dormir confortablement, vous allez donc vous couvrir avec une couverture. Cette dernière va piéger la chaleur émise par votre corps et la redistribuer dans l'espace restreint entre vous et la couverture, vous n'aurez plus froid. Les gaz à effet de serre dans la couche atmosphérique ont le même rôle que la couverture sur votre lit.

De même que si vous ajoutez d'autres couvertures sur votre lit, vous aurez de plus en plus chaud. Si on augmente la concentration des gaz à effet de serre dans l'atmosphère, on augmentera la température de la surface de la Terre. On parle alors d'effet de serre additionnel, c'est celui qui découle directement des activités humaines qui modifient la composition de l'atmosphère en émettant massivement des gaz à effet de serre, en particulier du $\mathrm{CO}_{2}$.

En conclusion de cette partie, nous avons donc posé les bases du système climatique. II s'agit d'une machine thermique complexe qui fonctionne grâce à l'énergie fournie par le soleil et grâce aux interactions et aux rétroactions entre ses différentes composantes. L'atmosphère joue un rôle important au niveau du bilan radiatif terrestre, en particulier à travers l'effet de serre.

\subsection{Bilan radiatif, effet de serre et gaz à effet de serre - F. Raffin et M. Saunois}

\subsubsection{Le bilan radiatif de la Terre ${ }^{60}$}

Revenons sur le bilan radiatif de la Terre, son équilibre thermique, comme évoqué précédemment par Marc Delmotte, en donnant quelques bases scientifiques pour comprendre le dérèglement climatique.

II faut d'abord se rappeler qu'en fonction de sa température, tout corps émet un rayonnement électromagnétique et que la longueur d'onde de ce rayonnement diminue lorsque la température augmente. C'est la loi établie par le physicien Wilhelm Wien ${ }^{61}$. Nous nous en rendons compte en observant le rayonnement visible. Plus c'est chaud, plus c'est bleu : un fer «porté au rouge» approche une température d'environ $800^{\circ} \mathrm{C}$ alors que la flamme jaune/bleue d'un chalumeau dépasse la température de $3000^{\circ} \mathrm{C}$. Si l'on considère maintenant le spectre du rayonnement électromagnétique sur une plus grande étendue, un corps qui émet des infrarouges sera forcément bien moins chaud qu'un corps qui émet des rayonnements visibles ou ultraviolets.

60. https://www.youtube.com/watch?v=J5yCXjpggE4

61. https://fr.wikipedia.org/wiki/Wilhelm_Wien 
Ainsi, le soleil qui a une température de surface élevée, aux alentours de $6000^{\circ} \mathrm{C}$, va émettre un rayonnement principalement dans le visible. La Terre qui, elle, a une température moyenne de $15^{\circ} \mathrm{C}$, émet principalement un rayonnement dans l'infrarouge (Fig. 7.4, courbes du haut). Par ailleurs, n'oublions pas que ces rayonnements transportent aussi de l'énergie, et cela vous le savez, quand vous vous mettez au soleil, vous vous réchauffez très vite.

L'atmosphère terrestre n'agit pas de la même façon sur les rayonnements qui arrivent sur la Terre et sur les rayonnements qu'émet la Terre en raison de leur différence de nature, visible ou infrarouge. Considérons le graphique au bas de la figure 7.4, qui nous donne l'absorption des rayonnements par l'atmosphère en fonction de leur longueur d'onde. Nous voyons que le rayonnement visible est, en grande partie, transmis par l'atmosphère (absorption quasi nulle). Heureusement, sans quoi, nous serions dans le noir. L'infrarouge, lui, est en grande partie absorbé (absorption proche de l'unité). Des bandes d'absorption apparaissent, où chacune des bandes correspond à un ou plusieurs gaz présent(s) dans l'atmosphère. Sous le spectre d'émission de la Terre, vous pouvez voir des bandes d'absorption liées à la vapeur d'eau, au $\mathrm{CO}_{2}$, au méthane $\mathrm{CH}_{4}$, au protoxyde d'azote $\mathrm{N}_{2} \mathrm{O}$, à l'ozone $\mathrm{O}_{3}, \ldots$

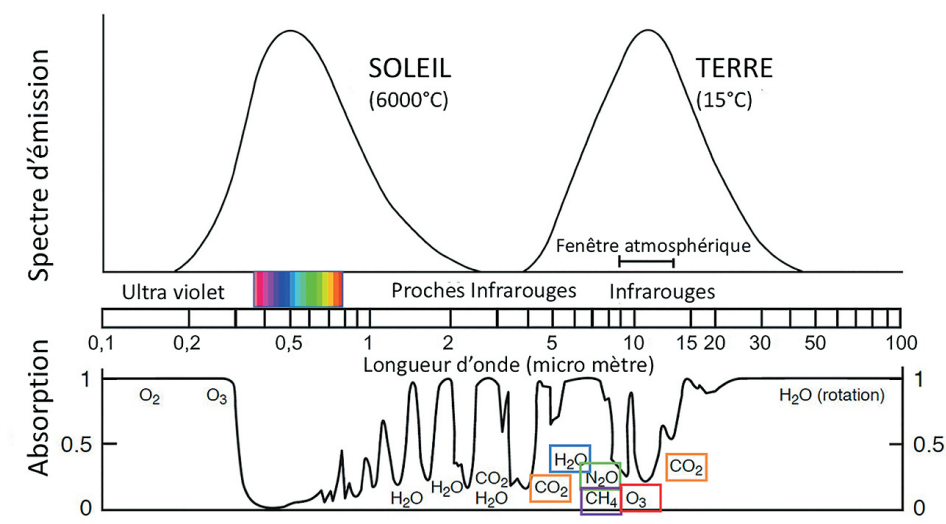

Figure 7.4: En haut: spectres d'émission du soleil et de la Terre. En bas: absorption de l'atmosphère en fonction de la longueur d'onde. Sont indiqués les principaux gaz à effet de serre et leur correspondance avec les bandes d'absorption en infrarouge. Source: Marielle Saunois.

L'énergie, qui arrive essentiellement sur Terre par le rayonnement visible du soleil, est soumise à un premier effet que l'on appelle l'effet parasol. En effet, en raison de la présence des nuages, d'aérosols réfléchissants et des surfaces brillantes (neige, glace), 30 \% du rayonnement solaire est réfléchi par l'atmosphère et la surface de la Terre. L'énergie émise par la Terre, sous la forme d'un rayonnement infrarouge, est, elle, soumise au fameux effet de serre. En effet, l'atmosphère ne laisse que très peu passer les infrarouges. Ceux-ci sont, en très grande partie, absorbés par les gaz à effet de serre, puis réémis par eux vers la Terre, dont la surface va se réchauffer en conséquence. On parle souvent péjorativement de cet effet de serre, mais sans lui, 
la température moyenne à la surface de la Terre serait de $-18^{\circ} \mathrm{C}$ et non pas de $15^{\circ} \mathrm{C}$. C'est lui qui nous réchauffe, un peu comme une couverture ou une couette dans notre lit pour reprendre la description de Marc Delmotte (voir section 7.1.3).

Le bilan radiatif de la Terre est la différence entre l'énergie solaire arrivant sur Terre et l'énergie émise par la Terre et repartant vers l'espace. C'est l'équilibre entre cette énergie reçue et l'énergie renvoyée qui maintient un climat viable sur la planète avec une température moyenne de $15^{\circ} \mathrm{C}$. Alors on comprend facilement qu'un déséquilibre entre les rayonnements entrant et sortant de l'atmosphère, par un changement d'albédo ou une modification des gaz à effet de serre, va modifier ce bilan radiatif et va avoir pour conséquence un dérèglement climatique.

\subsubsection{L'effet de serre additionnel lié aux activités humaines}

II nous faut maintenant distinguer l'effet de serre naturel, en l'absence d'activités humaines, de l'effet de serre additionnel, causé par ces mêmes activités humaines.

Les graphiques de la figure 7.5 représentent la contribution des différents gaz, respectivement à l'effet de serre naturel (à gauche) et à l'effet de serre additionnel (à droite). Le premier gaz à effet de serre naturel est la vapeur d'eau! Elle contribue en effet à environ $60 \%$ de cet effet de serre, suivie par le dioxyde de carbone $\mathrm{CO}_{2}$ pour $26 \%$, puis l'ozone $\mathrm{O}_{3}$, le méthane $\mathrm{CH}_{4}$ et le protoxyde d'azote $\mathrm{N}_{2} \mathrm{O}$. Les nuages participent aussi, de façon naturelle, à l'équilibre thermique de la Terre et au climat. Ils ont un pouvoir refroidissant, puisqu'ils réfléchissent le rayonnement solaire et donc contribuent à l'effet parasol. Mais, ils ont aussi un pouvoir réchauffant, car nous avons tous expérimenté qu'une nuit claire sans nuage est plus fraîche qu'une nuit nuageuse, ce qui montre bien l'effet de serre des nuages. En moyenne, les nuages ont plutôt tendance à refroidir le climat, mais les nuages fins et élevés comme les cirrus ont un effet de serre dominant.

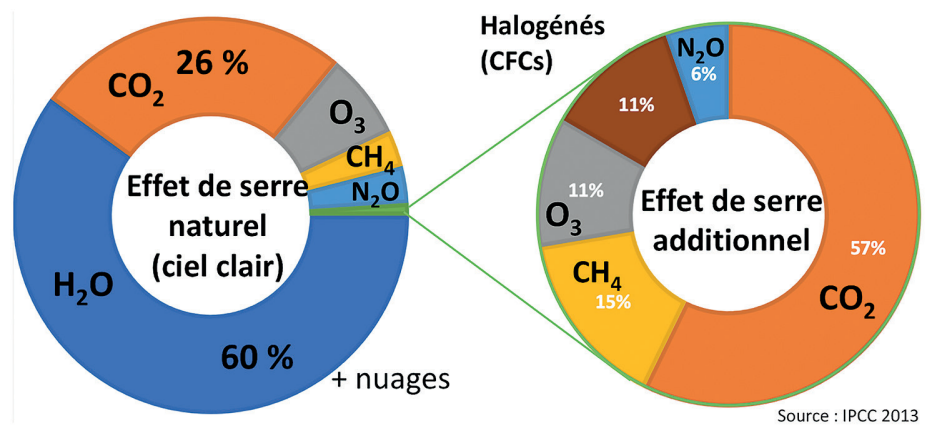

Figure 7.5: Contributions respectives des principaux gaz de l'atmosphère à l'effet de serre naturel (à gauche) et à l'effet de serre additionnel (à droite). Source: IPCC, $2013^{62}$. 
Les scientifiques sont désormais capables de déterminer les quantités de gaz présents dans l'atmosphère sur des temps géologiques, c'est-à-dire bien antérieurs au début du développement de l'agriculture et des industries. En particulier, si l'on regarde l'évolution des concentrations de trois gaz à effet de serre, le $\mathrm{CO}_{2}$, le méthane $\mathrm{CH}_{4}$ et le protoxyde d'azote $\mathrm{N}_{2} \mathrm{O}$, dans l'atmosphère depuis l'an zéro jusqu'en 2020. On constate que leurs concentrations sont restées quasi constantes sur les 1700 premières années, mais que depuis 1750, date prise comme référence pour la période pré-industrielle, elles ont toutes augmenté dans des proportions importantes (Fig. 7.6) :

- la concentration du $\mathrm{CO}_{2}$ a été multipliée par 1,4;

- celle du méthane par 2,6;

- et celle du protoxyde d'azote par 1,2.

Les activités humaines - industries, transports, agricultures - ont donc bien contribué à augmenter les concentrations de gaz à effet de serre dans l'atmosphère. Les humains ont même introduit dans l'atmosphère des gaz qui n'ont aucune origine naturelle. II s'agit en particulier de gaz composés de carbone, d'hydrogène, de fluor, et de chlore, les chlorofluorocarbones (CFC), les hydrochlorofluorocarbures (HCFC) ou encore les hydrofluorocarbones (HFC). Ces gaz synthétiques sont des gaz à effet de serre. Ils ont été produits à partir des années 1950 pour l'industrie du froid, de l'isolation, des nettoyants et de la propulsion. Leurs concentrations, mesurées à partir des années 1980, sont en augmentation pour la plupart d'entre elles, même si certaines tendent aujourd'hui à se stabiliser, voire à diminuer suite au protocole de Montréal visant à protéger la couche d'ozone stratosphérique ${ }^{63}$.

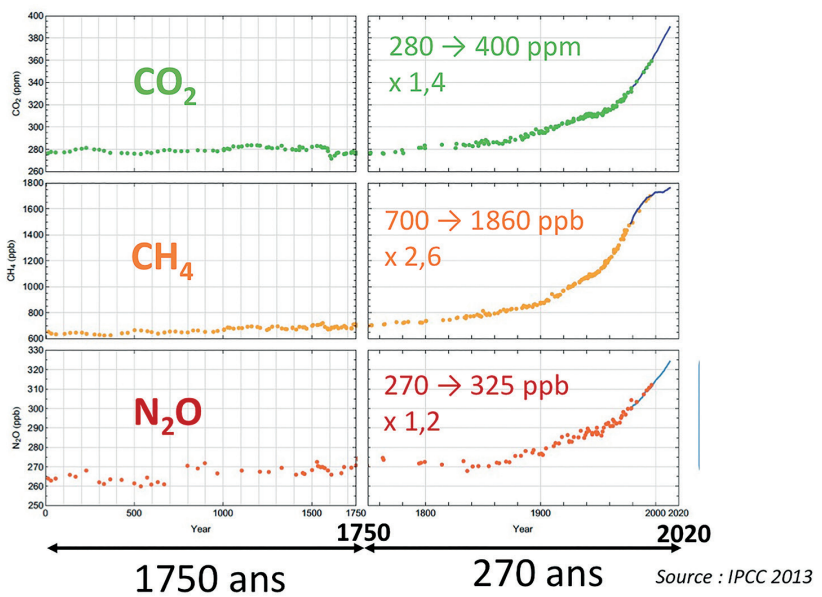

Figure 7.6: Évolutions sur les 2000 dernières années des concentrations de gaz majeurs participant à l'effet de serre additionnel $\left(\mathrm{CO}_{2}, \mathrm{CH}_{4}\right.$ et $\left.\mathrm{N}_{2} \mathrm{O}\right)$. Source: IPCC, $2013^{62}$.

63. a) https://fr.wikipedia.org/wiki/Protocole_de_Montréal

b) https://csl.noaa.gov/assessments/ozone/2018/twentyquestions/ 
C'est donc l'augmentation des concentrations de ces gaz à effet de serre, en lien avec les activités humaines, qui est responsable de ce qu'on appelle l'effet de serre additionnel. En calculant la manière dont les changements de composition depuis 1750 modifient l'effet de serre, les scientifiques estiment que (Fig. 7.5, à droite) :

- le $\mathrm{CO}_{2}$ est le premier gaz à effet anthropique, contribuant à $57 \%$ de l'effet de serre additionnel;

- suivi par le méthane (15\%), l'ozone (11\%), et le protoxyde d'azote (6\%). L'ensemble des composés halogénés (essentiellement les CFCs) contribue à hauteur de $11 \%$.

Ainsi, les scientifiques estiment la puissance par unité de surface associée à l'effet de serre additionnel à 2,29 W/m² entre 1750 et 2011. Rappelons pour la comparaison que la puissance reçue du rayonnement solaire par unité de surface, de jour et sous ensoleillement direct, est de l'ordre de $1000 \mathrm{~W} / \mathrm{m}^{2}$. Cet effet de serre additionnel, même faible, a ainsi entraîné une augmentation de la température moyenne à la surface de la Terre de 1,1 ${ }^{\circ} \mathrm{C}$ pour la décennie 2010-1019 par rapport à la décennie antérieure 1850-1900.

Si les émissions de gaz à effet de serre produits par les humains continuent à croître au même rythme qu'aujourd'hui, les modèles climatiques prédisent qu'en 2100 la température moyenne à la surface de la Terre pourrait augmenter de 2 à $4^{\circ} \mathrm{C}$.

\subsection{Les émissions de gaz à effet de serre par secteur et par région - M. Saunois}

Nous allons voir ici comment se répartissent les émissions des principaux gaz responsables de l'effet de serre additionnel (voir section 7.1.2) selon les secteurs d'activités et selon les pays développés ou en voie de développement.

Nous nous concentrerons sur le dioxyde de carbone, $\mathrm{CO}_{2}$, le méthane, $\mathrm{CH}_{4}$, et le protoxyde d'azote, $\mathrm{N}_{2} \mathrm{O}$, en oubliant volontairement l'ozone, $\mathrm{O}_{3}$, et les composés halogénés ou chlorofluorocarbones (CFC). En effet, on ne peut pas véritablement parler d'émission pour l'ozone, car il n'a pas de source directe, étant produit dans l'atmosphère via des réactions chimiques (voir section 2.3). Par ailleurs, les composés halogénés ne sont presque exclusivement émis que dans un seul secteur d'activité, celui de l'industrie.

Les secteurs d'activité émetteurs de $\mathrm{CO}_{2}$, de $\mathrm{CH}_{4}$ et de $\mathrm{N}_{2} \mathrm{O}$ peuvent être classés en 8 catégories (Fig. 7.7):

- L'extraction et la distribution des énergies fossiles (charbon, pétrole et gaz) qui sont ensuite elles-mêmes utilisées dans différents secteurs;

- Les transports;

- La production d'énergie; 
- Le chauffage des bâtiments pour le résidentiel et le tertiaire;

- Les industries parmi lesquelles les industries manufacturières, l'industrie du bâtiment et la production de ciment;

- L'agriculture avec l'élevage, la gestion des fumiers, la fertilisation des sols et la culture du riz;

- La gestion des déchets solides et liquides;

- L'exploitation de la biomasse incluant le changement d'utilisation des terres avec en particulier la déforestation et les feux de forêts.

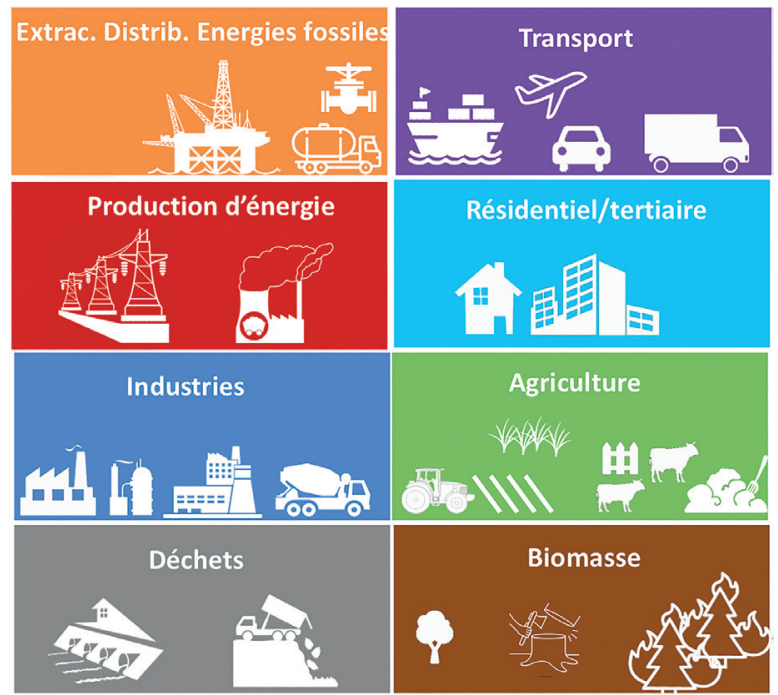

Figure 7.7: Secteurs d'activité émetteurs de $\mathrm{CO}_{2}$, de $\mathrm{CH}_{4}$ et de $\mathrm{N}_{2} \mathrm{O}$. Source: Marielle Saunois.

Les émissions de $\mathrm{CO}_{2}$ dans le monde sont estimées à 42 milliards de tonnes en 2018. Environ $84 \%$ sont dus à la combustion des énergies fossiles. Le reste vient du changement d'utilisation des terres et des sols, entre autres, de la déforestation. Parmi les trois sources d'émission, le charbon contribue pour $40 \%$, suivi de près par le pétrole (34\%), puis par le gaz (20\%) (valeurs issues du bilan mondial du carbone de 2019 - mise à jour annuelle disponible ${ }^{64}$ ).

La figure 7.8 décrit l'évolution des émissions de $\mathrm{CO}_{2}$ au cours des 60 dernières années pour les 6 premiers pays émetteurs actuels. Les émissions ont augmenté dans la plupart de ces pays, mais certains pays ou certaines régions ont vu leurs émissions diminuer comme les États-Unis ou l'Europe et ses 28 membres. En 2018 , les principaux pays émetteurs étaient la Chine, largement devant les États-Unis, I'Europe, l'Inde, la Russie et le Japon. À eux 6, ces pays couvrent $67 \%$ des émissions 
mondiales. Si, maintenant, on considère les émissions par habitant, ce sont bien les États-Unis qui émettent le plus de $\mathrm{CO}_{2}$ par habitant (Fig. 7.9). La Chine arrive aujourd'hui au même niveau que l'Europe. Finalement, si on répartit les émissions de $\mathrm{CO}_{2}$ dans les 8 catégories présentées, les principaux secteurs d'émissions de $\mathrm{CO}_{2}$ sont la production d'énergie, les industries et le transport.

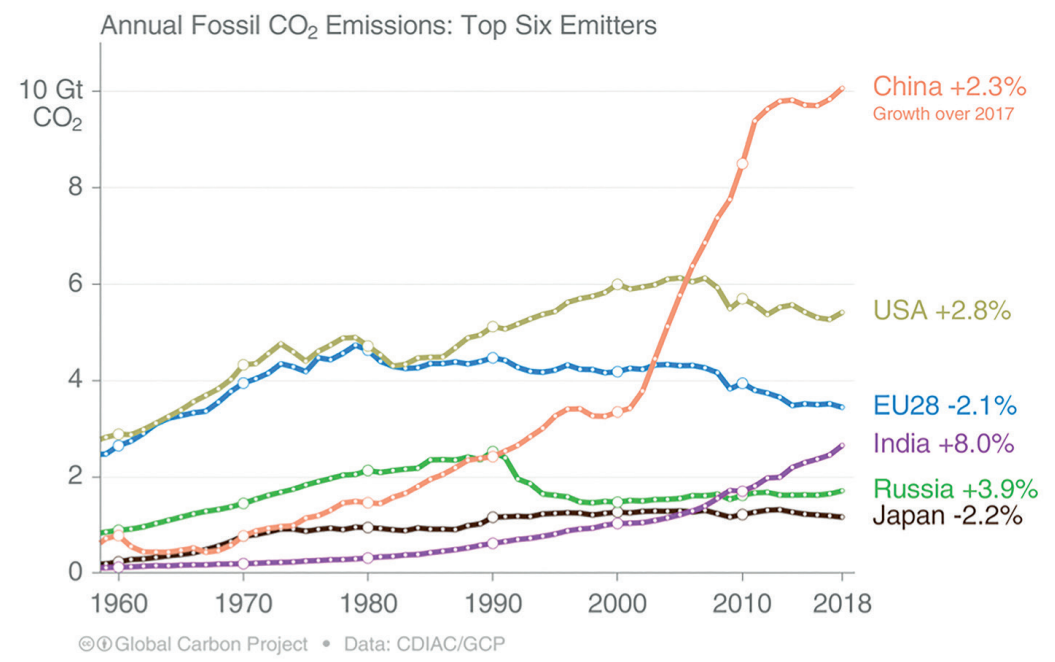

Figure 7.8: Évolutions des émissions de $\mathrm{CO}_{2}$ au cours des 60 dernières années pour les 6 principaux émetteurs mondiaux (Source: https://www.globalcarbonproject.org/carbonbudget/archive/2019/GCP_ CarbonBudget_2019.pdf).

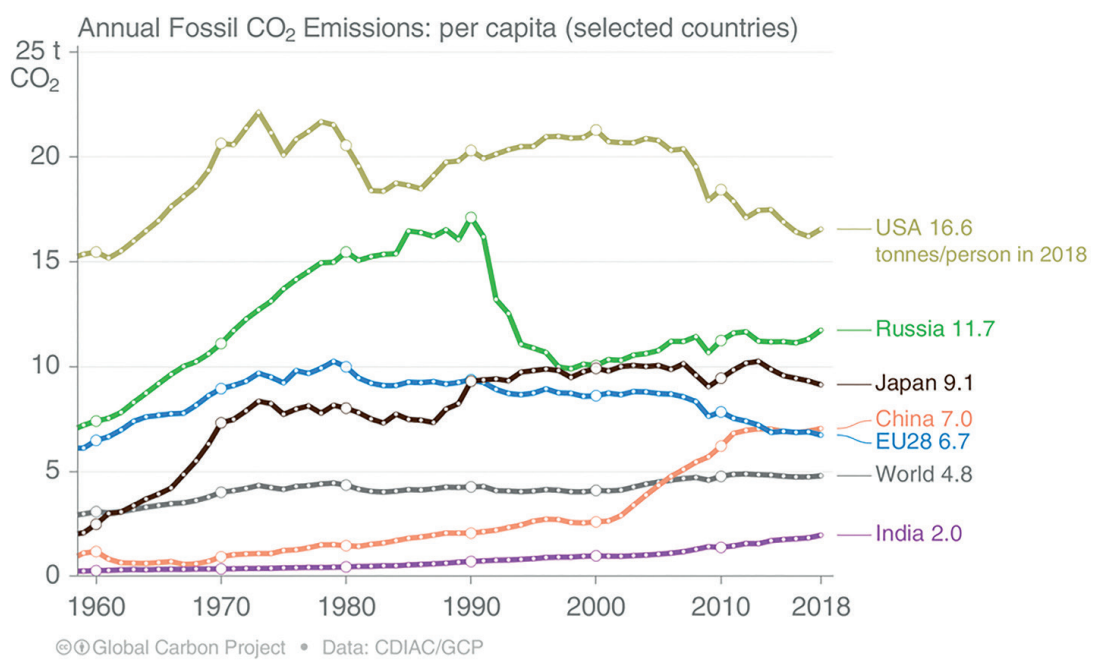

Figure 7.9: Évolutions des émissions de $\mathrm{CO}_{2}$ par habitant au cours des 60 dernières années pour les 6 principaux émetteurs mondiaux (Source: https://www.globalcarbonproject.org/carbonbudget/ archive/2019/GCP_CarbonBudget_2019.pdf). 
La répartition par secteur d'activité est fortement différente pour le méthane $\mathrm{CH}_{4}$. Assez peu de méthane est émis lors de la combustion des énergies fossiles. Par contre, du méthane est relâché dans l'atmosphère lors de leur extraction et de leur distribution. La contribution de ce secteur représente $24 \%$ des émissions anthropiques. Une contribution sensiblement équivalente est obtenue pour la gestion des déchets (20\%). Cela étant, le principal secteur d'émission du méthane à l'échelle mondiale est, de loin, l'agriculture qui contribue à près de la moitié (46\%) des émissions anthropiques de méthane dans le monde. Les émissions anthropiques de méthane dans le monde sont estimées à environ 350 millions de tonnes en 2017 (mise à jour régulière et plus d'information disponibles sur: https://www.globalcarbonproject.org/methanebudget).

La répartition par secteur pour le protoxyde d'azote témoigne, elle aussi, d'une part prépondérante de l'agriculture (55\%) et d'une contribution significative des feux de biomasse (24\%). La contribution du secteur industriel n'est, en revanche, que de $9 \%$. Les émissions mondiales de $\mathrm{N}_{2} \mathrm{O}$ sont de l'ordre de 10 millions de tonnes par an (mise à jour régulière et plus d'information disponible sur https://www. globalcarbonproject.org/nitrousoxidebudget/).

Finalement, si on met en regard les répartitions des secteurs émetteurs pour les principaux gaz à effet de serre, on voit que ces trois gaz ont, chacun, des secteurs dominants qui sont différents les uns des autres. Le méthane et le protoxyde d'azote sont émis par des secteurs d'activité qui n'auraient qu'une influence très réduite si on ne considérait que le $\mathrm{CO}_{2}$ : agriculture, déchets, extraction des énergies fossiles. En termes de masse, les quantités de gaz émises dans l'atmosphère chaque année par les activités humaines s'élèvent à environ 40 milliards de tonnes de $\mathrm{CO}_{2}, 350$ millions de tonnes de méthane, et 10 millions de tonnes de $\mathrm{N}_{2} \mathrm{O}$.

Avec ces chiffres, on constate que le $\mathrm{CO}_{2}$ est émis en quantité largement plus grande que les autres, et que les secteurs d'activités émettant du $\mathrm{CO}_{2}$ semblent ainsi les plus importants. Cependant, les gaz à effet de serre ne sont pas équivalents en termes de réchauffement climatique. Certains gaz ont une capacité plus forte à piéger le rayonnement infrarouge et donc à réchauffer le climat.

Il est donc nécessaire de prendre en compte cet aspect pour comparer les secteurs d'activité. Pour cela, on utilise un facteur de conversion approprié, qu'on appelle Pouvoir de Réchauffement Global ou PRG en abrégé. Le PRG d'un gaz est le forçage radiatif cumulé sur une durée donnée, c'est-à-dire la puissance radiative que le gaz renvoie vers le sol pendant cette durée et dont la valeur est mesurée relativement au $\mathrm{CO}_{2}$ sur la même durée.

Dans le tableau T1, le PRG est donné pour les principaux gaz à effet de serre sur une durée de 100 ans. Ainsi, pour le méthane, on estime un PRG de 28, ce qui signifie qu'un kilo de méthane a un impact 28 fois plus important sur l'effet de serre qu'un kilo de $\mathrm{CO}_{2}$. Le PRG du $\mathrm{N}_{2} \mathrm{O}$ est de 265, celui du CFC-11 est de plus de 4000. 


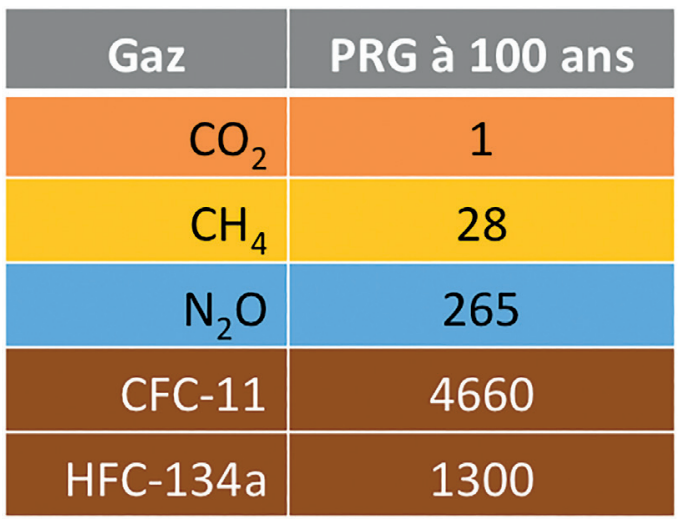

Tableau T1 Pouvoir de réchauffement global (PRG) des principaux gaz à effet de serre additionnel (Source: IPCC, 2013).

Ainsi en utilisant le PRG 100 pour calculer les émissions de gaz à effet de serre en équivalent $\mathrm{CO}_{2}$, on estime que les émissions de $\mathrm{CO}_{2}$, corrigées du PRG, contribuent effectivement à $76 \%$ des émissions de gaz à effet de serre, celles de méthane à $16 \%$; et celles de $\mathrm{N}_{2} \mathrm{O}$ à $6 \%$.

De même, si on applique ce calcul aux émissions totales de gaz à effet de serre en équivalent $\mathrm{CO}_{2}$ par secteur, la répartition fait apparaître l'ensemble des 8 secteurs considérés avec, bien sûr, une prépondérance des secteurs utilisant les énergies fossiles et émettant du $\mathrm{CO}_{2}$, mais montre aussi une contribution significative de l'agriculture et de l'extraction des énergies fossiles (Fig. 7.10).

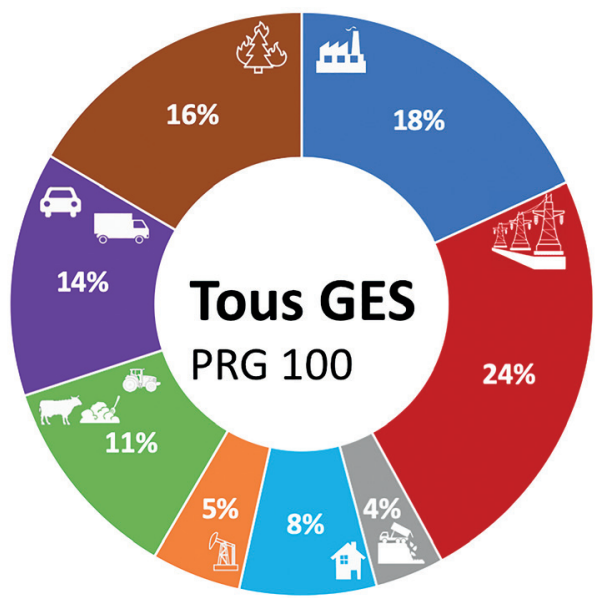

Figure 7.10: Répartition des gaz à effet de serre additionnel $\left(\mathrm{CO}_{2}, \mathrm{CH}_{4}\right.$ et $\left.\mathrm{N}_{2} \mathrm{O}\right)$ à l'échelle de la planète suivant les 8 secteurs d'activité considérés (Fig. 7.7) et tenant compte des pouvoirs de réchauffement global de chacun des gaz sur 100 ans. Source : EDGAR v432 - $2005^{65}$. 
L'analyse de cette répartition met en évidence les secteurs d'activité sur lesquels des efforts de réduction doivent être appliqués. Quelques-unes des solutions pour réduire les émissions seront exposées et discutées dans la dernière partie de cet ouvrage.

\subsection{De quelles façons notre gestion des écosystèmes terrestres contribue-t-elle au changement climatique? - N. de Noblet-Ducoudré}

L'objectif de cette partie est de montrer comment les usages que nous faisons de nos terres, contribuent au changement climatique.

Tout d'abord, que signifient «les usages que nous faisons de nos terres»? Depuis la sédentarisation des humains, il y a 8000 ans environ, nous avons défriché pour mettre en place des zones agricoles, des zones de pâturage pour nourrir le bétail. Nous avons construit des villes, et des routes. Nous avons déforesté pour exploiter le bois, et pour cultiver. Nous avons drainé des marécages pour en faire des zones habitables, récréatives ou agricoles. Dans les zones agricoles, nous labourons, nous fertilisons, nous arrosons, nous répandons des pesticides et des fongicides. Ce sont toutes ces activités qui sont regroupées sous le vocable «d'usages des terres». Aujourd'hui, plus de $70 \%$ de la surface terrestre libre de glace a été directement perturbée par les humains, par l'une de ces activités. C'est d'autant plus important que $50 \%$ de la population mondiale vit dans les zones urbaines, soit sur $1 \%$ des terres!

Toutes ces activités contribuent de deux façons au changement climatique.

Leur première contribution est d'émettre des gaz à effet de serre. Elles émettent, bien sûr, du dioxyde de carbone $\mathrm{CO}_{2}$, par exemple, lors d'une déforestation. Mais, elles émettent aussi deux autres gaz à effet de serre dont le pouvoir réchauffant est supérieur à celui du $\mathrm{CO}_{2}$. II s'agit du méthane, de formule chimique $\mathrm{CH}_{4}$, qui est en grande partie émis par l'élevage. Le troisième gaz est le protoxyde d'azote, encore appelé oxyde nitreux, de formule chimique $\mathrm{N}_{2} \mathrm{O}$. Les émissions de ce gaz résultent principalement de l'épandage d'engrais dans les zones agricoles. Si on cumule ces trois gaz à effet de serre, les usages que nous faisons de nos terres contribuent pour $23 \%$ aux émissions mondiales de gaz à effet de serre (Fig. 7.11).

Mais les écosystèmes contribuent aussi à piéger du $\mathrm{CO}_{2}$ par le mécanisme de la photosynthèse. La photosynthèse est le processus qui permet aux plantes, avec l'aide de la lumière, de transformer le $\mathrm{CO}_{2}$ de l'air et l'eau du sol en matière organique (c'est-à-dire en sucres) et en oxygène. Une partie du $\mathrm{CO}_{2}$, qui est fixé par photosynthèse, est relargué par la respiration des plantes et des sols. Cependant, si l'on fait le bilan mondial de la photosynthèse et de la respiration, on estime aujourd'hui 
qu'environ $29 \%$ des émissions totales de $\mathrm{CO}_{2}$ anthropique sont captées par les terres émergées, et donc stockées dans la biomasse des plantes et dans le sol. C'est ce qu'on appelle le puits biosphérique de carbone.

Si on fait le bilan net des puits et des sources de $\mathrm{CO}_{2}$ par les écosystèmes terrestres, on constate que $13 \%$ est émis mais $29 \%$ est capté. Les écosystèmes terrestres dans leur ensemble jouent donc aujourd'hui un rôle de puits de $\mathrm{CO}_{2}$ à l'échelle mondiale et contribuent, par ce biais, à ralentir le réchauffement climatique. Mais il faut faire attention, car ceci n'est pas vrai pour les émissions de $\mathrm{CH}_{4}$ et de $\mathrm{N}_{2} \mathrm{O}$. Les zones agricoles et de pâturage restent une source nette importante pour ces deux gaz à effet de serre.
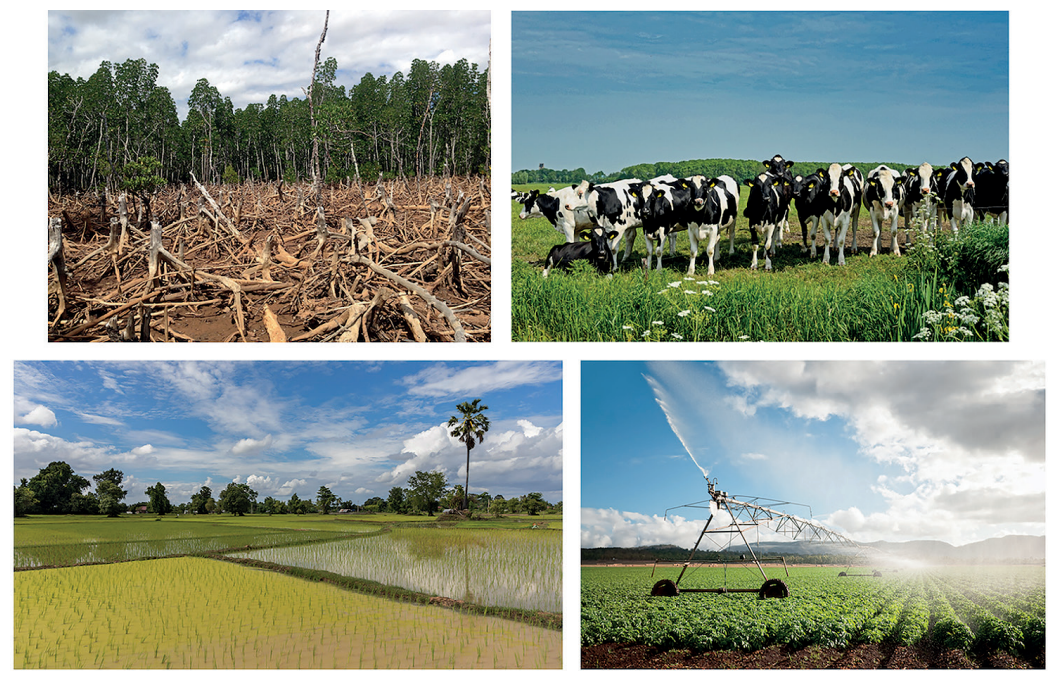

Figure 7.11: Vingt-trois pourcent des émissions mondiales de gaz à effet de serre sont liées aux activités agricoles. Exploitation des forêts, élevage et agriculture. Photos: wikimedia.commons et istock.

Nous avons vu précédemment que les usages que nous faisons de nos sols contribuent de deux façons au changement climatique. En fait, l'agriculture et la forêt n'échangent pas que des gaz à effet de serre avec l'atmosphère. Elles échangent aussi de l'eau et de la chaleur, c'est-à-dire de l'énergie.

Lorsque l'on transforme nos paysages, par déforestation, par exemple, ou par irrigation des champs, on perturbe ces échanges. Or, ces échanges sont importants parce qu'ils modifient la température du sol et de l'air, l'humidité de la masse d'air au-dessus de la surface et donc les précipitations. Pour illustrer ces échanges, prenons l'exemple d'une déforestation. Quand on coupe une forêt pour la remplacer, par exemple, par un champ, que se passe-t-il?

Une forêt est en général verte plus longtemps dans l'année. Plus dense, elle transpire plus aussi. En la coupant, on renvoie donc moins de vapeur d'eau vers 
l'atmosphère (Fig. 7.12). L'atmosphère s'assèche, ce qui peut conduire à une diminution des précipitations. Mais cette diminution des pluies ne se produit pas forcément là où la déforestation a eu lieu. Cette masse d'air plus sèche peut, en effet, être transportée par les vents loin de son origine.
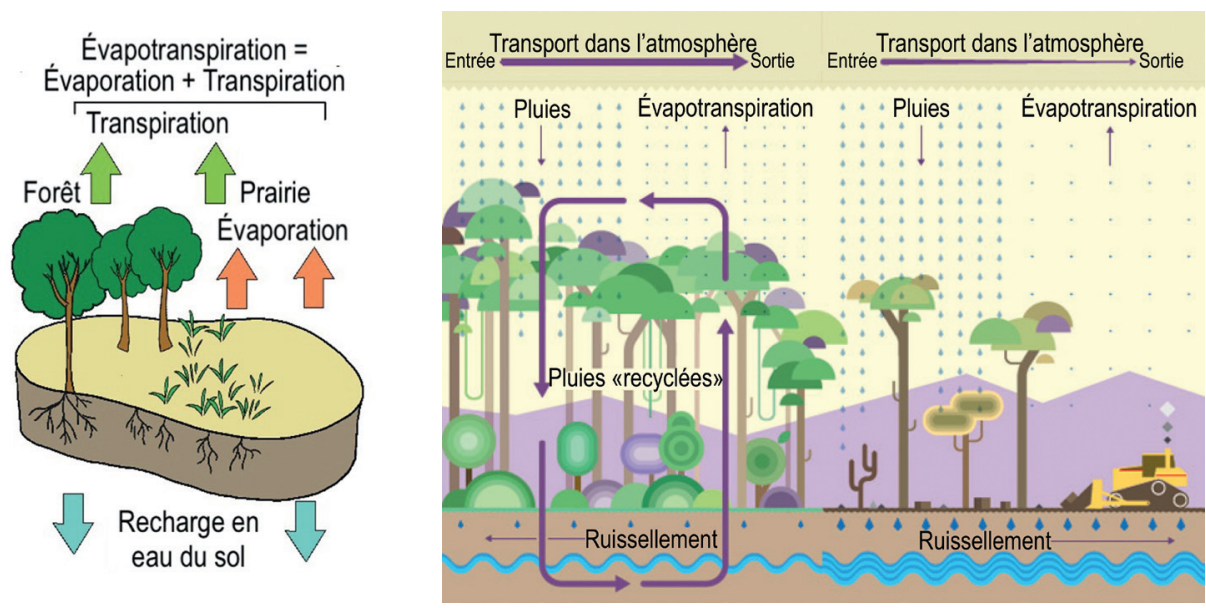

Figure 7.12: À gauche: mécanismes d'évaporation et de transpiration conduisant à l'évapotranspiration. Seuls la végétation et les êtres vivants dans leur ensemble transpirent et captent l'eau d'évaporation (pluie) et de condensation (rosée). L'eau dans un sol sans végétation ne peut que s'évaporer. Source: https://forestrypedia.com/evaporation-transpiration-and-evapotranspiration/ À droite: Illustration de l'importance de l'évapotranspiration des forêts, qui "pompent» l'eau de pluie infiltrée dans le sol et qui rechargent l'atmosphère en vapeur d'eau pour des pluies qui tomberont à d'autres endroits. En l'absence de forêts ou après déforestation, l'évapotranspiration est très réduite, l'eau tombée sur le sol a plus tendance à ruisseler, et les précipitations diminuent dû à l'atmosphère moins humide. Adapté de : Luiz E.O.C. Aragão, Environmental science: The rainforest's water pump, Nature 489 (7415), 217-8, September 2012 [1].

La transpiration n'est pas seulement un transfert de vapeur d'eau, c'est aussi un transfert de chaleur. Quand il y a transpiration, il y a refroidissement de la surface. On l'appréhende très bien de manière intuitive. Lors d'une randonnée, quand nous quittons un champ de blé, qui vient d'être fauché et qui est chaud, et que nous rentrons dans une forêt, nous ressentons de la fraîcheur. C'est de cela qu'il s'agit; la forêt prélève de l'énergie au sol pour transpirer et la température diminue.

Parlons maintenant du moment où la vapeur d'eau se condense en altitude dans l'atmosphère pour former de la pluie. La chaleur qui était piégée dans la vapeur d'eau est alors relâchée dans l'atmosphère. Lors d'une déforestation, puisque la transpiration diminue, on réchauffe donc la surface, mais on refroidit la haute atmosphère puisqu'il y a moins de vapeur d'eau à condenser.

Mais ce n'est pas tout! Lorsque nous coupons une forêt, nous augmentons l'albédo de la surface, c'est-à-dire sa capacité à réfléchir le rayonnement solaire incident. Une forêt est, en effet, souvent plus sombre que la zone agricole qui la remplace. Moins d'énergie absorbée se traduit par un refroidissement de la surface. 
Ainsi, si nous coupons des arbres, nous réchauffons le sol parce que la transpiration diminue, mais nous le refroidissons parce que son albédo augmente! Ces effets sont contradictoires. Quel en est le résultat net? De manière générale, en période estivale, la forêt sera plus fraîche tandis qu'en période hivernale, elle sera plus chaude.

Résumons, en modifiant le couvert végétal et en usant de nos terres, nous influons à la fois sur les émissions et les absorptions de gaz à effet de serre, et sur les échanges d'énergie et de vapeur d'eau entre la surface et l'atmosphère.

En modifiant la concentration en gaz à effet de serre de l'atmosphère, on affecte le climat à l'échelle mondiale, on joue donc sur l'intensité du réchauffement climatique global.

En perturbant les échanges d'eau et de chaleur, on affecte le climat à l'échelle régionale, là où le couvert végétal est modifié. On affecte également les régions voisines en modifiant la circulation des vents et la composition hydrique et thermique des masses d'air.

\subsection{Les indicateurs du changement climatique aux grandes échelles - M. Delmotte}

\subsubsection{Météorologie, climat et systèmes d'observation}

Avant de s'intéresser plus en détail au changement climatique, il est important de bien définir les notions dont on parle. En premier lieu, il est important de rappeler ce qu'on entend par météorologie et climat.

Dans l'esprit de tout un chacun, ces deux termes sont souvent peu différenciés. On les identifie au «temps», et ils ne sont pas toujours précisément définis, même dans les dictionnaires courants. Ils ont des points communs, mais ils se rapportent à des référentiels temporels et spatiaux qui sont différents dans les deux cas.

La météorologie se caractérise par une très grande variabilité dans le temps et dans l'espace. C'est la prévision du temps sur des échéances courtes et à l'échelle locale, c'est le temps qu'il fait. La notion de météorologie s'applique surtout sur des temps courts, de quelques heures à quelques jours, et sur des zones d'espaces réduites, quelques dizaines à quelques centaines de $\mathrm{km}^{2}$. Une prévision météorologique est d'autant plus fiable qu'elle porte sur une durée courte et une zone géographique réduite. Elle se caractérise par un certain nombre de paramètres physiques comme la température, les précipitations, le vent, la pression, l'humidité, etc.

Le climat est défini comme un état moyen de la météorologie, qui est calculé sur une période de référence de 30 ans. La variabilité du climat est peu marquée sur des périodes de temps caractéristiques de la vie humaine, de l'ordre de 50 à 100 ans. Le climat peut donc aussi se définir par type (tempéré, désertique, chaud et humide, continental), et caractérise de grande zones géographiques réparties selon les 
bandes de latitude. Le climat, c'est le temps qu'il devrait faire, celui auquel on fait référence quand on parle de moyennes annuelles, saisonnières ou mensuelles.

Une fois définies les notions de base, les composantes et le fonctionnement du système climatique, on peut alors s'intéresser à l'évolution du climat à grande échelle, temporelle ou spatiale et, en particulier, au changement climatique actuel.

Pour étudier l'évolution du climat, il faut être capable de suivre l'évolution temporelle d'une multitude de paramètres physiques, chimiques ou biologiques pertinents comme par exemple, la température dans l'air ou dans les océans, la pression, la composition chimique de l'atmosphère incluant notamment les concentrations en gaz à effet de serre, les vents, les courants marins, la couverture de neige et ou de glace des continents et des océans, la répartition de la végétation, etc.

Pour ce faire, on peut distinguer trois grandes approches:

- l'observation en temps réel;

- la reconstitution ou la reconstruction des variations passées du climat à partir d'archives climatiques, qu'elles soient naturelles ou d'origine humaine;

- la simulation à partir de modèles numériques du climat passé, présent ou futur, ce qui ne sera pas traité ici mais fera l'objet des sections 9.2 et 9.3.

Les observations se font in situ, à partir de capteurs ou d'instruments dédiés à un ou plusieurs types de mesures et souvent dépendants de la méthode mise en œuvre. Au départ, les sites d'observation ont été choisis en fonction d'objectifs scientifiques pour répondre à des questions précises.

Pour les études climatologiques, il faut disposer, autant que faire se peut, d'une couverture observationnelle à différentes échelles, locale, régionale, nationale et globale avec une bonne résolution spatiale et temporelle. Dans la pratique, il est évidemment plus compliqué de mettre en œuvre une telle couverture observationnelle sur les océans et dans les zones difficiles d'accès. Souvent, les scientifiques ont construit des réseaux d'observation nationaux, voire internationaux.

Ces réseaux mettent à disposition des méthodologies, qui sont validées par les scientifiques eux-mêmes et qui donnent lieu à des mesures homogènes avec une procédure garantie de contrôle de qualité, rendant alors possible la mutualisation des données. Le développement de tels réseaux d'observation, le perfectionnement des instruments et l'amélioration de leur précision permettent aujourd'hui de mieux appréhender les observations et de mieux comprendre la variabilité des systèmes naturels.

Au niveau européen, un réseau a ainsi été mis en place sous la forme d'une grande infrastructure de recherche pour suivre les concentrations des gaz à effet de serre. Ce réseau, appelé ICOS, regroupe des stations de mesure aussi bien pour l'atmosphère que pour l'océan et les écosystèmes terrestres. Les stations, qui sont 
gérées par chaque pays membre de l'infrastructure, doivent toutes, pour être labellisées, respecter un cahier des charges mis au point et approuvé par l'ensemble de la communauté scientifique. La méthodologie de mesure et de traitement des données est la même pour chaque type de station, que la mesure soit faite dans l'atmosphère, dans l'océan ou sur les écosystèmes terrestres.

Cela étant, les observations régulières, qui permettent de suivre l'évolution du climat, sont encore relativement récentes. Ainsi, les premières données météorologiques sur la température ne remontent qu'à 150 ans. À titre d'exemple, la figure 7.13 représente l'évolution de la température moyenne mesurée en France métropolitaine depuis un peu plus d'un siècle. On constate que cette température n'a cessé d'augmenter surtout à partir des années 1970.

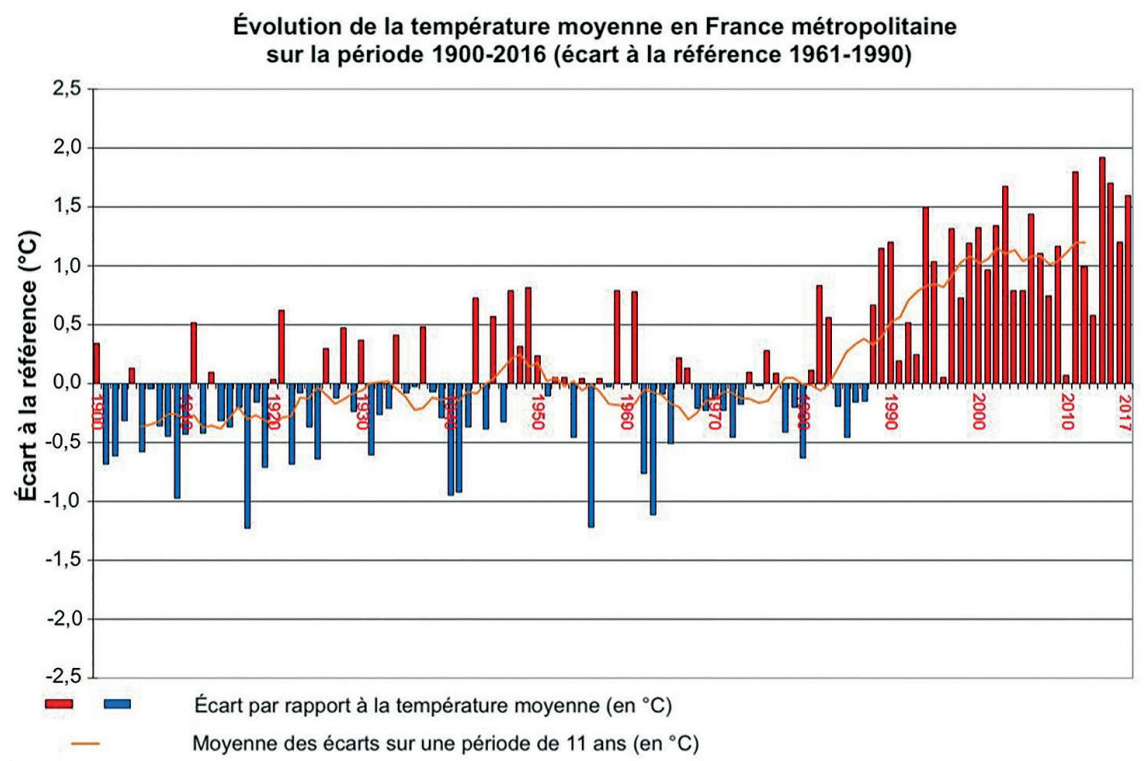

Figure 7.13: Évolution de la température moyenne mesurée en France métropolitaine sur la période 1900-2016 (écart à la référence 1961-1990). Les bâtonnets représentent les écarts à la référence pour chacune des années sur la période considérée. La courbe «lissée» en rouge est obtenue en prenant une moyenne des écarts sur 11 ans. Source : Météo France ${ }^{66}$.

\subsubsection{Observation des changements en cours}

Regardons un certain nombre d'observations qui montrent que les changements climatiques actuels sont détectables et illustrent cette conclusion du rapport du GIEC de 2013: "Le réchauffement du système climatique est sans équivoque,

66. http://www.donnees.statistiques.developpement-durable.gouv.fr/lesessentiels/indicateurs/e13. html 
et depuis les années 1950, beaucoup des changements observés sont sans précédents depuis des décennies jusqu'à des millénaires».

Qu'observe-t-on? Les températures augmentent non seulement de façon locale, comme nous venons de le voir avec la courbe de suivi des températures à Paris, mais elles augmentent aussi régionalement et globalement avec des disparités d'une zone géographique à l'autre. Le réchauffement global, qui est ainsi mesurable sur les continents comme dans les océans, est de plus d'un degré depuis le début de l'ère industrielle (sur les continents).

Les événements extrêmes se font de plus en plus fréquents et de plus en plus intenses. On constate une augmentation de la fréquence des canicules, des événements de pluie intense. C'est, par exemple, le cas des épisodes cévenols en France. Ces dernières années, l'intensité et la violence accrues des vents a été mise en évidence dans un certain nombre de cyclones. Les océans s'acidifient et la température des océans augmente y compris en profondeur avec des conséquences directes sur la vie des écosystèmes, et les coraux en particulier (voir sections 5.3.1 et 8.3).

On constate que le niveau de la mer monte, de plus de 20 cm depuis 1900 (Fig. 7.14).

Les zones de glace disparaissent ou reculent (Fig. 7.15). C'est le cas des glaciers de montagne et de la banquise dans l'hémisphère nord. Ainsi, les passages de Nord-Est et du Nord-Ouest sont parfois libres de glace, la calotte polaire du Groenland fond, la couverture de neige au sol est moindre et dure moins longtemps.

Les concentrations en gaz à effet de serre ont fortement augmenté et atteignent des niveaux qu'on n'avait pas connus au cours des 800000 dernières années. Le niveau actuel du $\mathrm{CO}_{2}$ atmosphérique est de 407 ppm mesuré en 2018. Pour mieux comprendre le changement climatique actuel et évaluer son impact potentiel ainsi que son intensité, il est utile de le mettre en perspective avec les variations passées du climat.
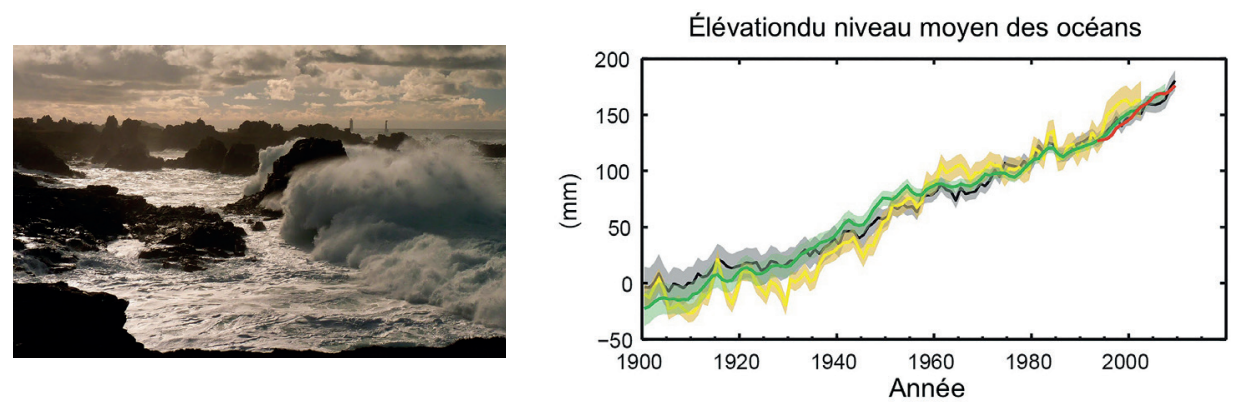

Figure 7.14: À gauche: la violence des tempêtes observées le long des côtes françaises (ici au large d'Ouessant). Source: @wikimedia.commons. À droite: l'évolution du niveau moyen des océans depuis 1900. Source: «Rapport IPCC WG1 (2013) AR5 résumé à l'intention des décideurs » 67. 

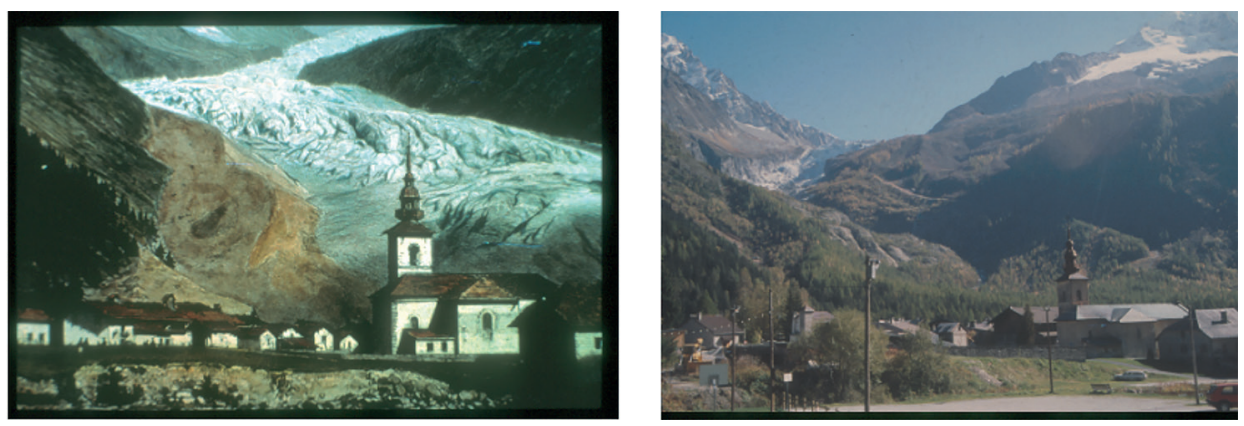

Figure 7.15: Illustration du recul du glacier des Bossons (vallée de Chamonix). À gauche: photo prise en 1864 (auteur inconnu). À droite: photo prise en 1995 (droits réservés).

\subsubsection{Archives paléoclimatiques et observations d'aujourd'hui}

Les reconstitutions paléoclimatiques permettent d'étendre l'étude des variations climatiques au-delà des périodes récentes, aussi loin que les archives le permettent. On peut distinguer deux types d'archives du climat, les archives historiques et les archives naturelles. Dans aucun cas évidemment, nous ne disposons d'une estimation directe d'un paramètre physique ou chimique comme par exemple la température. Nous verrons, en fait, dans la section 9.1 de cet ouvrage, comment sont exploitées ces archives climatiques.

Pour avoir une indication quantitative des températures d'été, on peut, par exemple, utiliser les dates de début des vendanges, qui sont un indicateur de la maturation du raisin et dépendent des conditions climatiques. De même, les périodes de famine ou d'épidémie sont souvent liées à des épisodes climatiques marqués, une sécheresse, une inondation ou une période de froid intense. En utilisant les écrits, les gravures, les fresques correspondant à une période donnée, on peut ainsi obtenir des indications sur les conditions climatiques prévalant à cette période en une zone donnée. II s'agit alors d'archives historiques.

Les archives naturelles gardent souvent, elles aussi, l'historique des conditions du milieu dans lequel elles se sont formées ou développées. Par exemple, la composition chimique de la neige et sa composition isotopique dépendent de la température à laquelle elle s'est formée. La croissance des arbres dépend de la température et du contenu en eau du sol, et cette dépendance se retrouve, entre autres, enregistrée par la taille des cernes de chaque arbre.

Dans ces archives naturelles, on trouve aussi trace de l'évolution du vivant qui dépend de l'environnement climatique. Les sédiments, qu'ils soient lacustres ou marins, renferment une multitude de traces du vivant, qui se sont accumulées au fond des lacs ou des océans au fur et à mesure de l'accumulation des couches sédimentaires. II s'agit de pollen, de petits coquillages ou de crustacés d'eau douce dans 
les lacs, de petits coquillages microscopiques - les foraminifères - dans les sédiments marins.

La démarche scientifique, développée ici pour les archives naturelles, est la suivante:

- selon le milieu que l'on souhaite étudier, atmosphère, océan, biosphère..., on va d'abord choisir l'archive la plus appropriée;

- une mission de collecte de l'archive sur le terrain est ensuite souvent nécessaire, laquelle prend soin de ne pas altérer la matrice naturelle;

- on procède à l'analyse de l'archive, souvent précédée d'une phase de préparation et/ou de traitement de l'échantillon qui passe par des analyses physiques, chimiques ou autres d'éléments conservés dans la matrice de l'archive;

- on passe ensuite à une phase d'interprétation des données pour transcrire ces données analysées en termes de paramètres climatiques, on parle de fonction de transfert qui nécessite elle-même de pouvoir définir une chronologie;

- on peut alors comparer les différentes séries paléo-climatologiques entre elles et avec les données récentes.

L'analyse des glaces polaires d'EPICA-DOME C en Antarctique (Fig. 7.16, à gauche) a ainsi permis de reconstruire plus de 800000 ans de climat passé, incluant l'évolution des concentrations en dioxyde de carbone et de méthane sur toute la période passée et permettant la comparaison avec les mesures courantes [2].
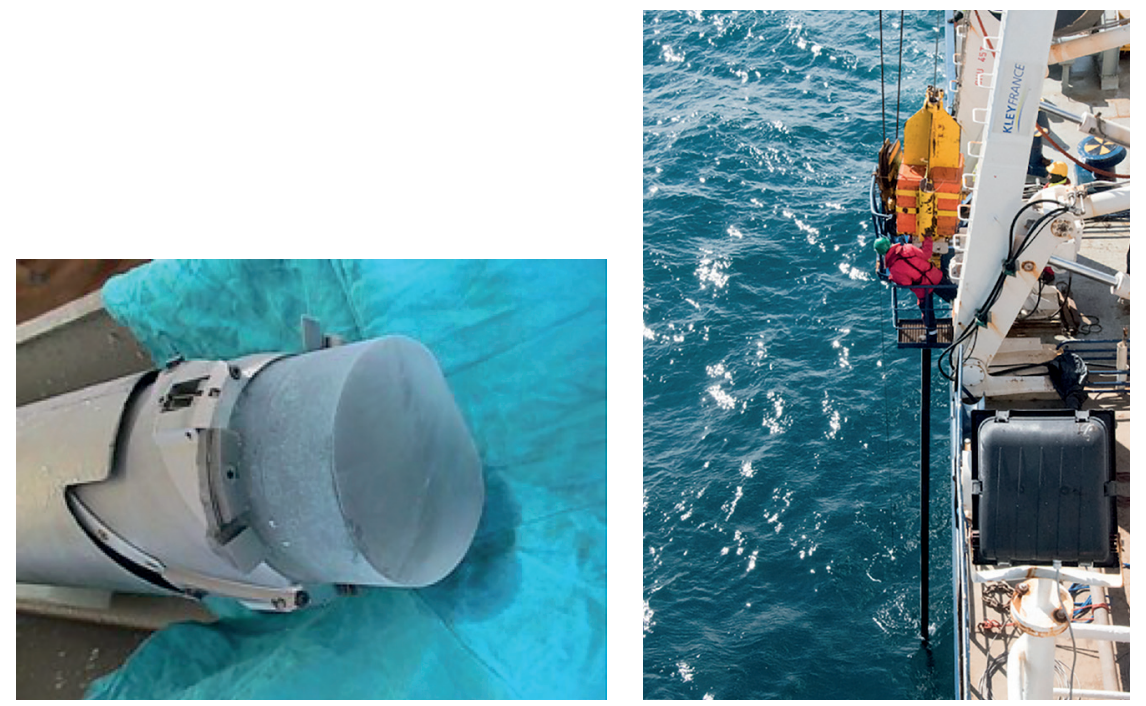

Figure 7.16: Reconstitutions du climat passé à partir d'archives naturelles. À gauche: l'analyse des glaces polaires en Antarctique (Source: CNRS Photothèque /Augustin Laurent). À droite: le carottage en mer pour l'extraction de carottes sédimentaires marines (Source: Ludovic Devaux, UMR CNRS EPOC). 
Les analyses de carottes sédimentaires marines (Fig. 7.16, à droite, dans le cadre de la mission MD218 CROTALE à bord du N.O. Marion Dufresne) permettent d'obtenir des informations, entre autres, sur l'évolution de la température de l'océan à différents niveaux de profondeur, sa salinité sur des échelles de temps qui peuvent couvrir plusieurs millions d'années [3].

Toutes ces informations paléoclimatiques sont essentielles pour mieux comprendre les changements actuels. Elles sont également cruciales pour la validation des modèles de climat qui sont utilisés pour simuler le climat futur.

\section{Références}

[1] L.E.O.C. Aragão. 2012. Environmental science: The rainforest's water pump, Nature 489 (7415), 217-8. DOI: https://doi.org/10.1038/nature11485.

Voir aussi : https://www.rtbf.be/info/societe/detail_ucl-decouverte-majeure-sur-Ievapotranspiration-des-forets?id=9418633.

[2] V. Masson-Delmotte, A. Landais. 2014. Explorer l'évolution du climat grâce aux glaces de l'Antarctique et du Groenland. Reflets phys. 41, 12-17. DOI: https://doi.org/10.1051/ refdp/201441012.

[3] V. Scao. 2013. Détermination des paléotempératures des océans à partir du rapport $\mathrm{Mg} / \mathrm{Ca}$ des foraminifères. Sciences de l'ingénieur [physics]. dumas-01420518. https:// dumas.ccsd.cnrs.fr/dumas-01420518. 


\section{Chapitre 8}

\section{Impacts des changements climatiques}

Depuis la fin du siècle dernier, le réchauffement climatique se manifeste non seulement par l'élévation de température moyenne, mais aussi par des sécheresses chroniques ou à répétition, des précipitations d'ampleur accrue, et des tempêtes de plus en plus dévastatrices. Ce chapitre présente des exemples de telles manifestations en France et dans différentes régions du monde, ainsi que leurs évolutions prévisibles pour les années à venir. II décrit aussi de façon plus précise les impacts du changement climatique sur les cultures et la végétation, sur les océans et les écosystèmes marins et sur la santé humaine. Comme nous le verrons, peut-être une menace forte et étendue porte-t-elle dès à présent sur les espèces marines en raison de l'acidification et de la désoxygénation des océans. Nous verrons aussi que l'élévation de température se traduit par un risque accru de mortalité pour les populations humaines. En Europe, les populations vivant dans les régions du sud sont les plus menacées. Le changement climatique est aussi susceptible d'augmenter l'incidence des maladies à transmission vectorielle dans des zones aujourd'hui tempérées.

\subsection{Manifestations du changement climatique en France et ailleurs dans le monde - S. Szopa}

Selon le rapport spécial du GIEC sur les conséquences d'un réchauffement à $1,5^{\circ} \mathrm{C}$, la température moyenne à la surface du globe a augmenté d'environ $1^{\circ} \mathrm{C}$ en France depuis 1850. En France, Météo France rapporte une hausse de la température moyenne observée de $1,7^{\circ} \mathrm{C}$ depuis 1900. La décennie 2010-2019 a été plus chaude de $0,19^{\circ} \mathrm{C}$ que la décennie $2000-2009$, elle-même plus chaude de $0,47^{\circ} \mathrm{C}$ que la décennie 1951-1990 (Fig. 8.1). La température moyenne s'est ainsi élevée de 0,66 C entre 1951 et 2019. Les cinq dernières années sont les plus chaudes jamais observées depuis 1850 et, avec un écart de $+2,3^{\circ} \mathrm{C}$ par rapport à la moyenne 1951-1990, l'année 2020 a été en France métropolitaine l'année la plus chaude depuis 1900. 


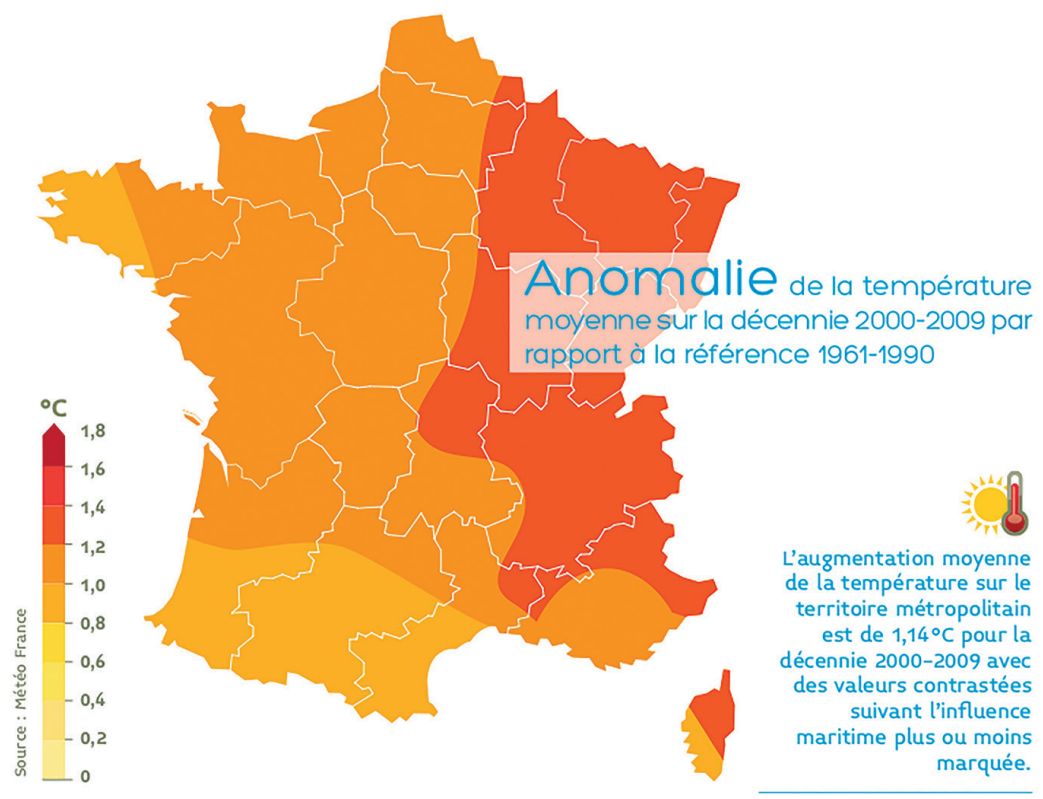

Figure 8.1: Anomalie de température: différence entre la moyenne de température de la décennie 2000-2009 et la moyenne de température de la décennie 1951-1990. Notons qu'il est nécessaire de moyenner sur au moins dix ans de données météorologiques pour pouvoir étudier les variations de climats. Source: Météo France ${ }^{68}$.

Cette élévation de température s'accompagne d'effets sur les précipitations, les vagues de chaleur, l'enneigement, les sécheresses, et augmente la fréquence des événements extrêmes.

Les précipitations ont évolué différemment d'une région à l'autre. Ainsi, sur la période 1959-2009, on constate généralement une hausse des précipitations annuelles dans la moitié nord et une baisse dans la moitié sud. Une tendance à plus de pluie au printemps et en automne sur toute la France et une baisse des cumuls sur les régions proches de la Méditerranée malgré une intensification des pluies extrêmes dans les régions méditerranéennes françaises.

Le pourtour méditerranéen fait partie des régions particulièrement vulnérables au changement climatique en raison de sa topographie particulière (mer fermée bordée de reliefs et au nord d'immenses déserts). Typiques de ce climat méditerranéen, les fortes précipitations automnales, liées à des orages d'automne induits par une mer encore chaude et une atmosphère plus froide, et pouvant être à l'origine de crues éclairs, se sont fait plus nombreuses et plus intenses ces dernières années comme l'indique la figure 8.2.

68. https://www.ecologie.gouv.fr/sites/default/files/ONERC_Brochure_impacts_en_France_PDF_ WEB.pdf 


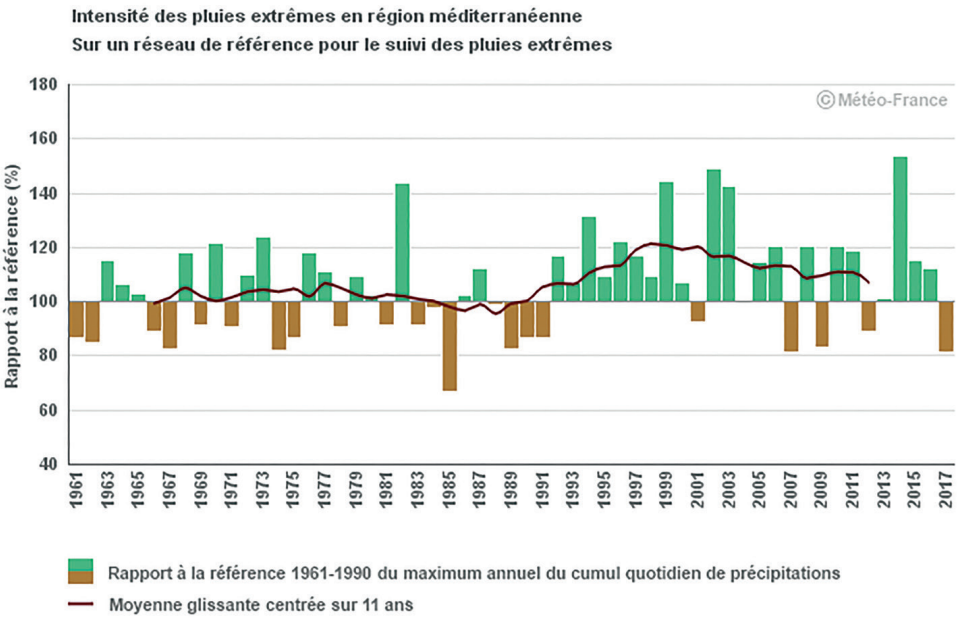

Figure 8.2: Intensité des événements pluvieux extrêmes méditerranéens de 1961 à 2017 par rapport à une moyenne sur une période de référence de 1961 à $1990^{69}$.

Depuis le milieu du $X X^{e}$ siècle, on observe aussi des évolutions de la fréquence et de l'intensité des événements extrêmes. Ainsi, on assiste à une augmentation du nombre de journées chaudes pour lesquelles les températures maximales sont supérieures à $25^{\circ} \mathrm{C}$. Les vagues de chaleur sont devenues plus fréquentes et plus intenses, par exemple, en 2019, les vagues de chaleur exceptionnelles ont provoqué des températures inédites de $46^{\circ} \mathrm{C}$ dans le sud de la France. L'évaporation des sols s'accentue, induisant des sécheresses plus fréquentes et plus intenses. Le nombre de jours de gel diminue et la durée de la période d'enneigement en moyenne montagne diminue également. En revanche, aucune tendance marquée ne se dégage sur l'évolution des tempêtes.

En France, I'ONERC (Observatoire National sur les effets du réchauffement climatique) suit une trentaine d'indicateurs mesurables et témoignant des effets du changement climatique pour en documenter la progression et adapter les politiques d'adaptation ${ }^{70}$.

À l'avenir, on s'attend à un renforcement de ces manifestations, comme l'illustre la figure 8.3, avec également une augmentation des risques dont l'occurrence est liée, en tout ou partie, aux paramètres climatiques comme les feux de forêt ou les inondations. Au-delà de 2050, l'intensification ou non de ces effets dépend, pour beaucoup

69. b) https://www.ecologie.gouv.fr/impacts-du-changement-climatique-atmosphere-temperatures-etprecipitations

70. a) https://meteofrance.com/changement-climatique/observer/le-changement-climatique-en-france b) https://www.ecologie.gouv.fr/impacts-du-changement-climatique-atmosphere-temperatures-etprecipitations

c) https://www.ecologie.gouv.fr/sites/default/files/ONERC_Brochure_impacts_en_France_PDF_ WEB.pdf 
d'entre eux, des politiques mondiales d'atténuation des émissions de gaz à effet de serre mises en place dans les décennies à venir. C'est moins le cas pour les risques liés à la montée des eaux océaniques, car cette variable répond de manière décalée dans le temps aux perturbations climatiques.

On note également que les territoires d'outre-mer vont, de par leur situation géographique, être affectés par des risques différents de ceux de la métropole. Pour les territoires situés dans les régions tropicales et régulièrement frappés par des cyclones tropicaux, aucune tendance certaine ne s'est dégagée quant à une augmentation de leur nombre au cours des dernières décennies (ONERC basé sur GIEC, 2014). En revanche, il est quasiment certain que l'intensité et la fréquence des cyclones les plus forts ont augmenté depuis 1970 dans le bassin Atlantique Nord, dans lequel se situent les Antilles. Pour ce qui concerne l'avenir, il subsiste encore de grandes incertitudes sur l'évolution à attendre des cyclones tropicaux, même pour les scénarios avec les plus fortes concentrations de gaz à effet de serre. II est probable que d'ici la fin du XXle siècle, le nombre de cyclones soit diminuera, soit ne changera pas, tandis que la force maximum des vents et les quantités de pluie associées devraient augmenter. Autrement dit, les cyclones risquent d'être plus dangereux (GIEC, 2014).

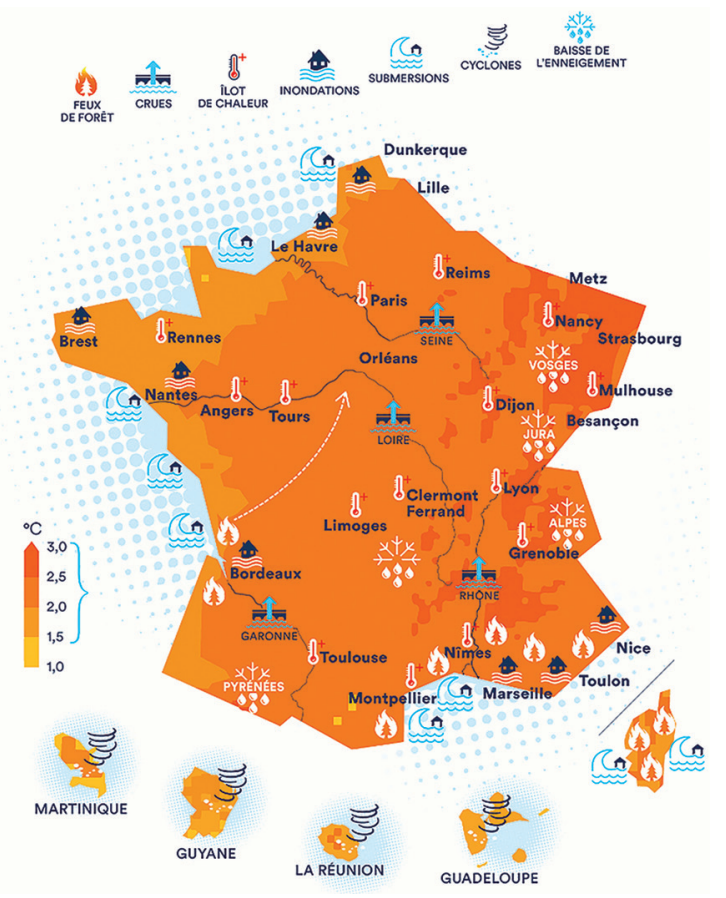

Figure 8.3: Carte des impacts liés au changement climatique déjà visibles et à venir en 2050. Source: Météo France ${ }^{71}$.

71. https://www.ecologie.gouv.fr/observatoire-national-sur-effets-du-rechauffement-climatiqueonerc 
Nous venons de voir très brièvement les effets du changement climatique en France, mais qu'en est-il ailleurs dans le monde? Tout d'abord, il faut noter que le réchauffement se manifeste de manière hétérogène. II est notamment plus fort au-dessus des continents et aux pôles (Fig. 8.4).

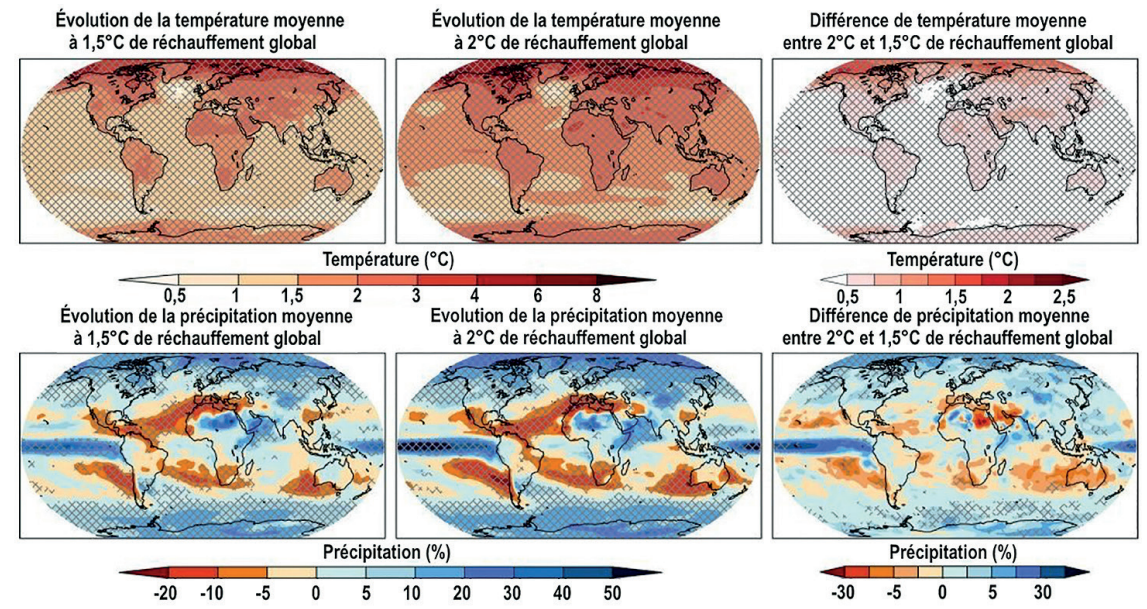

Figure 8.4: Projection des changements de température moyenne (en haut) et de précipitations moyennes (en bas) pour un réchauffement moyen planétaire de $1,5^{\circ} \mathrm{C}$ (à gauche) et $2^{\circ} \mathrm{C}$ (au milieu) en prenant pour référence la période préindustrielle (1861-1880). Les zones hachurées représentent les zones où plus de deux tiers des modèles (au moins 18 sur 26) s'accordent sur le signe du changement et sont donc les zones où les résultats sont considérés comme robustes. Source: SR1.5 IPCC, Chapitre $3^{72}$.

Les changements de précipitations vont également être hétérogènes avec des renforcements des précipitations sur les régions recevant déjà à l'heure actuelle beaucoup de précipitations, comme le nord de l'Europe ou certaines régions tropicales humides, et un renforcement de l'aridité dans des zones déjà sèches telles l'Afrique et le sud de l'Europe.

Certaines zones vont subir des changements plus importants que d'autres. C'est le cas des zones littorales ou des zones plates et de basse altitude, en Asie notamment, qui vont être particulièrement impactées par l'élévation du niveau marin favorisant des inondations. Or, plus de $50 \%$ de la population mondiale vit à moins de 100 km des côtes. Les îles sont aussi, à l'évidence, fortement menacées par la montée des eaux avec, d'ores et déjà, des migrations liées à cette montée des eaux ${ }^{73}$.

La fonte des glaciers menace également l'approvisionnement en eau potable de régions entières. C'est, en particulier, le cas de la région himalayenne où la neige et l'eau des glaciers sont la source principale d'eau des rivières asiatiques dont dépendent plus d'un milliard d'individus en Inde, en Chine et au Pakistan.

72. https://www.ipcc.ch/sr15/chapter/chapter-3/

73. http://storymaps.esri.com/stories/2017/climate-migrants/index.html 


\subsection{Comment le climat impacte-t-il les écosystèmes terrestres? - N. de Noblet-Ducoudré}

Nous avons vu précédemment comment l'Homme peut perturber le climat en perturbant les écosystèmes, à travers les usages qu'il fait des terres (voir section 7.4). Nous allons voir maintenant comment le changement climatique affecte les écosystèmes, et réfléchir aux conséquences que ces impacts peuvent avoir, en retour, sur le climat.

On a tendance à résumer le changement climatique au réchauffement global, mais ce n'est pas la variation progressive de la température moyenne annuelle qui va affecter la croissance des plantes. Les plantes et les êtres vivants dans leur ensemble sont sensibles aux variations saisonnières et diurnes de température, à la disponibilité en eau, aux événements extrêmes comme les canicules ou les inondations.

Examinons deux variables climatiques, la température et les pluies, et regardons comment leur évolution impacte les écosystèmes.

\subsubsection{Les changements de température}

- Le développement des espèces végétales est piloté par la température. L'apparition des nouvelles feuilles ou des fleurs, par exemple, dépend des conditions thermiques des semaines et des mois qui précèdent.

II y a quatre aspects du changement climatique qui sont importants pour les plantes: les hivers plus doux, les printemps et automnes plus chauds, les températures extrêmes en été qui sont parfois trop chaudes (Fig. 8.5).

- Le réchauffement climatique, avec ses hivers plus doux et ses printemps plus chauds, a avancé la reprise d'activité des êtres vivants au printemps. Dans nos jardins, nous voyons les bourgeons sortir plus tôt qu'il y a dix ans par exemple, et les plantes fleurir plus tôt. Dans le monde agricole, les activités démarrent plus tôt: le maïs, par exemple, était semé mi-mai dans les années 1970, alors qu'on le sème mi-avril aujourd'hui, voire plus tôt.

Mais les hivers trop doux peuvent aussi avoir des effets néfastes. Certaines plantes ont besoin d'un certain nombre de jours de froid pendant l'hiver, c'est ce qu'on appelle la dormance qui correspond à un état d'inactivité physiologique qui permet à ces plantes de survivre à l'hiver sous nos latitudes. Un manque de froid hivernal peut provoquer un retard dans la reprise d'activité de la végétation. C'est ce que l'on voit déjà pour certains arbres fruitiers, par exemple, les pommiers dans certaines régions de France où la levée de dormance se fait aujourd'hui 15 jours plus tard qu'en 1960. 


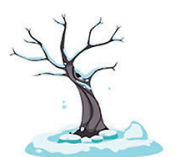

Hivers plus doux, Printemps plus chauds

Reprise d'activités plus précoce Levée de dormance des arbres plus tardive Semis du maïs avancé ...
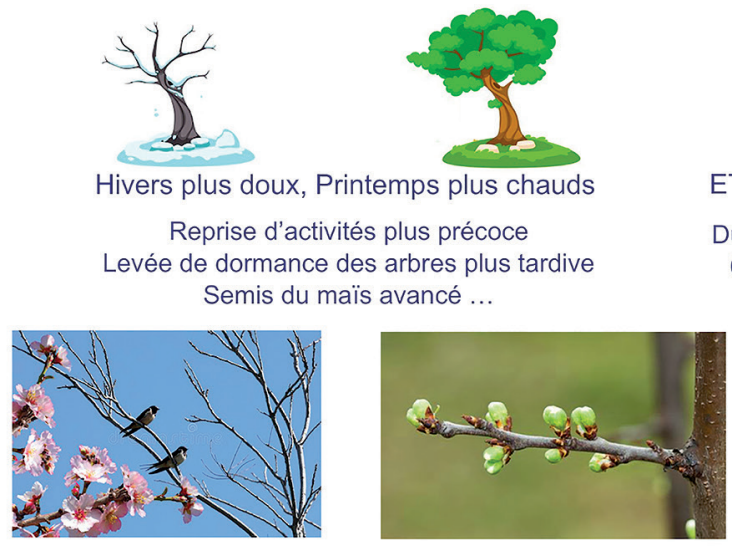

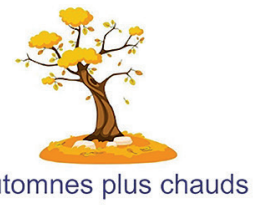

Durée de la saison de croissance plus longue (de 1 à 3 semaines dans les zones vertes) «Verdissement de la planète »

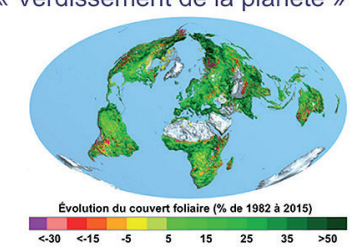

Figure 8.5: Influence du réchauffement climatique sur la végétation (Source: Nathalie de NobletDucoudré). Verdissement de la planète (Source: Boston University / Ranga Myneni).

- À l'automne, le réchauffement a tendance à retarder le départ des oiseaux migrateurs et la fin de l'activité végétale. C'est, entre autres, pour cela que l'on parle du verdissement de la planète! La durée de la saison de croissance de la végétation naturelle augmente. Elle augmente d'autant plus que l'on se trouve dans des régions froides, dans les zones boréales ou en altitude. Dans ces zones, le froid limite de moins en moins la croissance de la végétation. II existe même des régions où les forêts se mettent à pousser naturellement alors que les arbres avaient autrefois du mal à survivre.

- Par contre, si l'on s'intéresse à l'agriculture, au lieu de s'allonger, le cycle cultural est plus court aujourd'hui que dans le passé. Une culture a besoin d'une certaine quantité de chaleur pour effectuer son cycle de vie complet. Quand le climat se réchauffe, ce cycle s'accomplit plus vite... la culture mûrit plus vite.

- C'est ce qui explique pourquoi la date des vendanges est de plus en plus précoce. Une vigne, qui se récoltait pendant la $2^{\mathrm{e}}$ quinzaine de septembre en 1960, se récolte aujourd'hui au mois d'août!

Nous pourrions parler plus en détail des effets de la température sur les végétaux mais cela serait trop long. Ce qu'il nous faut retenir ici :

- il y a un contraste entre la végétation naturelle, dont les cycles de croissance s'allongent, et l'agriculture dont les cycles raccourcissent (Fig. 8.6);

- il peut y avoir contradiction entre une levée de dormance plus tardive à cause des hivers doux, et une floraison plus précoce à cause de printemps plus chauds. 


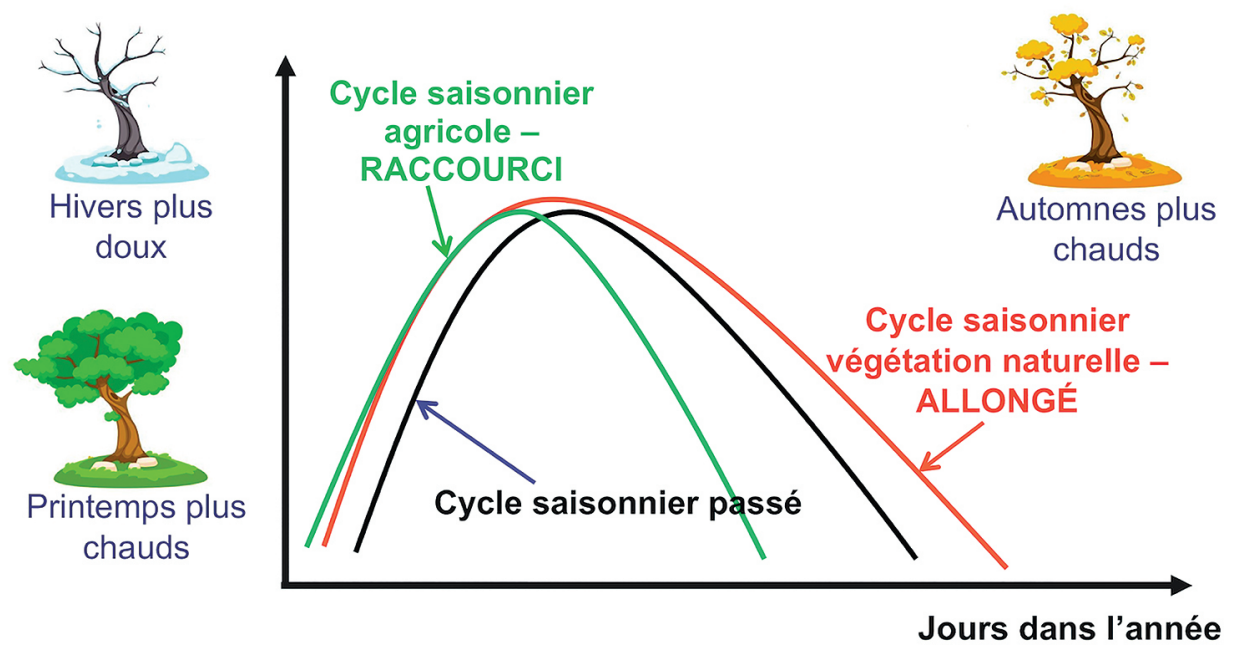

Figure 8.6: Évolution des cycles saisonniers avec le changement climatique. Source: Nathalie de Noblet-Ducoudré.

- Et enfin, les étés chauds sont plus fréquents et plus extrêmes. Or, les plantes comme les animaux, comme nous, peuvent souffrir de températures trop chaudes qui peuvent conduire au flétrissement pour la végétation et à des pertes de rendement très importantes en agriculture.

\subsubsection{Les changements de précipitation}

II y a deux aspects importants du changement climatique concernant les précipitations (Fig. 8.7):

- les cycles saisonniers sont souvent plus contrastés avec, en France par exemple, des hivers plus pluvieux et des étés plus secs. La sécheresse estivale est un problème pour la croissance des plantes;

- les événements pluvieux sont plus intenses. Une pluie trop intense peut fragiliser les plantes par un impact trop fort ou conduire à des inondations qui risquent de faire pourrir les jeunes pousses.

Dans les régions où les précipitations ne changent pas beaucoup, le sol s'assèche car l'évapotranspiration augmente en réponse au réchauffement climatique. Cela peut être problématique pour la croissance des plantes si les sols contiennent moins d'eau.

En conclusion, comme nous l'avons montré rapidement, le climat affecte le développement de la végétation en perturbant notamment son calendrier. 


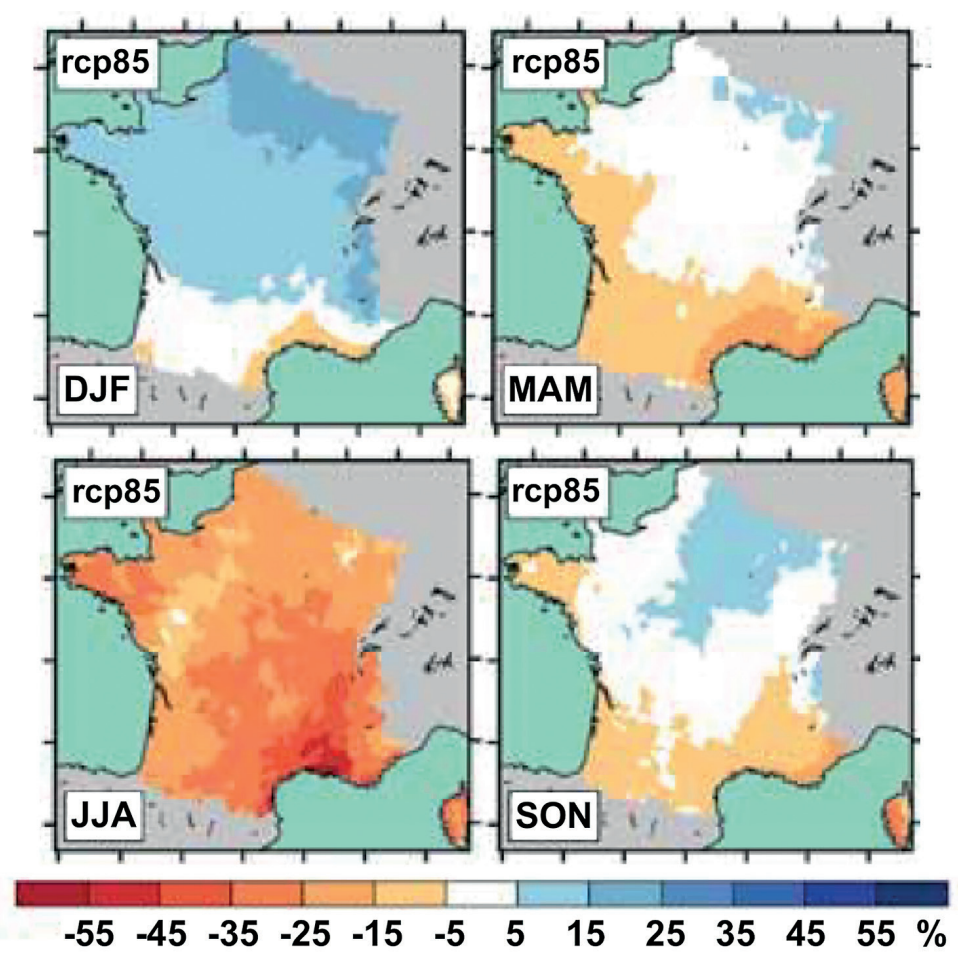

Figure 8.7: Évolution attendue des précipitations dans la France métropolitaine pour les 4 saisons, selon le scénario climatique le plus pessimiste (RCP8.5). Des hivers un peu plus humides (DJF: décembre, janvier, février), surtout dans le nord. Des étés beaucoup plus secs (JJA: juin, juillet, août), surtout dans le sud. Des changements un peu moins prononcés au printemps (MAM: mars, avril, mai) et en automne (SON : septembre, octobre, novembre). Source: [1].

Dans la section 7.4 de cet ouvrage, nous avions montré que la végétation impactait le climat, dû au fait qu'elle échangeait, entre autres, du $\mathrm{CO}_{2}$ et de la vapeur d'eau avec l'atmosphère. Ici, nous montrons que, si la végétation joue un rôle important sur le climat, celui-ci perturbe significativement, en retour, son évolution suivant les saisons. Par exemple, dans les régions plutôt froides en zone boréale, si le réchauffement climatique conduit au développement des arbres, alors ces arbres vont amplifier le réchauffement initial. À la fin, le climat de ces régions sera différent sous l'effet combiné des émissions de gaz à effet de serre ET de la réponse de la végétation.

\subsection{Acidification des océans et impacts sur les écosystèmes marins - L. Bopp}

L'océan a un rôle clé dans le système climatique (voir section 7.1.1). Ce rôle est particulièrement important dans le cas du changement climatique anthropique et ceci pour deux raisons majeures. L'océan absorbe la très grande majorité de 
l'énergie en excès dans le système climatique, énergie qui est engendrée par l'augmentation des gaz à effet de serre. Mais l'océan absorbe aussi une part importante de nos émissions de carbone. En absorbant chaleur et carbone, l'océan nous fait gagner du temps et nous évite une catastrophe climatique encore plus grande.

\subsubsection{Augmentation de la concentration de l'atmosphère en $\mathrm{CO}_{2}$ et acidification des océans}

Pour rappel, la concentration de $\mathrm{CO}_{2}$ dans l'atmosphère a augmenté de près de $50 \%$ depuis la révolution industrielle en raison des émissions produites par les activités humaines. Chaque année, l'océan absorbe près de $25 \%$ de ces émissions anthropiques, ralentissant ainsi la vitesse à laquelle le $\mathrm{CO}_{2}$ augmente dans l'atmosphère et perturbe le climat. Mais cette absorption n'est pas sans conséquence. On peut même dire que c'est une bien mauvaise nouvelle pour de nombreux organismes marins, car l'absorption de $\mathrm{CO}_{2}$ est responsable de l'acidification de l'océan.

Une fois dissous dans l'eau de mer, le $\mathrm{CO}_{2}$ forme un acide faible, l'acide carbonique de formule $\mathrm{H}_{2} \mathrm{CO}_{3}$, qui va pouvoir se dissocier en libérant des protons $\left(\mathrm{H}^{+}\right)$. En conséquence, plus l'océan absorbe de $\mathrm{CO}_{2}$, plus la concentration de $\mathrm{H}^{+}$augmente. C'est exactement ce que l'on mesure avec le $\mathrm{pH}$ (ou potentiel hydrogène) - attention quand le $\mathrm{pH}$ baisse, vous avez de l'eau de plus en plus acide et qui contient de plus en plus d'ions $\mathrm{H}^{+}$. Attention également, l'échelle de $\mathrm{pH}$ est une échelle logarithmique - à une baisse d'une unité pH correspond une multiplication par 10 des concentrations d'ions $\mathrm{H}^{+}$dans le milieu étudié.

La figure 8.8 met clairement en évidence la corrélation entre la diminution du pH mesuré dans l'océan Pacifique à la station marine ALOHA d'Hawaï (courbes du bas sur la figure) et l'augmentation de la pression partielle de $\mathrm{CO}_{2}$ mesurée à la fois dans l'eau de surface (courbe du haut reliant les points bleus) et dans l'air humide environnant (courbe du haut reliant les points rouges). Les mesures du $\mathrm{pH}$ (courbes reliant les points verts) ont été effectuées à trois profondeurs et comparées à des valeurs calculées à partir du taux d'alcalinité de l'eau et de la quantité dissoute de $\mathrm{CO}_{2}$ d'origine inorganique (courbes reliant les points ocres). La diminution du pH est bien vérifiée pour les mesures dans les eaux de surface et celles effectuées en moyenne profondeur. Le $\mathrm{pH}$ des eaux vers $1000 \mathrm{~m}$ de profondeur est relativement stable, mais l'acidité y est plus forte. De telles mesures ont démarré à la fin des années 1980 et se poursuivent régulièrement dans différentes stations de l'océan Pacifique et de l'océan Atlantique. Nous avons donc plus de 30 ans de recul sur l'évolution du pH, et le même signal se dégage dans l'Atlantique comme dans le Pacifique : le pH baisse ! C'est le signal de l'acidification de l'océan. 


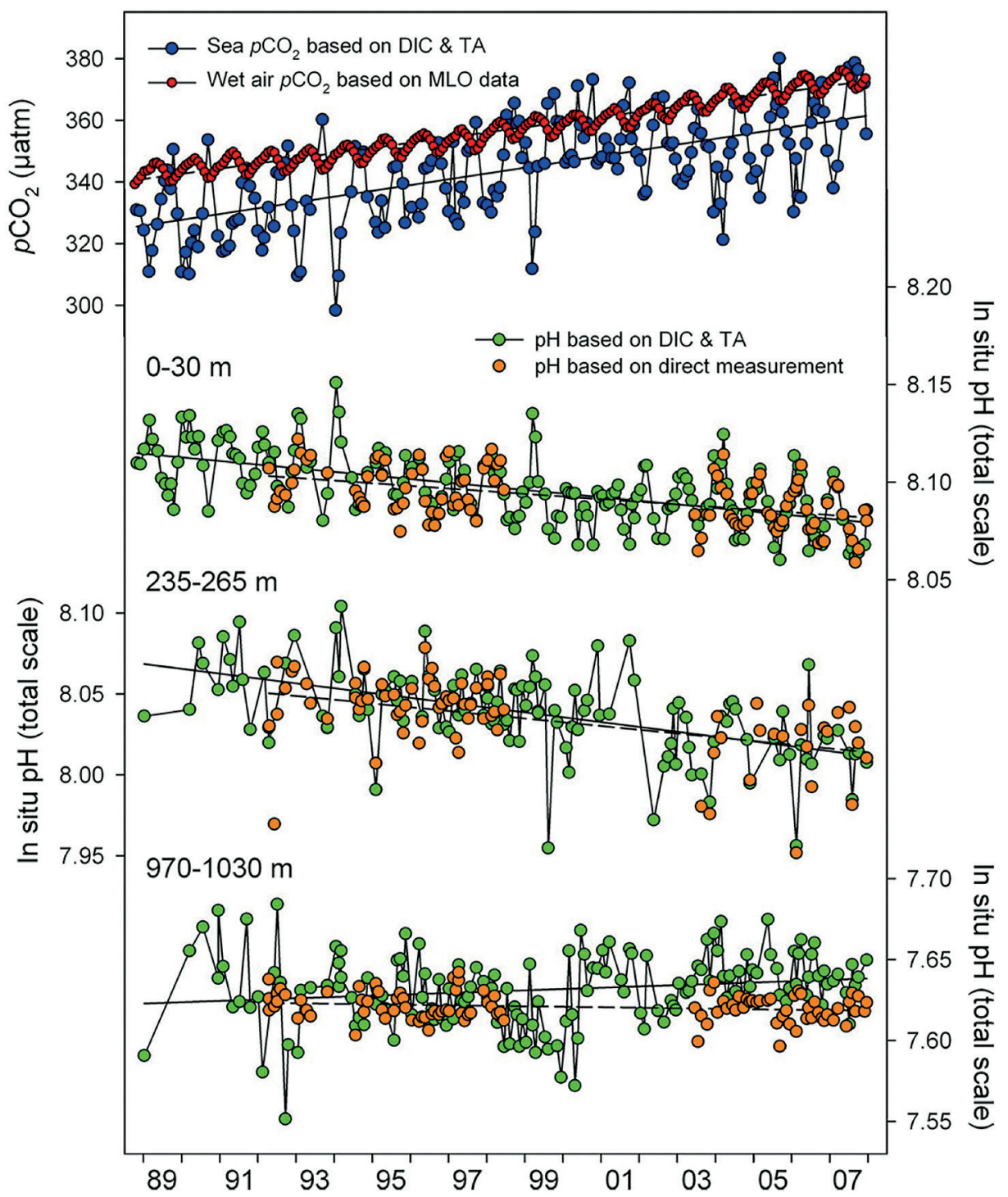

Figure 8.8: Corrélation entre la diminution du $\mathrm{pH}$ de l'océan (en surface et moyenne profondeur) et l'augmentation de la pression partielle de $\mathrm{CO}_{2}$ mesurée dans l'eau de surface et dans l'air humide environnant. Études et mesures effectuées à la station d'ALOHA d'Hawaï. Source: Figure 1 de la référence [2].

Comme on peut s'en douter, si nous continuons à émettre du $\mathrm{CO}_{2}$ au cours des prochaines années, l'acidification de l'océan se poursuivra. C'est ce que confirment les modèles climatiques, quel que soit le scénario d'émission de $\mathrm{CO}_{2}$, que l'on ne fasse rien pour réduire cette émission ( «business as usua/») ou que l'on parvienne à faire baisser ces émissions d'ici la fin du XXle siècle. 
À partir de 2050, cependant, les deux scénarios divergent pour aboutir à deux situations très contrastées en fin de siècle. Dans le cas où les émissions de $\mathrm{CO}_{2}$ continuent à croître, la température de surface augmente de plusieurs degrés et le $\mathrm{pH}$ baisse de 0,4 unité $\mathrm{pH}$ en 2100 . C'est une modification très importante de la chimie de l'eau de mer, qui correspond à une augmentation de la concentration des ions $\mathrm{H}^{+}$de 100 à $150 \%$ à la fin du XXle siècle. Les conséquences pour la vie marine pourraient être dramatiques. Dans le cas où nous maîtrisons nos émissions de carbone, la baisse du pH est bien moindre. Nous limitons non seulement le changement climatique et le réchauffement de notre planète, mais aussi l'acidification de l'océan.

\subsubsection{Conséquences de l'acidification sur les organismes marins}

Alors, pourquoi devons-nous nous inquiéter de cette acidification? Les organismes marins les plus menacés sont, sans doute, les espèces calcifiantes, celles qui fabriquent des squelettes, des tests ou des coquilles en calcaire ou carbonate de calcium.

La figure 8.9 représente ainsi des exemples d'organismes menacés tels que les coraux tropicaux d'eaux chaudes, les coraux profonds d'eaux froides, les oursins, les bivalves qui incluent notamment les espèces utiles à l'homme comme les moules ou les huîtres, des organismes microscopiques, comme des foraminifères ou des algues également microscopiques au nom barbare de coccolithophoridés.

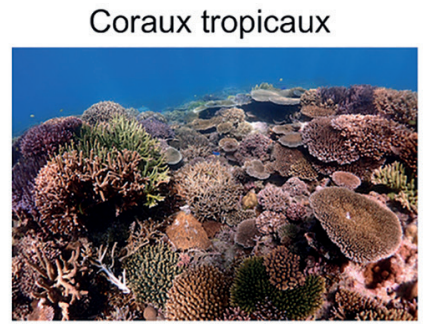

Foraminifères

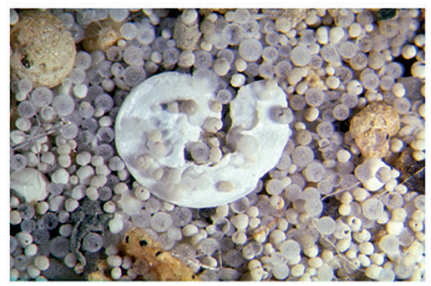

Ascidies

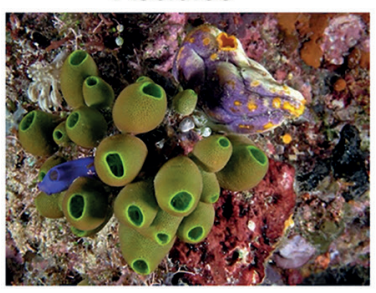

Bivalves

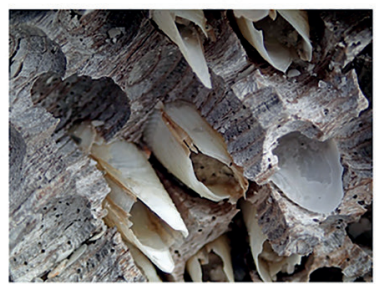

\section{Coccolithophoridés}

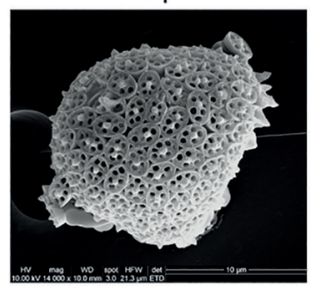

Oursins

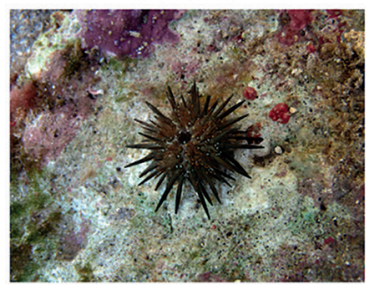

Figure 8.9: Organismes marins calcifiant. Sources: @lstock (coraux tropicaux, ascidies, coccolithophoridés). Wikimedia Commons (foraminifères, bivalves, oursins). 
Même si cela peut paraître contre-intuitif, plus l'océan contient de carbone, plus il est difficile pour ces organismes de se calcifier, c'est-à-dire plus il est difficile de synthétiser leurs coquilles ou leurs squelettes. Le paradoxe est que la calcification consiste justement à précipiter un ion calcium avec un ion carbonate qui contient du carbone.

En fait, quand le $\mathrm{CO}_{2}$ en excès se dissout dans l'eau de mer, il réagit avec de l'eau et des ions carbonates pour donner des ions hydrogénocarbonates $\mathrm{HCO}_{3}$-. Plus l'océan absorbe de $\mathrm{CO}_{2}$, plus la concentration d'ions carbonates diminue. II y a donc de moins en moins d'ions carbonate disponibles pour la calcification. Cette diminution des ions carbonates est une autre manifestation de ce que l'on appelle l'acidification de l'océan.

Beaucoup d'études scientifiques, menées sur des organismes marins différents, ont montré les impacts de l'acidification sur le comportement, la reproduction, et la survie de ces organismes. Beaucoup de scientifiques étudient, par exemple, la réponse des espèces coralliennes. Nous soulignerons ici l'importance d'un autre type d'organisme calcifiant: le ptéropode qui est un petit escargot de mer dont la coquille fait quelques millimètres seulement, et qui est à la base de nombreuses chaînes alimentaires dans les océans polaires de l'hémisphère nord et de l'hémisphère sud.

Le comportement d'un ptéropode dans de l'eau «normale» est, en effet, fondamentalement différent de celui dans une eau acidifiée, qui correspondrait à la situation à la fin du XXle siècle dans le cas d'un scénario où les émissions de $\mathrm{CO}_{2}$ ne cessent d'augmenter. Dans l'eau acidifiée, les mouvements du ptéropode sont difficiles et la coquille montre des signes de dissolution en raison de l'acidification. Cet état «végétatif» des ptéropodes dans un océan de plus en plus acide est donc aujourd'hui particulièrement alarmante sur l'évolution potentielle des écosystèmes marins associés à cette espèce.

\subsubsection{Autres causes de dégradation des océans}

Pour continuer avec les mauvaises nouvelles, il nous faut imaginer que l'acidification de l'océan n'est pas la seule menace qui pèse sur les écosystèmes marins. Au réchauffement de l'eau de mer que nous avons déjà mentionné et qui modifie l'aire de répartition de nombreuses espèces marines, il faut aussi ajouter un autre phénomène qui est celui de la désoxygénation de l'océan (Fig. 8.10). En effet, avec le changement climatique, l'océan perd de l'oxygène, parce qu'il est plus chaud et que le gaz $\mathrm{O}_{2}$ est moins soluble dans l'eau chaude. Cette désoxygénation peut aussi impacter de nombreuses espèces, en particulier, celles qui ont besoin d'oxygène pour la respiration. 


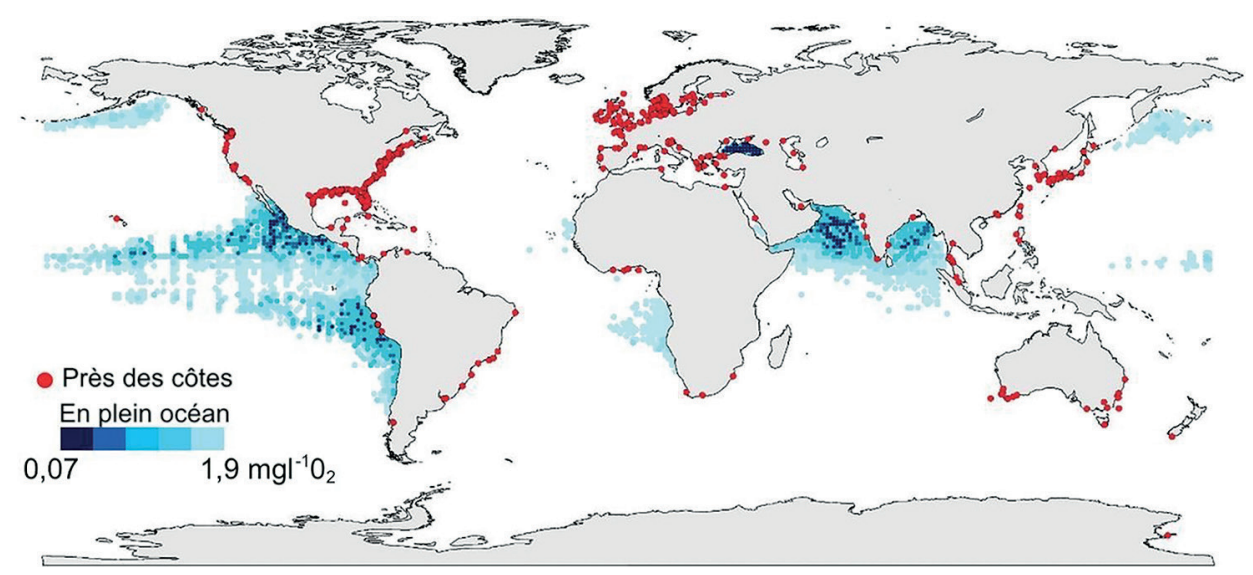

Figure 8.10: Zones océaniques déficitaires en oxygène (moins de $2 \mathrm{mg} / \mathrm{l}$ ), identifiées près des côtes (points rouges) ou en plein océan (points bleus). Source: GO2NE working group. Data from World Ocean Atlas 2013 and provided by R.J. Diaz.

Enfin, pour compléter le tableau des menaces qui pèsent sur les écosystèmes marins, il faut aussi tenir compte de pollutions, comme la pollution plastique, ou de la surpêche qui a déjà conduit à la disparition d'espèces marines dans certaines zones océaniques (voir section 5.3.1).

\subsubsection{Des solutions pour lutter contre l'acidification des océans?}

Quelles solutions faut-il adopter, en particulier, pour lutter contre l'acidification des océans? Certaines solutions pourraient être qualifiées de pis-aller ou même de fuite en avant! Des industriels ostréicoles de la côte Ouest des États-Unis, face à la baisse des rendements en lien, semble-t-il, avec l'acidification des eaux, se sont ainsi déplacés dans d'autres régions du monde, comme à Hawaii, où l'acidité naturelle de l'eau de mer est moins élevée.

D'autres solutions s'identifient plus à des mesures d'adaptation comme le fait d'aider ces mêmes industriels à mieux anticiper les variations naturelles d'acidité de l'eau de mer, pour leur éviter d'exposer les larves de bivalves à des eaux plus acides.

De façon plus radicale, il a été imaginé de corriger de façon artificielle l'augmentation de l'acidité de l'eau de mer en ajoutant à l'eau des composés alcalins, comme ce qui se pratique dans une piscine pour corriger le $\mathrm{pH}$. Cette solution peut sembler réaliste à une toute petite échelle, mais à l'échelle de l'océan mondial, les quantités d'additifs nécessaires seraient juste astronomiques...

Bien évidemment, la seule vraie solution à grande échelle consiste à limiter, puis réduire fortement nos émissions de $\mathrm{CO}_{2}$, ce qui permettrait d'atténuer à la fois le changement climatique et l'acidification de l'eau de mer. 


\subsection{Impacts des changements climatiques sur la santé humaine - R. Slama et C. Tran Kiem}

\subsubsection{Température et santé humaine - R. Slama}

La température a une influence claire et démontrée sur la santé humaine. Les connaissances épidémiologiques mettent en évidence une relation en « $U$ » entre la température et la mortalité toutes causes confondues, telle que représentée sur la figure 8.11. Autrement dit, on observe un accroissement de la mortalité aussi bien aux températures élevées qu'aux températures les plus basses et, entre ces températures extrêmes, une «zone de confort » où la mortalité est minimale.

Cette situation est résumée sur la figure 8.11 qui provient d'une méthodologie que les épidémiologistes et les statisticiens appellent l'approche des séries temporelles. Elle consiste à mettre en relation les températures pendant une ou plusieurs journées successives dans une ville donnée avec la mortalité dans la même ville le jour même, le lendemain et dans les quelques jours qui suivent, en corrigeant éventuellement l'effet de facteurs dits de confusion. Ce sont donc uniquement des effets à court terme de la température que l'on peut ainsi mettre en évidence.

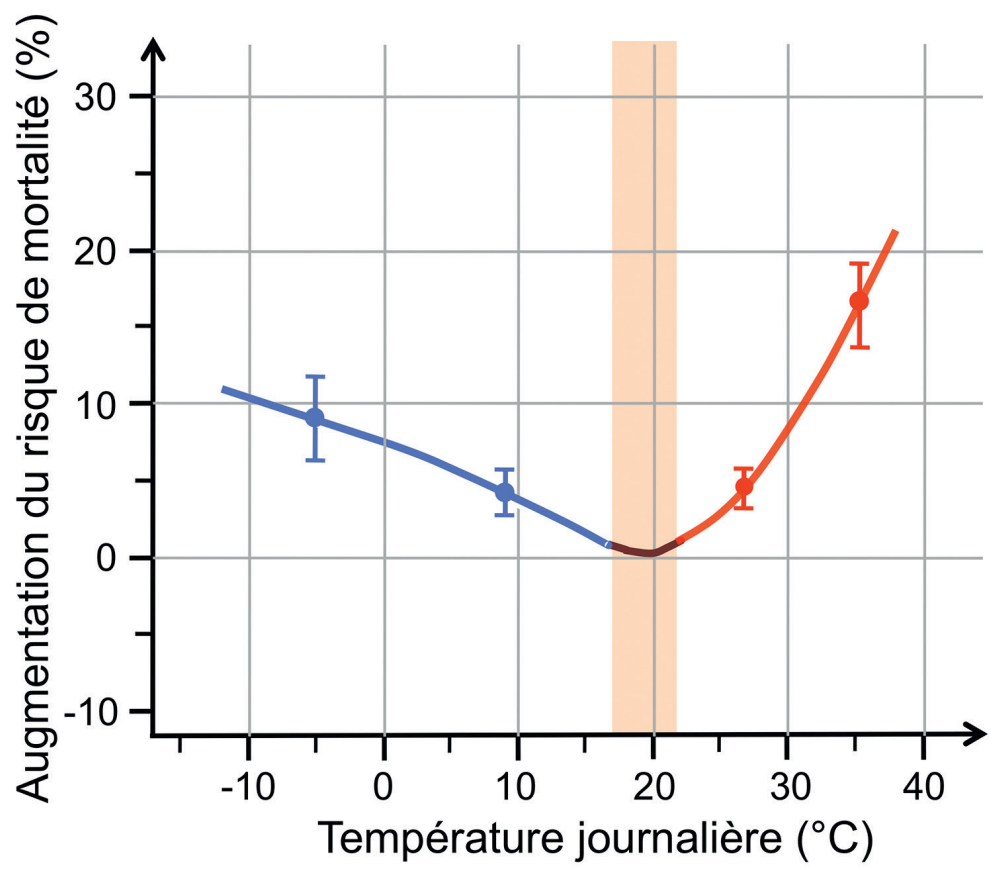

Figure 8.11: Courbe typique en «U» d'évolution de la mortalité toutes causes confondues en fonction de la température journalière. Source: D’après les résultats de [3]. 
II pourrait être tentant de chercher à comparer globalement la mortalité entre deux villes aux climats différents. En fait, cette comparaison serait assez difficile à faire, puisque les deux villes sont susceptibles de différer l'une de l'autre sur un grand nombre de facteurs pouvant influencer la santé. On serait en peine de déterminer la part effective de la température dans la différence de mortalité observée entre les deux villes. On écarte, au contraire, un grand nombre de biais avec l'approche «intraville» des séries temporelles, qui permet d'estimer plus aisément la relation à court terme entre température et mortalité. Cette approche prend, de plus, en compte un certain nombre de facteurs tels que les grandes tendances mensuelles ou annuelles dans la mortalité ainsi que la présence éventuelle d'épidémies, qui sont autant de «facteurs de confusion» potentiels.

Promenons-nous sur la planète et comparons les relations température-mortalité obtenues pour différentes villes (Fig. 8.12). On constate que la relation observée à Londres est très différente de celle observée à Stockholm.
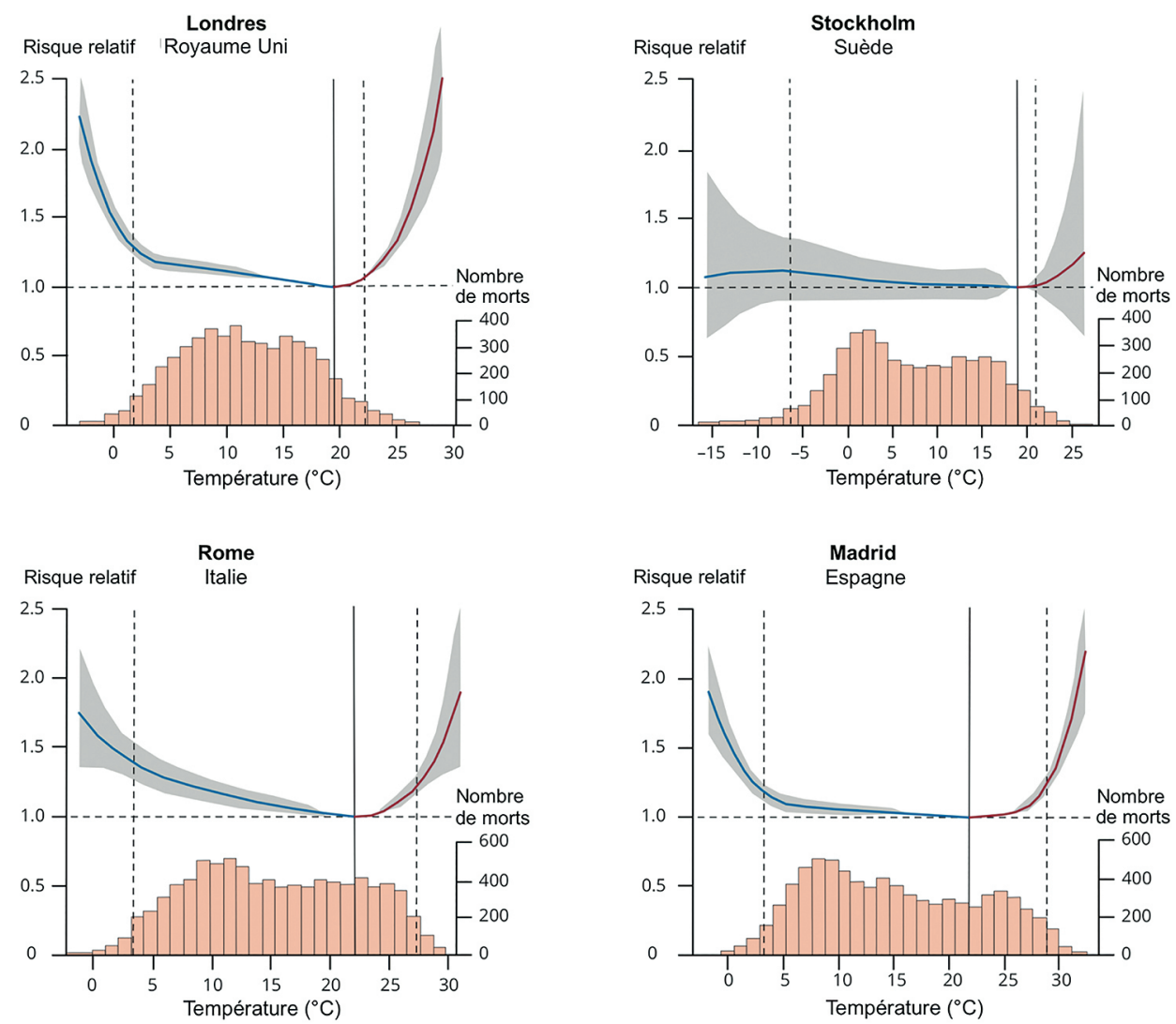

Figure 8.12: Relations température-mortalité pour différentes villes d'Europe. Source: European Environment Agency (EEA), 2019. Voir aussi [4]. 
En Suède, la mortalité augmente aux températures élevées, comme on s'y attend, mais la mortalité varie peu dans une gamme étendue de basses températures. Ces différences méritent qu'on s'y attarde pour essayer de les expliquer : une hypothèse est que les Suédois sont habitués à ces basses températures, et donc en souffrent moins que les habitants de Londres ou Madrid. Comment une telle adaptation pourrait s'expliquer? Probablement pas par des raisons génétiques, mais plutôt par des raisons sociétales liées au mode de vie (qualité de l'habitat, travail et temps passé en plein air...). Même si les températures s'abaissent fortement, ce fait est courant chez eux et leurs villes et modes de vie y sont adaptés. Les situations sont autres dans des villes comme Rome et Madrid. Dans une ville comme Madrid, la gamme de températures, où la mortalité est minimale, est relativement étendue, sans doute parce que les Madrilènes sont à la fois habitués à des étés très chauds et des hivers très froids.

Nous nous sommes déplacés d'une ville à l'autre. Restons maintenant dans un lieu donné et voyons s'il est possible de relier l'augmentation de mortalité aux températures élevées à une classe d'âge particulière ou à telle ou telle pathologie. Les travaux d'Antonio Gasparrini et ses collègues [5] montrent que si la mortalité est plus élevée, bien sûr, chez les personnes âgées que chez les personnes jeunes, l'augmentation de la mortalité aux températures élevées est observée parmi toutes les classes d'âge: la multiplication du risque de décès aux températures élevées est similaire aux différents âges de la vie. Ainsi, le problème n'est pas limité aux personnes âgées.

Quand on considère maintenant, de manière séparée, différentes pathologies ou causes de décès, on constate que le risque de décès est accru aussi bien pour les personnes souffrant de problèmes cardio-vasculaires que pour celles souffrant de problèmes respiratoires. Le risque de décès est également accru pour les autres causes de décès, y compris le suicide, mais avec une forme de la relation doseréponse qui diffère: elle n'est plus en $U$ mais monotone, avec un accroissement assez régulier du risque de décès par suicide des températures froides jusqu'aux températures élevées [6].

Cela suggère qu'il n'y a pas un mécanisme unique qui peut expliquer un effet lié aux températures, mais qu'il y a, selon les pathologies, différents mécanismes. Dans le cas de maladies cardiovasculaires, il est probable que le risque accru de mortalité est dû au fait qu'en période chaude, notre cœur travaille plus pour assurer la thermorégulation du corps, augmenter la transpiration, emmener le sang aux extrémités et favoriser la convection. Tout ceci fatigue les gens comme s'ils effectuaient un exercice d'endurance en permanence, augmentant ainsi le risque d'infarctus. Pour une autre cause de décès comme le suicide, un mécanisme supposé est que la température fait varier le niveau de sérotonine dans le corps. La sérotonine est une hormone impliquée dans le bien-être et un niveau déficitaire en sérotonine peut entraîner un état dépressif. En conséquence, les variations de sérotonine aux températures très élevées peuvent augmenter les états dépressifs et, par-là, le risque de suicide. 
Essayons maintenant de changer d'échelle de temps pour placer ces connaissances dans la perspective du changement climatique. Quel peut être l'impact du changement climatique et du décalage des températures vers les températures élevées sur la mortalité? L'impact global n'est, en fait, pas si facile à prévoir car si le nombre de décès attribuables aux températures chaudes va vraisemblablement augmenter, il est aussi possible que dans certaines zones, le nombre de décès attribuables aux températures froides - moins fréquentes - diminue (Fig. 8.13). Une équipe d'épidémiologistes londoniens a néanmoins pu faire une première estimation de cet impact à partir de projections sur le climat futur, faites par les climatologues [7].

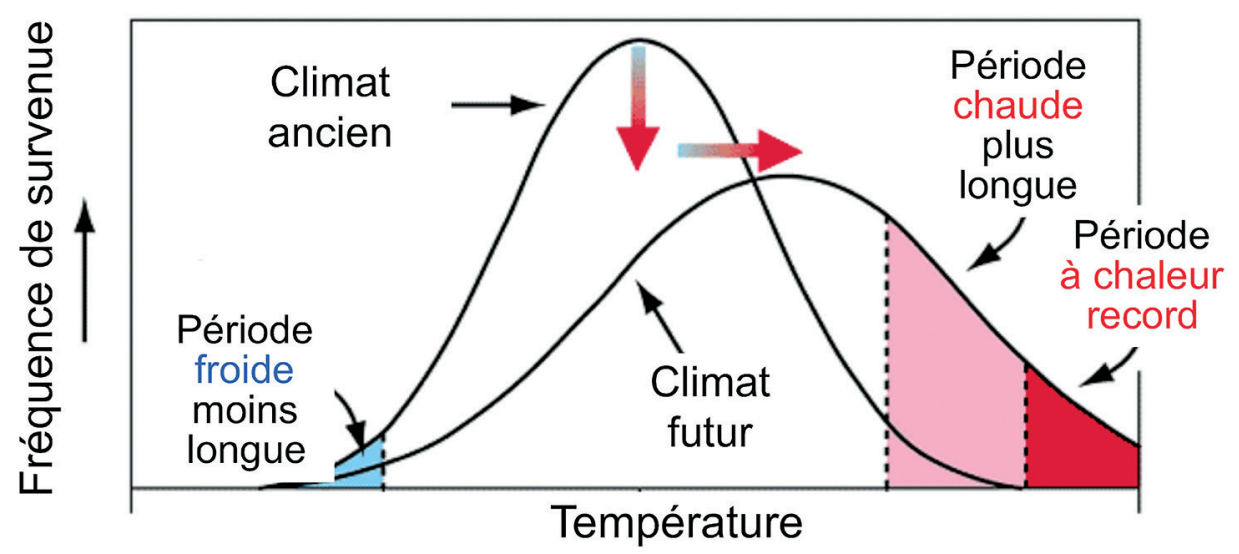

Figure 8.13: Évolution de la distribution annuelle de température prédite pour le climat futur. Avec la courtoisie des membres du GIEC.

Les épidémiologistes prédisent ainsi que dans les pays d'Europe du Nord, on peut s'attendre à une diminution de la mortalité liée aux basses températures en hiver et à une augmentation, dans l'ensemble moins importante ou similaire, de la mortalité liée aux températures chaudes en été. Ainsi, globalement, l'impact net sur la mortalité sur une année est quasi-nul dans le nord de l'Europe, typiquement dans les pays scandinaves.

Si on descend au sud de l'Europe, par exemple, dans le sud de la France, on assistera à une certaine diminution de la mortalité hivernale, car les températures en hiver seront moins rudes, mais on aura une augmentation beaucoup plus spectaculaire de la mortalité liée aux températures chaudes en été, qui fera bien plus que compenser la diminution de la mortalité hivernale (Fig. 8.14). Globalement, la mortalité aux températures extrêmes, dans notre pays comme dans d'autres pays du sud de l'Europe, risque d'augmenter considérablement dans l'hypothèse d'un changement climatique et en l'absence de mesures d'adaptation sociétale forte. 


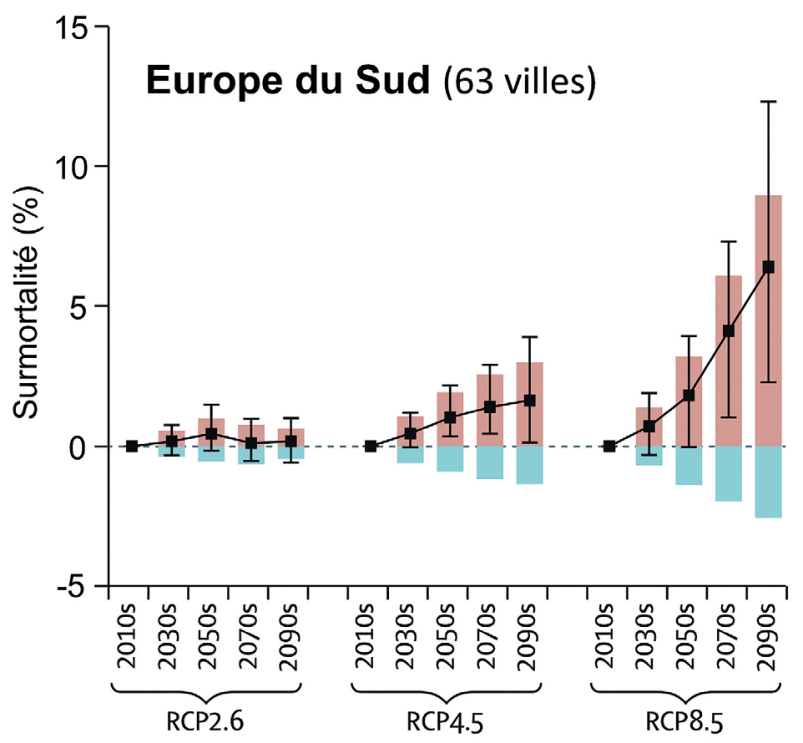

Figure 8.14: Évolution de la mortalité prédite pour 63 villes de l'Europe du Sud de 2010 à 2090. Les prédictions sont issues de trois modèles climatiques différents: RCP2.6, RCP4.5 et RCP8.5. Les barres en rose correspondent à l'augmentation de la mortalité associée aux températures plus chaudes en été, celles en bleu correspondent à la diminution de la mortalité associée aux températures moins froides en hiver. Les courbes en noir représentent les variations nettes de la mortalité dues au changement de température. Source [8].

L'exercice a été répété dans les différentes régions du globe et démontre que, notamment en Amérique du Sud, en Amérique centrale, en Asie du Sud-Est, la situation est similaire à celle de l'Europe du Sud, avec une augmentation de la mortalité due aux températures extrêmes.

Pour résumer et conclure, la température a un effet certain et bien démontré à court terme sur la mortalité. En modifiant les températures annuelles, le changement climatique va perturber le nombre de décès attribuables à la mortalité chaque année et dans certaines zones, notamment dans les zones tempérées ou les zones relativement chaudes aujourd'hui. Le bilan qu'on peut attendre, si on ne fait rien, est que la mortalité attribuable à la température va avoir tendance à augmenter dans l'hypothèse du changement climatique tel qu'on le décrit pour les prochaines décennies, en tout cas en-dehors des pays actuellement à climat froid.

\subsubsection{Maladies à transmission vectorielle - C. Tran Kiem}

Les maladies à transmission vectorielle sont des maladies infectieuses dont l'agent pathogène responsable est transmis par un organisme intermédiaire que l'on appelle vecteur ${ }^{74}$. Ces maladies sont susceptibles de se propager rapidement et il

74. https://www.santepubliquefrance.fr/maladies-et-traumatismes/maladies (voir «maladies à transmission vectorielle» sur le site) 
est donc légitime d'analyser l'influence possible du changement climatique sur ce type de maladie dans l'état actuel de nos connaissances.

De nombreuses espèces sont décrites comme porteuses d'agents pathogènes, par exemple certaines espèces de moustiques. Parmi les maladies vectorielles, on trouve notamment le paludisme transmis par le moustique Anophèle, le chikungunya dont le virus est transmis par les moustiques Aedes aegypti et albopictus et la maladie de Lyme transmise par les tiques. Dans le cas de la dengue, par exemple, lorsqu'un moustique Aedes pique un individu infecté, il peut être contaminé par le virus de la dengue qui se trouve dans le sang de l'individu infecté. Après une période d'incubation de quelques jours, le virus atteint les glandes salivaires du moustique, qui pourra le transmettre à un individu susceptible lors d'un prochain repas sanguin (Fig. 8.15).

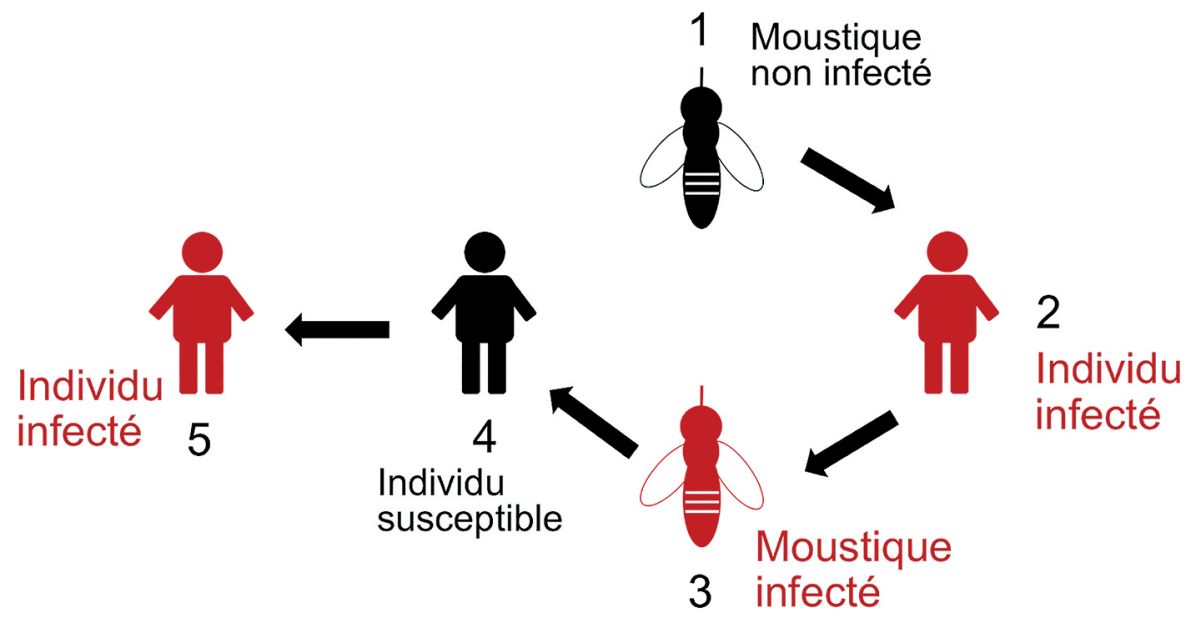

Figure 8.15: Exemple de cycle de transmission. Source: Cécile Tran Kiem.

L'Organisation mondiale de la Santé estime que ces maladies représentent plus de $17 \%$ du fardeau global des maladies infectieuses et que la moitié de la population mondiale vit dans des zones à risque [9]. L'organisation mondiale de la santé rapporte également que plus de 400000 décès dus au paludisme sont survenus en 2018 touchant des enfants de moins de 5 ans dans plus de $65 \%$ des cas. Le territoire français n'est pas exempt des risques associés aux maladies à transmission vectorielle. La maladie de Lyme, transmise par les tiques, a été à l'origine de plus de 65000 cas rapportés en 2018. Les moustiques Aedes sont régulièrement à l'origine d'épidémies de dengue ou de chikungunya dans les territoires d'Outre-Mer. Enfin, le moustique tigre Aedes albopictus a été détecté pour la première fois en 1999 en France métropolitaine et a colonisé la majorité des départements de France métropolitaine (Fig. 8.16). 


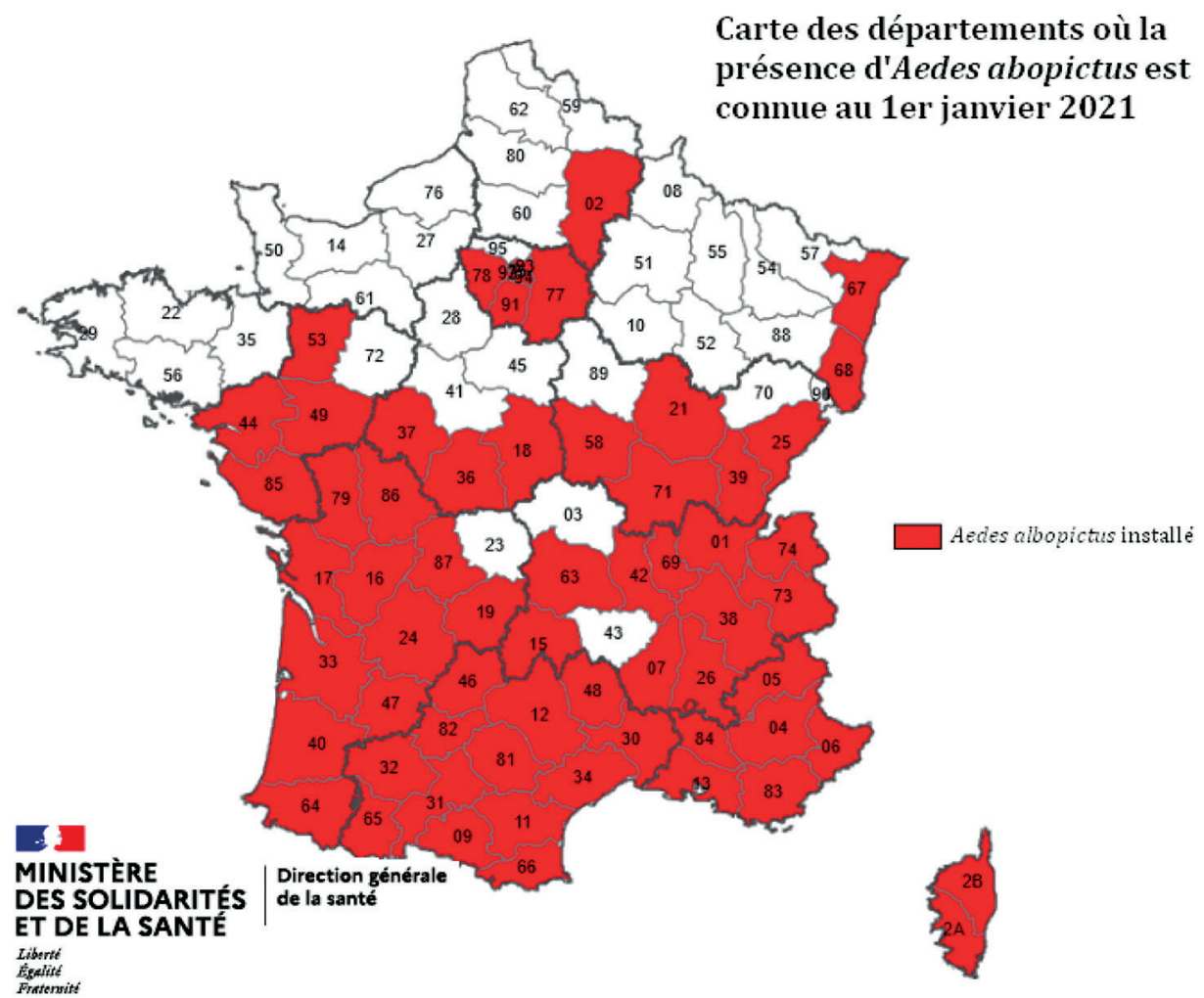

Figure 8.16: Départements où la présence du moustique tigre Aedes albopictus est connue (1er janvier 2021). Source: ministère de la Santé et des Solidarités ${ }^{75}$.

Un grand nombre de facteurs peut influencer la distribution des maladies à transmission vectorielle. Ainsi les modifications des pratiques agricoles, les changements démographiques ou encore la mobilité humaine sont des facteurs susceptibles de jouer un rôle dans l'émergence ou la réémergence de pathogènes. Le climat représente également l'un des nombreux déterminants de la distribution de ces maladies. Le graphe de la figure 8.17 représente les résultats d'une étude qui a cherché à quantifier la relation entre la température et certains traits de vie des moustiques Aedes [10] (on appelle traits de vie d'une espèce des caractéristiques liées à tous les organismes de cette espèce). Au-dessous de $13^{\circ} \mathrm{C}$ et au-dessus de $38^{\circ} \mathrm{C}$, on observe que la probabilité de survie est estimée nulle. Ces températures ne sont donc pas propices au développement de cette espèce ni à la transmission des maladies qu'elles propagent.

75. https://solidarites-sante.gouv.fr/sante-et-environnement/risques-microbiologiques-physiqueset-chimiques/especes-nuisibles-et-parasites/article/cartes-de-presence-du-moustique-tigreaedes-albopictus-en-france-metropolitaine 


\section{Survie de l'œuf à l'adulte}

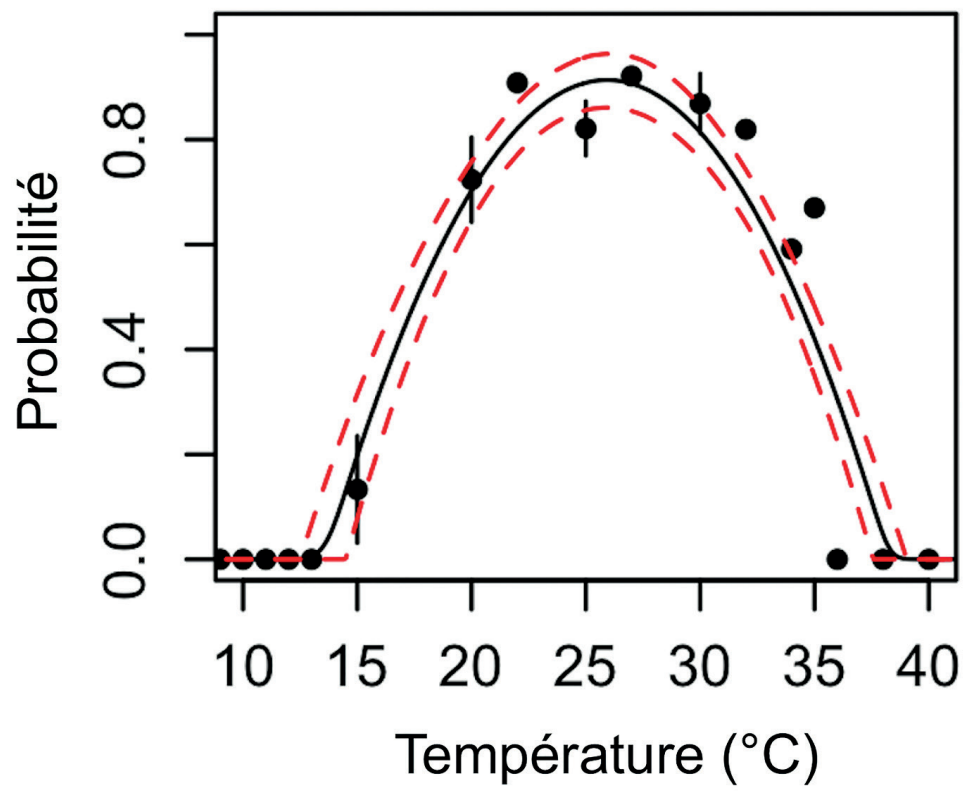

Figure 8.17: Probabilité de survie des larves de moustiques Aedes en fonction de la température. Source: E.A. Moredecai et al. [10].

D'autres variables environnementales telles que l'humidité ou le niveau de précipitation jouent également un rôle dans la transmission des maladies à transmission vectorielle, mais ces relations sont complexes et non linéaires. Par exemple, d'une part, un environnement trop sec ne permet pas le stade de développement aquatique de certains moustiques mais d'autre part, un fort niveau de précipitation est susceptible d'éliminer les gîtes larvaires. Différentes études se sont penchées sur les effets du changement climatique sur la transmission des maladies vectorielles. Celles-ci ont mis en évidence des relations complexes spécifiques à l'agent pathogène, à l'espèce vectrice mais également aux régions concernées [11, 12].

Pour conclure, les effets du changement climatique sur les maladies à transmission vectorielle sont complexes et impliquent de nombreux déterminants dont la compréhension demeure encore partielle. Le changement climatique est susceptible de modifier la distribution des maladies à transmission vectorielle et d'entraîner une augmentation de l'incidence de ces maladies dans des zones aujourd'hui plus tempérées. II est donc encore nécessaire d'en mieux comprendre les mécanismes afin de mieux évaluer les risques associés au changement climatique sur la santé humaine. 


\section{Références}

[1] G. Ouzeau, M. Déqué, M. Jouini, S. Planton, R. Vautard, M. Vrac. 2014. Le climat de la France au XXF siècle. Ministère de l'Écologie, du Développement Durable et de l'Énergie.

[2] J.E. Dore, R. Lukas, D.W. Sadler, M.J. Church, D.M. Karl. 2009. Physical and biogeochemical modulation of ocean acidification in the central North Pacific. PNAS 106(30), $123235-12240$.

https://www.pnas.org/content/pnas/106/30/12235.full.pdf.

[3] T. Li, R.M. Horton, Patrick, L. Kinney. 2013. Projections of seasonal patterns in temperature-related deaths for Manhattan, New York. Nature Climate Change 3, 717-721.

[4] A. Gasparrini, Y. Guo, M. Hashizume, P.L. Kinney, E.P. Petkova, E. Lavigne, ... B.G. Armstrong. 2016B. Temporal Variation in Heat-Mortality Associations: A Multicountry Study. Environ Health Perspect, 123(11), 1200-1207. doi: 10.1289/ehp.1409070 (2015B). Lien: https://ehp.niehs.nih.gov/doi/full/10.1289/ehp.1409070.

[5] A. Gasparrini, B. Armstrong, S. Kovats, P. Wilkinson. 2012. The effect of high temperatures on cause-specific mortality in England and Wales. Occup Environ Med 69: 56-61. Lien: http://www.ag-myresearch.com/uploads/1/3/8/6/13864925/2015_gasparrini_ehp.pdf.

[6] M. Burke, F. González, P. Baylis, S. Heft-Neal, C. Baysan, S. Basu, S. Hsiang. 2018. Higher temperatures increase suicide rates in the United States and Mexico. Nature Climate Change 8, 723-729.

[7] N. Watts, M. Amann, ..., A. Costello. 2018. The 2018 report of the Lancet Countdown on health and climate change: shaping the health of nations for centuries to come. Lancet 392, 2479-2514.

https://www.thelancet.com/journals/lancet/article/PIIS0140-6736(18)32594-7/fulltext? utm_source=kitchenertoday.com\&utm_campaign=kitchenertoday.com\&utm_medium= referral.

[8] A. Gasparrini, Y. Guo, F. Sera, A.M. Vicedo-Cabrera, V. Huber, S. Tong, et al. 2017. Projections of temperature-related excess mortality under climate change scenarios. The Lancet 1(9), E360-E367. DOI: https://doi.org/10.1016/S2542-5196(17)30156-0.

[9] D. Campbell-Lendrum, L. Manga, M. Bagayoko, J. Sommerfeld. 2015. Climate change and vector-borne diseases: what are the implications for public health research and policy? Philos Trans R Soc Lond B Biol Sci 370. Voir aussi : https://www.who.int/news-room/ fact-sheets/detail/vector-borne-diseases.

[10] E.A. Mordecai, J.M. Cohen, M.V. Evans, P. Gudapati, L.R. Johnson, C.A. Lippi, K. Miazgowicz, C.C. Murdock, J.R. Rohr, S.J. Ryan, et al. 2017. Detecting the impact of temperature on transmission of Zika, dengue, and chikungunya using mechanistic models. PLOS Neglected Tropical Diseases 11, e0005568.

[11] D. Roiz, S. Ruiz, R. Soriguer, J. Figuerola. 2014. Climatic effects on mosquito abundance in Mediterranean wetlands. Parasites \& Vectors 7, 333.

[12] M.E.J. Woolhouse, S. Gowtage-Sequeria. 2005. Host range and emerging and reemerging pathogens. Emerg Infect Dis 11, 1842-1847. 



\section{Chapitre 9}

\section{Reconstitutions paléoclimatiques, modèles et projections climatiques}

Ce chapitre est consacré aux modèles climatiques, à la façon dont ils sont élaborés et aux projections du climat futur qu'ils permettent de faire. Comme les modèles météorologiques, qui prévoient le temps pour demain, les modèles climatiques sont des modèles complexes qui reposent sur les équations de la thermodynamique des fluides. À la différence des modèles météorologiques, cependant, les modèles climatiques ne sont pas utilisés pour dire ce qu'il va se passer à une date donnée, mais nous fournissent des évolutions plausibles du climat sur des échelles de temps de plusieurs années, voire des dizaines d'années. II n'existe d'ailleurs pas un modèle, mais des modèles qu'on utilise avec différents scénarios d'émissions de gaz à effet de serre, qui résultent eux-mêmes de différents choix de société. La validation des modèles se fait en confrontant leurs résultats aux observations in situ et spatiales ainsi qu'à la connaissance des climats passés. Nous verrons que grâce à eux, il est possible d'estimer la quantité de $\mathrm{CO}_{2}$ restant à émettre pour ne pas dépasser des niveaux critiques de température moyenne à la surface du globe. Le chapitre se termine par une brève présentation du GIEC qui est chargé d'évaluer la science en lien avec le changement climatique à l'échelle internationale. 


\subsection{Reconstitutions paléoclimatiques de la variabilité climatique naturelle - J. Gherardi}

\subsubsection{Rappels sur le fonctionnement du système climatique aux différentes échelles de temps}

Nous avons vu que le climat de notre planète change à toutes les échelles, de temps et d'espace. Rappelons ici brièvement les principaux facteurs non anthropiques qui gouvernent l'évolution du système climatique de la Terre:

- le soleil lui-même, moteur du fonctionnement du système climatique, source d'énergie (voir section 7.1),

- l'albédo qui est le pouvoir réfléchissant d'une surface (voir section 7.2),

- l'effet de serre de son atmosphère, lié en particulier à la vapeur d'eau ainsi qu'à d'autres gaz présents en quantités moindres mais d'une efficacité de réchauffement très importante (le $\mathrm{CO}_{2}$, le méthane...) (voir section 7.2),

- la tectonique des plaques, c'est-à-dire la dérive des continents en lien avec les mouvements profonds de la terre, sur les échelles de temps géologiques (voir section 1.1).

Les forçages induits par les variations de ces différents facteurs sur les composantes du système climatique, l'océan, la glace, l'atmosphère, les surfaces terrestres et la végétation (Fig. 9.1) induisent des changements climatiques naturels qui peuvent être répartis en trois temps géologiques, comme on a pu l'apprendre dans la première partie de l'ouvrage (voir sections 1.1 et 1.2).

Complexité du système climatique

Des acteurs en perpétuelle interaction ...

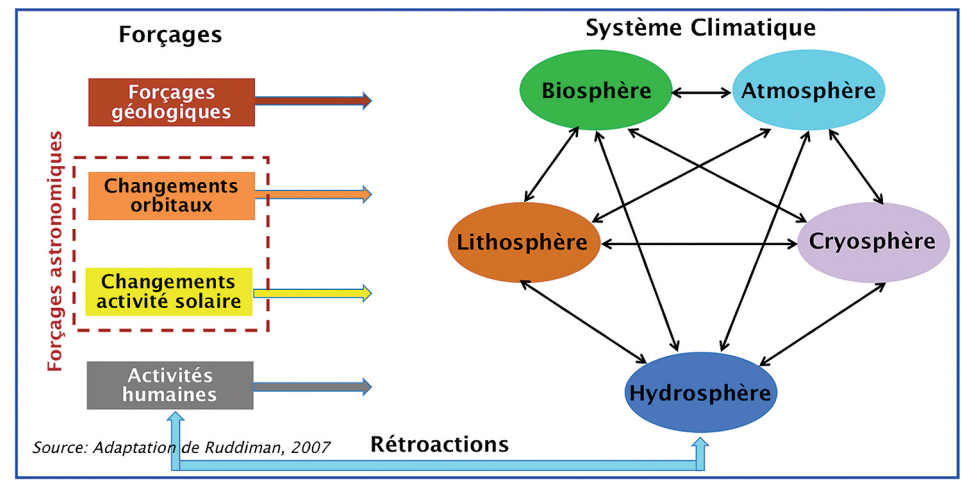

Figure 9.1: Diagramme montrant la complexité du système climatique. Avec la courtoisie de W.F. Ruddiman [1]. 
Nous ne rentrerons donc pas dans les détails. Rappelons-nous d'abord que le milliard d'années est l'échelle de temps sur laquelle les variations climatiques naturelles sont liées à la variation de luminosité du soleil (voir section 1.1). Rappelons ensuite que la deuxième échelle de temps, qui se chiffre en dizaines de millions d'années, est contrôlée par la tectonique des plaques, laquelle influence non seulement la teneur en $\mathrm{CO}_{2}$ de l'atmosphère via l'ouverture ou la fermeture de bassins océaniques, le soulèvement de chaînes de montagne et leur érosion, mais aussi le transport et la répartition de la quantité de chaleur via les enveloppes fluides que sont l'océan et l'atmosphère (voir section 7.1).

Enfin, nous avons vu que la dernière échelle de temps, qui s'étend de la dizaine à la centaine de milliers d'années, est directement liée à la quantité d'énergie solaire reçue par la Terre, celle-ci variant en fonction de trois paramètres orbitaux qui caractérisent les variations de l'orbite terrestre autour du soleil (voir Fig. 1.4, chapitre 1) ${ }^{76}$ :

- l'excentricité qui définit la forme de la trajectoire de la Terre autour du soleil, laquelle varie entre une orbite quasi circulaire et une orbite elliptique en oscillant de l'une à l'autre sur deux périodes principales de 413000 et 100000 ans respectivement;

- l'obliquité (l'inclinaison de l'axe de rotation terrestre par rapport à la perpendiculaire au plan de l'orbite) qui oscille entre $22^{\circ}$ et $24,5^{\circ}$ et amplifie ou réduit les saisons, notamment aux hautes latitudes, l'oscillation se faisant sur une période de 41000 ans;

- la précession des équinoxes, qui résulte des changements de la direction de l'axe de rotation terrestre par rapport aux étoiles et modifie la façon dont la Terre se positionne sur son orbite aux moments où interviennent les changements de saison. Cette modification se produit sur une période d'environ 25700 ans.

De façon résumée, les variations d'excentricité modulent le bilan d'énergie reçue sur une année à l'échelle du globe alors que l'obliquité et la précession ne modifient que la répartition d'énergie en latitude et la saisonnalité. Les variations combinées de ces trois paramètres entraînent donc des variations importantes d'ensoleillement durant l'été à $60^{\circ} \mathrm{N}$. L'astronome serbe, Milankovitch, a établi en 1940 la théorie selon laquelle ces variations sont à l'origine des cycles glaciaires et interglaciaires qui ont marqué le quaternaire. Le quaternaire est la période géologique dans laquelle nous nous trouvons depuis 2,5 millions d'années.

Mais comment a-t-il été possible de reconstituer les variations du climat sur ces périodes de temps si longues et lointaines? Comment mettre en évidence ces trois

76. https://planet-terre.ens-lyon.fr/ressource/milankovitch.xml 
rythmes climatiques naturels? À défaut d'observations, on s'en remet à l'étude des archives climatiques qui, comme nous l'avons vu dans la section 7.5, permettent de reconstituer les variations passées du climat de manière indirecte.

\subsubsection{Les archives climatiques et leur importance pour les modèles climatiques}

Reconstituer les variations du climat ancien passe donc par la reconstitution des paramètres climatiques et de leur évolution dans le temps. Ces paramètres sont différents selon la composante du système climatique que l'on va étudier. Cela peut être la température ou les précipitations, si on considère des archives continentales, mais cela peut aussi être la salinité, l'acidité ou la température de l'océan. Une approche indirecte est évidemment nécessaire, car on ne peut pas «mesurer» la température de la surface de l'océan il y a 25000 ans, par exemple. On parle donc d'indicateurs climatiques ou de "proxies» climatiques que les paléo-climatologues vont dénicher dans des archives très variées ${ }^{77} 78$.

L'histoire climatique se retrouve essentiellement dans quatre types d'archives différentes: les sédiments, marins, lacustres ou terrestres, la glace, les coraux et les arbres.

Sur les continents, le lœss, par exemple, est un indicateur très répandu en Europe et en Amérique du Nord. C'est un empilement de particules très fines transportées par le vent, qui se forme surtout pendant les phases froides et sèches des périodes glaciaires et qui enregistre ainsi l'alternance des phases glaciaire-interglaciaire.

Les pollens se conservent également très longtemps dans les sédiments lacustres ou dans les tourbières. La distribution et la quantité des différents pollens présents sur un site permettent d'évaluer les évolutions de température et de précipitation sur ce site, à la condition de connaître la distribution actuelle de ces mêmes pollens.

Pour reconstituer les climats anciens, on utilise donc ces proxies climatiques pour remonter à la température de l'eau ou de l'atmosphère, à la salinité des eaux et leur acidité, à l'intensité des précipitations, etc.

Que ce soit à partir de marqueurs vivants ou de facteurs physiques, les reconstitutions doivent être soigneusement validées et datées pour nous éclairer précisément sur les changements climatiques passés. II est donc indispensable de croiser les archives et comparer les résultats continentaux et océaniques pour que notre vision du système climatique et de son évolution soit complète.

C'est ainsi que l'analyse des sédiments marins a confirmé la théorie de Milankovitch. Plus spécifiquement, c'est l'analyse des squelettes calcaires des microorganismes,

77. https://www.climate.gov/maps-data/primer/past-climate

78. https://books.openedition.org/septentrion/52810?lang $=\mathrm{fr}$ 
les foraminifères vivant à la surface des océans que l'on retrouve dans les sédiments marins (Fig. 9.2), qui a permis de reconstituer la température de surface des océans et son évolution dans le passé.

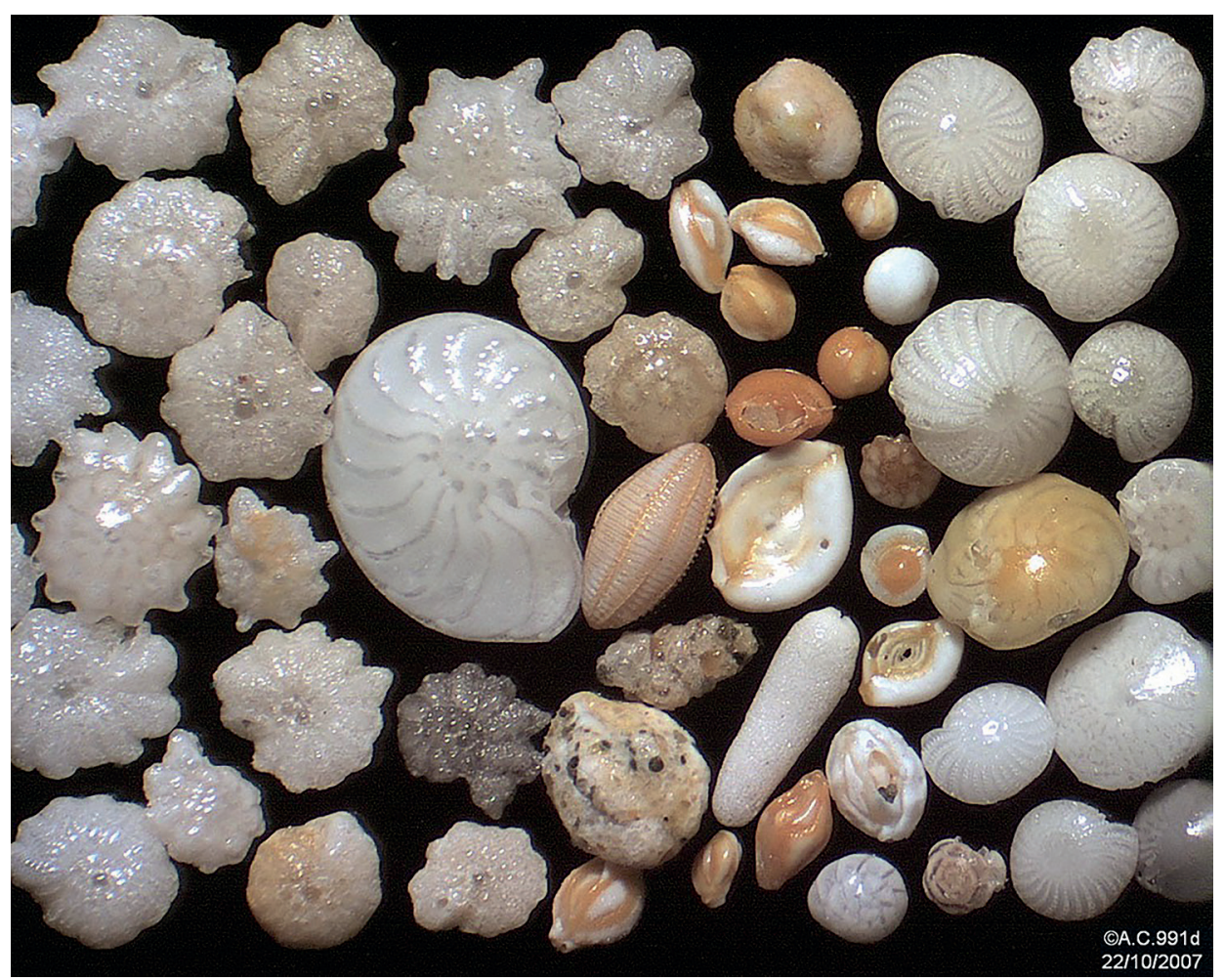

Figure 9.2: Illustration montrant la diversité des espèces de foraminifères présentes dans les océans. @A.C.991d sous licence Creative Commons.

La température de surface des océans est, en effet, un paramètre essentiel du climat, car elle contrôle les échanges d'eau et de chaleur avec l'atmosphère, et elle module aussi la solubilité des gaz et leur taux d'échange avec l'atmosphère. C'est aussi le paramètre le plus largement reconstruit à partir de différents indicateurs.

Les foraminifères sont ainsi des témoins climatiques précieux et précis. La reconstitution de l'évolution de température passe soit par la quantification de l'abondance d'une espèce par rapport à une autre, soit par l'analyse de la composition des squelettes. Le rapport magnésium sur calcium dans le squelette constitue un véritable paléothermomètre. Lors de l'élaboration de leur coquille calcaire, les foraminifères vont, en effet, incorporer du magnésium et du calcium dans un rapport qui est très précisément lié à la température (Fig. 9.3). Des calibrations sont établies selon les espèces pour être le plus précis possible. 


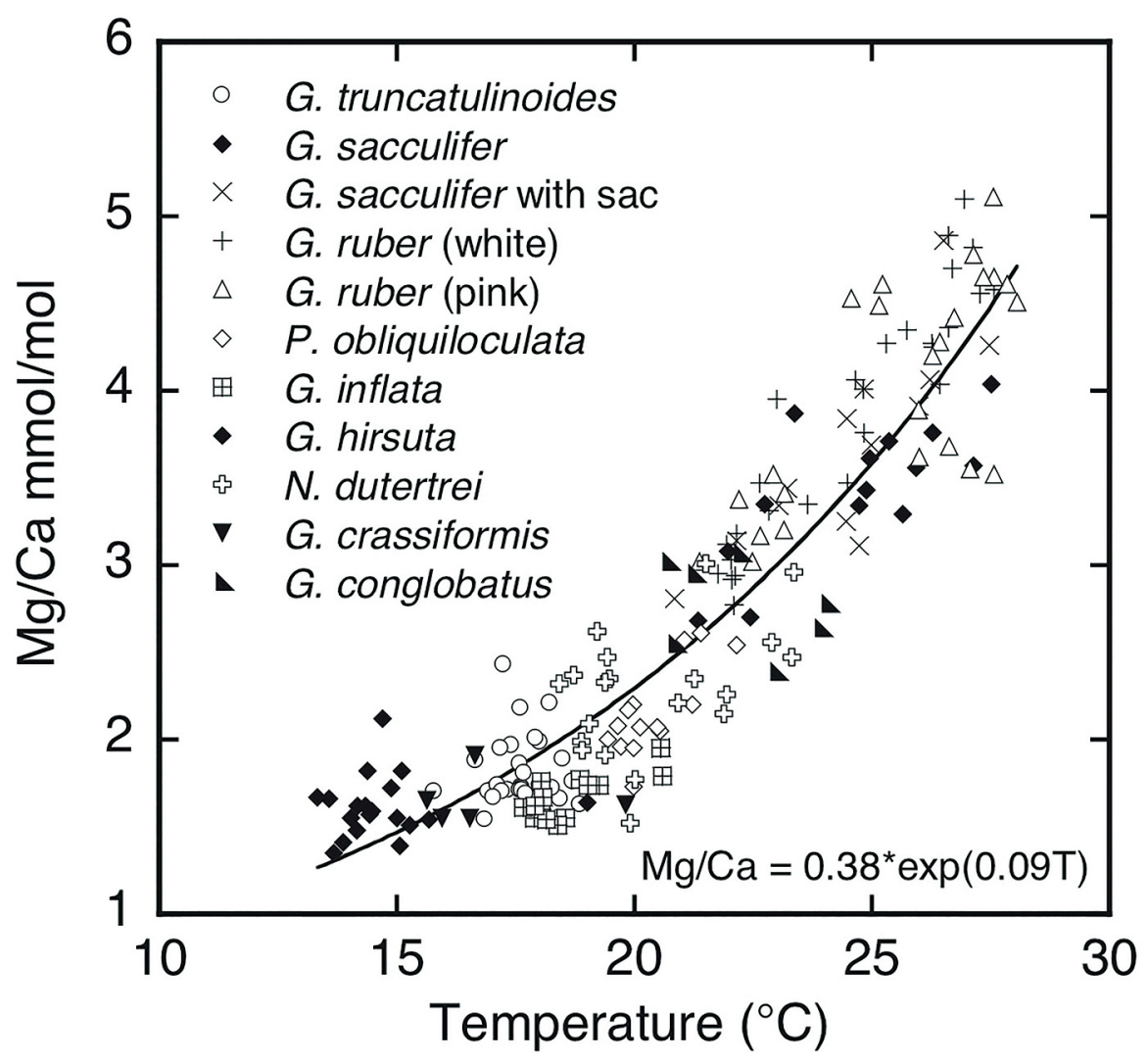

Figure 9.3: Évolution du rapport magnésium sur calcium en fonction de la température pour différentes espèces de foraminifères. Source: Figure 2 de Barker et al. (2005) [2].

On utilise aussi le thermomètre isotopique, c'est-à-dire l'analyse des proportions des différents isotopes de l'oxygène contenu dans la calcite des squelettes des foraminifères. Ces proportions dépendent à la fois de la température de l'eau de mer et de sa composition isotopique. L'utilisation combinée et comparée de ces différents proxies a permis de constituer des archives climatiques précises. Grâce à elles, on a ainsi pu démontrer que les variations des paramètres orbitaux étaient bien à l'origine des alternances entre périodes glaciaires et périodes interglaciaires (Fig. 9.4).

L'analyse des cycles glaciaires/interglaciaires via les archives climatiques a mis en évidence les interactions entre les différents forçages climatiques. Ainsi, si comme on l'a vu, les mouvements lents de la Terre autour du soleil sont le moteur des cycles glaciaires, la façon dont ces cycles sont marqués dépend aussi des modifications de la teneur atmosphérique en gaz à effet de serre. L'analyse des bulles d'air dans les glaces a, en effet, mis en évidence des variations de la concentration des gaz à effet de serre au cours des cycles glaciaires et le fait qu'elles amplifient les contrastes entre période glaciaire et période interglaciaire (courbes bleue et rouge de la Fig. 9.4). 


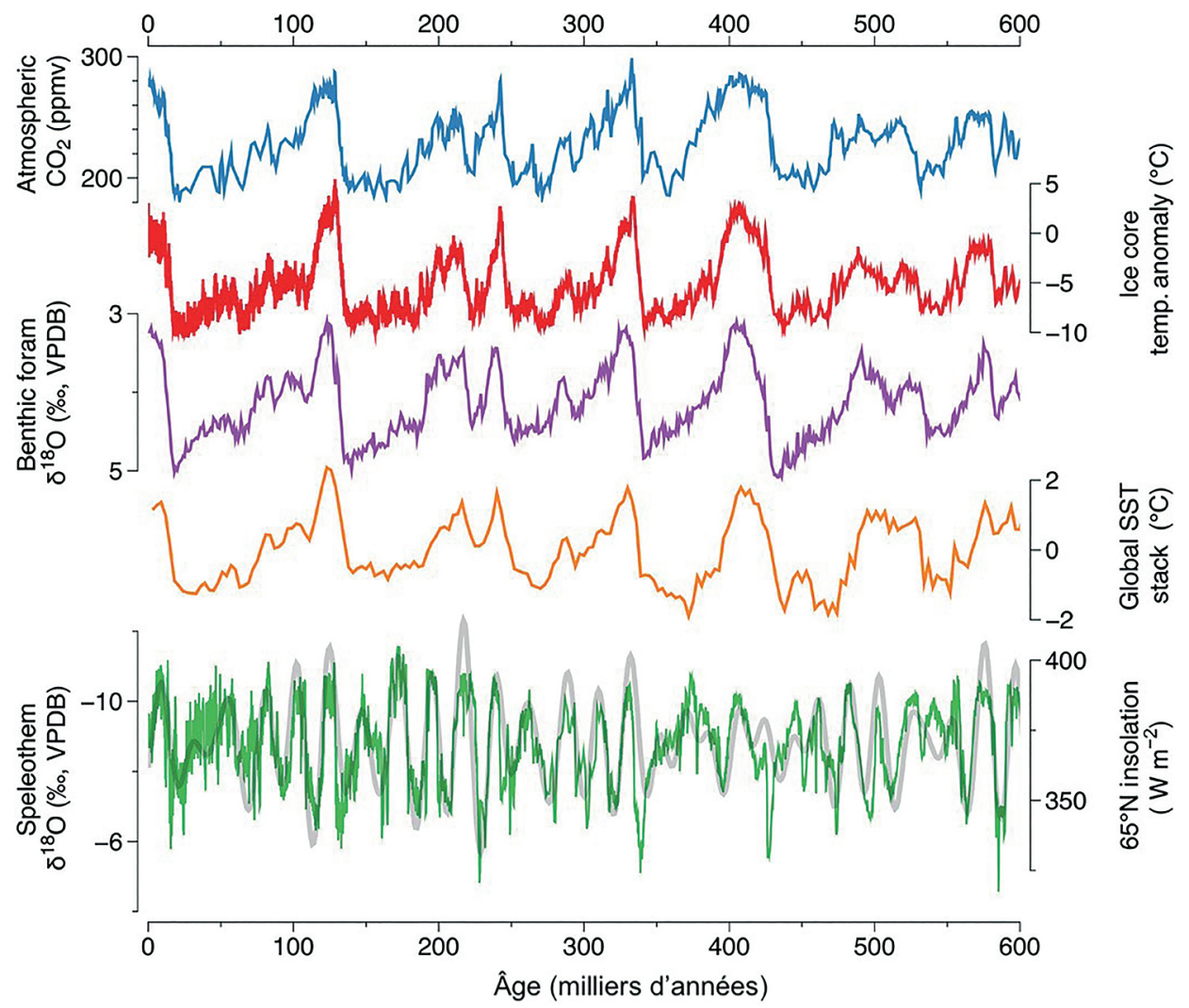

Figure 9.4: Combinaison de différents proxies pour l'étude des climats passés. En bleu : concentration de $\mathrm{CO}_{2}$ atmosphérique mesuré à partir de calottes de glace en Antarctique; en rouge : estimation de l'anomalie de la température de surface en Antarctique; en violet: proportion de l'isotope ${ }^{18} \mathrm{O}$ mesuré dans les foraminifères benthiques; en jaune: estimation de la température moyenne de surface des océans à partir de multiples enregistrements, dont le rapport $\mathrm{Mg} / \mathrm{Ca}$; en vert: évolution de la proportion de l'isotope ${ }^{18} \mathrm{O}$ dans les spéléothèmes (concrétion calcaire des grottes) mesurée en Chine; en gris: niveaux d'ensoleillement calculés à $65^{\circ} \mathrm{N}$ à partir des paramètres orbitaux. Source: Figure 1 de G. Liu et al. [3] .

Les glaces de l'Antarctique permettent aujourd'hui de reconstituer l'évolution du $\mathrm{CO}_{2}$ et du méthane atmosphérique jusqu'à 800000 ans. Les chercheurs s'efforcent désormais de remonter jusqu'à 1,2 millions d'années, ce qui permettrait d'étudier la transition entre une alternance de cycles glaciaires/interglaciaires rythmée à 100000 ans, donc contrôlée par l'excentricité et qui caractérise le dernier million d'années, et des oscillations glaciaires/interglaciaires sur une période plus courte de 40000 ans en phase avec les changements d'obliquité.

En conclusion, les archives climatiques, par leur diversité, permettent de remonter en confiance aux évolutions climatiques du passé et ce, sur des temps de plus en plus reculés. Les archives climatiques fournissent donc un banc d'essai indispensable pour tester la capacité des modèles du climat à représenter correctement ces évolutions climatiques du passé, en particulier dans les périodes de transition. 


\section{2. À quoi sert un modèle climatique? - J. Servonnat}

\subsubsection{Présentation des modèles climatiques}

Dans cette partie, nous allons donner les éléments pour comprendre en quoi consistent les modèles climatiques et comment ils sont utilisés. Prenons en exemple une question qui concerne la société: comment va évoluer le climat en réponse à une augmentation de la concentration de gaz à effet de serre? ou comment va évoluer la température du globe ou un phénomène particulier tel que les vagues de chaleur en Europe? Les modèles climatiques peuvent nous aider à répondre à ces questions.

L'idée avec un modèle climatique est de se créer une planète Terre virtuelle dont le fonctionnement repose sur les principes fondamentaux de la physique du climat et de tous ses compartiments introduits dans la section 7.1: l'atmosphère, l'océan, les surfaces continentales, la glace, les grands cycles biogéochimiques, dont celui du carbone, et leurs interactions mutuelles. Les modèles d'atmosphère et d'océan, par exemple, reposent sur les équations de la dynamique et de la thermodynamique des fluides de Navier Stokes, appliquées aux fluides géophysiques. Toutes ces équations sont résolues à différents temps et pour plusieurs milliers de points sur la Terre.

Dans ces modèles, il est possible de modifier, comme on le souhaite, des forçages climatiques, comme par exemple, imposer une augmentation de la concentration en gaz à effet de serre ou des changements de paramètres orbitaux. Au cours de la simulation, le modèle calcule l'évolution dans le temps des grandeurs physiques, biologiques et chimiques liées au climat (précipitations, températures, courants océaniques, vents, etc.) en réponse à ces forçages. Les calculs sont réalisés en tous points des grilles qui découpent en trois dimensions (longitudes, latitudes, altitudes/profondeurs), l'atmosphère, l'océan, les surfaces continentales et la glace de mer (Fig. 9.5).

Ce qu'il faut donc retenir à ce stade, c'est que ces modèles sont construits sur des lois physiques fondamentales. Pour faire simple, un modèle climatique, c'est une planète Terre virtuelle avec laquelle on peut faire des expériences comme, par exemple, faire varier les concentrations de gaz à effet de serre ou la quantité d'énergie qu'on reçoit du soleil ou encore introduire des éruptions volcaniques, et ce, non seulement dans la gamme de changements actuels, mais aussi bien au-delà. Les résultats du modèle nous permettent alors de voir comment le climat réagit en conséquence.

Nous pouvons trouver des illustrations complémentaires de ce que sont les modèles climatiques à partir de vidéos en libre accès ${ }^{79}{ }^{80}$. Nous pouvons également nous faire une première idée de projections climatiques à partir d'un modèle numérique

79. https://www.youtube.com/watch?v=toCFqOGVs54\&ab_channel=CSIRO

80. https://www.youtube.com/watch?v=FGB3mEll1ms\&t=159s\&ab_channel=ERISPROJECT - Exemple de simulation numérique à partir d'un modèle climatique 


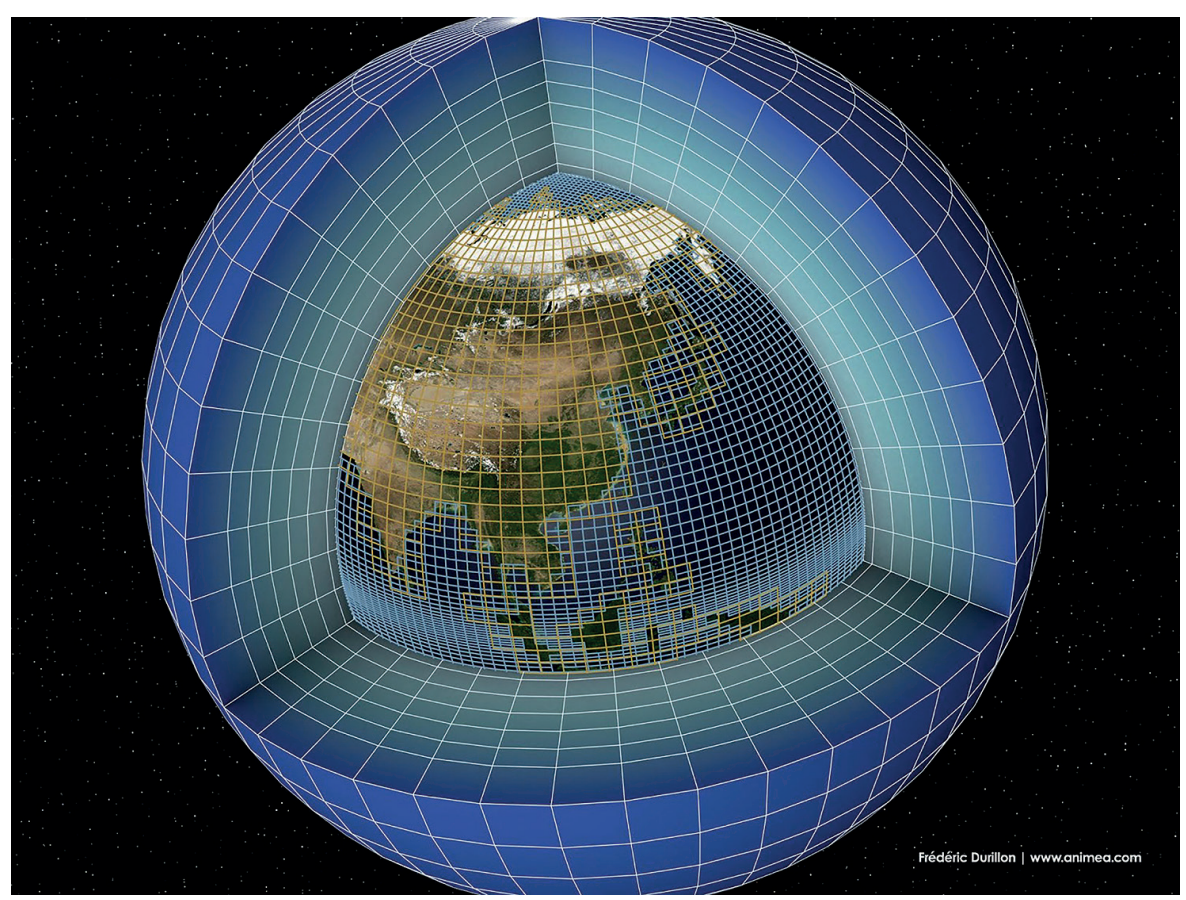

Figure 9.5: Type de maillage terrestre utilisé dans les modèles climatiques (Source: Frédéric Durillon, www.animea.com - tous droits réservés). Notons que l'échelle de l'atmosphère est dilatée verticalement pour mieux voir sa structure en trois dimensions.

qui simule le contenu en vapeur d'eau au-dessus de l'Amérique du Nord, dans la période entre avril et juin 2100, en supposant qu'aucune mesure n'ait été prise pour diminuer les émissions de gaz à effet de serre anthropiques d'ici la fin du XXle siècle (scénario socio-économique RCM8.5) ${ }^{81}$. Les résultats du modèle sont comparés à la situation qui a été observée pour la même période entre avril et juin 2011 au-dessus de l'Amérique du Nord. Une autre simulation à partir d'un deuxième modèle compare l'accumulation des nuages dans l'ensemble de l'atmosphère en 2090 à celle de l'année 1990 en supposant toujours qu'aucune mesure n'aura été prise pour diminuer les émissions de gaz à effet de serre anthropiques dans le siècle en cours (scénario socio-économique RCP8.5) ${ }^{82}$.

Tous ces modèles numériques, qui sont appelés GCM (Global Circulation Model) ou ESM (Earth System Model), sont de véritables prouesses scientifiques et techniques, car ils sont le résultat de plusieurs décennies de développement par des centaines de scientifiques. Ce que contient un modèle climatique à un moment

81. https://visgallery.ucar.edu/water-vapor-content-of-the-current-and-future-climate-of-northamerical

82. Illustration de la variabilité interne d'un modèle climatique: https://visgallery.ucar.edu/presentand-future-climate-in-a-global-model/ 
donné est une affaire de choix et de moyens techniques et humains. L'assemblage et le réglage d'un modèle est, en effet, extrêmement complexe, très coûteux en temps humain et en temps de calcul. À chacune des innombrables étapes de développement, les scientifiques ont à choisir parmi plusieurs options sur la base de critères scientifiques et techniques. Comme ils ne peuvent pas tout tester, les développeurs d'un modèle vont faire des choix, qui seront guidés par les sujets scientifiques dans leur domaine de spécialisation.

C'est pourquoi chaque groupe de modélisation dans le Monde a développé un modèle qui est différent des autres. On n'a donc pas une, mais des planètes Terre artificielles, très similaires sur les aspects généraux du climat, mais montrant des différences, notamment dans leurs réponses aux gaz à effet de serre. II existe des comparaisons entre ces modèles, qui permettent de centraliser les résultats de simulation et de les rendre accessibles à tous. Les exercices de comparaison, qui incluent également les projections futures, constituent une part importante des éléments de preuve sur lesquels s'appuient les rapports du GIEC.

\subsubsection{Variabilité interne et variabilité forcée de ces modèles}

Le but va maintenant être de mieux comprendre ce que nous disent ces modèles. Et pour cela, nous allons introduire les notions de variabilité interne, et de variabilité forcée (Fig. 9.6). Nous allons illustrer ces concepts à partir d'exemples idéalisés. Faisons ensemble une simulation climatique d'une centaine d'années.

Si l'on n'impose aucun forçage (aucune perturbation externe, Fig. 9.6a), le modèle simule quand même des variations (Fig. 9.6b). C'est ce qu'on appelle la variabilité interne, qu'on peut aussi appeler variabilité intrinsèque du système climatique. Elle est le résultat des circulations de fluides au sein de l'atmosphère et de l'océan et, plus généralement, elle est le résultat des interactions entre toutes les composantes du système climatique. Le seul moteur de cette variabilité interne est le déséquilibre thermique engendré par le rayonnement solaire, la Terre recevant notamment plus d'énergie à l'équateur qu'aux pôles (voir section 7.1).

On peut se faire une idée de ce qu'est la variabilité interne à partir d'un modèle simplifié où une Terre en rotation, supposée sans continents et donc uniquement recouverte d'eau, est simplement soumise à un flux solaire variable selon la latitude $^{83}$. L'atmosphère initialement à l'équilibre se met en mouvement, des structures apparaissent et se déplacent au cours du temps. Le même type de comportement aurait été obtenu pour les autres compartiments du système climatique : l'océan, la glace de mer, et les surfaces continentales.

83. https://visgallery.ucar.edu/water-vapor-content-of-the-current-and-future-climate-of-northamerical 


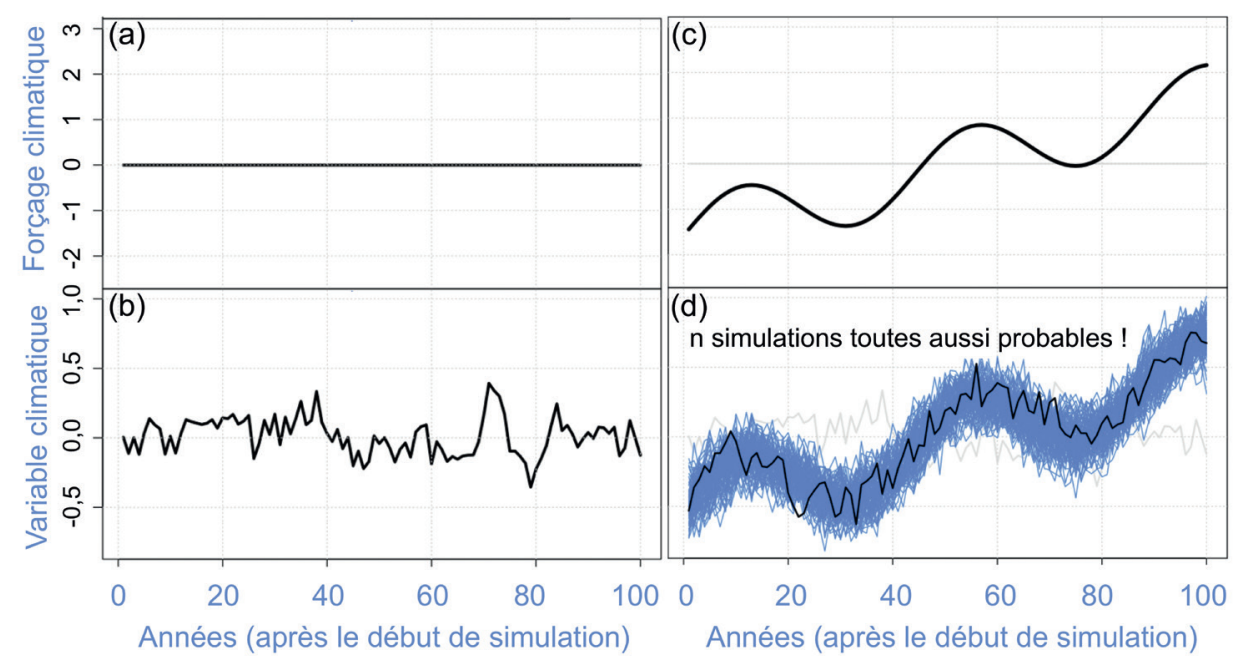

Figure 9.6: Variabilité interne et variabilité forcée d'un modèle climatique. (a) Forçage constant (nul) imposé au modèle; (b) variabilité interne seule dans ce cas de forçage (ou d'absence de forçage); (c) forçage radiatif variable imposé au modèle; (d) courbe grise : résultat du modèle ne montrant que la variabilité interne (comme sur (b)) et courbe noire: résultat complet du modèle incluant la variabilité interne et la réponse au forçage. Enveloppe bleue: superposition des résultats pour $n$ simulations différentes du modèle avec le même forçage radiatif. Source : Jérôme Servonnat.

La variabilité interne s'exprime à des échelles de temps qui vont du pas de temps du modèle, soit de quelques minutes pour les phénomènes atmosphériques à plusieurs centaines d'années pour la circulation océanique. Cette variabilité interne est par nature «chaotique» et de fait imprévisible.

Ajoutons maintenant un forçage idéalisé (Fig. 9.6c). Imaginons que ce signal représente des variations de la concentration de gaz à effet de serre. La simulation (Fig. 9.6d, courbe noire) fait toujours apparaître des oscillations aléatoires comme précédemment, mais ces oscillations sont maintenant modulées par les réponses du modèle au forçage qu'on lui fait subir. Le climat simulé par le modèle est la combinaison d'une variabilité forcée, due au forçage, et d'une variabilité interne qui, elle, est chaotique. Regardons d'abord la variabilité interne (Fig. 9.6d). Comme elle est chaotique, les éléments qui sont simulés, sont le fruit du hasard. Si nous changeons seulement un peu les conditions initiales du départ de la simulation, de l'ordre de l'incertitude incompressible sur cet état de départ, le modèle va simuler des événements différents, une histoire différente. Ceci n'est autre que le célèbre effet papillon. Si l'opération est répétée $n$ fois, à chaque fois, nous obtiendrons une séquence d'évènements légèrement différents, et toutes ces histoires sont toutes aussi probables les unes que les autres dans le monde du modèle (courbes bleues, Fig. 9.6d).

C'est pourquoi, on ne peut pas attendre de ces simulations qu'elles nous disent ce qu'il va se passer exactement à une date donnée. Étudier le climat, c'est étudier la distribution statistique du temps qu'il fait. II en est de même pour une simulation 
climatique. On privilégiera donc des analyses sur des périodes de plusieurs dizaines d'années, que ce soit pour mettre en évidence des différences de moyennes entre deux périodes ou, plus simplement, des tendances.

Considérons maintenant la variabilité forcée. Par définition, la variabilité forcée est la même d'une simulation à l'autre. On peut donc la caractériser, l'isoler en prenant en compte la moyenne de toutes les simulations. Cela peut nous permettre d'estimer la part de la variabilité simulée, qui est due à la variabilité forcée, et celle qui est due à la variabilité interne. C'est en suivant cette démarche que l'on peut, par exemple, estimer la contribution des gaz à effet de serre anthropiques aux changements climatiques des cent dernières années. Tous les modèles n'ont pas la même variabilité interne, ni systématiquement la même réponse à un même forçage. On est donc en présence de deux sources d'incertitude qu'il faut prendre en compte.

Cela étant, lorsque l'on compare les températures moyennes simulées par l'ensemble des modèles, tous simulent un réchauffement climatique avec l'augmentation de gaz à effet de serre, l'amplitude de réchauffement étant toutefois fortement différente d'un modèle à l'autre. Concernant les précipitations sur telle ou telle région, certains modèles vont simuler une augmentation et d'autres, une diminution. Et jusqu'ici, tous ces modèles donnent toujours des effets probables.

\subsection{3. Évaluation des modèles}

Quelle confiance accorder à tous ces modèles? Pour cela, il faut comparer le climat qu'ils simulent, avec ce qui est observé. C'est une démarche de base en physique. On observe un phénomène, on le théorise avec un modèle, et on vérifie si la théorie permet d'expliquer l'observation.

Il existe trois grandes formes d'évaluation. La première est ce qu'on pourrait appeler l'évaluation routinière. Au cours du développement d'un modèle climatique, les centres de modélisation réalisent des batteries de tests pour vérifier si le climat simulé par le modèle reproduit bien les grandes caractéristiques du climat récent. C'est en quelque sorte le contrôle qualité qui assure que le modèle reproduit bien le climat terrestre et qu'il permet également aux modélisateurs de s'assurer que les améliorations, qu'ils souhaitent apporter, permettent effectivement d'améliorer le modèle.

Comme deuxième type d'évaluation, on dispose de l'étude des paléoclimats. Le meilleur moyen pour savoir comment le climat évolue en réponse à des grandes variations de forçage, c'est d'étudier les climats du passé. On dispose, en effet, de reconstructions des variations climatiques passées et des forçages de ces mêmes époques. Le jeu pour le modélisateur est alors de voir s'il peut reproduire ces variations climatiques en imposant ces reconstructions de forçage au modèle. C'est un moyen de tester les réponses des modèles aux forçages.

Enfin, le troisième type d'évaluation est l'évaluation ciblée. Supposons que l'on s'intéresse à l'impact du changement climatique sur un phénomène en particulier 
comme, par exemple, les canicules en Europe ou la saisonnalité des précipitations sur une région d'Afrique. Les deux types d'évaluations précédentes ne suffisent pas à nous dire si ce climat régional est bien représenté. II nous faut impérativement disposer d'une évaluation ciblée de ces phénomènes dans les modèles pour avoir un regard critique sur leurs prédictions. Donc, soit on la trouve dans la littérature, soit on doit la faire nous-mêmes.

Ce regard critique nous permettra éventuellement d'exclure certains modèles d'une analyse parce qu'ils ne remplissent pas les critères que nous nous sommes fixés. Nous pourrions aussi envisager de pondérer la projection par la note obtenue par chaque modèle sur différents critères d'évaluation. L'évaluation ne nous permettra pas, en revanche, d'identifier un seul meilleur modèle qui pourrait être pris comme notre meilleure manière de nous projeter dans le futur. Pour faire simple, le système climatique est trop complexe pour être évalué de manière exhaustive. II y a trop d'incertitudes à plusieurs niveaux pour que l'on puisse démontrer qu'un seul modèle puisse suffire. Au contraire, la diversité de modèles est une richesse qu'il faut exploiter pleinement. Avec les modèles climatiques, on ne projette pas le futur qui va se produire, mais des futurs probables.

\subsection{Scénarios futurs et résultats - P. Braconnot}

\subsubsection{Rappels sur les éléments constitutifs de la modélisation climatique}

Afin d'anticiper l'évolution future du climat, il est d'abord nécessaire d'identifier les différents facteurs qui peuvent le modifier, ce que l'on appelle les forçages.

II s'agit, en particulier, d'anticiper l'impact des activités humaines, car les émissions croissantes de gaz à effet de serre, dont le dioxyde de carbone ou $\mathrm{CO}_{2}$, sont des facteurs majeurs de réchauffement. Les activités humaines émettent également des particules, les aérosols, qui ont un effet plus régional, majoritairement refroidissant mais aussi réchauffant pour certains comme les suies. Notre utilisation des terres, c'est-à-dire l'agriculture ou la déforestation, modifie aussi bien le climat que les cycles biogéochimiques, celui du carbone ou celui de l'azote, qui agissent en retour sur le climat. En résumé, pour anticiper les évolutions futures du climat, il faut donc faire des hypothèses quant à l'évolution des activités humaines qui émettent des gaz à effet de serre ou des aérosols et qui modifient l'usage des terres, c'est ce que l'on appelle des scénarios [4].

N'oublions pas que ces facteurs anthropiques se superposent à des facteurs d'origine naturelle comme la fluctuation de l'activité solaire ou des éruptions volcaniques qui refroidissent le climat sur plusieurs années, mais dont il n'est pas possible d'anticiper les manifestations sporadiques.

Le modèle de climat va permettre de calculer comment les changements de concentration des différents gaz à effet de serre et de la végétation vont modifier le 
bilan énergétique terrestre, car ces perturbations énergétiques sont la clef du changement climatique. Ces perturbations vont induire, à leur tour, des modifications de la circulation de l'atmosphère et de l'océan, des températures, des courants, des vents et des précipitations (Fig. 9.7).

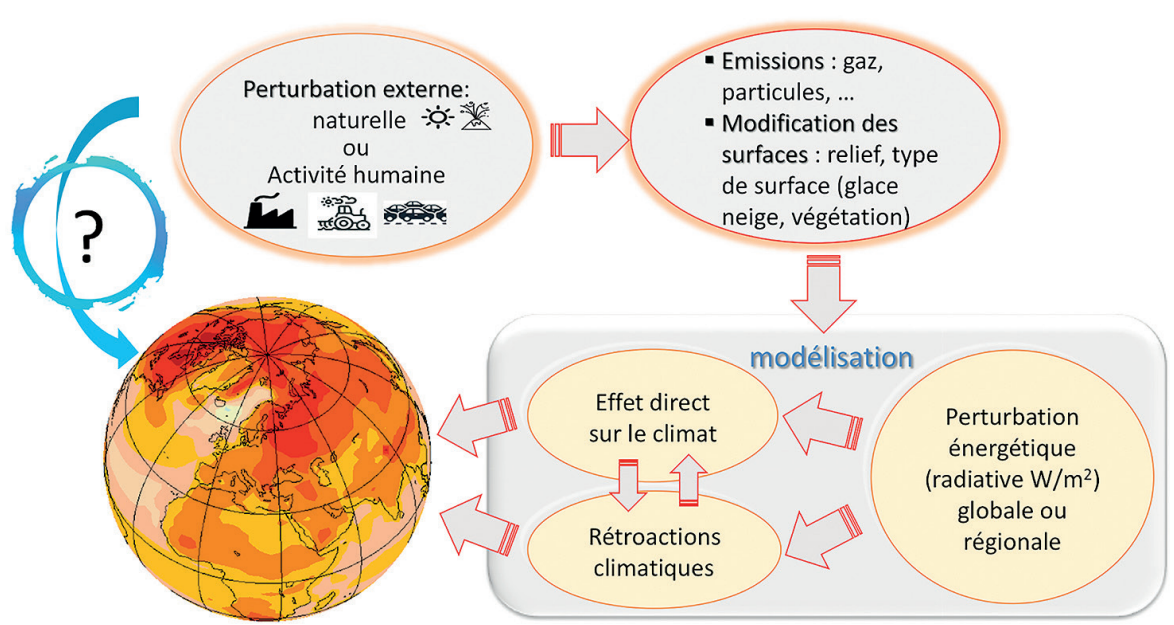

Figure 9.7: Schéma montrant la façon dont les perturbations externes d'origine naturelle ou anthropique sont prises en compte dans un modèle climatique. Source: Pascale Braconnot.

En résumé, la modélisation prend non seulement en compte les effets directs des perturbations liées aux activités humaines sur le climat, mais aussi les nombreuses rétroactions induites par les changements climatiques. Comme exemple de rétroaction, le réchauffement fait fondre la neige et fait donc apparaître des surfaces plus sombres, qui absorbent l'énergie solaire au lieu de la refléter. Ceci induit un réchauffement additionnel, on dit alors que la rétroaction est positive, car elle amplifie le réchauffement climatique.

\subsubsection{Construction des scénarios}

Les projections du climat futur font l'objet d'un sous-projet spécifique du programme international d'intercomparaison des modèles de climat (CMIP). Des experts en modélisation du climat collaborent avec des économistes afin d'établir ensemble différents scénarios.

Ces scénarios sont basés sur différentes hypothèses d'évolution de la population, de l'économie, de la demande en énergie, des technologies disponibles et des choix politiques en matière de climat et de pollution. L'objectif est de couvrir une large gamme de futurs possibles engendrant différents niveaux de gaz à effet de serre, différents usages des sols et donc différents climats qui vont être explorés par les modèles climatiques. On appelle ces scénarios trajectoires socio-économiques partagées ou SSPs (Shared Socioeconomic Pathways, en anglais). 
La figure 9.8 présente les cinq grandes familles de trajectoires socioéconomiques, classées suivant les défis socio-économiques à relever en termes d'atténuation et d'adaptation. Atténuer doit être compris comme : réduire le nombre de $\mathrm{W} / \mathrm{m}^{2}$ apportés au système climatique par l'activité humaine. S'adapter est mettre en place des solutions pour faire face au changement climatique. Les deux actions sont évidemment liées.

Des futurs très variés sont envisagés comme possibles, que ce soit d'une famille SSP à l'autre ou au sein d'une même famille. Ainsi, par exemple, le SSP5 est gourmand en énergie fossile alors que le SSP1 met en place des mesures pour remplir les objectifs de l'accord de Paris de 2015 sur le développement durable. Le SSP3 met l'accent sur les rivalités entre pays et le SSP4, sur les inégalités à l'intérieur d'un pays. Le SSP2 propose, quant à lui, une évolution intermédiaire.

Pour chaque famille, il existe, de plus, différentes possibilités de faire évoluer l'usage des terres ainsi que les émissions de gaz à effet de serre ou d'aérosols, et le forçage radiatif dépendant des détails du narratif choisi (Fig. 9.9). In fine, les perturbations de bilan radiatif (en anglais: RCP pour Radiative Concentrations Pathways) envisagées en 2100 dans les simulations du dernier programme CMIP6 s'étendent de 1,9 à $8,5 \mathrm{~W} / \mathrm{m}^{2}$. Ces chiffres correspondent à des trajectoires particulières issues des différentes familles socio-économiques [5].

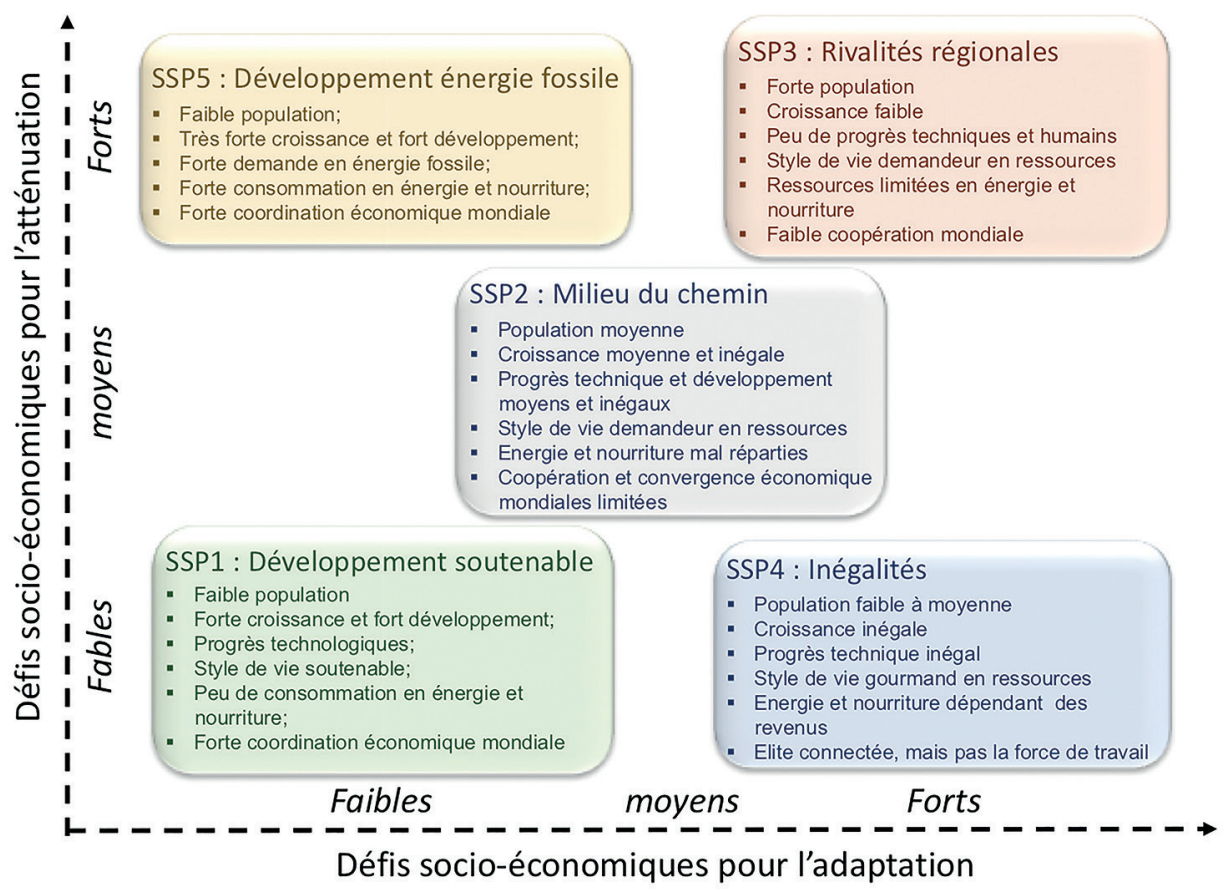

Figure 9.8: Familles de trajectoires socio-économiques (SSP) classées selon les défis à relever pour atténuer les changements climatiques ou pour s'y adapter. D'après O'Neil et al., 2016 [4]. 


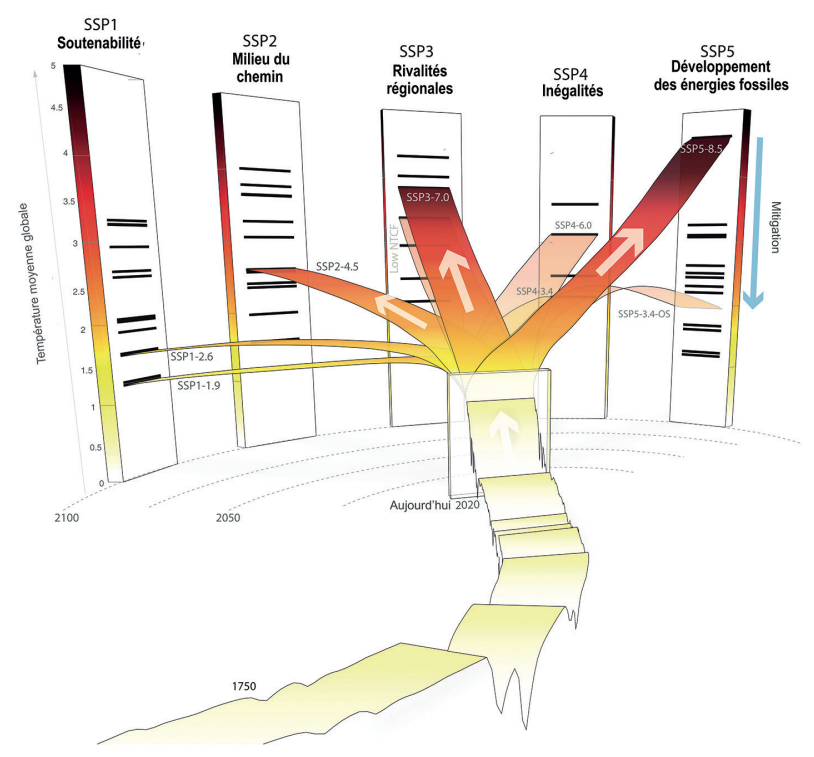

Figure 9.9: Schéma illustrant différents choix possibles de trajectoires d'évolution concernant l'usage des terres, les gaz à effet de serre ou les aérosols au sein des familles SSP (ici SSP1 et SSP3). Ces choix se traduisent in fine par différentes amplitudes des perturbations radiatives en 2100 (exprimées en $\mathrm{W} / \mathrm{m}^{2}$ ). Source: [5] sous Creative Commons License.

\subsubsection{Projections futures des scénarios et modèles climatiques}

Les modèles climatiques vont ainsi permettre d'explorer le climat futur selon les hypothèses choisies dans les différents scénarios, et l'on doit garder en mémoire que les résultats obtenus dépendent de ces hypothèses. Les hypothèses sont, en fait, des choix que l'humanité doit faire par la mise en place de politiques d'atténuation des changements climatiques. Les politiques d'adaptation aux changements climatiques doivent tenir compte, elles-mêmes, du fait que différents futurs sont possibles.

La figure 9.10 représente des résultats de simulation qui portent sur l'évolution de la température moyenne à la surface du globe et qui sont obtenus à partir des deux modèles climatiques français, celui de l'Institut Pierre Simon Laplace (IPSL [6]) et celui du Centre National de Recherches météorologiques (CNRM [7]). La partie la plus à gauche de la figure montre d'abord l'évolution de cette température calculée de 1880 à 1994 et comparée aux observations sur le terrain. On note le bon accord entre mesures et simulations pour cette période où l'élévation de température s'est «limitée» à $1^{\circ} \mathrm{C}$. La partie à droite de la figure correspond aux projections d'évolution calculées jusqu'à la fin du siècle par les deux mêmes modèles, mais pour quatre types de scénario. Quel que soit le scénario (mesures faibles ou fortes d'atténuation des changements climatiques), les résultats obtenus prévoient une augmentation de la température moyenne au-delà de $2^{\circ} \mathrm{C}$ environ. 


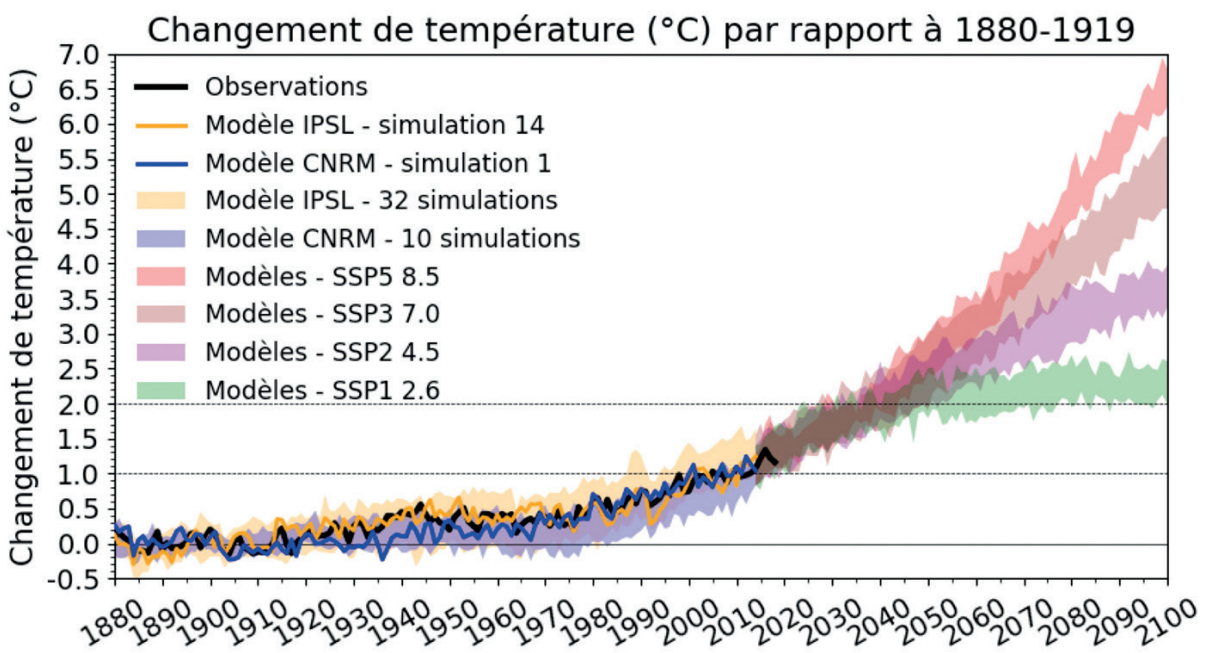

Figure 9.10: Évolution de la température moyenne de la planète. De 1880 à nos jours: évolution observée (courbe noire, 1880-2018) et évolutions estimées par un ensemble de simulations issues du modèle de l'IPSL (enveloppe jaune, 1880-2014) et du modèle CNRM-CM6 (enveloppe bleue, 1880-2014). On note que chacun des deux modèles simule bien la courbe observée (traits continus de couleur). Projections sur le 21e siècle: quatre scénarios sont envisagés: SSP1 2,6, SSP2 4,5, SSP3 7,0 et SSP5 8,5. Pour chaque scénario futur, l'enveloppe de couleur obtenue regroupe l'ensemble des simulations réalisées à partir des deux modèles. La période de référence choisie pour les projections est celle entre 1880 et $1919^{84}$.

\subsection{Combien de carbone nous reste-t-il à émettre? - S. Szopa}

\subsubsection{Objectif de réchauffement global}

Le budget carbone restant correspond à la quantité de $\mathrm{CO}_{2}$ qui peut encore être émise dans l'atmosphère avant de dépasser un certain seuil de température. C'est, en effet, en termes de température à ne pas dépasser qu'a été négocié l'accord de Paris, accord international sur le climat signé en 2015. Le réchauffement climatique est une problématique environnementale globale qui nécessite une action internationale coordonnée. Celle-ci implique donc d'abord de s'entendre sur le fait que le réchauffement actuel est bien lié aux activités humaines, pour ensuite se fixer un objectif commun et tenter d'avoir une stratégie qui limite le changement climatique. L'accord de Paris fixe comme objectif une limitation du réchauffement mondial entre $1,5^{\circ} \mathrm{C}$ et $2^{\circ} \mathrm{C}$ d'ici 2100 . Se fixer un objectif de réchauffement à ne pas dépasser permet également de dimensionner les politiques d'adaptation si, toutefois, les politiques compatibles avec cet objectif sont mises en place de manière tangible. 
Pour comprendre ce que signifie cet objectif, rappelons que le réchauffement actuel, tel qu'estimé à partir des observations dont nous disposons, est d'environ $1{ }^{\circ} \mathrm{C}$ par rapport à la température globale à l'ère préindustrielle.

L'accord de Paris mentionne donc deux seuils de température, qui peuvent sembler très proches l'un de l'autre. Pour comprendre les conséquences liées au dépassement de l'un ou l'autre des seuils, les États ont mandaté le GIEC de manière à ce qu'il coordonne la rédaction d'une synthèse des connaissances portant sur les conséquences des dépassements liés à ces seuils ainsi que sur les faisabilités techniques et économiques pour rester au-dessous de ces seuils. II s'agit du rapport spécial $1,5^{\circ} \mathrm{C}$ du GIEC qui a permis de démontrer qu'en termes d'impacts du changement climatique, chaque demi-degré compte ${ }^{85}$. Ce rapport a été établi grâce à un processus d'analyse de la littérature scientifique propre au GIEC (voir ci-après, section 9.5.2). Dans le résumé pour décideur du rapport IPCC SR1.5 ${ }^{87}$, les résultats des études d'impacts et des risques encourus sont synthétisés comme le montre la figure 9.12 (non exhaustive) ci-après.

Étant donné le niveau de réchauffement actuel de $1^{\circ} \mathrm{C}$, qui résulte des émissions passées de gaz à effet de serre, on constate, d'après la figure 9.11, que certains écosystèmes, comme les coraux d'eau chaude, sont d'ores et déjà affectés de manière irréversible ou persistante par le réchauffement actuel. La pêche et l'aquaculture sont aussi confrontées à des risques croissants dès $1^{\circ} \mathrm{C}$ de réchauffement. En revanche, pour d'autres systèmes comme les cultures de céréales, on peut raisonnablement penser qu'une augmentation à $2^{\circ} \mathrm{C}$, même si elle n'est pas sans conséquences sur ces cultures, n'engendrera qu'un risque modéré auquel il sera, sans doute, possible de s'adapter en partie.

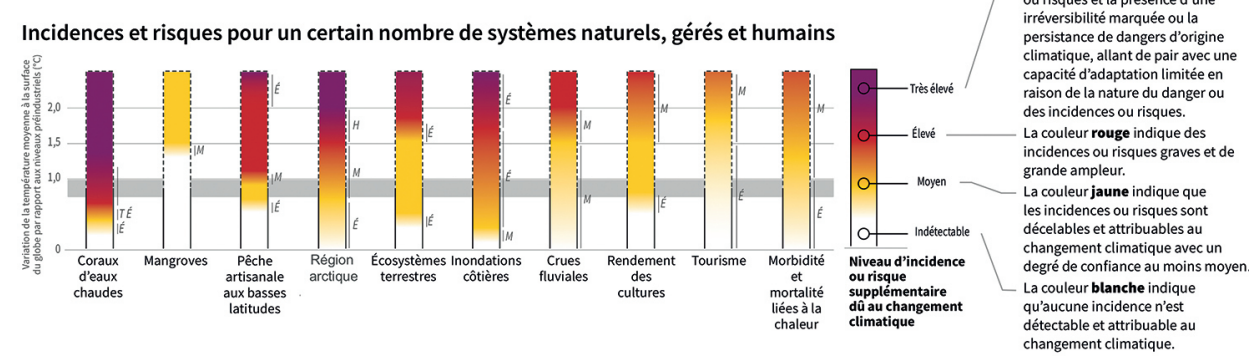

Figure 9.11: Impacts et risques encourus pour différents systèmes naturels selon le niveau d'augmentation de la température moyenne à la surface de la Terre (Extrait de la Figure SPM2, rapport IPCC 2018, SR1.5).

85. Rapport spécial IPCC 1.5

86. Rapport complet et figures téléchargeables à https://www.ipcc.ch/sr15/

87. Résumé pour décideurs en français:

https://www.ipcc.ch/site/assets/uploads/sites/2/2019/09/IPCC-Special-Report-1.5-SPM_fr.pdf 
On voit donc que les impacts diffèrent d'un domaine à l'autre et donc aussi, d'une région à l'autre. Le cap, que l'on se fixe, a forcément un caractère subjectif. On note également dans la figure 9.11 (à droite) que le GIEC a déterminé un degré de confiance pour les différents impacts et risques. Ceci est un point fondamental des travaux d'expertise du GIEC.

\subsubsection{Estimation du carbone restant à émettre}

Nous venons de voir que les impacts du réchauffement climatique vont dépendre du niveau de réchauffement. II reste maintenant à savoir quel niveau d'émission de $\mathrm{CO}_{2}$ est compatible avec cet objectif de température. Nous savons, en effet, que le $\mathrm{CO}_{2}$ atmosphérique est le principal gaz à effet de serre additionnel (voir section 7.2.2) et que pour limiter le $\mathrm{CO}_{2}$ atmosphérique, il est nécessaire de limiter nos émissions de $\mathrm{CO}_{2}$. Cela étant, pour fixer plus précisément les objectifs d'émission par pays ou par continent, encore faut-il connaître la relation entre émissions de $\mathrm{CO}_{2}$ et température de surface.

Pour rappel, une partie du $\mathrm{CO}_{2}$ émis vers l'atmosphère par les activités anthropiques va être captée par la végétation terrestre et l'océan. Comme les activités humaines émettent chaque année plus que ce que ces puits ne peuvent capturer, le $\mathrm{CO}_{2}$ s'accumule dans l'atmosphère (voir section 7.1). Dans l'état actuel du système Terre, il existe une relation de proportionnalité entre la quantité émise et la quantité de $\mathrm{CO}_{2}$ qui s'accumule dans l'atmosphère.

En connaissant les quantités de $\mathrm{CO}_{2}$ émises par les activités humaines année après année depuis le début de l'ère industrielle, il nous est possible de relier l'augmentation de température de surface à la quantité cumulée d'émissions de $\mathrm{CO}_{2}$ pour chaque année (par exemple, points noirs sur la Fig. 9.12, issus de résultats de modèles antérieurs à 2013, et fine courbe bleue issue de travaux de 2018).

Les modèles climatiques (voir section 9.2), qui prennent en compte les éléments du cycle du carbone, notamment les puits de carbone que sont l'océan et la végétation, ont permis de mettre en évidence que la température de surface augmente effectivement et de façon quasi linéaire avec la teneur atmosphérique en $\mathrm{CO}_{2}, \mathrm{y}$ compris pour des quantités élevées d'émissions cumulées de $\mathrm{CO}_{2}$. Ces modèles sont, en effet, capables de calculer la température de surface atteinte pour chaque niveau d'émissions cumulées de $\mathrm{CO}_{2}$, qu'il s'agisse des niveaux passés ou qu'il s'agisse de niveaux hypothétiques comme dans les scénarios futurs. La courbe rouge de la figure 9.12 représente ainsi une évolution de la température de surface, moyennée sur différents modèles considérés dans le rapport GIEC de 2013. Cette évolution quasi-linéaire a été utilisée pour prolonger la relation observée jusqu'en 2017 (courbe mauve dans le prolongement de la courbe bleue). 


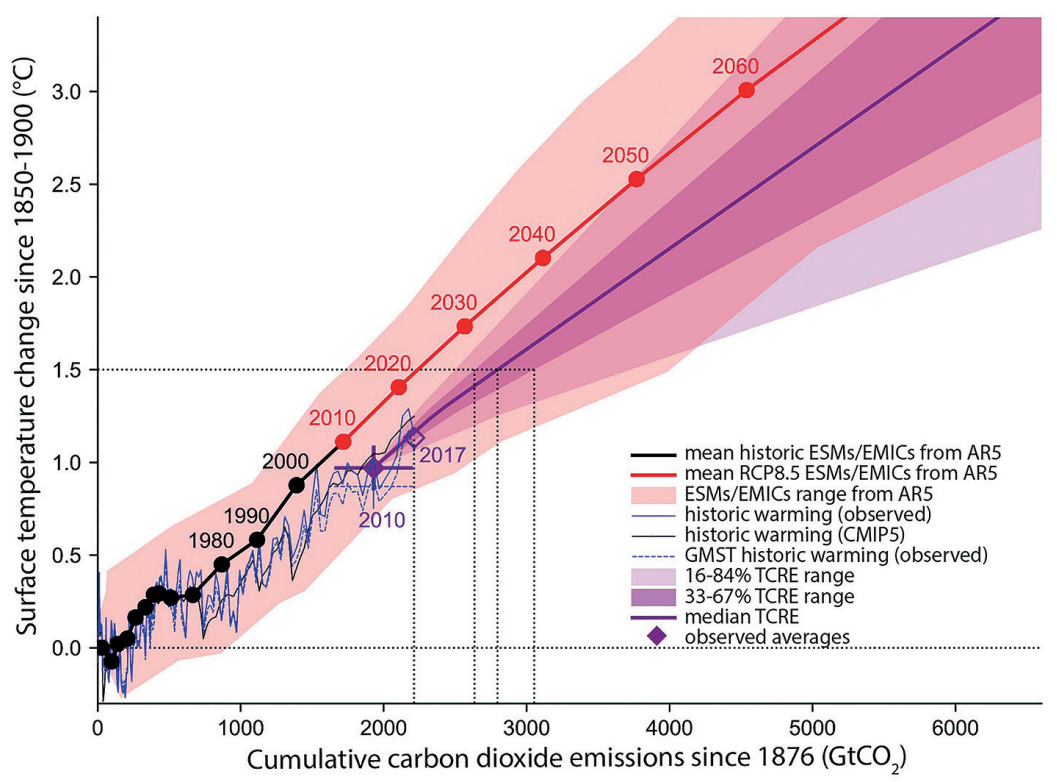

Figure 9.12: Réponse transitoire de la température moyenne de surface aux émissions cumulées de $\mathrm{CO}_{2}$ depuis 1876 [Figure 2.3 extraite du chapitre 2 du rapport IPCC, 2018, SR1.5].

Il a, par ailleurs, été démontré que la température atteinte à un niveau d'émissions cumulées de $\mathrm{CO}_{2}$ est proche de la température d'équilibre qui serait obtenue si l'on cessait (fictivement) d'émettre du $\mathrm{CO}_{2}$. Cette température se maintient ensuite, pendant longtemps, car une partie du $\mathrm{CO}_{2}$ d'origine anthropique demeure dans l'atmosphère pour des millénaires. En conséquence, le niveau de réchauffement auquel nous serons capables de stabiliser la température dépend du $\mathrm{CO}_{2}$ que l'on va ajouter à ce que l'on a déjà émis. De fait, rien n'empêche, physiquement, de stabiliser la température à son niveau actuel. C'est donc seulement notre capacité à nous affranchir des émissions de $\mathrm{CO}_{2}$ qui va déterminer le maximum de température globale que nous allons atteindre.

En conclusion, le niveau de réchauffement actuel est lié à l'ensemble du $\mathrm{CO}_{2}$ émis et cumulé sur des décennies. On réalise, d'après la figure 9.12, que pour ne pas dépasser un réchauffement de $1,5^{\circ} \mathrm{C}$, les émissions cumulées de $\mathrm{CO}_{2}$ doivent être inférieures à $2800 \mathrm{Gt}$. La marge d'incertitude étant toutefois de quelques centaines de gigatonnes. Or, le projet Global Carbon Project, qui réévalue chaque année le budget de carbone mondial, estime à $2400 \pm 238 \mathrm{Gt}$ le total des émissions de $\mathrm{CO}_{2}$ cumulées dans la période de 1850 à 2019 et liées à l'usage d'énergies fossiles, aux industries et aux changements d'usage des sols ${ }^{88}$ 89. En poursuivant le rythme actuel des émissions de $\mathrm{CO}_{2}$, soit environ $40 \mathrm{Gt}$ par an, les chiffres précédents nous

88. Rapport Le Quéré et al. (2018) : https://essd.copernicus.org/articles/10/2141/2018/

89. Global Carbon Project: https://www.globalcarbonproject.org 
permettent donc d'estimer que le seuil de $1,5^{\circ} \mathrm{C}$ sera éventuellement dépassé d'ici 10 ans.

II nous faut cependant souligner à nouveau que la figure 9.12 comporte des incertitudes dues à la dispersion des réponses des différents modèles de climat et qu'elle ne considère que le $\mathrm{CO}_{2}$ comme gaz à effet de serre. Cette figure fournit donc un ordre de grandeur de la quantité de $\mathrm{CO}_{2}$ restant à émettre et non une quantité absolue.

\subsubsection{Différentes options pour ne pas dépasser les seuils prescrits}

Dans le cadre du rapport SR1.5 du GIEC, de nombreux scénarios de modification des émissions de $\mathrm{CO}_{2}$ ont été analysés afin d'évaluer leur faisabilité, leur coût et leur impact sur le climat. Ces travaux ont montré que différentes trajectoires pour parvenir à l'objectif sont possibles (voir section 9.3), allant d'une réduction continue et progressive des émissions à une réduction différée mais qui est alors plus abrupte. Cependant, quelles que soient les trajectoires, des changements importants sont nécessaires dans tous les secteurs d'activité afin d'atténuer l'ampleur du réchauffement climatique, et chaque année compte pour la mise en place de ces changements.

Par ailleurs, toutes les trajectoires prévoient une élimination partielle du dioxyde de carbone produit (voir «les émissions négatives» en section 11.7), mais la quantité éliminée varie selon les trajectoires, tout comme les contributions relatives de la bioénergie avec captage et stockage du dioxyde de carbone (BECSC) et les éliminations réalisées dans le secteur de l'agriculture, des forêts et des autres utilisations des terres (AFAUT). Ces deux contributions mettent une pression supplémentaire sur l'usage des sols qu'il faudra donc accompagner.

\subsection{Présentation du GIEC et des rapports spéciaux - Équipe GIEC}

\subsubsection{Qu'est-ce que le GIEC?}

Nous avons sans doute tous entendu parler du GIEC (ou IPCC en anglais) qui a d'ailleurs déjà été mentionné à de nombreuses reprises dans cet ouvrage? Mais de quoi s'agit-il?

Le GIEC ou Groupe d'experts intergouvernemental sur l'évolution du climat (en anglais: Intergovernmental Panel on Climate Change) est l'organisme international chargé d'évaluer la science en lien avec le changement climatique ${ }^{90}$.

90. https://fr.wikipedia.org/wiki/Groupe_d\%27experts_intergouvernemental_sur_I\%27évolution_du_ climat 
Le GIEC a été créé en 1988 pour fournir régulièrement des évaluations relatives aux impacts et risques liés au changement climatique ainsi qu'aux options possibles pour y répondre, c'est-à-dire les moyens à mettre en œuvre dans les stratégies d'adaptation et de parade. Ces évaluations sont destinées aux:

- décideurs politiques;

- gouvernements;

- universités;

- et grand public.

Tous les pays membres de l'organisation météorologique mondiale (OMM) et des Nations Unies sont invités à rejoindre le GIEC. À l'heure actuelle, l'organisation compte 195 membres.

Le GIEC fonctionne en séances plénières régulières. II est divisé en 3 groupes de travail qui se concentrent sur des aspects spécifiques du changement climatique. Ces groupes de travail sont complétés par une équipe spéciale chargée de développer des méthodes pour estimer les émissions nationales de gaz à effet de serre et leur atténuation.

Si le GIEC produit principalement des rapports d'évaluation (par groupe de travail), il peut aussi rédiger des rapports spéciaux - qui traitent de sujets interdisciplinaires (avec plusieurs groupes de travail) - ainsi que des rapports méthodologiques.

Tous ces rapports sont non seulement écrits par des centaines d'éminents scientifiques, mais ils sont aussi examinés par des milliers d'experts venant du monde entier.

C'est pourquoi les rapports du GIEC peuvent être considérés comme la source la plus fiable de connaissance scientifique sur le changement climatique.

Le GIEC représente donc l'autorité scientifique à l'échelle mondiale dans le domaine du changement climatique.

\subsubsection{Comment sont produits les rapports du GIEC?}

On peut se demander en quoi des rapports écrits peuvent être utiles pour s'informer sur le changement climatique. En fait, plus qu'utiles, ils sont essentiels!

Les rapports du GIEC sont écrits par des centaines d'experts scientifiques provenant du monde entier. Ces experts sont sélectionnés sur la base de leur domaine d'expertise. Les équipes d'auteurs sont constituées de manière à garantir un équilibre géographique ainsi qu'un équilibre des genres. Ils rédigent les rapports sur la base du volontariat, en plus de leur travail de chercheur/professeur.

Des milliers d'autres experts contribuent également au processus en examinant les rapports et en s'assurant qu'ils reflètent bien l'état des connaissances de la communauté scientifique. 
Les rapports traversent plusieurs étapes de rédaction et de relecture, afin de garantir le plus haut niveau de rigueur scientifique. La relecture est une partie essentielle du processus du GIEC. Lors des différentes étapes, les experts relecteurs et les gouvernements sont invités à commenter les contenus des versions successives du rapport avant publication ainsi que leur cohérence.

Le résumé pour décideurs de chaque rapport est approuvé ligne après ligne par les délégations des gouvernements, en discussion avec les auteurs qui s'assurent de la véracité scientifique, avant d'être finalement accepté par le GIEC.

Ce processus long et minutieux garantit aux rapports d'évaluation du GIEC un contenu clair, scientifiquement rigoureux et objectif. C'est pourquoi, ils fournissent aux décideurs une base robuste pour la mise en place de politiques climatiques.

\section{Références}

[1] W.F. Ruddiman. 2007. The early anthropogenic hypothesis: Challenges and responses. Review of Geophysics 45. https://agupubs.onlinelibrary.wiley.com/doi/full/10.1029/ 2006 RG000207.

[2] S. Barker, I. Cacho, H. Benway, K. Tachikawa. 2005. Planktonic foraminiferal Mg/Ca as a proxy for past oceanic temperatures: a methodological overview and data compilation for the last glacial maximum. Quaternary Science Reviews 24, 821-834. https://www.whoi.edu/ cms/files/hbenway/2006/6/BarkerQSR(2005)_11406.pdf.

[3] G. Liu, X. Li, H-W. Chiang, H. Cheng, S. Yuan, S. Chawchai, S. He, Y. Lu, L.T. Aung, .... 2020. On the glacial-interglacial variability of the Asian monsoon in speleothem $d^{18} \mathrm{O}$ records. Science Advances 6(7), eaay8189. https://advances.sciencemag.org/content/6/7/ eaay8189.

[4] B.C. O'Neill, et al. 2016. The Scenario Model Intercomparison Project (ScenarioMIP) for CMIP6. Geosci. Model Dev. 9, 3461-3482.

[5] M. Meinshausen, et al. 2020. The shared socio-economic pathway (SSP) greenhouse gas concentrations and their extensions to 2500. Geosci. Model Dev. 13, 3571-3605. https://doi.org/10.5194/gmd-13-3571-2020, @ Author(s) 2020. This work is distributed under the Creative Commons Attribution 4.0 License.

[6] O. Boucher, et al. 2020. Presentation and evaluation of the IPSL-CM6A-LR climate model. Journal of Advances in Modeling Earth Systems 12, e2019MS002010.

[7] A. Voldoire, et al. 2019. Evaluation of CMIP6 DECK experiments with CNRM-CM6-1. Journal of Advances in Modeling Earth Systems 11, 2177-2213. 

Partie IV

\section{Comment répondre aux changements globaux}





\section{Chapitre 10}

\section{S'adapter aux changements, de la réduction de la vulnérabilité à l'augmentation de la résilience}

Ce chapitre présente ce qu'implique notre adaptation aux changements globaux, en particulier au changement climatique. Nous verrons d'abord en quoi consiste un processus d'adaptation. Étant donné l'ampleur du changement en cours, nous verrons que l'adaptation passe nécessairement par une transformation profonde de notre société. En fait, il n'y a pas de distinction franche entre le processus d'adaptation au changement climatique et les actions d'atténuation de ce changement, que nous verrons dans le prochain chapitre. S'adapter implique de mener des actions de planification à tous les niveaux, local, régional et international en visant en particulier à réduire les vulnérabilités à la variabilité climatique. S'adapter nécessite aussi de trouver les moyens de gouvernance appropriés à tous les niveaux, car ce n'est qu'au prix d'un investissement certain à la fois individuel et collectif que pourra s'épanouir une gouvernance politique efficace de l'environnement. À la fin du chapitre sont abordés des exemples pratiques d'adaptation possible dans le domaine de la santé et dans celui de l'agriculture; comment réduire les risques de mortalité imputables aux températures élevées et comment adapter les cultures aux sécheresses à répétition. En fait, plus que des solutions, ce sont des réflexions auxquelles le lecteur est invité tout au long de ce chapitre. 


\subsection{L’adaptation: déclinaisons et enjeux - J.P. Vanderlinden}

\subsubsection{Stimuli climatiques, vulnérabilité, capacité adaptative et transformation}

Les changements climatiques sont bien là. II nous faut en limiter l'ampleur, mais il est sûr que, dans une certaine mesure, il nous faudra aussi nous y adapter. Atténuer les changements est une nécessité, s'adapter est une contingence.

D'après la définition du GIEC (2001): "L'adaptation, c'est l'ajustement des systèmes naturels ou humains en réponse à des stimuli climatiques ou à leurs effets, afin d'atténuer les effets néfastes ou d'exploiter des opportunités bénéfiques. »

Quelles sont la nature et la temporalité des stimuli dont on parle? Cette question est fondamentale, car souvent on se trompe en pensant que l'on s'adapte à des changements de moyenne: température plus chaude (ou plus froide), précipitations qui augmentent en moyenne (ou qui diminuent), etc. Ce n'est qu'une vision partielle de choses. Le défi d'adaptation le plus important se rapporte à la variabilité climatique, c'est-à-dire la fréquence des évènements extrêmes qui nous menacent. Un degré de plus en moyenne à Brest est sans doute supportable, mais des canicules dix fois plus fréquentes, c'est une menace d'une autre ampleur. Moyenne et variabilité sont donc deux des stimuli auxquels nous devons nous adapter. II existe un troisième stimulus, plus compliqué à appréhender et fondamental pour l'être humain. Le changement climatique crée de nouvelles incertitudes. Nous devons prendre des décisions aujourd'hui pour un avenir qui nous est obscurci par le caractère jamais vu auparavant des changements climatiques d'origine humaine. Si aujourd'hui le progrès des connaissances sur le climat nous a permis d'acquérir un certain nombre de certitudes, il n'en demeure pas moins que de nouvelles incertitudes ont vu le jour et qu'il faudra en prendre compte. Le troisième stimulus associé au changement climatique, qui n'impacte que les humains, est donc celui associé à de plus grandes incertitudes sur l'avenir, des incertitudes augmentées par l'ajout d'incertitudes fortes sur l'évolution précise du climat.

La définition proposée par le GIEC inclut une notion d'opportunité à saisir. II s'agit d'une question fondamentale. Certains d'entre nous sont certainement mieux placés que d'autres pour bénéficier de la réduction des impacts des changements climatiques. L'adaptation pourrait alors renforcer les inégalités. Or, les inégalités sont sources de vulnérabilités et la première opportunité est justement de réduire ces vulnérabilités. Une dynamique de réduction de la vulnérabilité à la variabilité actuelle du climat a bien été enclenchée et nous en verrons un exemple dans ce chapitre (voir section 10.3).

Pour nous adapter, il nous faut non seulement anticiper la météorologie de demain, mais aussi développer nos capacités adaptatives pour ne pas nous laisser 
dépasser. Un tel travail de préparation prend différentes formes, notamment celle de la planification comme le décrira Charlotte Da Cunha dans la suite de ce chapitre (voir section 10.4).

Finalement, et c'est sans doute encore plus fondamental que les points précédents: il nous faut changer nos modes de vie! II faut que toutes et tous, personnes et institutions, intégrions la question climatique dans nos prises de décision et que ces questions menaçant l'humanité deviennent prioritaires. II nous faut nous transformer, ce que commentera Yorghos Remvikos dans la suite de cette section (voir section 10.2).

\subsubsection{Le processus d'adaptation dans un contexte d'incertitudes et de certitudes}

L'adaptation est un processus, pas un état. Face aux incertitudes et à l'évolution du monde, nous ne sommes pas adaptés, nous nous adaptons.

Si l'on repart de la notion de «stimuli climatiques», pour pouvoir s'adapter, il faut être en mesure de capturer les signaux, les trier, les interpréter. Le fondement de l'adaptation, c'est la connaissance que l'on peut acquérir sur un environnement changeant. Ceux qui, étudiant la composition de l'atmosphère, ont découvert l'accumulation de $\mathrm{CO}_{2}$ dans l'atmosphère terrestre, un peu par surprise, n'avaient pas l'intention initiale de tirer la sonnette d'alarme. Par contre, ils étaient curieux et en mesure de capturer, grâce à des moyens significatifs, différents signaux. Sans avoir accès aux signaux propres aux stimuli, il n'y a pas de moyen de s'adapter. En bref, sans observations, sans recherches, sans séries chronologiques portant sur différentes variables, il n'y a pas de signal, pas d'observation de stimuli, pas d'adaptation.

Ensuite, Iorsqu'un stimulus a pu être identifié, interprété, et jugé suffisamment saillant pour que cela appelle un ajustement, il faut disposer des ressources pour cet ajustement. Dans des systèmes économiques qui fonctionnent à flux tendus, il n'y a généralement pas de ressources en sus et l'ajustement prend du temps ou ne se fait pas. II faut donc, au contraire, des systèmes économiques qui permettent de disposer de moyens pour s'adapter à tout moment, une gouvernance et une conception de la coordination capables de relever nos défis collectifs (voir plus loin section 10.5).

II faut, par ailleurs, être conscient que le processus d'adaptation se déroule dans un contexte d'incertitudes. Aux incertitudes liées au climat lui-même s'ajoutent des incertitudes liées à la société. Par exemple, sur le plan juridique, une décision prise pour l'adaptation risque-t-elle d'être contestée face à une cour de justice, est-elle réglementaire? Notre responsabilité sera-t-elle engagée si une décision entraîne des conséquences négatives imprévues? Pourra-t-on me poursuivre pour inaction? II y a des incertitudes morales: les normes morales sont-elles partagées par tous les concernés? L'acceptabilité des décisions d'adaptation est-elle menacée par des questions d'ordre moral? Aussi bien les sphères juridiques que morales relèvent de la façon dont la société, le collectif, fera face à la décision. Cela nous amène aux 
incertitudes sociétales. Risque-t-on des mobilisations, des paniques, des amplifications sociales, ou des dénis collectifs avec une prise de risque inconsidérée?

En bref, l'adaptation au changement climatique ne soulève pas que des questions de sciences du climat, mais aussi des questions juridiques, morales, sociétales, institutionnelles, etc. Les sources d'incertitude, qui sont parfois fort locales, génèrent aussi des solutions très liées au contexte de mise en œuvre. Cela étant, face aux incertitudes, il y aura néanmoins des certitudes. Ainsi, en dépit des difficultés, l'adaptation est intégrée progressivement dans les dynamiques de planification qui se développent au sein de structures privées et publiques. Une autre certitude est que les choix faits aujourd'hui auront une influence durable sur les années à venir. II nous faut donc agir aujourd'hui de façon raisonnée. On sait par ailleurs que l'adaptation aux changements climatiques aura des impacts sur les différents secteurs de l'activité humaine. Ces impacts sont nombreux, multifactoriels, et multiformes.

Dans tous les cas, une approche intégrée de l'adaptation (verticalement et horizontalement) s'impose. Comme nous l'avons mentionné, l'adaptation est dépendante des lieux et des contextes. En matière d'adaptation, chaque solution, chaque approche, devra être taillée sur mesure.

\subsection{L'adaptation en tant que transformation profonde - Y. Remvikos}

\subsubsection{Le concept d'adaptation et ses différentes acceptions}

Revenons un instant sur le concept d'adaptation. Charles Darwin a été l'un des premiers à l'utiliser en proposant l'adaptation comme une réponse des espèces aux pressions changeantes des milieux, réponse indissociable de ce qu'il appellera la sélection naturelle. Le vivant ne fait cependant pas que subir les changements, il y contribue aussi, l'humain en étant l'exemple par excellence. Des auteurs du début du $X X X^{e}$ siècle ont, quant à eux, prétendu que l'humanité n'était plus adaptée aux besoins de l'économie industrielle, autrement dit, qu'il fallait adapter la société à l'économie. Cet autre usage "anthropocentré» du concept d'adaptation a accompagné la montée de l'idéologie néolibérale avec les conséquences qu'on lui connaît sur l'environnement. II a aussi renforcé, de fait, la distinction entre, d'un côté, ce qui appartient à la Nature et de l'autre, les humains qui s'en sont «volontairement» exclus depuis le XVIIe siècle (voir section 1.3).

D'autres approches encore laisseraient à penser que l'adaptation se fait dans un monde susceptible d'atteindre un équilibre stable. Or, comme l'affirmait l'écologue Crawford Stanley Holling dans un article de 1973, «l'état par défaut des écosystèmes est le changement et non l'équilibre, encore moins la stabilité» [1]. Précédemment dans cet ouvrage (voir section 4.1.2) Pierre-Henri Gouyon invoquait un «équilibre 
dynamique» pour décrire la biodiversité. De même, nous avons vu que le climat changeait en permanence depuis des millions d'années (voir sections 1.1 et 1.2), que cela soit dû à des forces hors de notre portée comme la variabilité de l'énergie solaire, la tectonique des plaques ou les mouvements de la Terre sur son orbite, ou que cela soit le résultat actuel des activités humaines. Vivre dans un monde en devenir est une compréhension qu'il nous faut ainsi adopter, même si un monde changeant affecte notre capacité à prévoir son évolution et rend forcément fragiles les propositions de planification de l'adaptation.

Enfin, une partie de la littérature tend à présenter l'adaptation, tantôt comme un processus, donc l'ajustement au changement, parfois comme le résultat même de l'action d'adapter, voire comme la finalité de cette action. Il est évident que cette définition, mêlant moyens et fins, n'est pas acceptable. Retenons donc que l'adaptation est un processus qui doit être (ré)activé en permanence et qu'il n'y pas d'adaptation aux conditions ambiantes sans tenir compte de l'adaptation des milieux eux-mêmes et de leurs altérations permanentes, notamment celles dues à nos propres activités. Quant aux finalités de l'adaptation, elles sont à trouver dans notre sécurité, bien sûr, mais aussi dans la soutenabilité, la résilience, le bien-être ou même tout à la fois.

\subsubsection{Le réchauffement climatique, un défi auquel l'humanité tout entière doit faire face}

Est-ce que le réchauffement climatique est un problème en soi, auquel il nous faut nous adapter? Poser la question en ces termes revient, en prenant une métaphore du domaine médical, à traiter un symptôme plutôt que diagnostiquer la maladie. Nous ne pouvons pas nous adapter au réchauffement climatique, encore moins tenter de l'atténuer, sans porter notre attention sur les principales causes qui en sont à l'origine. Sans pour autant oublier la variabilité naturelle du rayonnement solaire, nous admettons aujourd'hui que le réchauffement climatique actuel, brutal au regard des évolutions passées qui se sont faites sur des millions d'années, est principalement dû aux activités humaines et aux choix récents de développement (libération d'énormes quantités de gaz à effet de serre) que nous avons nous-mêmes opérés. Les principales causes se trouvent ainsi dans nos modes de production et de consommation et plus généralement, dans nos modes de vie, dispendieux et peu soucieux des contraintes et du fonctionnement de la biosphère.

C'est donc, sans doute, un autre rapport à la Terre, qu'il nous faudrait considérer, autre que celui qui s'est imposé dans l'Anthropocène des dernières centaines d'années, avec l'exploitation généralisée et sans limite de l'environnement, justifiée par la nécessité de la croissance. Peut-être y a-t-il des inspirations à trouver dans des cultures qui, parfois, depuis longtemps, entretiennent un rapport à la Terre bien différent de celui développé dans la culture occidentale.

Doit-on par ailleurs, à ce stade, faire une distinction franche entre le processus d'adaptation au changement climatique et celui d'atténuation de ce changement en 
considérant que toute action, qui vise à la réduction des émissions des gaz à effet de serre, relève de l'atténuation alors que l'adaptation est limitée aux tentatives de réduction des impacts du changement climatique? La séparation peut sembler artificielle et le processus d'adaptation pourrait être interprété comme un simple ajustement du système de société sans le remettre en question. Est-ce vraiment la solution de préserver la structure du système actuel face au défi d'ampleur que pose le réchauffement climatique à toute l'humanité?

$\mathrm{Si}$, en revanche, l'adaptation porte sur une transformation des modes de vie, en maîtrisant les effets de nos activités, en choisissant ces activités, en étant soucieux des limites de notre planète, alors elle serait aussi une force de réduction des émissions des gaz à effet de serre, menant à un système différent, une société conçue sur des principes renouvelés, non seulement sobre, mais aussi peut-être moins inégalitaire, plus juste. Le processus d'adaptation au réchauffement climatique, considéré ainsi comme une transformation profonde, nous invite donc à réfléchir sur le monde, que l'on souhaite voir venir, et à ne pas subir que les tendances imprimées par l'économie mondialisée.

\subsection{L’adaptation en tant que réduction des vulnérabilités à la variabilité climatique actuelle - J.P. Vanderlinden}

Nous abordons ici l'adaptation au changement climatique, vue sous l'angle de la réduction des vulnérabilités à la variabilité climatique actuelle.

\subsubsection{Vulnérabilité et exposition}

Commençons par domestiquer les concepts d'exposition et de vulnérabilité en les illustrant à partir d'événements climatiques marquants, plus ou moins récents.

La tempête Xynthia de 2012 a été la plus meurtrière en France pour le siècle présent. Les personnes qui vivaient en zone inondable étaient exposées à l'eau. Dans les maisons où l'eau montait rapidement, la mobilité était fortement réduite, et il était difficile d'échapper à la noyade. Dans le cas plus récent de la pandémie Covid19, certaines personnes ont, au contraire, pu échapper au contact du virus grâce au mesures de confinement et/ou à la pratique de gestes barrières : ces personnes n'ont pas été exposées. Le concept d'exposition permet donc d'identifier des leviers particuliers d'action pour extraire les victimes potentielles, humaines ou non humaines, des lieux ou des conditions qui les exposent au danger.

Le concept d'exposition permet aussi d'analyser des situations très diverses où l'environnement changeant interagit négativement avec les humains et non-humains. Ainsi, les vagues de chaleur, notamment celle de 2003 en France, ont exposé plus particulièrement les habitants des grandes villes vivant sous les toits, celles et ceux 
qui devaient se déplacer dans des îlots de chaleur pour faire leurs courses, aller au travail ou vivre, tout simplement. La pollution des océans par les plastiques menace toute la faune qui y est exposée, notamment les espèces pélagiques qui traversent les champs de macro et méso déchets plastiques.

L'exposition à un risque climatique peut ainsi être définie, de manière générale, comme la présence de personnes, de moyens de subsistance, d'espèces ou d'écosystèmes, de ressources et de services environnementaux, d'éléments d'infrastructure ou de biens économiques, sociaux ou culturels, dans un lieu susceptible de subir des dommages.

Passons maintenant au concept complémentaire de vulnérabilité, c'est-à-dire la propension des humains ou non-humains et des écosystèmes à être affectés par un événement dangereux, une situation dangereuse, lorsqu'ils y sont exposés. Dit autrement, la vulnérabilité est la propension ou prédisposition à subir des dommages. Ainsi, les personnes âgées et/ou celles souffrant de fragilité (on a pu aussi parler de comorbidité) ont été et sont encore parmi les plus vulnérables, que ce soit dans le cas des vagues de chaleur, de la pandémie Covid-19 ou même dans le cas d'inondations comme celle due à la tempête Xynthia. Pour ce dernier cas, il était, par contre, plus difficile d'anticiper la vulnérabilité des personnes atteintes de fragilité respiratoire ou cardiaque.

Si l'on reprend l'exemple de la pollution des océans par les plastiques, des espèces peuvent devenir vulnérables dès lors que leur régime alimentaire inclut la consommation de méduses, car ces dernières ont la capacité de capturer les particules de microplastique. La pollution aux plastiques est également un danger pour les espèces marines habituées à se déplacer sur de grandes distances, car les exigences énergétiques de ces migrations les affaiblissent, les vulnérabilisent, les plastiques exacerbant alors leur faiblesse.

Dans tous les cas cités, il est alors possible d'imaginer des actions pour rendre les personnes ou les espèces non-humaines moins vulnérables, en ciblant les éléments les rendant vulnérables.

\subsubsection{Conceptualisation d'un risque climatique}

Voyons maintenant comment théoriser les concepts d'exposition et de vulnérabilité dans un contexte de changement climatique en envisageant, par exemple, un choc météorologique comme une tempête côtière qui provoque une inondation. Pour conceptualiser une telle situation (Fig. 10.1), il nous faut d'abord préciser la variabilité climatique qui est à l'origine de cet événement inhabituel et déterminer si elle est d'origine naturelle ou anthropique. Dans l'exemple, choisi, la tempête est associée à une dépression météorologique, provoquant une surcote de la marée et des précipitations intenses. 
Pour qu'un tel événement nous donne des raisons de nous y intéresser, il faut, bien entendu, que quelque chose soit exposé. Donc, en poursuivant notre conceptualisation, il nous faut considérer l'exposition à l'événement : y a-t-il des personnes, des objets, des écosystèmes, qui seront exposés «dans le chemin de l'eau »? L'exposition seule ne suffit pas, non plus, à engendrer un risque. II n'y aura de risque que s'il y a vulnérabilité, le troisième "pétale» de cette conceptualisation du risque climatique dans la figure 10.1. C'est donc la conjonction d'un événement, d'une exposition et d'une vulnérabilité, qui engendre le risque.

En fait, le contexte de ce risque n'est pas que climatique. L'exposition et la vulnérabilité s'inscrivent aussi dans un contexte social, économique, politique... dans un contexte de développement. C'est le développement de la société qui nous donnera, en retour, des options pour nous adapter en jouant sur la réduction des vulnérabilités ou des expositions. Le développement d'une société n'est pas indépendant des risques climatiques majeurs qu'elle peut subir. Une catastrophe climatique, réalisée ou prévisible, contraint certaines possibilités de développement de la société. À l'inverse, le choix d'options de développement peut, selon les cas, augmenter ou diminuer les risques d'avoir un climat qui engendre un tel événement «catastrophique».

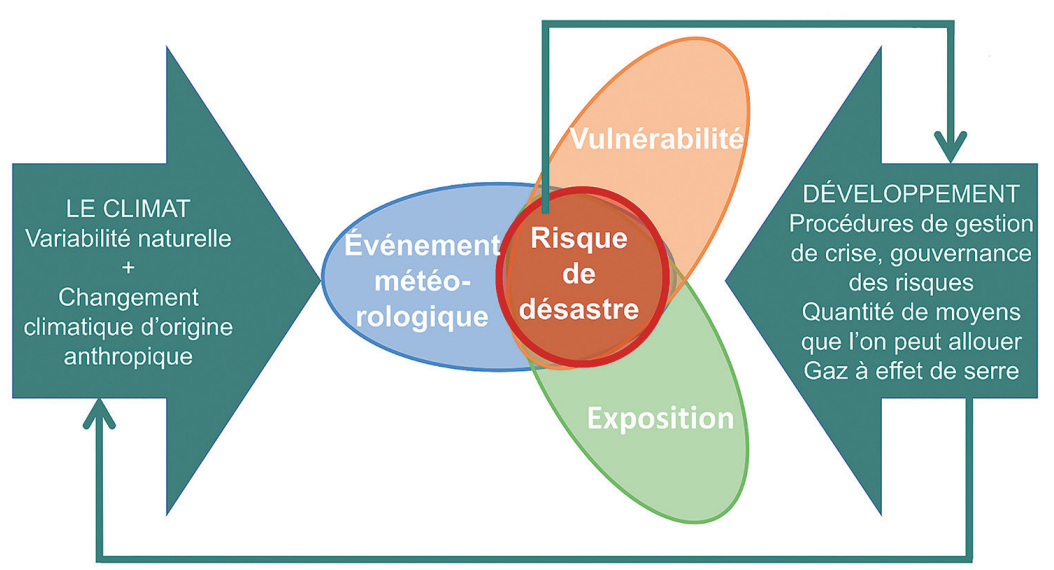

Figure 10.1: Diagramme schématique reliant les notions d'exposition, de vulnérabilité et de développement dans le cas d'un événement climatique susceptible d'engendrer un risque important (d'après IPCC 2014, page 3).

\subsubsection{Identification des facteurs de vulnérabilité et des leviers d'actions, l'exemple d'Ho Chi Minh Ville}

Nous allons maintenant illustrer par une étude de cas qu'il est possible de passer d'une conceptualisation de la vulnérabilité / résilience à un travail sur le terrain destiné à identifier des leviers d'adaptation. Cette étude concerne la ville d'Ho Chi Minh Ville au Vietnam et les inondations qu'elle subit. Nous décrirons d'abord la 
situation, puis nous analyserons l'exposition et la vulnérabilité des personnes, pour aboutir à une cartographie des risques à partir d'indicateurs préalablement choisis.

Le centre d'Ho Chi Minh Ville et son quartier financier sont traversés par le fleuve Saigon qui se trouve dans la zone d'influence des marées et qui, combiné à la rivière Dong Nai, amène les précipitations d'un bassin versant gigantesque. Outre le fleuve Saigon et la rivière Dong Nai, de nombreux canaux traversent aussi Ho Chi Minh Ville. Cette situation a poussé le gouvernement Vietnamien à développer des modalités de gestion du risque d'inondation en faisant appel à des partenariats pour une partie des études.

Ho Chi Minh ville connaît une très forte croissance démographique et un développement rapide, qui créent inévitablement des inégalités de richesse importantes. Si le centre-ville, produit de l'histoire, notamment de l'histoire coloniale, concentre des richesses depuis fort longtemps, il fait néanmoins face à des développements d'habitats précaires, de l'autre côté du fleuve (Fig. 10.2, haut), et se situe à quelques pas de développements immobiliers gigantesques.
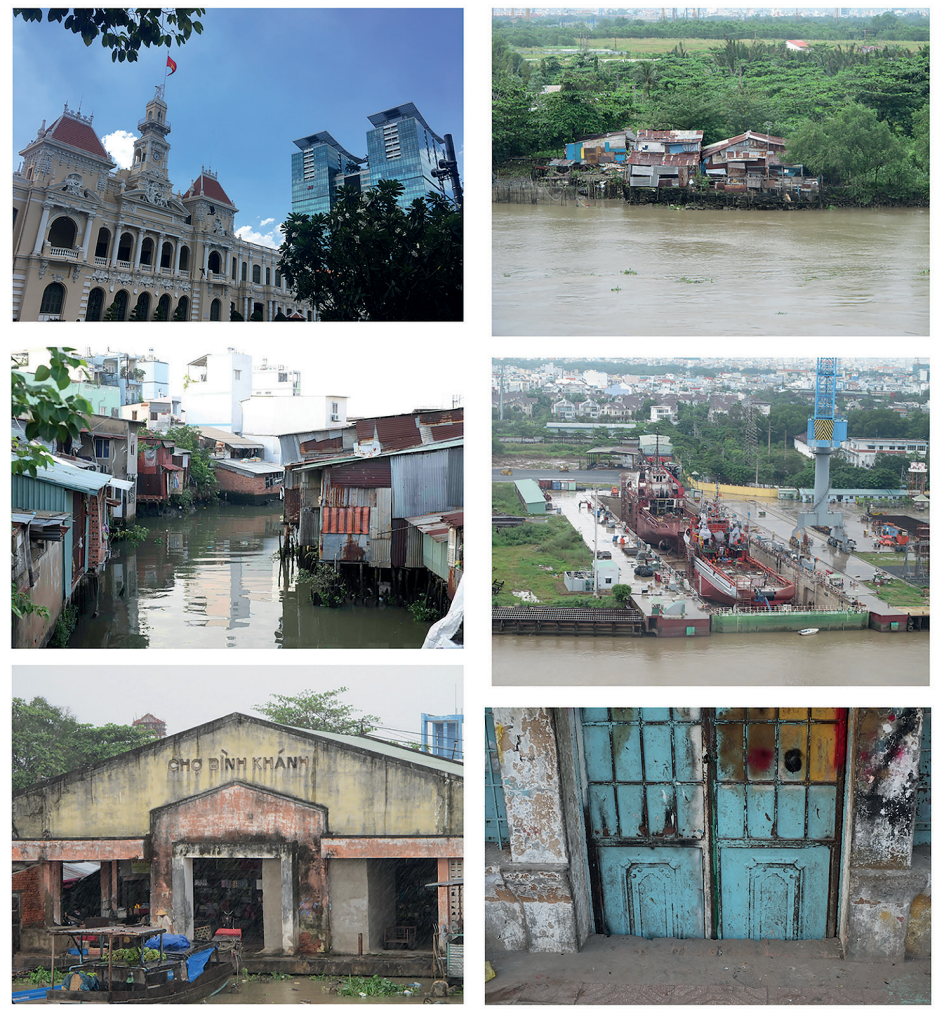

Figure 10.2: Illustrations du risque élevé d'inondation à Ho Chi Minh Ville. De haut en bas et de gauche à droite: - centre-ville, - habitats précaires de l'autre côté du fleuve, - canaux traversant la ville, zone industrielle, - édifice patrimonial, - maison s'enfonçant dans le sol. Source: photo: Jean-Paul Vanderlinden (CC BY-NC-SA 4.0). 
Avec la croissance démographique, les canaux dans la ville sont effectivement devenus de plus en plus occupés par les habitats précaires des nouveaux arrivants (Fig. 10.2 milieu, à gauche). Ceux-ci en sont parfois chassés, pour permettre le curage et le réaménagement des canaux et donc, contribuer à une meilleure gestion des inondations. Ces actions dépossèdent donc, en retour, les plus pauvres de leur logement.

Dans l'enchevêtrement humide que constitue Ho Chi Minh Ville, il y aussi de l'industriel lourd (Fig. 10.2 milieu, à droite) et des édifices patrimoniaux toujours en usage, qui se trouvent au ras de l'eau et dont la fragilité ne fait aucun doute (Fig. 10.2 bas, à gauche). Certains lotissements pavillonnaires très étendus se trouvent même sous le niveau de l'eau. Les gens surélèvent alors leur maison pour éviter l'eau lors des inondations. De son côté, le gouvernement surélève les rues pour éviter qu'elles ne soient inondées, et l'eau coule alors dans les maisons. Non seulement des rues sont surélevées, mais des maisons s'enfoncent naturellement dans le sol (Fig. 10.2 bas, à droite). Et au milieu de tout cela, chacun et chacune essaient de se débrouiller.

C'est dans ce contexte que le projet de gestion des inondations s'est développé et que l'analyse exposition vulnérabilité / inondation a été menée. En suivant la conceptualisation présentée précédemment (Fig. 10.1), l'analyse s'est déroulée en quatre étapes: d'abord, la spécification de l'événement, ensuite l'analyse d'exposition, puis l'analyse de vulnérabilité, et enfin la combinaison des trois pour cartographier les risques.

Trois principaux paramètres ont été identifiés pour caractériser les inondations, les spécifier:

- la vitesse de l'eau, à la fois parce qu'une partie de la population est vulnérable à pied et qu'une partie des constructions ne résiste pas bien à l'érosion;

- la hauteur d'eau, car plus l'eau monte, plus le nombre d'éléments touchés est important, et plus les infrastructures de protection risquent d'être dépassées;

- la durée de l'inondation, car certains des habitats ne sont plus salubres en cas d'inondation prolongée alors qu'une grande part de l'activité économique, le petit commerce, se fait au niveau de la rue.

L'identification de ces trois caractéristiques prioritaires nous a permis, en combinant un modèle numérique d'élévation et un modèle hydrologique, de créer des évènements de référence plus ou moins violents et de cartographier les caractéristiques associées à la vitesse de l'eau, la hauteur d'eau et la durée d'inondation. On obtient alors des cartes qui combinent les caractéristiques de l'inondation avec ce que l'on observe sur le terrain en termes d'habitat, de bâtiment précieux, d'infrastructures critiques (hôpitaux, réserves de carburant, prisons et autres éléments) et de population. Autrement dit, on obtient des cartes d'exposition. 
Après avoir compilé les informations sur l'exposition aux inondations, il nous faut déterminer les vulnérabilités associées. L’analyse de vulnérabilité est aussi complexe que celle sur l'exposition. Elle implique la récolte d'un grand nombre de données, puis la construction d'indicateurs synthétiques qui sont, en quelque sorte, une mesure de la vulnérabilité. Dans le cas d'Ho Chi Minh Ville, trois indicateurs ont été utilisés, se concentrant sur des dimensions statistiquement indépendantes: la vulnérabilité due aux infrastructures (ex: maison insalubre), la vulnérabilité due à la démographie (ex: présence de personnes plus fragiles), et la vulnérabilité sociale (éducation, économie, pouvoir détenu). À nouveau, on établit une carte (Fig. 10.3) :

\section{Une cartographie des vulnérabilités}
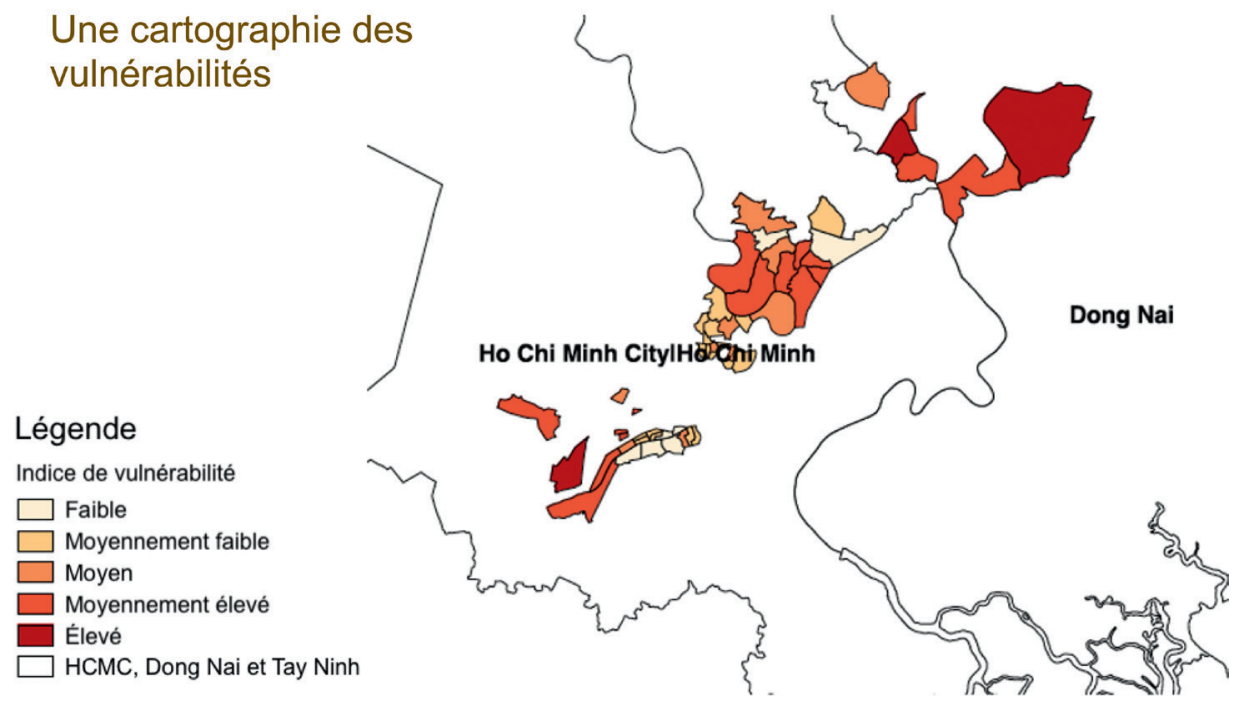

Figure 10.3: Cartographie des vulnérabilités aux inondations dans la région d'Ho Chi Minh City. Source : Jean-Paul Vanderlinden.

L'étape suivante est de combiner les cartes de vulnérabilité aux cartes d'exposition. Nous aboutissons alors à une troisième série de cartes qui représentent les niveaux de dangerosité en termes d'exposition ET de vulnérabilité selon l'un des trois indicateurs de spécification de l'inondation, par exemple la hauteur d'eau.

À quoi cela sert-il du point de vue de l'adaptation? Souvenons-nous que l'adaptation est notamment la réduction des vulnérabilités actuelles. L'analyse, que nous venons de présenter, permet de cibler les interventions possibles et de comparer, par exemple, différentes options. Quel quartier faut-il protéger en priorité? Vaut-il mieux travailler sur l'exposition que sur la vulnérabilité? Les infrastructures critiques sont-elles suffisamment protégées? Les réponses à ces questions se trouvent sur les cartes de la figure 10.4, qui représentent respectivement les niveaux de risque selon la position sociale, la salubrité des infrastructures et la fragilité des personnes en cas de hauteur d'eau élevée. Établir ces cartes a nécessité 3 ans de travaux d'une 
équipe franco-vietnamienne de près d'une vingtaine de personnes ! L'adaptation doit commencer maintenant - et n'est pas nécessairement bon marché!
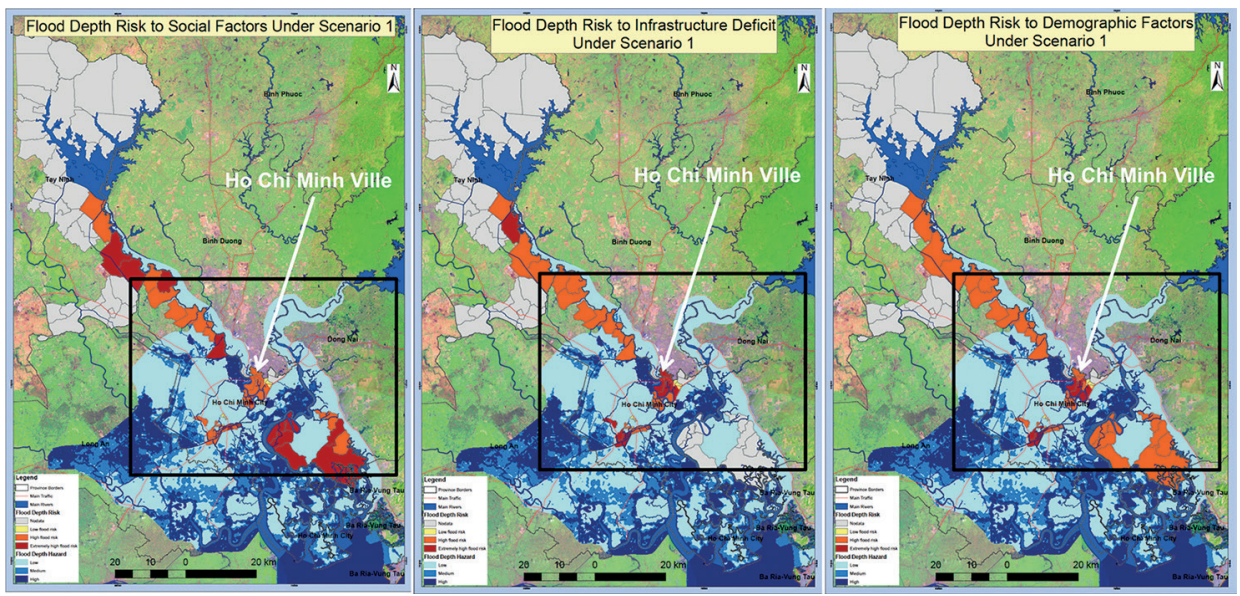

Figure 10.4: Cartographies des risques associés à la hauteur d'eau selon les trois indicateurs de vulnérabilité dans la Province d'Ho Chi Minh Ville. De gauche à droite: facteurs sociaux; déficit d'infrastructures; facteurs démographiques. Le risque d'inondation, indiqué en bleu, va croissant du bleu clair au bleu foncé. Les risques associés à la hauteur d'eau vont croissant du jaune au rouge en passant par l'orange. Les zones en ocre clair sont celles pour lesquelles il n'y a pas de données. Source [2].

\subsection{Planification territoriale de l'adaptation - Ch. da Cunha}

\subsubsection{Historique des plans d'adaptation et de leur mise en place}

Les politiques internationales et nationales relatives au climat ont fait leur apparition dès les années 1990, peu après la publication du premier rapport du GIEC. À la fin de ces années, les premières villes, de grandes villes pour la plupart, ont ainsi réalisé leurs premiers plans climat.

En 1993, ICLEI (International Council for Local Environmental Initiatives) a lancé le programme "Cities for Climate Protection», l'idée étant de fournir une assistance technique aux villes qui voulaient mettre en place une planification de l'action climatique.

Jusqu'à la moitié des années 2000 environ, cette planification s'est principalement focalisée sur des actions d'atténuation du réchauffement climatique et beaucoup moins sur des stratégies d'adaptation au changement climatique.

En 2007, la Convention des Maires - un mouvement de coopération européen associant des autorités locales et régionales - a lancé son initiative «Mayors Adapt». 
En 2013, la Commission européenne adopte la première stratégie de l'Union Européenne sur l'adaptation au changement climatique. On y trouve des actions concrètes, y compris une proposition d'intégration de ces actions au sein d'autres politiques européennes.

En France, la première stratégie nationale d'adaptation parait en 2006, suivie, en 2011 du premier plan national d'adaptation au changement climatique (le PNACC) qui propose, de façon plus détaillée, un ensemble de 84 actions exprimées en 242 mesures [3]. Le deuxième PNACC, sorti en 2018, se concentre sur les Solutions d'Adaptation Fondée sur la Nature (SAFN) et I'Outre-mer.

En 2015, la loi Grenelle 2 rend obligatoire l'adoption des plans climat-énergie territorial (PCET). La loi sur la transition énergétique pour la croissance verte ajoute la dimension «air» dans la planification climatique (PCAET). L'adoption d'un PCAET est désormais obligatoire pour l'ensemble des intercommunalités de plus de 20000 habitants.

Ces PCAET n'imposent pas de directives contraignantes sur l'adaptation, mais des injonctions formelles de "réfléchir » aux effets du changement climatique. On voit ainsi se mettre en place une généralisation des réflexions régionales sur l'adaptation [4].

À défaut d'afficher directement des objectifs d'adaptation, la première Convention Citoyenne pour le Climat, constituée en octobre 2019, a également mené des réflexions sur nos manières de consommer, de travailler et de nous déplacer dans un monde changeant auquel il va falloir s'adapter.

\subsubsection{Mise en place des stratégies locales d'adaptation}

Les politiques d'adaptation des villes doivent tenir compte des changements à venir, mais aussi des actions qui sont déjà en cours et qu'elles doivent intégrer. L'articulation des politiques d'adaptation avec les autres politiques publiques est ainsi primordiale (Fig. 10.5).

Les actions planifiées ne doivent pas être redondantes avec celles déjà en place dans d'autres plans, notamment ceux en lien avec l'environnement. Ainsi, la plupart des PCAET sont réfléchis de manière à s'articuler avec le Schéma de Cohérence Territorial, le Plan Local de l'Habitat, le Plans de Déplacements Urbains, et d'autres programmes spécifiques comme le Programme «Défi Zéro Gaspillage », la charte du PNR (Parc Naturel régional), la compétence GEMAPI (Gestion des milieux aquatiques et prévention des inondations) dans les zones côtières, etc.

Les approches d'adaptation diffèrent des approches classiques de la planification urbaine, où les tendances passées servent souvent de base pour la prise de nouvelles décisions. Une stratégie d'adaptation nécessite de prendre en compte les risques et les opportunités futures. Certaines villes procèdent ainsi à des évaluations détaillées des risques associés au changement climatique. D’autres se concentrent plus sur un type de vulnérabilité ou sur certains secteurs économiques. 
Les PCAET en France se basent sur l'analyse des vulnérabilités actuelles à partir de données régionales ou locales disponibles, essentiellement celles émanant de Climat HD et de la DRIAS-les futurs du climat. Ils créent ainsi des scénarios locaux de risques et d'opportunités.

\begin{tabular}{|c|c|c|}
\hline $\begin{array}{l}\text { 1. Articulation } \\
\text { avec les autres } \\
\text { politiques } \\
\text { publiques }\end{array}$ & $\begin{array}{l}\text { La planification des actions } \\
\text { d'adaptation ne peut pas faire } \\
\text { doublon avec des actions en } \\
\text { place dans les divers plans en } \\
\text { lien avec l'environnement }\end{array}$ & $\begin{array}{l}\text { Les PCAETS réalisent ainsi } \\
\text { une action pour réfléchir } \\
\text { à cette articulation } \\
\text { (SCOT, PLU, PDU et autres } \\
\text { spécificités locales) }\end{array}$ \\
\hline $\begin{array}{l}\text { 2. Prise en compte } \\
\text { des risques et } \\
\text { opportunités } \\
\text { futures }\end{array}$ & $\begin{array}{l}\text { Analyse de la vulnérabilité } \\
\text { à l'état actuel et } \\
\text { données régionales et locales } \\
\text { disponibles (météoHD, DRIAS) } \\
\text { pour créer des scénarios } \\
\text { locaux d'évolution des } \\
\text { risques et opportunités }\end{array}$ & $\begin{array}{l}\text { Spéculations sur les risques } \\
\text { et opportunités dans la } \\
\text { nouvelle génération de plans } \\
\text { (depuis 2020) }\end{array}$ \\
\hline
\end{tabular}

Figure 10.5: Enjeux d'une politique d'adaptation.

\subsubsection{Cas d'étude : New-York city}

Nous prenons ici l'exemple de New York City (NYC) de manière à illustrer la façon dont des mesures institutionnelles d'adaptation peuvent être prises et les processus qui sont utilisés pour leur élaboration. La ville de New York est construite sur trois îles, Long Island, Manhattan, et Staten Island, et se trouve entourée de quatre rivières, dont seule, la Bronx River, est une rivière d'eau douce. Un chercheur, Eric Sanderson, en 2009, a recréé des cartes de ce qu'était New York au XVIIIle siècle [5]. L'image reconstituée de la figure 10.6 représente ainsi les forêts de Times Square, les prairies de Harlem et les zones humides du centre-ville. On réalise à partir de cette image reconstituée que la ville d'aujourd'hui est construite sur une zone humide et donc potentiellement très vulnérable.

La tempête Sandy, et les dégâts qu'elle a causés en 2012, a été un tournant dans la prise de conscience des New-yorkais vis-à-vis des risques climatiques, accélérant du même coup le processus de planification. Le caractère urbain de New York, sa vulnérabilité et son interconnexion ont, en effet, amplifié l'impact de l'inondation due à la tempête Sandy. Une grande partie des infrastructures essentielles de la ville se trouvaient dans des zones inondées. Les installations et les services ont été complètement ou partiellement fermés pendant l'inondation et, pour certains d'entre eux, pendant de longues périodes après l'inondation. Les perturbations de réseaux de distribution, comme celui de l'électricité, ont affecté, en retour, les services de santé, les transports et les télécommunications, etc. La désorganisation s'est ainsi étendue bien au-delà des zones inondées. Autour des zones inondées, de nombreux habitants n'ont pas pu se rendre à leur travail et n'ont pas eu accès aux services. 


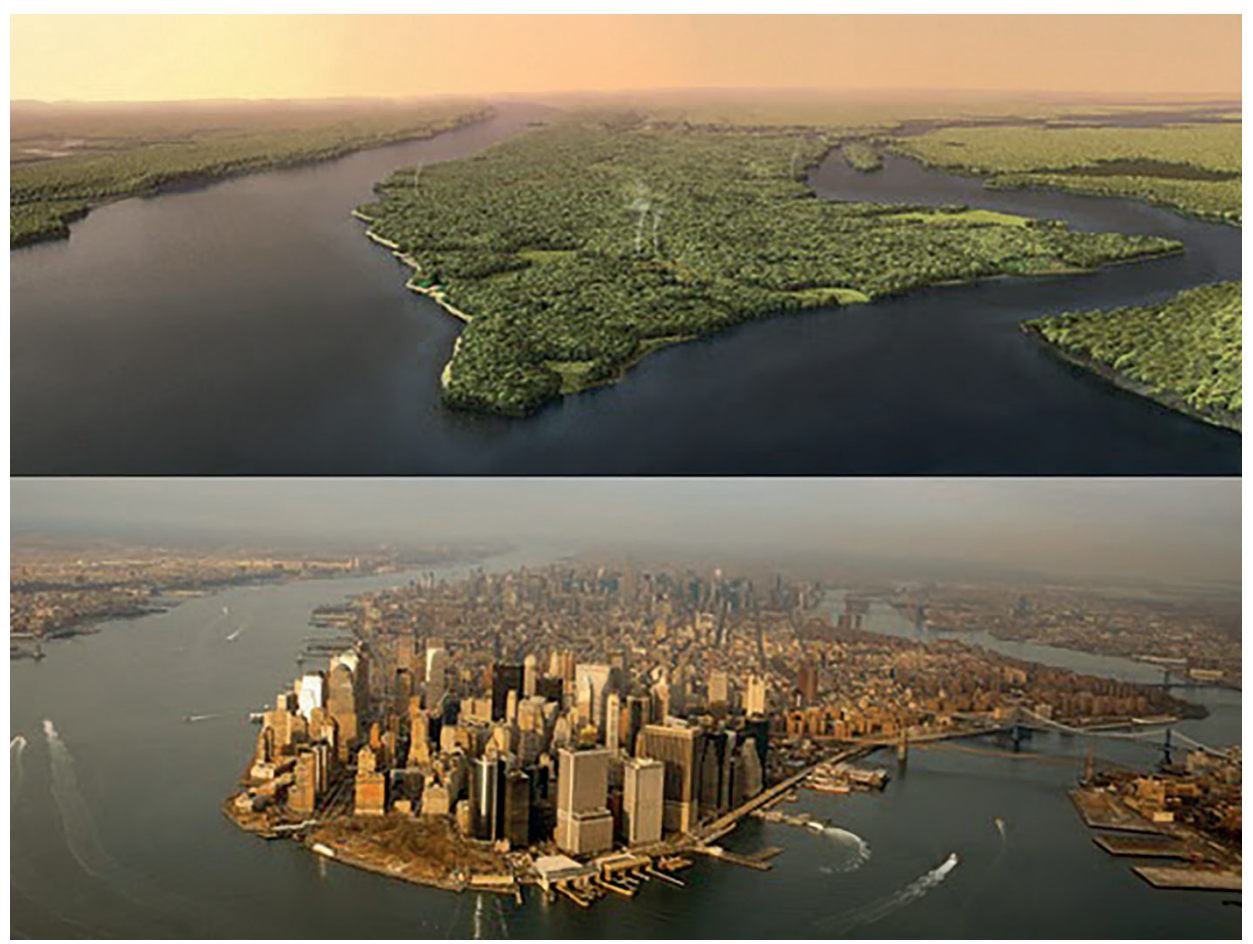

Figure 10.6: En haut: Image reconstituée de Manhattan il y a 400 ans. En bas: Image de Manhattan aujourd'hui. Source: Modern Era Aerial Photo @ Stephen Amiaga, PPA, PAPA

La planification de l'adaptation a été intégrée dans un processus de planification plus global et préexistant à NYC. L'objectif sous-jacent est de rendre la ville «résiliente». Les plans d'adaptation anticipent donc les changements à venir, qu'ils soient quotidiens ou liés à des événements extrêmes, Sandy devenant l'exemple de ce qui pourrait arriver plus souvent dans le futur.

Mais les plans d'adaptation visent aussi et, avant tout, l'amélioration de la qualité de vie de tous les New-yorkais. Des changements majeurs sont prévus sur le littoral en supplément des mesures d'adaptation du tissu urbain existant. Ils englobent des mesures «vertes» de restauration des écosystèmes (Fig. 10.7), mais aussi des mesures qui portent sur les comportements des New-yorkais ou leurs capacités d'action, comme la création de jardins communautaires, des projets de fermes urbaines et la participation des New-yorkais eux-mêmes aux projets de restauration de sites naturels. La caractéristique la plus frappante est certainement l'importance consacrée à la participation de la société civile, impliquant des réseaux mixtes (publics et privés), des ONG et des organisations communautaires qui sont très fortes à NYC. 


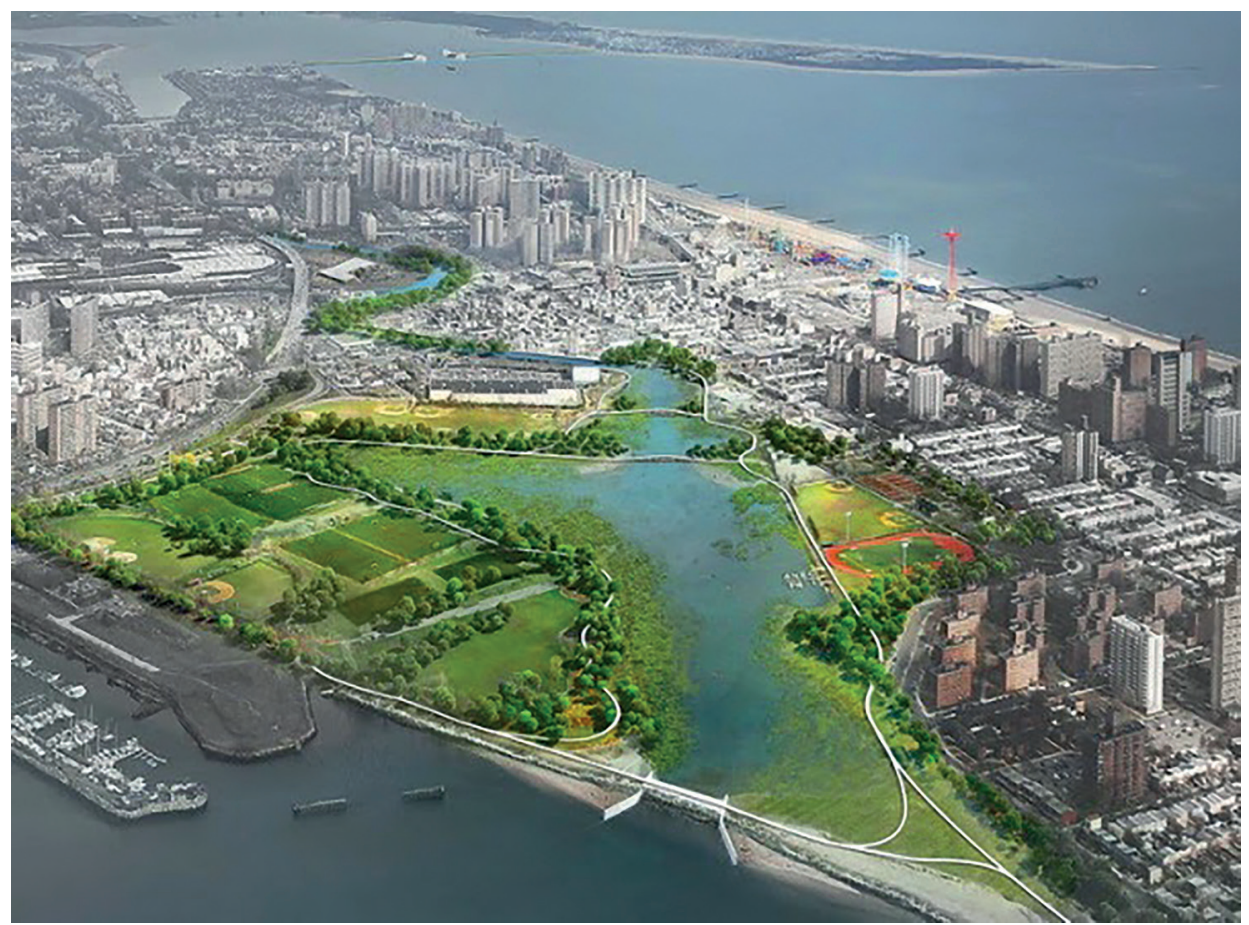

Figure 10.7: Projet de réaménagement/verdissement des berges en zone humide derrière Coney Island pour contrôler les eaux de crue (https://www.gothamgazette.com/environment/4255-no-retreatfrom-the-coastline). Photo: Mayor's office - tous droits réservés.

La caractéristique la plus frappante de ces mesures est certainement l'importance accordée à la participation de la société civile, laquelle inclut des réseaux mixtes (publics et privés), des ONG et des organisations communautaires, très influentes à NYC.

L'exemple de Sandy à NYC ou de Xynthia sur la côte Atlantique n'est pas sans rappeler les effets de la crise du Covid-19, que l'on vit actuellement, quand on le regarde sous l'angle de l'événement extrême. Ce type d'événement nous apporte des éléments nouveaux qu'il faut analyser pour faire évoluer nos sociétés, notamment par le biais de la planification.

\subsubsection{Défis restants pour la planification de l'adaptation au niveau local}

Quatre principaux défis seront à relever pour réussir les plans d'adaptation (Fig. 10.8) [6, 7].

Le premier défi réside dans le transfert de connaissances. En France, si l'Agence de la Transition Écologique (ADEME) joue évidemment un rôle central dans la construction des PCAET pour ce qui concerne leur structuration et la généralisation 
de certaines actions, le transfert de connaissances d'une intercommunalité à l'autre est rarement explicité dans les plans, et les actions les plus originales ne sont pas forcément reprises. II apparaît nécessaire de comprendre pourquoi les municipalités ne tirent pas plus parti des retours sur expériences ainsi que des évaluations et des données des autres plans, du moins de façon explicite. On s'attend, par ailleurs, à ce que les agences régionales, les municipalités et les universités contribuent de façon plus significative au transfert de connaissances.

Le deuxième défi porte sur la participation de l'ensemble des parties prenantes, laquelle est essentielle pour une planification réussie de l'adaptation au climat. Même si la majorité des collectivités relève ce défi, peu d'entre elles y arrivent pleinement. Par exemple, la prise en compte des populations vulnérables reste très limitée. La collaboration avec le monde universitaire est souvent négligée. La participation des citoyens doit être encouragée. L'éducation populaire et les initiatives de science citoyenne pourraient notamment offrir davantage de possibilités de participation du public (voir section 10.5).
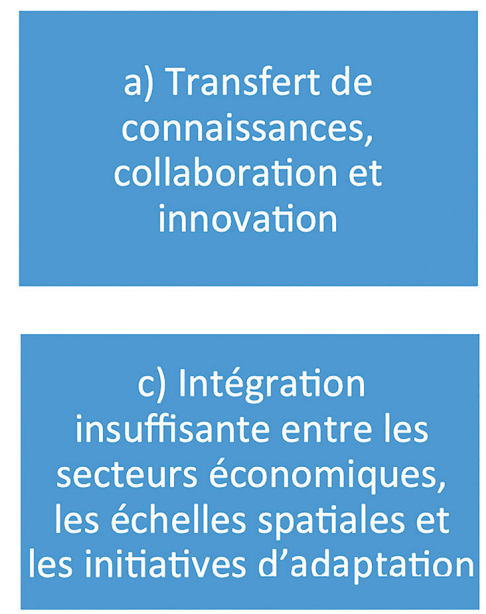
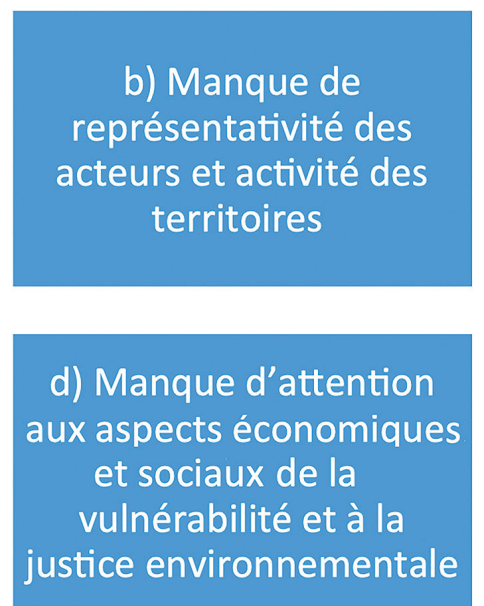

Figure 10.8: Défis à relever pour réussir les plans d'adaptation aux changements climatiques

Le troisième défi est d'arriver à une meilleure intégration verticale entre les différentes échelles spatiales de la planification (territoire national, région, ville, quartier) ainsi qu'à une meilleure intégration horizontale entre les différents secteurs économiques, entre les services techniques des mairies ou encore entre les initiatives d'adaptation. II est urgent pour les politiques publiques d'encourager et de faciliter l'intégration des différentes actions d'adaptation, en particulier celles qui apparaissent analogues d'un document de planification urbaine ou territoriale à l'autre.

Le quatrième défi et non des moindres est de s'appuyer sur une analyse plus approfondie des trois dimensions de la vulnérabilité humaine: l'exposition, la sensibilité et la capacité d'adaptation et de s'attaquer non seulement aux aspects 
environnementaux mais aussi aux aspects sociaux et économiques de la préparation au changement climatique. Une attention plus explicite devrait être accordée à l'éradication du sans-abrisme ou du mal-logement, à la réduction de la pauvreté, à la sécurité alimentaire urbaine, à l'accès à des prix abordables pour les soins de santé, à la multimodalité et à la sécurité publique et, de manière plus générale, à la continuité des services publics [8].

\subsection{Enjeux de gouvernance - R. Brett}

\subsubsection{Décision politique et protection de l'environnement}

Nombre de philosophes l'ont mis en évidence: le cadre politique et institutionnel contemporain ne permet pas d'apprécier les catastrophes écologiques à leur juste mesure ni de permettre l'élaboration de solutions adaptées aux défis du XXle siècle. La situation qui voit les partis politiques et leurs dirigeants principalement soucieux de satisfaire aussi rapidement que possible les intérêts de leurs électeurs dans la perspective d'une élection toute proche, rend hypothétique la poursuite d'une réflexion et d'une action politique sur le long terme comme l'exige pourtant la résolution des défis écologiques. Ainsi que l'écrivait Michel Serres dans son Contrat naturel [9] : «Pour sauvegarder la Terre ou respecter le temps, au sens de la pluie et du vent, il faudrait penser vers le long terme, et, pour n'y vivre pas, nous avons désappris à penser selon ses rythmes et sa portée».

L'exercice du pouvoir par un petit nombre d'élu(e)s au cours d'un mandat limité à cinq ans ne peut donc être entièrement satisfaisant. Conviendrait-il pour autant qu'un gouvernement de spécialistes, dont la légitimité découlerait moins d'une élection que de compétences scientifiques reconnues, soit établi pour une durée indéterminée afin de prendre les décisions qui s'imposent? II semble qu'une telle "technocratie» ne soit évidemment pas souhaitable en raison des risques qu'elle ferait planer sur l'ensemble des libertés. II ne faut du reste pas oublier que toute décision politique, même lorsqu'elle revêt un haut degré de technicité - comme la mise sur le marché d'un nouveau pesticide par exemple - est le fruit de la représentation que s'en font les décideurs et donc leurs électeurs. En outre, technique et science sont en partie des constructions sociales qui traduisent certaines représentations du monde nécessitant d'être questionnées et contextualisées. En ce sens, on ne peut que suivre Catherine et Raphaël Larrère lorsqu'ils affirment, dans Du bon usage de la nature - Pour une philosophie de l'environnement [10], que «L'idée de construction sociale du risque (...) exige que la nature et sa science soient portées à l'intérieur de la société, qu'elles deviennent objets d'un débat public».

L'approche renouvelée des modalités de prise de décisions qui engagent le futur de la société impose tout d'abord de tirer toutes les conséquences des 
enseignements des sciences à l'occasion de l'adoption des règles qui régissent le collectif, telles que la loi ou le règlement par exemple et nécessite, ensuite, que soit inclut le plus grand nombre d'individus dans le processus d'élaboration de la norme.

S'il ne convient pas de s'en remettre aux scientifiques pour prendre les décisions, il importe néanmoins de mieux intégrer les contraintes écologiques - contemporaines et surtout à venir - identifiées par ces derniers, dans les politiques publiques et les normes juridiques. Inclure le long terme dans les décisions ne revient d'ailleurs pas à décider du long terme, mais consiste plutôt à organiser des procédures par lesquelles la société pourra, par exemple, aborder rationnellement ce que la raison ne peut encore trancher, à savoir des questions scientifiques non résolues.

À l'heure actuelle, les processus normatifs organisent davantage la conciliation d'impératifs contradictoires au lieu de permettre la véritable intégration du souci écologique dans l'action publique. Le Droit tente souvent d'arbitrer entre le développement économique et la protection de l'environnement plutôt que de considérer l'environnement comme la toile de fond du développement économique sans laquelle rien n'est possible. Ce faisant, il tente de faire coexister les intérêts au lieu de les faire converger.

La Charte de l'environnement adossée à notre Constitution'11 illustre parfaitement cette ambiguïté puisqu'après avoir rappelé en son préambule que «la préservation de l'environnement doit être recherchée au même titre que les autres intérêts fondamentaux de la Nation» - procédant ainsi à une égalitarisation peu pertinente des intérêts - elle énonce en son article 6 que les politiques publiques concilient la protection et la mise en valeur de l'environnement, le développement économique et le progrès social.

\subsubsection{L'importance de la participation de tous à la prise de décision}

Les processus normatifs devraient donc intégrer davantage les contraintes scientifiquement établies. Néanmoins l'instauration d'un tel dialogue entre scientifiques et décideurs (élus ou administrations) nécessiterait que soit menée une réflexion profonde sur le statut du chercheur et sur la place de la recherche dans la société. Mais même déployé dans de saines conditions, ce dialogue aurait pour effet de rendre le reste de la société totalement aphone. Si l'on admet avec Jürgen Habermas (Droit et démocratie [11]) que la légitimité d'une norme est moins à rechercher dans son contenu que dans la procédure aboutissant à son adoption, il y a effectivement lieu de repenser les modalités d'intervention des

91. Loi constitutionnelle $n^{\circ} 2005-205$ du 1er mars 2005 :

https://www.legifrance.gouv.fr/contenu/menu/droit-national-en-vigueur/constitution/chartede---environnement 
individus et des groupes sociaux organisés - ce que l'on appelle la société civile dans les processus normatifs qui les concernent, dans le sens de leur plus grande participation.

En France, c'est par rapport aux questions environnementales que la démocratie participative s'est développée le plus. II ne faut pas y voir le fait du hasard, bien loin d'être un sujet exclusivement technique, l'environnement est d'abord un sujet social: ce qui nous entoure, nous environne, c'est ce qui nous construit. Ce qui se joue dans notre rapport à l'environnement, c'est notre humanité, notre façon d'être au monde et d'exister. Il est donc naturel, si l'on ose dire, que nous aspirons plus ou moins intensément à décider de notre cadre de vie. Le droit se fait l'écho de cette préoccupation: l'article 7 de la Charte de l'environnement énonce ainsi que «Toute personne a le droit (...) de participer à l'élaboration des décisions publiques ayant une incidence sur l'environnement».

Mais de Débats publics en Conventions citoyennes, il ne semble pas que les pouvoirs publics aient encore trouvé les bonnes formules permettant d'associer efficacement la population à l'exercice du pouvoir, dans la double perspective de l'amélioration de la qualité de l'environnement et de l'acceptation des normes. Les récentes difficultés que les gouvernants ont pu connaître à Sivens, Notre-Damedes-Landes ou encore à l'occasion du mouvement des "gilets jaunes» en sont des exemples dramatiques. Et même, lorsque «la formule» semble fonctionner, ces décideurs ne paraissent pas réellement prêts à donner toute sa portée à l'expression citoyenne, ainsi que le démontre le destin des propositions de la Convention citoyenne sur le climat.

II importe de souligner que la montée en puissance de l'expert et la réappropriation de la chose publique par les gouvernés ne font en aucun cas disparaître les représentants traditionnels, politiques ou administratifs, qui demeurent les véhicules incontournables permettant à la volonté générale et à l'intérêt général de s'incarner. Sans prétendre du tout les remplacer, ces deux mouvements ont uniquement pour effet de les remettre à leur juste place.

On insistera enfin sur l'importance de la conscientisation des individus, quel que soit leur âge. En effet, les citoyens ne peuvent délibérer rationnellement à propos de sujets aussi complexes que les sujets écologiques sans avoir bénéficié au préalable d'une éducation à l'environnement qui leur permette, sinon de maîtriser avec précision l'ensemble des données d'un problème spécifique, au moins d'en comprendre les enjeux, tenants et aboutissants. Ce n'est qu'au prix d'un investissement certain à la fois individuel et collectif qui aurait pour but la compréhension des équilibres naturels que pourrait s'épanouir une gouvernance politique de l'environnement efficace et apaisée qui ouvrirait la voie à la construction d'une authentique Cité écologique (Serge Audier [12]). 


\subsection{Domaine de la santé : adaptation aux températures élevées - R. Slama}

L'adaptation des sociétés humaines au réchauffement climatique est abordée ici dans le domaine de la santé, prolongeant ainsi l'analyse de l'impact sanitaire des températures élevées, qui a été faite précédemment (voir section 8.4.1).

\subsubsection{Situations à New York et en France durant les dernières décennies}

Comme nous l'avons vu, la relation dose-réponse, qui relie la température et la mortalité n'est pas la même dans des villes comme Stockholm et Londres. On ne peut pas incriminer des différences génétiques entre les populations de Londres et de Stockholm pour expliquer l'essentiel de cette différence, comme d'ailleurs entre les populations de toutes les grandes villes. D'une part, les différences génétiques sont très limitées d'une ville à l'autre, surtout au sein d'un même continent, et d'autre part, ce sont surtout des raisons sociétales, liées au mode de vie, qui expliquent les écarts observés.

Pour aborder la question de l'adaptation au changement climatique du point de vue de la santé, il est pertinent d'étudier l'évolution de la mortalité au cours du temps pour une ville donnée. C'est une étude de ce type qu'ont menée des épidémiologistes américains dans la ville de New York, où l'on dispose d'un registre de la mortalité sur une très longue période. Ils ont ainsi pu établir des relations dose-réponse entre températures élevées et mortalité comme celles rapportées sur la figure 10.9 tout au long du XXe siècle. Chaque courbe de la figure correspond à une décennie entre 1900 et 2010. L'examen de la hauteur des courbes pour une température donnée nous indique la sensibilité de la population new-yorkaise à différentes périodes, selon le niveau. Nous constatons qu'à une température donnée, la mortalité (ou plus précisément, le «sur-risque» de mortalité par rapport aux températures «confortables»), a très fortement décru au cours du XXe siècle. Par exemple, à $30^{\circ}$, le risque de décès était multiplié par deux dans les années 1900 (une augmentation de 100\%), alors que dans les années 2000, il n'est plus multiplié que par 1,1 à 1,2 (une augmentation de 10 à $20 \%$ ).

Cette décrue spectaculaire n'est évidemment pas due au fait que les New-yorkais aient muté génétiquement et que leurs organismes se soient adaptés aux températures élevées. II faut beaucoup plus de temps à des mutations génétiques pour se répandre dans une population, quelle que soit la façon dont elle se produit, même dans un contexte d'immigration comme à New York. D'autres mécanismes sont en jeu à l'échelle de la société, sur lesquels nous reviendrons. 


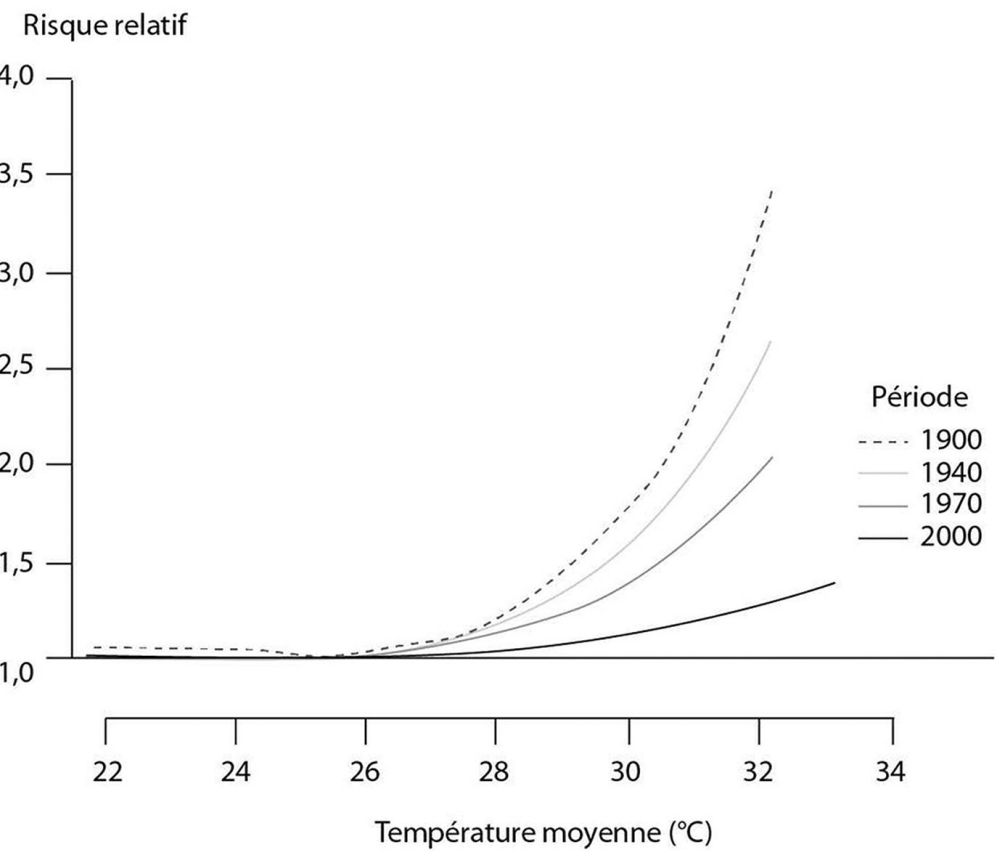

Figure 10.9: Évolution du risque de mortalité en fonction de la température au cours du XXe siècle dans la ville de New York. Source: Rémy Slama, Le mal du dehors, l'influence de l'environnement sur la santé, Quae éditions, 2017, Paris [13].

Demandons-nous tout d'abord si la même évolution a eu lieu en France. C'est à Alain Jacques Valleron [14], de l'Académie des sciences, que nous devons une étude similaire, mais sur une période moins longue. En France, nous ne disposons, en effet, d'un enregistrement des causes de décès, faite de façon fine par I'INSERM, que depuis 1968. Le temps écoulé est toutefois suffisamment long pour se faire une idée de l'évolution. Les résultats de l'étude sont synthétisés dans le tableau 10.1. Lorsque l'on considère séparément trois périodes entre 1968 et 2009, l'étude met en évidence que le risque individuel de mortalité à la température de $25^{\circ} \mathrm{C}$, rapporté à celui à la température de référence de $18^{\circ} \mathrm{C}$, a quelque peu évolué au cours du temps. De 1,18 dans la première période, le rapport est passé à 1,15 dans la dernière période. La variation n'est pas spectaculaire, car on aboutit seulement à une diminution de trois points en pourcentage. Néanmoins, l'évolution va bien dans le même sens que celle observée à New York sur une période beaucoup plus grande et dans une société très différente.

Peut-on alors conclure que l'impact sanitaire des températures élevées a diminué au cours des dernières décennies en France comme à New York? C'est, en fait, plutôt le contraire qui est vrai, à savoir que globalement, on souffre davantage des températures élevées. Pourquoi? II faut d'abord préciser ce qu'on entend par impact sanitaire: il s'agit du nombre de cas d'une pathologie donnée ou de décès attribuables à un facteur donné (ici la température). Or la relation dose-réponse, comme celles 
représentées sur la figure 10.9 pour New York, ne suffit pas à l'estimer; il faut la combiner avec la distribution de la température tout au long de l'année. Si on regarde plus attentivement le tableau, on constate qu'en France, le nombre de jours avec des températures très élevées (les vagues de chaleur) a très fortement augmenté au cours du temps: il a presque triplé des années 1970 aux années 2000. L'impact sanitaire - le nombre de décès attribuables aux vagues de chaleur - s'obtient en combinant l'effet individuel de la chaleur avec la fréquence des jours «chauds». Ici, bien qu'au niveau individuel, le risque de mortalité diminue un peu, l'augmentation forte de l'événement étudié - la canicule - entraîne une augmentation probable de l'impact populationnel.

\begin{tabular}{|c|c|c|c|c|c|c|c|}
\hline Périodes & $\begin{array}{l}\text { Température } \\
\text { moyenne } \\
\text { en été }\left({ }^{\circ} \mathrm{C}\right)\end{array}$ & $\begin{array}{l}\text { Température } \\
\text { moyenne } \\
\text { en hiver }\left({ }^{\circ} \mathrm{C}\right)\end{array}$ & $\begin{array}{c}\text { Jours de } \\
\text { vague de } \\
\text { Chaleur }\end{array}$ & $\begin{array}{l}\text { Jours de } \\
\text { vague de } \\
\text { froid }\end{array}$ & $\begin{array}{l}\text { Température } \\
\text { de mortalité } \\
\text { minimale }\end{array}$ & $\begin{array}{c}\text { Jours de } \\
\text { température } \\
>\text { température } \\
\text { minimale }\end{array}$ & $\begin{array}{c}\text { Rapport } \\
\text { entre taux de } \\
\text { mortalité à } \\
25^{\circ} \mathrm{C} \text { et } 18^{\circ} \mathrm{C}\end{array}$ \\
\hline $\begin{array}{c}1968 \\
-1981\end{array}$ & $\begin{array}{r}17,6 \\
\pm 1,4\end{array}$ & $\begin{array}{r}4,2 \\
\pm 1,8\end{array}$ & $\begin{array}{l}6,8 \\
\pm 6\end{array}$ & $\begin{array}{r}8,2 \\
\pm 2,4\end{array}$ & $\begin{array}{c}17,5^{\circ} \mathrm{C} \\
\pm 1,4\end{array}$ & $\begin{array}{c}825 \\
\pm 228\end{array}$ & $\begin{array}{c}1,18 \\
\pm 0,12\end{array}$ \\
\hline $\begin{array}{r}1982 \\
-1995\end{array}$ & $\begin{array}{r}18,6 \\
\pm 1,5\end{array}$ & $\begin{array}{c}4,6 \\
\pm 1,8\end{array}$ & $\begin{array}{c}5,8 \\
\pm 4,0\end{array}$ & $\begin{array}{r}22,6 \\
\pm 3,5\end{array}$ & $\begin{array}{c}17,8^{\circ} \mathrm{C} \\
\pm 1,5\end{array}$ & $\begin{array}{c}962 \\
\pm 243\end{array}$ & $\begin{array}{c}1,16 \\
\pm 0,08\end{array}$ \\
\hline $\begin{array}{r}1996 \\
-2009\end{array}$ & $\begin{array}{l}19,2 \\
\pm 1,5\end{array}$ & $\begin{array}{r}5,0 \\
\pm 1,7\end{array}$ & $\begin{array}{r}16,7 \\
\pm 5,5\end{array}$ & $\begin{array}{r}8,9 \\
\pm 2,9\end{array}$ & $\begin{array}{c}18,2^{\circ} \mathrm{C} \\
\pm 1,6\end{array}$ & $\begin{array}{c}994 \\
\pm 262\end{array}$ & $\begin{array}{r}1,15 \\
\pm 0,08\end{array}$ \\
\hline
\end{tabular}

Tableau 10.1: Évolution du risque individuel de mortalité lié aux températures chaudes pour 3 périodes en France. D'après l'étude de Jacques Valleron [14].

\subsubsection{Pratiques à mettre en œuvre pour l'avenir}

Revenons à New York et interrogeons-nous sur les causes de l'atténuation de l'effet d'une température donnée au cours du siècle passé, c'est-à-dire l'aplatissement des relations dose-réponse de la figure 10.9. Nous avons écarté l'hypothèse d'une modification rapide de l'organisme. En fait, c'est la société qui s'est adaptée. Si la société new-yorkaise et la société américaine dans son ensemble ont réussi à s'adapter aux températures élevées, c'est en sachant mieux guérir les maladies et troubles que la chaleur rend plus fréquents, et en protégeant mieux les habitants des chaleurs élevées, notamment en généralisant les climatisations et les climatiseurs individuels, au domicile et dans les véhicules. Or, cette dernière solution augmente fortement la chaleur en dehors des habitats et contribue à créer des îlots de chaleur. Les personnes qui n'ont pas les moyens d'avoir des climatiseurs et celles qui sont dans la rue vont alors subir des températures plus élevées. On risque donc accroître les inégalités sociales et la sensibilité de certaines catégories sociales aux évènements climatiques. À cette première conséquence néfaste s'ajoute le fait que les 
climatiseurs consomment beaucoup d'énergie. Dans un pays comme les États-Unis où l'électricité est en grande partie produite à partir des sources d'énergie fossiles, cette consommation électrique se traduit ainsi par de fortes émissions de $\mathrm{CO}_{2}$. En outre, les premiers climatiseurs installés, qui sont pour certains toujours en fonctionnement, émettent aussi des chlorofluorocarbones, qui contribuent à la fois au réchauffement climatique et à la destruction de la couche d'ozone. La figure 10.10 illustre l'augmentation régulière de $\mathrm{CO}_{2}$ émis aux États-Unis depuis les années 2000, et la généralisation des climatiseurs.

En fait, en s'adaptant localement aux effets du changement climatique, on a amplifié ce phénomène à l'échelle globale. C'est une course en avant qui n'est absolument pas tenable à moyen terme à l'échelle de la planète. L'exemple des ÉtatsUnis ne pourrait être transposé à l'ensemble des pays du globe. Si tous les pays se mettaient à émettre autant de gaz à effet de serre que les États-Unis, ils rendraient très rapidement le climat encore plus rapidement intenable qu'il n'est sur la trajectoire suivie actuellement.

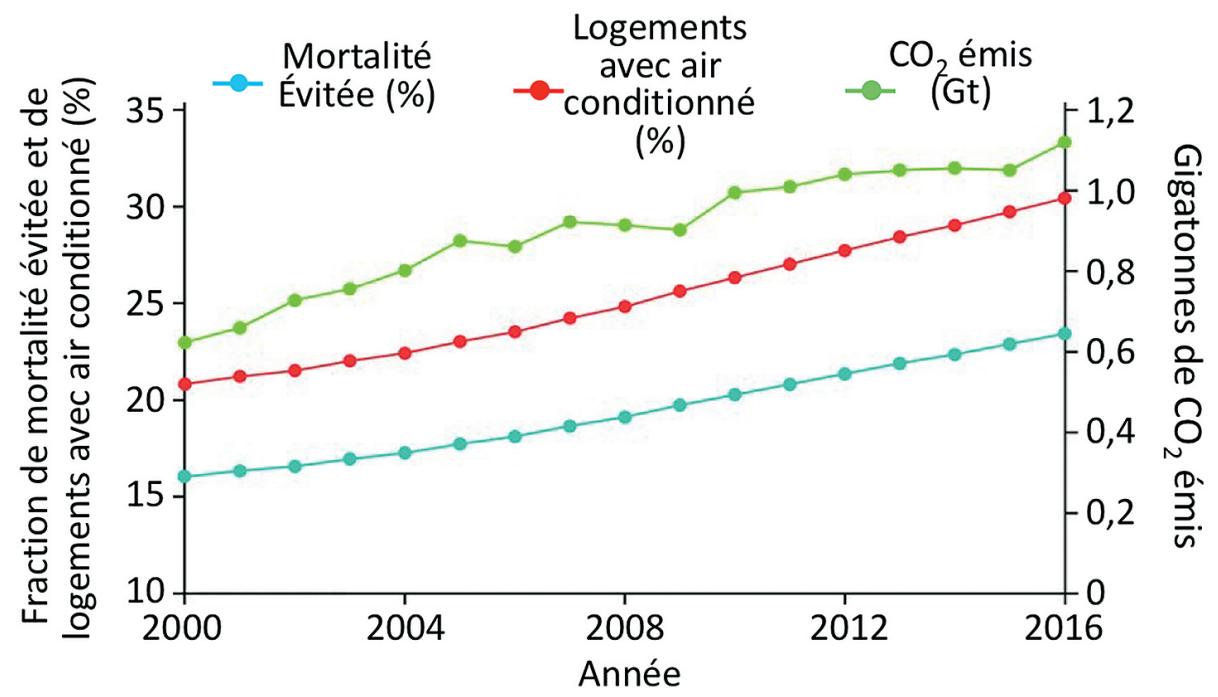

Figure 10.10: Évolutions récentes aux États-Unis - du pourcentage de mortalité épargnée par l'utilisation de climatiseurs (en bleu clair), - du pourcentage de logements pourvus de climatiseurs (en rouge) - et des quantités de gaz carbonique émis dans l'atmosphère [15].

II faut donc trouver d'autres solutions pour se protéger des températures élevées. On peut imaginer de nouvelles technologies plus ou moins complexes, mais on peut aussi se tourner vers des solutions du passé, à des époques et dans des lieux où les humains subissaient déjà des températures élevées de façon chronique. Pour s'adapter, les modes de vie de ces populations tenaient compte de certains rythmes circadiens en évitant des activités très physiques et fatigantes dans les périodes de pleine chaleur. Transposé à notre époque et en France, ce serait 
notamment favoriser les pauses méridiennes et travailler à des horaires différents de nos horaires classiques de bureau.

On peut aussi s'inspirer de l'urbanisme qu'adoptaient, par exemple au XIIle siècle, les Omeyyades en Andalousie avec les jardins de l'Alhambra (Fig. 10.11) : construire (ou réhabiliter) des villes en exploitant à la fois des espaces verts et des espaces bleus, c'est-à-dire, avec un recours très fort à l'eau pour permettre, dans une certaine mesure, de tempérer l'atmosphère locale. II faut imaginer l'équivalent moderne des jardins de l'Alhambra. On peut aussi reproduire avec les moyens d'aujourd'hui les architectures qu'on trouvait par exemple sur les îles grecques, il y a plusieurs siècles, avec des habitations peintes tout en blanc et des fenêtres relativement petites. C'est d'ailleurs une solution qui commence à être adoptée dans une ville comme New York où l'on peint les toits des immeubles en blanc. Ce choix d'un albédo plus propice à réfléchir une fraction importante de l'énergie que nous envoie le soleil, pourrait d'ailleurs être étendu aux rues en macadam très sombre qui absorbe fortement la chaleur.

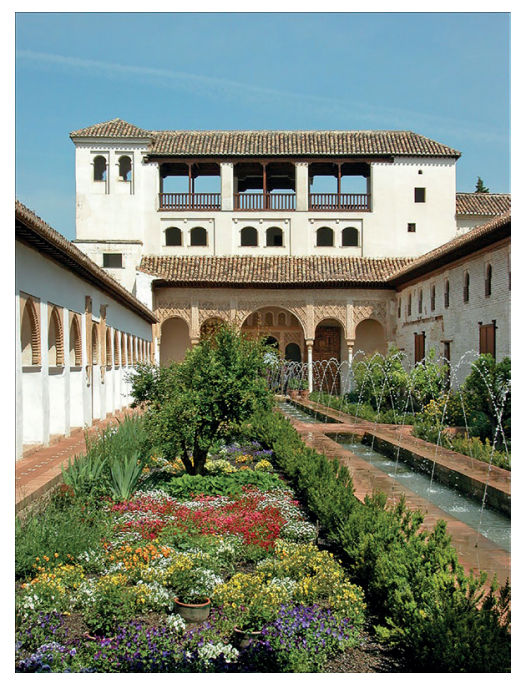

Figure 10.11: Vue des jardins de l'Alhambra réalisés au XIII siècle en Andalousie - Source: Jean-Pierre Dalbéra (Creative Commons 2.0). https://commons.wikimedia.org/wiki/File:LAlhambra_de_Grenade_ (8356975975)_(2).jpg.

En résumé, nous voyons qu'il y a dans certaines sociétés, qu'elles soient riches et modernes ou qu'elles soient anciennes et ingénieuses, tout un ensemble des solutions qui pourraient nous permettre de mieux nous adapter aux températures élevées en modifiant l'urbanisme de nos villes. D'autres solutions, comme celles basées sur l'usage intensif de climatisation individuelle, et alimentée par des énergies émettrices de gaz à effet de serre, risquent au contraire d'accroître le changement climatique. Dans la recherche des solutions d'adaptation, il est ainsi indispensable de ne pas nous limiter à une seule facette du problème, mais de considérer simultanément les aspects sociétaux, les aspects environnementaux et les aspects sanitaires. 


\subsection{Domaine de l'agriculture : adaptation aux sécheresses - J.-C. Bureau}

\subsubsection{Le problème des ressources en eau}

En 2009, une équipe internationale de 26 chercheurs, menés par Johan Rockström du Stockholm Resilience Centre et Will Steffen de l'Université nationale australienne, publie un article dans les revues Nature et Ecology and Society [16] dans lequel elle identifie neuf limites planétaires à ne pas dépasser si l'humanité veut pouvoir se développer dans un écosystème sûr, c'est-à-dire évitant les modifications brutales, non-linéaires, potentiellement catastrophiques et difficilement prévisibles de l'environnement (Fig. 10.12): le réchauffement climatique, l'acidification des océans, les flux biogéochimiques, l'ozone stratosphérique, etc. Parmi les neufs «limites», plusieurs d'entre elles concernent assez directement l'agriculture en étant aussi les plus proches d'être dépassées, voire même d'être réellement au-delà des capacités d'adaptation de la planète. C'est notamment le cas de la pollution azotée et de l'effondrement de la biodiversité.

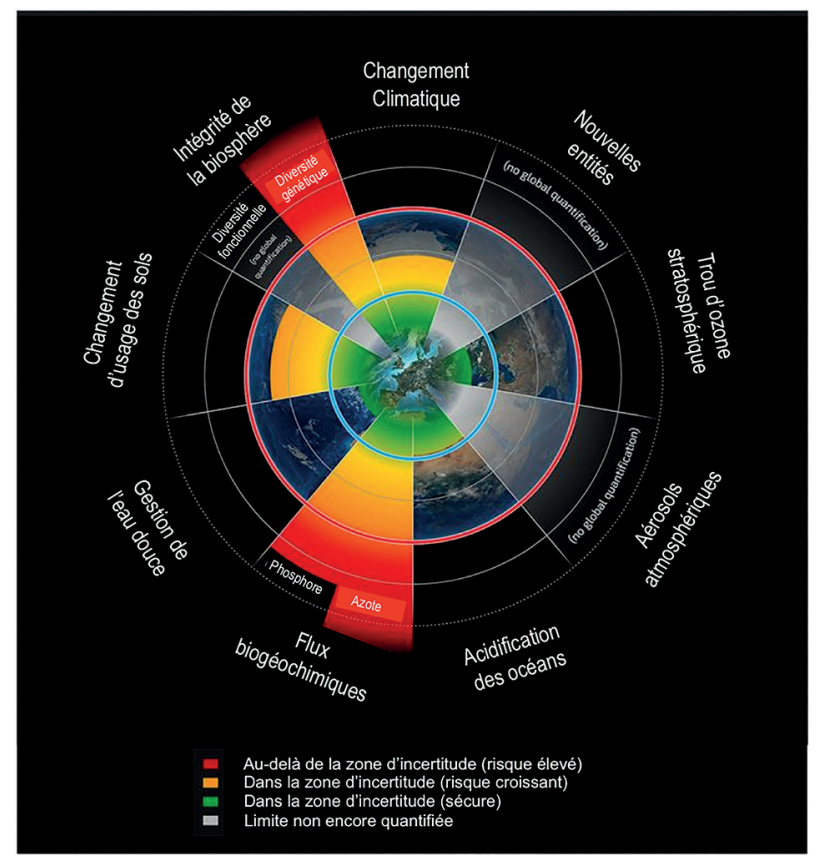

Figure 10.12: Les limites planétaires. Image inspirée de [17] - Design: Globaïa.

La gestion de l'eau douce est une autre limite sur laquelle intervient l'agriculture. L'agriculture est, en effet, le premier secteur qui prélève de l'eau douce en quantité. Selon le cycle naturel de l'eau (voir section 2.4), une partie de l'eau douce, qui 
se forme dans l'atmosphère par évaporation des océans ou par transpiration des végétaux, finit par se condenser et retourner à la terre grâce aux précipitations... mais pas nécessairement au moment et à l'endroit où l'on souhaiterait que l'eau revienne. Dans un pays d'agriculture comme la France, les sécheresses et les événements extrêmes sont de plus en plus fréquents, et il devient de plus en plus difficile de cultiver certaines plantes sans irrigation, en particulier dans le sud de la France. À titre d'exemple, les cultures de maïs, très répandues dans le sud-ouest, souffrent souvent d'un déficit de pluie, car les semailles se font assez tardivement au printemps, et il est alors nécessaire d'utiliser l'irrigation et les réserves en eau du sol.

Or, l'irrigation se fait souvent au détriment des rivières, de plus en plus fréquemment à sec ou avec un débit insuffisant pour diluer les pollutions. Un assèchement, même temporaire, nuit gravement à la biodiversité, car la base de la chaîne alimentaire est constituée d'insectes qui ont une phase aquatique. Au niveau mondial, environ la moitié de l'eau utilisée pour l'irrigation agricole vient de nappes phréatiques fossiles [18]. Celles-ci connaissent des problèmes de baisse de niveau et d'épuisement dans de nombreuses régions du monde, tout particulièrement dans le sous-continent indien, l'Asie centrale, la Chine, et une partie de l'Afrique [19]. Les difficultés de recharge de ces nappes se multiplient aussi en France où l'irrigation doit, de plus en plus souvent, être limitée ou interdite en été.

En France, les agriculteurs tentent de réduire les prélèvements dans les rivières et nappes souterraines en construisant des bassins de rétention de l'eau de pluie pour irriguer. Les effets ne sont pas toujours favorables à l'environnement, car dans de tels bassins, l'eau se réchauffe et fait potentiellement le lit de bactéries, dont des cyanobactéries toxiques, qui peuvent se diffuser en aval du versant. En bref, l'irrigation, bien que de plus en plus nécessaire, a souvent des effets pervers.

II existe des solutions techniques pour économiser l'eau d'arrosage, ce qu'on appelle le goutte-à-goutte, mais ces solutions sont souvent coûteuses.

\subsubsection{S'adapter aux sécheresses grâce à de nouvelles cultures et des cultivars ${ }^{92}$}

À court terme, un moyen simple d'action pour s'adapter aux sécheresses à répétition consiste à changer les plantes qu'on cultive. Ainsi, plutôt que de cultiver du maïs qui demande de grandes quantités d'eau dans les mois de juin et juillet, on peut passer à des cultures plus économes en eau, surtout en été. Certains agriculteurs dans le sud-ouest de la France commencent effectivement à cultiver le sorgho à la place du maïs (Fig. 10.13). Le sorgho est une céréale originaire d’Éthiopie, résistante à la sécheresse et qui est, de ce fait, largement cultivée en Afrique, en Inde et en Amérique du Sud, aussi bien pour ses graines dans l'alimentation humaine que pour son fourrage

92. http://www.vivreici.be/videos/detail_secheresse-l-agriculture-va-devoir-s-adapter?videold $=1568948$ 
dans l'alimentation animale. Cela étant, le maïs irrigué, plus rentable reste encore largement dominant. L'évolution vers des cultures moins consommatrices d'eau comme le sorgho est freinée par le fait que les agriculteurs paient peu l'eau prélevée dans les nappes et reçoivent des subventions aux infrastructures d'irrigation.

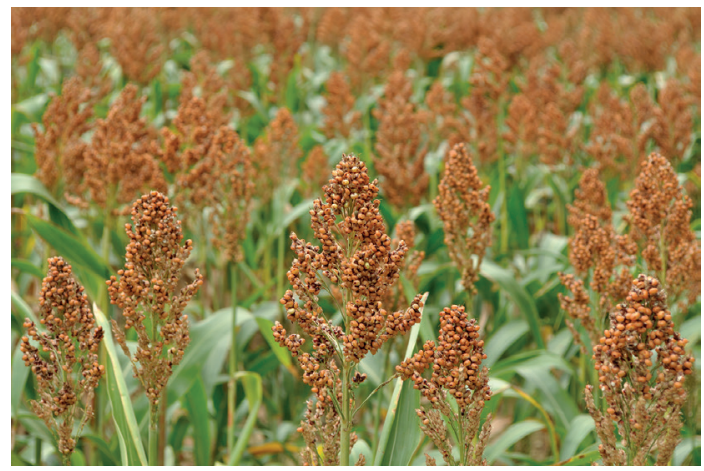

Figure 10.13: Culture de sorgho en Haute Garonne (France). Source : Gyrostat (Creative commons 4.0).

Parmi les mesures d'adaptation aux sécheresses, on peut aussi recourir à d'autres façons de les cultiver. De nouvelles démarches agronomiques sont explorées dans ce sens comme le fait d'associer plusieurs plantes sur la même terre de culture ou de favoriser la présence de matière organique dans les sols. On parle alors de méthodes culturales.

Sur le long terme, il semble nécessaire d'adapter les cultures en sélectionnant des plantes plus aptes à résister à la sécheresse. Pour ce faire, certains pays misent surtout sur la modification du génome, ce qu'on appelle le génie génétique, considéré par eux comme un secteur d'avenir pour l'adaptation des plantes aux sécheresses. En France, les résistances sociales au génie génétique sont fortes. À plus long terme aussi, une solution consiste à pratiquer la sélection génétique des plantes cultivées sans recourir, pour autant, à des modifications génétiques. L'industrie agricole et les centres de recherches en agronomie testent, en effet, en permanence de nouveaux croisements entre des variétés de plantes ou de fruits pour arriver à créer des produits qui réunissent les qualités requises. On parle de cultivars, mot emprunté à la langue anglaise pour désigner une variété cultivée (CULT/vated VARiety). La création de nouveaux cultivars est longue, car il faut parfois plusieurs dizaines d'années pour s'assurer qu'aux principales qualités requises (présentement, l'adaptation à la sécheresse), soient aussi réunies les conditions de production régulière et uniforme, de résistance aux maladies et aux intempéries, etc. Pour proposer des cultivars adaptés aux sécheresses à répétition, on peut, par exemple, sélectionner dans un panel de variétés, celles qui sont les plus précoces dans la saison et voir si cette précocité se traduit effectivement par un avantage compétitif. On peut aussi rechercher des plantes dont les racines s'enfoncent profondément dans la terre pour puiser l'eau et régénèrent en même temps le sol en y stockant du carbone. Comme 
nous l'avons vu précédemment (section 2.6.2), le stockage du carbone favorise la fertilité du sol sur le long terme. Un sol riche en matière organique joue aussi un rôle d'éponge qui permet en retour de stocker plus d'eau. De nombreuses recherches de ce type sont aujourd'hui menées en parallèle avec le développement des nouvelles méthodes de culture.

\subsubsection{Des changements de culture à l'agroforesterie, l'exemple du Sahel}

D'après la vidéo produite par le CIRAD:(Centre de coopération internationale en recherche agronomique pour le développement) : https://www.youtube.com/watch?v=kCYG2e9Xt60

Diversifier les cultures et réfléchir à leur impact sur plusieurs années, c'est aussi planter des arbres en les associant aux cultures et aux pâturages, ce qu'on appelle l'agroforesterie. Dans les régions sèches en particulier, l'agroforesterie fait partie des quelques techniques utiles pour affronter les questions du changement climatique, que ce soit les questions de mitigation ou les questions d'adaptation.

Les systèmes agroforestiers offrent de nombreux avantages, certains connus depuis toujours, d'autres révélés récemment par la science. Un premier avantage évident est de créer des zones d'ombre, notamment pour revitaliser les pâturages soumis à rude épreuve en période de sécheresse. Un deuxième avantage est de permettre le piégeage du carbone en grande quantité dans les sols, car la biomasse d'un arbre contient beaucoup plus de carbone que les plantes céréalières. Un autre avantage encore tient au phénomène d'évapotranspiration des arbres, qui permet aux forêts de propager les pluies (voir section 7.4).

Un exemple emblématique des bienfaits de l'agroforesterie est celui du Sahel, région d'Afrique particulièrement soumise aux sécheresses où l'arbre nommé Faidherbia albida (Fig. 10.14) est véritablement clé en matière d'adaptation aux changements climatiques.

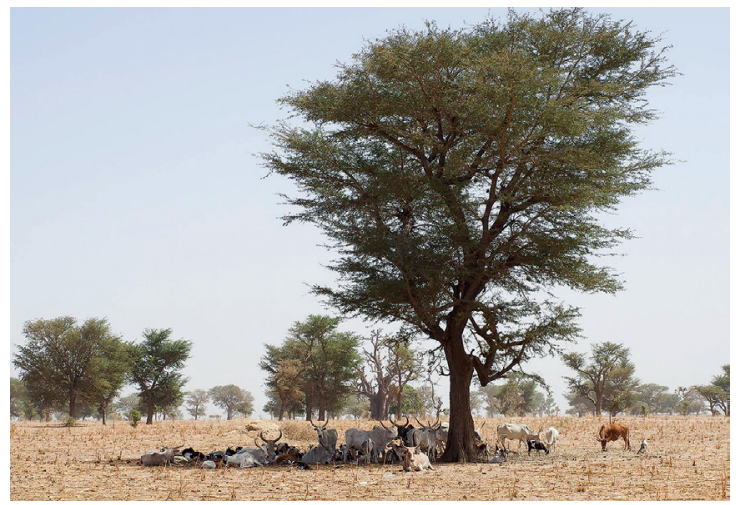

Figure 10.14: Image d'un faidherbia albida au Sénégal abritant un troupeau de chèvres. Source: NoahElhardt (Creative commons 4.0 International). 
Cet acacia améliore la fertilité des sols, fournit du bois aux populations et du fourrage au bétail pendant la saison sèche. II a cette caractéristique extraordinaire de phénologie inversée, qui favorise le développement de ses feuilles en saison sèche, juste au moment où les éleveurs ont besoin de fourrage. Le Faidherbia albida est aussi capable d'absorber l'azote de l'air et de le restituer aux cultures. Grâce à ses racines très profondes, non seulement il va profiter des ressources en profondeur pour sa propre croissance, mais il est aussi capable de remonter une partie de ces ressources d'eau et de minéraux vers la surface.

Un autre bénéfice rendu par ce type d'arbre vient d'être révélé par des scientifiques du Cirad (Centre de coopération internationale en recherche agronomique) dans le cadre d'un partenariat international; la présence de l'arbre en relativement grande densité dans un champ limite les attaques de chenilles aux cultures. Pour comprendre ce phénomène, les chercheurs ont croisé des données écologiques avec l'analyse du paysage. Les chercheurs en sont venus à la conclusion que ces arbres seraient un refuge pour des oiseaux, des chauves-souris et des insectes, ennemis naturels des chenilles. L'agroforesterie permettrait donc aussi de protéger naturellement les cultures contre les ravageurs. Cette découverte est un argument supplémentaire pour inciter les agriculteurs à conserver les arbres dans leurs champs.

En résumé, l'agroforesterie cumule de nombreux avantages tels que le stockage du carbone, l'adaptation au changement climatique, le maintien de la fertilité des sols et la protection des cultures. Cette solution, qui est un modèle d'adaptation pour les pays tropicaux, peut aussi être (re)transplantée dans nos pays tempérés. Faisons juste appel à notre mémoire en nous souvenant, par exemple, de l'agroforesterie des bocages: une pratique que l'on a un peu perdu et qui mériterait d'être retrouvée.

\section{Références}

[1] C.S. Holling. 1973. "Resilience and stability of ecological systems". Annual Review of Ecology and Systematics. Vol.4:1-23, (1973).

[2] IPCC. 2014. Summary for policymakers. In: Climate Change 2014: Impacts, adaptation, and vulnerability. Part A: Global and sectoral aspects. Contribution of Working Group II to the Fifth Assessment Report of the Intergovernmental Panel on Climate Change [Field, C.B., V.R. Barros, D.J. Dokken, K.J. Mach, M.D. Mastrandrea, T.E. Bilir, M. Chatterjee, K.L. Ebi, Y.O. Estrada, R.C. Genova, B. Girma, E.S. Kissel, A.N. Levy, S. MacCracken, P.R. Mastrandrea, L.L. White (eds.) ]. Cambridge University Press, Cambridge, United Kingdom and New York, NY, USA, pp. 1-32.

[3] ONERC. 2017. Vers un $2^{\mathrm{e}}$ plan d'adaptation au changement climatique pour la France. Enjeuxet recommandations. La documentation française, Paris, pp.229. ISBN: 978-2-11-145442-2.

[4] E. Richard. 2014. L'action publique territoriale face au défi de l'adaptation: déterminants et effets de la prise en compte des changements climatiques à l'échelle régionale. VertigO la revue électronique en sciences de l'environnement Hors-série 20. 10.4000/vertigo.15283. 
[5] E.W. Sanderson, M. Boyer (Illustrator). 2009. Mannahatta: A Natural History of New York City. Abrams Books. ISBN 0810996332.

[6] E. Lioubimtseva, C. da Cunha. 2020. Local climate change adaptation plans in the US and France: comparison and lessons learned in 2007-2017. Urban Climate 31, 100577. doi: https://doi.org/10.1016/j.uclim.2019.100577.

[7] D. Reckien, M. Salvia, O. Heidrich, J.M. Church, F. Pietrapertosa, S. De Gregorio-Hurtado, V. D’Alonzo, A. Foley, S.G. Simoes, E. Krkoška Lorencová, H. Orru, K. Orru, A. Wejs, J. Flacke, M. Olazabal, D. Geneletti, E. Feliu, S. Vasilie, C. Nador, Krook- A. Riekkola, M. Matosović, P.A. Fokaides, B.I. loannou, A. Flamos, N.-A. Spyridaki, M.V. Balzan, O. Fülöp, I. Paspaldzhiev, S. Grafakos, R. Dawson. 2018. How are cities planning to respond to climate change? Assessment of local climate plans from 885 cities in the EU-28. Journal of Cleaner Production 191, 207-219. https://doi.org/10.1016/j.jclepro.2018.03.220.

[8] I. Anguelovski, L. Shi, E. Chu, D. Gallagher, K. Goh, Z. Lamb, K. Reeve, H. Teicher. 2016. Equity impacts of urban land use planning for climate adaptation: critical perspectives from the Global North and South. Journal of Planning Education and Research 36, 333-348. $10.1177 / 0739456 \times 16645166$.

[9] M. Serres. 2020. Le contrat nature/ (1990). Flammarion, coll. «Champs essais».

[10] C. Larrère et R. Larrère. 2009. Du bon usage de la nature - Pour une philosophie de l'environnement. Flammarion, coll. «Champs essais».

[11] J. Habermas. 1997. Droit et démocratie. Gallimard, coll. «Nrf essais».

[12] S. Audier. 2020. La Cité écologique - pour un éco-républicanisme. La découverte, coll. "Sciences humaines».

[13] Rémy Slama. 2017. Le mal du dehors, l'influence de l'environnement sur la santé, Quae éditions, Paris.

[14] Todd, Valleron. 2005. Env Health Perspect. https://ehp.niehs.nih.gov/doi/10.1289/ehp. 1307771.

[15] N. Watts, et al. 2019. $9^{\text {th }}$ Report of the Lancet Countdown on Health and Climate Change, p. 41.

http://orca.cf.ac.uk/126885/1/The\%202019\%20Report\%20of\%20the\%20Lancet\%20 Countdown\%20-\%20final\%20accepted.pdf

[16] J. Rockström, W. Steffen, K. Noone et al. A safe operating space for humanity. Nature 461, 472-475 (2009). https://doi.org/10.1038/461472a.

[17] W. Steffen, K. Richardson, J. Rockström, S.E. Cornell, I. Fetzer, E.M. Bennett, R. Biggs, S.R. Carpenter, W. de Vries, C.A. de Wit, C. Folke, D. Gerten, J. Heinke, G.M. Mace, L.M. Persson, V. Ramanathan, B. Reyers, S. Sörlin. Planetary boundaries: Guiding human development on a changing planet. Science 347(6223), 1259855. DOI: 10.1126/science.1259855.

Voir aussi: https://coffeebreaksciencefr.wordpress.com/2015/04/01/en-bref-mars-2015partie-ii-pollution-plastique-et-limites-planetaires/

[18] S. Jasechko, D. Perrone. 2021. Global groundwater wells at risk of running dry. Science 23, 418-421. https://science.sciencemag.org/content/372/6540/418.

[19] S.W.D. Turner, M. Hejazi, C. Yonkofski, S.H. Kim, P. Kyle. 2019. Influence of groundwater extraction costs and resource depletion limits on simulated global nonrenewable water withdrawals over the twenty-first century. Earths Future 7, 123-135.

doi:10.1029/2018EF001105. https://agupubs.onlinelibrary.wiley.com/doi/pdfdirect/10.1029/ 2018EF001105 



\section{Chapitre 11}

\section{Agir sur les causes des changements globaux}

Agir contre le réchauffement climatique impose des mesures fortes dans tous les secteurs pour atteindre des émissions nettes de $\mathrm{CO}_{2}$ nulles d'ici à 2060, avec l'objectif de limiter les hausses des températures à $1,5^{\circ} \mathrm{C}$, voire plus certainement $2^{\circ} \mathrm{C}$, pour la fin du XXIe siècle. L'obtention de la neutralité carbone, si elle s'accompagne d'une forte réduction des autres gaz à effet de serre, permettra de stabiliser la température, mais n'arrêtera pas les effets du réchauffement sur les composantes lentes du système climatiques (élévation des mers, réchauffement océanique, fonte des calottes polaires) dont elle ne pourra que limiter l'ampleur. Autrement dit, comme nous l'avons indiqué en introduction du chapitre 10, les actions d'atténuation du changement climatique ne peuvent être totalement dissociées des actions d'adaptation à ce changement. La situation est différente pour ce qui concerne l'érosion de la biodiversité, car il n'y a pas vraiment de sens à nous adapter à la dégradation continue de notre environnement naturel. La lutte contre l'érosion de la biodiversité impose de revoir drastiquement notre rapport au vivant (voir section 4.4) et nos modes d'appropriation des terres et des mers (voir section 5.1). Si on assiste déjà à des pertes d'espèces conséquentes, le grand péril déjà bien présent réside dans la perte des effectifs de populations entières, qui augmente leurs risques d'extinction (voir section 5.6). Tout au long de ce chapitre, nous présentons des actions possibles pour préserver la biodiversité et freiner le réchauffement climatique: comment réduire la pression anthropique sur les écosystèmes, comment agir sur l'agriculture et l'alimentation, comment agir sur l'habitat, les transports, les modes de consommation et la production d'énergie, comment agir directement sur les émissions de $\mathrm{CO}_{2}$. Loin d'être des prescriptions, ces actions sont, en fait, celles que l'on retrouve dans la littérature, ouvrages et médias, traitant des mêmes sujets. Nous souhaitons juste que leur présentation dans ce chapitre invite à des réflexions en réalisant que des compromis seront toujours à faire dans nos choix sociétaux, économiques et technologiques. 


\subsection{Réduire la pression anthropique sur les écosystèmes: limiter la dégradation et la fragmentation des habitats - J. Lecomte et N. Frascaria}

\subsubsection{Permettre la libre évolution}

La crise de la Covid-19 nous a magistralement montré que les êtres vivants non-humains ont de grandes capacités à se réapproprier quasi instantanément les espaces libérés par les humains. En effet, durant la période de confinement du printemps 2020 de nombreux citadins ont été surpris de réentendre les oiseaux dont le chant est la plupart du temps masqué par les bruits de la ville, mais aussi de voir nombre d'animaux, plus habitués à se cacher et vivre en dehors des villes, déambuler au sein même de zones largement anthropisées. Ainsi des renards, des cerfs et des sangliers ont été observés en train d'arpenter les rues de grandes villes occidentales. Des groupes de singes ont envahi des quartiers de la ville de Medellin en Colombie, des otaries ont déambulé dans le port de Mar el Plata en Argentine et des kangourous ont cheminé dans la ville d'Adélaïde en Australie. La partie n'est donc pas perdue pour la biodiversité si tant est qu'on lui «lâche la bride». Permettre la libre évolution des non-humains au sein d'espaces protégés suffisamment grands et nombreux et les admettre au sein même d'espaces anthropisés en ville et en campagne, c'est en effet leur permettre rapidement de développer différentes formes d'adaptation face aux perturbations (Fig. 11.1). En retour, il nous faut accepter différentes formes de naturalité [1] et de diplomatie envers les non-humains [2]. Edward Wilson dans un de ses derniers ouvrages formule qu'il faudrait garantir $50 \%$ d'espaces en protection sur notre planète pour stopper l'érosion de la biodiversité [3].

Mais pour illustrer les réponses offertes par la libre évolution face à l'érosion de la biodiversité et les modalités de sa mise en œuvre, nous vous invitons ci-après à la lecture des paragraphes de conclusion d'un ouvrage intitulé 20000 ans: ou la grande histoire de la nature [4].

"Dans la cacophonie des nouvelles apocalyptiques [concernant la biodiversité] qui nous assourdissent quotidiennement, il y a quelques bonnes nouvelles. La forêt revient et le mieux à faire est de ne surtout rien faire. II faut lui laisser le temps et l'opportunité de vieillir et d'évoluer à sa guise et sans contraintes. Dans son sillage, elle a entraîné la réapparition de nombreuses espèces qui avaient disparu [en France] depuis longtemps. Voilà une liste réjouissante: pic noir, cigogne noire, chouette de Tengmalm, hibou grand-duc, phoque gris, loutre, castor, cerf, bouquetin, chamois, marmotte, quatre espèces de vautours, aigle royal, balbuzard pêcheur, faucon pèlerin, saumon atlantique, alose, esturgeon, mérou brun, cachalot, rorqual, fou de Bassan, lynx boréal, loup gris, ours brun, et tant d'autres encore... La loi de protection de la nature votée en 1976 (voir section 1.5 de cet ouvrage) fut le 
point de basculement de notre histoire. Les magnifiques paysages dont nous nous enorgueillissons à juste titre n'étaient plus de tristes décors d'opéra complètement vides. Mais voilà que les acteurs reviennent peu à peu. Les solutions pour accélérer le processus de ré-ensauvagement de notre pays sont testées et approuvées. Dans le même temps, ce sont nos campagnes qui se vident à grande vitesse de toute la faune et la flore sauvages qui s'y étaient adaptées au fil des siècles et qui faisaient tout le charme de nos terroirs. La rationalisation et la simplification sont encore les maitres mots de l'agriculture intensive et industrielle. C'est le prochain défi majeur à relever. Parviendrons-nous à enrayer la chute précipitée avant qu'il ne soit trop tard? Avant que nos campagnes deviennent des déserts stériles. Là aussi on connaît les solutions [ ]. La France sauvage est en perpétuel devenir. La nature évolue sans cesse et s'adapte en permanence à toutes les situations, avec ou sans l'homme. II ne s'agit pas, dès lors, de revenir à un hypothétique état passé (et lequel?) mais de décider quel visage nous voulons pour la France d'aujourd'hui et de demain. Quels espaces de liberté sommes-nous prêts à laisser à la nature pour qu'elle puisse s'adapter, réagir, proposer des combinaisons nouvelles et surtout garder tout son potentiel évolutif et créatif? Il faut préserver la part d'imprévu de la nature,
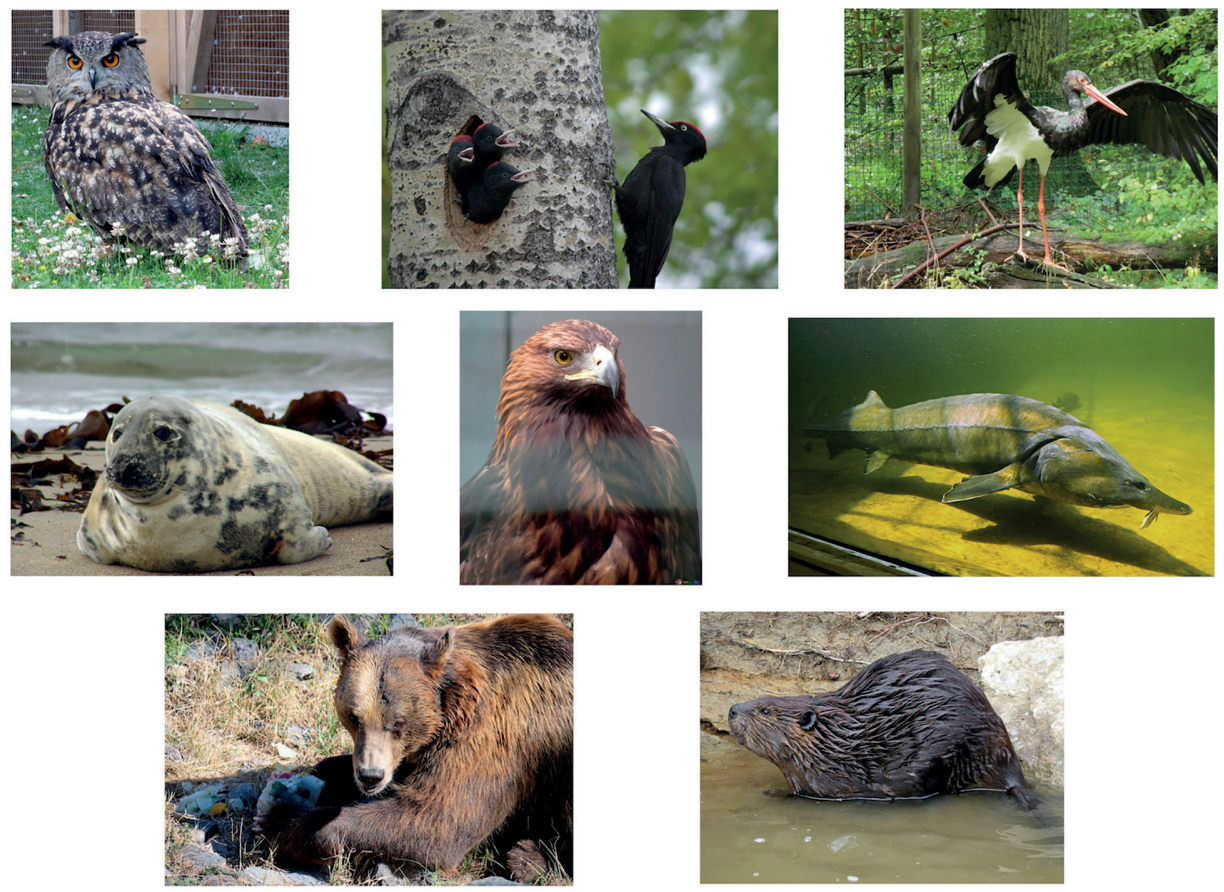

Figure 11.1: Quelques espèces qui ont pu bénéficier de politiques de protection de la nature. De haut en bas et de gauche à droite: le hibou grand-duc, le pic noir, la cigogne noire, le phoque gris, l'aigle royal, l'esturgeon, l'ours brun, le castor. Images sous licence Creative Commons ou libérée de droits d'auteur - Source: Stefdn, Alastar Rae, Gzen92, Andreas Trepte, torange.biz, Musicaline, Flickr. 
sa capacité à rebondir. Déjà le chevreuil forme des troupeaux dans les grandes plaines cultivées: le faucon crécerelle, le faucon pèlerin et le hibou grand-duc nichent et chassent dans nos plus grandes villes: les chamois s'ébattent au bord de la Méditerranée, dans le maquis de l'Estérel: c'est du jamais vu depuis 20000 ans! Ils ne nous attendent pas: ils proposent d'eux-mêmes de nouvelles façon d'habiter le monde et le pratiquer. Ils bousculent nos habitudes. Accueillir leurs propositions et tenter, à notre tour, de nous y adapter, quitte à faire des contre-propositions si certaines d'entre elles nous gênent, de manière à faire monde commun. Il nous faut également accepter l'idée de refuges et de corridors qui permettent aux espèces d'aller et venir, en fonction des fluctuations environnementales. Il y a tant de choses à expérimenter pour parvenir à trouver une nouvelle alliance avec le monde sauvage. Il ne tient qu'à nous de permettre à l'aventure de se poursuivre...".

\subsubsection{Accompagner la libre évolution}

Cette section s'appuie sur la vidéo produite par l'Agence régionale de la biodiversité (ARB) en Île-de-France et intitulée: "Les Trames écologiques: des bénéfices multiples pour les humains» - https://dai.ly/x7/crry

Les activités humaines impactent les milieux naturels, notamment en les fragmentant (voir section 5.1). Les espèces ne peuvent plus faire leur cycle de vie, se reproduire ou se déplacer. Si cette dégradation est trop importante et que l'on souhaite aboutir rapidement à un nouveau fonctionnement pour un écosystème dégradé sans attendre sa restauration naturelle, il est possible d'intervenir en le restaurant ou le réhabilitant. Les humains deviennent alors les pilotes de ces actions de restauration qui doivent s'adapter à chaque type d'écosystème. Les corridors écologiques ou «trames» sont un exemple de restauration volontaire. La notion de «trame», issue du Grenelle de l'environnement en 2007, recouvre l'ensemble des solutions pour empêcher cette fragmentation en reconnectant les espaces qui ont été séparés du fait des activités humaines. II s'agit ainsi de mettre en place des corridors écologiques qui permettent cette reconnexion. Les trames sont dites verte, bleue, brune ou noire en référence aux milieux terrestres, milieux aquatiques, au sol et aux milieux obscurs, respectivement.

L'urbanisation et les pratiques intensives de l'agriculture consomment et fragmentent les milieux naturels. Les espèces se retrouvent ainsi prisonnières et fragilisées. Leur survie dépend alors d'un réseau continu de corridors et de réservoirs de la biodiversité. Ces trames apportent aussi des bénéfices importants aussi aux humains (Fig. 11.2). 


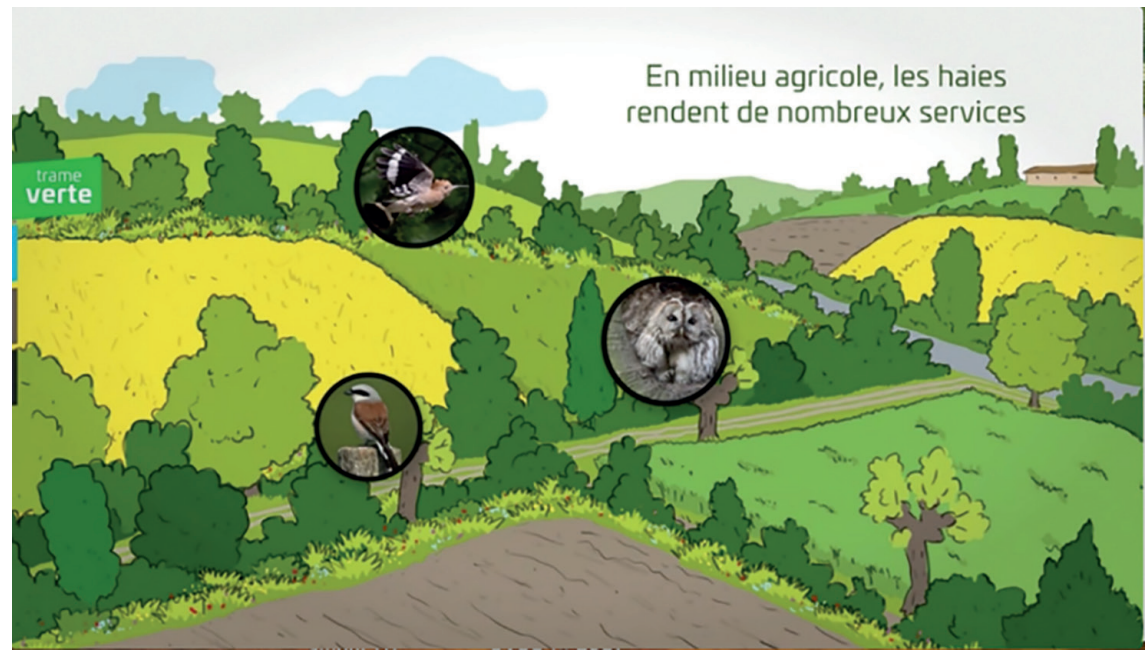

Figure 11.2: En milieu agricole, les haies limitent l'érosion des sols, protègent les cultures du vent, accueillent une faune utile aux agriculteurs, offrent du bois de chauffe local et enfin limitent la dissémination des pesticides. Illustration extraite de la vidéo produite par l'Agence régionale de la biodiversité (ARB) en Ille-de-France.

La présence d'un cours d'eau, connecté à des zones d'expansion de crues, limite, par ailleurs, les risques d'inondation. Les méandres du cours d'eau et le boisement de ses berges améliorent la qualité de l'eau. II offre un milieu naturel propice aux loisirs, à l'éducation, l'observation, la détente. Les zones humides régulièrement alimentées par les cours d'eau aussi sont très utiles aux humains (Fig. 11.3).

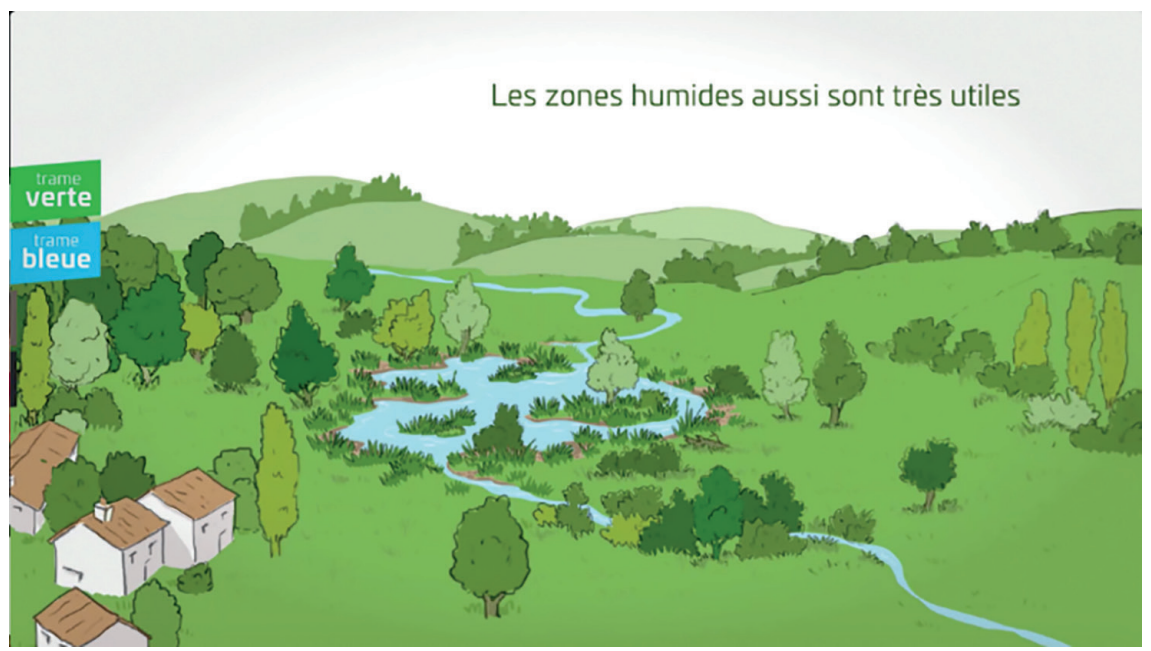

Figure 11.3: Les zones humides absorbent l'excès d'eau en période de crue, constituent des réserves d'eau lors des sécheresses, épurent l'eau grâce aux végétaux, réduisent l'effet de serre en piégeant du carbone dans les sols. Illustration extraite de la vidéo produite par l'Agence régionale de la biodiversité (ARB) en île-de-France. 
Dans les zones urbanisées, la nature des trames vertes joue aussi un rôle essentiel pour les humains (Fig. 11.4). En ville, la végétation améliore le cadre de vie des habitants, lutte contre l'effet d'îlot de chaleur urbain, rafraîchit la ville, réduit les pollutions et améliore notre santé.

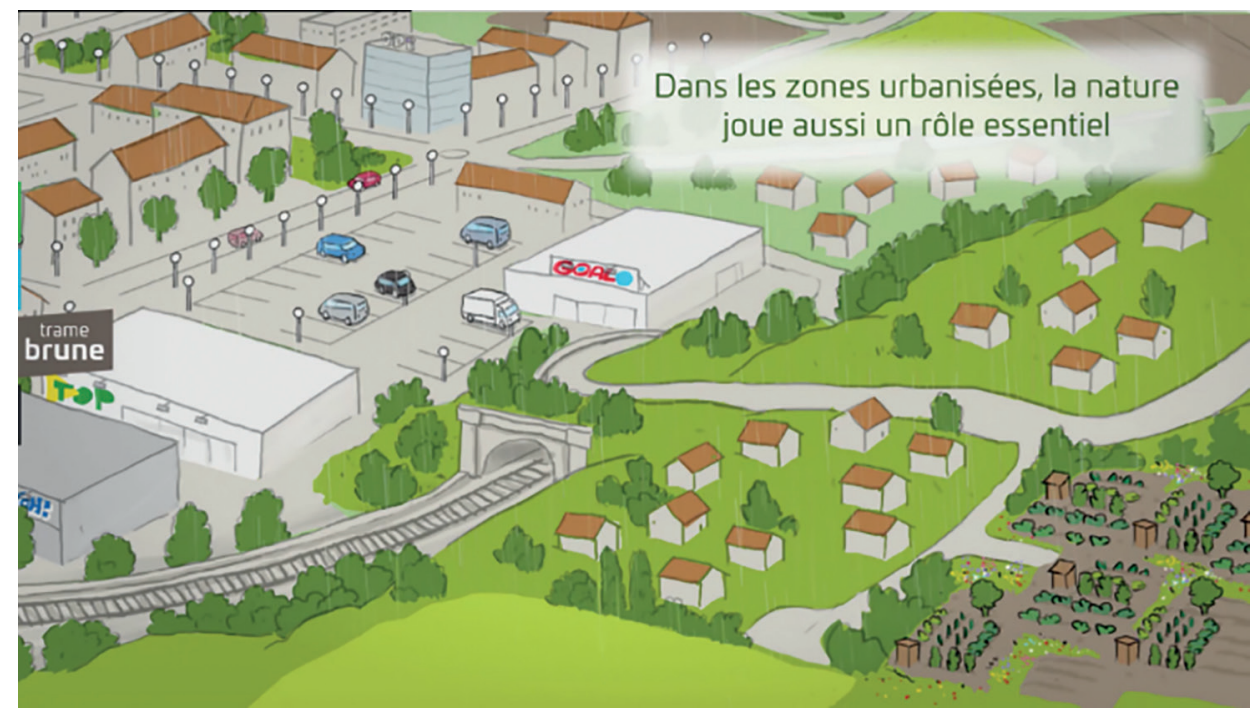

Figure 11.4: La pleine terre absorbe et filtre les eaux pluviales. Elle permet aussi la production de fruits et légumes. Illustration extraite de la vidéo produite par l'Agence régionale de la biodiversité (ARB) en ile-de-France.

La pollution lumineuse y perturbe les populations animales autant qu'elle nous affecte, alors que l'alternance jour/nuit est essentielle au sommeil et à la santé de même qu'elle permet d'observer le ciel étoilé.

En conclusion et pour reprendre les propos de Cristiana Pasça Palmer, secrétaire exécutive de la Convention sur la diversité biologique, "Une biodiversité saine est l'infrastructure essentielle qui soutient toutes les formes de vie sur terre, y compris la vie humaine». Pour mémoire, $23 \%$ des terres ont connu une réduction de leur productivité en raison de la dégradation des sols, - plus de $75 \%$ des cultures vivrières mondiales dépendent de la pollinisation et la disparition des pollinisateurs représente une perte potentielle de 235 à 577 milliards de dollars US -, et $70 \%$ de médicaments anticancéreux ou synthétiques sont inspirés par la nature. Ces quelques exemples, qui rappellent les conséquences de l'érosion de la biodiversité et dont certains ont été présentés au cours de l'ouvrage, nous invitent tous à être les acteurs pour restaurer et préserver ce tissu vivant essentiel et interconnecté! 


\subsection{Agir sur le levier agricole : agroécologie, système alimentaire et réduction du gaspillage - J.-C. Bureau et T. Brunelle}

L'agriculture est aujourd'hui un des secteurs de l'activité humaine pour lequel plusieurs limites planétaires de développement durable ont été dépassées (voir Fig. 10.12). Des solutions sont néanmoins possibles pour tenter de revenir en deçà de ces limites, réduire les pollutions et atténuer le réchauffement climatique. Dans ce qui suit, nous considèrerons en particulier la réduction des gaz à effets de serre spécifiques à l'agriculture et le changement des modes de culture pour favoriser une meilleure qualité des sols. Ces évolutions ne peuvent être engagées efficacement que si nous changeons en même temps nos modes de consommation alimentaire tout en réduisant le gaspillage de nourriture. Ces aspects seront également discutés.

\subsubsection{Réduction des gaz à effet de serre dans l'agriculture}

L'agriculture n'est certes pas la source essentielle des émissions de gaz à effet de serre (voir section 7.2), mais elle est néanmoins un contributeur important par ses émissions de gaz spécifiques comme le méthane $\left(\mathrm{CH}_{4}\right)$ et le protoxyde d'azote $\left(\mathrm{N}_{2} \mathrm{O}\right)$.

Le méthane est essentiellement émis par les ruminants, les ovins et les bovins, que ce soit pour la production de viande ou pour l'élevage laitier. L'émission de méthane est, en fait, liée au processus de rumination des herbes, qui permet aux animaux de digérer la cellulose et s'accompagne d'un dégagement de méthane. L'ampleur du phénomène devient très importante dès lors que l'on a affaire à de grands troupeaux d'élevage comme en Australie, aux États-Unis, en Inde et, toute proportion gardée, comme en Irlande et dans certaines parties de la France. L'ampleur du phénomène est aussi renforcée par le fait que le méthane a un pouvoir réchauffant qui est environ 28 fois supérieur à celui du gaz carbonique $\left(\mathrm{CO}_{2}\right)$. Réduire les émissions de méthane dans l'agriculture ne peut se faire qu'en réduisant significativement le nombre des animaux d'élevage, ce qui suppose en retour que nous adoptions une alimentation plus végétale. Nous reviendrons sur ce point ultérieurement (voir section 11.2.3).

Le protoxyde d'azote est le second gaz à effet de serre par ordre d'importance qu'émet l'agriculture. Bien que ses émissions soient moins importantes que celles du méthane, son pouvoir réchauffant dans l'atmosphère est encore plus élevé puisqu'il est environ 260 fois supérieur à celui du gaz carbonique (voir section 7.3). Les émissions de protoxyde d'azote ont deux origines. II provient, d'une part, de déjections animales et d'autre part, des engrais azotés qui sont épandus dans les zones de culture intensive, que ce soit des engrais issus des déjections animales (les lisiers) ou des engrais industriels. Réduire les émissions de $\mathrm{N}_{2} \mathrm{O}$, c'est donc en premier lieu réduire les déjections animales, ce qui suppose, comme pour le méthane, de réduire 
le nombre des animaux d'élevage, non seulement celui des ruminants, mais aussi celui des porcs et des volailles. Cependant, réduire les émissions de $\mathrm{N}_{2} \mathrm{O}$, c'est aussi réduire la fertilisation des sols par des engrais azotés.

La réduction des engrais azotés pose les problèmes plus généraux des modes de culture agricole et de ce qu'on appelle la cascade de l'azote (Fig. 11.5). II est bon de se rappeler qu'historiquement, à la fin du XIXe siècle, on pensait que la population humaine serait à tout jamais limitée à quelques centaines de millions d'individus, car les quantités d'engrais azotés disponibles pour les cultures et la production agricole étaient elles-mêmes limitées au guano et aux déjections animales. Or, c'est grâce à la synthèse de l'ammoniac que des chimistes ont réussi à capturer l'azote de l'air de façon stable, à l'utiliser pour fabriquer des amino-nitrates et à nourrir une population mondiale qui est passée progressivement du milliard d'êtres humains à plus de sept milliards aujourd'hui. Si ce procédé a donc eu un impact très positif sur l'alimentation humaine, on s'aperçoit néanmoins, un siècle plus tard, que plus de la moitié de cet azote, qu'on utilise sous forme d'engrais azotés, ne profite pas véritablement aux plantes. L'utilisation d'engrais se traduit, en revanche, par des pollutions de l'air, de l'eau et des sols (voir sections $2.3,2.4$ et 2.5 ) et d'autres effets complexes qui participent à la cascade de l'azote; tenter de régler une des pollutions amène souvent à en recréer d'autres par des processus qui sont des cascades chimiques de transformation. Ce problème de flux biogéochimiques, celui de l'azote en particulier, est l'une des principales limites planétaires au développement durable (voir Fig. 10.12). Le traiter nécessite de revenir à des méthodes culturales qui reposent beaucoup plus sur les cycles biologiques «naturels», comme nous allons l'évoquer dans ce qui suit.

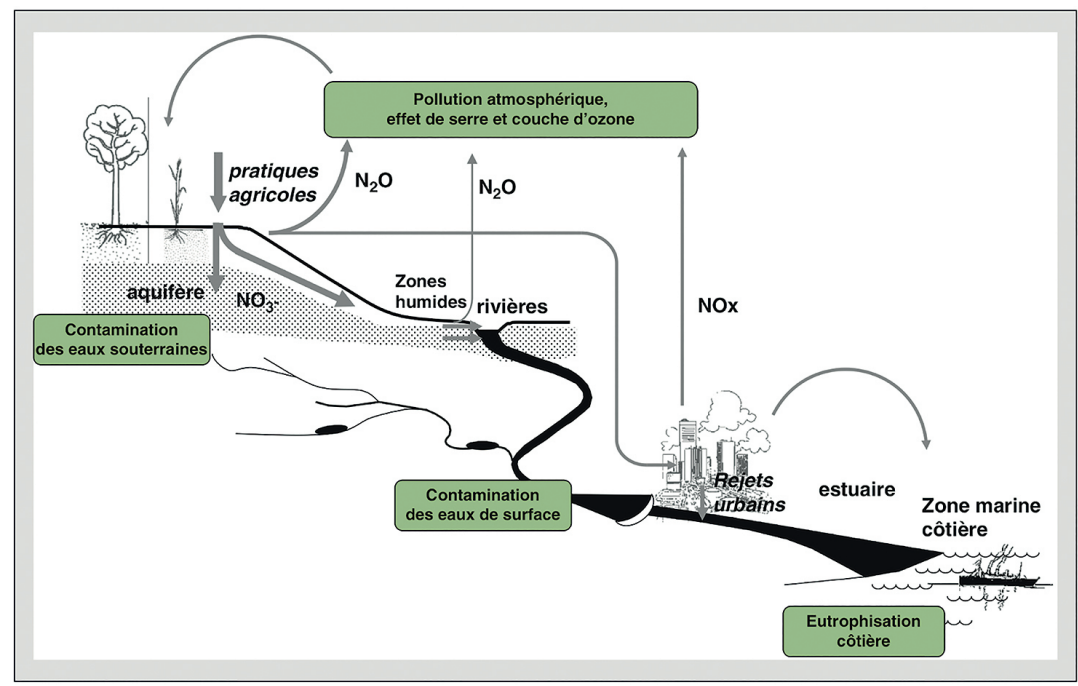

Figure 11.5: Schéma de principe montrant le cheminement de l'azote depuis les sols agricoles jusqu'aux zones marines côtières à travers le continuum aquatique, les espaces urbains et le réservoir atmosphérique, et les problèmes environnementaux occasionnés par les pertes d'azote réactif à toutes les étapes de ce cheminement. Source: G. Billen et al. [5]. 


\subsubsection{L'agroécologie et l'agriculture bio}

Comme nous venons de l'évoquer, l'utilisation massive d'engrais et la cascade de l'azote sont des menaces sérieuses pour l'humanité en termes de pollutions. Des exemples emblématiques de pollutions sont ceux de zones maritimes du golfe du Mexique ou de la mer Baltique où l'on assiste à une eutrophisation complète des eaux. Comme l'a par ailleurs évoqué Christine Hatté dans un précédent chapitre (voir section 2.6), l'appauvrissement en carbone des sols dues aux pratiques d'agriculture intensive se traduit par une érosion des sols, qui risque de conduire à terme à des pertes importantes de rendement agricole sur une grande partie de la planète (voir Fig. 2.22). Dans ce contexte, l'agroécologie est une façon de concevoir des systèmes de production qui s'appuient sur les fonctionnalités offertes par les écosystèmes eux-mêmes en visant à la fois à diminuer les pressions sur l'environnement et à préserver les ressources naturelles. Autrement dit, il s'agit d'utiliser au maximum la nature comme facteur de production en maintenant ses capacités de renouvellement.

Limiter les engrais azotés peut ainsi consister à associer à des cultures gourmandes en azote des cultures de légumineuses qui sont capables de capter l'azote de l'air à travers les nodules de leurs racines et de le restituer au sol pour en faire profiter les autres cultures. Augmenter les stocks de carbone dans les sols, c'est d'abord arrêter d'en perdre en cessant progressivement les pratiques où les parties aériennes des plantes ne sont pas rendues au sol et où le labour jusqu'à $30 \mathrm{~cm}$ de profondeur contribue à une respiration massive des sols (par oxydation) ainsi qu'à une perte de leur biodiversité. II est, à l'inverse, souhaitable de favoriser le couvert permanent en limitant le labour et en alternant les cultures, ce qui favorisera en retour la biodiversité et les apports d'autres nutriments au sol. Limiter le labour ne signifie cependant pas de s'en passer totalement, car le non-labour s'accompagne fréquemment d'emplois d'herbicides pour venir à bout d'adventices ( «les mauvaises herbes») envahissantes, notamment dans les grandes régions céréalières françaises.

Par ailleurs, au lieu de sélectionner systématiquement des plantes et des céréales où le grain est privilégié aux dépens des racines et des parties foliaires qui constituent des puits de carbone, un retour à un équilibre plus naturel entre les différentes composantes, grain, feuilles et racines, pourrait enrichir les sols de culture en carbone, sans pour autant diminuer les rendements au point que les cultures ne soient plus rentables.

De manière générale, l'agroécologie réintroduit ainsi de la diversité dans les systèmes de production agricole, et le rôle de la biodiversité comme facteur de production est renforcé, voire restauré. Parmi ces changements de méthode culturale, on assiste au développement de l'agriculture «bio» qui s'impose peu à peu auprès d'un certain nombre de consommateurs. L'agriculture bio présente, en effet, 
l'intérêt de ne plus utiliser de produits chimiques ou d'en utiliser peu, de réduire l'utilisation des engrais, d'associer des cultures complémentaires sur le même sol, de procéder à une rotation des cultures suivant les saisons et/ou de favoriser les cultures qui s'étalent sur un grand nombre d'années.

Cela étant, il faut rester prudent quant à un passage généralisé et rapide de l'agriculture conventionnelle à l'agriculture bio, en Europe par exemple, car sans pesticides ni engrais, les rendements à court/moyen terme de l'agriculture bio sont un peu plus faibles que ceux de l'agriculture conventionnelle, même si, dans des conditions expérimentales, on arrive à avoir des rendements similaires. II serait regrettable que pour compenser cette baisse éventuelle de rendement, on accroisse les exportations, ce qui reviendrait à délocaliser les cultures, ou que l'on abatte les arbres pour disposer de plus de surface à cultiver. Un passage ambitieux à l'agriculture bio doit nécessairement se traduire par un changement de la demande et par des changements de comportements alimentaires, notamment par un meilleur stockage des produits alimentaires, par une diminution du gaspillage et par l'adoption d'une alimentation moins riche en viande et qui tire plus parti du végétal.

\subsubsection{Modes de consommation alimentaire et réduction de gaz à effet de serre}

Nous présentons ici les modes de consommation alimentaire et leurs effets sur les émissions de gaz à effet de serre. Nous allons voir comment manger différemment pour lutter contre le réchauffement climatique.

On sait qu'il y a une grande variabilité de la consommation alimentaire selon les différentes régions du monde et que cette variabilité concerne surtout la consommation de viande. Pour illustrer ce point, le graphique de la figure 11.6 représente les niveaux de consommation de viande de ruminant (de viande rouge) exprimés en calories par habitant et par jour dans les différentes régions du monde avec en rouge, les niveaux actuels, et en jaune, les niveaux projetés à 2050. Nous constatons, par exemple, que le Brésil est la région du monde où les niveaux de consommation de viande de ruminant sont les plus élevés en atteignant 140 calories par habitant et par jour, alors que le niveau moyen est moins de 10 calories par habitant et par jour en Inde. Certes, I'Inde est un cas particulier en raison de restrictions religieuses sur la consommation de viande dans ce pays. Cela étant, si nous considérons le niveau de consommation par habitant de l'Afrique sub-saharienne, nous voyons qu'elle est deux à trois fois inférieure à celles des régions les plus consommatrices que sont, par exemple, l'Amérique latine, les régions de l'ex-URSS et les pays occidentaux. 


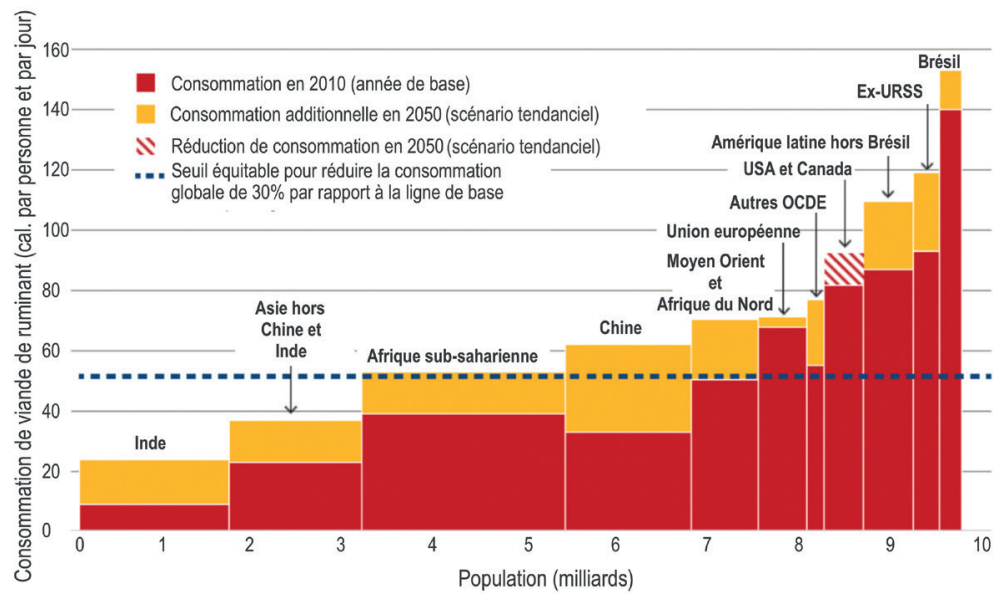

Figure 11.6: Consommation de viande en calories par personne et par jour suivant les différentes régions du monde. En rouge, les données actuelles. En jaune, les consommations estimées en 2050. Source: GlobAgri-WRR model with source data from FAO (2017a); UNDESA (2017); FAO (2011b); and Alexandratos and Bruinsma (2012) [6].

Alors faut-il réduire notre consommation de viande? Autrement dit, doit-on choisir entre un régime végétarien, c'est-à-dire sans viande et avec des légumes, ou un régime végan où là on n'a plus du tout de produits animaux, y compris œufs et lait? Pour répondre à cette question, considérons les coefficients d'émission, exprimés en kilogrammes d'équivalent $\mathrm{CO}_{2}$, pour un kilogramme de denrée alimentaire produite (Fig. 11.7).

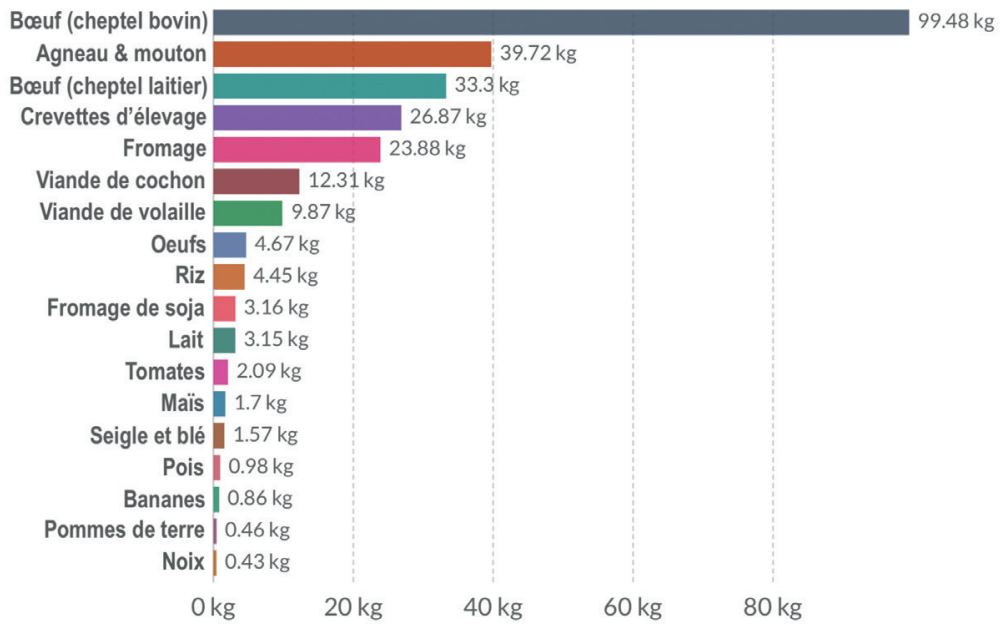

Figure 11.7: Émissions de gaz à effet de serre pour 100 grammes de protéine. Les gaz à effet de serre sont mesurés en kilogramme équivalent dioxyde de carbone ( $\mathrm{kg}$ eq. $\mathrm{CO}_{2}$ ). Les gaz à effet de serre non $\mathrm{CO}_{2}$ sont donc inclus en étant pondérés par leur impact de réchauffement relatif. Source: Poore J. \& Nemecek T. Additional calculations by Our World in Data. OurWorldlnData.org /Environmental-impactsof-food. CC BY [7]. 
Nous constatons que la viande de bœuf a un coefficient d'émission très important avec environ cent kilogrammes d'équivalent $\mathrm{CO}_{2}$ pour un kilogramme de nourriture produite. De même, des coefficients élevés sont estimés pour la viande de mouton et la viande d'agneau ainsi que pour un produit laitier tel que le fromage avec plus de vingt kilogrammes d'équivalent $\mathrm{CO}_{2}$ pour un kilogramme de nourriture produite. À l'inverse, les coefficients sont bien plus faibles pour les produits végétaux avec des valeurs inférieures à cinq kilogrammes d'équivalent $\mathrm{CO}_{2}$ pour un kilogramme de nourriture produite. Un point particulièrement intéressant à noter est qu'il y a des différences substantielles de coefficients d'émission pour les différents types de viandes et notamment, entre viandes rouges et viandes blanches. Les viandes blanches, porcs, poulets, ont des coefficients d'émission qui sont relativement plus élevés que les produits végétaux, mais bien moins élevés que la viande rouge ou que certains produits laitiers. Plusieurs études montrent qu'il suffirait de substituer une fois par semaine la viande blanche à la viande rouge pour réduire déjà substantiellement nos émissions liées à notre consommation alimentaire [8] .

Pour mieux comprendre les coefficients d'émission qui sont présentés sur la figure 11.7, il est nécessaire d'identifier les principales sources d'émission le long de la chaîne d'approvisionnement. Les études réalisées dans ce but indiquent que pour la viande de bœuf, dont le coefficient est le plus élevé, la principale source est le méthane produit par les ruminants lors de leur processus de digestion. C'est ce qu'on appelle la fermentation entérique. Ce type d'émission est, en fait, important pour tous les ruminants. Ceci explique les coefficients élevés rapportés sur la figure 11.7 pour tous les produits issus de ces mêmes ruminants, que ce soit la viande de bœuf, le lait, les fromages et la viande de mouton ou d'agneau. Une autre source d'émissions pour les bovins est associée à l'usage des sols. On sait, en effet, que l'élevage de bovins et, de façon générale, l'élevage de ruminants, est très gourmand en terres, que ce soit des terres de pâturage ou des terres de cultures pour l'alimentation des animaux. La création de terres dédiées au pâturage peut, par exemple, conduire à une déforestation locale comme on le rencontre dans le cas de grands troupeaux d'élevage.

Les études indiquent, par ailleurs, que les émissions de gaz à effet de serre dues aux transports représentent une part très faible pour la plupart des produits qui sont considérés sur la figure 11.7. Cela va à l'encontre de ce que l'on entend souvent, par exemple, à propos des circuits courts. II y a un véritable consensus scientifique sur le fait que les circuits courts ou l'alimentation locale ne sont effectivement pas des leviers importants de réduction d'émission de gaz à effet de serre [9]. À l'inverse, les circuits courts et l'alimentation locale sont à encourager, car ils incitent à maintenir la production agricole sur notre territoire.

Considérons maintenant les régimes alimentaires dans leur ensemble au-delà des produits agricoles, comme il a été présenté. La figure 11.8 représente le potentiel d'atténuation des émissions de gaz à effet de serre pour différents régimes alimentaires types, en supposant que l'ensemble de la population les adopte. 


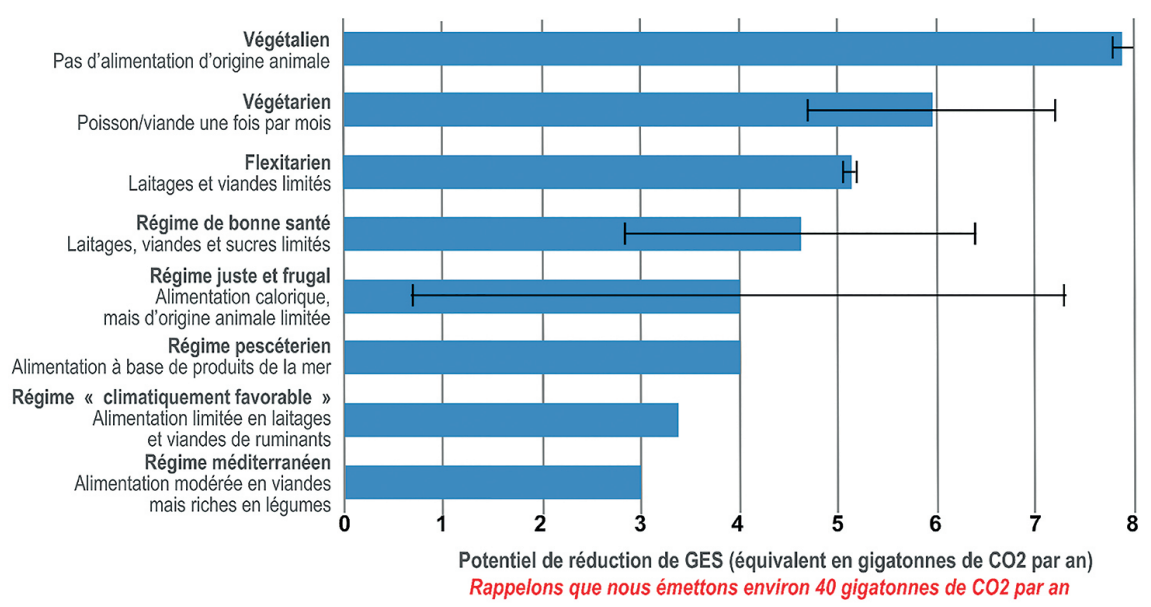

Figure 11.8: Réduction des gaz à effet de serre envisageable en 2050 par le changement de régime alimentaire selon différents scénarios examinés dans la littérature. Les barres d'erreur correspondent à des effets additionnels, possibles ou non, de stockage de carbone selon l'occupation des sols. Les données sans barre d'erreur sont issues d'une étude unique. Source: IPCC report 2019, p. 488 [10].

Le régime alimentaire qui permet les plus fortes réductions d'émission est le régime végétalien, qui est le régime alimentaire où l'on ne mange aucun produit animal, y compris le lait et les œufs. Ensuite, viennent les régimes végétariens et flexitariens. La consommation de viande est exclue du régime végétarien alors que le régime flexitarien ne l'exclut pas mais consiste néanmoins en une réduction importante de la viande. Probablement le résultat le plus important rapporté sur la figure 11.8, est le fait que les régimes de type méditerranéen ou ce qu'on appelle encore les régimes climatiquement favorables, c'est-à-dire des régimes où l'on consomme moins de viande, mais où on continue tout de même à en consommer, permettent des réductions significatives d'émission. Le niveau de réduction pour ces régimes atteint près de $10 \%$ par rapport au régime alimentaire standard, soit environ 4 gigatonnes de $\mathrm{CO}_{2}$ en moins par an par rapport aux 40 gigatonnes d'émission totale de gaz à effet de serre chaque année.

Pour réduire nos émissions de gaz à effet de serre, il n'est donc pas nécessaire de devenir tous végans! II est préférable de privilégier une réduction raisonnable de notre apport en viandes et en particulier en viandes rouges. Cette réduction se traduira d'une manière ou d'une autre par une diminution du nombre d'animaux élevés pour la viande, en particulier des troupeaux de ruminants.

\subsubsection{Gaspillage alimentaire et émissions de gaz à effet de serre}

Une bonne partie de notre consommation alimentaire est perdue ou gâchée. En réduisant la perte de produits alimentaires, on peut aboutir aussi à d'importantes réductions d'émission de gaz à effet de serre. C'est ce qu'illustre le graphique sur la 
figure 11.9 qui représente les différentes pertes et gaspillages alimentaires dans les grandes régions du monde.

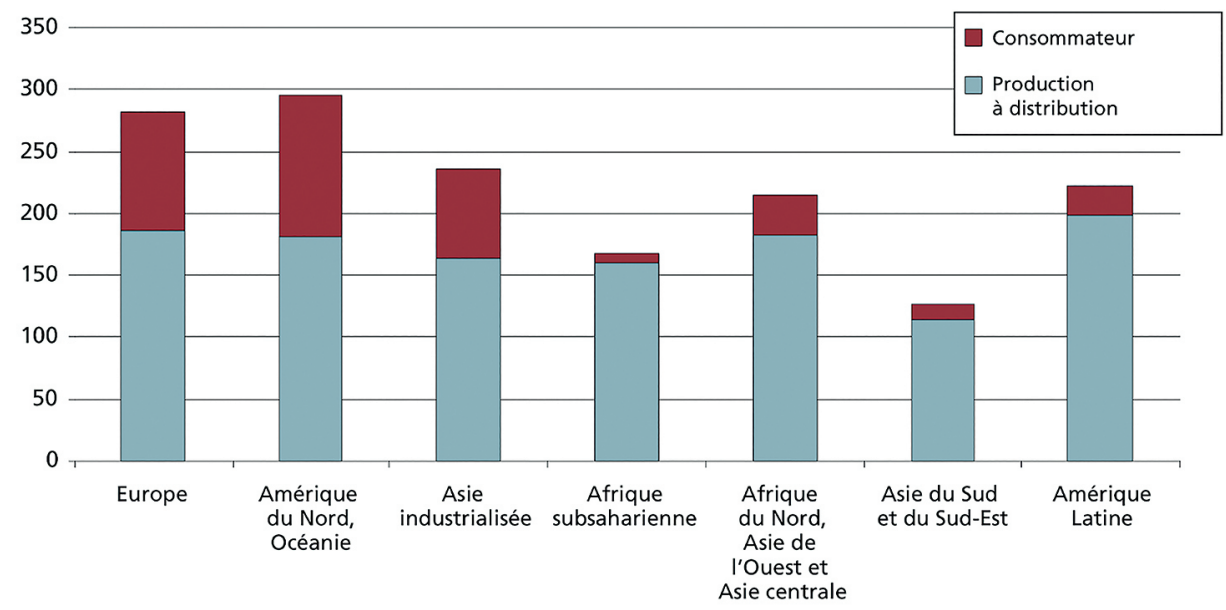

Figure 11.9: Pertes et gaspillages alimentaires en kilogrammes par habitant, par an et par région, aux stades de la consommation et de l'avant consommation. Source: FAO, Organisation des Nations Unies pour l'Alimentation et l'Agriculture ${ }^{93}$.

En bleu sont rapportées les pertes de production jusqu'à la vente au détail et en rouge, les pertes au niveau du consommateur. Ces pertes sont exprimées en kilogrammes par personne et par an. On constate qu'en Europe, il y a presque deux cent soixante-quinze kilos de produits perdus ou gaspillés en moyenne par personne. Les niveaux sont bien inférieurs en Afrique sub-saharienne, par exemple, où les pertes sont d'environ cent soixante kilogrammes en moyenne par personne. L'écart se situe essentiellement dans les pertes au niveau de la consommation. Certes, ces chiffres sont entachés d'une grande incertitude, car ils ont été estimés à partir d'un certain nombre d'hypothèses relativement simplistes. Cependant, ils fournissent une indication réaliste des écarts entre les différentes régions du monde. D'un point de vue global, on estime à $30 \%$ le pourcentage total de pertes et de gaspillages de produits alimentaires dans le monde.

Ce sont, en fait, les fruits et les légumes qui les produits alimentaires que l'on gaspille le plus, car ce sont aussi les produits les plus périssables. Le graphique de la figure 11.10 représente le pourcentage des pertes de production initiale suivant les différentes régions du monde. Contrairement au graphique précédent, on constate globalement peu d'écarts entre les différentes régions. Dans les régions industrialisées, les fruits et légumes sont essentiellement perdus au stade de la production. On constate aussi 15 à 30\% de gaspillages sur l'ensemble des achats par les 
consommateurs. Dans les régions en voie de développement, les pertes principales se situent au niveau de la transformation des produits et de leur distribution.

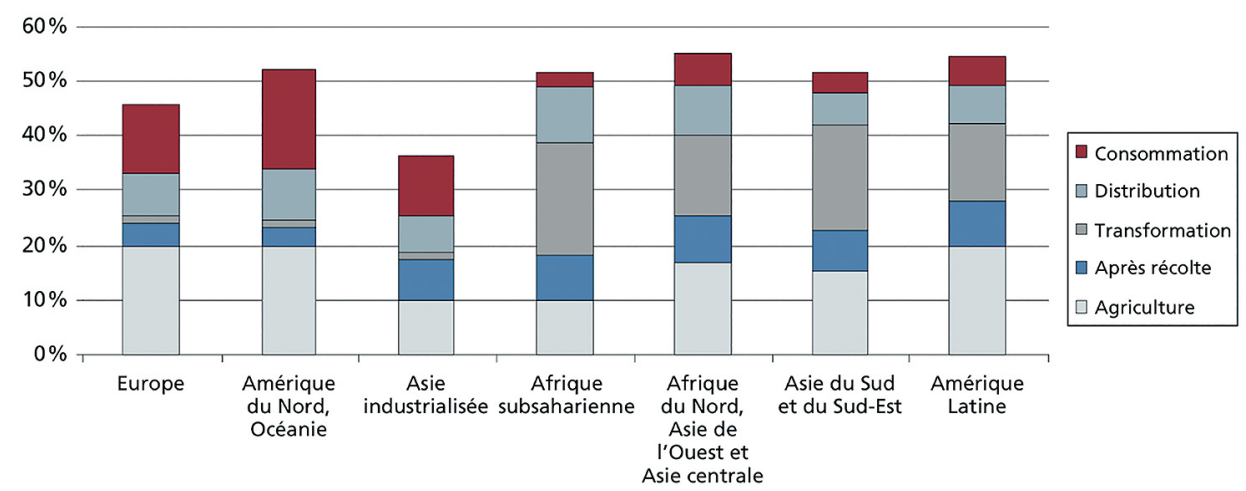

Figure 11.10: Production initiale perdue ou gaspillée aux différents stades de la chaîne alimentaire pour les fruits et légumes dans le monde. Source: FAO, Organisation des Nations Unies pour l'Alimentation et l'Agriculture ${ }^{95}$.

À l'opposé des fruits et légumes, ce sont les produits laitiers qui sont les moins perdus et les moins gaspillés. La raison principale en est que ce sont des produits moins périssables et surtout plus faciles à conserver. Le pourcentage de production initiale, perdue ou gaspillée, est typiquement de 10 à $20 \%$ et intervient principalement au niveau de la consommation.

\subsection{Solutions techniques pour réduire les émissions individuelles de gaz à effet de serre - F. Richecœur}

Nous décrirons dans cette section certaines des solutions techniques qui peuvent être mises en œuvre pour réduire les émissions de gaz à effet de serre (GES) que nous produisons individuellement dans la vie de tous les jours. Rappelons qu'entre 1990 et 2017, la France a diminué de 15\% ses émissions de gaz à effet de serre et ce, malgré l'augmentation dans le même temps de celles induites par les transports. Le dioxyde de carbone $\left(\mathrm{CO}_{2}\right)$, provenant majoritairement de l'utilisation d'énergies fossiles, représente $70 \%$ des émissions de GES en France. Trois secteurs prédominent comme dans beaucoup de pays occidentaux: le logement résidentiel, les transports et l'agriculture (Fig. 11.11). Dans ce qui suit, nous nous focaliserons principalement sur les deux premiers secteurs et n'apporterons sur l'agriculture, déjà traitée par ailleurs (sections 10.7 et 11.2), que des commentaires sur les techniques d'analyse des sols et des cultures. 


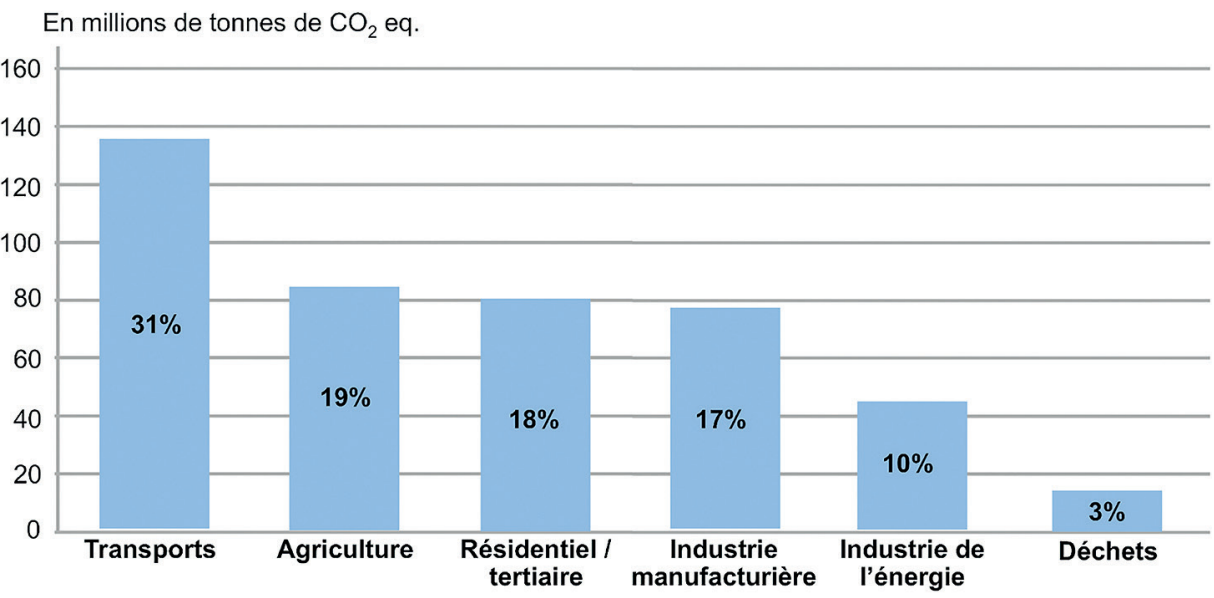

Figure 11.11: Répartition sectorielle des émissions de gaz à effet de serre en France en $2019^{94}$.

\subsubsection{Les technologies pour chauffer et isoler l'habitat individuel}

Au-delà de la phase de construction des logements, l'habitat individuel contribue fortement à l'émission de gaz à effet de serre, principalement le $\mathrm{CO}_{2}$, à travers les solutions de chauffage encore majoritairement utilisées aujourd'hui. L'enjeu est de parvenir à décarboner le chauffage en combinant plusieurs actions. Les émissions de $\mathrm{CO}_{2}$ peuvent être vues comme le produit de trois termes: les émissions de $\mathrm{CO}_{2}$ par unité d'énergie utilisée, l'énergie nécessaire par mètre carré de logement et la surface de logement:

1

$$
\text { Quantité de } \mathrm{CO}_{2}=\frac{\text { Quantité de } \mathrm{CO}_{2}}{\begin{array}{l}
\text { Énergie de chauffage } \\
\text { nécessaire }
\end{array}}
$$

2 Énergie de chauffage nécessaire

Surface des logements
3

\section{Surface des logements}

Réduire les émissions consiste à réduire chacun des trois termes. Commençant par le dernier terme, une première action évidente est d'adopter des logements dont la surface est raisonnable et adaptée aux usages. Intéressons-nous maintenant au premier terme et à l'énergie utilisée. Toutes les sources d'énergie n'ont pas la même empreinte carbone. Pour un kWh destiné au chauffage, la quantité de $\mathrm{CO}_{2}$ émise peut varier pratiquement du simple au double:

94. https://ree.developpement-durable.gouv.fr/themes/defis-environnementaux/changementclimatique/emissions-de-gaz-a-effet-de-serre/article/les-emissions-des-gaz-a-effet-de-serredu-secteur-residentiel 
- gaz naturel : 0,198 kg de $\mathrm{CO}_{2}$ par kWh;

- électricité : 0,213 kg de $\mathrm{CO}_{2}$ par kWh (méthode «saisonnalisée » EDF-ADEME);

- mazout: 0,264 kg de $\mathrm{CO}_{2}$ par $\mathrm{kWh}$.

Une réduction significative des émissions est obtenue en remplaçant toutes les chaudières au fioul/mazout par des dispositifs de production de chaleur utilisant le gaz naturel ou l'électricité. Ces derniers systèmes ont vu leurs rendements augmenter ces dernières années. Le rendement est le rapport entre l'énergie utilisée en entrée du dispositif et l'énergie de chauffage produite.

Les chaudières au gaz naturel fonctionnent sur un principe simple: le gaz est brûlé, les flammes obtenues chauffent de l'eau qui circule dans un réseau de canalisation alimentant des radiateurs qui transfèrent la chaleur depuis l'eau vers l'air de la pièce. II existe néanmoins trois types de chaudière:

- Les chaudières traditionnelles où l'énergie thermique issue de combustion du gaz est utilisée pour chauffer l'eau à haute température (de 60 à $90^{\circ} \mathrm{C}$ ). Leur rendement est compris entre 0,6 et 0,9 ;

- Les chaudières basse température qui sont conçues pour chauffer l'eau à $50^{\circ} \mathrm{C}$. Elles atteignent des rendements entre 0,9 et 0,95 ;

- Les chaudières à condensation, qui permettent d'atteindre des rendements théoriques supérieurs à 1,0 entre 1,05 et 1,1. II est effectivement possible d'avoir des rendements supérieurs à l'unité en utilisant une grande partie de l'énergie chimique contenue dans le combustible, puis en récupérant en plus, par condensation, l'énergie contenue dans l'air ambiant.

Plusieurs technologies de chauffage électrique sont apparues au cours des cinquante dernières années. L'idée la plus simple pour concevoir un chauffage électrique est d'utiliser l'effet Joule en chauffant une résistance qui convertit l'énergie électrique en chaleur. Depuis les convecteurs de première génération jusqu'aux panneaux radiants actuels, les matériaux et circuits créés ont permis d'améliorer l'efficacité et le confort de ces systèmes, mais leur rendement ne peut pas dépasser l'unité. Le meilleur rendement est obtenu en utilisant une pompe à chaleur qui peut théoriquement fournir trois fois plus de chaleur qu'elle ne consomme d'électricité. Ceci est dû au fait que l'électricité n'est pas transformée en énergie thermique mais elle est utilisée pour transférer à l'intérieur du bâtiment la chaleur contenue dans l'air extérieur. Même quand il fait froid dehors, l'air contient de l'énergie thermique! Le fonctionnement de la pompe à chaleur consiste donc à réchauffer l'air intérieur en refroidissant l'air extérieur (voir section 11.6 pour plus de détails).

Terminons maintenant par le deuxième terme de l'équation d'émission, qui quantifie l'énergie thermique nécessaire pour chauffer un mètre carré de logement. C'est en améliorant l'isolation du logement que l'on peut réduire ce terme. Spontanément, 
la chaleur se propage du milieu chaud (l'intérieur du logement) vers le milieu froid (l'air extérieur en hiver). L'objectif de l'isolation est d'empêcher ce transfert de l'énergie thermique à travers les murs et les menuiseries en créant des barrières thermiques telles que:

- le double vitrage qui emprisonne une lame de gaz à très faible conduction de chaleur;

- la laine de roche ou de verre qui emprisonne le volume d'air en limitant les transferts vers l'extérieur.

Une maison passive très bien isolée n'a besoin que de $15 \mathrm{kWh} / \mathrm{m}^{2} / \mathrm{an}$, ce qui la rend plus performante que:

- la maison dite «à basse consommation» (50 kWh/m²/an);

- la maison bioclimatique (entre 40 et $50 \mathrm{kWh} / \mathrm{m}^{2} / \mathrm{an}$ );

- la maison dite «à Très Haute Performance énergétique (THPE)» (moins de $\left.40 \mathrm{kWh} / \mathrm{m}^{2} / \mathrm{an}\right)$.

En conclusion, réduire les émissions de gaz à effet de serre, dues au chauffage des logements individuels, est possible en combinant plusieurs actions sur la taille des logements, l'utilisation de sources d'énergie à très faible émission et une isolation performante ${ }^{96}$. II est possible d'avoir une isolation optimale, mais cela demande une mise en œuvre spéciale et bien souvent une plus grande complexité technique avec, notamment, des matériaux spécifiques ou une gestion fine des flux d'air qui nécessite la ventilation du logement.

\subsubsection{Les technologies pour les transports}

Lorsque l'on parle d'émissions de GES dans les transports, on considère que $96 \%$ des gaz émis sont constitués de $\mathrm{CO}_{2}$. Pour bien comprendre le problème des émissions, il est nécessaire de regarder les émissions respectives des différents modes de transport (Fig. 11.12) [11-13]. La majorité des émissions provient des voitures particulières et des véhicules légers, dû principalement au fait que ce sont les véhicules les plus nombreux. On note également que le nombre de kilomètres parcourus par personne est en constante augmentation. Les émissions du secteur aérien représentent $2 \%$ des émissions en France si l'on ne considère que les vols intérieurs. Cette proportion monte à $4 \%$ en prenant en compte les vols extérieurs. Le transport ferroviaire étant majoritairement électrifié, et l'électricité produite en France n'émettant que très peu de gaz à effet de serre, la contribution du train est négligeable. 
Véhicules particuliers

Véhicules utilitaires

Poids lourds

Deux-roues

Non routier

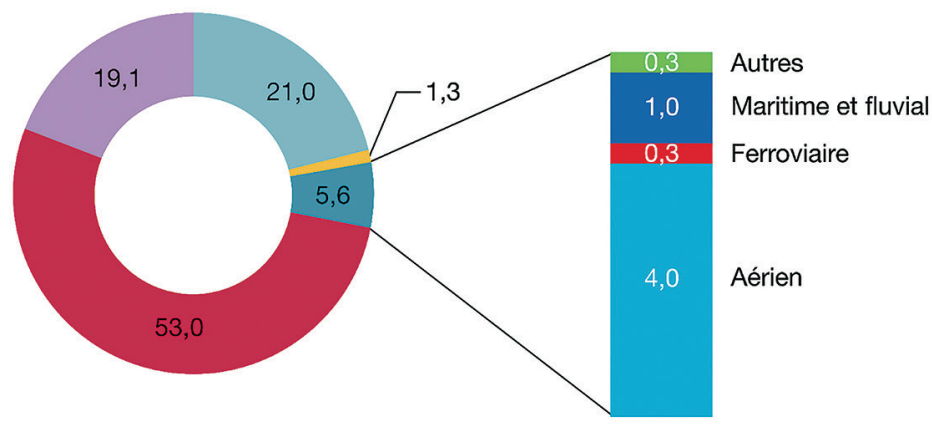

Figure 11.12: Répartition des émissions de Gaz à Effet de Serre (GES) des transports en France en 2018 en $\%{ }^{95}$

La contribution du transport routier a fortement augmenté depuis 1990. Les deux raisons principales viennent du fait que la population augmente et que chacun parcourt plus de kilomètres (Fig. 11.13, rectangles à droite). Heureusement, certaines technologies ont permis de réduire une partie des émissions grâce à l'amélioration des rendements des moteurs thermiques et au développement de nouveaux modes de propulsion bas carbone (Fig. 11.13, rectangles à gauche).

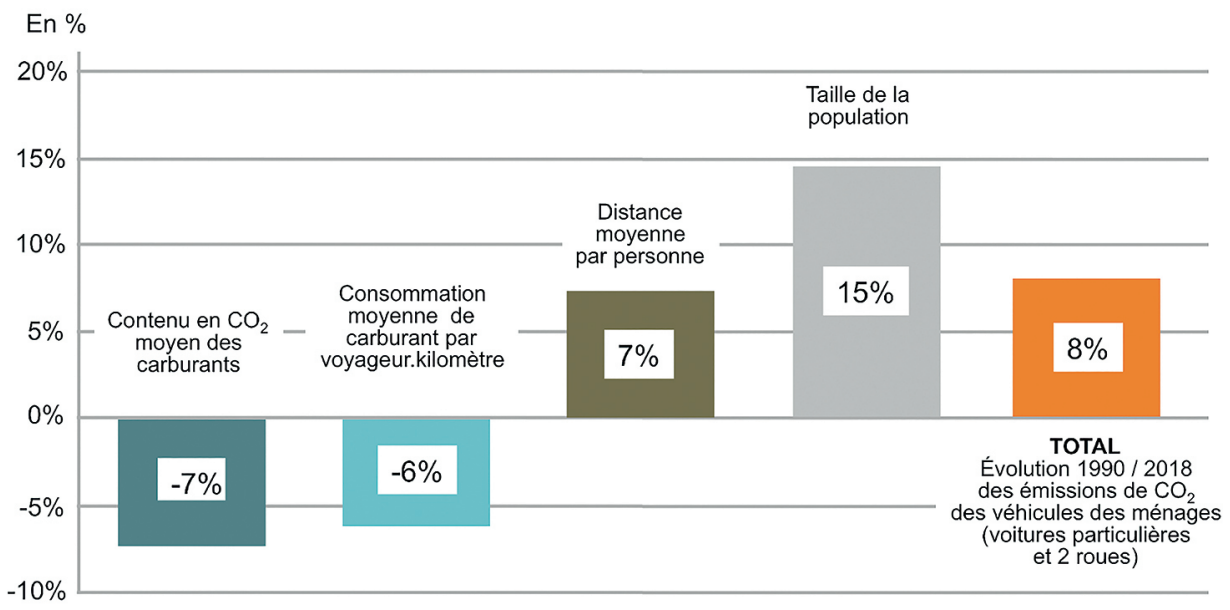

Figure 11.13: Facteurs explicatifs de l'évolution des émissions de $\mathrm{CO}_{2}$ des véhicules que possèdent les ménages - France - $1990 / 2018^{96}$.

Parmi les améliorations technologiques effectuées dans le transport routier, les ingénieurs ont commencé par améliorer l'existant, c'est-à-dire optimiser les moteurs thermiques. Le transport routier a pu se démocratiser grâce au développement de

95. https://www.statistiques.developpement-durable.gouv.fr/edition-numerique/chiffres-clesdu-climat/11-emissions-de-ges-des-transports 
la production de masse et l'utilisation de l'essence dans les moteurs. Quand un véhicule doit emporter son propre carburant (automobile, avion, bateau, etc.), l'essence est effectivement le candidat le plus performant. En termes d'énergie produite par unité de masse transportée, les performances de l'essence (10 $000 \mathrm{Wh} / \mathrm{kg})$ sont, par exemple, sans commune mesure avec celles d'une batterie au lithium ( $200 \mathrm{Wh} / \mathrm{kg}$ ). On cherche donc à maximiser le rendement d'un moteur, c'est-à-dire le pourcentage d'énergie du carburant que le moteur va pouvoir utiliser pour propulser le véhicule. Le rendement augmente avec la température et la pression dans le moteur. On doit donc comprimer l'air, par exemple avec l'utilisation du turbo, et réaliser la combustion la plus efficace pour augmenter la température (Fig. 11.14). Seulement, il faut que les matériaux résistent. Actuellement, les rendements des moteurs thermiques ne dépassent pas $33 \%$, dit autrement, $67 \%$ de l'énergie contenue dans le carburant est dissipée en chaleur dans l'atmosphère. La thermodynamique nous dit qu'il est très difficile de faire mieux (le rendement de Carnot).

Dans le secteur de l'automobile, pour franchir un cap et tendre vers l'absence d'émissions, il faut une rupture dans la motorisation, c'est-à-dire utiliser d'autres technologies. Un premier saut a été tenté avec les voitures hybrides, mais le gain en termes d'émission est finalement marginal par rapport à l'augmentation de la complexité qu'entraînent à la fois la gestion de deux moteurs et la récupération d'énergie au freinage. La voiture tout électrique a actuellement le vent en poupe, car elle permet de n'avoir localement aucune émission de GES et, en France en particulier, la production électrique bas-carbone aboutit globalement à des émissions très faibles. Techniquement, l'utilisation de moteur électrique est aussi plus simple que celle d'un moteur thermique. II y a moins d'entretien, mais c'est, en revanche, toute une logistique industrielle et des habitudes de consommation à modifier pour permettre de produire des moteurs et des pièces en masse, de recharger les batteries, de réparer, recycler, partager...
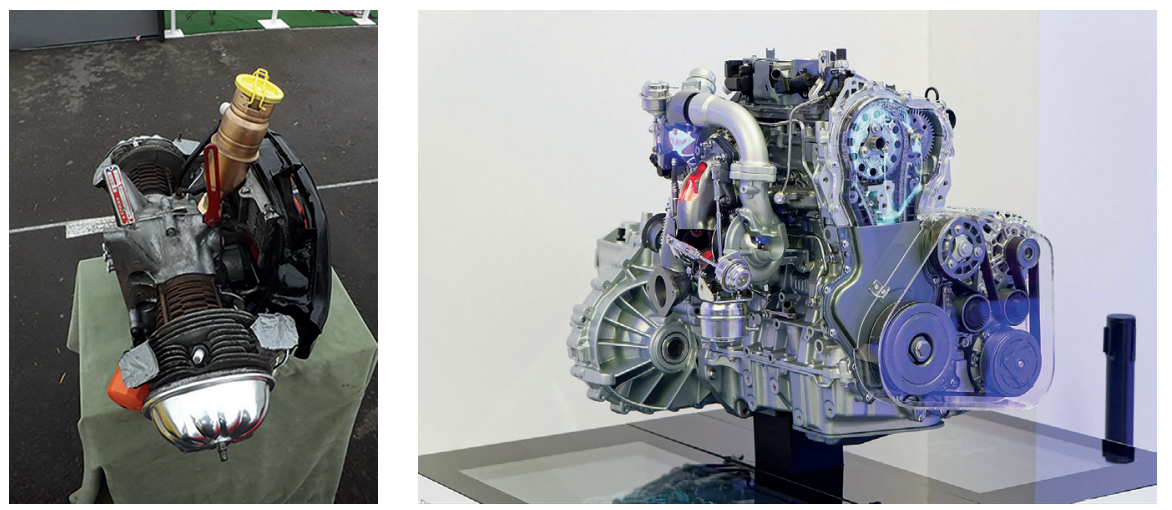

Figure 11.14: Évolution de la complexité des moteurs thermiques pour augmenter le rendement. À gauche: Citroen A-series 2CV 602cc Engine. Source: Wikimedia Commons (source: Andrew Bone). À droite: Renault moteur energy dCi 160 twin turbo EDC. Source: Wikimedia Common (source: Thesupermat). 
Dans le secteur aérien, la chute du trafic aérien pendant le confinement du printemps 2020 en France a fortement impacté l'industrie de l'aéronautique qui, malgré une contribution relativement faible aux émissions globales, est perçue comme un contributeur important du changement climatique. Depuis de nombreuses années, cette industrie s'est lancée dans la conception de l'avion bas-carbone grâce à la combinaison de technologies et d'usages vertueux. À l'heure actuelle, il n'est pas possible de faire voler un avion transportant plusieurs centaines de passagers sur plusieurs milliers de kilomètres avec des moteurs électriques. Comme dans l'automobile, les émissions de gaz à effet de serre augmentent, car de plus en plus de personnes voyagent en avion et parcourent de plus en plus de kilomètres. Les technologies permettent seulement de réduire les émissions par kilomètre parcouru et par personne, en réduisant la consommation des moteurs: roulage électrique, moteur avec soufflante non carénée (open rotor) (Fig. 11.15), groupe auxiliaire de puissance (APU) électrique, procédure d'approche et réduction du poids total avec des nouveaux matériaux. Le maître mot est d'utiliser les moteurs thermiques avec des biocarburants ou de l'hydrogène uniquement pour ce qui ne peut pas être assuré par une autre technologie à zéro carbone.

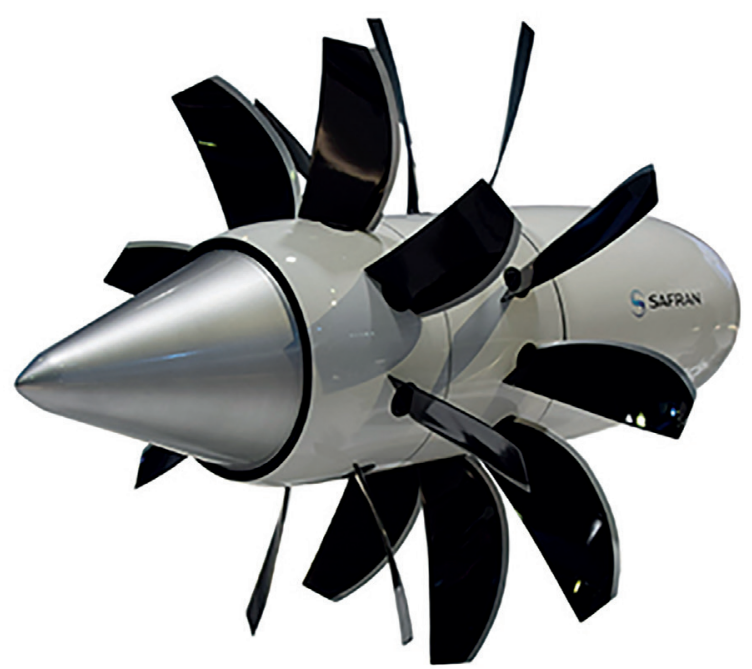

Figure 11.15: Safran open rotor mockup, Paris Air Show 2017. Source: Wikimedia Commons (source: Tiraden).

En conclusion, les solutions techniques existent pour réduire, voire annuler, dans certains cas, les émissions de GES par kilomètre et par passager, mais ce sont les usages croissants des transports routiers, qui ont le plus gros impact. La stratégie d'évolution n'est pas facile à planifier, car les transports sont des «industries du temps long», où les systèmes fonctionnent pendant plusieurs dizaines d'années. II faudrait, pour ce faire, une planification très élaborée, mais qui serait difficile à tenir dans un environnement fluctuant et incertain. 


\subsubsection{L'hydrogène, le combustible de demain pour l'énergie et les transports?}

L'hydrogène est régulièrement cité comme une solution pour réduire les émissions de dioxyde de carbone dans les transports. L'hydrogène présente effectivement des avantages très séduisants: un kilogramme d'hydrogène possède autant d'énergie que trois kilogrammes d'essence, la combustion d'hydrogène n'émet pas de $\mathrm{CO}_{2}$, l'hydrogène est stockable. Ces caractéristiques permettent d'imaginer deux usages principaux pour l'hydrogène dans le secteur de l'énergie: le brûler pour produire de la chaleur ou le faire réagir dans une pile à combustible pour produire de l'électricité. Les fusées fonctionnent déjà avec des moteurs brûlant de l'hydrogène et les piles à combustible sont utilisées dans des trains ou des véhicules expérimentaux.

Le stockage et l'utilisation de l'hydrogène nécessitent cependant de modifier beaucoup de dispositifs industriels, mais c'est encore la production d'hydrogène qui est actuellement la plus problématique. En effet, quand on parle d'hydrogène, on ne parle pas de l'atome d'hydrogène, que l'on retrouve en très grande quantité sur Terre sous la forme d'eau et de méthane, mais de la molécule de dihydrogène. La molécule de dihydrogène ne se trouve pas à l'état naturel, il faut la produire. À l'heure actuelle, le dihydrogène est essentiellement produit à partir de molécules de méthane desquelles on vient arracher les atomes d'hydrogène (vaporeformage) ${ }^{96}$. Cet arrachage nécessite de l'énergie qui, pour l'instant, est fortement émettrice de $\mathrm{CO}_{2}$ : pour produire un kilogramme d'hydrogène, $10 \mathrm{~kg}$ de $\mathrm{CO}_{2}$ sont émis ! Le bilan n'est donc pas positif. Les procédés électrolytiques semblent, en revanche, plus favorables pour produire l'hydrogène de façon décarbonée et économique, et contribuer aux objectifs que la France s'est fixée en matière de développement des énergies renouvelables et de réduction des consommations d'énergie fossile ${ }^{97}$.

En conclusion, l'hydrogène est considéré comme un vecteur énergétique potentiellement de grand intérêt, c'est-à-dire un moyen efficace de stocker de l'énergie et de la restituer quand on le veut et à l'endroit que l'on souhaite. Pour généraliser son usage, il sera cependant nécessaire de le produire en grande quantité et sans émission de GES, de développer les infrastructures de stockage, et d'adapter les dispositifs d'utilisation de l'hydrogène (moteurs ou piles à combustibles).

\subsubsection{Les technologies pour l'agriculture}

Les émissions de gaz à effet de serre de l'agriculture sont spécifiques, car majoritairement composées d'autres molécules que le $\mathrm{CO}_{2}$ et issues de processus biologiques. Les émissions de protoxyde d'azote $\mathrm{N}_{2} \mathrm{O}$ sont principalement issues de l'épandage d'engrais minéraux et organiques, d'excrétions dans les pâturages, de la

96. https://fr.wikipedia.org/wiki/Vaporeformage

97. Plan de développement de l'hydrogène pour la transition énergétique: https://www.ecologie.gouv. $\mathrm{fr} /$ sites/default/files/Plan_deploiement_hydrogene.pdf 
décomposition des résidus de culture, de l'épandage des boues et des composts. Les bovins sont responsables de $91 \%$ des émissions de méthane $\mathrm{CH}_{4}$, liées au processus digestif et de $57 \%$ des émissions de méthane $\mathrm{CH}_{4}$ liées aux déjections.

Les technologies utilisées dans l'agriculture (tracteurs, moissonneuses, etc.) ont permis, soit d'intensifier des actions qui étaient autrefois réalisées manuellement, soit d'artificialiser des pratiques comme l'arrosage, la production d'engrais, le désherbage chimique, etc. Ce sont des technologies développées pour le rendement et la production, et non pas au service d'une meilleure compréhension de l'agriculture et de la façon de réduire ses émissions de gaz à effet de serre. L'environnement vivant est très complexe, comme dans le domaine de la santé, la technologie est d'une aide très précieuse, mais il faut ici l'utiliser non seulement pour produire mais aussi pour comprendre. Satellites, analyses de sols, mesures et obtentions de données sont indispensables et doivent être déployés à grande échelle. Plus encore que de technologie, c'est aujourd'hui de sciences dont il y a besoin pour mieux comprendre l'agriculture dans toute sa complexité et son fonctionnement systémique [14].

\subsubsection{Conclusion}

Le logement, les transports et l'agriculture sont les trois secteurs qui contribuent majoritairement aux émissions de gaz à effet de serre en France. Les acteurs de ces secteurs sont sensibles aux enjeux climatiques et de transition énergétique, ils travaillent depuis de nombreuses années à réduire les émissions de gaz à effet de serre dans leurs activités en optimisant leur fonctionnement par le développement de technologies. Ces développements ont considérablement réduit les émissions par unité produite (kilomètre parcouru, mètre carré de logement, kilo de céréale produit, etc.), mais en même temps les usages ont fortement augmenté jusqu'à annuler les gains technologiques. De plus, l'intégration de nombreuses techniques complexifie les systèmes en les rendant plus difficiles à fabriquer et à maintenir, et en augmentant leur impact sur l'environnement, soit de manière directe par l'utilisation de matériaux rares ou difficiles à extraire, soit de manière indirecte en changeant les conditions de travail. La trajectoire à suivre est donc une simplification technologique grâce à une meilleure compréhension des fonctionnements et à une réduction des usages (kilomètres parcourus, tailles des logements, en particulier). Bien évidemment, cette trajectoire est à suivre en développant la production d'énergie de plus en plus décarbonée (voir section 11.4).

\subsection{Produire une énergie décarbonée: les énergies renouvelables (EnR) - J.-M. Lourtioz}

Les énergies renouvelables sont souvent considérées comme étant au cœur même de la transition énergétique et de la transition écologique, mais que représentent-elles, en fait, aujourd'hui parmi les énergies que nous produisons et que 
nous consommons et quelles perspectives offrent-elles pour notre avenir et l'avenir de la planète? Nous essaierons, dans cette section, de donner quelques éléments de réponse à ces questions.

\subsubsection{Les différents types d'énergie renouvelable}

Par définition une source d'énergie est dite renouvelable si son renouvellement naturel est assez rapide pour qu'on puisse la considérer comme inépuisable à l'échelle du temps humain. C'est le cas du bois qui se renouvelle à la vitesse de croissance des arbres dès lors que cette croissance est respectée. Ce n'est, par contre, le cas ni du charbon, ni du pétrole ni du gaz dont les réserves ont mis des centaines de millions d'années pour se constituer.

L'énergie renouvelable la plus utilisée aujourd'hui reste encore la biomasse, le bois en particulier, mais aussi les déchets ménagers, végétaux ou animaux qui servent au chauffage avec une partie convertie en électricité. La matière organique, après fermentation ou transformation, sert également à la production de biogaz et de biocarburants pour le transport.

Deuxième énergie renouvelable en termes d'utilisation, l'énergie hydraulique où l'énergie potentielle de l'eau est convertie en électricité. L'hydroélectricité est bien présente en France, les plus gros producteurs étant la Chine, le Canada, le Brésil et les États-Unis. Sa répartition est globalement homogène par continent dans le monde.

Vient en troisième position l'énergie éolienne (Fig. 11.16), où l'énergie mécanique est convertie en électricité. Éoliennes sur terre, mais aussi en mer, ce qui peut être une chance pour des pays comme la France qui, avec 11 millions km² d'espace maritime, se place au deuxième rang mondial après les États-Unis.

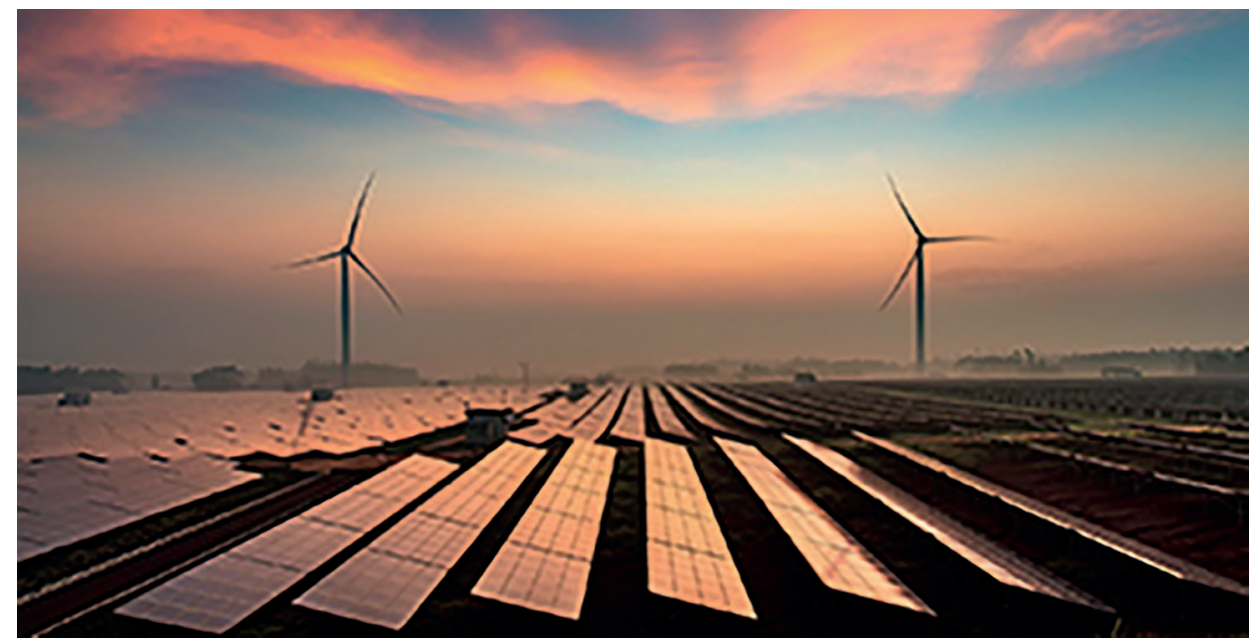

Figure 11.16: Illustration des «nouvelles» énergies renouvelables: l'éolien et la conversion photovoltaïque de l'énergie solaire. Photo @istock. 
Enfin, on peut regrouper arbitrairement toutes les énergies renouvelables obtenues par extraction d'énergie thermique, que ce soit du sol (la géothermie), de l'air extérieur (les pompes à chaleur) ou du soleil pour être utilisée en chauffage ou convertie en électricité (le photovoltaïque - Fig. 11.16). Concernant la géothermie, nous sommes tous familiers de sa forte présence en Islande, où les sources d'eau chaude sont particulièrement nombreuses, mais elle est aussi bien présente en France, notamment sur le site de Paris Saclay où l'eau chaude est extraite du plateau de l'Albien (Fig. 11.17).

Outre le fait que toutes les énergies mentionnées précédemment sont renouvelables, donc non épuisables, elles ne présentent pas de risque majeur pour la santé humaine et produisent peu ou pas d'émission de gaz à effet de serre dans leur utilisation. Ceci est illustré sur la figure 11.18 pour ce qui concerne les émissions de $\mathrm{CO}_{2}$. II ne faut cependant pas oublier qu'en contrepartie, leur développement implique l'investissement de nouvelles infrastructures qui, elles, ont un coût et nécessitent en grande partie l'utilisation d'énergie carbonée pour leur mise en place.

Les énergies renouvelables répondent, pour l'essentiel, aux critères d'acceptabilité par nos sociétés même si leur installation peut parfois amener des controverses en termes de pollution visuelle, sonore ou d'occupation d'espaces. Les oppositions de la population à telle ou telle installation, quelle qu'elle soit, doivent nécessairement être prises en compte et la décision retenue doit être le résultat d'une délibération démocratique (voir section 10.5).

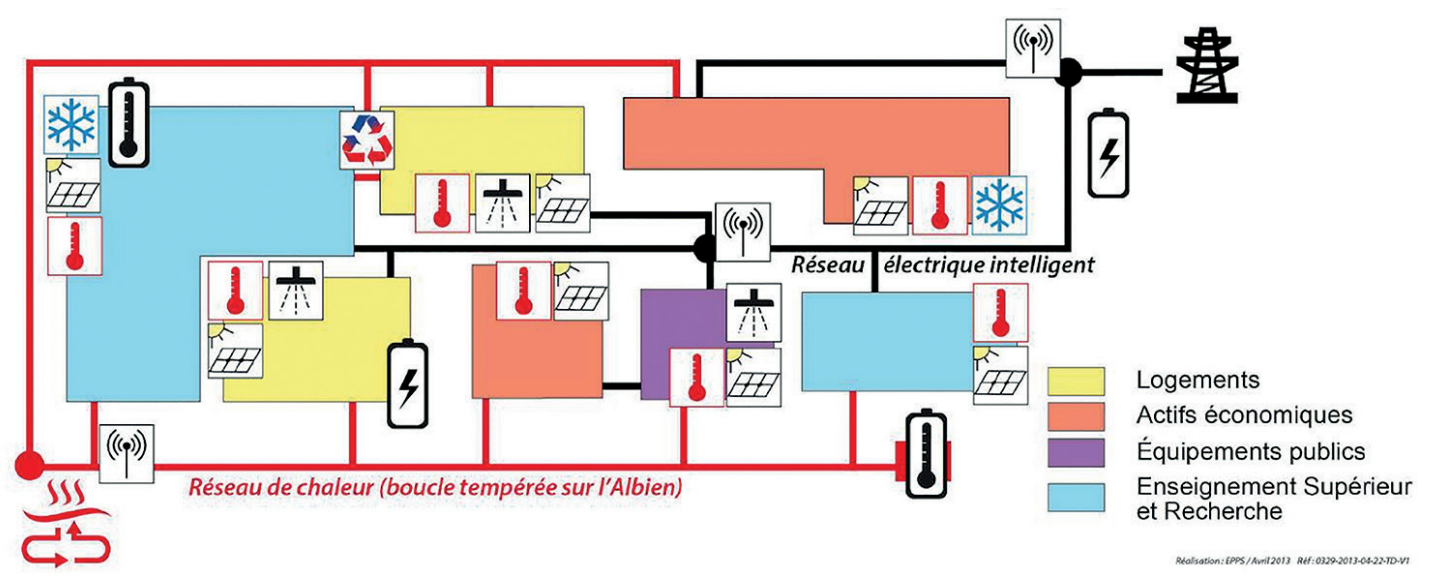

Figure 11.17: Schéma du réseau de chaleur sur le site Paris Saclay. Source: Établissement Public d’Aménagement Paris Saclay. 


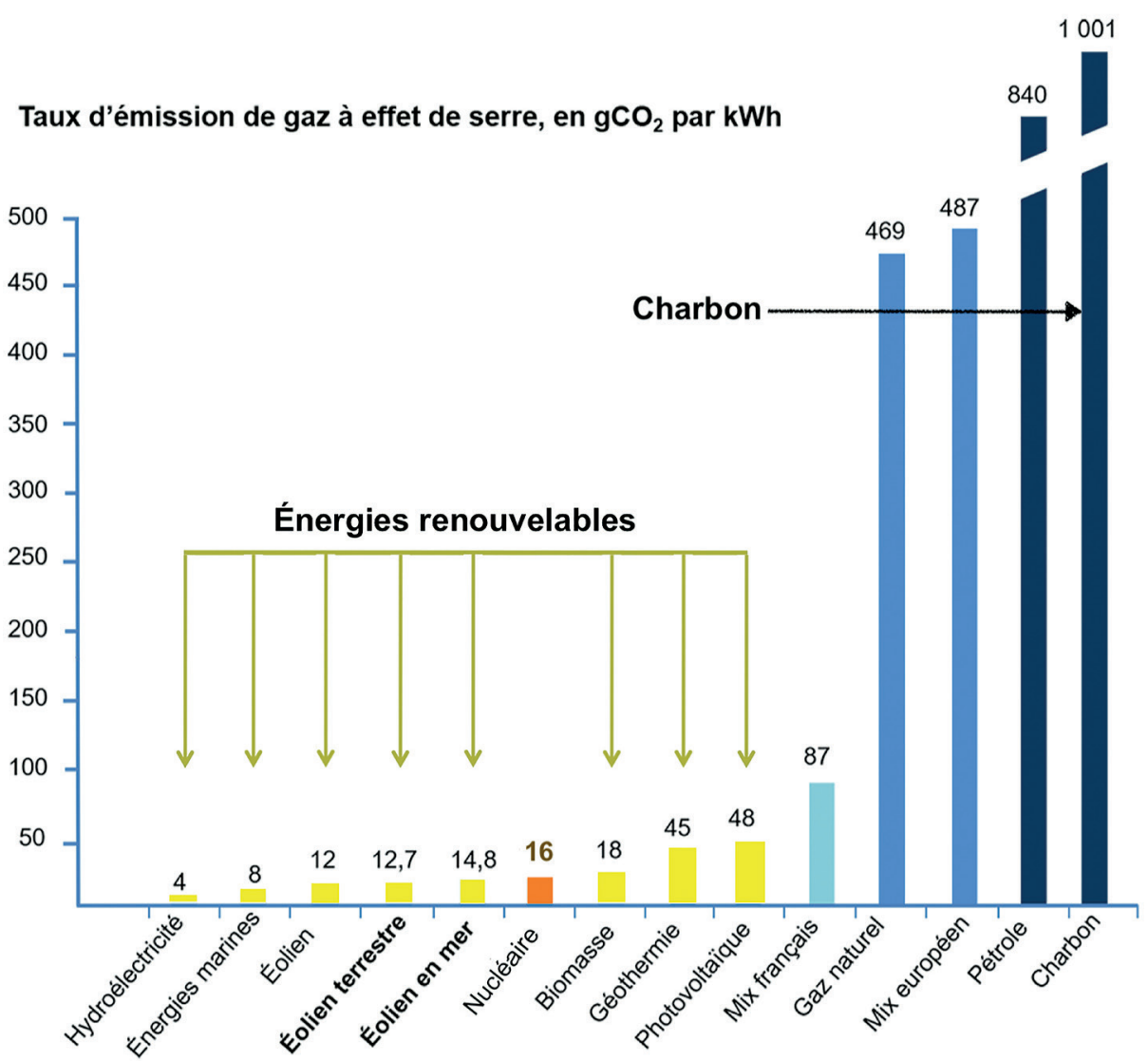

Figure 11.18: Émissions en équivalent $\mathrm{CO}_{2}$ par kWh, comparées par source d'énergie. Source: rapport GIEC 2011, Ademe (2015), Ecoinvent (données 2011).

\subsubsection{Place des énergies renouvelables en France et dans le monde}

En 2019, la part des énergies renouvelables dans la production mondiale d'énergie était estimée à $18 \%$ en prenant en compte le fait qu'elles ne présentent pas les pertes de conversion des énergies fossiles ${ }^{98}$ (Fig. 11.19, à gauche). La valeur estimée passe à $15 \%$ sans cette correction. On peut, en effet, noter que la conversion de l'éolien, du solaire et de l'hydraulique en électricité est directe alors qu'en revanche, la transformation des combustibles fossiles en électricité passe par l'usage et le chauffage d'un liquide caloporteur. Quelle que soit la façon dont l'estimation est faite, la biomasse traditionnelle (essentiellement le bois) reste dominante avec une part comprise entre 6,5 et $7 \%$. Les «nouvelles» énergies renouvelables (éolien, solaire thermique 
ou photovoltaïque) apparaissent encore modestes avec une part d'environ 2,5\%. En revanche, leur progression récente est spectaculaire, car les capacités mondiales de production de l'éolien et du solaire ont été multipliées par un facteur 5 de 2010 à 2019 (Fig. 11.19, à droite).
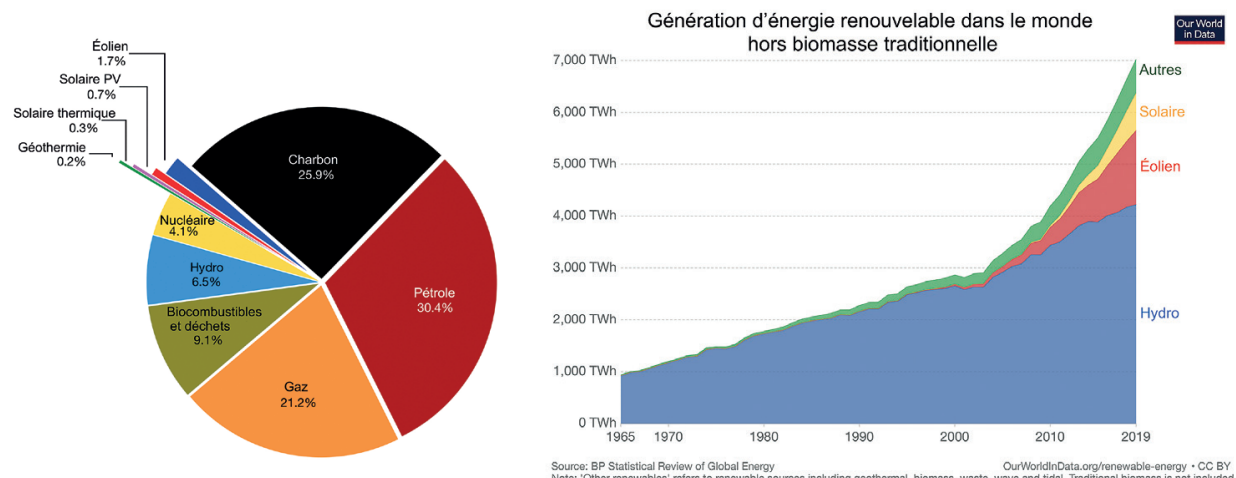

Figure 11.19: À gauche: part des différentes sources d'énergie dans la production mondiale d'énergie en 2019. Le calcul des énergies primaires est basé sur la «méthode de substitution» qui prend favorablement en compte le fait que les énergies renouvelables ne présentent pas les pertes de conversion des énergies fossiles dans la production d'électricité ${ }^{100}$. À droite : évolution de la génération d'énergies renouvelables hors biomasse traditionnelle de 1965 à 2019. Ce sont ici les chiffres bruts non corrigés par la méthode de substitution. Source: BP Statistical Review of Global Energy ${ }^{100}$.

Toutes les énergies renouvelables hormis la biomasse traditionnelle sont en constante progression dans le monde même si dans le bilan des sources d'énergie, cette progression est un peu masquée par l'augmentation simultanée de la demande globale d'énergie et donc de la consommation d'énergies fossiles, qui en résulte (voir Fig. 2.5, section 2.1.5). La croissance des énergies renouvelables n'est, par ailleurs, pas homogène partout dans le monde. Aujourd'hui, c'est en Europe que les EnR représentent la part la plus importante de la consommation énergétique. L’Amérique du Sud s'en sort également bien avec ses grandes infrastructures hydro-électriques, notamment au Brésil où $42 \%$ de la consommation énergétique est d'origine renouvelable. En comparaison, les États-Unis n'atteignent que $10 \%$ de leur consommation énergétique en énergie renouvelable. La Chine et l'Inde plafonnent quant à elles à 10 et $11 \%$ respectivement.

Néanmoins, la baisse des coûts des énergies renouvelables, leur amélioration technologique continue et la prise de conscience écologique devraient pousser les énergies renouvelables vers le haut partout sur la planète dans les années qui viennent. Selon l'Agence Internationale de l'Énergie (AIE) ${ }^{99}$, d'ici 2024, l'énergie renouvelable devrait être le secteur avec la croissance la plus rapide au sein de la production d'électricité pour satisfaire environ 30\% de la demande globale en électricité. Ce positionnement des EnR dans la production d'électricité s'illustre déjà en France où 
elles ont été pour 21,5\% dans la production d'électricité en France métropolitaine en 2019 (Fig. 11.20).

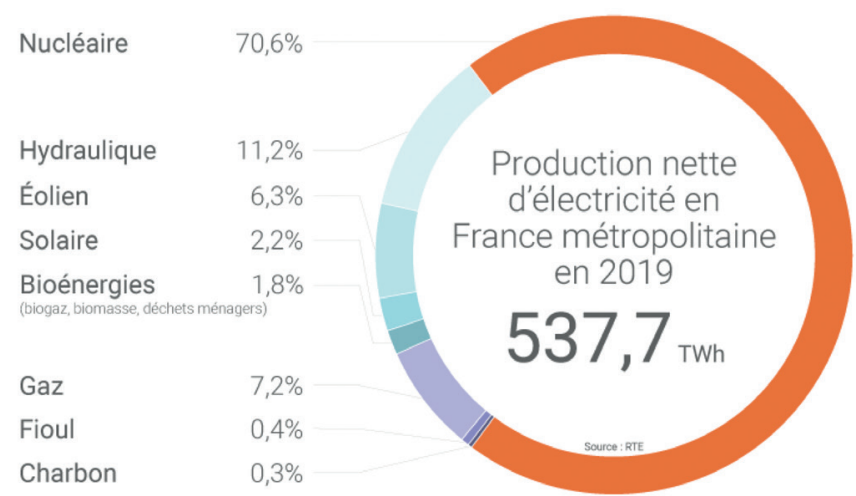

Figure 11.20: Le mix de production électrique en France métropolitaine est dit «décarboné» à près de $92 \%$. Source: RTE (Réseau de Transport d'Electricité) ${ }^{100}$.

Plus globalement, l'Agence Internationale s'attend à ce que, d'ici 4 ans, les énergies renouvelables couvrent jusqu'à $20,5 \%$ de la consommation énergétique (contre $17-18 \%$ aujourd'hui). En Chine et en Inde, elle prévoit notamment des hausses respectives de $16 \%$ et $51 \%$ de la consommation énergétique d'origine renouvelable. En France, la Loi de Transition Énergétique ${ }^{101}$ prévoit d'atteindre 32\% d'énergies renouvelables dans le mix énergétique du pays d'ici 2030.

\subsubsection{Limites au développement des nouvelles énergies renouvelables}

Si les prévisions sont plus que favorables aux énergies renouvelables, on ne peut toutefois omettre quelques freins à leur développement. D'une part, il faut du temps, plusieurs années, pour que de nouvelles sources d'énergie se développent au meilleur niveau technologique et dans les meilleures conditions d'acceptation sociale. D'autre part, comme nous l'avons déjà mentionné, ce développement implique des coûts d'investissement, parfois élevés, et des nouvelles infrastructures qui nécessitent l'utilisation d'énergie carbonée pour leur installation. Comme pour toute nouvelle infrastructure, leur durabilité peut poser question et il faut, dès le départ, envisager des méthodes de recyclage des matériaux en parallèle. Enfin, et c'est sans doute la principale difficulté: les nouvelles sources d'énergie renouvelable sont des sources intermittentes. Le vent ne souffle pas toujours et le soleil n'éclaire pas la nuit.

100. https://www.connaissancedesenergies.org/la-production-delectricite-en-france-metropolitainetous-les-chiffres-cles-de-2019-200212-0.

Voir aussi : https://www.actu-environnement.com/media/pdf/news-35995-chiffres-cles-enr-2020.pdf 101. https://www.ecologie.gouv.fr/loi-transition-energetique-croissance-verte 
Pour pallier cette intermittence, il faut stocker l'énergie produite lorsque celle-ci ne peut être utilisée immédiatement afin de pouvoir la restituer ultérieurement. II existe, en fait, de nombreux moyens de stocker l'énergie ${ }^{102}$ allant du stockage mécanique au stockage chimique ou électrochimique. Le stockage mécanique consiste, par exemple, à utiliser des stations de pompage ${ }^{103}$, qui transforment l'énergie électrique en énergie de gravitation. Une solution élégante du stockage chimique est de fabriquer de l'hydrogène par électrolyse ${ }^{104}$, lequel peut lui-même être utilisé comme source d'énergie (voir section 11.3.3). Le stockage dans des batteries électrochimiques (par exemple, lithium - ion) est sans doute le plus connu aujourd'hui à défaut d'être nécessairement le plus efficace. En fait, le stockage de l'électricité est actuellement un domaine en pleine extension, stimulé en partie par le développement des énergies renouvelables. Sont notamment à l'étude ce qu'on appelle des réseaux électriques «intelligents», capables de gérer l'hétérogénéité des sources de production ainsi que leur décentralisation et de les agréger pour répondre de façon optimale à la demande d'électricité ${ }^{105}$. Une architecture de réseau intelligent est schématisée sur la figure 11.21.

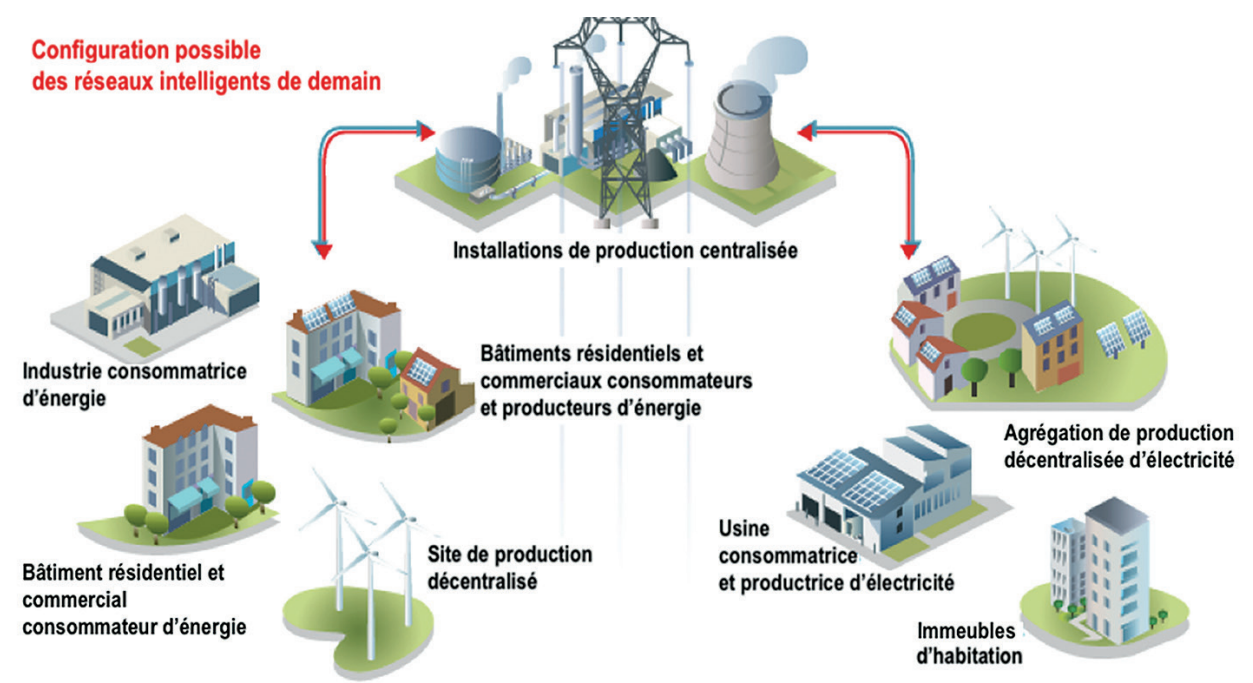

Figure 11.21: Évolution possible de l'architecture et des fonctionnalités pour les réseaux électriques de demain. Source @ ADEME/P.P.\&A./Scriptoria ${ }^{107}$.

102. https://particulier.edf.fr/fr/accueil/guide-energie/electricite/stockage-energie-electricite.html 103. https://www.youtube.com/watch?v=UL9kyQPa76Q\&feature=youtu.be

104. https://www.pv-magazine.fr/2020/03/05/lancement-de-projets-de-production-dhydrogeneassociant-energie-solaire-et-batterie-en-espagne/

105. https://www.ademe.fr/expertises/energies-renouvelables-enr-production-reseaux-stockage/ passer-a-laction/transport-lenergie/reseaux-electriques-intelligents 


\subsubsection{Focus sur la conversion photovoltaïque de l'énergie solaire}

Nous savons tous que le soleil est la principale source d'énergie de notre planète. Or, si le flux d'énergie solaire représente, en moyenne, plus de 5000 fois l'énergie globale que l'humanité consomme actuellement ${ }^{106}$, le solaire photovoltaïque ne contribue même pas à $1,5 \%$ de l'énergie que nous produisons. En fait, avec les panneaux photovoltaïques actuels en silicium, dont le rendement de conversion lumière - électricité est d'environ $20 \%$, produire $100 \%$ de notre énergie à partir de la conversion photovoltaïque du rayonnement solaire imposerait de couvrir en panneaux solaires une surface équivalente à $75 \%$ du territoire français. Avec une ambition plus mesurée, ne cherchant à répondre qu'à $10 \%$ de la demande mondiale en énergie et anticipant une optimisation réaliste du rendement des panneaux d'un facteur 2, la surface à couvrir se limiterait à moins de $4 \%$ du territoire français, soit aussi environ 1 à $2 \%$ des surfaces urbanisées de par le monde. Des rendements de conversion lumière - électricité s'élevant à plus de $47 \%$ ont été effectivement rapportés en 2019 par des équipes de chercheurs ${ }^{107}$. Même si le passage à la production industrielle n'est jamais direct, ces chiffres sont prometteurs pour l'avenir.
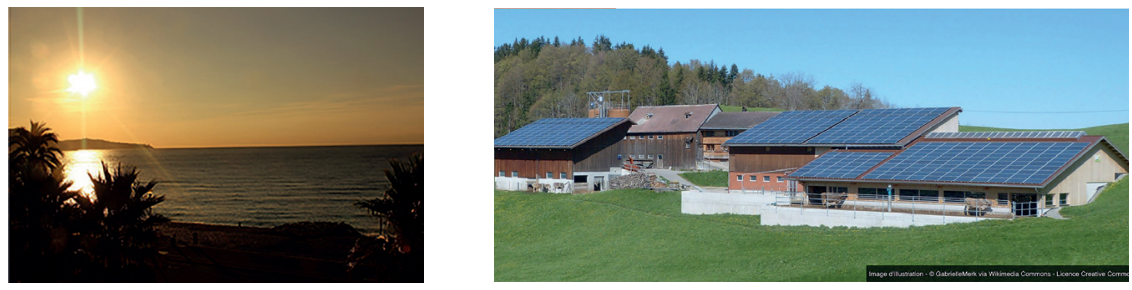

Figure 11.22: Le soleil est, de loin, la principale source d'énergie sur Terre, mais son exploitation directe ne représente encore qu'une part réduite de la production totale d'énergie. À gauche: lever de soleil. Crédit photo: Chtiperdu (CC-BY-SA-3.0). À droite: panneaux photovoltaïques d'habitations. Source photo: GabrielleMerk (CC-BY-SA-4.0).

\subsubsection{Conclusion}

Les énergies renouvelables offrent la perspective de produire une énergie qui préserve nos ressources naturelles en réduisant les émissions de gaz à effet de serre. Seules, elles ne pourront pas tout résoudre, mais contribueront pour

106. La puissance reçue du soleil au zénith par unité de surface et en l'absence de nuages est typiquement de l'ordre de $1000 \mathrm{~W} / \mathrm{m}^{2}$. Cependant le soleil n'éclaire pas la nuit, l'intensité de l'éclairement dépend des saisons, de la latitude, du créneau horaire concerné et de la présence ou non de nuages si bien que sur une année, la puissance effective reçue par $\mathrm{m}^{2}$ est d'environ $200 \mathrm{~W}$. II « suffit » alors de multiplier ce chiffre par la surface du globe terrestre et par le nombre d'heures dans une année pour trouver la quantité d'énergie que le soleil fournit annuellement. La surface du globe supposé sphérique se calcule à partir de la formule : $4 \pi R^{2}$ où $R=6371 \mathrm{~km}$ représente le rayon du globe. Tout calcul fait, on obtient une énergie de : 892 millions de térawatts heure (TWh), alors que la consommation d'énergie par l'humanité est actuellement de 170000 térawatts heure (TWh).

107. https://en.wikipedia.org/wiki/Solar_cell_efficiency 
beaucoup à la transition énergétique si on les associe à d'autres sources décarbonées comme le nucléaire, dont il est question dans la section 11.5 et pourvu qu'il soit sécurisé, ou à des sources fossiles moins carbonées comme le gaz naturel, sachant que les réserves de gaz comme celles du pétrole et du charbon ne sont pas infinies. Sans doute, le plus important est que le développement des énergies renouvelables s'accompagne de transformations profondes de nos modes de vie et de consommation (voir sections 10.2 et 11.6).

\subsection{L'énergie nucléaire, une autre source d'énergie décarbonée : principes, avantages et limites - L. Audouin}

\subsubsection{Principes de l'énergie nucléaire ${ }^{108}$}

Le terme "énergie nucléaire» est utilisé pour décrire la production d'électricité par le biais de réactions nucléaires dites de fission. S'il est en théorie possible d'utiliser d'autres réactions nucléaires (notamment la fusion, la source d'énergie des étoiles, ou bien la radioactivité), il n'existe aucune mise en application industrielle de ces processus, pour des raisons de complexité technologique ou de simple faisabilité à grande échelle : la fission est la seule voie exploitée.

\section{Principe du réacteur}

La fission est une réaction nucléaire particulière, dans laquelle un noyau se fragmente en deux noyaux (Fig. 11.23). Ce processus est fortement exo-énergétique (de l'ordre de $200 \mathrm{MeV}$, soit 200 millions d'électrons-volts, autrement dit 1 million de fois plus qu'une réaction chimique individuelle) et s'accompagne de l'émission de plusieurs neutrons.

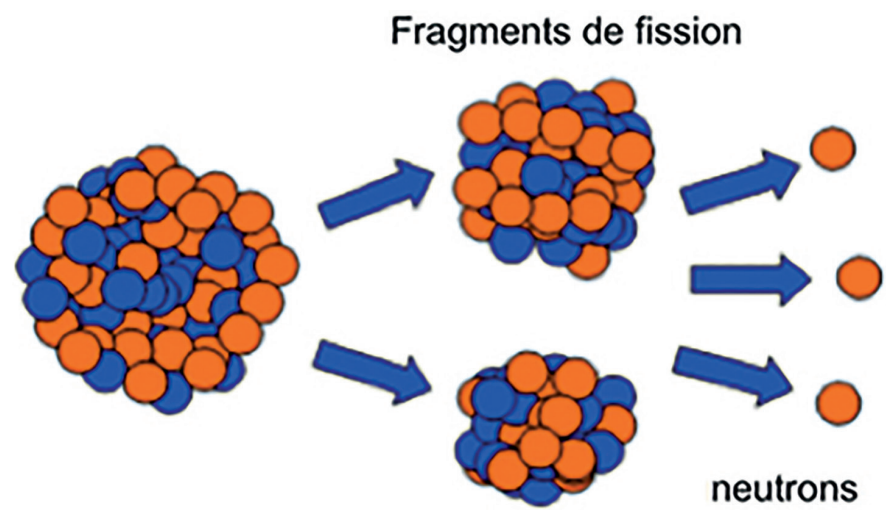

Figure 11.23: Désintégration d'un noyau atomique lourd en deux noyaux plus légers. Source: L. Audouin.

108. https://www.planete-energies.com/fr/medias/dossiers/comprendre-l-energie-nucleaire 
Or, quelques noyaux se caractérisent par une très forte probabilité de fissionner lorsqu'ils interagissent avec un neutron (on les qualifie de «fissiles»). On peut ainsi créer une réaction en chaîne: les fissions libèrent des neutrons, qui entraînent d'autres fissions, etc. (Fig. 11.24). La clé de la conception d'un réacteur nucléaire est de parvenir à provoquer mais aussi stabiliser cette réaction en chaîne: le nombre de noyaux fissionnant doit être constant dans le temps (à l'inverse d'une bombe nucléaire, où il s'agit de provoquer le maximum de réactions dans un temps très bref).

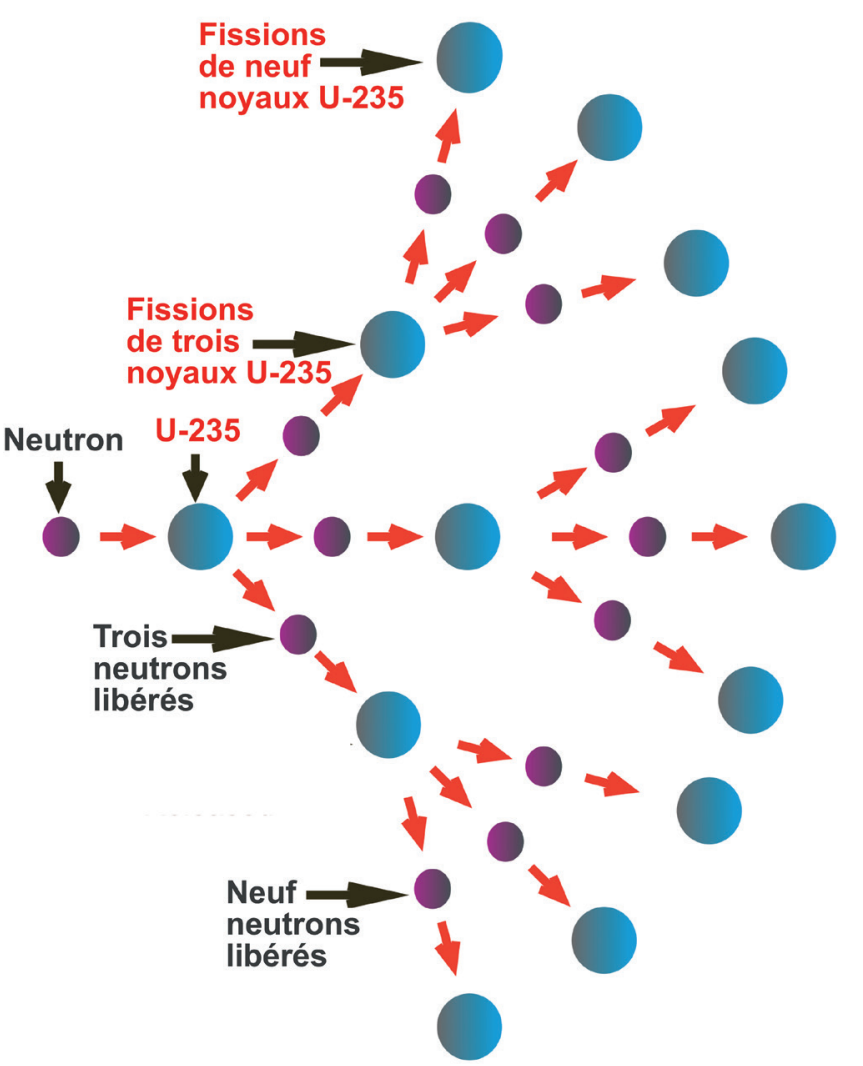

Figure 11.24: La réaction en chaîne au sein des réacteurs nucléaires. Source: SeekPng.com_ reaction-png_1234970.

L'énergie libérée par les fissions se retrouve immédiatement sous forme de chaleur, laquelle est extraite du "cœur» du réacteur via un fluide (eau le plus souvent, mais des gaz ou des métaux liquides peuvent être utilisés) pour être convertie en électricité, à l'image de ce qui est pratiqué dans une centrale à gaz ou charbon. Pour des raisons de complexité et de rentabilité, l'utilisation directe de la chaleur du réacteur pour des usages classiques (chauffage) n'est pas possible directement. 


\subsubsection{Centrales nucléaires}

\section{Dimensionnement et intégration dans un réseau électrique}

Bien qu'il soit techniquement possible de faire des réacteurs nucléaires de toutes dimensions, en pratique, les réacteurs électrogènes civils sont des dispositifs de grande puissance, de 300 à 1650 MW électriques. Quelques dizaines de réacteurs sont donc suffisants pour produire la quantité d'électricité consommée par un pays de taille moyenne comme la France. Les réacteurs peuvent seulement être construits à proximité d'un grand fleuve, ou de la mer, qui vont jouer un rôle de "source froide » pour le cycle thermodynamique utilisé pour la production d'électricité (Fig. 11.25).

Un réacteur nucléaire est une source dite «pilotable» au même titre que les réacteurs thermiques: il est possible de faire varier la puissance produite en fonction des besoins, jusqu'à $\pm 5 \%$ par minute, soit un passage de 0 à la pleine puissance en 20 minutes au mieux (les centrales à gaz sont les plus agiles, avec $20 \% / \mathrm{min}$ ). Cette caractéristique est intéressante à la fois pour suivre les variations de consommation au fil du temps, mais aussi accompagner l'insertion dans le mix de production de ressources non pilotables (éolien, solaire).

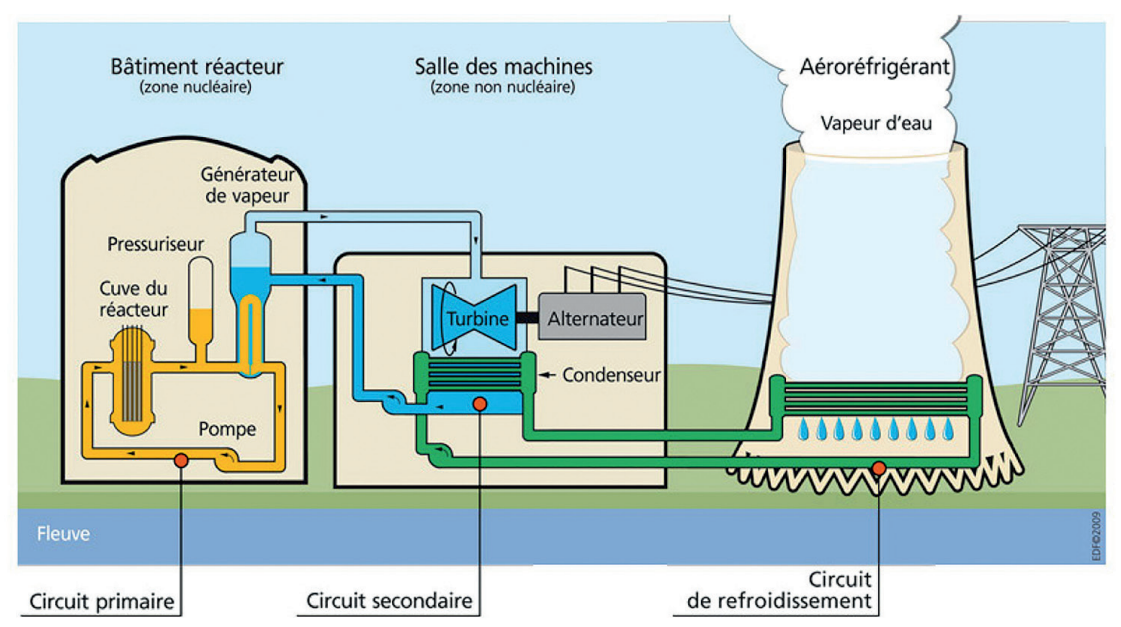

Figure 11.25: Schéma de principe d'une centrale nucléaire avec aéroréfrigérant. Source: IRSN (Institut de Radioprotection et de Sûreté Nucléaire) EDF@2009

\section{Équation économique}

La structure de coût de l'énergie nucléaire (Fig. 11.26) est assez comparable à celle des énergies renouvelables: un très lourd investissement initial (qui est modélisé en France à $35 \%$ du coût de l'électricité) et des frais d'investissement et opérationnels conséquents (55\%). Le combustible nucléaire ne représente qu'environ $9 \%$ du coût de l'électricité. 


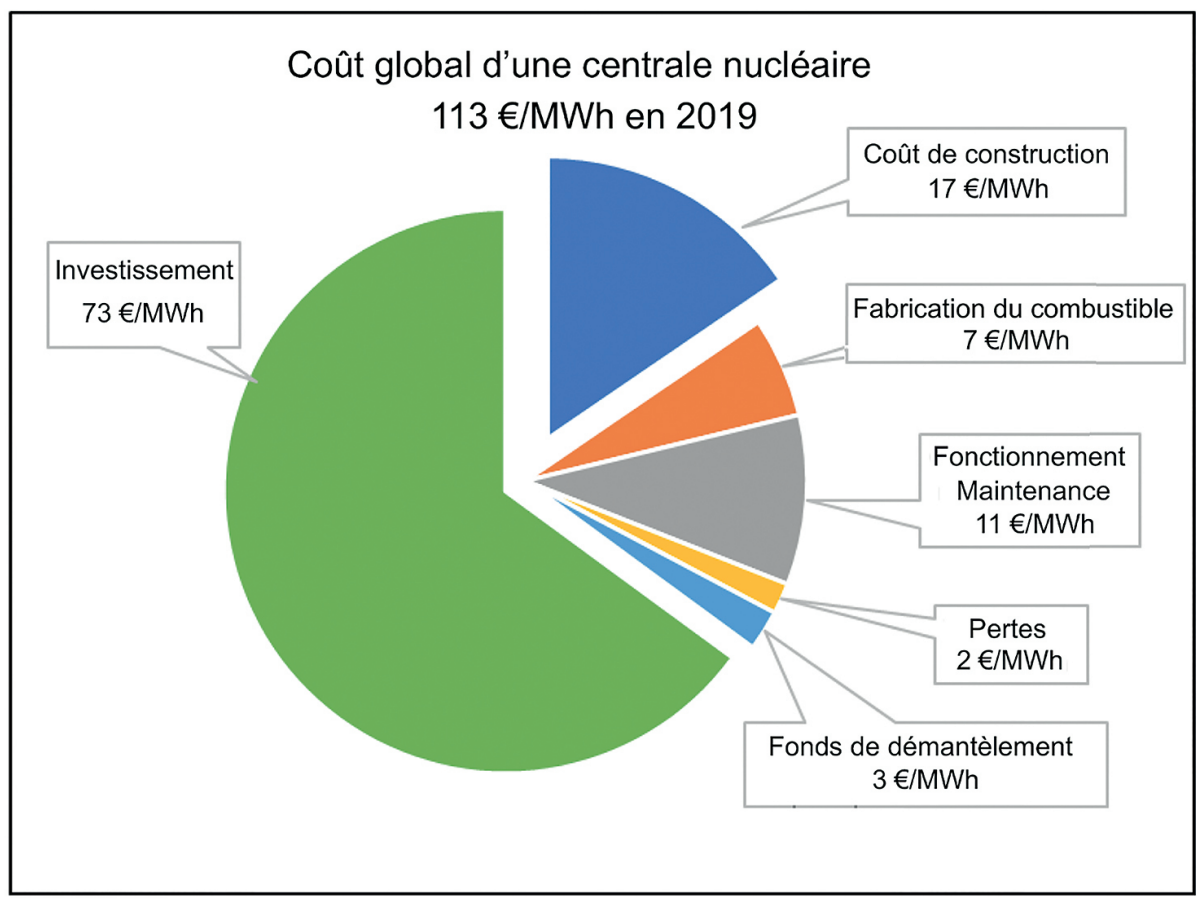

Figure 11.26: Structure de coût de l'électricité dans le projet britannique Hinkley Point ${ }^{109}$.

Les derniers $10 \%$ correspondent au provisionnement pour la gestion du combustible usagé et pour le démantèlement des installations à la fin de leur exploitation. L'équation économique de l'électricité nucléaire évolue rapidement ces dernières années, avec des investissements de sûreté qui croissent rapidement, des coûts de construction de centrale mal maîtrisés et des incertitudes fortes sur les sommes réelles qui seront nécessaires pour une gestion sûre du combustible usagé sur de très longues périodes, bien supérieures à la vie des réacteurs. Historiquement, le nucléaire était particulièrement compétitif, avec des prix estimés à $33 € / M W h$; on se situe aujourd'hui au-delà de $110 € / M W h$ pour les réacteurs actuellement construits.

\subsubsection{Une énergie à faible émission de $\mathrm{CO}_{2}$}

\section{Émissions de $\mathrm{CO}_{2}$}

Lors de son fonctionnement, un réacteur n'émet pas de gaz à effet de serre. En revanche, des GES sont émis lors de la construction, notamment du fait des larges quantités de béton et d'acier utilisées; de plus, l'extraction/raffinage du minerai et

109. https://medium.com/generation-atomic/the-hinkley-point-c-case-is-nuclear-energy-expensivef89b1aa05c27 (licence CC BY-SA 4.0) 
la fabrication du combustible sont coûteuses énergétiquement. Les estimations du contenu carbone de l'énergie nucléaire sont très variables en fonction du mode de calcul et du mix énergétique du pays étudié: les rapports du GIEC retiennent une valeur moyenne internationale de 12 à $16 \mathrm{~g}$ eq. $\mathrm{CO}_{2}$ par kWh (Fig. 11.27). Le nucléaire se range donc dans la catégorie des énergies très peu émettrices de $\mathrm{CO}_{2}$.

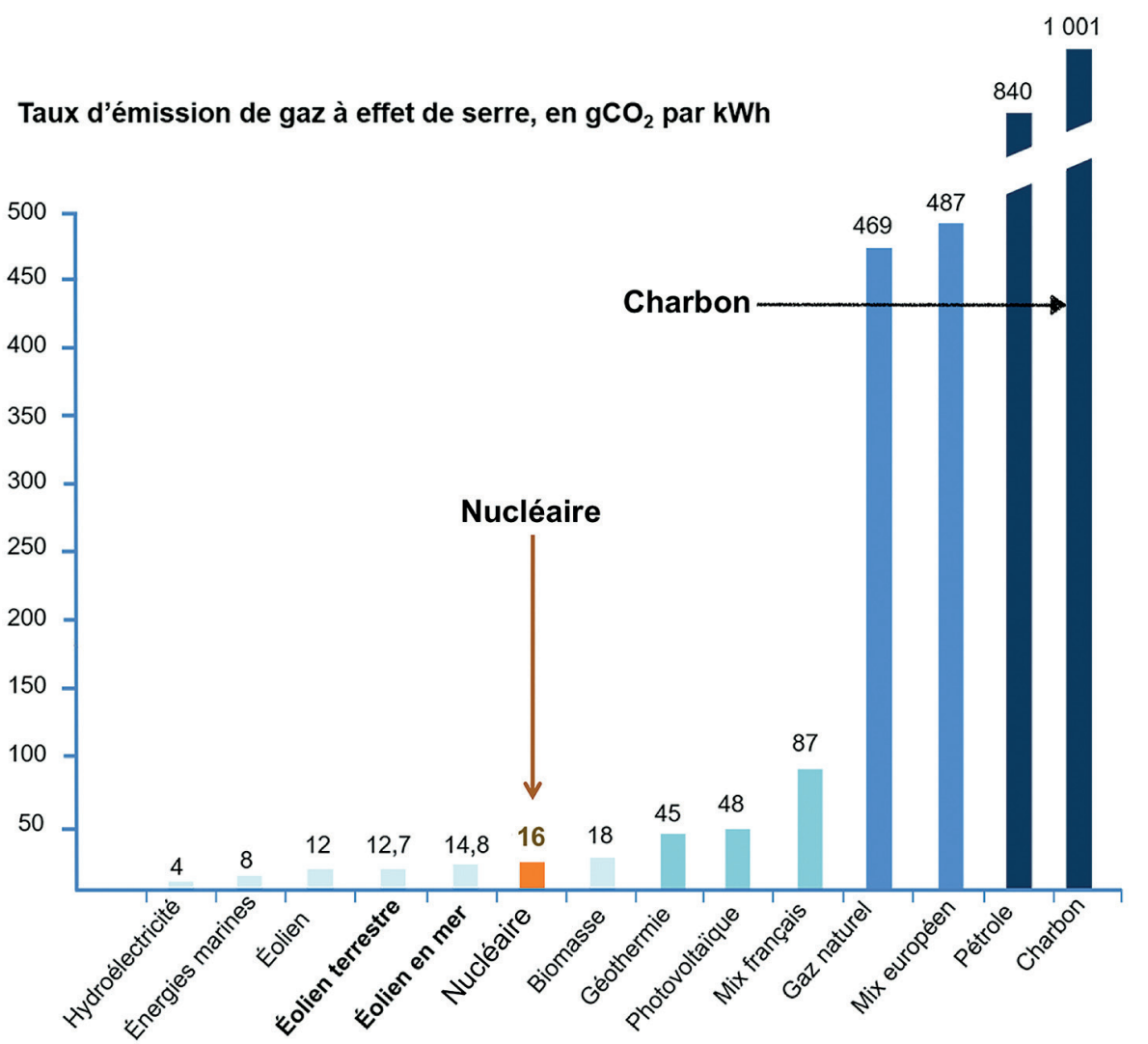

Figure 11.27: Émissions en équivalent $\mathrm{CO}_{2}$ par kWh, comparées par source d'énergie. Source: rapport GIEC 2011, Ademe (2015), Ecoinvent (données 2011).

\section{Une énergie à larges réserves}

L'énergie nucléaire est basée sur l'utilisation de minerais présents sur Terre: ce n'est pas une énergie renouvelable. L'Uranium est présent en quantité finie, avec des stocks exploitables estimés entre 15 et 25 MT, soit environ 150 ans au rythme de consommation actuel (70 kT/an). Les réserves sont très inégalement réparties (principalement Asie centrale, Afrique centrale, Canada, Australie), ce qui pourrait en faire un enjeu stratégique (Fig. 11.28). 


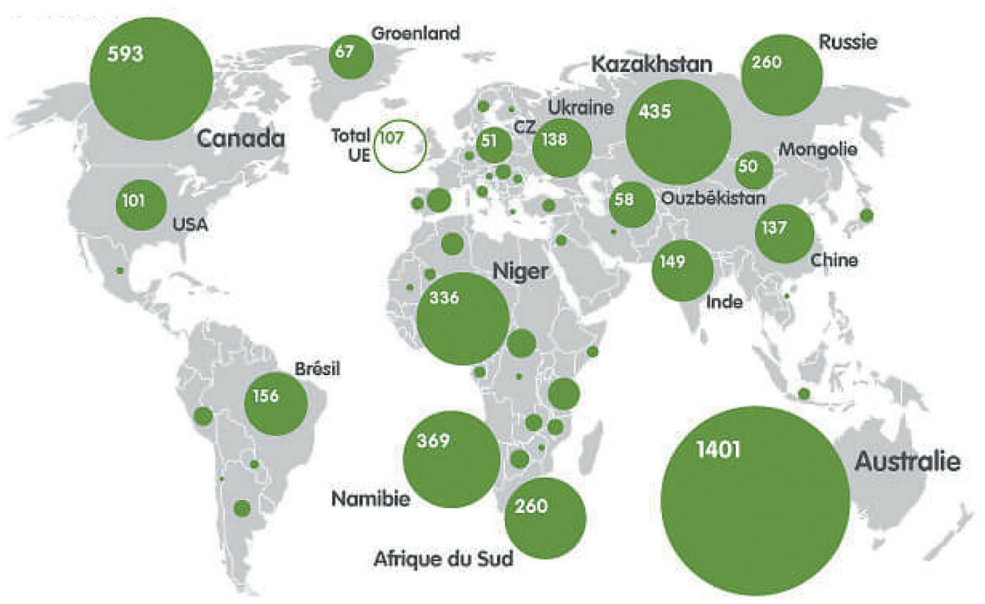

Figure 11.28: Estimation des réserves d'uranium par zone géographique en 2017. Source: OCDE/AIEA «Red Book» 2028 ${ }^{110}$.

II n'existe qu'un seul noyau fissile dans la nature: ${ }^{235} \mathrm{U}$. Cet isotope est très minoritaire dans l'uranium présent sur Terre, il ne représente que 0,7\% du total. Certaines technologies de réacteurs permettent d'utiliser comme principal noyau fissile ${ }^{239} \mathrm{Pu}$, un noyau artificiel, formé dans les réacteurs via la capture d'un neutron par ${ }^{238} \mathrm{U}$, l'isotope majoritaire présent dans la nature. Si un tel combustible présente de fortes contraintes (technologie plus complexe, démultiplication des flux de matières hautement dangereuses), son utilisation permettrait de multiplier les réserves de plus d'un ordre de grandeur (ou de préserver plusieurs millénaires de ressources, même dans l'hypothèse d'une forte augmentation de la production mondiale d'énergie nucléaire). Un autre axe de recherche, plus exploratoire, porte sur l'utilisation de ${ }^{233} \mathrm{U}$ (formé à partir de ${ }^{232} \mathrm{Th}$, plus abondant que l'Uranium dans la nature).

\subsubsection{Radioactivité et déchets nucléaires}

\section{La radioactivité}

Certains noyaux, dits radioactifs, ont la propriété d'émettre spontanément un ou plusieurs corpuscules: noyau de ${ }^{4} \mathrm{He}$, électron ou positron; on parle respectivement de radioactivité a, $\beta$ - ou $\beta+$ (Fig. 11.29). Cette émission peut être suivie de celle d'un ou plusieurs photons. La radioactivité est en soi un événement totalement aléatoire, mais sa probabilité dans le temps est parfaitement fixe et unique pour une espèce nucléaire donnée: on peut donc prévoir le comportement d'un large ensemble d'une espèce nucléaire. On peut montrer qu'il faut un temps fixe, appelé période radioactive, pour que la moitié des noyaux d'un échantillon donné ait émis un corpuscule. Le noyau subsistant («noyau fils») peut lui-même être radioactif, conduisant à une

110. https://www.kernenergie.ch/fr/uranium-matiere-premiere-_content---1--1085.html 
chaîne de désintégration; mais longue ou courte, une telle chaîne se termine nécessairement par un noyau stable.

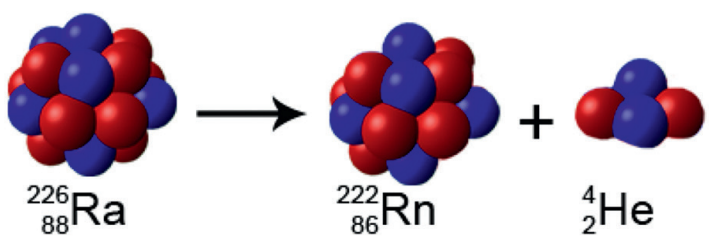

Figure 11.29: Désintégration alpha du Radium-226 en radon 222. La désintégration génère de plus un noyau d'Hélium 4 et un rayonnement radioactif, composé d'énergie et de particules de matière. Source: Wikilivres

\section{Radioactivité et organismes vivants}

Les corpuscules émis lors d'une désintégration radioactive ont la propriété de perturber la matière par leurs collisions sur ses constituants (Fig. 11.30). De très fortes irradiations peuvent perturber un organisme vivant au point de conduire à son décès à court terme. Les irradiations de plus faible intensité n'ont pas d'impact immédiat majeur, mais peuvent conduire à l'apparition de pathologies comme les leucémies et les cancers, après plusieurs années voire plusieurs décennies. Leur survenue est aléatoire, la probabilité étant liée à quantité d'irradiation reçue et au débit de dose.

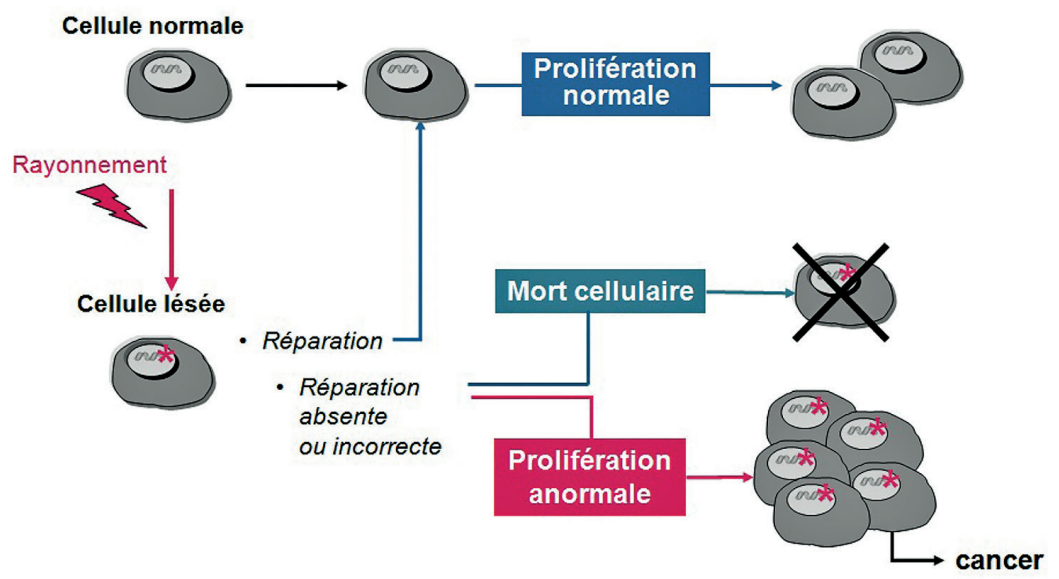

Figure 11.30: Les conséquences possibles (non équi-probables!) d'une interaction d'une particule ionisante avec un tissu organique ${ }^{111}$. Source : CEA /Direction des Sciences du Vivant.

II faut noter que la radioactivité est présente naturellement dans l'environnement. Les activités industrielles mettant en jeu des matériaux radioactifs (typiquement, la production d'électricité par le nucléaire) ne doivent jamais conduire à 
exposer le public à une irradiation supérieure à une fraction de celle due à la radioactivité naturelle.

\section{Déchets nucléaires}

Comme tout processus industriel, l'énergie nucléaire produit des déchets dont une partie présente un danger pour l'homme et le vivant (Fig. 11.31). La spécificité du nucléaire est que ce danger n'est pas seulement chimique, mais principalement dû à la radioactivité. Le combustible nucléaire, après son utilisation dans un réacteur, est particulièrement létal : il doit être strictement confiné. Les procédés de fabrication du combustible, les réparations de réacteurs et surtout leur démantèlement vont également produire des déchets plus ou moins radioactifs.

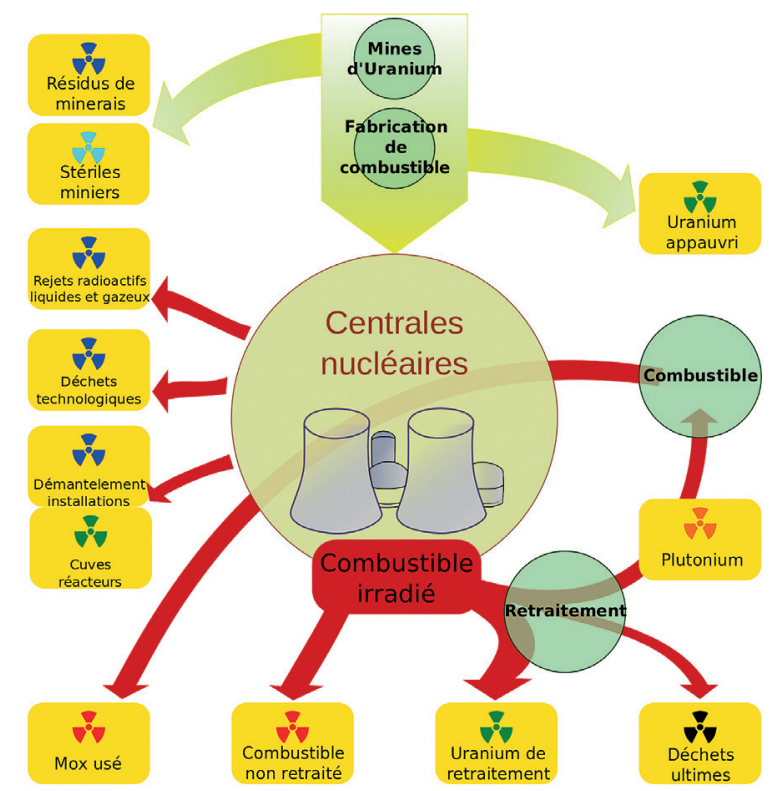

Figure 11.31: Origines des déchets sur l'ensemble du cycle de production nucléaire. À noter qu'en France, la fraction potentiellement réutilisable n'est pas classée comme déchet, même en l'absence d'usage présent. Source : Biem CC BY-SA 4.0.

La radioactivité s'atténue dans le temps, mais les durées peuvent être très longues. Les déchets nucléaires doivent faire l'objet d'un conditionnement et d'un stockage spécifiques, sur des échelles de temps adaptées à leur période radioactive : quelques années pour certains, mais des millions pour d'autres. Leur diversité chimique rend ce conditionnement délicat, et la sûreté d'un confinement sur des échelles de temps géologiques reste un défi technique et scientifique de premier ordre.

La plupart des pays utilisant l'énergie nucléaire envisagent aujourd'hui de créer pour les déchets les plus dangereux des sites de stockage en profondeur, dans 
lesquels les roches agiraient en barrière de dernier ressort entre les déchets et la biosphère. Malgré de nombreuses actions de recherche en la matière, aucun pays n'a cependant mis en service un tel site de stockage.

\section{Accidents, risques, acceptabilité}

Comme pour toute activité industrielle de grande ampleur, un accident peut avoir des conséquences environnementales et sanitaires sérieuses et concerner des zones étendues. Le danger n'est pas celui d'une explosion à la façon d'une bombe (rigoureusement impossible) mais celui de la dispersion de grandes quantités de matières plus ou moins radioactives, créant une contamination durable de vastes étendues, les cas extrêmes (et pour autant difficilement comparables) étant les accidents de Tchernobyl en 1986 (Fig. 11.32) et de Fukushima en 2011. L'estimation de l'impact sanitaire réel de tels événements reste un défi pour les scientifiques, davantage pour des questions de suivi des publics concernés que de description des phénomènes : l'apparition de pathologies est décalée d'années voire de décennies, et peut rarement être imputée sans ambiguïté à l'exposition à la radioactivité. Le caractère invisible et complexe de la radioactivité, son association (pour partie réelle, pour partie fantasmée) avec des technologies à buts militaires et une pesante culture du secret contribuent à renforcer l'inquiétude des populations et a fortement dégradé au fil du temps l'acceptabilité de l'énergie nucléaire par les opinions publiques.

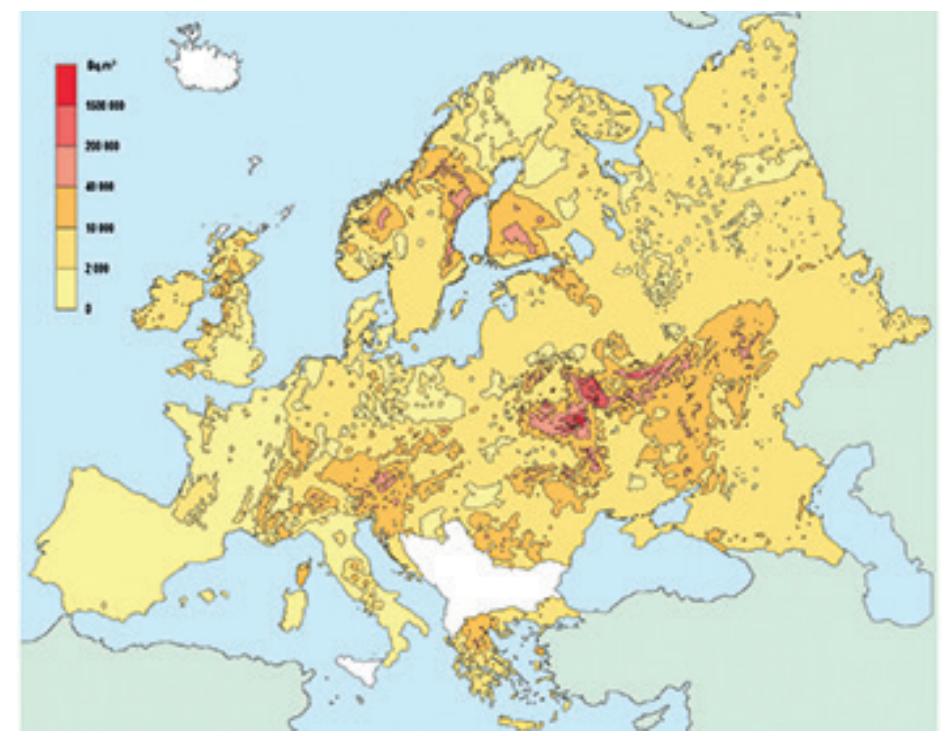

Figure 11.32: Dépôts de ${ }^{137} \mathrm{Cs}$ liés à l'accident de Chernobyl (1986). Source: IRSN ${ }^{112}$.

112. https://www.irsn.fr/FR/connaissances/Installations_nucleaires/Les-accidents-nucleaires/accidenttchernobyl-1986/2016-Tchernobyl-30ans-apres/Pages/5-Tchernobyl-2016-30ans-apres-accidentFrance-Europe-impacts.aspx\#.YWiNLi3pP_Q 


\subsubsection{Le nucléaire, une source modeste à l'échelle mondiale; et demain ? ${ }^{113}$}

En 2019, 10,3\% de l'électricité mondiale a été produite par les 457 réacteurs nucléaires en service dans le monde. Ces deux chiffres sont en légère diminution sur les vingt dernières années. Du fait de sa complexité et de son coût d'investissement important, le nucléaire n'est développé que dans les pays industrialisés. La quasi-totalité des réacteurs se situe dans l'OCDE et en Chine, où ils représentent en moyenne autour de $20 \%$ de la production électrique. La France est un cas tout à fait singulier avec près de $80 \%$ d'électricité produite par le nucléaire. Un recours fortement accru au nucléaire à l'échelle mondiale se heurterait à deux difficultés: sa complexité technologique, qui le réserve aux pays avancés, et les limites des ressources, qui imposeraient une transition vers des technologies encore plus complexes - donc coûteuses et potentiellement risquées.

On voit donc que le nucléaire, du fait de son très faible contenu carbone et de réserves potentiellement abondantes, peut constituer un outil pour produire l'électricité sans émission de gaz à effet de serre. Mais, entre un prix de revient orienté à la hausse qui menace sa compétitivité, l'environnement technique qu'il exige, les inconnues qui pèsent sur la gestion des déchets et une acceptabilité en fort recul, il peut difficilement être la seule solution au défi climatique.

\subsection{Changer nos modes de vie : sobriété, efficacité et substitutions dans notre consommation énergétique - F.-M. Bréon}

\subsubsection{Sobriété dans nos modes de vie}

Le changement climatique est engendré par les émissions de dioxyde de carbone et d'autres gaz à effet de serre. La plus grande partie des émissions de $\mathrm{CO}_{2}$ sont liées à la consommation des énergies fossiles. Historiquement, ce fut d'abord le charbon, puis le pétrole et enfin le gaz fossile. Cet ordre s'applique aussi aux quantités de $\mathrm{CO}_{2}$ émises. Stabiliser le climat nécessite donc de diminuer très fortement la consommation de ces énergies fossiles ${ }^{114}$.

Une partie de la solution passe nécessairement par une forme de sobriété. On entend par là un changement important de notre mode de vie. Voici quelques exemples de comportements plus sobres que nous pourrions adopter sans, bien sûr, que cette liste ne soit exhaustive:

113. https://www.lemondedelenergie.com/avenir-nucleaire-energie/2020/12/27/.

Voir aussi : https://www.ccomptes.fr/fr/publications/la-filiere-epr

114. F.-M. Breon. Réchauffement climatique. https://www.humensciences.com/livre/Rechauffementclimatique/51.

On y aborde plus en détail les questions évoquées ici. 
- réduire les déplacements en voiture et le nombre de kilomètres parcourus;

- limiter la vitesse maximale sur autoroute car, même si le temps de trajet augmente, la consommation d'énergie nécessaire peut être significativement réduite;

- réduire la surface des logements, ou densifier les logements existants;

- diminuer la température de confort de ces mêmes logements en hiver, et l'augmenter en été pour limiter l'appel à la climatisation;

- prendre des douches plutôt que des bains;

- diminuer la production et la consommation d'objets de toutes sortes. Pour cela, on peut imaginer le partage d'un certain nombre d'objets qui n'ont pas un usage quotidien dans chaque foyer (Fig. 11.33);

- on pourrait aussi limiter le nombre d'écrans par foyer, de même que de nombreux autres appareils électroniques, etc.

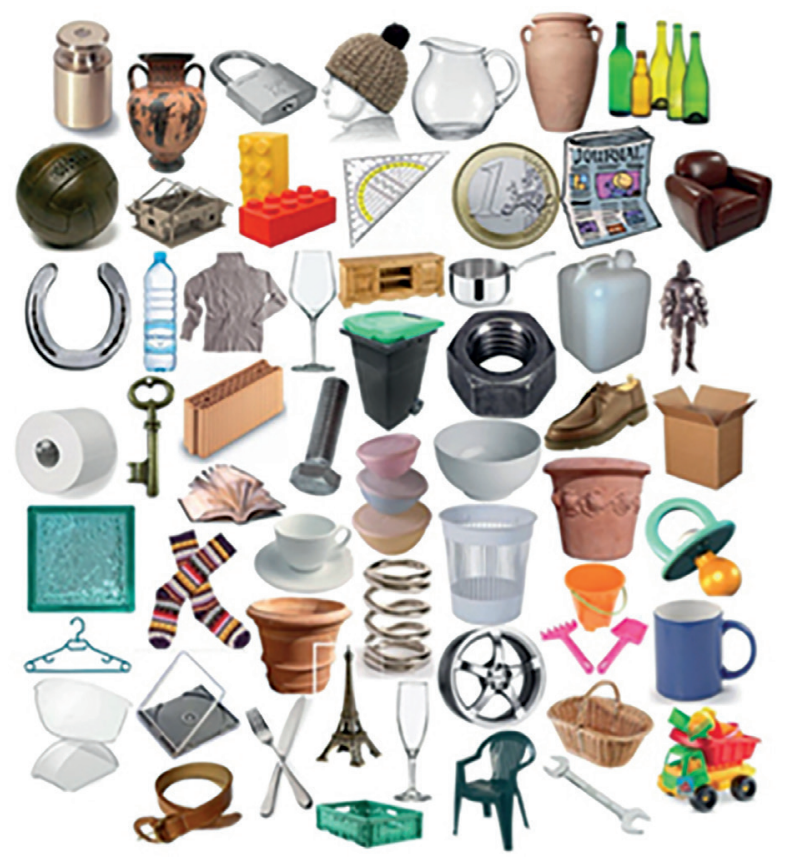

Figure 11.33: Les objets en tout genre qu'on accumule au fur et à mesure de notre vie de tous les jours. Source: web-ac-reims.

Toutes les modifications, que nous venons de suggérer, ont un impact immédiat, concret, quotidien sur notre façon de nous déplacer, de nous alimenter, de nous habiller, de nous distraire, etc. Les gouvernements peuvent orienter ces comportements individuels, par exemple, en taxant les pratiques énergivores ou mieux, par 
l'incitation fiscale. Mais, il est clair que les modifications de comportement passent d'abord par la compréhension de chacun face aux changements globaux et au changement climatique, en particulier. Chacun d'entre nous est tout aussi capable d'ajouter, de façon volontaire, bien d'autres actions à celles qui ont été mentionnées.

La sobriété ${ }^{115}$ s'applique non seulement aux individus, mais aussi aux entreprises, quel que soit leur secteur d'activité, ainsi qu'aux institutions publiques et aux administrations. Le monde de la recherche, y compris celui des laboratoires de recherche sur le climat, n'échappe pas au champ d'application des mesures de sobriété. La recherche sur le climat a, en effet, un coût énergétique: c'est un joli paradoxe! La modélisation (voir section 9.2) nécessite l'utilisation d'ordinateurs puissants, sur de grandes périodes. Or, non seulement ces machines consomment de grosses quantités d'énergie et nécessitent d'être refroidies, mais leur fabrication même n'est pas exempte de coûts énergétiques. Par ailleurs, les chercheurs se déplacent, que ce soit pour recueillir des échantillons sur le terrain ou pour échanger leurs résultats avec d'autres collègues. Devrait-on au vu de sa finalité sanctuariser la recherche sur le climat? Même si le sujet peut faire débat, les efforts doivent être partagés et il n'y a pas de raison objective de favoriser la recherche climatique plutôt que la santé, l'éducation ou le secteur de l'alimentation. La sobriété doit s'appliquer à tous les pans de la société, et c'est à l'État de la mettre en place.

\subsubsection{Gain d'efficacité dans le résidentiel et les transports}

Clairement, le passage à la sobriété dans nos modes de vie ne peut se faire que très progressivement, car il implique une transformation profonde de nos sociétés (voir section 10.2). Cependant, la sobriété énergétique n'est pas uniquement celle des usages. II est possible de diminuer notre consommation d'énergie grâce à l'amélioration de processus, sans que cela n'ait un impact notable sur notre mode de vie ${ }^{116}$. L'isolation des bâtiments est l'exemple même des progrès que l'on peut accomplir dans le secteur du résidentiel. La consommation d'énergie liée au chauffage représente, en effet, près du tiers des usages énergétiques en France. Or, les technologies d'isolation ont fortement progressé ces vingt dernières années et on sait désormais construire des bâtiments qui, à confort égal, consomment beaucoup moins d'énergie. Ceci a été décrit précédemment par Franck Richecœur (section 11.3). Des normes ont été instaurées qui visent à interdire de construire des «passoires thermiques». L'enjeu se porte désormais sur les bâtiments anciens qui devraient être également isolés, ce qui pose à la fois des problèmes de financement et de recours à des professionnels compétents.

115. C. Florian. De la sobriété énergétique à la sobriété matière: un concept clé pour la transition mais aux contours encore mal définis. https:/lapc-climat.fr/de-la-sobriete-energetique-a-la-sobrietematiere-un-concept-cle-pour-la-transition-mais-aux-contours-encore-mal-definis/.

116. La vision du gouvernement français sur la définition de l'efficacité énergétique. https://www. ecologie.gouv.fr/action-france-lefficacite-energetique. 
L'autre grand chantier concerne les transports. Les efforts ont surtout porté sur la technologie par le développement de moteurs plus efficaces. Mais le gain d'efficacité a été partiellement compensé par une augmentation du poids des véhicules. La consommation moyenne des voitures ces dernières années a même augmenté du fait de la part croissante des gros SUV (Sport Utility Vehicle) dans le parc automobile ainsi que du nombre croissant de kilomètres parcourus en moyenne par les automobilistes. Ceci vous a été également commenté par Franck Richecœur (voir section 11.3). II semble ainsi nécessaire de passer à des mesures supplémentaires pour la mise en place de normes environnementales qui limitent la masse des voitures. La limitation de la vitesse, déjà évoquée pour les aspects de sobriété (section 11.6.1), pourrait aussi conduire à la fabrication de voitures allégées, moins exigeantes en termes de matériaux de structure, et donc d'avoir des consommations plus faibles au km parcouru.

On peut aussi chercher à limiter le transport des marchandises et donc à consommer des biens qui sont produits localement plutôt qu'à l'autre bout du monde. II faut, cependant, être attentif à cette mesure, car la production locale est parfois plus énergivore que le transport, même sur de longues distances. Ainsi, il peut être plus efficace de faire venir des légumes du sud de la France ou même d'Espagne que de chercher à les faire pousser sur place sous des serres qui seraient chauffées au gaz. Consommer local doit s'accompagner d'une réflexion sur les modes de production des produits alimentaires et leur saisonnalité.

\subsubsection{Une meilleure efficacité dans notre consommation d'énergie : le tout électrique?}

En résumé des paragraphes précédents, il faut viser à une consommation plus sobre et des technologies optimisées pour consommer moins d'énergie pour le même service. Mais la marge de progression n'est pas infinie. Nous sommes face à un défi immense ${ }^{117}$, car la stabilisation du climat nécessite une diminution énorme des émissions de $\mathrm{CO}_{2}$ et autres gaz à effet de serre. On sait que la nature et certaines technologies peuvent absorber une partie de nos émissions et l'objectif affiché est de n'émettre pas plus que cette absorption. On parle alors de «neutralité carbone». Bien évidemment, cela demande des transformations profondes de notre société dont la mise en place prendra du temps.

En France, nous nous sommes fixés l'objectif d'arriver à la neutralité carbone à l'échéance 2050. Pour y arriver, il faudra donc se passer de plus en plus de combustibles fossiles. Nous avons vu qu'en termes de sources primaires d'énergie, l'exploitation de la biomasse et le développement de nouvelles énergies renouvelables figuraient parmi les solutions envisagées (section 11.4). Le bois des forêts et des

117. Résumé du rapport du GIEC SR15. Traduction Française par Citoyens pour le Climat: https://drive. google.com/file/d/1mH_lyAmLujMiSwZ8MmxiuDRKu32vrCv5/view lavec une présentation des scénarios envisageables pour le futur). 
broussailles est d'ailleurs utilisé pour le chauffage ou la cuisson depuis que les humains ont domestiqué le feu. On peut évidemment envisager d'autres usages énergétiques de la biomasse, y compris pour la production d'électricité. La biomasse peut elle-même être considérée comme une source d'énergie renouvelable, mais à la condition que l'on ne coupe pas les forêts plus vite qu'elles ne repoussent. La biomasse produite par déforestation ne répond évidemment pas au critère de soutenabilité!

Une autre option possible est l'usage plus intensif de l'électricité comme source secondaire d'énergie. L'électricité est, en effet, un outil extraordinaire pour être substitué aux combustibles fossiles, car elle peut s'appliquer à pratiquement tous les usages $^{118}$. Elle permet de se déplacer dans des trains ou dans des voitures équipées de batteries. Elle permet de fabriquer des objets, de s'éclairer, de faire fonctionner les appareils numériques ou encore de se chauffer. En revanche, elle a un gros défaut, car elle se «conserve» mal. Par exemple, pour stocker l'énergie contenue dans un litre d'essence, il faut une batterie de la technologie lithium-ion d'environ 50 kilogrammes. Clairement, il est plus facile de transporter son énergie sous forme liquide que dans une batterie, ce qui limite donc l'usage de l'électricité si nous souhaitons garder le niveau de mobilité dont nous bénéficions aujourd'hui avec les véhicules individuels. Néanmoins, les concepteurs de batteries ont accompli de grands progrès au cours de ces dernières années, et il est raisonnable d'envisager un futur proche dans lequel les déplacements terrestres seront majoritairement électriques. À l'inverse, à moins d'une rupture technologique majeure, cela ne semble pas crédible pour le transport aérien. La masse des batteries nécessaires n'est pas compatible avec les avions tels que nous les connaissons.

En ce qui concerne le chauffage, l'électricité est souvent présentée comme une aberration. Le chauffage électrique est d'ailleurs interdit dans de nombreux pays. L'électricité est considérée comme une énergie noble et la transformer en chaleur alors même qu'elle a souvent été générée à partir de chaleur - avec un rendement d'ailleurs assez mauvais - semble pour le moins absurde. Cependant, l'électricité peut être utilisée pour chauffer un bâtiment sur le principe inverse du réfrigérateur: la pompe à chaleur qui va extraire les calories de l'air extérieur ou du sol pour les injecter dans la pièce. Cette pompe fonctionne donc à l'électricité et permet un rendement global impressionnant (Fig. 11.34). Pour une calorie apportée par l'électricité, deux ou plus viennent de l'extérieur et peuvent donc être considérées comme renouvelables. Cette technologie, qui existe depuis longtemps et qui se répand actuellement, fait nettement gagner en efficacité et rend l'électricité attractive même pour le chauffage. La pompe à chaleur permet ainsi de regagner le rendement énergétique qui a été perdu au moment de la génération de l'électricité.

118. Chaîne YoutTube de «Le Reveilleur» (sur climat et énergie) : https://www.youtube.com/channel/ UC1EacOJoqsKaYxaDomTCTEQ. 


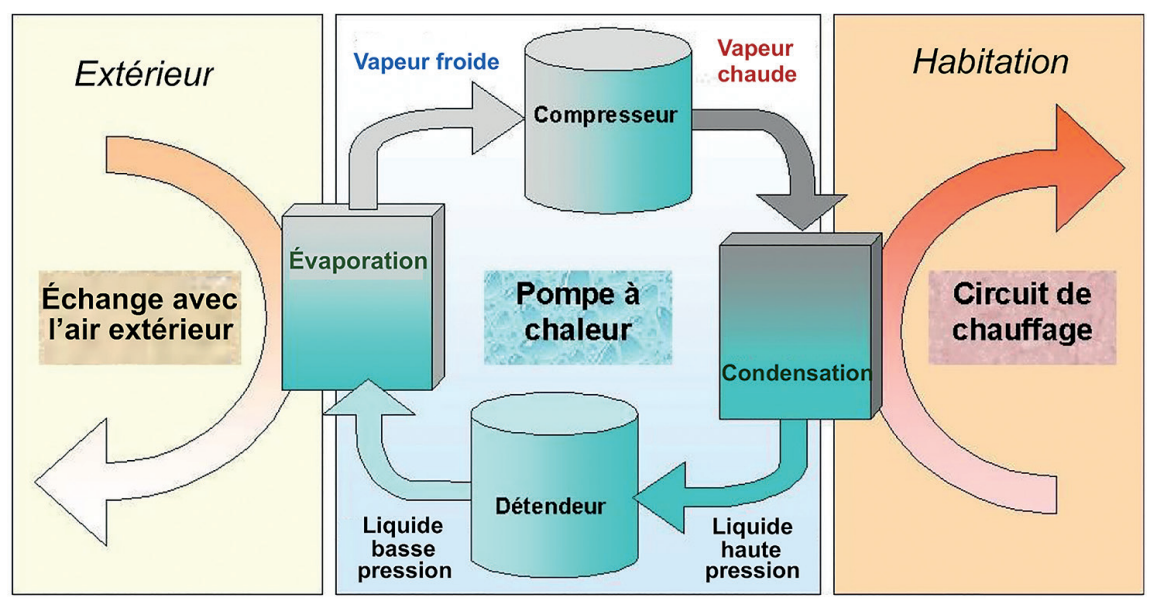

Figure 11.34: Schéma de principe d'une pompe à chaleur. Le liquide frigorigène (le même que dans un réfrigérateur) est vaporisé lors de l'échange avec l'air extérieur. Un compresseur comprime la vapeur et élève sa température. La vapeur chaude cède sa chaleur au circuit de chauffage de l'habitation. Le liquide obtenu par condensation est alors refroidi grâce à un détendeur, et un nouveau cycle peut recommencer. Source: Luna04 at fr.wikipedia.

II nous faut cependant revenir sur le fait que l'électricité n'est pas une source primaire d'énergie, mais qu'elle est obtenue par conversion d'une énergie primaire (chaleur, mouvement, lumière, gravité, chimique, nucléaire...) en énergie électrique. II faut donc d'abord la «fabriquer» afin de permettre les divers usages, que nous avons mentionnés. Aujourd'hui, une grande majorité de l'électricité produite dans le monde repose sur la combustion du charbon et, dans une moindre mesure, sur celle du gaz. Si nous voulons la neutralité carbone, il faudra nous en passer, ce qui nécessite de développer soit les énergies renouvelables, soit le nucléaire ${ }^{119}$.

Insistons quelque peu sur ce point en guise de conclusion. Limiter le changement climatique nécessite de diminuer fortement nos usages des énergies fossiles. On fait vite l'amalgame en affirmant qu'il faut donc diminuer notre consommation d'énergie, et donc notre consommation d'électricité. C'est une erreur puisque l'électricité peut être produite avec de très faibles émissions de $\mathrm{CO}_{2}$, que ce soit par des énergies renouvelables (section 11.4) ou du nucléaire (section 11.5). On peut donc parfaitement imaginer un futur dans lequel notre consommation totale d'énergie diminue, mais avec un transfert de nombreux usages fossiles vers de l'électrique, et donc une augmentation de la consommation de cette forme d'énergie.

Contrairement à ce qui est souvent affirmé, la lutte contre le changement climatique ne passe pas nécessairement par la diminution de la consommation électrique, mais par une sobriété énergétique, dans laquelle, potentiellement, l'électricité produite de manière décarbonée, prendrait une part majoritaire.

119. Résumé du rapport du GIEC SR15. Traduction Française: https://drive.google.com/file/d/1mH_ IyAmLujMiSwZ8MmxiuDRKu32vrCv5/view. 


\section{7. Émissions négatives de $\mathrm{CO}_{2}-\mathrm{C}$. Even}

Les émissions négatives font partie de ce que l'on appelle la "géo-ingénierie». Ce sont des procédés à grande échelle, qui visent à modifier le climat de manière volontaire, en particulier pour atténuer le réchauffement climatique dû aux activités humaines.

\subsubsection{Géo-ingénierie : traitements directs et indirects du réchauffement climatique}

La géo ingénierie se subdivise en deux grandes catégories de techniques:

(1) Celles qui visent à traiter directement le réchauffement climatique lui-même. Le but est alors d'aboutir à une diminution du rayonnement solaire incident sur la Terre et de refroidir ainsi la surface de celle-ci. Une façon d'obtenir ce résultat est d'augmenter la part du rayonnement solaire qui est renvoyé dans l'espace. Plusieurs processus peuvent être imaginés dans ce but, comme l'illustre la figure 11.35. Par exemple, l'un des processus (b) consiste à injecter des petites particules d'aérosols dans la haute atmosphère. Accumulées sous forme de nuages, elles réfléchiront en partie la lumière solaire incidente et réduiront, de ce fait, l'intensité solaire qui arrive à la surface de la Terre.

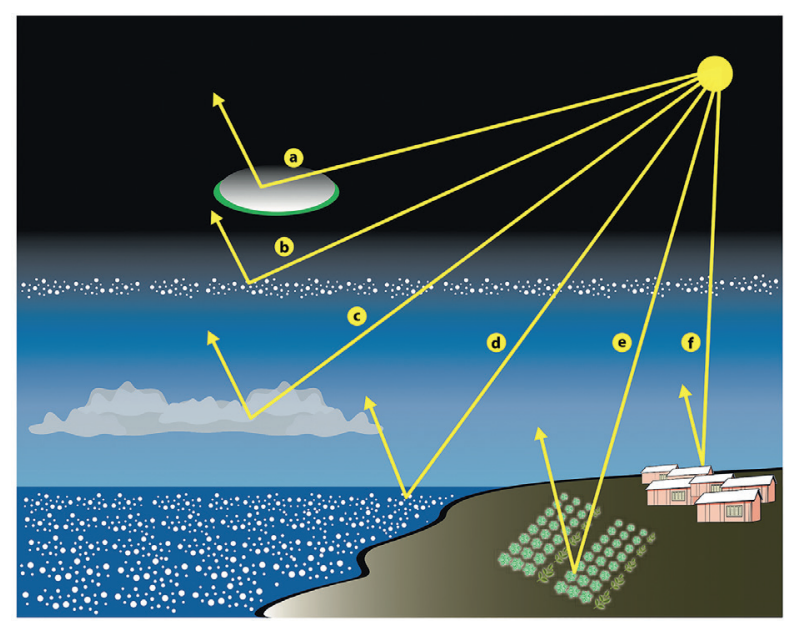

Figure 11.35: Processus imaginés pour réfléchir le rayonnement solaire et éviter qu'il ne soit absorbé à la surface de la Terre. (a): utilisation de satellites dans l'espace, (b): injection d'aérosols dans la stratosphère, (c) : «ensemencement» de nuages au-dessus de l'océan, (d): accroissement de la réflectivité de l'océan, (e): plantation de végétation réfléchissante, (f) : application de peinture blanche sur les toits et sur d'autres infrastructures de bâtiment. Source : [15].

Le principal avantage potentiel de ces techniques de refroidissement est leur rapidité. Elles pourraient agir à l'échelle de quelques années seulement avec une baisse de la température de quelques degrés en une décennie. Leur principal inconvénient est que, par exemple dans le cas des aérosols, il faudrait en réinjecter très régulièrement 
dans l'atmosphère. Si pour une raison ou pour une autre ce n'était pas fait, un réchauffement très rapide et de grande ampleur s'ensuivrait. À ceci s'ajoute la possible dangerosité des particules d'aérosols pour la santé humaine (voir section 2.3).

(2) La deuxième catégorie de techniques vise à agir sur la cause du réchauffement climatique, à savoir la trop grande quantité de gaz carbonique dans l'atmosphère, non pas par une baisse de nos émissions de $\mathrm{CO}_{2}$ (qui est de toute façon nécessaire), mais par des procédés de capture du $\mathrm{CO}_{2}$ en excès dans l'atmosphère. On parle ainsi d' émissions négatives», par opposition aux émissions (positives) qui consistent à ajouter du $\mathrm{CO}_{2}$ dans l'atmosphère. Ces techniques indirectes agiront plus lentement que celles visant au refroidissement direct, à l'échelle de plusieurs dizaines d'années au lieu de quelques années, mais elles auront le grand mérite de s'attaquer directement à la cause du réchauffement.

Le GIEC envisage dès à présent la réalisation d'émissions négatives dans différents scénarios de modélisation climatique (voir section 9.4.3), bien que pour l'instant aucune technique d'émissions négatives n'ait été mise en place à grande échelle. La figure 11.36 représente les émissions annuelles de $\mathrm{CO}_{2}$ qui sont prévues pour la fin de ce siècle selon différents scénarios, partant des émissions actuelles d'environ 40 Gt pour les années 2020 (courbe noire). Sont aussi indiqués sur la figure les niveaux d'augmentation de température moyenne correspondant aux différents scénarios.

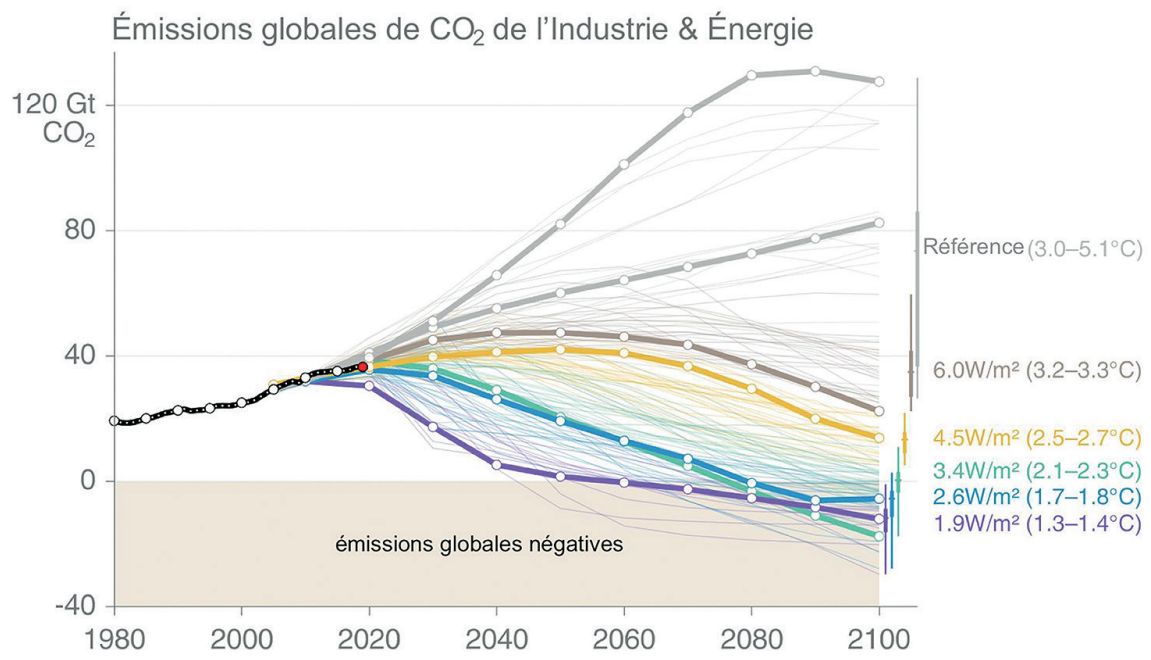

Figure 11.36: Évolutions des émissions globales de $\mathrm{CO}_{2}$ prévues jusqu'à la fin du siècle suivant les différents scénarios envisagés dans les modèles climatiques. Sont mis en correspondance les niveaux d'augmentation de la température moyenne qui seront atteints en 2100 par rapport à la température de l'ère préindustrielle. La courbe noire correspond à l'évolution enregistrée depuis le début du siècle jusqu'à 2019. Les autres courbes en traits gras correspondent aux scénarios considérés plus particulièrement dans le programme CMIP6 (programme d'intercomparaison entre modèles climatiques). Source: Global Carbon Project ${ }^{120}$. 
On constate que les scénarios, pour lesquels l'augmentation de température à l'horizon 2100 reste limitée à moins de $2{ }^{\circ} \mathrm{C}$ par rapport à l'ère préindustrielle, sont ceux qui incluent des techniques d'émissions négatives à grande échelle. La zone en ocre clair correspond aux scénarios pour lesquels les émissions globales de $\mathrm{CO}_{2}$ sont négatives.

\subsection{2. Émissions négatives par capture du $\mathrm{CO}_{2}$ atmosphérique [16]}

Les émissions négatives se subdivisent aussi en deux catégories:

(1) Celles qui consistent en un captage direct du $\mathrm{CO}_{2}$ atmosphérique par des procédés physico-chimiques. Par exemple, l'adsorption du $\mathrm{CO}_{2}$ atmosphérique sur un solide et son absorption par une solution liquide sont parmi les procédés chimiques envisagés. II nous faut souligner que ces techniques sont très énergivores, car il faut traiter de grandes quantités d'air afin d'extraire des quantités non négligeables de $\mathrm{CO}_{2}$. En effet, on rappelle que celui-ci ne représente qu'environ 0,04\% de l'atmosphère. Le $\mathrm{CO}_{2}$ ainsi récupéré pourra être, soit utilisé à des fins industrielles, soit stocké géologiquement. Ces techniques sont actuellement en cours de développement dans certaines usines d'Europe ou d'Amérique du Nord, par exemple.

(2) Les techniques de capture indirecte du $\mathrm{CO}_{2}$ atmosphérique, par le procédé biologique de photosynthèse. Rappelons à cet égard que la photosynthèse est à la base de la vie. En effet, c'est grâce à elle que les plantes peuvent pousser en produisant leur propre nourriture en présence de lumière et ce, à partir du $\mathrm{CO}_{2}$ de l'air et de l'eau venant du sol ou des précipitations. Les plantes sont elles-mêmes à la base de la chaîne alimentaire. La réaction chimique de la photosynthèse est la suivante:

$$
\mathrm{CO}_{2}+\mathrm{H}_{2} \mathrm{O}+\text { lumière } \rightarrow \mathrm{CH}_{2} \mathrm{O}+\mathrm{O}_{2}
$$

dans laquelle $\mathrm{CH}_{2} \mathrm{O}$ est la formule d'un sucre élémentaire, qui va servir à nourrir la plante. L'oxygène de l'air est produit uniquement par photosynthèse et renouvelé en permanence. En fait, il n'y a pas que les plantes qui réalisent la photosynthèse, mais aussi certains micro-organismes unicellulaires appelés micro-algues, qui comprennent aussi des bactéries et que l'on trouve dans l'eau, douce ou salée. Au total, environ la moitié de la photosynthèse est réalisée par les plantes et l'autre moitié par les microorganismes.

Revenons aux techniques d'émissions négatives basées sur la photosynthèse, qui sont essentiellement les suivantes:

- L'afforestation et la reforestation, qui consistent à planter des forêts, soit là où il n'y en avait pas avant (afforestation), soit là où il y en avait auparavant (reforestation). Ce sont les techniques les plus faciles à mettre en œuvre et les mieux maîtrisées. Cependant, leur effet sur le climat est controversé: en effet, l'afforestation dans les régions tempérées et boréales pourrait réduire l'albédo et résulter en un réchauffement net malgré la capture de carbone. 
Par contre, l'afforestation dans les régions tropicales tendrait à refroidir le climat grâce à la formation de nuages qui augmenteraient l'albédo, ainsi que par le captage du carbone.

- La fertilisation des océans : l'idée est de «booster » la photosynthèse réalisée par les micro-organismes marins, grâce à l'addition de nutriments dans l'océan, notamment du fer. Des études portant sur cette technique montrent que son potentiel de séquestration de carbone reste limité. De plus, une forte production de biomasse à la surface des océans cacherait la lumière pour les organismes marins vivant en-dessous et les empêcherait de réaliser eux aussi la photosynthèse. Cette technique aurait donc sans doute un fort impact sur la biodiversité marine.

- Les BECCS (BioEnergy with Carbon Capture and Storage), amplement cités par le rapport du GIEC d'août 2019 "Climate change and land» ${ }^{121}$. De quoi s'agit-il? On fait pousser de la végétation (plantes ou arbres), ce qui permet d'absorber du $\mathrm{CO}_{2}$ atmosphérique. On l'utilise ensuite comme combustible, par exemple pour alimenter une centrale thermique, ce qui fournit de l'électricité et/ou de la chaleur. Le $\mathrm{CO}_{2}$ réémis lors de la combustion est capté et stocké géologiquement. Cependant, d'après le rapport du GIEC cité ci-dessus, dans l'objectif d'un réchauffement de $1,5^{\circ} \mathrm{C}$ en 2100 , il faudrait utiliser jusqu'à 7 millions de $\mathrm{km}^{2}$ de terres arables, ce qui représente environ $11 \%$ de la surface agricole mondiale actuelle. De plus, le besoin en eau pour le même objectif serait de deux fois la consommation mondiale d'eau pour l'agriculture. On voit ainsi que la mise en œuvre de cette technique à grande échelle apparaît comme largement irréaliste.

En conclusion, vraisemblablement, aucune des techniques d'émission négative citées ne sera appliquée à grande échelle seule. On peut, par contre, imaginer qu'une combinaison de techniques pourra être utilisée. Enfin, il ne faut pas oublier que si les scénarios du GIEC prévoient des émissions négatives, la réduction de nos émissions (positives) reste l'objectif primordial.

\section{Références}

[1] F. Sarrazin, J. Lecomte. 2021. Transition évolutive et naturalité, in Protéger l'environnement. De la science à l'action (eds. Mermans, E. \& Dussault, A.-C.). Éditions Matériologiques.

[2] B. Morizot. 2016. Les diplomates. Wildproject.

[3] E.O. Wilson. 2016. Half-earth: our planet's fight for life. WW Norton \& Company.

121. Rapport du GIEC d'août 2019 «Climate change and land» : https://www.ipcc.ch/site/assets/uploads/ sites/2/2019/09/SR15_Summary_Volume_french.pdf 
[4] S. Durand. 2018. 20000 ans: ou la grande histoire de la nature. Éditions Mondes Sauvages.

[5] G. Billen, J. Garnier, M. Benoît, J. Anglade. 2013. La cascade de l'azote dans es territoires de grande culture du nord de la France. Cah. Agric 22 (4).

[6] World resources report: creating a sustainable food future - A Menu of Solutions to Feed Nearly 10 Billion People by 2050, Synthesis report p.17 (Dec. 2018).

https://research.wri.org/sites/default/files/2019-07/creating-sustainable-foodfuture_2_5.pdf

[7] H. Ritchie, M. Roser. 2020. - "Environmental impacts of food production". Published online at OurWorldInData.org. Retrieved from: https://ourworldindata.org/environmentalimpacts-of-food [Online Resource].

[8] C.L. Weber, H.S. Matthews. 2008. Food-miles and the relative climate impacts of food choices in the United States. Environ. Sci. Technol. 42, 3508-3513. https://doi.org/10.1021/ es702969f.

[9] T. Brunelle, M. Coat, V. Viguié . 2017. Demand-side mitigation options of the agricultural sector: potential, barriers and ways forward. OCL 24. https://doi.org/10.1051/ocl/2016051.

[10] IPCC Report 2019, Climate Change and Land: an IPCC special report on climate change, desertification, land degradation, sustainable land management, food security, and greenhouse gas fluxes in terrestrial ecosystems, p. 488. https://www.ipcc.ch/site/ assets/uploads/2019/11/SRCCL-Full-Report-Compiled-191128.pdf

[11] M. Khouchane. 2014. Réduction des émissions de gaz à effet de serre dans les transports. Éditions Universitaires Européennes.

[12] A. Madani, C. Bryant. 2019. L'adaptation aux changements climatiques dans le transport terrestre, "Regards croisés». Editions Econotrends Ltd.

[13] S. Porte. 2020. Le Dernier Avion - L'impact du trafic aérien sur l'environnement: mondialisation, tourisme, santé, dérèglement climatique - Voyager moins pour voyager mieux. Tana Editions.

[14] S. Pellerin, L. Bamière, L. Pardon. 2015. Agriculture et gaz à effet de serre - Dix actions pour réduire les émissions. Éditions Quae.

[15] K. Caldeira, et al. 2013. "The Science of geoengineering". Annu. Rev. Earth Planet. Sci. 41, 231--56.

[16] J.C.M. Pires. 2019. Negative emissions technologies: A complementary solution for climate change mitigation., Science of the Total Environment 672, 502--514 (2019). 


\section{Chapitre 12}

\section{Aller vers une transition écologique juste}

V. Masson-Delmotte, A. Vallet, S. Szopa et J.-M. Lourtioz

Au cours de cet ouvrage, nous avons évoqué à différentes reprises l'importance d'une transformation profonde de la société pour nous adapter et faire face aux changements d'ampleur que connaît notre planète. Nous avons, entre autres, souligné la nécessité d'une réduction des vulnérabilités face au changement climatique (section 10.3), la dimension participative que doit revêtir la gouvernance en matière de politique environnementale (section 10.5) et l'exploitation de nos outils juridiques pour corriger ou réduire les effets négatifs associés au droit de propriété, à la liberté contractuelle ou à la liberté du commerce et de l'industrie sur l'environnement (section 1.5). Dans ce chapitre, nous montrons comment les questions d'éthique, de justice sociale et d'équité participent effectivement à la transition écologique en prenant pour exemples la transition bas carbone, les stratégies d'adaptation et d'atténuation au changement climatique et la gestion de la biodiversité et des services écosystémiques. Nous apporterons notamment des éléments d'éclairage sur les questions suivantes:

- Qu'entend-on par justice climatique ou environnementale, par la notion de «transition juste»?

- Quelles sont les inégalités que peuvent engendrer ou renforcer les politiques environnementales?

- Quels sont les grands principes d'une transition écologique juste et perçue comme juste?

- Quels outils et solutions permettent de mieux prendre en compte les perspectives d'équité et les inégalités dans la mise en œuvre de la transition écologique? 


\subsection{Qu'entend-on par «transition écologique juste »?}

Pour introduire le concept de transition écologique juste, il convient au préalable de rappeler brièvement ce qu'impliquent les notions de justice, d'équité et d'impartialité.

La justice garantit les droits des personnes à partir des règles morales et juridiques d'impartialité et d'équité de traitement en s'appuyant notamment sur l'éthique et les valeurs de la société. Par extension, la justice environnementale, dont fait partie la justice climatique, relie développement et droits humains de sorte que les politiques relatives au changement climatique soient abordées dans une optique qui préserve les droits des plus vulnérables et répartisse avec équité et impartialité les responsabilités, les efforts et les avantages. En matière de changement climatique, cela concerne les actions visant à réduire les émissions de gaz à effet de serre, les actions d'adaptation au changement climatique, et la question des pertes et dommages [1-8].

L'équité suppose effectivement une répartition impartiale, sans favoritisme ni discrimination, des mesures et des efforts qui sont prévus pour faire face au changement climatique comme pour préserver la biodiversité. Ceci nécessite d'analyser au préalable la façon dont les coûts, les impacts ou les avantages des mesures envisagées seront répartis dans et par la société, que ce soit entre groupes sociaux, entre générations ou selon le genre.

On peut ainsi définir plusieurs niveaux de la société pour lesquels justice, équité et impartialité doivent être garantis en termes de stratégie et de politique environnementales. Tout d'abord, à un niveau individuel, on parlera, par exemple, d'une justice entre les ménages riches et les ménages pauvres. Sur un plan plus collectif, on veillera à ce que les différentes générations et les différentes communautés (par exemple, les communautés indigènes) soient traitées de façon juste et équitable, sans oublier que l'avenir des générations futures est à considérer en priorité. Concernant les acteurs du monde économique, certains secteurs d'activité pourront revendiquer de ne pas subir un coût démesuré dans la mise en œuvre de la transition écologique, par exemple, les salariés pourront demander à être traités équitablement selon les secteurs d'activité. À l'échelle internationale, nous reviendrons dans la suite sur les notions de justice et d'équité entre différents pays et différentes régions du monde.

De fait, le terme de justice a de multiples acceptions selon le contexte et pour les acteurs concernés, les justices revendiquées vont être différentes. Le concept de justice et son application à l'environnement sont, par nature, multidisciplinaires. On peut ainsi se placer indifféremment du point de vue économique (distribution équitable des impacts, des risques, des responsabilités et des bénéfices, etc.), du point de vue politique (justice dans la participation des acteurs aux processus de décision) ou du point de vue socioculturel (justice dans la reconnaissance des systèmes de valeurs, les préoccupations et les préférences de chacun). 
À défaut d'une définition unique, nous proposons de définir la «transition écologique juste» comme un "processus juste et équitable pour aller vers une société post-carbone et un développement résilient et soutenable».

Cette acceptation est pertinente aussi bien pour les délibérations entre les différents pays qu'à l'intérieur d'un pays, d'un secteur d'activité ou au sein des différents groupes sociaux qui cherchent à construire ces transformations. Les différents objectifs de développement durable (ODD proposés par l'ONU en 2015, Fig. 12.1) visent à prendre en compte les enjeux économiques, sociaux ou environnementaux pour mieux évaluer les conséquences des différents choix.
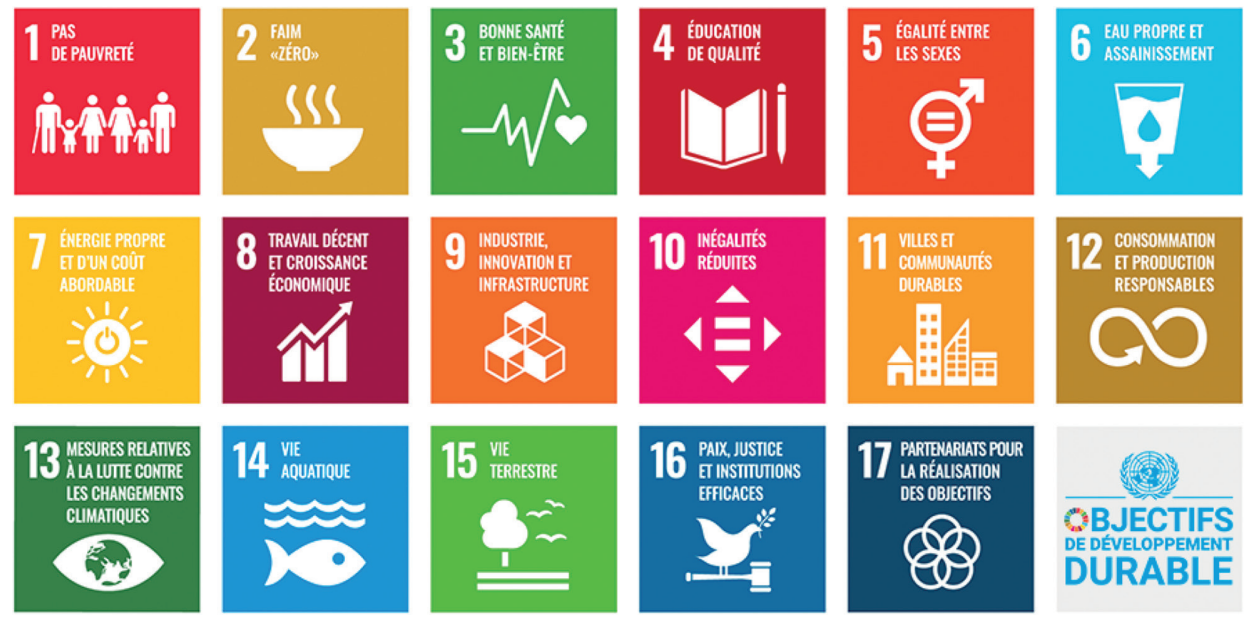

Figure 12.1: Objectifs du développement durable (ODD). Source: Unesco (https://fr.unesco.org/sdgs).

La notion de transition juste sur les aspects climatiques a émergé dans les années 1990 du fait de groupes de réflexion, universitaires notamment, des ONG, de partis politiques et de mouvements de la société. Cette notion est mentionnée dans l'accord de Paris de 2015 sur le climat et fait partie des éléments pris en compte dans le cadre de la convention climat des Nations Unies ou des projets d'action de l'Union Européenne. Elle est explicitement abordée dans les récents rapports du GIEC [9] et a été spécifiquement intégrée en France dans la stratégie nationale bas carbone et dans la lettre de mission pour la convention citoyenne pour le climat, en particulier sur les questions de justice sociale.

Ces enjeux autour de la justice et de l'équité se retrouvent aussi dans tous les textes internationaux en lien avec la biodiversité comme les rapports de l'IPBES sur les questions de biodiversité et de services écosystémiques [10]. On les retrouve également dans les prises de paroles des communautés natives et indigènes qui revendiquent leurs droits à interagir, bénéficier, entretenir la nature de façon équitable par rapport aux autres acteurs du territoire. 


\subsection{Inégalités distributives : un juste partage des externalités positive et négative de l'environnement?}

Les sources d'inégalités environnementales sont nombreuses. Nous les abordons ici selon quatre grands thèmes:

(i) responsabilités concernant les émissions de gaz à effet de serre;

(ii) impacts et conséquences des changements climatiques;

(iii) efforts pour la conservation de la biodiversité et des services écosystémiques;

(iv) bénéfices reçus du fonctionnement des écosystèmes.

\subsubsection{Responsabilités d'émissions de gaz à effet de serre}

Les inégalités vis-à-vis des émissions de gaz à effet de serre peuvent se décliner à différentes échelles: à l'échelle internationale, par pays, par habitant et en considérant plus spécialement la situation en France. Pour rappel, dans le monde, le premier secteur d'activité qui contribue au réchauffement climatique et perturbe le bilan radiatif de la terre est le secteur de l'énergie, du fait de la combustion des énergies fossiles (voir sections 2.1 et 7.3). Des émissions conséquentes de gaz à effet de serre sont également dues à l'utilisation des terres (voir section 5.1), en particulier pour la production alimentaire (voir section 11.2.3), avec des impacts très forts sur la biodiversité.

\section{Émissions annuelles totales de $\mathrm{CO}_{2}$ suivant les régions du monde}
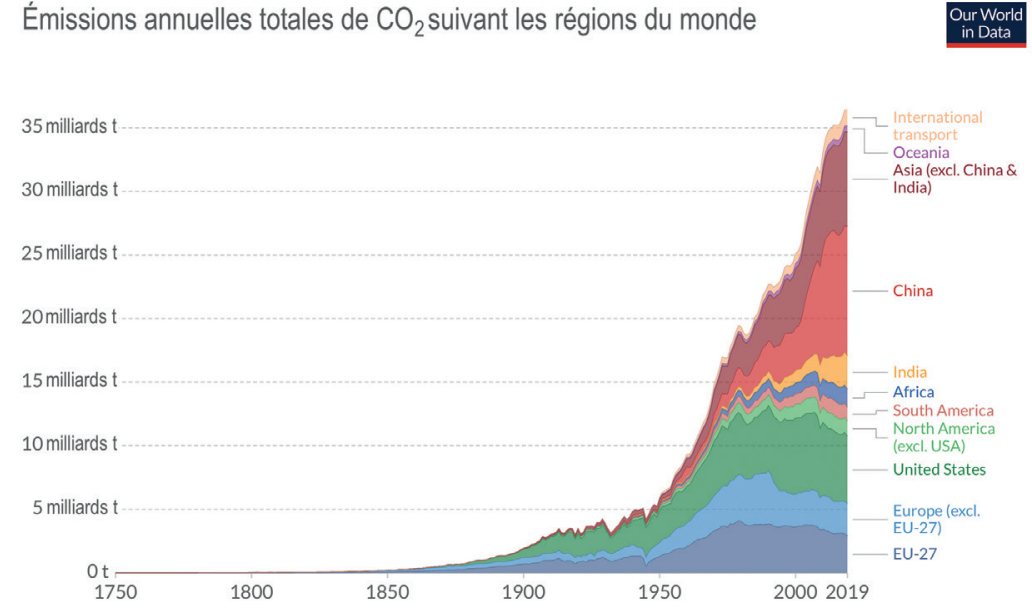

Figure 12.2: Évolution des émissions annuelles totales de $\mathrm{CO}_{2}$ depuis 1750 et suivant les régions du monde. Source: Our World in Data (https://ourworldindata.org/co2-and-other-greenhouse-gasemissions). 
Depuis le début de l'ère industrielle, la liste des pays les plus émetteurs de $\mathrm{CO}_{2}$, par leur utilisation d'énergies fossiles et leur production de ciment, a beaucoup évolué (Fig. 12.2). Par exemple, en 1950, la responsabilité du cumul des émissions était principalement le fait des États-Unis et de l'Europe, alors que ces dernières années, les émissions de ces pays ont stagné ou légèrement diminué tandis que celles de la Chine, par exemple, ont fortement augmenté de même que celles d'autres pays d'Asie comme le Japon ou la Corée du Sud. Ceci nous interroge quant à une responsabilité commune par rapport à l'effet cumulatif du $\mathrm{CO}_{2}$ sur l'évolution du climat. On voit que les perspectives sont différentes selon que l'on prend en compte les responsabilités historiques cumulées ou bien les responsabilités actuelles. Pour donner quelques ordres de grandeur, les sept principaux contributeurs aux émissions de gaz à effet de serre (Chine, États-Unis, Union Européenne, Inde, Russie, Japon et... l'aviation internationale, qui n'est pas un pays, mais qui se place au $7^{\mathrm{e}}$ rang mondial des émetteurs), sont aujourd'hui responsables de $65 \%$ des émissions de $\mathrm{CO}_{2}$ dans le monde et le $\mathrm{G} 20$ (les vingt économies les plus importantes dans le monde) a contribué à près de $80 \%$ de ces émissions.

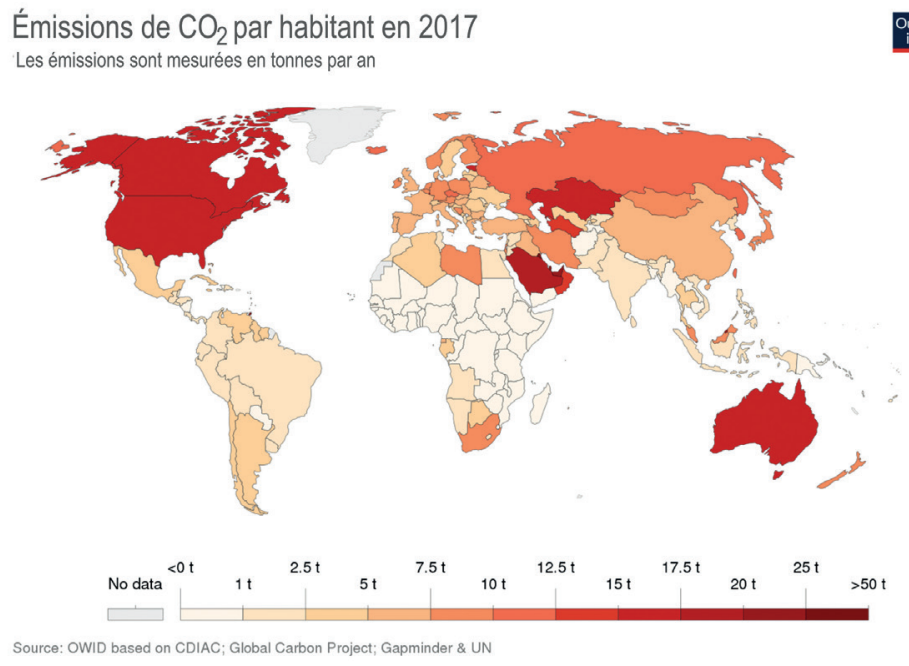

Figure 12.3: Émissions de $\mathrm{CO}_{2}$ par habitant et par an dans le monde en 2017. Source: Our World in Data https://fr.wikipedia.org/wiki/Fichier:CO2_emissions_per_capita,_2017_(Our_World_in_Data).svg.

Par habitant, le panorama est un peu différent (Fig. 12.3). Des pays comme la Chine, qui apparaissent globalement comme de très gros émetteurs, ont une population très importante et, de ce fait, des niveaux intermédiaires d'émission par habitant. Ramenées par habitant, les émissions reflètent davantage le niveau de vie de chaque pays et son mix énergétique. Ainsi, les émissions de gaz à effet de serre par habitant sont particulièrement importantes en Amérique du Nord, en Australie, dans les pays du golfe Persique ou en Russie. Dans le cadre de l'accord de Paris sur le climat, différents pays se sont engagés en 2015 à réduire leurs émissions. Les cinq pays du G20, qui n'arrivent pas à tenir leurs engagements, bien qu'ils soient parmi les 
économies les plus puissantes de la planète, sont l'Australie, le Brésil, le Canada, la Corée du Sud et les États-Unis d'Amérique. Or, non seulement, ces pays contribuent à une part importante des émissions de $\mathrm{CO}_{2}$ à un niveau global, mais sont également ceux ayant les émissions par habitant les plus élevées ${ }^{122}$.

La situation en France est assez différente, car notre électricité est essentiellement décarbonée, en raison des proportions importantes de l'hydroélectricité et de l'énergie nucléaire dans la production électrique. Les secteurs qui émettent le plus de gaz à effet de serre en France sont les transports (30\%), les bâtiments (20\%), puis viennent ensuite l'agriculture, l'industrie et d'autres secteurs (voir section 11.3) ${ }^{123}$. Notons que ce qui relève des modes de vie individuels, à savoir les voitures individuelles et le chauffage des logements, représente plus de la moitié des émissions du secteur des «transports» ou du secteur résidentiel, respectivement. C'est une caractéristique que l'on retrouve dans le monde entier: deux tiers des émissions dans le monde relèvent des modes de vie des ménages.

La France apparaît plutôt parmi les bons élèves au niveau mondial lorsque l'on regarde son empreinte carbone qui est de l'ordre de 6,6 tonnes d'équivalent $\mathrm{CO}_{2}$ par personne et par an à condition de ne prendre en compte que les émissions produites sur le territoire national. Ces émissions ont baissé à partir des années 1990, mais stagnent ces dernières années, exception faite de 2020 en raison des confinements. Néanmoins, l'empreinte carbone des Français est quasiment doublée si l'on considère le transport international et le bilan net du commerce mondial, obtenu en soustrayant les émissions liées à ce que l'on exporte et en ajoutant celles liées à ce que l'on importe. L'empreinte carbone s'élève alors pour la France à environ 11 tonnes d'équivalent $\mathrm{CO}_{2}$ par personne et par an, ce qui s'explique par la part importante des importations de biens manufacturés que nous achetons. Notons, en outre, que ces émissions ne prennent pas en compte l'effet de nos importations sur les modifications d'usage des terres dans d'autres régions, par exemple, la déforestation importée, celles-ci restant difficiles à estimer avec précision.

II y a également une grande dispersion de l'empreinte carbone individuelle selon les catégories de revenus des ménages et les analyses récentes, faites pour le Haut Conseil pour le Climat, font état d'un facteur 4 environ en France entre l'empreinte carbone des ménages, qui font partie des $10 \%$ les plus modestes, et celle des ménages parmi les $10 \%$ les plus aisés. À titre de comparaison, une étude d'OXFAM de 2020 montre qu'au niveau mondial, les 1\% de personnes les plus riches émettent deux fois plus de gaz à effet de serre que les $50 \%$ de personnes les plus pauvres.

La question des inégalités s'inscrit donc aussi bien entre individus qu'entre pays, et les émissions de gaz à effet de serre sont bien en relation avec le mode de vie et le niveau de vie.

122. UNEP Emission Gap Report. https://www.unep.org/emissions-gap-report-2020

123. a) Section A3, du rapport 2020 du Haut Conseil pour le climat, p. 34, www.hautconseilclimat.fr.

b) Rapport 2021 du Haut Conseil pour le climat, Renforcer l'atténuation, engager l'adaptation, https://www.hautconseilclimat.fr/wp-content/uploads/2021/06/HCC-rappport-annuel-2021.pdf. 
Le niveau de réchauffement actuel atteint $1,1^{\circ} \mathrm{C}$ en moyenne au-dessus des continents et de l'océan pour la dernière décennie, par rapport à la période 1850-1900, et résulte du déséquilibre du bilan d'énergie de la Terre du fait des activités humaines. Le niveau futur du réchauffement va dépendre en premier lieu du cumul des émissions passées, présentes et futures de $\mathrm{CO}_{2}$, ainsi que des émissions des autres facteurs qui agissent sur le climat. Pour limiter son ampleur, on dispose d'une certaine marge de manœuvre que l'on peut exprimer en «budget carbone compatible» (voir section 9.4). De plus en plus de pays prennent des engagements pour atteindre la neutralité carbone à différents horizons temporels, que ce soit la France, l'Union Européenne dans son ensemble, la Corée du Sud, la Chine ou le Japon. Ceci va de pair avec une meilleure compréhension de la manière dont le climat fonctionne et de la nécessité d'une volonté collective à limiter les émissions de $\mathrm{CO}_{2}$, si on veut stabiliser le réchauffement.

Si on regarde les «budgets carbone restants», se pose la question de leur répartition équitable et impartiale selon les différents pays. Pour répondre aux questions de justice et d'équité, il faudrait prendre en compte les responsabilités historiques ou actuelles des uns et des autres ainsi que leur capacité à agir, qui est en fait très hétérogène en termes de ressources financières, technologiques et de compétences. En France, la stratégie de réduction des émissions de $\mathrm{CO}_{2}$ se traduit par un budget carbone qui est un objectif d'émissions sur plusieurs années. La France a cependant échoué à tenir son premier budget et a dû revoir à la baisse son engagement pour le second. La trajectoire initiale avait été construite sur la base d'une taxe carbone qui allait être croissante, mais cette taxe a été gelée suite aux mouvements « des gilets jaunes». En 2019, le gouvernement a constitué une Convention Citoyenne pour le Climat afin de recueillir leurs propositions pour mettre en œuvre les objectifs de baisse des émissions de gaz à effet de serre, et respecter les budgets carbone d'ici à 2030, dans un esprit de justice sociale.

\subsubsection{Impacts et conséquences des changements climatiques}

L'ampleur des risques climatiques est évidemment liée à l'ampleur du réchauffement. Cependant, ces impacts se font d'ores et déjà sentir sur des systèmes fragiles comme les récifs de coraux tropicaux et la glace de mer de l'Arctique (voir sections 5.3 et 8.3), mais aussi par la récurrence d'événements extrêmes dont des vagues de chaleur de plus en plus intenses sur les continents, l'intensification des pluies torrentielles dans de nombreuses régions et une intensification des sécheresses avec des questions de sécurité humaine et de sécurité des ressources en eau (voir section 2.4). Sont en particulier affectées de grandes régions clés pour la production alimentaire mondiale comme les greniers à blé. II est aussi nécessaire d'envisager les risques de changements abrupts et irréversibles, comme, par exemple, une déstabilisation des glaces de l'Antarctique qui pourrait contribuer à la montée du niveau des mers sur plusieurs siècles avec des enjeux sur toutes les régions littorales. Les impacts des changements climatiques englobent donc à la fois 
des tendances graduelles, des effets chroniques et des effets aigus via des événements extrêmes plus fréquents et plus intenses.

Cela étant, les risques ne résultent pas que des situations que nous venons d'évoquer, et de leur probabilité, mais aussi des conditions d'exposition à ces situations et de la vulnérabilité des écosystèmes, des individus et des communautés qui y sont exposés (voir section 10.3) et des stratégies de gestion de risque et d'adaptation. Ce n'est, par exemple, pas la même chose, en termes d'impacts, de vivre dans une plaine, sur une terre basse en littoral ou dans les régions de haute montagne. Par ailleurs, les systèmes de mutualisation de risques (protection sociale, assurance) permettent de limiter les conséquences individuelles des aléas climatiques. La capacité à s'adapter à un climat qui change, dépend des ressources individuelles, et d'actions structurelles, plus difficiles à mettre en œuvre dans les pays les plus pauvres. Des ressources dépend, par exemple, la capacité à construire des ouvrages de protection pour gérer des crues ou des risques de submersion côtière. Tout ceci pose la question de la limite aux capacités d'adaptation, lesquelles sont donc fonction du contexte, de l'accès aux technologies, des ressources et de faculté d'un individu, d'une communauté ou d'un écosystème à faire face à des conditions qui changent fréquemment. Des effets en cascade peuvent aussi se produire: le climat change, ce qui entraîne des effets physiques ou biogéochimiques, lesquels affectent en retour les écosystèmes. Certaines sociétés vont y être exposées alors que d'autres ne seront exposées qu'à des effets plus ponctuels, de moindre ampleur et avec moins de risques en cascade.

Le rapport SR1.5 du GIEC [9] a combiné spatialement 14 indicateurs d'impacts (relatifs à l'eau, l'énergie, la chaleur extrême, les rendements agricoles, etc.) pour mettre en évidence les "points chauds» du globe associés aux risques climatiques les plus prononcés. L'intensité des risques est particulièrement marquée dans un certain nombre de régions tropicales, notamment en Asie du Sud-Est, en Afrique de l'Ouest ou dans les régions du pourtour méditerranéen. Ce sont aussi des régions où il y a une population vulnérable très dense, avec des revenus très faibles. Ainsi, le sud de l'Afrique et la région méditerranéenne vont devoir faire face à des problèmes de ressources en eau et de chaleur extrême. La chaleur très intense en Afrique de l'Ouest et au Sahel aura des conséquences potentiellement sévères sur les rendements des cultures et la productivité du bétail avec des enjeux en termes de sécurité alimentaire. De manière générale, les régions tropicales auront à faire face à la dégradation des ressources issues de la pêche. Les petits états insulaires en développement seront en outre menacés par la montée du niveau des mers et la dégradation des récifs de coraux.

D'autres écosystèmes, à d'autres endroits du globe, sont également menacés, comme dans le cas du recul de la glace de mer en Arctique et ses conséquences sur les écosystèmes marins ou le dégel des sols de l'Arctique et ses conséquences sur la végétation. Les vagues de chaleur plus intenses en mer tendront à aggraver le blanchiment des coraux et à dégrader ces écosystèmes avec des effets en cascade 
sur d'autres espèces marines. Outre l'Arctique, on voit aussi apparaître des «points chauds » dans les régions de haute montagne avec le recul des glaciers, qui peut non seulement entrainer des risques locaux mais aussi des problèmes de ressources en eau plus en aval.

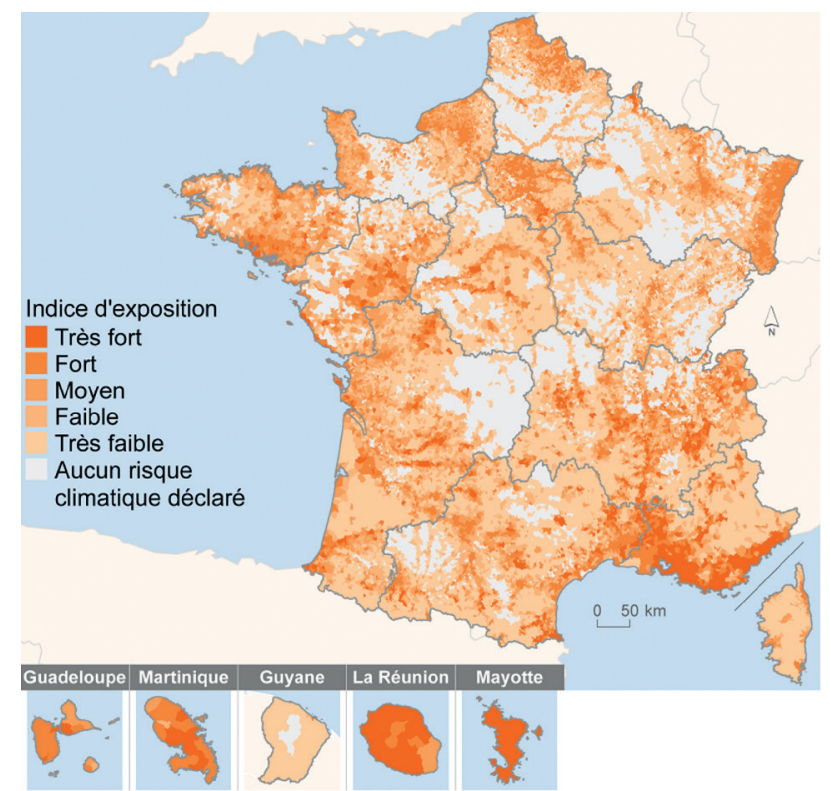

Figure 12.4: Carte des expositions aux impacts climatiques (inondations, avalanches, tempêtes et cyclones, feux de forêt, mouvements de terrain) établie en 2016 pour la France métropolitaine et les départements ultramarins. Source: MTES, Gaspar, 2017 - Insee, RP, 2014 - @IGN, BD Carto ${ }^{\circledR}, 2016$, Traitements: SDES, 2019.

Sur le territoire français (métropole et outre-mer), les inégalités face aux changements climatiques sont également présentes avec les indices d'exposition particulièrement forts dans certains territoires d'outre-mer, sur le pourtour de la méditerranée, autour des régions de montagne et le long des cours d'eau (Fig. 12.4). Deux tiers de la population sont exposés à des inondations ou des mouvements de terrain. Près de $16 \%$ des communes sont concernés par les feux de forêts et des mouvements de terrain, cette fois, plutôt liés aux conditions sèches. À cela s'ajoutent les risques accrus d'avalanche en haute montagne ou de tempêtes tropicales et d'ouragans dans les territoires d'outre-mer. Environ $18 \%$ de la population française est ainsi soumise à des niveaux de risque relativement importants lorsque l'on croise les différents types de risques. En revanche et fort heureusement, il y a des exceptions, des territoires peu vulnérables, surtout situés en zone rurale et qui concernent $20 \%$ des communes et environ $5 \%$ de la population ${ }^{124}$.

124. Rapport 2021 du Haut Conseil pour le climat, Renforcer l'atténuation, engager l'adaptation, https:// www.hautconseilclimat.fr/wp-content/uploads/2021/06/HCC-rappport-annuel-2021.pdf 
On réalise ainsi que les expositions et les vulnérabilités sont très contrastées à la fois au niveau international, d'un pays à l'autre, mais aussi à l'intérieur d'un même pays, ce qui souligne les enjeux de coopération et de solidarité posés par le changement climatique.

\subsubsection{Efforts pour préserver la biodiversité et les services écosystémiques}

Comment juger de la répartition inégale des efforts que doivent fournir les pays pour protéger la biodiversité et les écosystèmes à l'échelle globale de notre planète? La biodiversité peut se mesurer grâce à un indicateur qu'on appelle la richesse spécifique et qui décrit le nombre d'espèces présentes dans un milieu: les mammifères, les reptiles, les amphibiens, les oiseaux, etc. La biodiversité est impactée par différentes menaces, qui découlent principalement des activités humaines et qui sont identifiées dans le rapport d'évaluation de l'IPBES publié en 2019. Ces menaces concernent, par exemple, le changement d'utilisation des terres, la déforestation, la surexploitation des ressources, la pollution, les espèces invasives et aussi le changement climatique lui-même (voir sections 5.1, 5.2, 5.3, 5.4 et 5.5). Certains territoires ont la chance d'avoir une richesse spécifique très élevée, mais sont en même temps soumis à des menaces très intenses: on les définit comme des points chauds lou « hotspots») de la biodiversité (Fig. 12.5).

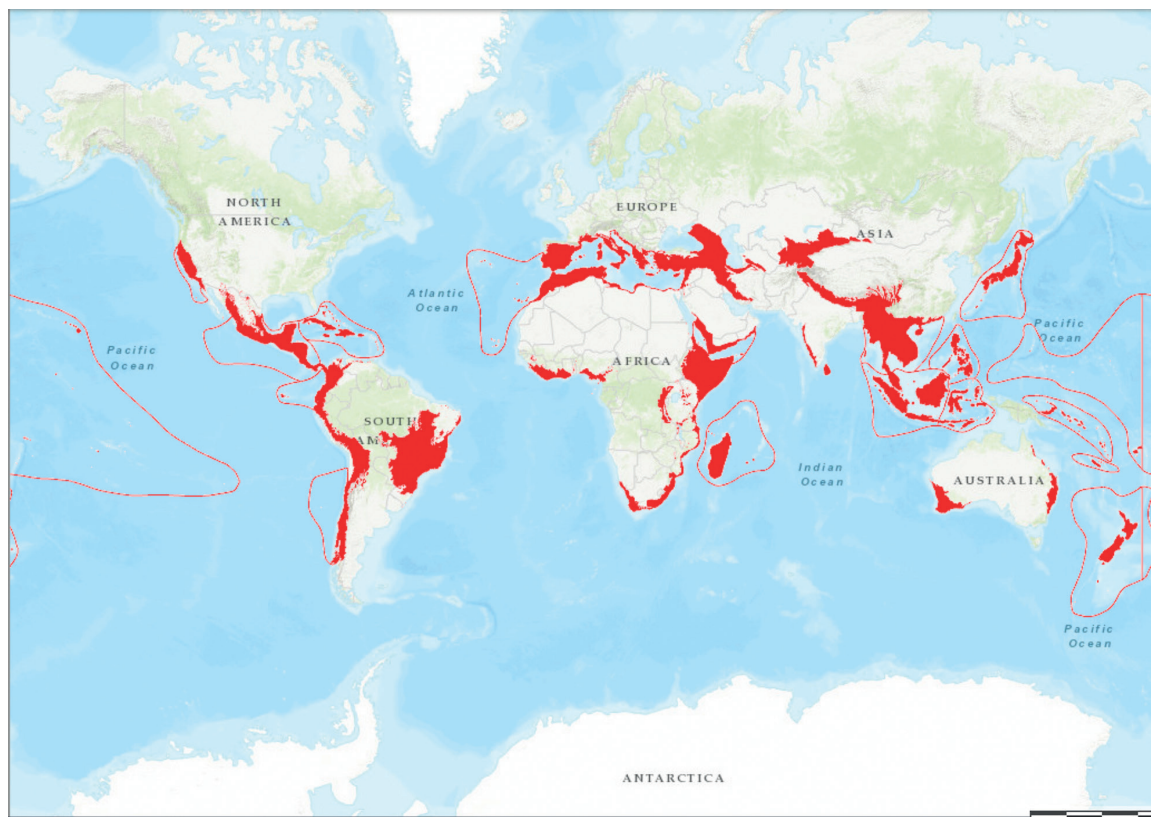

Figure 12.5: Hotspots de la biodiversité (en rouge sur la carte), qui correspondent aux régions avec de forts enjeux, car recélant une grande diversité d'espèces, mais aussi avec de fortes menaces sur cette diversité. Source: Conservation Synthesis, Center for Applied Biodiversity Science at Conservation International http://www.biodiversityhotspots.org/xp/Hotspots/resources/maps.xml [11] - tous droits réservés. 
Ces hotspots de biodiversité peuvent être terrestres, mais également marins. Leur répartition selon les différentes régions du monde résulte à la fois de la distribution de la richesse spécifique en biodiversité et de la distribution des menaces. Ainsi, la richesse spécifique pour trois grands taxons: les oiseaux, les mammifères et les amphibiens, est assez bien corrélée, en termes de distribution spatiale, à celle des forêts denses, la forêt amazonienne, les forêts d'Afrique centrale, au Congo principalement, et les forêts d'Asie du Sud. La densité de population est généralement considérée comme une bonne "mesure» des menaces sur cet environnement. Or, ces menaces sont réparties de façon très hétérogène. Ainsi, dans le bassin amazonien, il y a très peu de menaces car il y a une densité de population assez faible, ce qui explique que l'Amazonie ne soit pas dans son intégralité considérée comme un hotspot de la biodiversité au sens strict, même si elle est concernée par des enjeux en termes de conservation. Les hotspots de biodiversité se situent essentiellement en Amérique latine, en Asie du Sud-Est, dans le bassin méditerranéen et en Afrique sur toute la côte est notamment. En conséquence, tous les pays situés dans ces régions ont plus d'efforts à fournir que les autres pour préserver leur biodiversité.

Si l'on se focalise plus particulièrement sur les "gros» animaux, ce qu'on appelle la mégafaune, et que l'on considère la surface des aires où ils sont protégés et les dépenses qui sont liées à leur préservation, on constate que les efforts mis en œuvre pour protéger cette biodiversité ne sont pas forcément répartis sur les hotspots de biodiversité (Fig. 12.6). Par exemple, le Brésil qui était un des pays où la richesse spécifique était l'une des plus élevées, se situe dans une fourchette moyenne
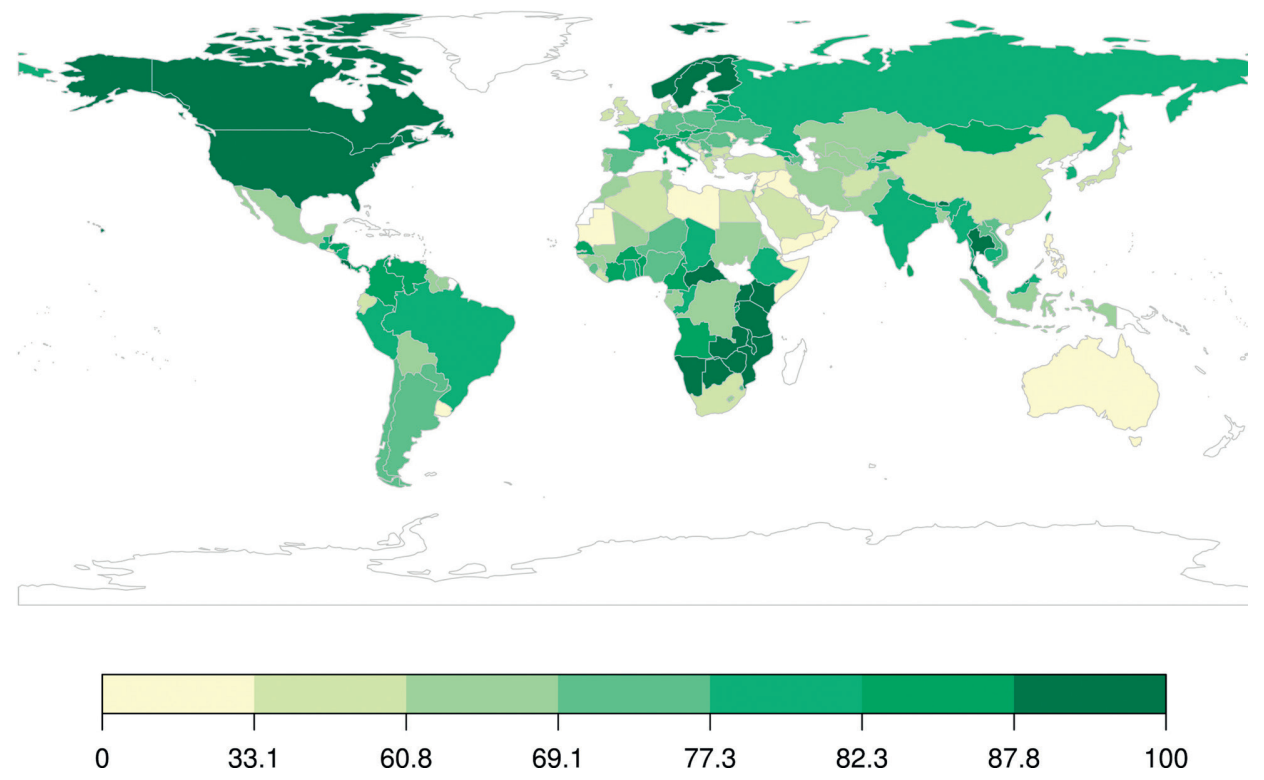

Figure 12.6: Répartition des efforts consentis dans les différentes régions du monde pour protéger la mégafaune. Source: Lindsey et al., 2017 [12]. 
en termes d'efforts pour préserver la mégafaune. Les pays de l'axe andin, également identifiés comme hotspots, sont parmi ceux qui fournissent les efforts les plus faibles dans ce domaine. En revanche, en Afrique, sur la côte est, de nombreux efforts de conservation sont mis en œuvre. De même, bien qu'il y ait très peu de hotspots de biodiversité en Amérique du Nord, des efforts conséquents ont été consentis pour la préservation de la mégafaune.

Les écarts d'un pays à l'autre ou d'une région à l'autre s'expliquent par l'ampleur de l'effort à fournir pour protéger la biodiversité et le coût que cet effort représente en termes de choix politique pour le pays ou la région en question. Un exemple récent est celui du Brésil et du président Bolsonaro dont le choix politique en faveur du développement de l'agro-industrie s'est traduit par une position ouvertement anti-conservation. L'obligation de protéger la biodiversité, imposée par la diplomatie internationale, était alors perçue et dénoncée comme une atteinte à la souveraineté nationale.

\subsubsection{Bénéfices reçus du fonctionnement des écosystèmes}

Une autre illustration des inégalités en matière de biodiversité concerne la répartition des bénéfices tirés du fonctionnement des différents écosystèmes, que l'on appelle aussi services écosystémiques (voir section 4.5). II existe trois grandes catégories de services écosystémiques: les services d'approvisionnement comme la production de biens (production de bois, production d'eau douce, etc.), les services de régulation (régulation du climat, séquestration de carbone, protection contre les inondations, etc.) et les services dits culturels qui sont plutôt de l'ordre de la satisfaction intellectuelle (l'inspiration, la beauté scénique, le lien avec les activités pratiques de sport en extérieur, etc.). La facilité d'accès à ces services pour les uns et la difficulté d'accès pour les autres peuvent être sources d'inégalités importantes.

Un exemple parmi d'autres concerne le partage des ressources en eau, qui peut être générateur de conflits entre pays, comme il a été mentionné dans la section 1.5 de cet ouvrage traitant des droits internationaux. Les conflits peuvent aussi se manifester à un niveau plus local entre communautés urbaines et communautés rurales dispersées, comme illustré sur la figure 12.7 dans la région d'Abancay, dans les Andes péruviennes où l'accès aux ressources en eau a été à l'origine de conflits importants [13]. Un projet intégrant la protection, la restauration et la gestion des écosystèmes où se trouve la source d'eau de la ville y a fait l'objet d'une coopération entre le Pérou, la France et l'Union Européenne. De tels exemples sont nombreux et concernent aussi bien les pays en voie de développement que les pays développés. De manière générale, les bénéfices tirés des écosystèmes sont distribués de façon inéquitable dans la société. 
Légende

$\Delta$ Pics

- Implantations humaines

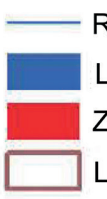
Rivières

Lacs

Zones furbanisées Limites du site étudié

\section{Altitude (m)

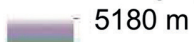 \\ $1613 m$}
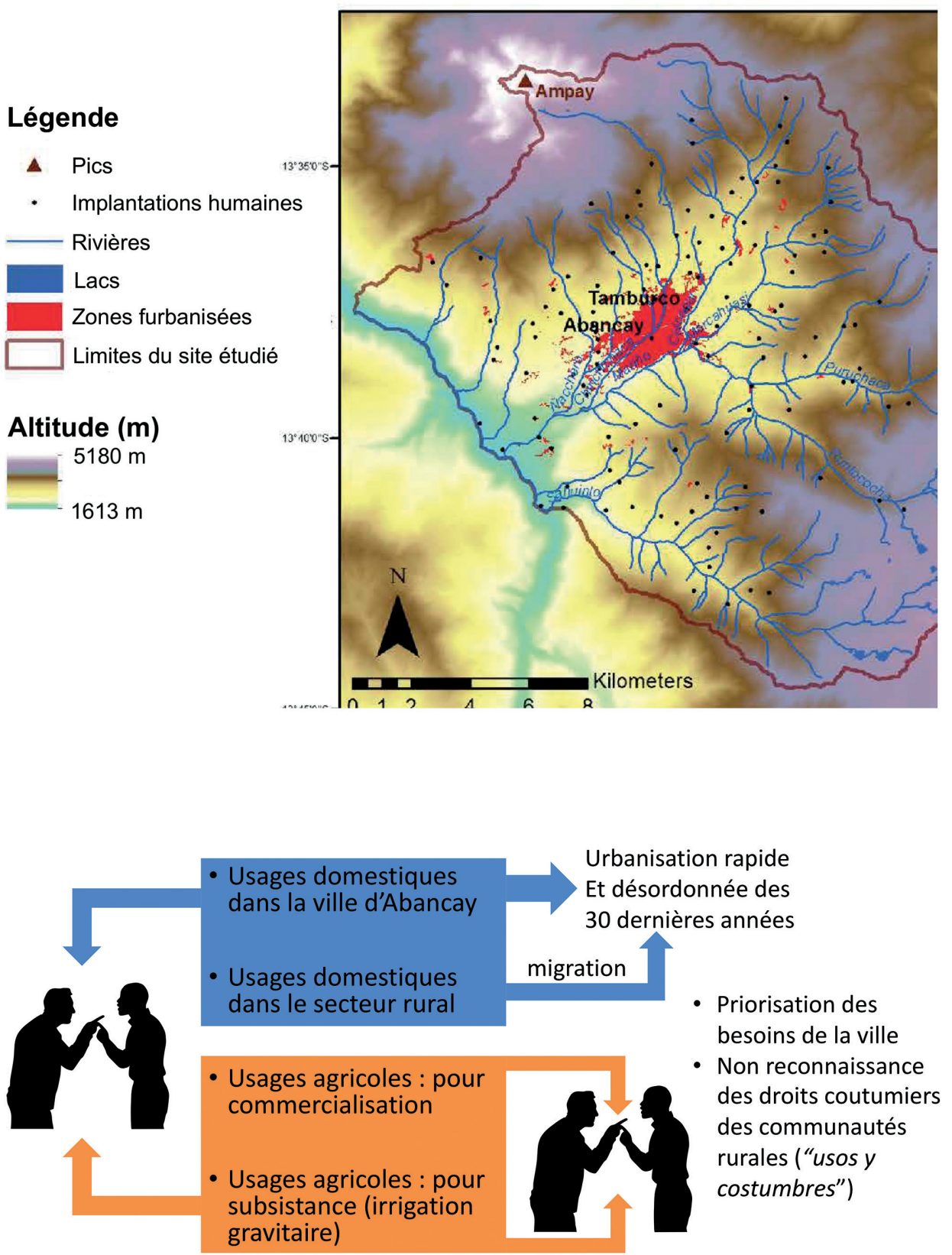

Figure 12.7: Conflits pour le partage de l'eau douce dans la région d'Abancay au sein des Andes péruviennes. Source image: Améline Vallet. 


\subsection{La dimension participative dans les prises de décision en politique environnementale}

\subsubsection{L'exemple des négociations internationales sur le climat}

Lors des négociations internationales sur le climat, comme celles qui ont conduit à l'accord de Paris ${ }^{125}$, chaque pays compte théoriquement pour une voix et peut bloquer un consensus entre les autres pays. La conduite d'un accord résulte de négociations permanentes, mais aussi de rapports de force qui évoluent au cours du temps. Si l'on examine de près la rédaction de l'accord de Paris, on peut lire en filigrane qu'il est le résultat de compromis avec des pays qui sont, soit dans des situations spécifiques de responsabilité, soit dans des situations de vulnérabilité. Par exemple, dans le préambule, il est mentionné que "les pays peuvent être touchés non seulement par les changements climatiques, mais aussi par les effets des mesures prises pour faire face à ces changements». II s'agit, dans ce cas, d'une mention émanant de pays très dépendants des énergies fossiles.

Un autre point de l'accord porte sur les impératifs d'une transition juste pour la population active avec mention des enjeux de reconversion pour les salariés de secteurs d'activité amenés à se transformer. Un autre point encore concerne les peuples autochtones, soucieux de l'intégrité des écosystèmes, de la protection de la biodiversité, reconnue par certaines cultures comme la terre nourricière, et des questions de justice climatique. Enfin, dans la rédaction de l'accord, des principes sont énoncés faisant référence à l'équité, à des responsabilités communes mais différenciées et à des capacités également différenciées eu égard aux différentes conditions nationales. L'accord de Paris résulte donc de tous ces compromis et d'une recherche de consensus entre des pays qui ont des configurations très différentes. Il témoigne aussi des rapports de forces entre les différents pays.

\subsubsection{L'exemple de la gouvernance des services écosystémiques}

Des rapports de force similaires se retrouvent dans la gouvernance des services écosystémiques, par exemple, à des échelles locales comme nous l'avons évoqué précédemment pour le partage de l'eau et les conflits autour de ses usages (Fig. 12.7). On peut montrer qu'il existe un déséquilibre, une asymétrie, entre les bénéfices reçus des écosystèmes par certains acteurs d'une part, et leur capacité à gérer ces mêmes services, d'autre part [13]. II y a aussi des inégalités non seulement dans la distribution des bénéfices, mais aussi dans la participation à la prise de décision, car tout le monde n'est pas capable d'exercer la même influence à ce 
niveau. Le sentiment d'injustice va donc être renforcé par l'asymétrie entre la distribution des services et la participation effective à la décision.

On observe la même asymétrie dans les actions d'atténuation des changements climatiques, car les pays les plus émetteurs de $\mathrm{CO}_{2}$ ne sont pas ceux pour lesquels l'exposition au risque climatique est la plus élevée. Ce sentiment d'injustice va se retrouver non seulement au niveau international mais aussi parfois à l'intérieur d'un pays, à l'intérieur d'une communauté, voire d'un territoire. Les contextes sont différents, mais présentent des caractéristiques communes. Des solutions sont, chaque fois, à trouver pour une prise de décision juste et équitable.

\subsection{Le rôle de l'action publique contre les inégalités environnementales}

L'action publique pour la transition écologique peut se traduire par :

- des incitations économiques (la taxe carbone);

- l'accompagnement d'actions volontaires des acteurs économiques et sociaux;

- des approches réglementaires;

- la mise en œuvre de projets;

- des programmes d'information.

Ces grands modes d'action ne doivent pas faire oublier la stratégie globale des États qui, parfois, est malheureusement absente. L'inaction peut être aussi responsable d'injustices.

Plusieurs critères permettent d'analyser la pertinence des actions publiques:

- leur efficacité environnementale;

- leur efficacité économique (leur coût);

- leur impact social par la redistribution;

- leur faisabilité politique;

- leur facilité de mise en œuvre.

\subsubsection{Des incitations économiques pour lutter contre le changement climatique?}

S'agissant de la réduction des émissions de gaz à effet de serre, deux grands types d'actions sont généralement mis en œuvre. L'un consiste à faire payer le pollueur, par exemple via une fiscalité sur le carbone. L'autre consiste à accompagner les secteurs les plus émetteurs de gaz à effet de serre vers le changement, notamment par des politiques d'emploi qui favorisent la reconversion et la formation au service de secteurs plus porteurs de la transition écologique. 
Cependant, quand les actions (ou les inactions) engendrent trop d'inégalités, elles entraînent, en retour, un refus violent de la société. Cela a été, par exemple, le cas de manifestations de la part de communautés indigènes contre des atteintes à leur environnement, contre la privatisation et la monétisation de certaines ressources naturelles comme les semences.

En France, le mouvement des gilets jaunes à l'automne 2018 s'est inscrit également dans une dynamique semblable (Fig. 12.8). Faire payer les acteurs économiques pour les dommages, qu'ils créent à l'environnement, avait pourtant bien fonctionné pour agir contre la pollution de l'eau. En revanche, la tarification du carbone, qui a été proposée, s'est avérée très inégalement répartie avec un effort important sur les ménages qui se déplaçaient avec leur véhicule particulier. Pour les catégories de populations les moins aisées et qui n'avaient pas d'autres alternatives, cette augmentation de la fiscalité a été perçue comme injuste et menaçant leurs fragiles équilibres budgétaires. Par ailleurs, la fiscalité sur le carbone était le résultat de différentes logiques comme, par exemple, le fait de protéger certaines branches économiques exposées à la compétition internationale, mais c'était aussi le résultat de luttes de pouvoir dans la société entre des acteurs économiques de différents secteurs. Aujourd'hui, cette fiscalité a été gelée, sans qu'une autre approche ne la remplace dans le secteur des transports pour la stratégie bas carbone en France.

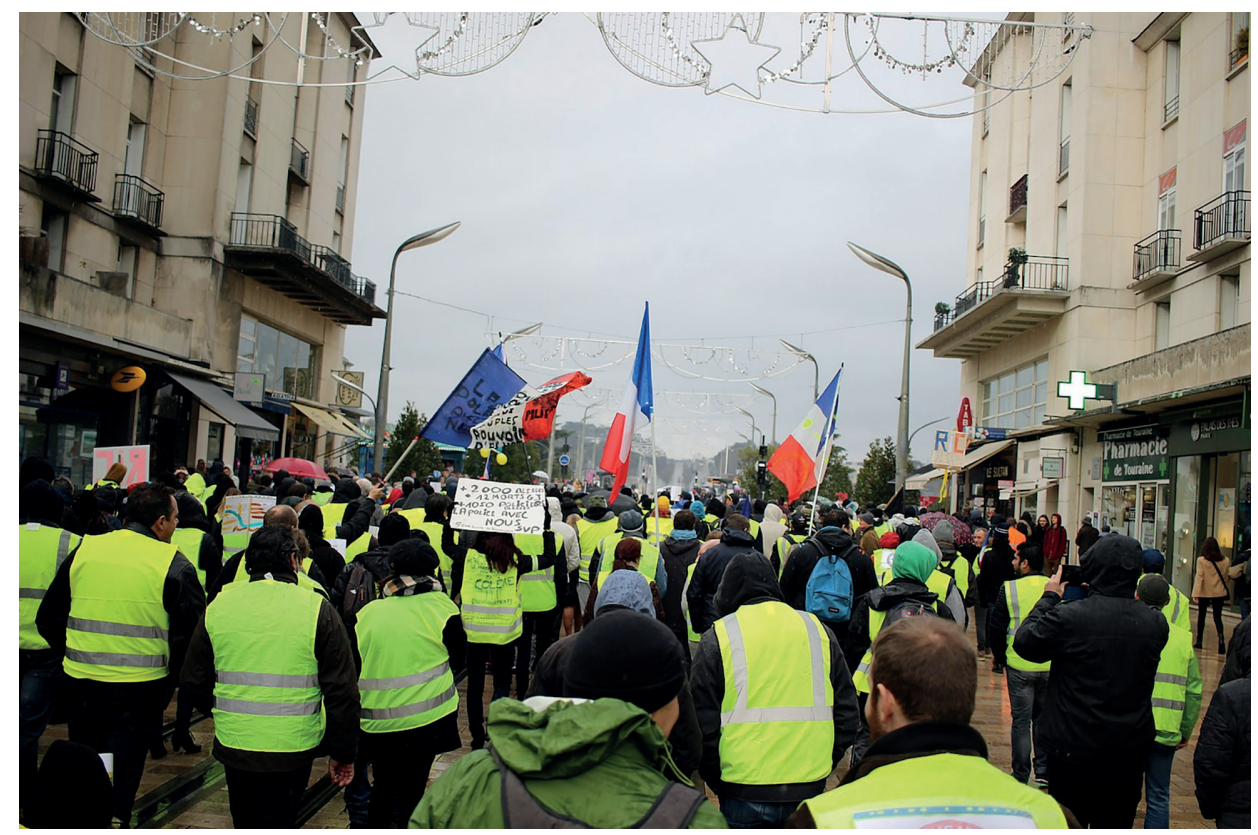

Figure 12.8: Manifestations des gilets jaunes à Tours - Acte X (janvier 2019). Source: Wikimedia commons CC BY-SA 4.0. 
Avant le mouvement des gilets jaunes, il y avait déjà eu un certain nombre de signaux d'alerte, qui n'avaient pas été pris en compte, et les manifestations ont mis en lumière les difficultés de l'État à construire les conditions d'une transition juste. De nouvelles procédures de délibération ont ensuite été mises en place avec, en 2019, la création de la Convention Citoyenne pour le Climat. L'objectif annoncé était de tenir les engagements de la France pour la baisse de ses émissions de gaz à effet de serre en priorisant l'esprit de justice sociale; les baisses d'émissions que permettraient le projet de loi issu de ses propositions ne sont pas encore à la hauteur des engagements de la France, ni de la révision à la hausse de l'engagement de l'Union européenne.

Différentes solutions sont envisageables pour construire une transition juste et réduire les inégalités actuelles ou futures [2] ${ }^{125}$. Par exemple, lutter contre la précarité énergétique, les logements indignes ou situés à proximité de zone exposées à la pollution, participent aussi à la transition écologique. Prendre en compte l'effet de l'augmentation du prix des biens et des services suite à la mise en place de taxes ou de normes, peut permettre de limiter les effets potentiellement inégalitaires des politiques climatiques. Refondre la fiscalité plus largement en lien avec une taxe carbone doit viser à limiter les exonérations des plus riches tout en accompagnant les plus modestes et en garantissant la transparence sur l'utilisation des recettes fiscales.

Les actions doivent aussi être envisagées à différents niveaux, par exemple, en donnant un accès plus équitable aux ressources et aux infrastructures et donc en repensant l'aménagement du territoire (voir section 10.4). De même, s'il faut évidemment compenser des dommages provoqués par les changements climatiques et éviter un creusement supplémentaire des inégalités, il faut aussi accélérer la mise en œuvre de stratégies d'adaptation qui intègrent d'emblée la dimension de la justice, ce qui est encore très peu pratiqué (voir sections 10.3 et 10.4) 126. $^{126}$ Un enjeu important, comme nous l'avons déjà mentionné à plusieurs reprises, est celui de la participation aux prises de décisions. Le fort contexte de défiance vis-à-vis des institutions renforce, en effet, l'importance des processus participatifs et démocratiques, mais aussi celle de la «redevabilité » de l'État concernant les processus qu'il engage. L'État est le garant de la mise en œuvre de politiques publiques sur le climat et la biodiversité et donc de leur équité. II est aussi l'arbitre des décisions dès lors qu'il n'y a pas de consensus dans la société.

\subsubsection{Agir avec des outils réglementaires pour la préservation de la biodiversité?}

Dès la fin du XVIIIle siècle, puis massivement au XIXe, des espaces naturels protégés ont été créés pour préserver la nature. Bien souvent, les décisions ont été prises unilatéralement par les gouvernements sans consulter les populations locales qui résidaient dans les espaces destinés à être protégés. Ces créations ont entraîné trois types d'impacts sociaux et économiques: des expulsions et des déplacements 
forcés, - des dommages économiques en interdisant ou en restreignant l'utilisation des terres et des ressources naturelles -, des pertes de bien-être en restreignant l'accès aux lieux spirituels ou aux lieux à valeur symbolique importante. Le nombre de personnes déplacées pendant la création des parcs naturels est estimé entre 900000 et 14 millions. Rien qu'en Inde, le nombre de personnes expulsées est évalué entre 100000 et 600000 . L'utilisation de tels outils de politique publique réglementaire pour des objectifs écologiques va forcément affecter en priorité des personnes déjà vulnérables. Pour rendre les contraintes moins importantes, il faut alors repenser la création des zones naturelles protégées et leur gestion au quotidien de façon beaucoup plus participative. D'une gouvernance autoritaire, basée sur une expertise technique, il faut passer à une gouvernance partagée qui sera beaucoup plus inclusive et plus participative [14].

La création d'un espace naturel protégé selon le mode décisionnel «top-down» (vertical) est restée l'approche la plus courante jusqu'au début du XXe siècle. Aujourd'hui, l'approche de la gouvernance partagée est mise en avant pour permettre la participation d'un plus grand nombre d'acteurs différents dans la prise des décisions locales et favoriser l'intégration de différentes formes de connaissances, non seulement de nature scientifique sur la diversité interne de taxons ou sur la richesse de la biodiversité, mais aussi des connaissances locales, par exemple, sur les plantes médicinales et leur utilisation ${ }^{126}$. Ce partage va donc permettre de connecter les acteurs locaux, qui sont directement impactés par la création de l'espace naturel protégé, et le gestionnaire, l'institution publique à l'échelle nationale [15]. On va aussi recréer des connexions entre différents niveaux de prise de décision et pouvoir planifier à long terme la multifonctionnalité des territoires. En prenant en compte les effets de moteurs externes, que sont la globalisation, l'urbanisation et le changement climatique, il sera possible d'assurer à tous les acteurs, y compris ceux qui se trouvent dans l'espace naturel protégé, une vision à long terme de la façon dont évoluera leur bien-être et leur activité économique.

On retrouve cette dimension participative dans les enjeux climatiques. Pour des situations nouvelles et inédites, il y a nécessité d'expérimenter, d'innover et de tirer parti des perspectives qu'apportent les uns et les autres en fonction de leurs conditions de vie, de leurs lieux de vie et de leurs revenus. C'est sans doute la meilleure façon de construire une réflexion sur ce qui peut être juste.

\subsection{Grands principes d'une transition écologique juste et perçue comme juste?}

Nous avons vu que la transition écologique juste revêt de nombreuses facettes qu'il faut considérer dans la gestion des problèmes climatiques et d'érosion de la biodiversité. Une plus grande justice est à trouver dans les responsabilités des 
émissions de gaz à effet de serre et des impacts du changement climatique. II est aussi nécessaire de distribuer de façon plus équitable les bénéfices tirés des services écosystémiques et de répartir les efforts à fournir pour préserver la biodiversité. Une participation renforcée de tous les acteurs dans la prise de décision et la mise en place des outils réglementaires est un des éléments clé, mais de nouveaux modes d'actions sont aussi à inventer, qui nous concernent tous en tant que citoyens.

Les grands principes d'une transition écologique juste sont de garantir l'accès aux ressources et le droit au développement au plus grand nombre avec une attention particulière portée aux jeux de pouvoir, aux inégalités sociales et aux situations de vulnérabilité dans nos sociétés. Pour cela, nous faut-il sans doute reconsidérer nos propres valeurs pour aboutir à des modes de consommation, de production et d'interaction avec la nature qui soient plus responsables et plus soutenables? Des effets d'échelle sont aussi à prendre en compte, du niveau national à un niveau de proximité au bénéfice des populations locales, mais aussi du niveau national à un niveau plus global pour éviter que des dommages ne soient causés à des populations vulnérables à distance.

Enfin, un regard croisé doit être porté sur les conséquences des actions qui seront entreprises pour endiguer le réchauffement climatique et préserver la biodiversité. Ainsi, abandonner une énergie fossile comme le charbon sera bénéfique à la réduction du $\mathrm{CO}_{2}$ atmosphérique, à la qualité de l'air et de l'eau et donc de notre santé, mais aura des effets négatifs pour l'emploi de ceux qui en dépendent ou pour ceux qui y ont investi leurs capitaux. Des reconversions seront nécessaires et des aides devront y être apportées. De même, avoir une alimentation plus saine avec plus de protéines végétales et moins de produits animaux aura des avantages en matière de santé publique, de pression sur les terres et les écosystèmes, et d'émissions de gaz à effet de serre, mais développer des pratiques d'afforestation pour stocker du carbone ou de monocultures pour produire de l'énergie à partir de la biomasse peut exacerber les tensions pour l'usage des terres et de l'eau, entraîner la dépossession de terres pour certaines populations autochtones, avec des effets négatifs en matière de sécurité alimentaire et de droits humains ${ }^{127}$. II est donc essentiel de pouvoir analyser tous ces aspects afin de prendre des décisions informées et de ne laisser personne «sur le carreau» dans la transition écologique, climatique et énergétique.

\section{Références}

[1] M. Robinson. 2015. Pour une justice climatique. France Forum 57, https://www.institut jeanlecanuet.org/content/pour-une-justice-climatique.

[2] Travaux du CESE (Conseil Économique, Social et Environnemental). 2016. La justice climatique: enjeux et perspectives pour la France.

127. https://www.ipcc.ch/srccl/ 
https://www.lecese.fr/travaux-publies/la-justice-climatique-enjeux-et-perspectives-pourla-france

[3] R.D. Bullard. 1990. Dumping in Dixie: race, class, and environmental quality. Boulder, États-Unis d'Amérique, Westview Press Boulder, San Francisco, Oxford.

[4] S.L. Cutter. 1995. Race, class and environmental justice. Progress in Human Geography 19(1), 111-122.

[5] D. Schlosberg. 2007. Defining environmental justice. Theories, movements, and nature. Oxford University Press, $238 \mathrm{p}$.

[6] V. Deldrève, J. Candau. 2014. "Produire des inégalités environnementales justes?», Sociologie 5(3), 255-269. DOI : 10.3917/socio.053.0255. URL: https://www.cairn.info/revuesociologie-2014-3-page-255.htm.

[7] T. Sikor, J. Fisher, R. Few, A. Martin, M. Zeitoun. 2013. The Justices and Injustices of Ecosystem Services. Ed. Thomas Sikor, pp. 201-214.

[8] D. Schlosberg, L.B. Collins. 2014. From environmental to climate justice: climate change and the discourse of environmental justice. Wiley Interdisciplinary Reviews: Climate Change 5(3), 359-374.

[9] IPCC Special Report. 2018. Global warming of $1.5^{\circ} \mathrm{C}$. www.ipcc.ch/report/SR15.

[10] IPBES Global Assessment Report on Biodiversity and Ecosystem Services. 2019. https:// ipbes.net/global-assessment.

[11] R.A. Mittermeier, P. Robles-Gil, M. Hoffmann, J.D. Pilgrim, T.B. Brooks, C.G. Mittermeier, J.L. Lamoreux, G.A.B. Fonseca. 2004. Hotspots revisited: Earth's biologically richest and most endangered ecoregions. CEMEX, Mexico City, Mexico 390 p. https://databasin.org/ datasets/23fb5da1586141109fa6f8d45de0a260/.

[12] P.A. Lindsey, G. Chapron, L.S.Petracca, D. Burnhame, M. W.Hayward, P. Henschel, A.E. Hinks, S.T. Garnetti, D.W. Macdonald, E.A. Macdonald, W.J. Ripple, K. Zander, A. Dickmane. 2017. Relative efforts of countries to conserve world's megafauna. Global Ecology and Conservation 10, 243-252. https://doi.org/10.1016/j.gecco.2017.03.003.

[13] A. Vallet, B. Locatelli, C. Barnaud, D. Makowski, Y.Q. Conde, H. Levrel. 2020. Power asymmetries in social networks of ecosystem services governance. Environmental Science \& Policy 114, 329-340.

[14] S. Lele, P. Wilshusen, D. Brockington, R. Seidler, K. Bawa. 2010. Beyond exclusion: alternative approaches to biodiversity conservation in the developing tropics. Sustainability 2(1-2), 94-100.

[15] S. Sarkki, T. Parpan, M. Melnykovych, et al. 2019. Beyond participation! Social innovations facilitating movement from authoritative state to participatory forest governance in Ukraine. Landscape Eco/34, 1601-1618. https://doi.org/10.1007/s10980-019-00787-x. 


\section{Conclusion}

En partant d'une perspective historique, cet ouvrage montre que l'humanité a, notamment depuis le début de l'ère industrielle, un impact grandissant sur les flux d'énergie et de matière de notre planète et sur le vivant, ce qui en retour conditionne la survie de nos sociétés et du monde tel que nous le connaissons. Mieux comprendre les mécanismes et conséquences du changement climatique et de l'érosion de la biodiversité nous est alors apparu central à développer dans cet ouvrage pour illustrer la manière dont ces deux enjeux majeurs sont interconnectés et comment ils sont reliés à nos modes de vie et de consommation. Si l'aggravation du réchauffement climatique et de l'érosion de la biodiversité fait peser des menaces sérieuses sur l'avenir de nos sociétés, elle questionne tout autant nos modèles de développement économique que notre rapport au vivant.

La lutte contre l'érosion de la biodiversité impose de préserver les entités de la biodiversité et leur potentiel évolutif, à la fois dans des espaces dédiés et au sein même de nos espaces anthropisés. Ceci implique de revoir nos modes d'appropriation des terres et des mers, lesquels entraînent la réduction des effectifs des populations d'espèces, voire la disparition à tout jamais d'une partie d'entre elles, et altèrent aussi la capacité des écosystèmes à s'adapter aux changements climatiques.

La lutte contre le changement climatique ou son atténuation passe par la préservation des écosystèmes forestiers et la mise en place de nouveaux schémas énergétiques visant à limiter les gaspillages, à améliorer l'efficacité énergétique, et à faire évoluer les comportements de consommation. De nouveaux modes de production d'énergie sont aussi à développer en opposition au schéma actuel dominant à l'échelle mondiale, principalement basé sur les énergies fossiles, en relevant les défis associés à leur pilotage et leur exploitation. Le réchauffement climatique déjà en cours impose concomitamment la mise en place d'actions d'adaptation.

La transition écologique passera donc nécessairement par des transformations profondes de nos modes de développement et de nos relations au vivant. Sur la base de connaissances issues des rapports les plus récents du GIEC et de I'IPBES, qui sont développées dans les trois premières parties de l'ouvrage, nous avons souhaité offrir dans la dernière partie un cadre de réflexions pour une transition vers 
un développement soutenable. Cette transition est une opportunité pour repenser nos façons de consommer, de produire, de travailler, de nous déplacer et de vivre ensemble sur notre planète aux limites finies tout en réduisant les inégalités sociales. En ce sens, la transition écologique est plus encore une transition socio-écologique.

Jean-Michel Lourtioz, Jane Lecomte, Sophie Szopa 


\section{Glossaire}

\section{Abiotique}

Un milieu abiotique est un milieu où la vie est impossible. Un facteur abiotique d'un milieu naturel est un facteur indépendant des êtres vivants.

\section{Acidification des océans}

Phénomène associé à l'augmentation de la concentration de $\mathrm{CO}_{2}$ dans l'eau des océans, entraînant la présence de l'acide $\mathrm{H}_{2} \mathrm{CO}_{3}$ et celle d'ions $\mathrm{H}^{+}$qui menacent la croissance d'espèces marines calcifiantes comme les coraux.

\section{Afforestation}

Plantation d'arbres sur une surface restée dépourvue d'arbres depuis longtemps.

\section{Agriculture biologique / Bio}

Mode de production de cultures et d'élevage respectueux des équilibres naturels et excluant l'usage d'engrais, d'OGM et d'intrants (produits non présents initialement dans le sol).

\section{Agriculture intensive}

Mode de production agricole caractérisé par l'usage d'intrants pour maximiser la production par rapport aux moyens humains et matériels mis en œuvre.

\section{Agroécologie}

Conception de systèmes de production agricole, qui s'appuient sur les fonctionnalités offertes par les écosystèmes et visent à diminuer les pressions sur l'environnement et à préserver les ressources naturelles.

\section{Aire de répartition}

Zone géographique où une espèce vivante est présente. 


\section{Albédo}

Fraction de la lumière que réfléchit un corps non lumineux.

\section{Animisme}

Attitude consistant à attribuer aux choses une âme analogue à l'âme humaine.

\section{Anthropique}

Dû à l'existence et à la présence d'humains.

\section{Anthropocène}

Époque géologique créée «à propos» pour caractériser l'avènement de l'humain comme principale force de changement sur Terre.

\section{Anthropocentrisme}

Attitude qui tend à faire de l'humain le centre du monde.

\section{Atmosphère}

Enveloppe gazeuse entourant la Terre.

\section{Baril}

Volume de référence servant à mesurer les quantités de pétrole produites et qui vaut environ 159 litres.

\section{Barrière géographique}

Rivière, montagne, vallée, océan, glacier... qui divisent l'aire de répartition d'une espèce en plusieurs zones.

\section{Bilan radiatif de la Terre}

Bilan qui dresse l'inventaire de l'énergie reçue par la Terre, provenant principalement du Soleil, et de l'énergie qu'elle renvoie vers l'espace.

\section{Bio-accumulateurs}

Organismes qui ont la capacité d'absorber et concentrer certaines substances chimiques. 


\section{Biocapacité}

Capacité totale de ressources renouvelables que la biosphère peut offrir pour répondre aux besoins humains.

\section{Biocapacité locale}

Capacité d'une zone biologiquement productive à offrir des ressources renouvelables et à absorber les déchets découlant de leur consommation, notamment la séquestration du $\mathrm{CO}_{2}$.

\section{Biodiversité}

Notion désignant à la fois la diversité des espèces vivantes, la diversité génétique au sein de chaque espèce et la diversité des écosystèmes regroupant différentes espèces.

\section{Biodiversité domestiquée}

Ensemble des espèces et sous-espèces (races, variétés) domestiquées par les humains et ayant été soumises à sa sélection.

\section{Biodiversité ordinaire}

Notion désignant la diversité des espèces à la fois communes et abondantes dans un écosystème donné.

\section{Biodiversité remarquable}

Ensemble d'espèces considérées comme remarquables pour la biodiversité, ou menacées et jugées importantes pour un écosystème ou encore représentative d'un habitat naturel ou de l'état de l'écosystème.

\section{Bioénergie}

Ensemble des énergies produites à partir de matière biologique: bois, cultures agricoles, déchets organiques, etc.

\section{Biomasse}

Ensemble des matières organiques d'origine végétale, animale, bactérienne ou fongique, utilisables comme source d'énergie. 


\section{Biosphère}

Ensemble des organismes vivants et de leurs milieux de vie, sur terre, dans les océans et dans l'air.

\section{Biotique}

Relatif au monde vivant. Un facteur biotique est un facteur lié aux êtres vivants (par opposition à abiotique).

\section{Biotope}

Milieu biologique défini par des caractéristiques physiques et chimiques relativement uniformes permettant des conditions de vie homogènes.

\section{Bourre}

Terme utilisé par Buffon, naturaliste du XVIIII siècle, pour désigner des espaces de terre, sauvages et «indisciplinés».

\section{Calotte fennoscandienne}

Calotte glaciaire qui recouvrait la Fennoscandie, région comprenant essentiellement la Finlande et la péninsule Scandinave.

\section{Calotte glaciaire}

Type de glacier formant une étendue de glace continentale (d'eau douce) de grandes dimensions.

\section{Calotte Laurentide}

Calotte glaciaire qui recouvrait une grande partie du continent nord-américain, s'étendant du Canada à la région des Grands Lacs, lors de la dernière glaciation.

\section{Carbonates}

Sels associant l'ion $\mathrm{CO}_{3}^{--}$à un ou plusieurs groupe(s) chimiques. Le plus connu est le carbonate de calcium qui constitue l'élément essentiel des coquilles de nombreux organismes. Plus généralement, les carbonates sont des minéraux très répandus à la surface du globe. 


\section{Carbone intermédiaire}

Terme utilisé par Christine Hatté (section 2.6.4) pour désigner le carbone qui est stocké dans le sol pendant des temps de durée intermédiaire de quelques décennies.

\section{Carbone labile}

Terme désignant le carbone et la matière organique stockés dans le sol, qui sont rapidement dégradables et assimilables par les micro-organismes.

\section{Carbone stable}

Terme désignant le carbone qui reste stocké dans le sol pendant des durées de plusieurs siècles ou de plusieurs millénaires.

\section{CFC I HCFC I HFC}

Chlorofluorocarbures / hydrocarbofluorocarbures / hydrofluorocabures.

\section{Chimie verte}

Aussi appelée chimie durable ou chimie écologique ou chimie renouvelable, c'est une chimie qui prévoit la mise en œuvre de principes pour réduire et éliminer l'usage ou la génération de substances néfastes pour l'environnement.

\section{Chlordécone}

Insecticide organochloré toxique, écotoxique et persistant qui a été originellement utilisé dans les Antilles pour la culture des bananes.

\section{Chromatographie}

Méthode physico-chimique qui sert à séparer les différentes substances présentes dans un mélange.

\section{Circulation thermohaline}

Circulation des eaux océaniques engendrée par les différences de densité de l'eau de mer, elles-mêmes dues aux différences de température et/ou de salinité des eaux.

\section{Climat}

Distribution statistique des conditions atmosphériques sur une période donnée. 


\section{Compensation écologique}

Mesure visant à compenser ou contrebalancer les effets de «perte nette de biodiversité » lors d'un aménagement ou de la réalisation d'un projet potentiellement créateur de nuisances.

\section{Coopération au développement}

Transfert des ressources d'un pays à l'autre.

\section{Couche d'ozone}

L'ozone est présent dans l'atmosphère à différentes altitudes. La couche d'ozone désigne généralement la couche de ce gaz, située dans la haute atmosphère et qui nous protège des rayons ultraviolets dangereux pour notre santé.

\section{Croissance complexifiante multidimensionnelle}

Notion qui s'oppose à une croissance uniquement mesurée par l'augmentation du produit national. La complexification illustre un double mouvement de diversification et d'intégration qui permet à la société de croître en se réorganisant sans perdre sa cohérence. La croissance multidimensionnelle prend aussi en compte la qualité des relations établies entre les hommes au sein de la sphère humaine et avec leur environnement naturel.

\section{Croissant fertile}

Région du Proche-Moyen-Orient en forme de croissant avec des plaines fertiles irriguées par le Jourdain, l'Euphrate, le Tigre et le Nil, où l'agriculture néolithique serait née.

\section{Cryosphère}

Désigne les régions gelées de la Terre: glaciers, calottes glaciaires et platesformes de glace, icebergs, banquise, glace de lac et de rivière, ainsi que pergélisol et sol gelé saisonnier.

\section{Cultivar}

Variété d'une espèce végétale obtenue artificiellement pour être cultivée. 


\section{Cycle de l'eau}

Parcours naturel de l'eau (liquide, vapeur, solide) entre les grands réservoirs d'eau sur Terre: océans, atmosphère, lacs, cours d'eau, nappes d'eaux souterraines et glaciers.

\section{Cycle exosphérique du carbone}

Terme utilisé par Gilles Ramstein (section 1.1) pour désigner la façon dont le carbone présent dans l'atmosphère sous forme de $\mathrm{CO}_{2}$ retombe dans l'océan et le manteau terrestre pour être ensuite réémis dans l'atmosphère lors d'éruptions volcaniques.

\section{«Dead zones» eutrophisées}

Zones aquatiques dont la pollution, souvent due aux engrais azotés et/ou phosphatés, entraîne la disparition d'un grand nombre d'espèces vivantes par désoxygénation de l'eau.

\section{Décantation gravitaire}

Procédé qui permet d'éliminer le sable et d'autres particules présentes dans les eaux d'égout en les laissant tomber par gravitation au fond d'un bassin de décantation.

\section{Défaillances de marché}

Situation dans laquelle le marché échoue à produire ou allouer optimalement des ressources économiques. En l'occurrence, dans la section 1.6.3, il s'agit de la non prise en compte des biens publics.

\section{Déficit écologique}

Écart entre la quantité de ressources naturelles utilisées par les humains et la quantité de ressources que la nature peut régénérer.

\section{Densité-dépendance}

Corrélation entre la densité d'une population et son taux de croissance. Généralement, le taux diminue lorsque la densité augmente. Mais, l'effet inverse peut se produire dans le cas de populations à faible effectif (voir Effet Allee). 


\section{Dérive génétique}

Évolution d'une population ou d'une espèce causée par des évènements génétiques aléatoires, notamment la perte de diversité dans le cas de populations de petite taille.

\section{Développement durable / soutenable}

Conception de développement qui s'inscrit dans une perspective de long terme. «Soutenable» est repris de l'anglais «sustainable» pour bien signifier que le développement doit répondre aux besoins des générations du présent sans compromettre la capacité des générations futures à répondre aux leurs.

\section{Dioxyde de carbone $/ \mathrm{CO}_{2}$}

Autre nom du gaz carbonique dont la molécule de base est constituée d'un atome de carbone et de deux atomes d'oxygène.

\section{Distillat}

Produit d'une distillation.

\section{Diversité écosystémique}

Variété des écosystèmes où les espèces vivantes interagissent avec leur environnement et entre elles.

\section{Diversité génétique}

Degré de variétés des gènes au sein d'une même espèce.

\section{Diversité spécifique}

Nombre d'espèces différentes.

\section{Dust bowl}

Région des États-Unis où sévissaient, dans les années 1930, de redoutables tempêtes de poussière provoquées par la sécheresse et l'érosion.

\section{Économie «extractive»}

Économie basée sur le rendement à court terme et l'exploitation massive des ressources naturelles. 


\section{Économie de la prédation}

Économie des premiers humains «chasseurs-cueilleurs» basée sur la chasse et la pêche.

\section{Éconosphère}

Ensemble des rouages de l'économie de marché.

\section{Écosystème}

Unité écologique de base formée par le milieu (biotope) et les organismes qui y vivent (biocénose).

\section{Écotoxicologie}

Étude des substances toxiques pour l'environnement et les humains et de leurs effets.

\section{Effet Allee}

Effet caractérisant des populations à faible effectif où une faible densité de population est corrélée à un fort taux de croissance de cette population.

\section{Effet Allee anthropogénique}

Effet Allee qui se manifeste lorsqu'une espèce est déclarée rare, sa rareté la valorisant auprès des humains qui cherchent à la collectionner ou la protéger. L'espèce reste cependant toujours menacée d'extinction.

\section{Effet de serre (naturel / additionnel)}

Mécanisme par lequel certains gaz de l'atmosphère absorbent le rayonnement infrarouge émis par la Terre, qui n'est donc plus renvoyé vers l'espace. Ce mécanisme, qui est semblable à celui d'une serre traditionnelle, maintient la température de l'atmosphère et celle du sol à un niveau plus ou moins élevé. L'effet de serre naturel est dû à la vapeur d'eau. L'effet de serre additionnel est dû à des gaz que nous émettons comme le $\mathrm{CO}_{2}$.

\section{Effet parasol}

Effet protégeant la Terre d'une partie du rayonnement provenant du soleil en la renvoyant vers l'espace. Y contribuent la réflexion de la surface de la Terre (voir albédo) et la réflexion/diffusion du rayonnement par l'atmosphère. 


\section{Empreinte écologique}

Indicateur ou mode d'évaluation environnementale qui comptabilise la pression exercée par les humains sur les ressources naturelles.

\section{Enclosures}

Terme anglais faisant référence à la délimitation des parcelles de terre destinées aux cultures ou à l'élevage. Cette pratique entamée en Angleterre dès le XVIe siècle est considérée comme un marqueur du droit de propriété, contribuant aussi au développement du capitalisme.

\section{Énergie}

Grandeur physique qui caractérise l'apport nécessaire au changement d'état d'un système (l'élévation de sa température, son changement de volume ou sa transformation profonde).

\section{Énergie de flux}

Ressources énergétiques que l'on ne peut stocker, mais dont on peut capter le flux (rayonnement solaire, mouvements de l'air - éolien, des eaux maritimes - marées, etc.).

\section{Énergie de stock}

Ressources énergétiques que l'on exploite à partir de leurs stocks (charbon, pétrole, gaz, uranium, eau stockée dans un barrage, etc.).

\section{Énergie finale}

Énergie fournie directement au consommateur sous forme de gaz, d'essence, de fioul, de bois, d'électricité... Elle se chiffre par une facture du fournisseur d'énergie.

\section{Énergie fissile}

Ressource énergétique que l'on exploite par fission (désintégration) de ses composants ultimes (ex: l'uranium).

\section{Énergie fossile}

Ressource énergétique que l'on exploite à partir de ses stocks, lesquels ont souvent mis des millions d'années à se former par fossilisation d'organismes vivants (charbon, pétrole, gaz). 


\section{Énergie utile}

Part de l'énergie finale réellement exploitée pour répondre à un service précis (éclairage, chauffage, transport, etc.). Dans le déplacement en voiture, le passage de l'énergie finale à l'énergie utile fait intervenir le rendement du moteur et la transformation du mouvement des pistons en rotation des roues.

\section{Épisode cévenol}

Épisode de pluies abondantes avec risque d'inondation, qui se déroule généralement en début d'automne et qui affecte principalement les Cévennes et le Piémont Cévenol dans le sud de la France.

\section{Érosion}

Usure et transformation que les eaux et les actions atmosphériques font subir à l'écorce terrestre.

\section{Érosion silicatée}

Érosion des minéraux silicatés (à base de silicium et d'oxygène) qui constituent l'essentiel de la matière rocheuse.

\section{Espèce}

Ensemble d'individus animaux ou végétaux, semblables par leurs formes adultes et embryonnaires et par leur génotype, vivant au contact les uns des autres, s'accouplant entre eux et demeurant féconds entre eux

\section{Espèce endémique}

Sous-ensemble des espèces présentes naturellement dans une aire géographique sans que l'homme en soit la cause.

\section{Espèce exotique envahissante}

Espèce introduite délibérément ou accidentellement dans une aire distincte de son aire d'origine et qui a tendance à proliférer au détriment des espèces natives/ indigènes de cette aire.

\section{Espèce native / indigène}

Espèce animale ou végétale qui évolue au sein de son aire de répartition naturelle et/ou de son aire de dispersion potentielle, et qui n'a donc pas été importée ni transplantée. 


\section{Espèce rare}

Espèce végétale ou animale dont le nombre d'individus est très faible.

\section{Espèce remarquable}

Espèce rare et/ou figurant sur la liste rouge des espèces menacées, qui de surcroît suscite des mesures de conservation dès lors qu'elle est emblématique d'une région.

\section{Éthique environnementale}

Éthique relative à la protection de l'environnement, principalement formalisée dans le monde anglo-américain et australien au XXe siècle.

\section{Eutrophisation}

Processus par lequel des nutriments, notamment des matières azotées ou phosphatées, s'accumulent dans un milieu ou un habitat.

\section{Évapotranspiration}

Quantité d'eau qui est transférée à l'atmosphère en s'évaporant par le sol, les nappes liquides et la transpiration des végétaux.

\section{Excentricité orbitale}

Façon dont l'orbite décrite par un objet céleste autour de son centre d'attraction s'écarte d'une orbite circulaire.

\section{Exsudation racinaire}

Substances organiques, plus ou moins fluides, qui suintent des racines des plantes et des arbres.

\section{Externalités environnementales}

Coût environnemental, notamment associé à des dommages environnementaux, qui n'est pas internalisé (supporté) financièrement par la chaîne de production et de distribution. 


\section{Facteur d'équivalence}

Facteur multiplicatif pour passer d'un hectare à un hectare global en prenant en compte «l'utilité» de la surface pour les humains par rapport à une surface de référence.

\section{Facteur de rendement}

Facteur multiplicatif permettant de prendre en compte le rendement de production dans la biocapacité locale d'une surface.

\section{Facteur forçant du climat}

Perturbation d'origine extérieure au système climatique qui impacte son bilan. (voir forçage)

\section{Feuillaison}

Renouvellement annuel des feuilles des arbres.

\section{Fissile}

Qui tend à se diviser, se fendre, se désintégrer comme les atomes d'uranium. La désintégration des atomes d'uranium produit l'énergie des centrales nucléaires.

\section{Forçage (naturel / anthropique)}

Action d'une perturbation d'origine extérieure au système climatique qui impacte son bilan. Cette action peut être naturelle, venant du soleil ou du volcanisme, ou d'origine humaine par les activités émettant des gaz à effet de serre.

\section{Forçage radiatif}

Différence entre la puissance radiative reçue et la puissance radiative émise par le système climatique. Si l'énergie reçue est supérieure à l'énergie émise, le forçage radiatif est positif. Dans le cas contraire, il est négatif.

\section{Gaz à effet de serre}

Gaz contribuant à l'effet de serre atmosphérique (voir effet de serre). 


\section{Génotoxicité}

Effets de substances et de rayonnements qui peuvent compromettre l'intégrité physique ou fonctionnelle du matériel génétique d'un individu ou d'une espèce.

\section{Génotype}

Information portée par l'ensemble du matériel génétique (génome) d'un individu.

\section{GIEC}

Groupe d'experts intergouvernemental sur l'évolution du climat. IPCC en anglais: Intergovernmental Panel on Climate Change.

\section{Goulot d'étranglement génétique}

Lorsqu'une population est fortement réduite sur au moins une génération, le faible nombre de reproducteurs restants ne transmet qu'une fraction de la diversité génétique initiale aux générations ultérieures.

\section{Gouvernance}

Manière de gouverner, d'exercer le pouvoir.

\section{Hectare global}

Unité de surface utilisée pour quantifier l'empreinte écologique des humains, qui prend en compte le type de surface, son utilité pour les humains et son rendement de production de ressources naturelles.

\section{Holocène}

Période interglaciaire dans laquelle l'humanité se développe depuis un peu plus d'une dizaine de milliers d'années.

\section{Homogénéisation biotique}

Processus par lequel deux communautés écologiques ou plus, distribuées spatialement, deviennent de plus en plus similaires au fil du temps. Processus qui résulte en grande partie du remplacement d'espèces natives (ou spécialistes) par des espèces généralistes introduites (ou favorisées) par les activités humaines. 


\section{Homogénéisation fonctionnelle}

Phénomène corrélé à une dégradation directe ou indirecte d'un écosystème par suite de la disparition d'espèces spécialistes au profit d'espèces généralistes: la perte des interactions positives établies sur une longue durée entre espèces spécialistes altère le fonctionnement de l'écosystème.

\section{Homogénéisation génétique}

Perte de diversité génétique au sein d'une espèce.

\section{Homogénéisation taxonomique}

Similarité accrue dans la composition de communautés écologiques résultant de l'invasion des quelques mêmes espèces.

\section{ICOS}

Integrated Carbon Observation System: infrastructure de recherche qui observe les flux des gaz à effet de serre en Europe et dans les régions adjacentes.

\section{Illuviation}

Accumulation progressive de diverses substances dans un sol sous l'action de l'écoulement vertical ou horizontal des eaux.

\section{Indice de développement humain (ou IDH)}

Indice statistique composite pour évaluer le taux de développement humain des pays du monde. Trois critères: le PIB par habitant, l'espérance de vie à la naissance et le niveau d'éducation des enfants de 17 ans et plus.

\section{Intériorité}

Caractère de ce qui est intérieur intime et non exprimé.

\section{Intrants (agricoles)}

Ensemble des produits qui ne sont pas naturellement présents dans le sol et qui y sont rajoutés afin d'améliorer le rendement d'une culture. 


\section{IPBES}

Intergovernmental Science-Policy Platform on Biodiversity and Ecosystem Services - En français: Plateforme intergouvernementale scientifique et politique sur la biodiversité et les services écosystémiques.

\section{Joule}

Unité de mesure de travail, d'énergie et de quantité de chaleur.

\section{Jour de dépassement de la Terre}

Date de l'année, calculée par l'ONG américaine Global Footprint Network, à partir de laquelle l'humanité est supposée avoir consommé l'ensemble des ressources que la planète est capable de régénérer en un an.

\section{Keynésianisme}

Nom générique donné aux écoles de pensées (issues de l'économiste britannique John Maynard Keynes) selon lesquelles les marchés laissés à eux-mêmes ne conduisent pas à l'optimum économique, l'État devant jouer un rôle dans le domaine économique pour pallier les défaillances des marchés.

\section{kWh}

Unité de mesure d'énergie couramment utilisée dans la consommation électrique et qui correspond à une puissance de 1000 watts consommée pendant une heure.

\section{Latérite}

Roche rouge ou brune riche en fer et en alumine.

\section{Lessivage}

Phénomène d'entraînement par l'eau de substances fixées sur des particules fines à travers les sols.

\section{Liberté contractuelle}

Libre choix des termes d'un contrat et du cocontractant.

\section{Lithosphère}

Couche externe de la croûte terrestre constituée de plaques mobiles. 


\section{Mécanique céleste}

Description du mouvement d'objets astronomiques tels que les étoiles et les planètes à l'aide de théories physiques et mathématiques.

\section{Mécanismes sociotechniques}

Mécanismes relevant de la sociologie des techniques, qui traite des interactions entre «la société» et «la technique».

\section{Mégafaune}

Ensemble des espèces animales de grande taille incluant arbitrairement les mammifères les plus lourds. Pour la faune du sol, il s'agit d'animaux de taille supérieure à $80 \mathrm{~mm}$.

\section{Métaux lourds}

Selon la définition historique, ce sont le mercure, le plomb et le cadmium. Parlant de pollution, on préfère aujourd'hui la notion d'éléments traces métalliques qui englobent tous les métaux toxiques dont les métaux radioactifs.

\section{Météorologie}

Étude scientifique des phénomènes atmosphériques à relativement court terme comparé à la climatologie qui porte sur le moyen / long terme.

\section{Méthane}

Gaz de formule moléculaire $\mathrm{CH}_{4}$ (un atome de carbone associé à 4 atomes d'hydrogène). Constituant du gaz naturel et combustible d'origine fossile, il est aussi l'un des principaux gaz intervenant dans l'effet de serre additionnel.

\section{Modèle de Kleber}

Modèle proposé par Markus Kleber (Université de l'État d’Oregon) pour décrire les interactions entre la matière organique des sols et la matière minérale.

\section{Modèles de chimie-transport}

Code informatique qui réunit un ensemble d'équations représentant le transport et la chimie d'espèces chimiques, et qui permet de quantifier l'évolution d'un panache de polluants en fonction du temps sur différents domaines. 


\section{Modélisation}

Conception d'un modèle permettant de décrire un phénomène plus ou moins complexe.

\section{Nappe phréatique}

Nappe d'eau que l'on rencontre à faible profondeur et qui alimente les puits et les sources en eau potable. C'est la nappe la plus exposée à la pollution en provenance de la surface.

\section{Niche écologique}

Milieu occupé par une espèce, incluant ses relations avec les autres espèces et son mode d'alimentation.

\section{Obliquité terrestre}

Angle d'inclinaison entre l'axe de rotation de la Terre et la perpendiculaire au plan de son orbite autour du soleil.

\section{Optimum médiéval}

Période de climat inhabituellement chaud localisée sur les régions de l'Atlantique nord et qui a duré du Xe au XIVe siècle.

\section{Palafittes}

Construction lacustre du néolithique récent, il y a environ 10000 ans.

\section{Paléoclimatologie}

Science qui étudie les climats passés et leurs variations.

\section{Paramètres orbitaux}

Paramètres requis pour identifier de manière unique le mouvement orbital d'une planète. Les 3 paramètres considérés dans l'ouvrage pour l'orbite terrestre sont: l'excentricité de l'orbite, l'inclinaison de l'axe de rotation de la Terre et la précession de l'axe de rotation autour d'une direction perpendiculaire au plan de l'orbite. 


\section{Perche du Nil}

Poisson d'eau douce de la famille des Latidae qui évoluait originellement dans le Nil. Son développement a été favorisé par les humains du fait de sa grande taille et de son bon apport en protéines comme produit alimentaire.

\section{Périhélie}

Point de l'orbite d'une planète qui est le plus proche du Soleil.

\section{Perturbateurs endocriniens}

Substances chimiques d'origine naturelle ou artificielle, étrangères à l'organisme, qui peuvent interférer avec le fonctionnement de notre système hormonal en induisant des effets néfastes pour nous même ou nos descendants.

\section{Pesticide}

Substance utilisée pour lutter contre des organismes considérés comme nuisibles. Désigne à la fois les insecticides, les fongicides, les herbicides et les parasiticides.

\section{Petit Âge glaciaire}

Période climatique froide principalement localisée sur l'Atlantique nord ayant approximativement eu lieu du début du XIVe à la fin du XIXe siècle.

\section{Phénotype}

Ensemble des traits observables d'un organisme ou d'un individu.

\section{Photosynthèse}

Processus par lequel les plantes vertes et les arbres synthétisent des matières organiques grâce à l'énergie lumineuse, en absorbant le gaz carbonique de l'air et en rejetant de l'oxygène.

\section{Phylogénie}

Étude des liens de parenté entre les êtres vivants et ceux qui ont disparu: entre individus, entre populations d'une même espèce et entre espèces différentes. 


\section{Physicalité}

S'oppose à l'intériorité dans la description des êtres. Elle est l'ensemble des expressions visibles et tangibles propres à une entité et qui résultent des caractéristiques physiologiques intrinsèques à cette entité.

\section{Physiocrates}

Fondateurs supposés, à la fin des années 1750, de la science économique et précurseurs du libéralisme économique.

\section{Phytopathogène}

Se dit d'un agent provoquant une maladie des végétaux.

\section{Phytoplancton}

Ensemble des végétaux microscopiques et unicellulaires qui flottent dans les eaux marines de surface et dérivent au gré des courants.

\section{PIB (Produit Intérieur Brut)}

Indicateur économique qui permet de quantifier la valeur totale de la richesse annuelle produite par les agents économiques résidant à l'intérieur d'un territoire.

\section{PM2,5 et les PM10}

Abréviations désignant des particules microscopiques de tailles inférieures à 2,5 et $10 \mu \mathrm{m}$, respectivement.

\section{Pollution chimique}

Pollution due aux substances chimiques rejetées sur terre, dans l'air et dans les océans.

\section{Pollution lumineuse}

Pollution due essentiellement à l'excès de lumière artificielle émise la nuit par les centres urbains avec des conséquences dommageables sur la faune, la flore, la fonge, les écosystèmes et la santé humaine.

\section{Pouvoir de Réchauffement Global (PRG)}

Facteur de conversion qui permet de comparer l'influence de différents gaz à effet de serre sur le système climatique, le gaz carbonique étant le gaz de référence. 
ppm

Sigle de partie par million désignant une fraction d'un millionième de masse, de volume, etc.

\section{Précession climatique}

Effet dû à la précession de l'axe de rotation de la Terre (voir paramètres orbitaux) et qui entraîne un décalage régulier de la position des équinoxes, moments de l'année où le soleil est pile à la verticale au-dessus de l'équateur.

\section{Productivité nette de l'écosystème}

Quantité de biomasse végétale produite par unité de temps et par unité de biomasse déjà présente dans un écosystème.

\section{Produits phytosanitaires}

Produits chimiques utilisés pour soigner, protéger, aider à la croissance des végétaux ou encore pour en prévenir les maladies.

\section{Propagules}

Structures de dissémination (propagation) et de reproduction d'espèces.

\section{Protistes}

Êtres vivants unicellulaires à noyau distinct (eucaryotes, par opposition aux bactéries ou aux procaryotes, dont la cellule ne possède pas de noyau).

\section{Puits de carbone}

Réservoir naturel ou artificiel qui absorbe le $\mathrm{CO}_{2}$ et séquestre le carbone sur un temps long par rapport à la durée de séjour du $\mathrm{CO}_{2}$ dans l'atmosphère.

\section{Pyramide des âges}

Représentation graphique de la répartition par sexe et âge de la population à un instant donné.

\section{Radioactivité}

Phénomène physique par lequel des noyaux atomiques instables se transforment spontanément en d'autres atomes en émettant simultanément des particules de matière et de l'énergie. 


\section{Ratio de séquestration}

Rapport qui est utilisé pour calculer l'empreinte écologique et qui permet de comparer le taux de séquestration de carbone d'une surface donnée à celui d'une même surface de référence.

\section{Ravinage}

Érosion plus ou moins importante du sol par les eaux de ruissellement.

\section{Reboisement}

Action de planter des arbres sur un terrain nu (antérieurement boisé ou non).

\section{Rendement}

Rapport de l'énergie utile, que fournit une machine, à l'énergie qui lui a été fournie.

\section{Réseau trophique}

Ensemble de chaînes alimentaires reliées entre elles au sein d'un écosystème et par lesquelles l'énergie et la biomasse circulent.

\section{Respiration autotrophique}

Émission de dioxyde de carbone par les plantes et la végétation.

\section{Respiration hétérotrophique}

Émission de dioxyde de carbone par les micro-organismes du sol qui permettent la décomposition de la litière et de la matière organique du sol.

\section{Ressources fossiles}

Combustibles riches en carbone (essentiellement des hydrocarbures) issus de la méthanisation d'êtres vivants morts et enfouis dans le sol depuis plusieurs millions d'années.

\section{Ressources halieutiques}

Ressources vivantes (animales et végétales) des milieux aquatiques marins ou d'eau douce que les humains exploitent par la pêche ou l'aquaculture. 


\section{Salinité}

Teneur en sel d'un milieu, notamment d'un milieu aqueux.

\section{Scénario}

Terme utilisé dans l'ouvrage pour modéliser les évolutions possibles de la démographie mondiale (section 3.2) ou du climat terrestre (section 9.3).

\section{Sciences participatives}

Formes de production de connaissances scientifiques auxquelles des acteurs non scientifiques et non professionnels participent de façon active et volontaire, qu'il s'agisse d'individus ou de groupes.

\section{Sécheresse}

État d'un environnement confronté à un manque d'eau significativement long et important pour qu'il ait des impacts sur la flore, la faune et les sociétés.

\section{Services écosystémiques}

Bénéfices offerts aux sociétés humaines par les écosystèmes.

\section{Smog}

Brume brunâtre et épaisse provenant d'un mélange de polluants atmosphériques.

\section{Sommet de la Terre de Rio de Janeiro}

Conférence des Nations unies sur l'environnement et le développement, qui s'est tenue à Rio de Janeiro au Brésil du 5 au 30 juin 1992, réunissant 120 chefs d'État et de gouvernements et 189 pays.

\section{Soutenabilité des économies}

Capacité à faire face à ses engagements budgétaires sans qu'il soit nécessaire d'ajuster dans le futur la politique budgétaire.

\section{Souveraineté étatique}

Principe général qui confère aux États le pouvoir d'exercer tous les droits reconnus par le droit international. En matière d'environnement, on parle néanmoins 
de souveraineté territoriale limitée, ou d'utilisation non dommageable du territoire (voir section 1.5.1 de l'ouvrage).

\section{Spectrométrie de masse}

Technique physique d'analyse permettant de détecter et d'identifier des molécules d'intérêt par mesure de leur masse, et de caractériser leur structure chimique.

\section{Stochasticité démographique}

Méthode d'analyse démographique qui cherche à prendre en compte la dimension aléatoire des évènements dans l'étude de l'évolution spatiale et temporelle d'une population.

\section{Stress oxydant}

Agression des constituants des cellules biologiques, qui se caractérise par un déséquilibre entre la production d'espèces réactives de l'oxygène et les capacités cellulaires antioxydantes.

\section{Surexploitation}

Prélèvement d'une ressource naturelle, qui dépasse sa capacité à se reconstituer naturellement.

\section{Surfaces bioproductives}

Surfaces terrestres susceptibles de produire une offre continue en ressources renouvelables. Dans le calcul de l'empreinte écologique, on distingue cinq types de surfaces bioproductives: les champs cultivés, les pâturages, les forêts, les pêcheries, les terrains construits.

\section{Système climatique}

Ensemble des interactions entre l'atmosphère, l'océan, la cryosphère, la lithosphère et la biosphère de la Terre, qui sous l'effet du rayonnement solaire, détermine le climat de la planète.

\section{Système endocrinien}

Ensemble des organes qui ont la capacité de relâcher des hormones dans le sang. 


\section{Taxonomie}

Branche des sciences naturelles, qui a pour objet de décrire la diversité des organismes vivants et de les regrouper en entités appelées taxons afin de les identifier, les décrire, les nommer et les classer.

\section{Taylorisme}

Méthode introduite par Frederick Winslow Taylor d'organisation scientifique du travail industriel grâce à l'utilisation maximale de l'outillage et la suppression des gestes inutiles.

\section{Tectonique des plaques}

Modèle scientifique expliquant la dynamique globale de la lithosphère terrestre à partir du concept de dérive des continents introduit par Alfred Wegener.

\section{The Paradox of the Faint Young Sun}

Contradiction apparente entre la présence d'eau liquide sur Terre dès les premiers âges et la faible luminosité du Soleil à cette époque (environ $30 \%$ plus faible qu'aujourd'hui).

\section{Théorie du pic pétrolier}

Théorie qui prévoit le moment où l'extraction mondiale de pétrole aura atteint son niveau maximal avant de connaître un déclin dû à l'épuisement progressif des réserves contenues dans le sous-sol terrestre.

\section{Tour à flux}

Système qui permet de quantifier les échanges de $\mathrm{CO}_{2}$, d'eau et d'énergie entre l'atmosphère et un écosystème donné.

\section{Toxicité aigüe}

Toxicité induite dans un court laps de temps par l'administration d'une ou plusieurs doses d'un produit ou mélange toxique.

\section{Toxicité chronique}

Développement d'effets néfastes résultant de l'exposition à long terme à un contaminant ou à un facteur de stress. 


\section{Transition démographique}

Passage d'un régime traditionnel où la fécondité et la mortalité sont élevées à un régime où la natalité et la mortalité sont faibles.

\section{UICN}

Union internationale pour la conservation de la nature.

\section{Unité comptable}

Élément d'une entité ou d'une action, pour laquelle on tient une comptabilité qui lui est propre.

\section{Utilitarisme}

Doctrine en philosophie politique ou en éthique sociale qui prescrit d'agir (ou de ne pas agir) de manière à maximiser le bien-être collectif, entendu comme la somme ou la moyenne de bien-être de l'ensemble des êtres sensibles et affectés.

\section{Vague de chaleur}

Suite de plusieurs jours consécutifs pendant lesquels sont observées des températures anormalement élevées.

\section{Vortex / spirale d'extinction}

Notion utilisée en écologie dans des modèles de populations d'espèces pour décrire ou catégoriser la dynamique et les causes de leur extinction. 



\section{Enjeux de la transition écologique}

\section{Enseigner la transition écologique aux étudiants de licence à l'université}

Sous la direction de Jean-Michel LOURTIOZ, Jane LECOMTE et Sophie SZOPA assistés de Catherine EVEN et Guillaume ROUX

et ouvrage, aux nombreuses illustrations, donne une vision transversale des changements environnementaux d'échelle mondiale que connaît notre planète aux limites finies. Son objectif est, en particulier, de faire comprendre les mécanismes et conséquences du réchauffement climatique et de l'érosion de la biodiversité ainsi que leurs relations avec nos modes de vie et de consommation.

Pour l'essentiel, il reprend l'un des premiers enseignements numériques dispensé à grande échelle en 2020 sur les « Enjeux de la transition écologique » auprès de plusieurs milliers d'étudiants de licence de toutes disciplines de I'Université Paris-Saclay.

À l'instar de ce cours, cet ouvrage s'articule en quatre parties. Partant d'une perspective historique des évolutions du climat et de la biodiversité, les trois premières parties montrent l'impact grandissant de l'humanité sur les flux d'énergie et de matière de la planète et sur les trajectoires évolutives du vivant. Cet impact conditionne en retour la survie de nos sociétés et du monde tel que nous le connaissons.

La quatrième et dernière partie de cet ouvrage offre un cadre de réflexions pour une transition vers un développement soutenable. Cette transition peut être une opportunité pour repenser nos façons de produire, de travailler, de consommer, de nous déplacer, tout en visant l'équité sociale pour un « bien vivre ensemble » partagé sur la planète.

Pour répondre à l'objectif de transversalité de l'ouvrage, plus de quarante enseignant(e)s-chercheurs(euses) et chercheurs(euses) d'horizons différents y ont contribué, mêlant savoirs des sciences de la nature aux sciences humaines et sociales en passant par le droit, l'économie, la gestion, l'agronomie et la médecine.

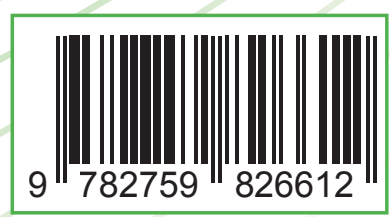

